PHYSICAL SCI.LIB.

TC

824

C2

A2

NO. 216 


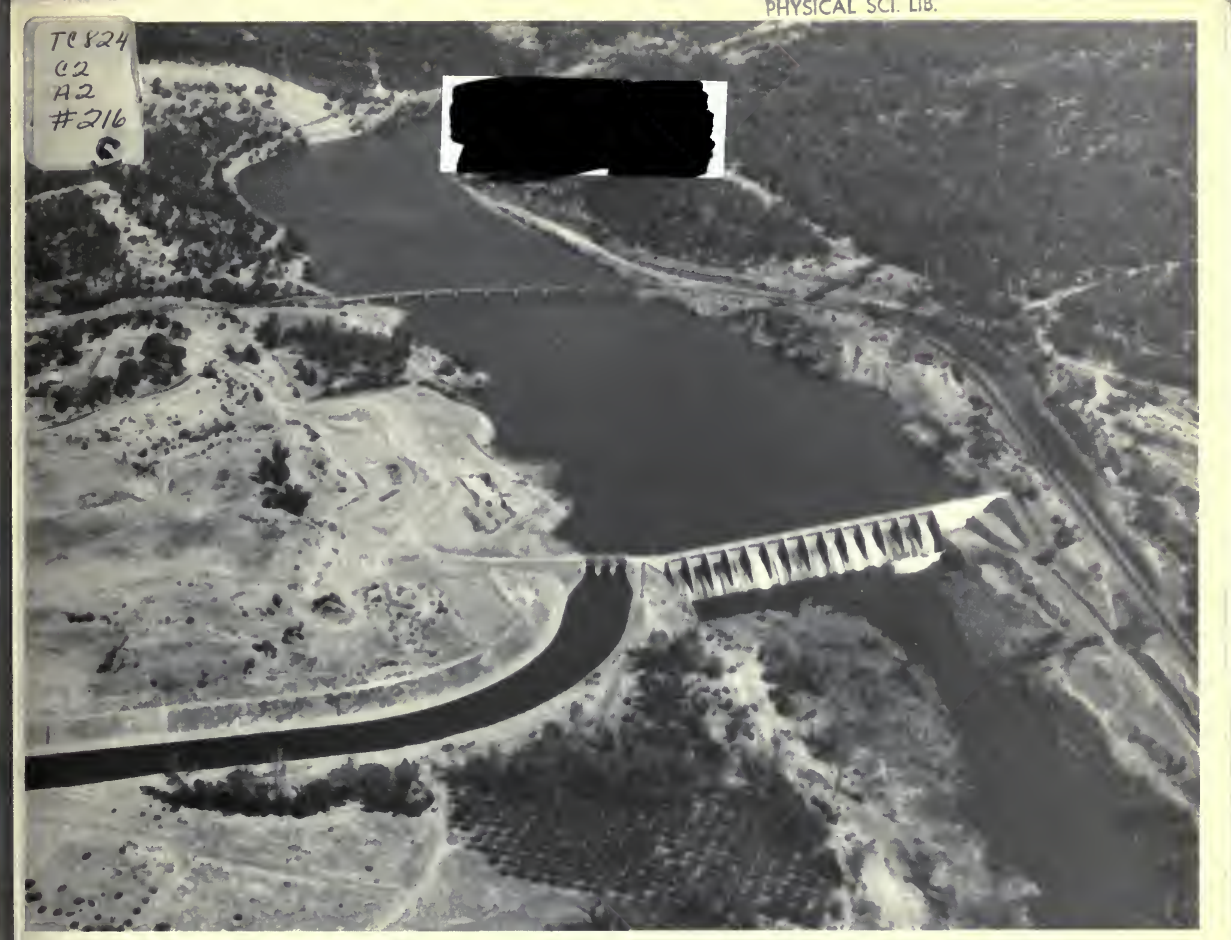

State of California

The Resources Agency

Department of

Water Resources

1982

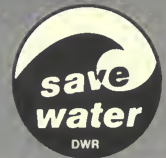

Inventory of

Instream Flow Requirements

Related to Stream Diversions

Bulletin 216

December 1982 


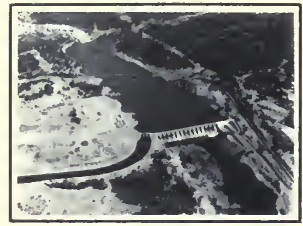

ON THE COVER: Thermalito Diversion Dam (center), Thermalito Power Canal (left), the Feather River (foreground), and Lake Oroville (background) are all features of the State Water Project near Oroville. At Thermalito Diversion Dam, water from Lake Oroville is channeled to both the power canal and the Feather River. Hydroelectric power is generated at the canal. Flows released to the Feather River support fisheries and wildlife. 
Department of

Water Resources

Bulletin 216

\section{Inventory of Instream Flow Requirements Related to Stream Diversions}

December 1982

Huey D. Johnson

Secretary for Resources

The Resources Agency
Edmund G. Brown Jr. Governor

State of California
Ronald B. Robie Director Water Resources 
FOREWORD

The quantity and quality of water flowing in stream channels greatly influence fish and wildife nopulations, riparian (streamside) vegetation, esthetics, and recreational values along rivers. Since excessive diversion of water from a stream can essentially kill $1 t$, legal measures in many areas require that a certain amount of water be left, or bypassed, in a stream, or at least returned to the stream after it has been used for other purposes, such as power production. In recent years, the bypass of instream flows has been legally required as a condition to acquiring appropriative water rights, Federal Energy Regulatory Commission licenses, and to a lesser depree, Bureau of Land Management and U. S. Forest Service Special Use Permits, etc.

Until now, finding out just what the specific streamflow requirements are has sometimes been difficult because the data are stored in various locations by a variety of agencies. This inventory has been compiled to streamline data retrieval by providing a single reference document that summarizes data on streamflow requirements for over 500 stream locations.

The information was gathered from the Lnvironmental Services Branch of the Department of Fish and Game, the State Water Resources Control Board, the National Marine Fisheries Service, the U. S. Forest Service, the U. S. Fish and Wildlife Service, and the Department of Water Resources. Thousands of water rights were reviewed and checked for accuracy. Mapping was based on water rights documents, national forest maps, and topographic sheets of the U.S. Geological Survey.

This inventory of instream flows should be useful to a wide variety of people, including fish blologists, watermasters, game wardens, water and power project planners, water rights consultants, lawyers, land use managers, and water users. These and others interested in water development and stream habitat maintenance should find this compilation of particular value.

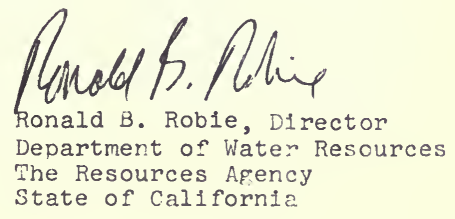


State of California

EDMUND G. BROWN JR., GOVERNOR

The Resources Agency

HUEY D. JOHNSON, SECRETARY FOR RESOURCES

Department of Water Resources

PONALD 3. ROBIE, Director

CHARLES R. SIFOEMAKER

Deputy Director

MARY AIJIE MARK

Deputy Director
M. CATHARIIE BERGREN

Assistant Director
ROBERT W. JAMES Deputy Director

GERALD H. MERAL Deputy Director

\section{DIVISIOIN OF PLANNING}

Donald E. Owen ................. Chlef Arthur C. Gooch . : Chief, Measurements and Supply Branch Donat B. Brice... . Chief, Water Reclamation and Supply Section Andrew S. Lee ......... Chief, Instream Use Unit

This report was prepared by

Charles W. Plke ............. Associate Planner Dean R. Powell ........... Student Assistant

with assistance from

Kenneth R. Yoshikawa . . . . . . . . . . Draftsperson

and

Lindsay P. Deauville . . . . . . . . Student Volunteer Jennifer K. Dickson ........... Student Volunteer Rovert B. Leonard ............ Student Volunteer Catherine C. McEfee ............ Student Volunteer Thomas A. Salzano ........... Student Volunteer Allison L. Stramler .......... Student Volunteer John A. Vaskovich . . . . . . . . . Student Volunteer Margaret :hitfield ........... Student Volunteer Gregory A. Wikler 
CONTENTS

FOREWORD

$\underline{\text { Page }}$

1. 11

INTRODUCTION

HOW INSTREAM FLOW REQUIREMENTS ARE DETERMINED AND ENFORCED 3

HOW TO USE TABLES AND MAPS 7

Method 1 - Geographic Location known 7

Method 2 - Water Rights Application Number Known 8

Method 3 - Hydrologic Unit or Identity Number Known 8

INDEXES TO TABLES AND MAPS ?

Index 1 - Water Rights Application Number Referenced to 11 IFR Identity Number

Index 2 - Hydro Units/IFR Ident1ty Number Referenced to 23 Map Page

Maps - Watershed Index Map of California 26

Locations of Instream Flow Requirements by 27 Hydrologic Unit

TABLE OF INSTREAM FLOW REQUIREMENTS RELATED TO STREAM DIVERSIONS Abbreviations and Notes 112

Sacramento Hydrologic Basin 113

San Joaquin Hydrologic Basin 151

Tulare Lake Hydrologic Basin 130

San Francisco Bay Hydrologic Basin 187

North Coast Hydrologic Basin 217

North Lahontan Hydrologic Basin 277

Central Coast Hydrologic Basin 236

South Lahontan Hydrologic Basin 236 
State of California

Department of Water Resources

CALIFORNIA WATER COMMISSION

SCOTT E. FRANKLIN, Chairperson, Newhall

ALEXANDRA C. SIILLMAN, Vice Chairperson, Arcata

Thomas k. Beard ............... Stockton Roy E. Dodson . . . . . . . . . . . . San Diego Danlel M. Dooley . . . . . . . . . . . . . . Visalia Harrison C. Dunning . . . . . . . . . . . . . Dav1s Merrill R. Goodall . . . . . . . . . . . Claremont Donald L. Hayash1 . . . . . . . . . . . San Francisco Charlene H. Orszag . . . . . . . . . . . Sherman Oaks

Orville L. Abbott

Executive Officer and Chief Engineer

Tom Y. Fujimoto

Assistant Executive Officer

Ine California Water Commission serves as a policy advisory body to the Director of Water Resources on all California water resources matters. The nine-member citizen cormission provides a water resources forum for the people of the State, acts as a liaison between the lefislative and executive branches of state Government, and coordinates Federal, State, and local water resources efforts. 
Copies of this bulletin at $\$ 5.00$ each may be ordered from:

State of Colifornia

DEPARTMENT OF WATER RESOURCES

P.O. Box 388

Sacromento, California 95802

Make checks poyable to STATE OF CALIFORNIA

California residents add sales tox. 



\section{INTRODUCTION}

This report is an inventory of locations in California where water is legally required to flow past stream diversions for the benefit of fisheries, wildlife, and recreation. Streamflow quantities and the periods when they are required are described.

Information presented on tables and maps includes: diversion locations by waterway, section, township, and range; water rights apolicants; water rights application numbers; Federal Energy Regulatory Commission (FERC) project numbers, and diversion names.

Information on streamflow requirements can be found by using two indexes. These indexes cross reference (I) the water rights application number to the location identity number, and (2) hydrologic units numbers to a map displaying site location.

Step-by-step procedures explain how best to use the indexes to retrieve data.

Although great effort has been made to provide a complete and up-to-date inventory, its accuracy cannot be guaranteed. Water rights change. New rights are created while old ones may be cancelled. Owners may change. Changes in physical characteristics such as points of diversion, place of use and amount of diversion may contribute to modification of instream flow requirements. Original water rights documents, FERC licenses, or special use permits should be consulted as necessary if absolute accuracy is required. 
HOW INSTREAM FLOW REQUIREMENTS ARE

DETERMINED AND ENFORCED

\section{DEFINITION}

Instream flows are waters flowing in a natural stream channel. These flows occurring in a stream (hence, "instream flows") vitally support fisheries, wildlife, recreation, and streamside vegetation. At dams, pumps, and other water diversion structures, bypass flows or releases of water into the stream below these structures are often required to sustain instream values. The contractually or legally required amount of water and its timing past the diversion structure to maintain instream values is the "instream flow requirement".

AUTHORITY FOR INSTREAM FLOW REQUIREMENTS

Protection of instream uses is a matter of State and Federal law. The California Water Code provides that fish, wildlife, and recreational uses are beneficial uses of water. The California Fish and Game Code declares that the protection and conservation of fish and wildlife resources are of utmost public interest, and recognizes the importance of commercial and sport uses as well as esthetic, scientific, and educational uses. The federal Fish and Wildife Coordination Act (F\&WCA) requires federal agencies to consider instream and environmental values in profects under the administrative or regulatory furisdiction of federal agencies.

In grantinf water rights permits, the State Water Resources Control Board (SWRCB) may impose terms and conditions on the diversion and use of the water. Most permit terms and conditions specify requirements relating to: the quantity of water and times when it may be diverted; quantities of water and times when $1 t$ must bypass the point of diversion for downstream water users; water quality; and stream protection and mitigation measures. These latter measures include fishway structures and instream flow requirements. Generally, a permit condition may be subsequently modified for instream protection only if the SWRCB has previously reserved furisdiction specifically for tinat purpose.

The Department of Fish and Game's (DFG) role in the water rights permit application process is to evaluate the stream and the amount of water required for the preservation and enhancement of fish and wildilfe resources. Frequently ( 70 to 80 percent of the water rights applications), DFG relies on the process of protesting these applications to gain leverage in obtaining suitable bypass flows. DFG resolves the protests by negotiating a settlement with the water rights applicant. These settlements are considered and often recognized by the SWRCB when determining the terms and conditions governing, the diversions and use of water under the permit. 
The California Environmental Quality Act (CEQA) of 1972 requires an environmental impact report to be prepared for projects which will have a significant adverse impact upon the environment, so that environmental values can enter into the decision-making process. In response to $C E Q A$, project sponsors frequently emnloy consulting biologists to assess needs and impacts on fisheries. Prior to CEQA, these assessments were not always conducted.

Projects sponsored by or within the administrative or regulatory jurisdiction of federal agencies must comply with the National Environmental Pollcy Act (NEPA) and F\&WCA. NEPA requires that the impacts of projects on the environment (including streams) must be assessed. The F\&WCA requires that any federal agency involved in project planning or licensing must first consult with the U. S. Fish and Wildife Service and with the appropriate state fish and wildIIfe agency on ways to conserve, develop, and improve wildilfe resources. The agencies are required by F\&WCA to accommodate the conservation of wildilfe resources insofar as is consistent with the primary purposes of the project.

The Federal Energy Regulatory Commission (FERC) has the power to impose terms and conditions upon the licensing and relicensing of hydroelectric projects. Under NEPA, the F\&WCA, and the Federal Power Act, exemptions from FERC licensing for hydroelectric power projects may not be granted if the project sponsor does not have written concurrence from state fish and wildife agencies and the U. S. Fish and Wildilfe Service. Many FERC license terms and conditions pertained to the release of water for fisheries purposes and for the protection and enhancement of other instream uses.

Agencies such as the U. S. Forest Service and the Bureau of Land Management, which administer federal lands, require special use permits for commercial endeavors on federal lands. The special use permits may contain conditions and terms requiring instream flow releases from stream diversions.

DETERMINING INSTREAM REQUIREMENTS

Typically, the quantity and timing of these bypass flows are determined by technical studies of the stream's aquatic habitat and recreation resources, coupled with negotiations between the diverters and those agencies responsible for maintaining the resources (1.e., Callfornia Department of Fish and Game, the U. S. Fish and Wildilfe Service, the Bureau of Land Management, and the U. S. Forest Service). The negotiated agreement usuaily becomes part of the permit or license.

Instream flow requirements are not necessarily consistent within a watershed or even on the same stream. Very few agreements have provisions for altering terms and conditions at a later date. Several methods of assessing the biological requirements of aquatic life have been used over the years. Even the more recent improved methods do not always provide the same quancitative results. 
The technical aquatic assessment is only one stage of the process setting specific bypass flow regimes. Apreements require negotiations which may yield flow schedules different from the technical recommendations. Similarly, administrative agencies mav place more or less weight upon the aquatic streamflow needs versus offstream diversion uses.

A factor contributing to the variability of instream flow requirements is that during the past decade, laws and regulations requiring: consideration of instream flows have been strengthened, and administrative officials have become more sensitive to instream needs.

ENFORCEMENT

Enforcement of instream flow releases is similar to enforcement of other water rights, terms and conditions. Other water users are relied upon to volce protests of possible violations of water rights, terms, and conditions, and bring them to the attention of the SWRCB staff. Section 764, Title 23 of the California State Administrative Code specifles the enforcement of terms and conditions for water rights. Section 764 states:

"Any person affected by a violation of any term or condition of a permit may file a complaint in writing with the board. The complaint shall contain the following:

(a) Name and address of the complainant.

(b) The nature of the alleged violation.

(c) The manner in which the complainant is affected.

(d) A statement of whether complainant is required by Part 5.1 (commencing with Section 5100) of Division 2 of the Water Code to file with the board a statement of his water diversion and use, and if complainant is so required, a statement:

(1) that complainant has flled with the board a statement of water diversion and use; or

(2) setting forth the reason for complainant's failure to file such a statement of water diversion and use.

(e) The specific action which complainant requests.

Any person may complain of a violation of a condition intended for the benefit of the public." 
Section 764.5 continues:

"If after investigation, the Board's Division of Water Rights finds that a violation has occurred which might be cause for revocation of the permit or other action by the Board, the matter will be referred to the Board for hearing. If the Division finds that no such violation has occurred, the complainant will be notified and no further action will be taken unless the complainant requests a hearing by the Board, in that event the Board will determine whether to hold a hearing based on information supplied by the complainant and the Board's staff. If, after notice in accordance with Water Code Sections 1410 and 1410.1 and a hearing, if a hearing is requested by permittee, the Board finds a violation has occurred, the Board may revoke the permit or take such other action as appears appropriate under the circumstances. The provisions of this article apply to licenses in the same manner as to permits."

If the SWRCB determines that a violation of terms or conditions of a water right is occurring, Water Code Sections 1831-1836, 1840, 1845, and 1850-51 provide for SWRCB administrative sanctions prior to the revocation of the water right. The SWRCB may issue cease and desist orders, petition the courts for infunctive relief, and, that falling, levy fines up to $\$ 500$ per day.

The SWRCB is not responsible for enforcement of all streamflow requirements. As discussed earlier, other agencies have existing agreements for streamflow requirements or have jurlsdiction to obtain such requirements in permits and licenses. Protests for noncompllance with bypass flows and conditions should also be addressed to the appropriate agency such as the Federal Energy Regulatory Agency, Bureau of Land Hanagement, U. S. Forest Service, Department of the Interior, or the California Department of Fish and Game. 


\section{HOW TO USE TABLES AND MAPS}

The information inventoried in this report is displaved in two principal formats:

1. A "Table of Instream Flow Requirements Related to Stream Diversion", identifies each requirement by assipning an Identity Number which locates each diversion by hydrologic basin. The Instream Flow Requirements (IFR) are listed in alnhanumeric sequence by Identity iNumber. This means that all IFRs for a hydrologic unit (watershed) are grouped topether.

2. Detalled maps, "Locations of Instream Flow Requirements by Hydrologic Unit", Dages 26-110, display the geopraphic location of each IFR. IFRs are deplcted by large dots with one- or twodigit numbers adjacent. The dots are correctly drawn on the maps with respect to township, range and section. Township and range coordinates are noted on the maps. In situations where more than one IFR is located in a section (particularly in the Russian and Napa dralnages), only one dot is shown per section with the several identity number suffixes listed. The combination of the hydrologic unit code plus the suffix produces the Identity Number by which IFRs are listed in the table.

Additionally, an index is provided to cross reference water rights application numbers to IFR Identity Numbers.

Here are three methods of using these tables, maps, and indexes:

Method 1 - Geographic Location Known

Step 1: Turn to the watershed index map of the State (page 26). This index map shows the State's mafor streams and hydrologic basins and indicates the nages of more detailed hydrologic units. For example, the Sacramento River Basin is represented by detalled hydrologic unit maps on pages 27 through 39. A diversion situated on the upper Sacramento River near Shasta Lake is mapped on page 37 .

Step 2: Murn to the detalled hydrologic unit map, in this example, page 37. If the desired diversion site is the McCloud Reservoir, then the site lies within the hydrologic area designated as A22.A3.

Step 3: Each diversion point is depicted with a large dot. Adjacent to the dot is a one- or two-digit number. mhat number is used as a suffix to the hydrologic unit code to produce the site 1dentity number (for example, A22. A3-1 for McCloud Dam). 
Step 4: Refer to the tables of Instream Flow Requirements table (beginning on page 1ll) where all the site Identity Numbers are alphanumerically listed with their corresponding flow agreements (for example, A22.A3-1 is on page 147).

Method 2 - Water Rights Application Number Known

Index 1 (pages 11 through 22) cross-references SWRCB water rights application numbers to Identity Number. For example, application number 13746 is referenced to Identity Number A06.B2-1. Using the Identity Number, turn to the table of Instream Flow Requirements (page 122) to find the instream flow requirement.

Method 3 - Hydrologic Unit Code or Identity Number Known

To find the location of the IFR site on the maps, follow steps 1 and 2 .

Step 1: Turn to Index 2, Hydro Units/Site Ident1ty Number Referenced to Map" (pages 23 through 25). Remember that the first five digits of Identity Numbers are the same as the hydrologic unit codes. Index 2 lists the hydrologic unit codes and the pages of the maps where they appear. (e.g., A06.00 is on page 30). As explained under Method 1 , the complete Identity Number can be determined from the detailed map.

Step 2: Refer to the table (beginning on page 111) to learn the Instream Flow Requirement ( $A 06.32-1$ is on page 122). 
INDEXES TO TABLES AND MAPS 

INDEX 1

WATER RIGHTS APPLICATION NUMBER REFERENCED TO IFR IDENTITY NUMBER

Water Rights Application Number

1341

1342

1345

1346

1389

1614

1920

1920

1920

2372

2372

2372

$2652 \mathrm{~A}$

3850

4703

5648

5648

$5648 \mathrm{~F}$

6470

6594

6594

6737

6737

6737

6737

6737

6737

6737

6737

6737
I FR

Identity

Number

B 14. DO- 2

B 14. DO-3

B14. E1-2

B 14. $\mathrm{CO}-1$

G0 1. $\mathrm{CO}-1$

A 10 . B0-1

C0 3. B 3-1

C03.B3-2

CO 2. B3-3

A $10 . \mathrm{C} 3-1$

A $10 . \mathrm{C} 3-2$

A $10 . C 3-3$

A09. A4-6

WO $3 . \mathrm{BO}-3$

CO3. B3-4

B09.B 1-1

B09. B 1-2

B0 4 . CO- 15

B0 8. J0-1

F11.F3-1

F11. F3-2

B0 $4 . \mathrm{CO}-1$

BO 4 . $\mathrm{CO}-2$

B0 $4 . \mathrm{CO}-3$

BO 4. $\mathrm{CO}-4$

$\mathrm{BO} 4 . \mathrm{C} 0.5$

BO 4. $\mathrm{CO}-6$

B0 $4 . \mathrm{CO}-7$

BO 4. $\mathrm{CO}-8$

BO 4 . $\mathrm{CO}-9$
Water Rights

Application

Number

6737

6737

6737

6737

6737

6737

6737

6737

8495

10122

10338

10338

10588

11115

11115

11122

11192

11193

11199

11281

11315

11327

11327

11389

11407

11596

11788

11792

11792

$11792 \mathrm{~A}$
IFR

Identity

Number

B0 4. $\mathrm{C} 0-10$

B0 4 . CO-11

BO 4 . $\mathrm{C} 0-12$

B0 4 . $\mathrm{CO}-13$

B0 4. $\mathrm{C} 0-14$

B0 $4 . \mathrm{CO}-16$

B0 4. C0 -15

B0 4. $\mathrm{C} 0-17$

A $11 . C 2-1$

B09. E 1-1

B 14. A 1-2

B $14 . \mathrm{CO}-2$

A 19. C0-1

B 14. D 0-1

B 14. DO-7

F06.D0-4

A 1 1. D1-1

A 11.D1-2

$A 02$. $B 0-1$

B21. B 2-3

F14.A1-39

F 14.B4-10

F14.B4-11

$\mathrm{A} 04 . \mathrm{CO}-1$

F06.B3-1

A $10 . A 2-2$

B0 6. $\mathrm{CO}-3$

B0 5. B0-1

BO 5. $\mathrm{CO}-1$

B0 5. $\mathrm{CO}-2$ 
Water Rights

Application

Number
IFR

Identity

Number

11966

12193

12243

12243

12243

12330

12340

12389

12490

12490

12493

12509

12624

12724

12726

13095

13156

13156

13206

13304

13372

13533

13633

13707

13708

13746

13775

13778

13778

13778

13778

13886

13928
F11.F1-2

F15. A0-3

CO3.B3-1

C03.B3-2

CO3.B3-3

F14.A1-22

Y02.B 1-2

A0 4.D 4-2

B09. E1-4

B09. E1-5

B $10 . \mathrm{CO}-8$

F15. A0 -6

A06. C4-1

$\mathrm{C} 03 . \mathrm{B3}-4$

C03.B $3-5$

A23.D4-1

B03. B0-1

B0 3. BO-2

F06.A3-6

F15. AO-2

A05.B 1-1

F14.B5-31

F14.C1-23

B0 4. A3-4

B0 4.A3-1

A0 6. B2- 1

F01.00-1

C06.B2-1

C06.B2-2

C06. B2-3

C06.B2-4

T05. B0-1

B 14.DO-6 water Rights

Application

Number

13929

13956

13958

13982

14079

14112

14127

14274

14316

14433

14444

14444

14519

14546

14602

14691

14743

14785

14804

15017

15201

15404

15406

15407

15424

15444

15502

15504

15505

15507

15508

15510

15511

15563
IFR

Identity

Number

B 14.D0-8

A 10. E 2-1

F14.B3-2

G05. BO-2

F 14.B3-3

A11. B3-1

B 10.B 1-1

A 10.D1-5

A07.D0-6

X19.07-1

A08. D0-1

A0 8. DO-2

F09.A0-1

A0 7. D0 -4

A 23. D 1-1

F11.C2-2

A23.A2-1

A22. A3-2

A09. A 1 - 1

A0 7. D0 - 5

B03.B $0-1$

F14.B2-5

A07.D0-5

A20. $\mathrm{BO}-3$

A 19.B 1-1

F11.A1-2

G05.A0-11

G05.AO-12

G05.AO-13

G05. A0-4

G05.A0-5

G05. AO-3

G05.BO-1

A 10. A3-1 


\section{Water Rights} Application Number

\begin{tabular}{|c|c|}
\hline 15574 & $\mathrm{~A} 08 \cdot \mathrm{C} 0-1$ \\
\hline 15574 & $A 10 . D 1-2$ \\
\hline 15574 & $A 10 . D 1-3$ \\
\hline 15574 & A10.E1-1 \\
\hline 15710 & $\mathrm{~A} 07 . \mathrm{D} 0-4$ \\
\hline 15717 & $A 22 . A 3-1$ \\
\hline 15719 & A23.A3-1 \\
\hline 15777 & $\mathrm{~B} 04 \cdot \mathrm{CO}-18$ \\
\hline 15779 & F14.C1-1 \\
\hline 15975 & $A 04 . A 2-1$ \\
\hline 15975 & $\mathrm{AO} 4 \cdot \mathrm{CO}-1$ \\
\hline 15975 & $\mathrm{~A} 04 . \mathrm{C} 0-1$ \\
\hline 16102 & $\mathrm{~B} 14 . \mathrm{D} 0-5$ \\
\hline 16103 & B 14. E 1-1 \\
\hline 16104 & $\mathrm{~B} 10 . \mathrm{C} 0-5$ \\
\hline 16108 & F13.A3-1 \\
\hline 16109 & F13.A3-2 \\
\hline 16130 & E06.DO-9 \\
\hline 16161 & A22. A $4-1$ \\
\hline 16163 & $\mathrm{~F} 14 . \mathrm{B} 4-8$ \\
\hline 16170 & $\mathrm{~B} 10 . \mathrm{C} 0-6$ \\
\hline 16173 & $\mathrm{~B} 10 . \mathrm{C} 0-7$ \\
\hline 16187 & $\mathrm{~B} 11 . \mathrm{A} 0-1$ \\
\hline 16188 & $\mathrm{~A} 13 \cdot \mathrm{B} 0-1$ \\
\hline 16189 & $\mathrm{~A} 13 \cdot \mathrm{B} 0-1$ \\
\hline 16219 & B21.B2-2 \\
\hline 16347 & F14.C3-17 \\
\hline 16430 & F14.C3-15 \\
\hline 16454 & $\mathrm{~F} 09 \cdot \mathrm{A} 0-2$ \\
\hline 16586 & T0 $4 . A 3-3$ \\
\hline 16750 & F06.A5-6 \\
\hline 16757 & $\mathrm{~T} 10 . \mathrm{B} 2-1$ \\
\hline 16768 & F06.C1-1 \\
\hline
\end{tabular}
Identity Number
Water Rights Application

Number

16854

17055

17116

17198

17232

17271

17291

17292

17317

17345

17375

17376

17400

17526

17527

17536

17590

17624

17809

17811

17838

17851

17913

17978

17997

18011

18028

18052

18084

18087

18087

18087

18087
IFR

Identity

Number

C0 6.B $4-1$

E07.B2-3

T10.A 7-1

E02.DI -6

F14.C1-29

F14.C2-9

F09.DO-1

T10.B1-1

E0 1. A3-1

F13.B 0-1

A19. B 1-1

A 19. B 1-1

W0 3. B0-1

B13. A 1-2

F05.C6-1

F10.D0-1

E02.DO-7

F14.C1-34

F11.C3-2

F03. A 1-3

T0 4. A3-2

T04. A3-1

T0 4. A2- 1

F14.B5-35

F05.D2-3

A10. D1-4

B0 4. A3-2

F 13.E0-11

A0 6. C2-1

A06. C2-2

A0 6. C2-5

A06. C3-1

A06. C3-2 
Water Rights Application Number

\begin{tabular}{|c|c|}
\hline 18087 & $\mathrm{~A} 06 . \mathrm{C} 3-3$ \\
\hline 18087 & $\mathrm{~A} 06 . \mathrm{C} 5-1$ \\
\hline $18104 \mathrm{~A}$ & E06. E0-84 \\
\hline $18104 \mathrm{~B}$ & E06. E0-71 \\
\hline 18136 & F $11 . G 2-1$ \\
\hline 18144 & E06.E0-66 \\
\hline 18213 & T09.H1-2 \\
\hline 18227 & $\mathrm{C} 03 . \mathrm{B} 3-6$ \\
\hline 18291 & $A 06 . D 2-1$ \\
\hline 18295 & G05.A0- 10 \\
\hline 18375 & T10.C1-1 \\
\hline 18409 & $\mathrm{G} 06 . \mathrm{B} 0-2$ \\
\hline 18470 & A $10 . E 3-1$ \\
\hline 13470 & A $10 . E 3-2$ \\
\hline 18508 & E02.B3-2 \\
\hline 18652 & $\mathrm{~B} 10 . \mathrm{C} 0-9$ \\
\hline 13677 & F11.F1-4 \\
\hline 13702 & F11. C $3-3$ \\
\hline 18714 & B13. A2-1 \\
\hline 18733 & B $13 . \mathrm{B} 0-1$ \\
\hline 18844 & A1 1. C5-1 \\
\hline 18395 & $\mathrm{~B} 05 . \mathrm{C} 0-4$ \\
\hline 19145 & A23.B2-1 \\
\hline 19149 & B09.D $0-1$ \\
\hline 19149 & B09.D0-2 \\
\hline 19149 & B09. D0-3 \\
\hline 19149 & B09.D0-5 \\
\hline 19149 & B09.DO-7 \\
\hline 19171 & $\mathrm{~F} 01.00-2$ \\
\hline 19238 & T04.A1-1 \\
\hline 19304 & В09. B2-1 \\
\hline 19309 & A23.E3-1 \\
\hline 9351 & F14.B $4-14$ \\
\hline
\end{tabular}
Identity Number
Water Rights Application Number

\section{1}

19423

19423

19423

19423

19551

19629

19653

19801

19841

19841

19841

19841

20072

20072

20350

20357

20117

20469

20540

20541

20545

20711

20866

20920

21009

21024

21079

21106

21121

21148

21151

21151
IF $R$

Identity Number

A20.B0-1

B10. C0-1

B10. $\mathrm{CO}-2$

B $10 . \mathrm{CO}-3$

B 10. $\mathrm{CO}-4$

E02. DO-6

G05. A0-2

A 11 .E3-1

T07.00-1

A $10 . C 2-1$

A 10.D1-1

A 10.D2-2

A $10 . D 2-3$

A $10 . D 2-1$

A 10.D3-2

B0 4. A2-1

E06.E0-61

A 11.E4-1

F06.D0-3

F14.C1-32

F14.C1-33

W0 3 . BO-2

E02.D0-11

F06.A5-3

F14.B2-1

B0 7 . D 2-1

C02.BO-1

A10.C4-1

F14.C1-25

F14.C3-14

F14.B2-3

A09. A4-1

A09. A4-3 
Water Rights Application Number

21156

21170

21184

21256

21364

21438

21443

21607

21608

21674

21676

21833

21945

21955

21991

22038

22039

22042

22061

22095

22110

22110

22156

22266

22277

$22287 \mathrm{~A}$

22287B

22321

22324

22325

22331

22359

22372
IF $\mathrm{R}$

Identity

Number

F14.B2-4

E14.B2-2

F14.C1-24

E13. E0-12

F06.B5-4

W20. F0-1

A $11 . \mathrm{C} 3-1$

F06.DO-2

F06.D0-2

F 14.B5-37

A19.82-1

F13.H1-1

A0 6.D2-2

G07.00-3

F15. A0-1

E14.B3-4

A0 7. DO-2

A03. A4-21

A 12 . $\mathrm{B} 0-2$

E06. E0-47

A06.B2-2

A06.B2-3

F14.B5-36

G07. O0-2

E14.C1-27

E05.A2-1

F05.A2-1

$\mathrm{A} 07 . \mathrm{DO}-3$

C06. $\mathrm{AO}-2$

F14.B6-1

F06.B5-5

F14.B $4-7$

$\mathrm{Z} 01 . \mathrm{B} 0-1$
Water Rights

Application

Number

22377

22412

22445

22461

22463

22503

22539

22573

22634

22661

22689

22704

22711

22739

22785

22807

22822

22831

22835

22841

22870

22920

22929

22949

23003

23025

23038

23039

23047

23079

23114

23118

23120
IFR

Identity

Number

E13.H5-1

T0 4. A2-4

E14.B5-34

W20.EO-2

E13.E0-13

A21.B2-1

X22. F1-1

G06. BO-3

F06. $\mathrm{C} 2-3$

E14.34-6

F15.A0-5

T10.B6-1

E06. EO-80.

A03.A4-18

E06.E0-72

F14.B 4-5

G06. B0 - 1

P06.B5-3

E06. E0-44

E06.E0-57

F13. E0-14

E07.B2-4

F14.84-9

E06. E0-62

F03.A $1-5$

F05. Н2-1

F11. E1-1

F14.C1-20

A $10 . B 0-2$

F06.A5-2

T08.00-3

Y02. B 1-1

E13.EO-7 


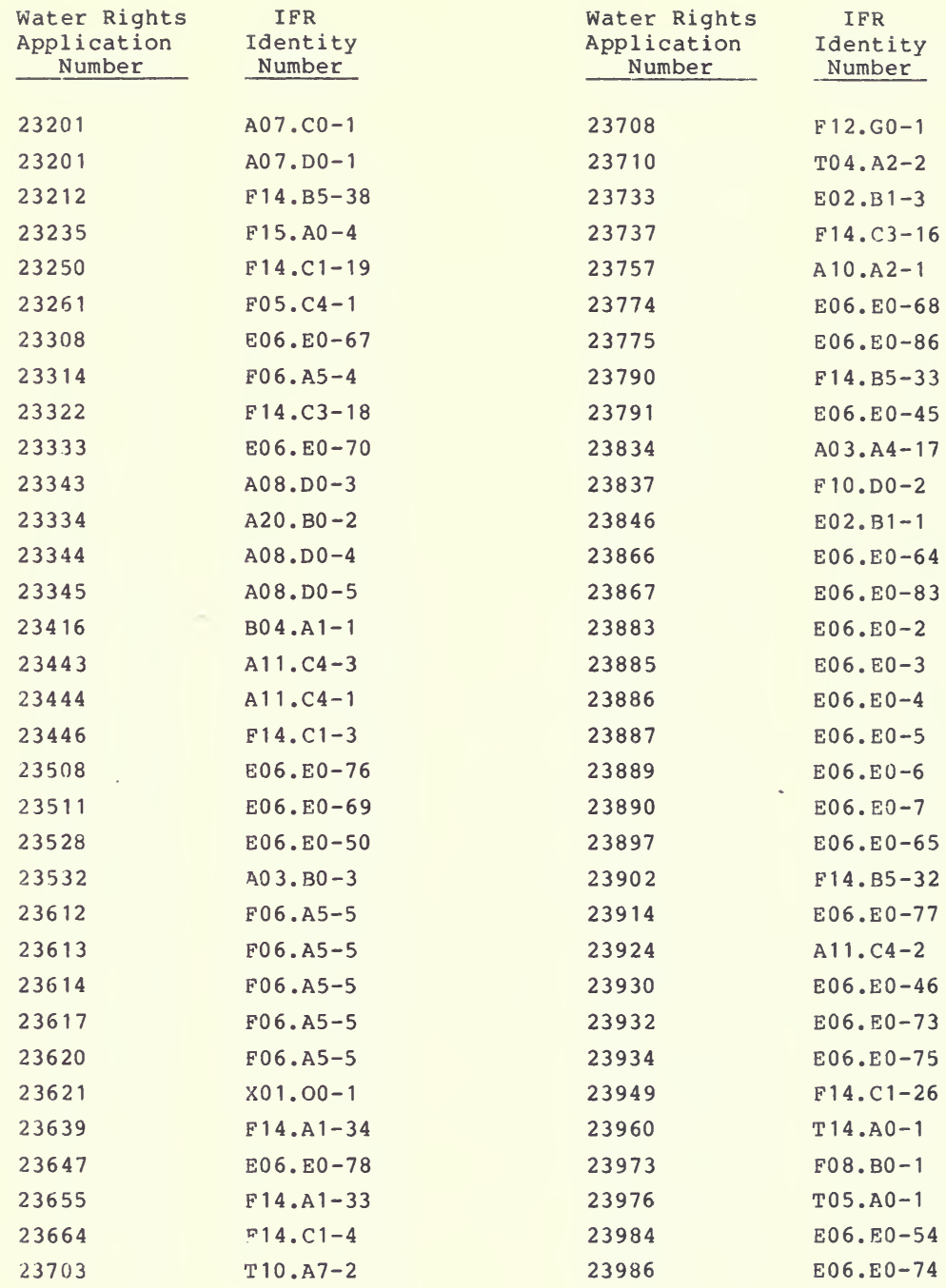


Water Rights Application Number

\begin{tabular}{|c|c|}
\hline 23987 & $\mathrm{E} 06 . \mathrm{E} 0-48$ \\
\hline 24010 & F14.C3-1 \\
\hline 24013 & F14.C1-36 \\
\hline 24014 & F14.C1-2 \\
\hline 24018 & E06.EO-1 \\
\hline 24020 & $\mathrm{E} 06 . \mathrm{F} 0-85$ \\
\hline 24022 & $\mathrm{E} 06 . \mathrm{E} 0-56$ \\
\hline 24023 & E06.DO-2 \\
\hline 24024 & E0 6.DO - 10 \\
\hline 24043 & $\mathrm{E} 06 . \mathrm{E} 0-53$ \\
\hline 24044 & F14.C3-5 \\
\hline 24045 & $\mathrm{~A} 03 . \mathrm{A} 4-6$ \\
\hline 24051 & F14.B5-1 \\
\hline 24072 & E06.E0-33 \\
\hline 24076 & $\mathrm{E} 06 . \mathrm{EO}-40$ \\
\hline 24085 & $\mathrm{~B} 04 . \mathrm{A} 1-2$ \\
\hline 24098 & A1 1. D5-5 \\
\hline 24099 & A $11 . D 5-6$ \\
\hline 24104 & F14.C3-11 \\
\hline 24105 & F05.B3-1 \\
\hline 24124 & E06.E0-39 \\
\hline 24125 & $\mathrm{E} 06 . \mathrm{E} 0-38$ \\
\hline 24126 & F $14 . C 3-12$ \\
\hline 24127 & F06.A2-1 \\
\hline 24129 & $\mathrm{E} 06 . \mathrm{E} 0-63$ \\
\hline 24139 & $\mathrm{~F} 14 . \mathrm{C} 1-16$ \\
\hline 24140 & F14.C1-17 \\
\hline 24141 & F14.C1-18 \\
\hline 24147 & $\mathrm{E} 06 . \mathrm{E} 0-26$ \\
\hline 24172 & T0 4.A2-5 \\
\hline 24200 & F05.EO-1 \\
\hline 24203 & F14.B $4-2$ \\
\hline 24209 & F $14 . C 2-7$ \\
\hline
\end{tabular}

Identity

Number
Water Rights Application Number

24238

24240

24244

24266

24268

24269

24269

24270

24271

24272

$24277 A \& B$

24278

24285

24292

24296

24296

24296

24296

24296

24297

24297

24297

24297

24297

24307

24303A

24309

$24310 A \& B$

$24311 \mathrm{~A}$

$24311 \mathrm{~B}$

24318

24320

24323
IFR

Identity Number

F06. D0 -8

EO2. $\mathrm{CO}-4$

F14.C3-13

F14. B5-28

F14.A1-18

F14.B5-29

F14.B5-43

F14.A1-19

F14.B3-1

A03. A4-1

E06. EO-34

E06.D0-6

E06.E0-36

E06. E0-30

A0 3. BO- 5

A03 . B 0-6

A03. B0-9

A03. B 0-15

A0 3. B0-6

A03. $\mathrm{BO}-6$

A03. B0-7

A03. B 0-8

A0 3. BO-9

A03. B 0-10

E06.E0-32

E06. E0-55

E06.E0-28

F14.B5-30

F14.B5-48

F14.B $5-48$

F14.C2-6

G04. $\mathrm{BO}-3$

F13. E0-6 
Water Rights

Application

Number

$24325 A$

24327

24332

24335

24336

24345 A

24348

24358

24359

24364

24366

24375

24395

24398

24405

24416

24433

24442

24443

24456

24468

24481

24488

24489

24492

24503

24504

24505

24518

24522

24525

24526

24526

24527
IFR

Identity

Number

F14.C1-37

E06. E0-27

E06.E0-29

B10. $\mathrm{CO}-10$

A0 4.D 4-1

E06.E0-59

E06.E0-41

E06.E0-35

F14.C1-15

F13.F3-1

A03. A 4-7

F05.D2-2

E06.E0-37

E07.B2-2

F14.C3-10

A06. D4-3

E06. E0-9

E06.E0-31

E06.E0-15

E06.E0-14

E06.DO-3

F14.C2-2

F14.C3-3

F14.C3-19

F13. E0-1

E06.DO-4

E06.D0-1

E06.E0-16

F14.B 5-6

F14.C1-7

F13.E0-3

F13. E0-3

E13.E0-8

E06.E0-17
Water Rights

Application

Number

24531

24538

24558

24570

24570

24570

24570

24576

24591

24592

24606

24617

24635

24664

24664

24666

24670

24673

24675

24677B

24678

24679

24686

24688

24689

24691

24693A

24694

24701

24721

24725

24729

24741
IFR

Identity

Number

E06.D0-5

A03. $\mathrm{BO}-4$

F06.C2-1

T04. A2-6

T04.A2-7

T0 4. A2-8

T04.A2-9

F13. E0-2

E06.E0-19

E06.E0-18

F 14.C $1-36$

F14. C1-8

A03. BO - 10

A0 3. $A 4-12$

A03. A $4-20$

T04. A 1-3

F06.B 2-1

A0 3. A 4-8

F06.B 5-2

E06.E0-79

F 14.B 5-4

F14.B5-44

T08.00-2

E14.B4-3

F06.A3-2

F14.C1-21

E06.E0-20

F06. C2-2

B 14. E1-4

A03. A4-11

F 14.C3-4

B0 4. A3-3

F14.C1-9 
Water Rights Application Number

24759

24761

24762

24762

$24763 \mathrm{~A}$

24788

24789

24790

24791

24795

24820

24827

24837

24839

24851

24864

24865

24866

24868A

24877

24882

24887

24889

24890

24891

24892

24893

24894

24898

24899

24905

24908

24909
IF R

Identity

Number

F08.BO-2

F14.A1-1

F14.A1-25

F14.A1-29

F14.C1-35

EO2.DO-5

F 14.C1-31

F14. B5-40

F 14.B5-5

F14.C2-3

F14.C1-28

F14.A1-2

F06.A3-3

F06.A5-1

A03.A4-9

F14. B5-7

F14.A1-3

E06.E0-21

F13.E0-15

F14. B5-8

F14.A1-28

F14. A1-4

F14.A1-7

F14. B5- 10

F 14.A1-26

F14.A1-6

F14.B5-45

F14. B5-9

F14.C1-11

F14.B5-42

F14.A1-5

F14. A 1-8

F 14 . B 5-11 water Rights

Application

Number

24911

24912

24914

24916

24917

24919

24921

24924

24925

24928

24931

24932

24935

24943

24947

24951

24954

24958

24962

24963

24967

24968

24969

24972

24983

24989

24993

24995

24996

25000

25002

25005

25010
IFR

Identity

Number

F05.A3-4

F14. B5-12

T 10. B 4-1

Е06. E0-22

E06.E0-23

F14. B5-13

F 14.A1-9

F14. B4-1

F 14.B 5-47

E01. A2- 1

F 14.A1-15

F14.B5-20

F 14.B5-19

F13. E0-4

F 14.A1-14

F14.B5-18

F11.A1-1

F14.B5-17

F 14.A1-12

F14.A1-11

F 14.A1-10

F14. A1-30

F14.A1-30

F14.B5-16

A09.A4-2

F06.B5-1

F06.B5-15

F14.C2-4

F 14.C3-6

A23. C1-1

T 10.A3-1

F14.B5-14

F 14.A1-17 
Water Rights

Application

Number

25012

25015

25021

25025

25028

25032

25060

25061

25063

25064

25066

25068

25076

25077

25078

25084

25110

25112

25138

25154

25157

25169

25173

25185

25186

25207

25207

25242

25268

25268

25279
IFR

Identity

Number

F14.A1-16

F14. B5-21

F14.A1-27

F14.B5-39

F14.B5-41

F14.B5-22

F13. E0-5

F13. E0-5

A0 6. C2-4

A2 1. B 1-1

F05.B4-1

F15.D0-1

F14.A1-24

E06. E0-24

E06.E0-24

F14.B5-27

F14.B5-26

A22. A2-1

F11.C2-1

F14.B5-25

F14.B5-24

CO1.E0-1

F13.D0-1

W2 $8 . B 0-1$

E06.E0-51

F14. B5-23

F14.B5-23

F03.A1-6

F14.C1-12

F14.C1-12

B 14. A1-3 water Rights

Application Number

25291

25293

$25294 A$

25302

25303

25306

25307

25317

25318

25321

25334

25337

25338

25344

25345

25358

25363

25367

25368

25368

25368

25368

25393

25394

25407

25420

25432

25432

25433

25447

25448

25449
IFR

Identity

Number

G04.B - -2

E06. E0-58

E06.E0-60

E02. DO-3

E02. D 0-8

G04. $\mathrm{BO}-1$

F06.A3-1

E06. E0-8

E02. DO-14

F14.C1-22

F14.C3-2

E02. DO-1

E02.DO-2

F14.B5-3

G04. BO-4

E01. A3-2

F 14.A $1-13$

F14.A1-20

W03.AO-1

W0 3. AO-2

W03. $A 0-3$

W0 3. AO-4

F14.A1-21

F14.A1-31

E02.B2-1

G04. $\mathrm{B} 0-3$

A03.A4-3

A03. A 4 - 5

A03.A4- 19

E06.E0-42

E06.E0-10

E06.E0-11 
Water Rights

Application Number

25455

25461

25462

25463

25464

25465

25467

25468

25469

25470

25485A

25506

25512

25530

25544

25562

25568

25571

25596

25598

25630

25647

25652

25654

25655

25655

25656

25657

25658

25659

25666

25674

25676
IFR

Identity

Number

E06. E0-43

E02. B1-2

E02. B3-1

$\mathrm{E} 02 . \mathrm{B} 2-4$

E02. B2-4

$\mathrm{E} 02 . \mathrm{B} 2-4$

E02. B 1-6

E02. B1-5

E02. B1-4

E02. B2 -3

E06.E0-52

A06. B2 -4

B 1 4. A 1-1

B0 4 . B0-1

E03. $\mathrm{A} 1-4$

T0 4 . B0-3

T0 4.A 1-2

F05. C1-1

F14.C1-5

E02. D0-4

E0 6.E0-12

F15. A0 -7

T0 4 . B0-2

T0 4 B0-2

T0 4. B0-8

T0 4. B0-9

T0 4. B0-2

T0 . B0-7

T0 4. B0-9

T0 4.B0-9

F11.F1-3

F14.A2-3

E02.D0-12
Water Rights

Application

Number

25682

25691

25697

25700

25709

25712

25730

25731

25734

25740

25749

25750

25765

25770

25776

25790

25812

25815

25822

25830

25833A

25846

25850

25866

25868

25877

25880

25888

25897

25917

25935

25943

25950
IFR

Identity

Number

F14.35-46

F14.C2-10

F05.B3-2

E06. E0-13

T0 4. B0-1

E06.E0-81

E02. B 3-4

E02. B 3-4

E02. $83-5$

F14.C3-20

F14.C1-6

E02.B3-6

A03.A4-4

E07.B 3-1

A0 3.A $7-1$

F05.C3-1

E07.B2-1

A06.B2-5

F 14.C1-13

F14.A2-1

E06.E0-49

F06.C1-2

F14.C1-30

A05.B1-3

E02.DO-9

E02. C0-3

F14.C2-8

F14.C1-14

A09. A2-1

G08. D0-1

E06.E0-25

F06.D0-1

E02.D0-10 
Water Rights Application Number

\begin{tabular}{|c|c|}
\hline 25958 & Т0 $4 . \mathrm{B} 0-4$ \\
\hline 25967 & $\mathrm{~A} 12 . \mathrm{B} 0-1$ \\
\hline $26003 A$ & $\mathrm{~A} 03 \cdot \mathrm{B} 0-1$ \\
\hline 26012 & B0 4. A4-1 \\
\hline 26016 & E $14 . C 2-5$ \\
\hline 26022 & $\mathrm{~F} 14 . \mathrm{C} 3-7$ \\
\hline $26036 \mathrm{~A}$ & E06. E0-82 \\
\hline 26055 & F14.C3-8 \\
\hline 26063 & $A 05 . B 1-4$ \\
\hline 26113 & F14.A2-2 \\
\hline 26125 & $\mathrm{EO} 2 . \mathrm{CO}-5$ \\
\hline 26126 & $\mathrm{E} 02 . \mathrm{CO}-5$ \\
\hline 26150 & พ03. BO-4 \\
\hline 26160 & $A 09 . A 4-5$ \\
\hline 26168 & $\mathrm{~A} 03 . \mathrm{BO}-2$ \\
\hline 26177 & $\mathrm{~A} 03 . \mathrm{A} 4-2$ \\
\hline 26192 & w01. o0-1 \\
\hline 26233 & $\mathrm{~F} 14 . \mathrm{A} 1-32$ \\
\hline 26236 & E13.CO-1 \\
\hline 26255 & F11. C3-1 \\
\hline 26313 & $A 03 . A 4-14$ \\
\hline 26323 & $\mathrm{~F} 11 . \mathrm{C} 2-3$ \\
\hline 26401 & F06.A3-5 \\
\hline 26408 & $A 10 . E 4-1$ \\
\hline 26414 & F14.B5-49 \\
\hline 26452 & $A 03 . A 4-15$ \\
\hline 26460 & $\mathrm{C} 06 . \mathrm{A} 0-1$ \\
\hline 26479 & $\mathrm{~F} 06 . \mathrm{A} 2-2$ \\
\hline 26517 & A06.D 4-1 \\
\hline 26517 & $\mathrm{~A} 06 . \mathrm{D} 4-2$ \\
\hline 26534 & B 14. DO-4 \\
\hline 26534 & B 14. D0-9 \\
\hline 26542 & B $14 . E 1-3$ \\
\hline 26656 & $\mathrm{~A} 03 . \mathrm{A} 4-13$ \\
\hline
\end{tabular}

Identity

Number

T04. B0-4

A12. $\mathrm{BO}-1$

3. BO-

. A4 -

. $C 2-5$

. $\mathrm{C} 3-7$

.E0-82

. $33-8$

. B 1-4

4. $A 2-2$

$2 . \mathrm{CO}-5$

2. $\mathrm{CO}-5$

3. $\mathrm{BO}-4$

9. A4-5

3. $\mathrm{BO}-2$
Water Rights

Application Number

$--$

$--$

$--$

Raker Act.

Raker Act.

Raker Act.

- -

$--$

$--$

--

Riparian

Riparian

Riparian

Riparian

Riparian

Riparian

Riparian

Riparian
IF Identity Number

A05.A1-1

A23.E3-2

A03.A4-16

A09. A4-4

A05.B 1-1

A0 5. B 1 - 2

A06.B5-1

A06.B5-2

A 11.D 4-1

A 1 1. D4-2

A11.D5-2

A11. D5-3

A 23. B 1-1

A23. B1-2

B09. D0-4

B09. D0 - 6

B09. E 1-1

B09. E 1-3

B 10.D1-1

B10.D1-2

B 10. E0-1

CO 4 . B 2-1

C0 4 . B 3-1

CO 4 . $\mathrm{B} 3-2$

C05.A $1-1$

F05.D2-1

F06.A1-1

F14.C2-1

G05.A0-6

G05. $\mathrm{AO}-7$

G05.A0-8

G05. A0-9

G05.B $0-3$

G05. B0-4

G07.00-1

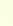


Index 2

Hydro Units/IFR Identity Number Referenced to Map Page

Hydro Unit

Code/ Map

IFR I.D. No Page

$\mathrm{AOI}$.

A02.

A03.

A. 04 .

A05.

A06.

A07.

A08.

A09.

Al0.

A11.

A12.

A13.

AI4.

A15.

A16.

A17.

A18.

A19.

A20.

A21.

A22.

A23.AI-C 4

A23.D1-E 4

A24.

BOI.

B02.

B03.

BO4.

B05.

B06.

B07.

B08.

B09.

B10.

BII.

B12.

B13.

BI4.
27

27

28

28

29

30

31

31

32

32

33

33

34

34
35

36

36

35

36

37

37

39

39

40

40

40

41

41

42

42

43

44

44

45

45

45

46
Hydro Unit

Code/ Map

IFR I.D. No Page

COI.AO-BO
COI. CO-GO
COI.HO
COI.JO-MO
COI. .NO

47

48

47

48

49

COI.PO

COI.QO-TO

COI.UO

COI.VO-WO

COI.X

C02.

C03.

c04.

C05.

c06.

C07.

EOI.

E02.

E03.

E04.

E05.

E06.AO-DO

E06.E0

E07.

FOI.

F02.

F03.

F05.A

F05.B-D

F05.E-H

F05.J

F06.

F07.

F08.

F09.

FI0.

FII. A-D

Fll.E-G

F12.

47

49

50

49

50

47

51

51

52

52

50

54

53

55

55

56

57

58

59

59

59

60

61 ilydro Unit

\begin{tabular}{l} 
Code/ \\
IFR I.D. No \\
\hline
\end{tabular}

F13.

FI4.A-B

PI4.C

inl 15 .

63

70

71

72

GOI.

G02.

73

G03.

G04.

G05.

73

73

73

74

G06.

G07.

G08. A

G08. B-D

G09.

74

74

75

76

76

G10.

G11.

G12.

G13.

T04.

m05.

506.

m07.

T08.

76

77

77

77

78

18

78

79

79

TO9. AO-GO

TO9.HO-JO

79

T09.KO

mlo.

TII.

80

ol

82

83

m12.

83
T13.

T14.

T15.

62

63

64

65

UOI.

U02.

U03.

vo 4 .

U05.

83

83

83

84

84

84

66
66

67

68

67 
Index 2 (Continued)

Hydro Un1ts/IFR Ident1ty Number Reference to Map Page

Hydro Unit

\begin{tabular}{l} 
Code/ \\
IFR I.D. No \\
\hline
\end{tabular}

W01.

W02.

W03. A0-BO

W03. CO-DO

W04.

W05.

W06.

W07.

W08.

W09.A1-A3

W09.BI-B4

W09.CI-D4

W10.

WII.

W12.

W13.

WI 4 .

WI5.

W16.

W17.

W18.

W19.

W20.

W2I.

W22.

W23.

W24.

W25.

W26.

W27.

W28. A-D

W28. E-G

W28.J

W29.

$\mathrm{X} 01$.

$\mathrm{X} 02$.

$\mathrm{X} 03$.

$\times 04$.

$\mathrm{X} 05$.
Map

Page

86

86

86

87

88

88

88

87

87

89

90

91

92

92

92

93

93

93

93

93

93

93

94

94

87

87

95

95

96

97

97

93

90

90

98

98

98

98

98
Hydro Un1t

Code/ Map

IFR I.D. No Page

$\mathrm{x} 06$

98

X07. $\quad 98$

$\times 08 . \quad 98$

X09. $\quad 99$

X10. 100

XI1. $\quad 101$

X12. 101

X13. 102

X14. 102

XI5.A0-DO 103

XI5.E0 $\quad 104$

X16. 103

X17.A 103

X17.B-D 99

$\mathrm{X} 18$.

99

X19.

$\times 20$.

$\mathrm{x} 21$.

$\mathrm{X} 22$.

$\mathrm{X} 23 . \mathrm{AO}$

105

106

106

106

107

$\mathrm{X} 23 . \mathrm{B} 0$

106

$\times 24$.

$\times 25$.

$\times 26$.

$\times 27$.

106

107

104

104

Y01. 108

Z01. $\quad 109$

Z02. 109

Z03. $\quad 109$

204. 110

205. 110

Z06. $\quad 110$

Z07. 110

Z08. 110

209. 110

Z10. 110

Z11. 110 



\section{WATERSHED INDEX MAP}

LOCATION OF INSTREAM FLOW REQUIREMENTS BY HYDROLOGIC UNITS

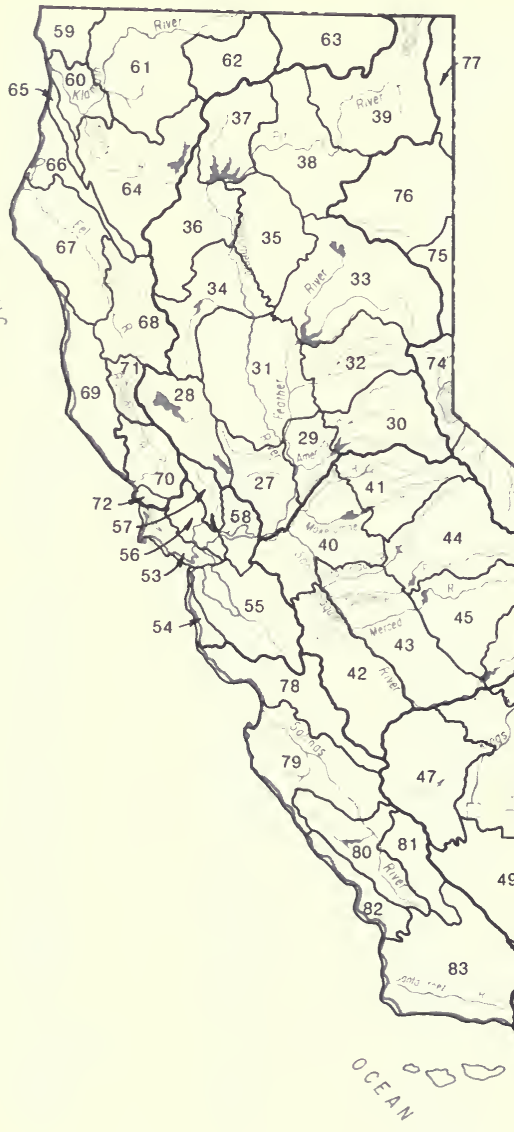
EACH NUMBERED AREA REPRESENTS
A SINGLE DETAILED MAP OF SEVERAL
SMALL HYDROLOGIC UNITS. THE NUMBERS REPRESENT THE PAGES WHERE THE DETAILED MAPS ARE DISPLAYED. EACH DETAILED MAP SHOWS THE LOCATIONS OF INSTREAM FLOW REQUIREMENTS. 


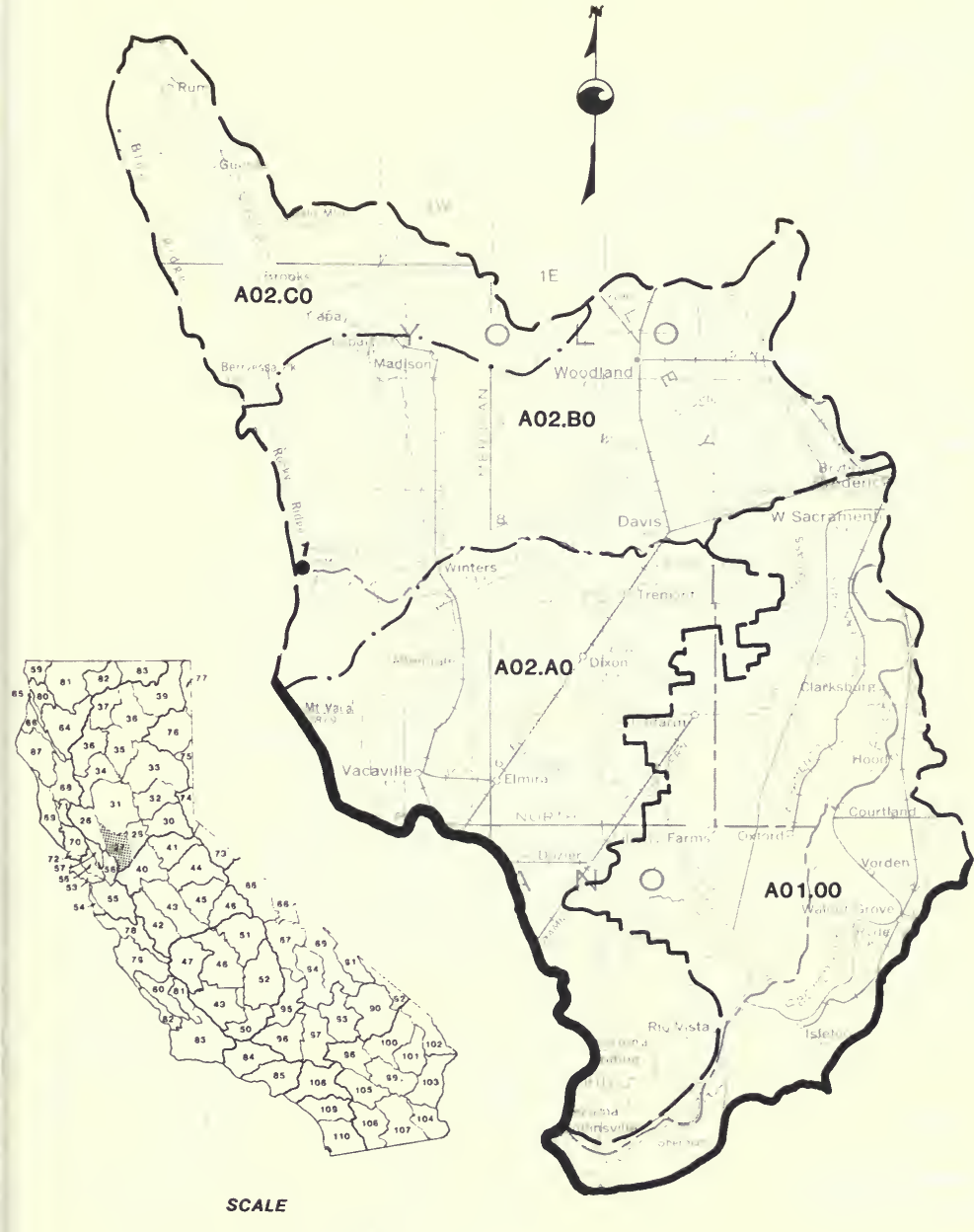

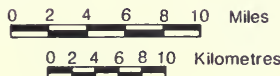




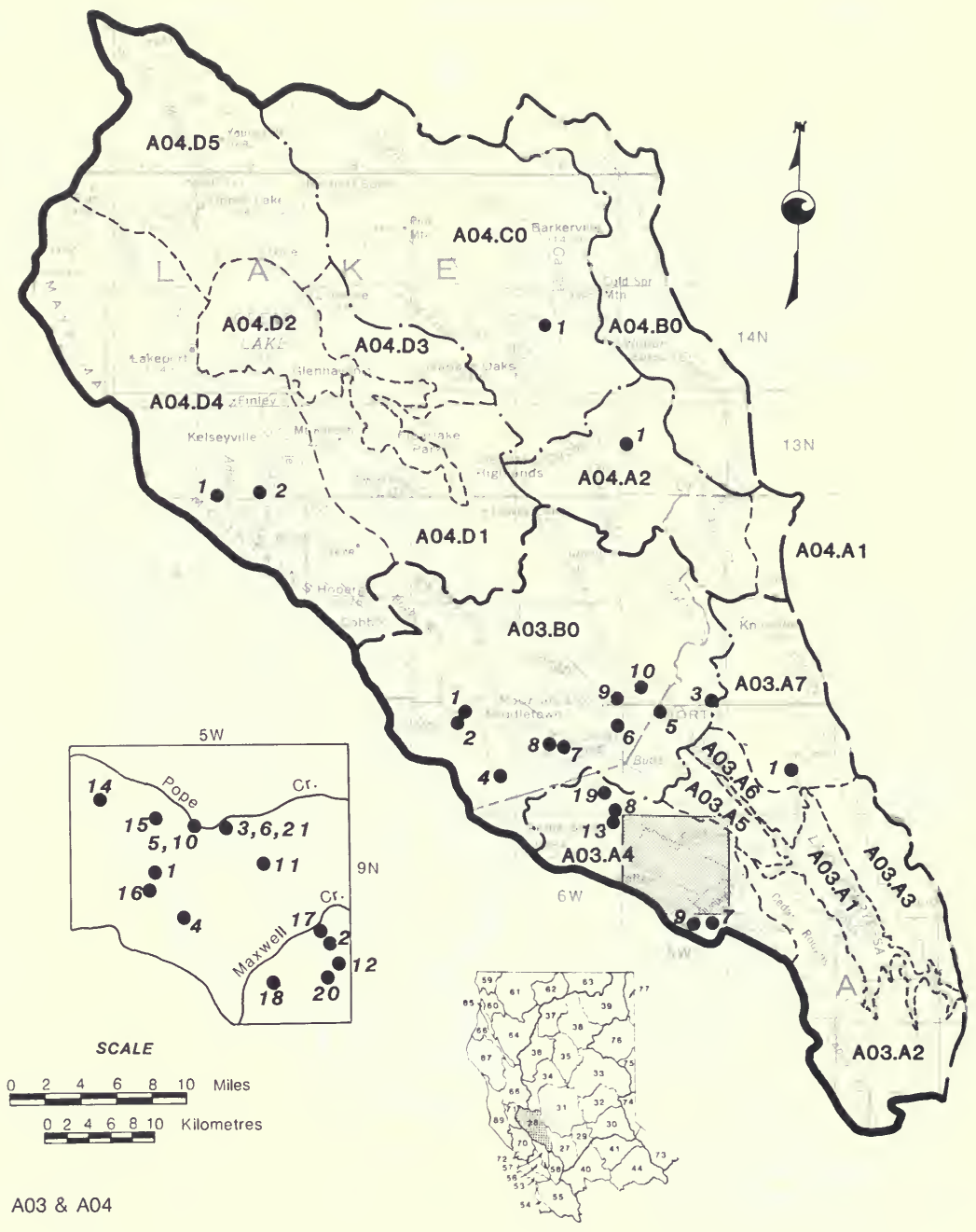




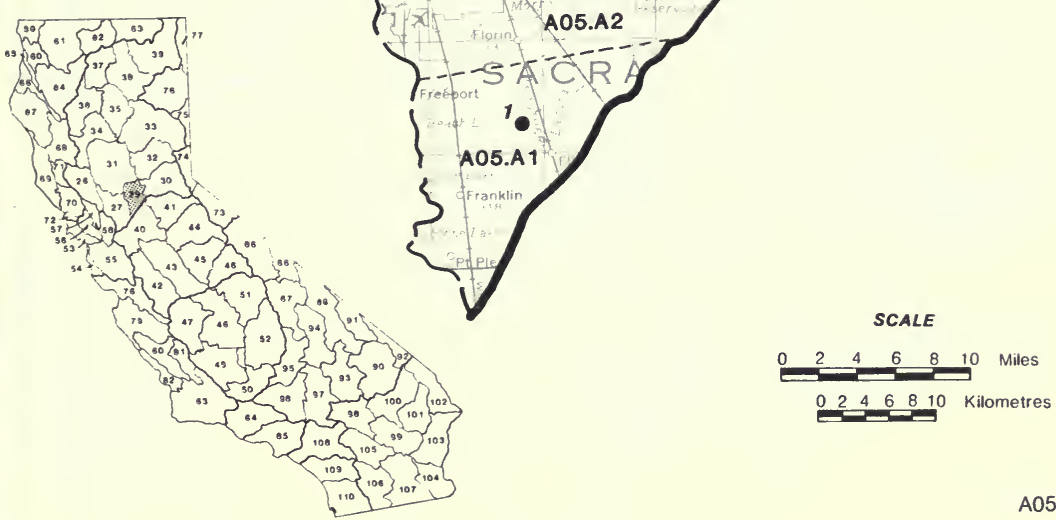




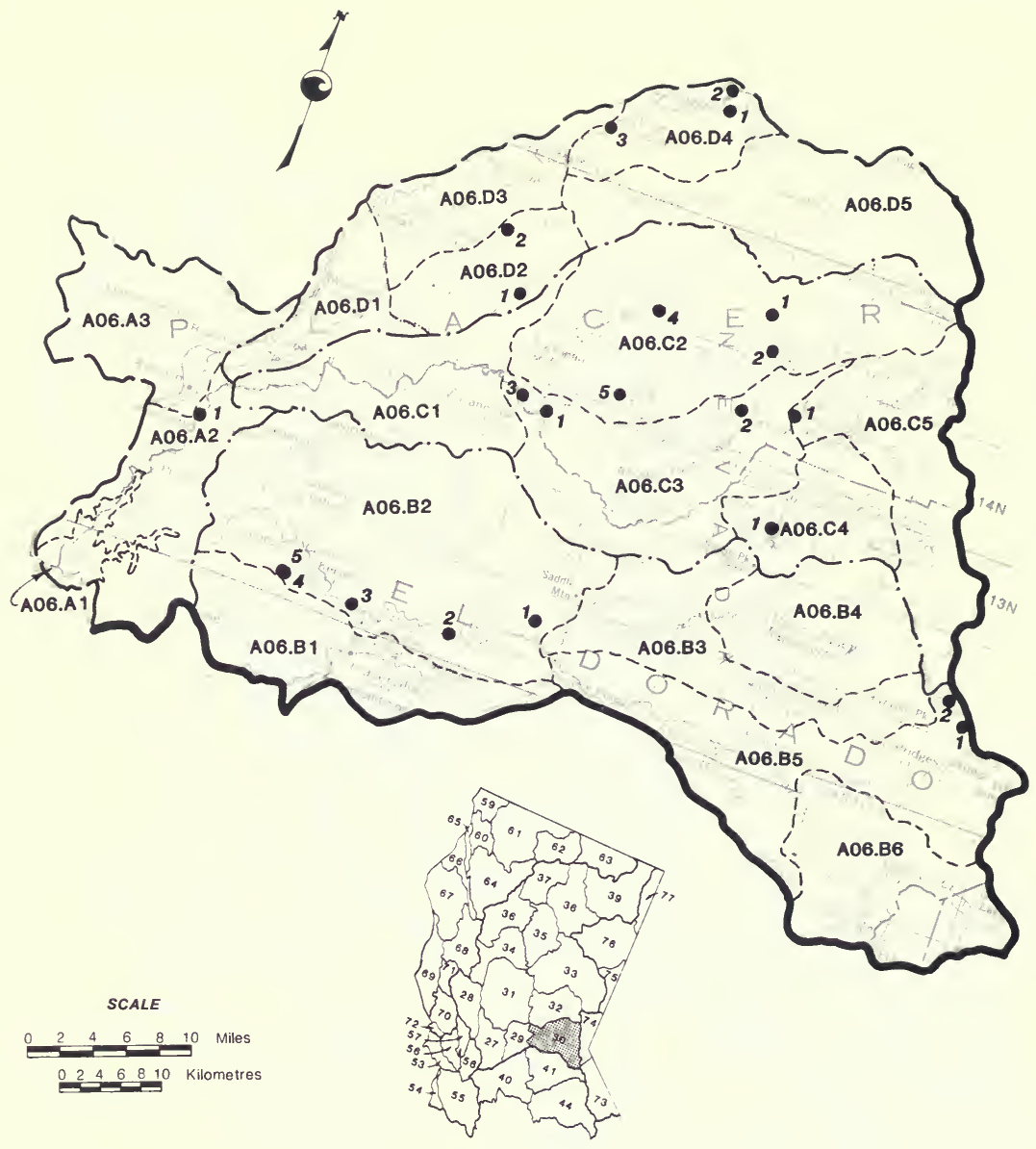




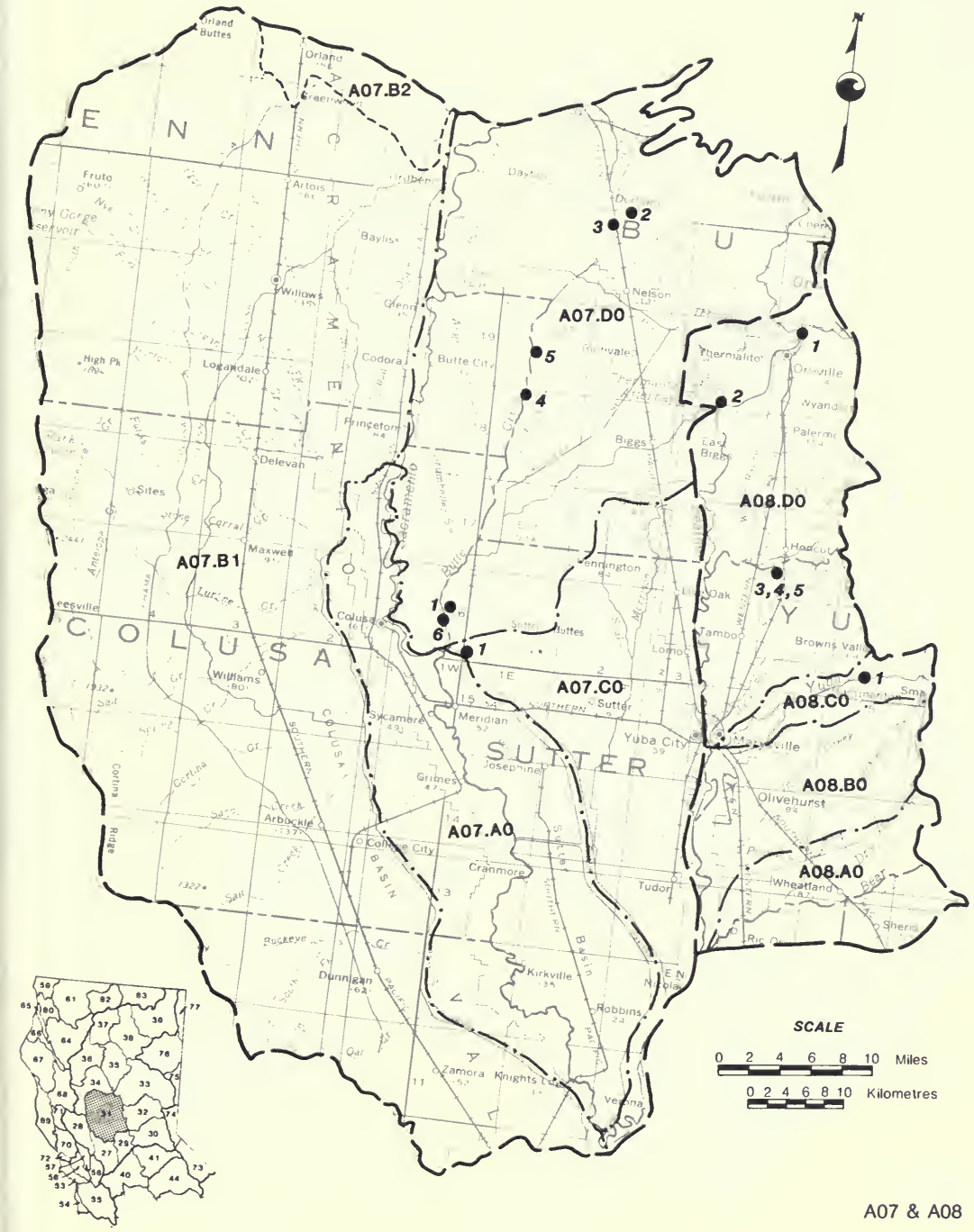




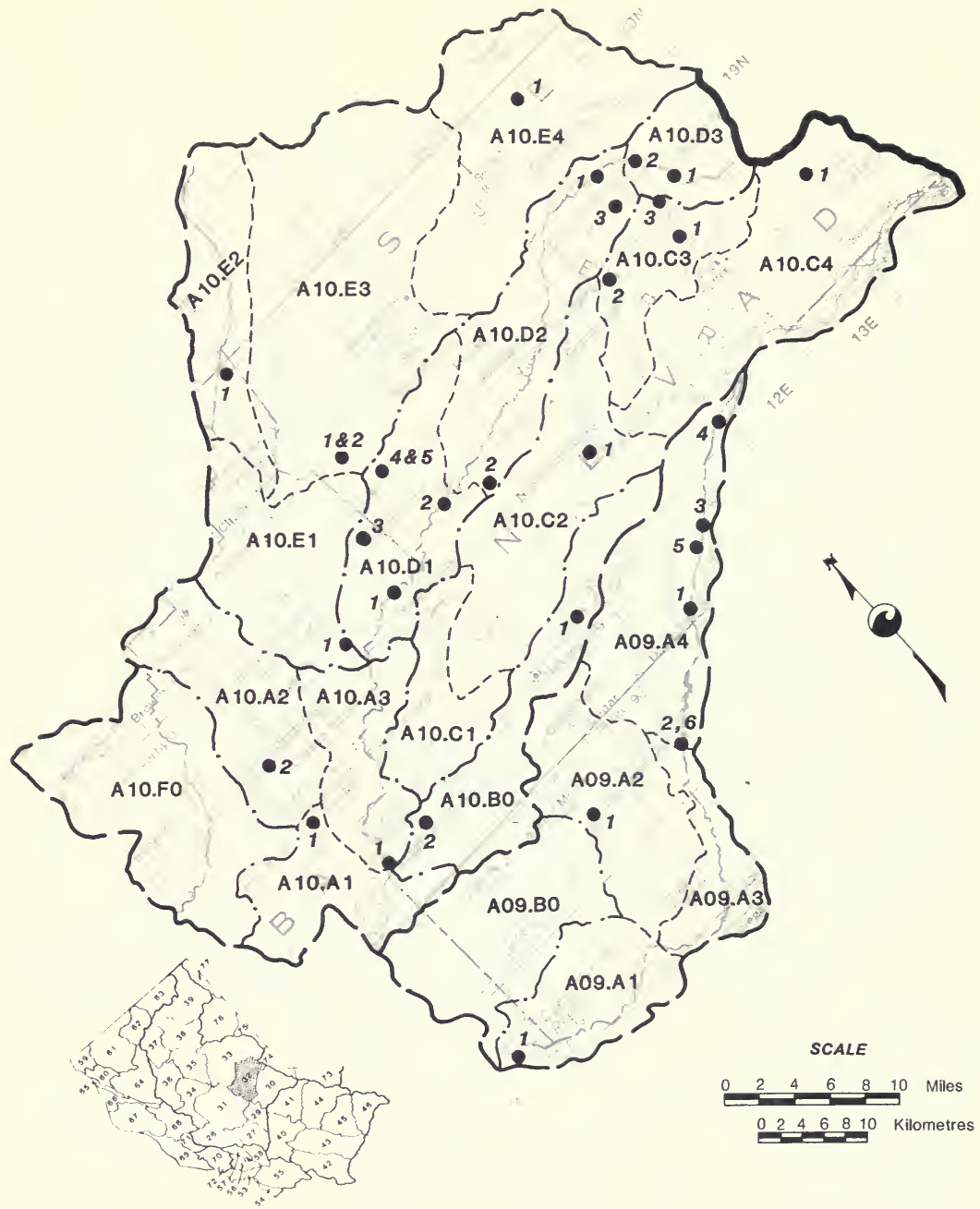

$A 09 \& A 10$ 


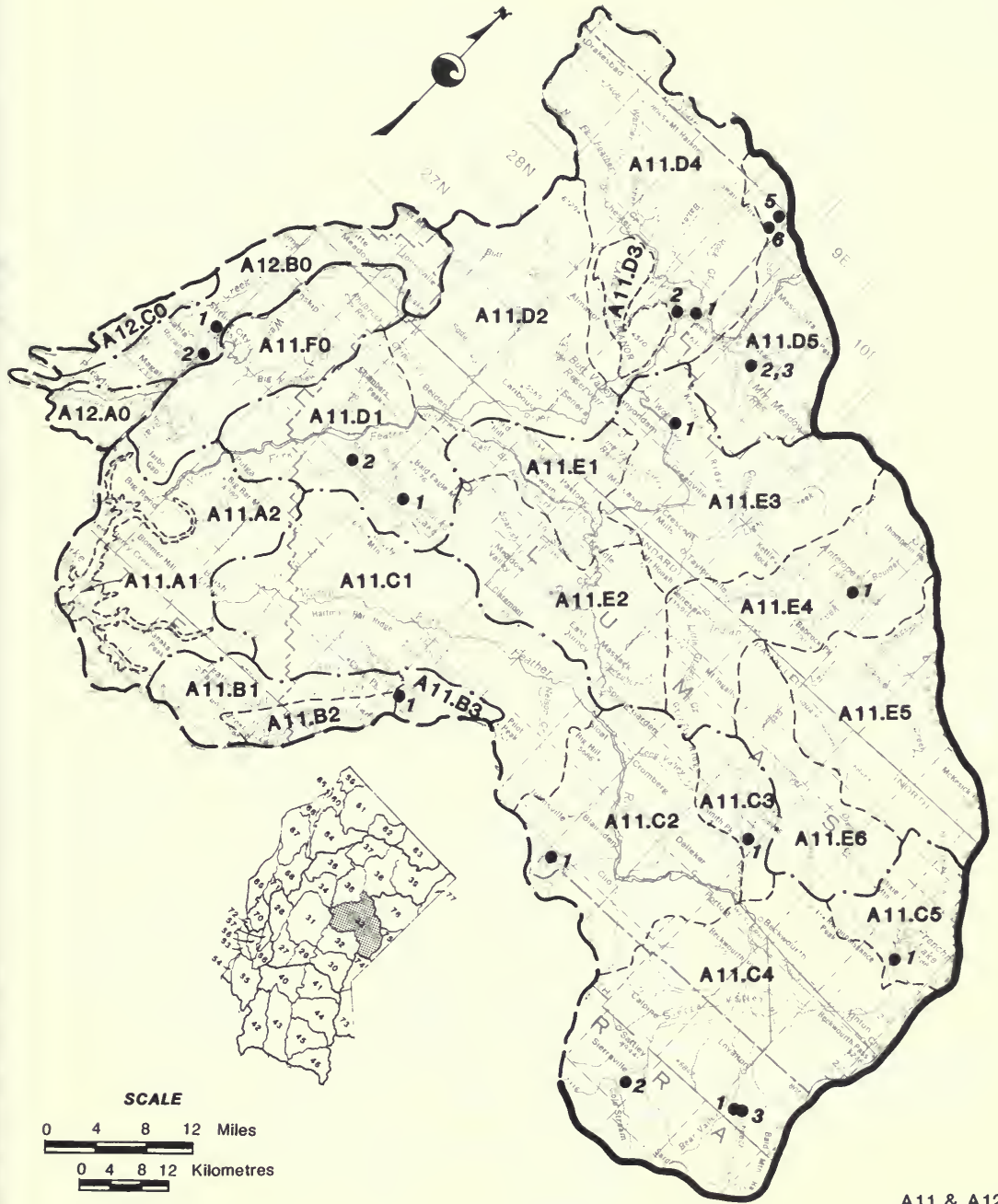

A11 \& A12 


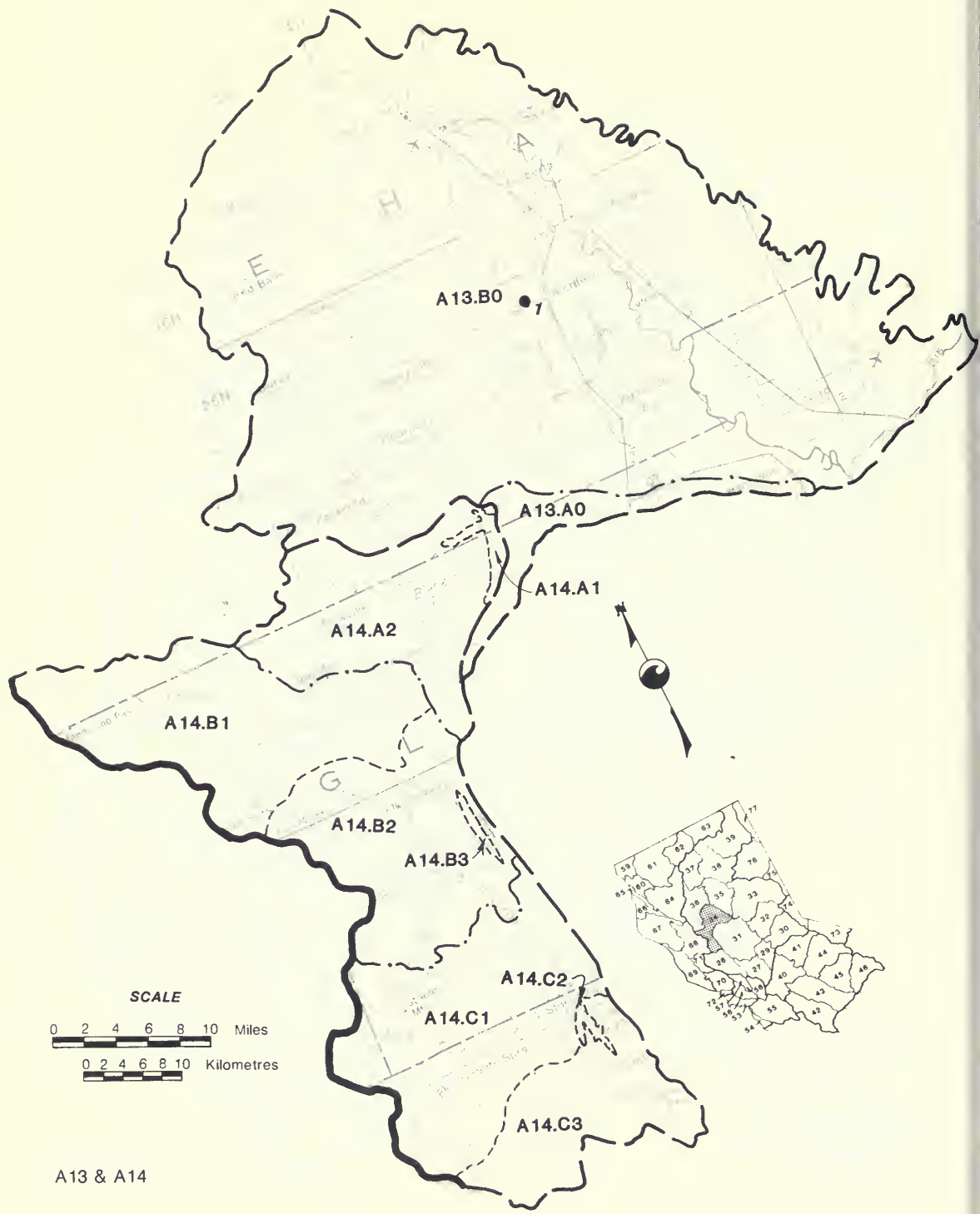

34 


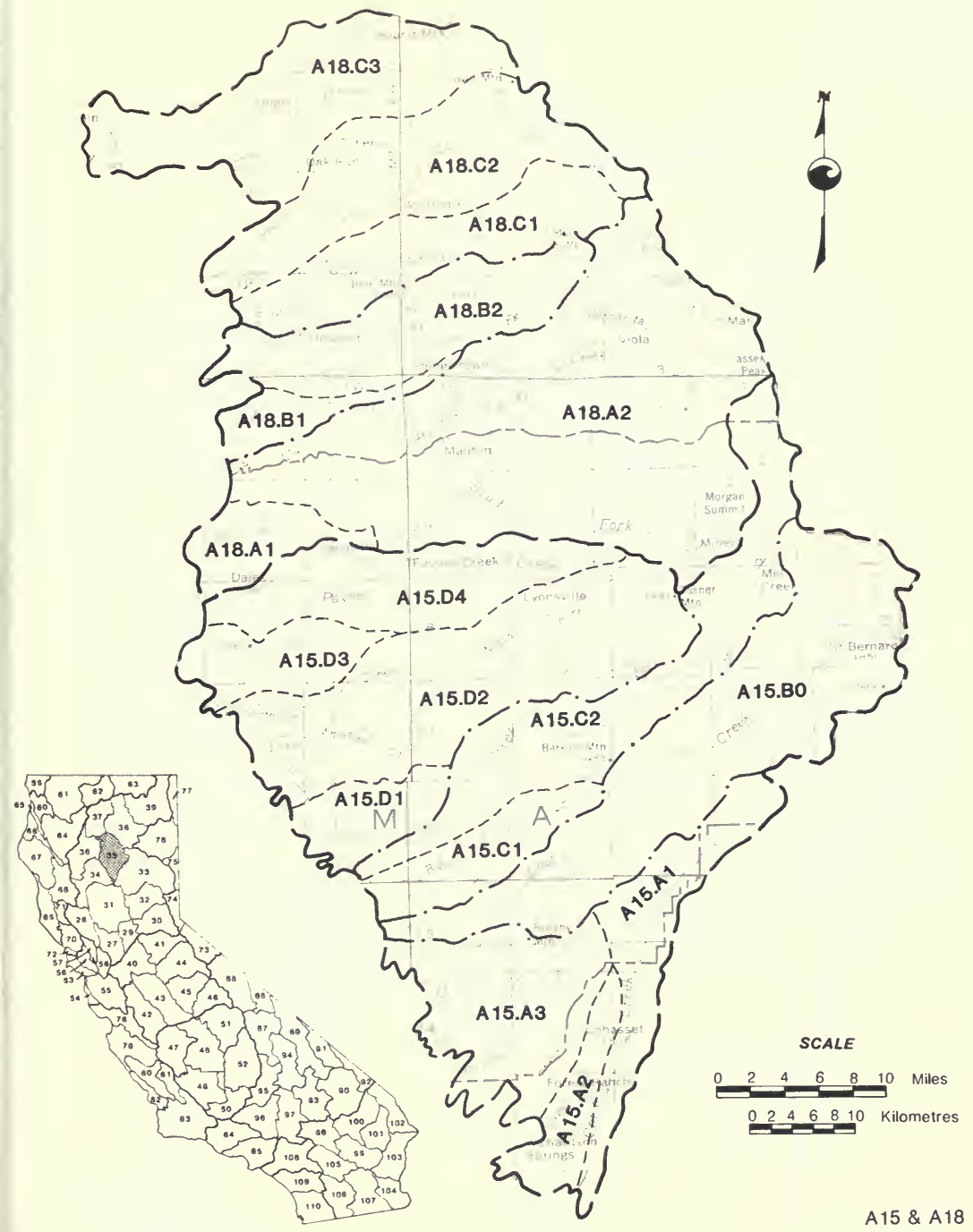




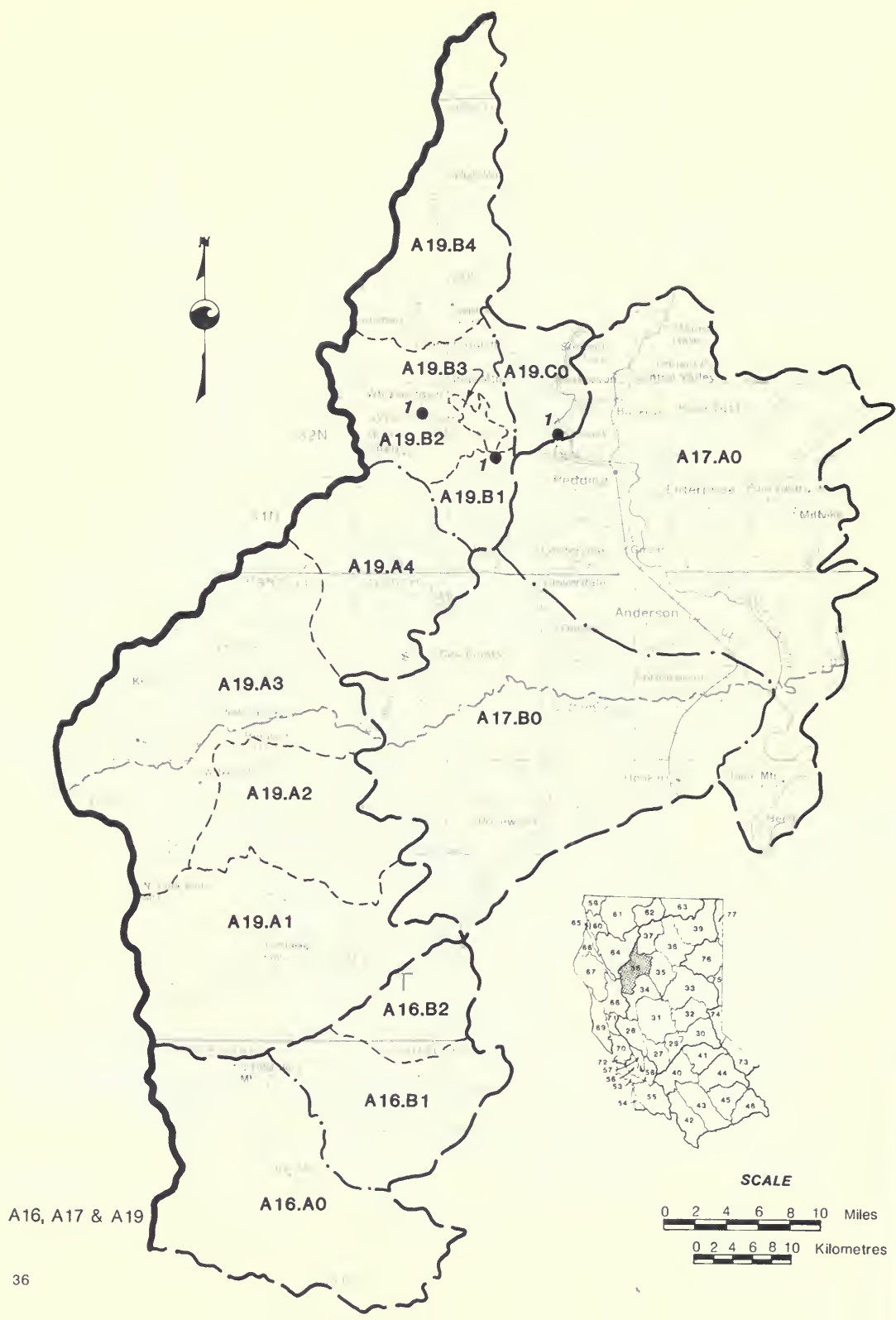




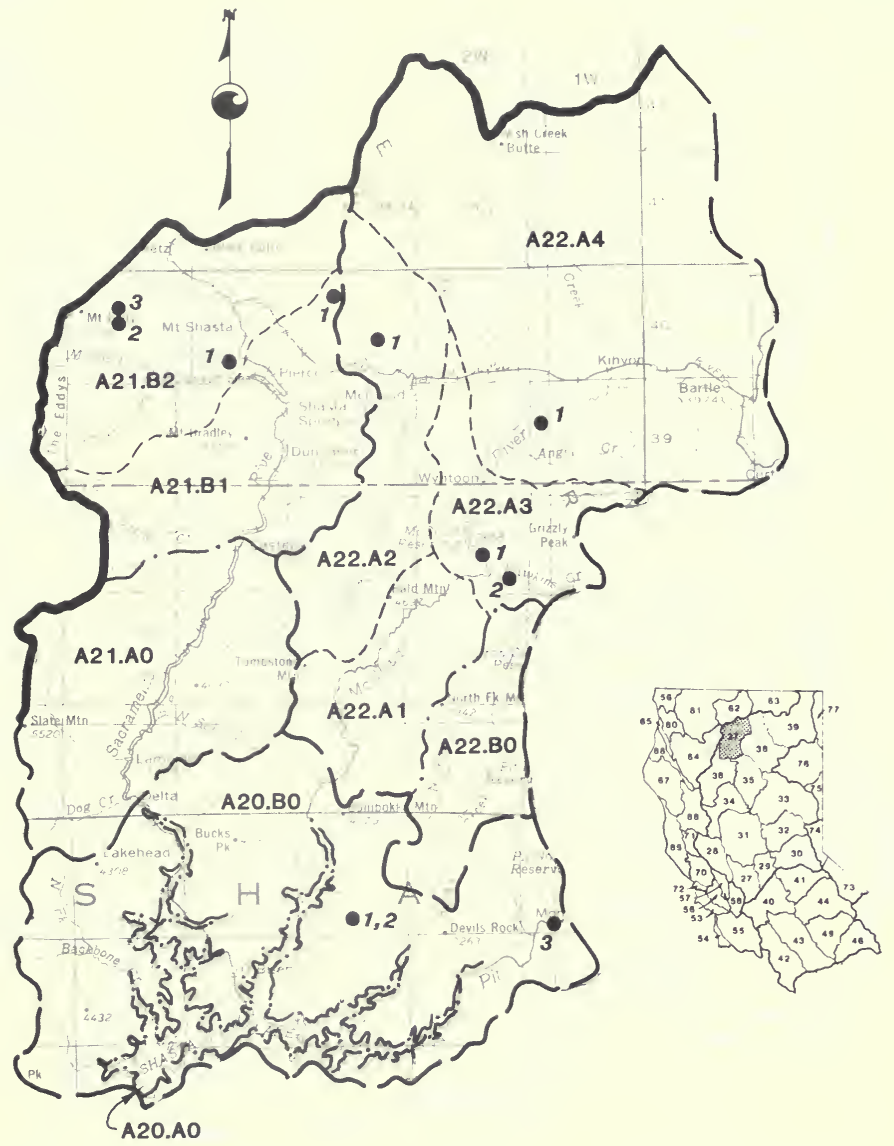

SCALE 


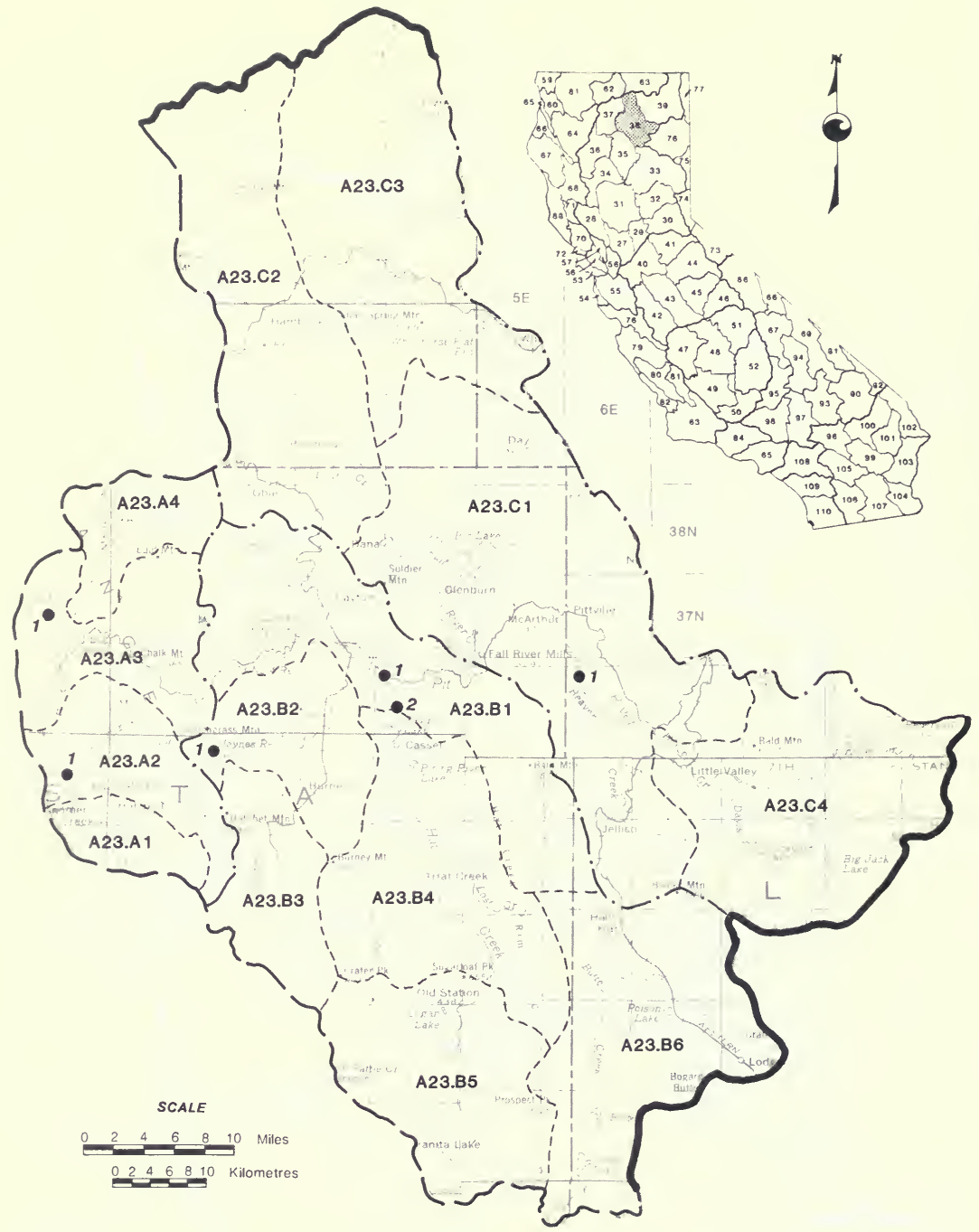

A23.A, B \& C 


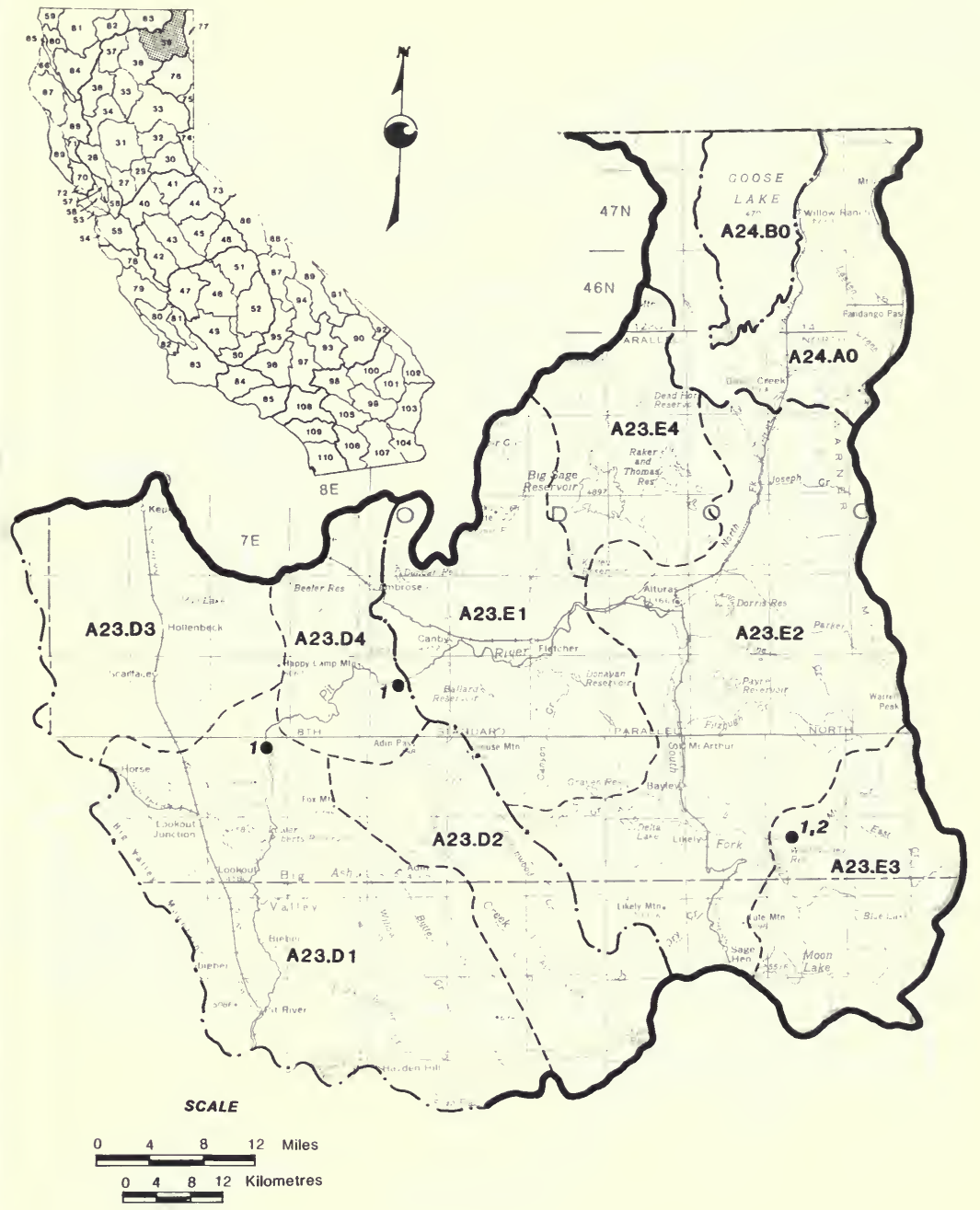

A23.D, E \& A24 


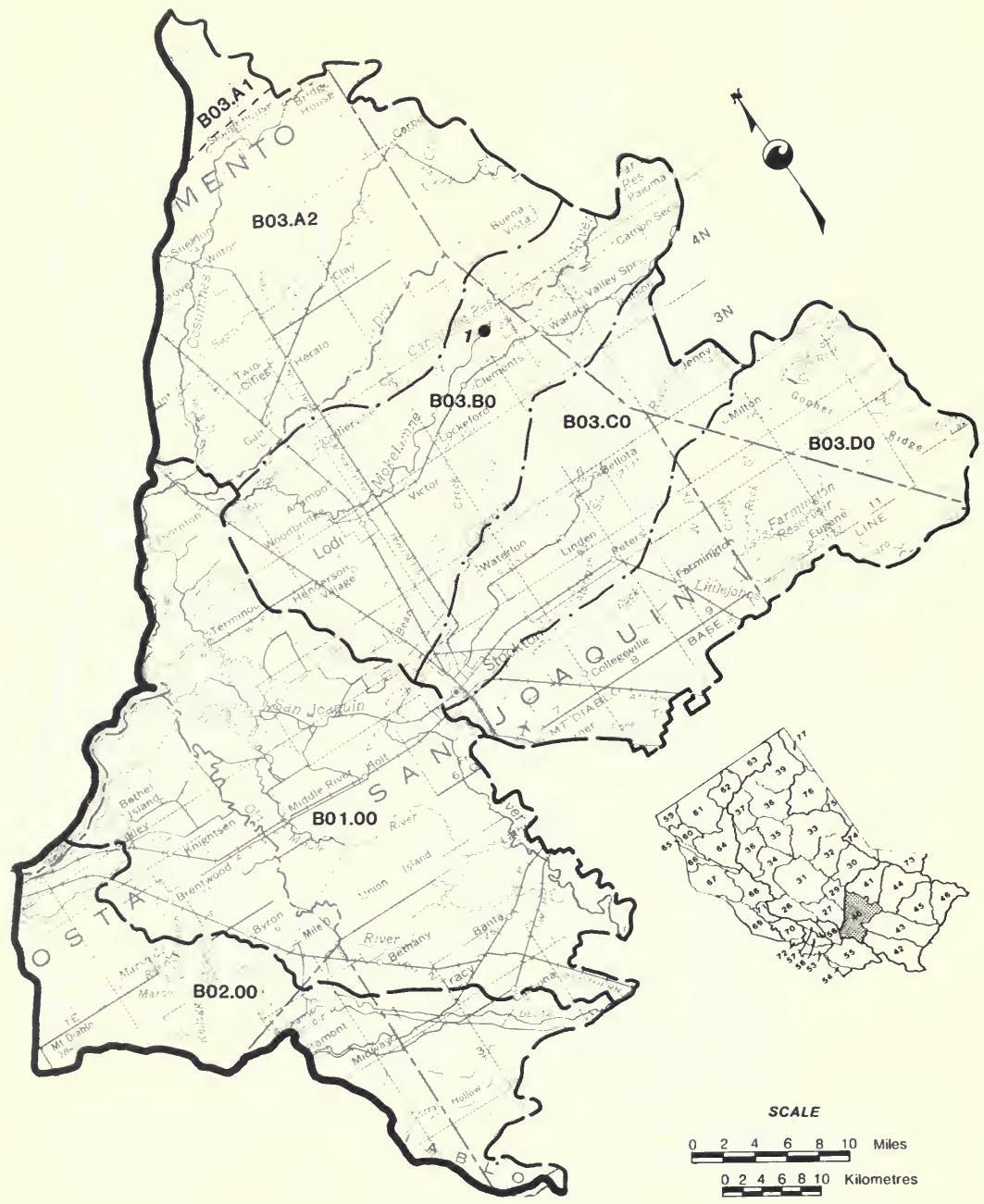

$B 01, B 02$ \& $B 03$ 


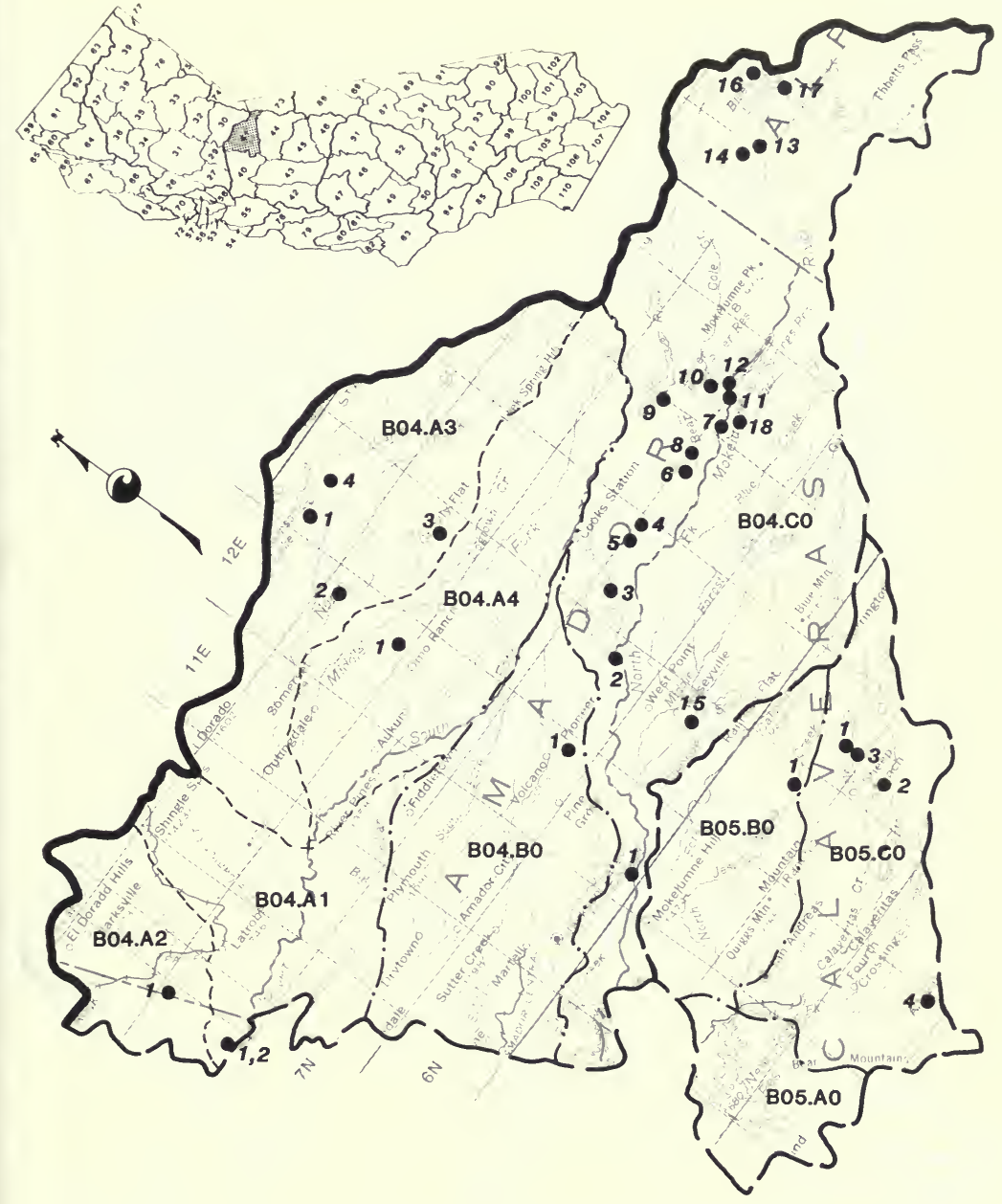

SCALE 


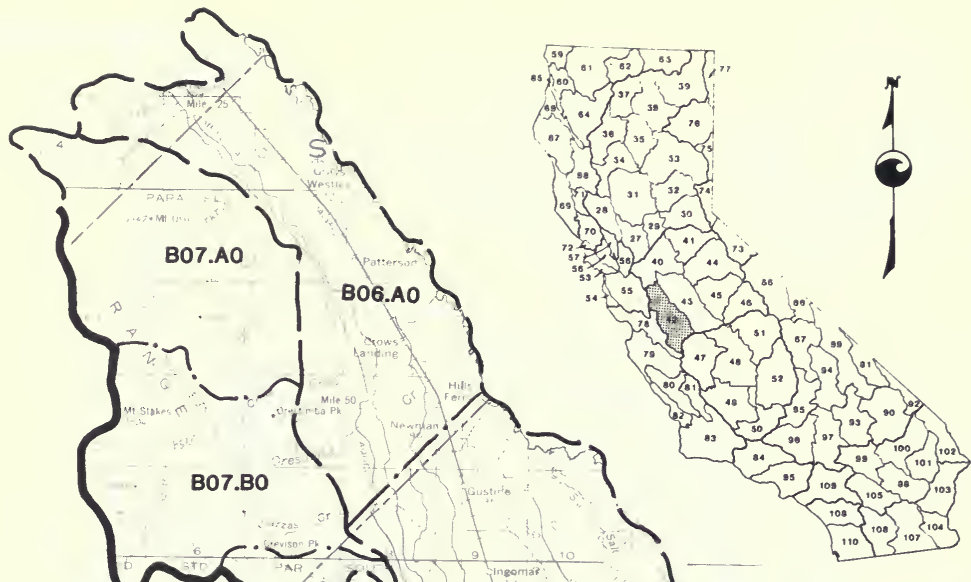

SCALE

$\begin{array}{lllllll}0 & 2 & 4 & 6 & 8 & 10 & \text { Miles }\end{array}$

0246810 Kilometres

-

B06 \& B07

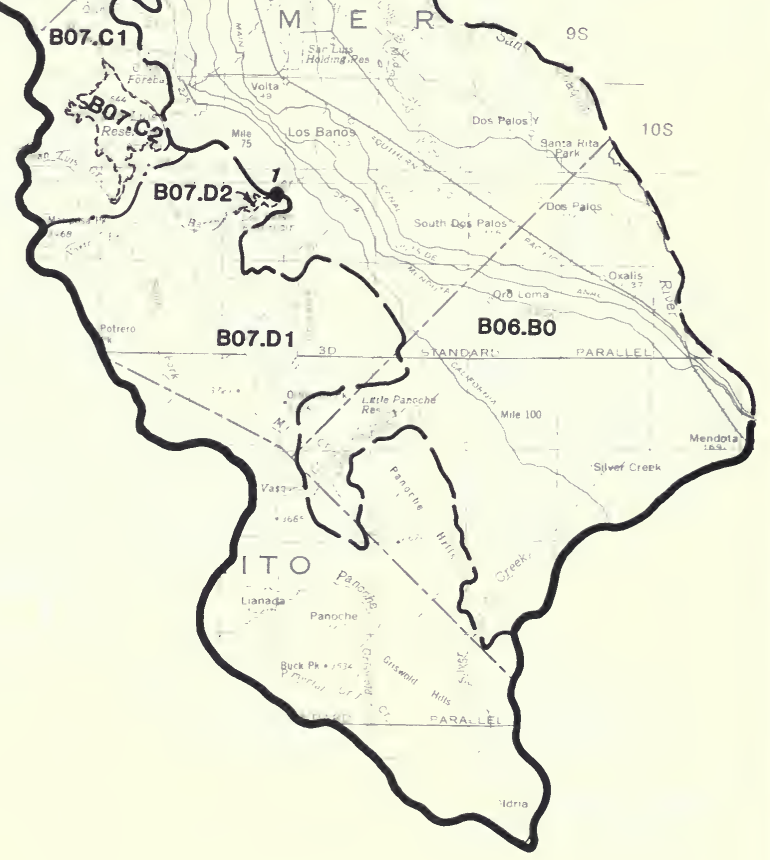




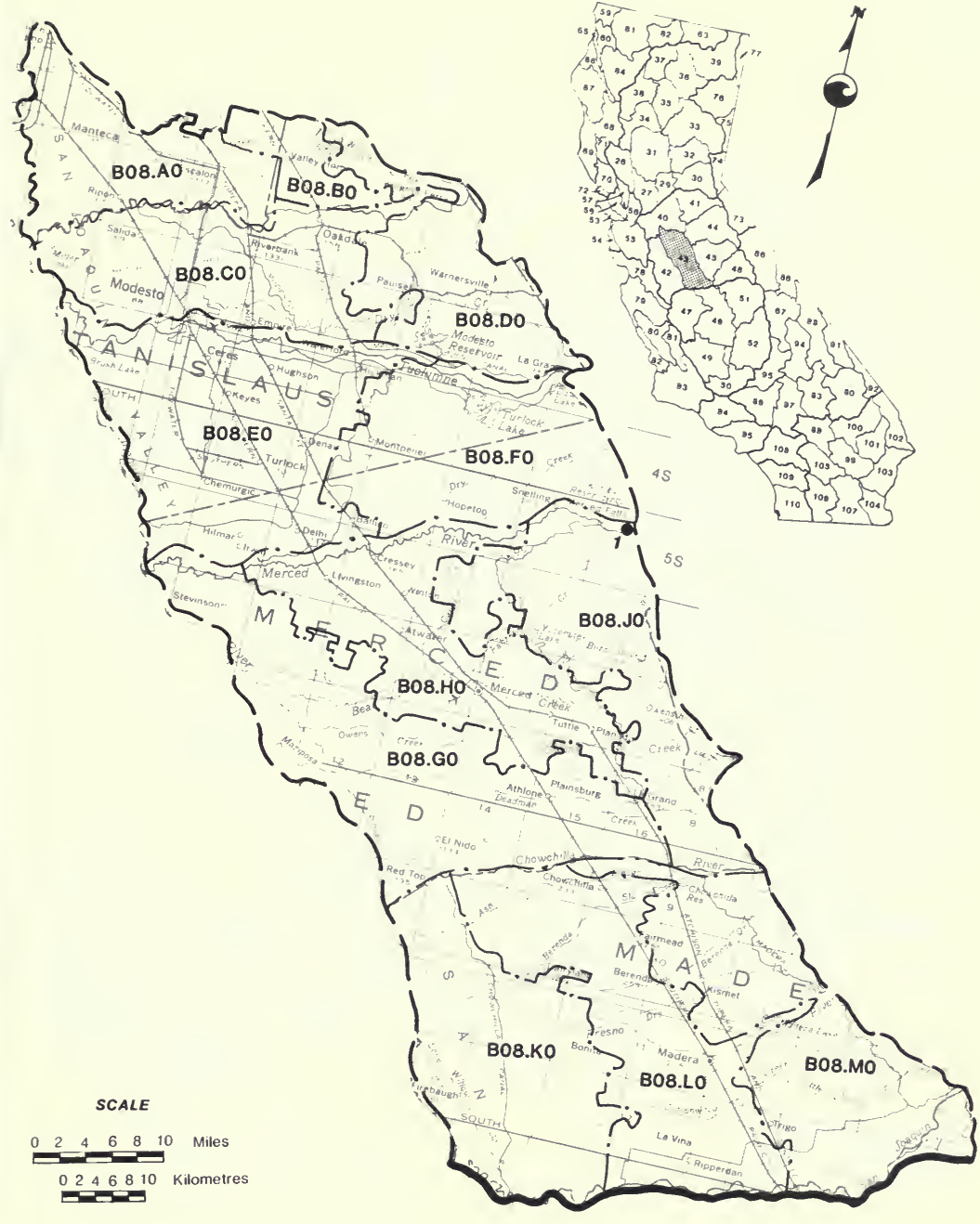

B08 


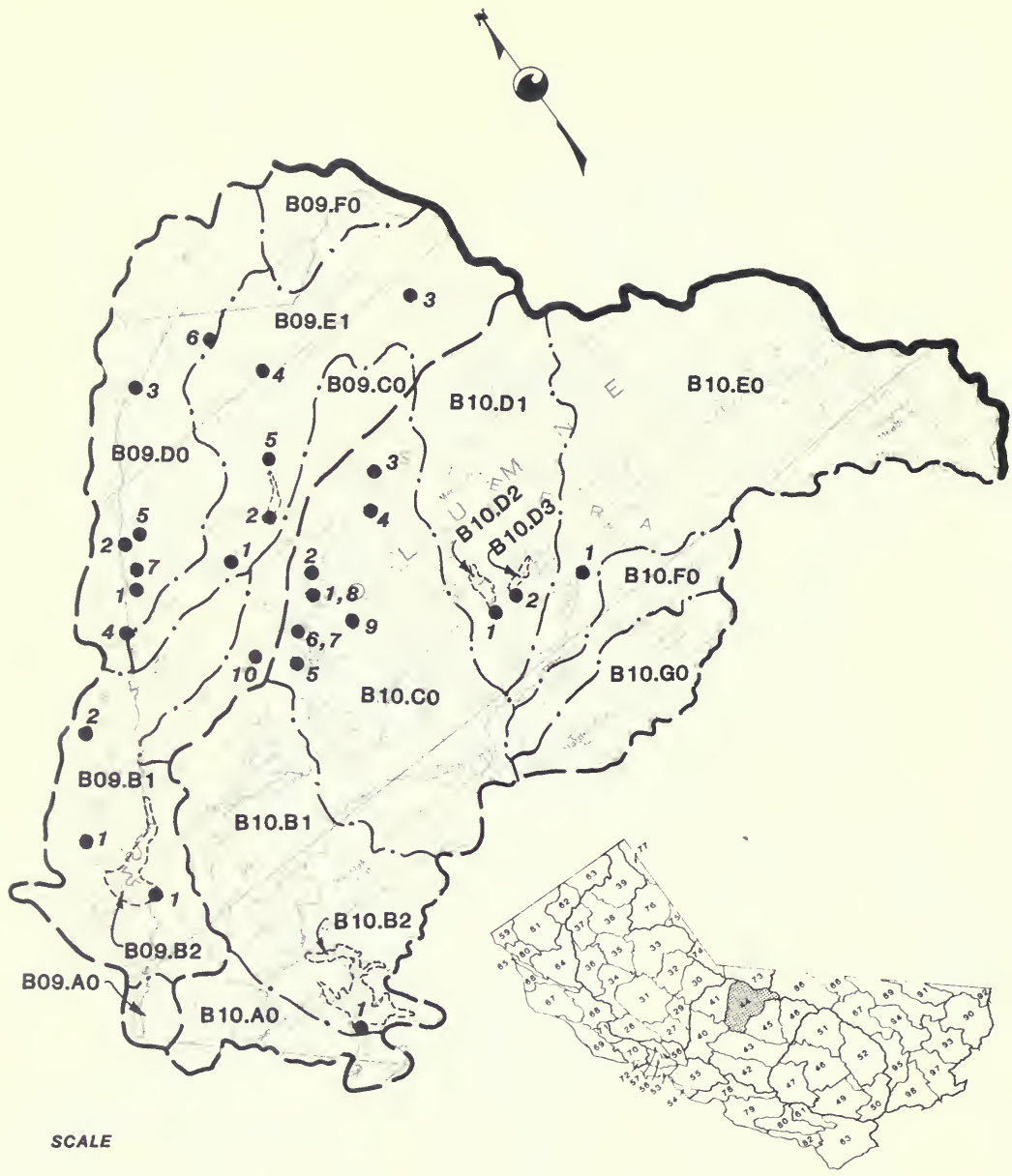

$\begin{array}{lllllll}0 & 2 & 4 & 6 & 8 & 10 & \text { Miles }\end{array}$

0246810 Kilometres

B09 \& B10 


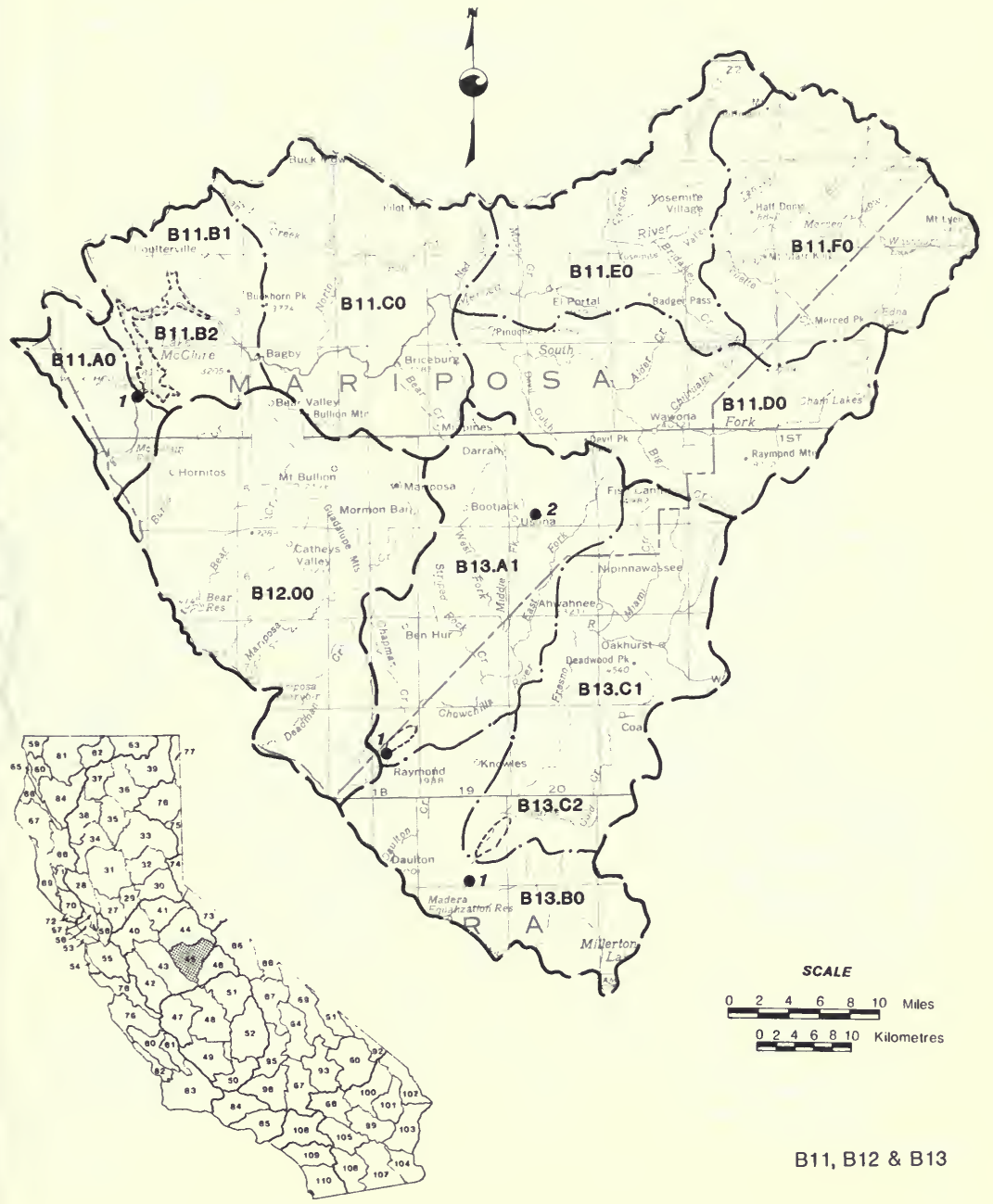




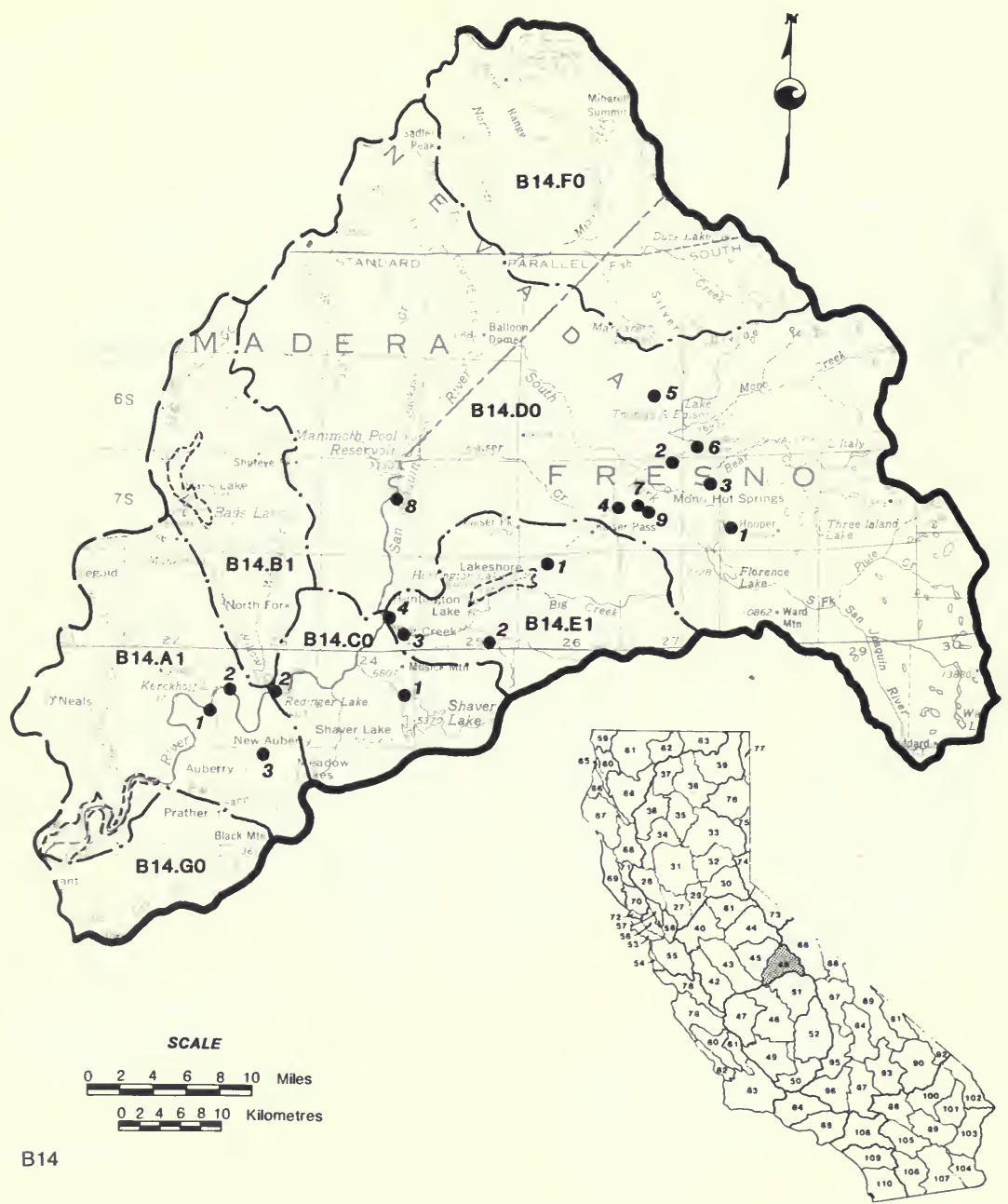




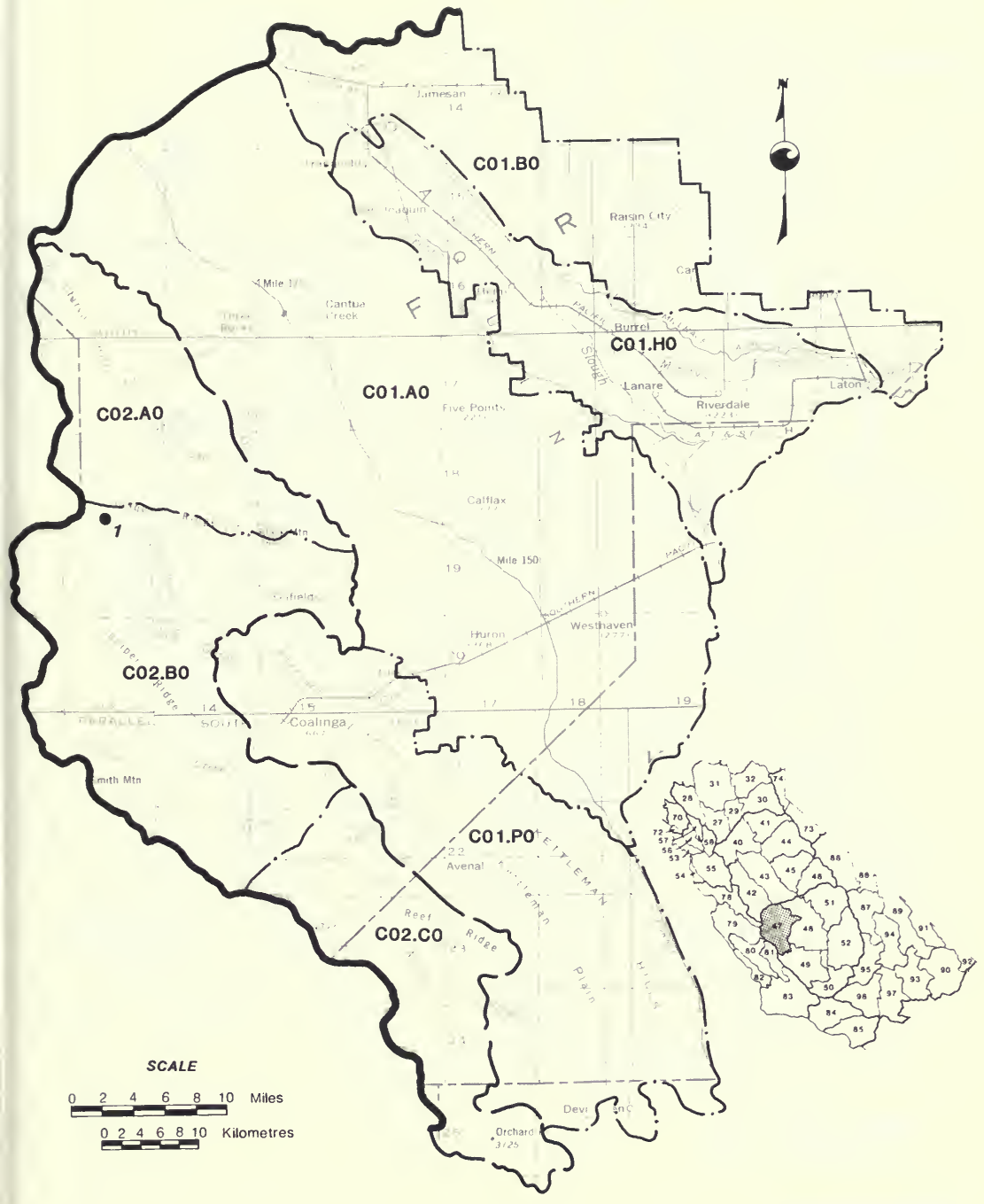

C01.A, B, H, P \& CO2 


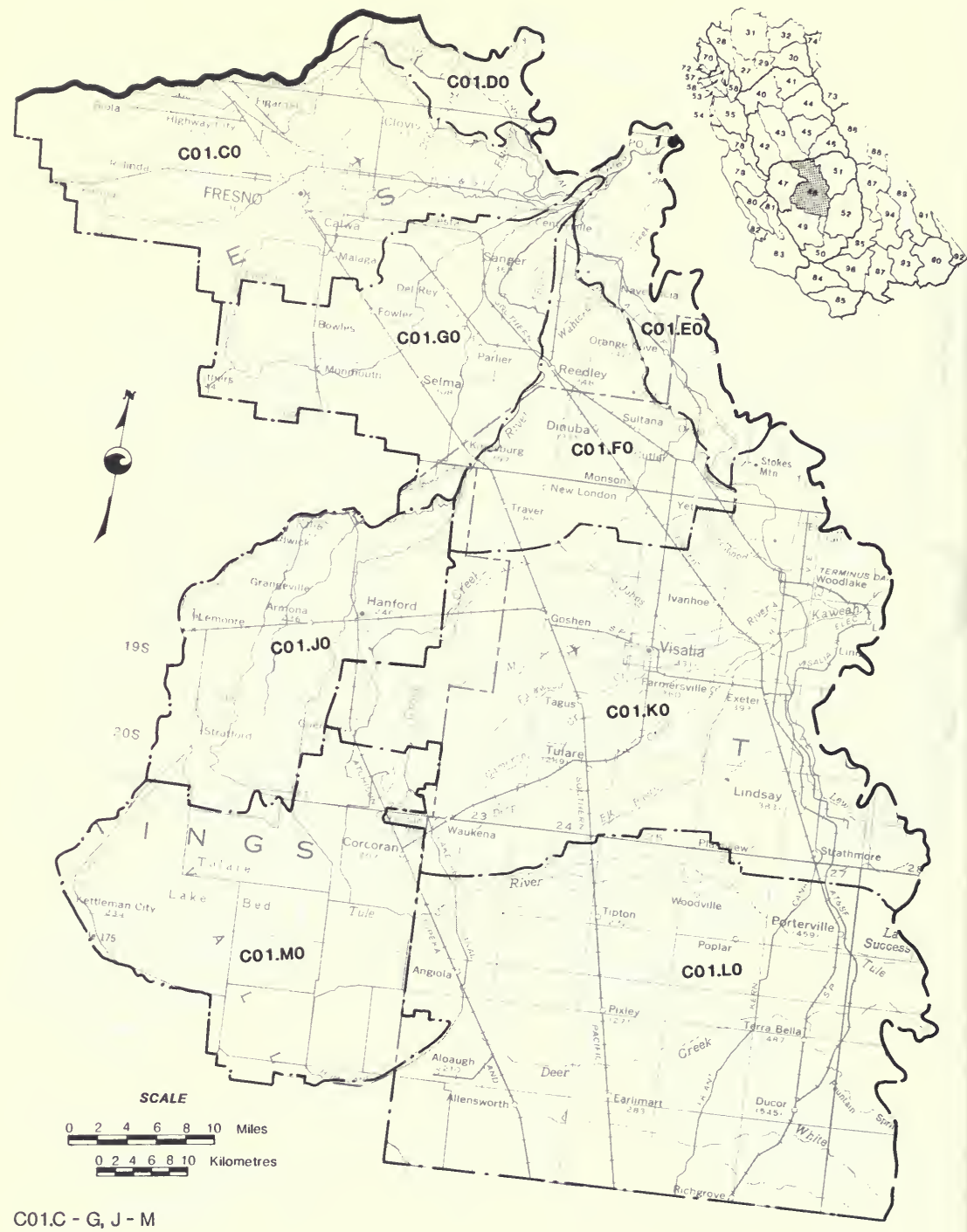




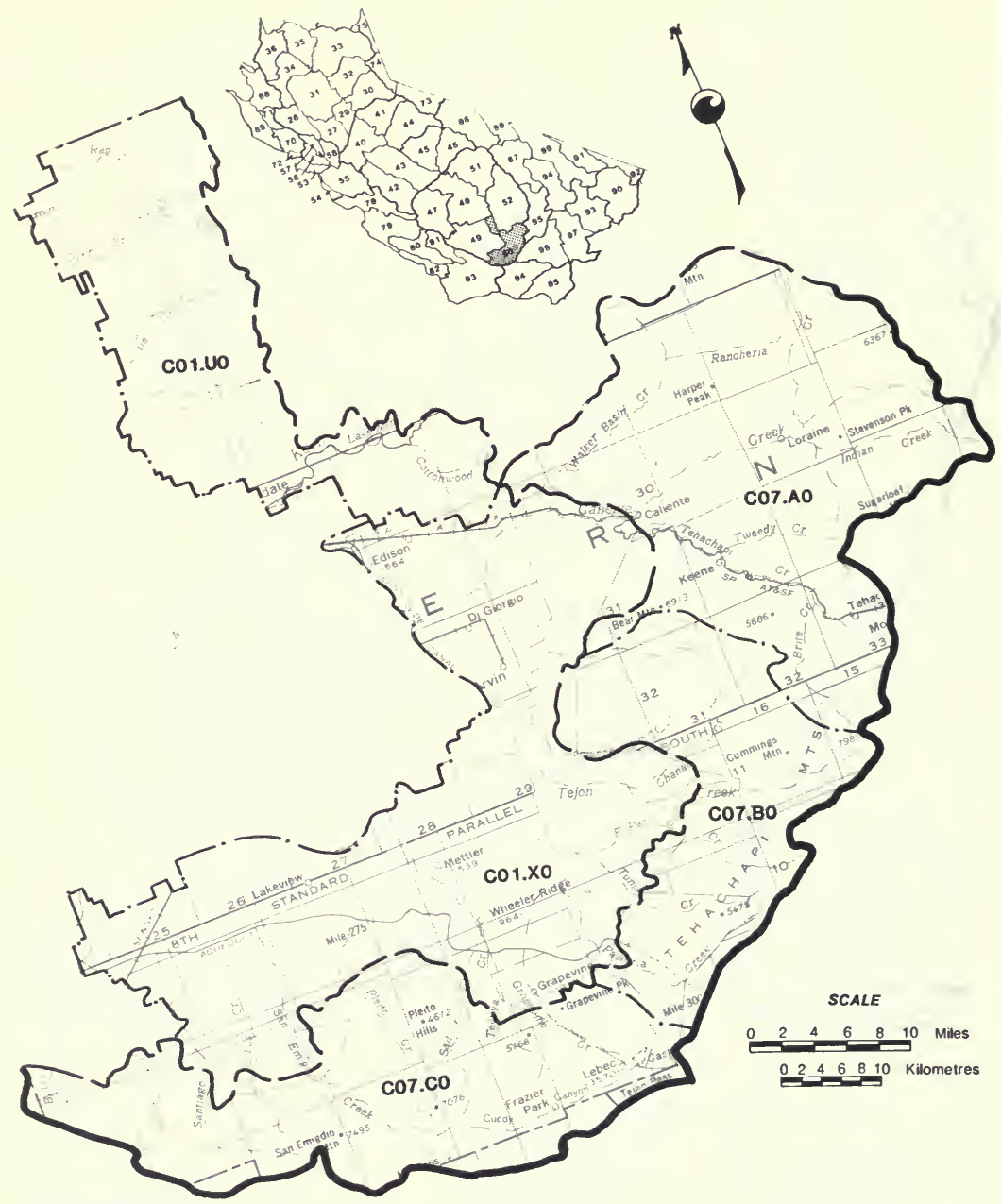

C01.U, X \& C07 


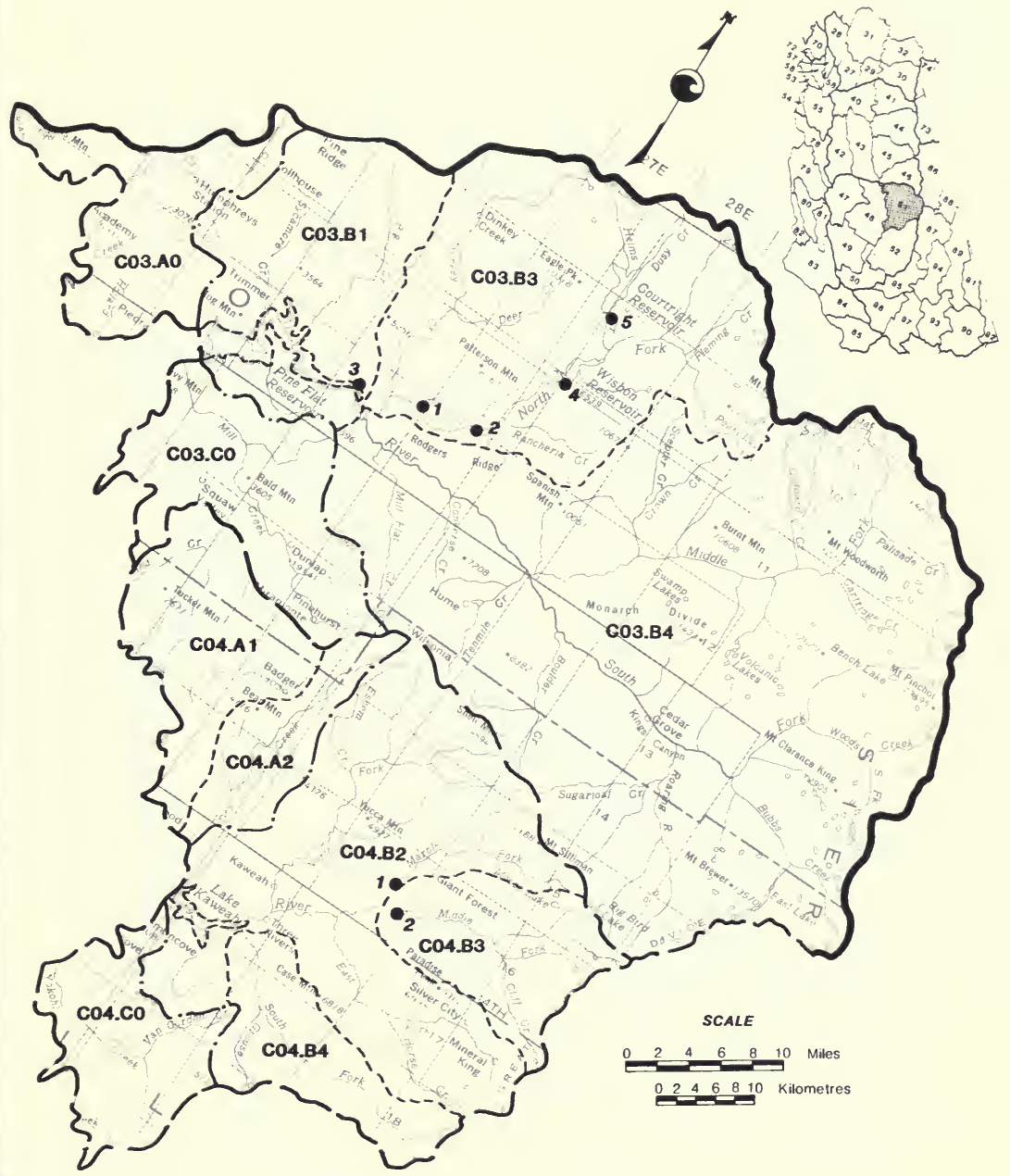

CO3 \& CO4 


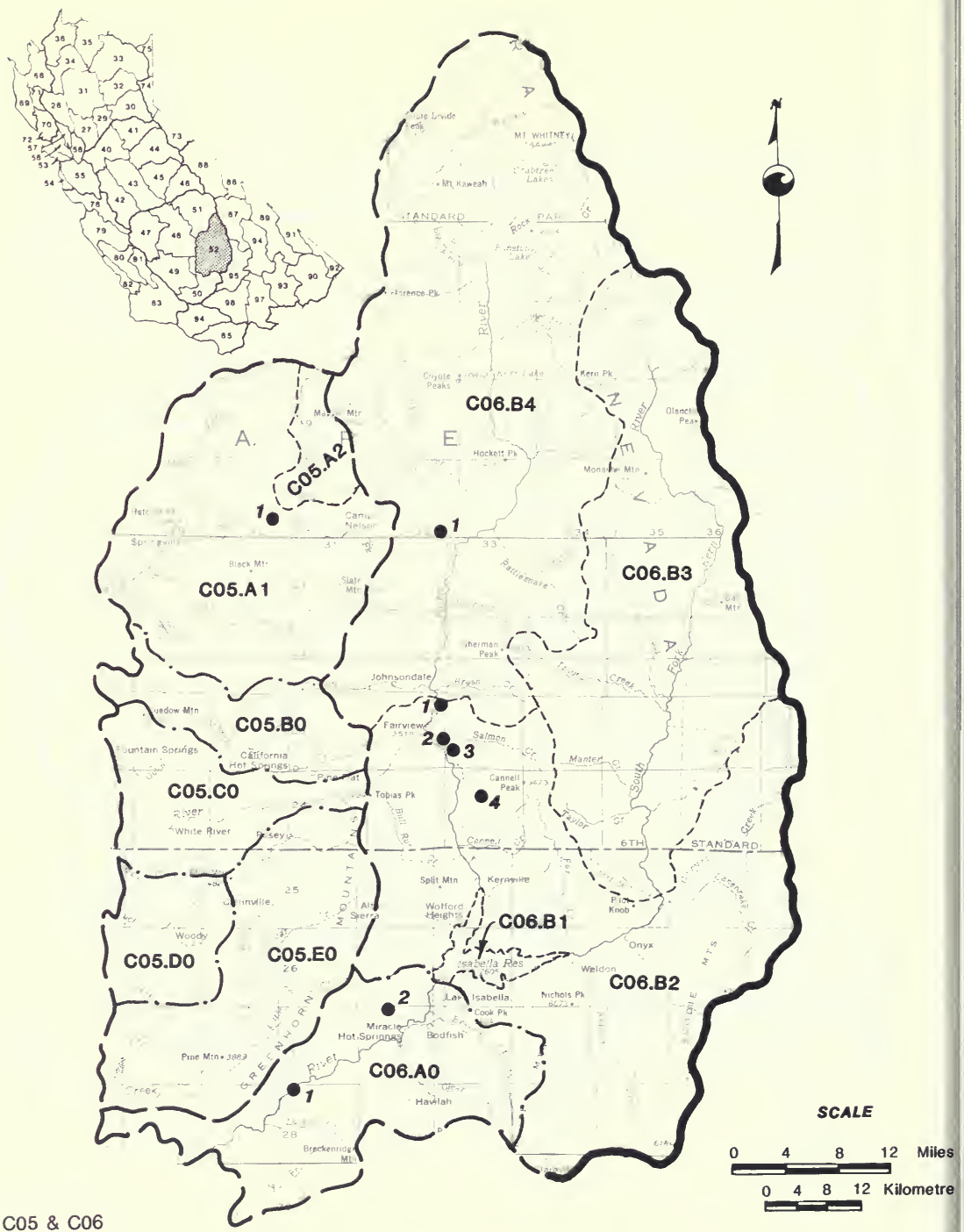




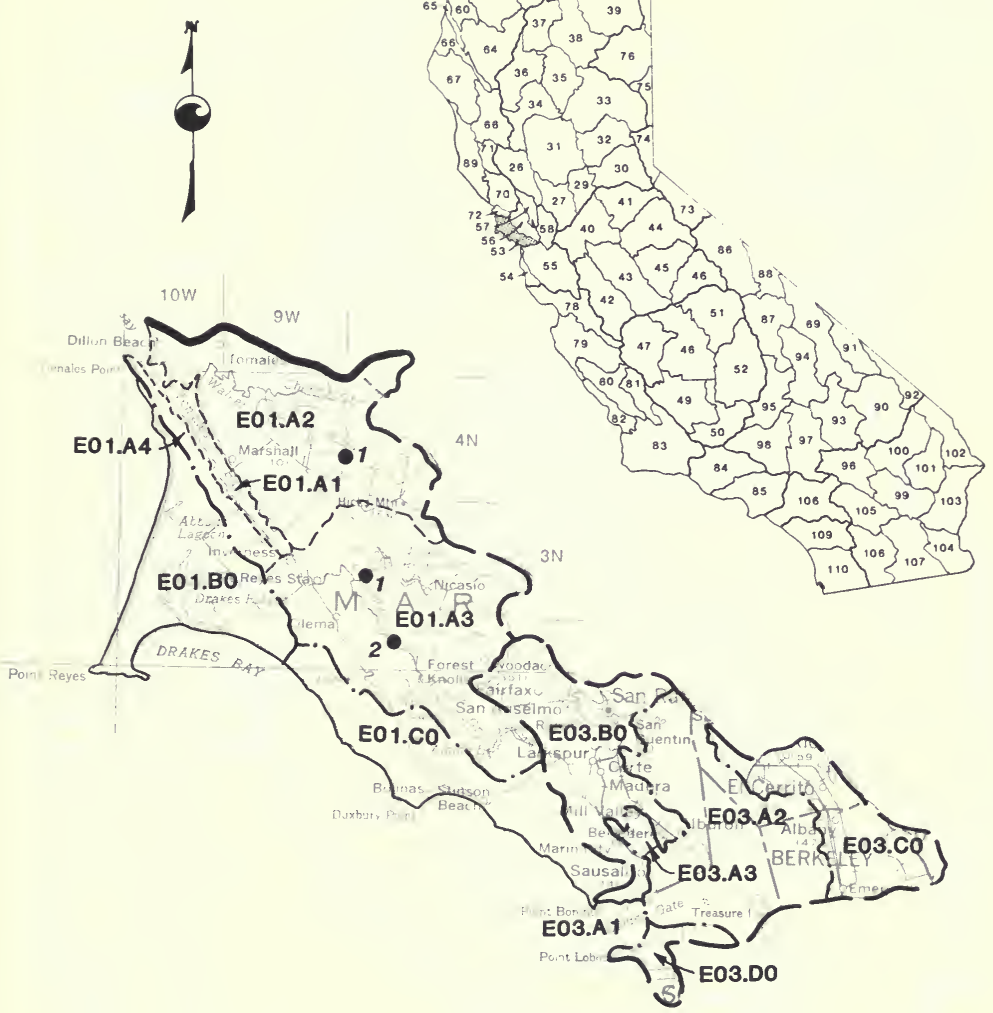

SCALE

$\begin{array}{lllll}2 & 4 & 6 & 8 & 10\end{array}$ Miles 


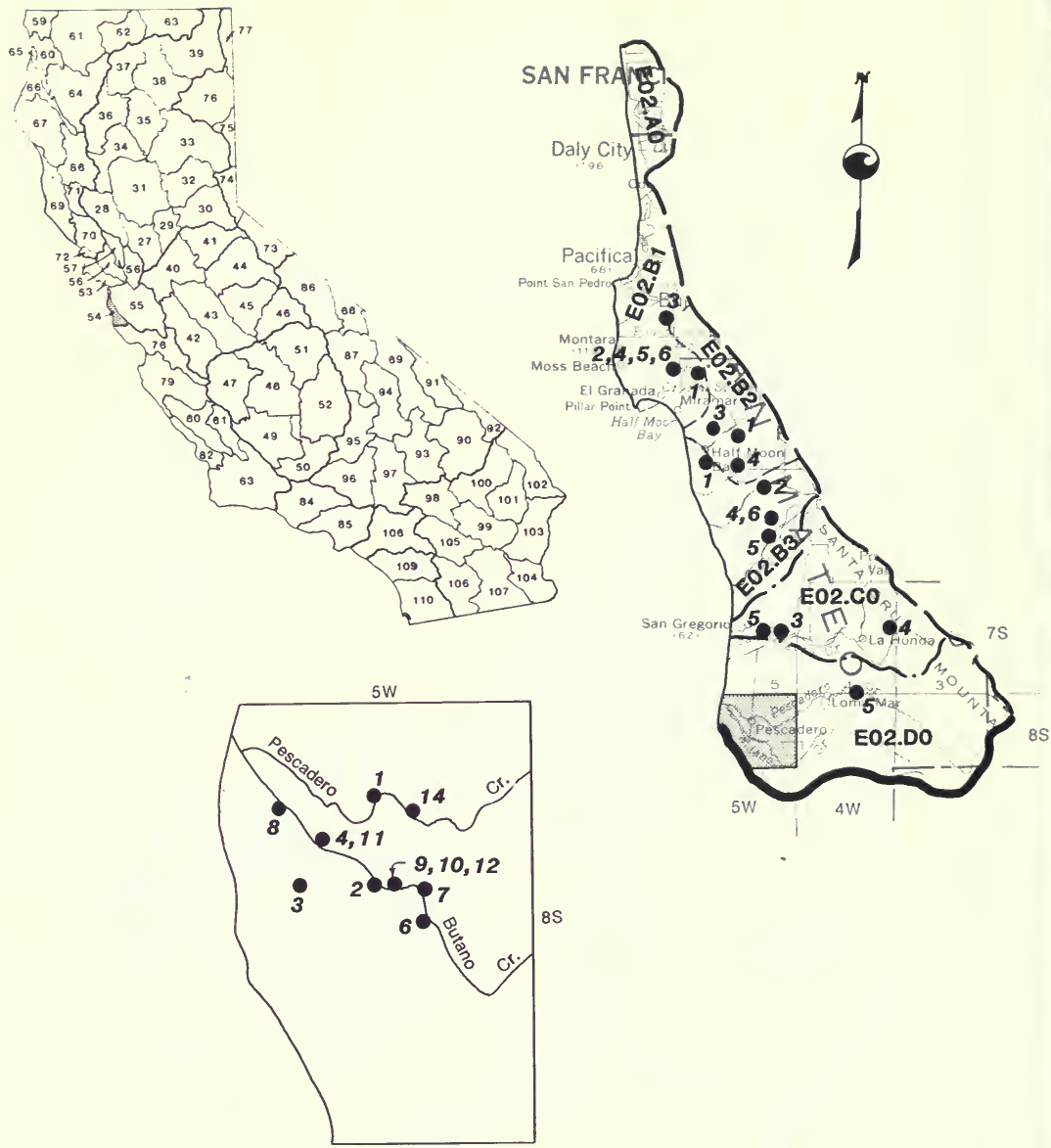

SCALE

\begin{tabular}{lllllll}
0 & 2 & 4 & 6 & 8 & 10 & Miles \\
\hline
\end{tabular}

$\begin{array}{lllllll}0 & 2 & 4 & 6 & 8 & 10 & \text { Kilometres }\end{array}$ 


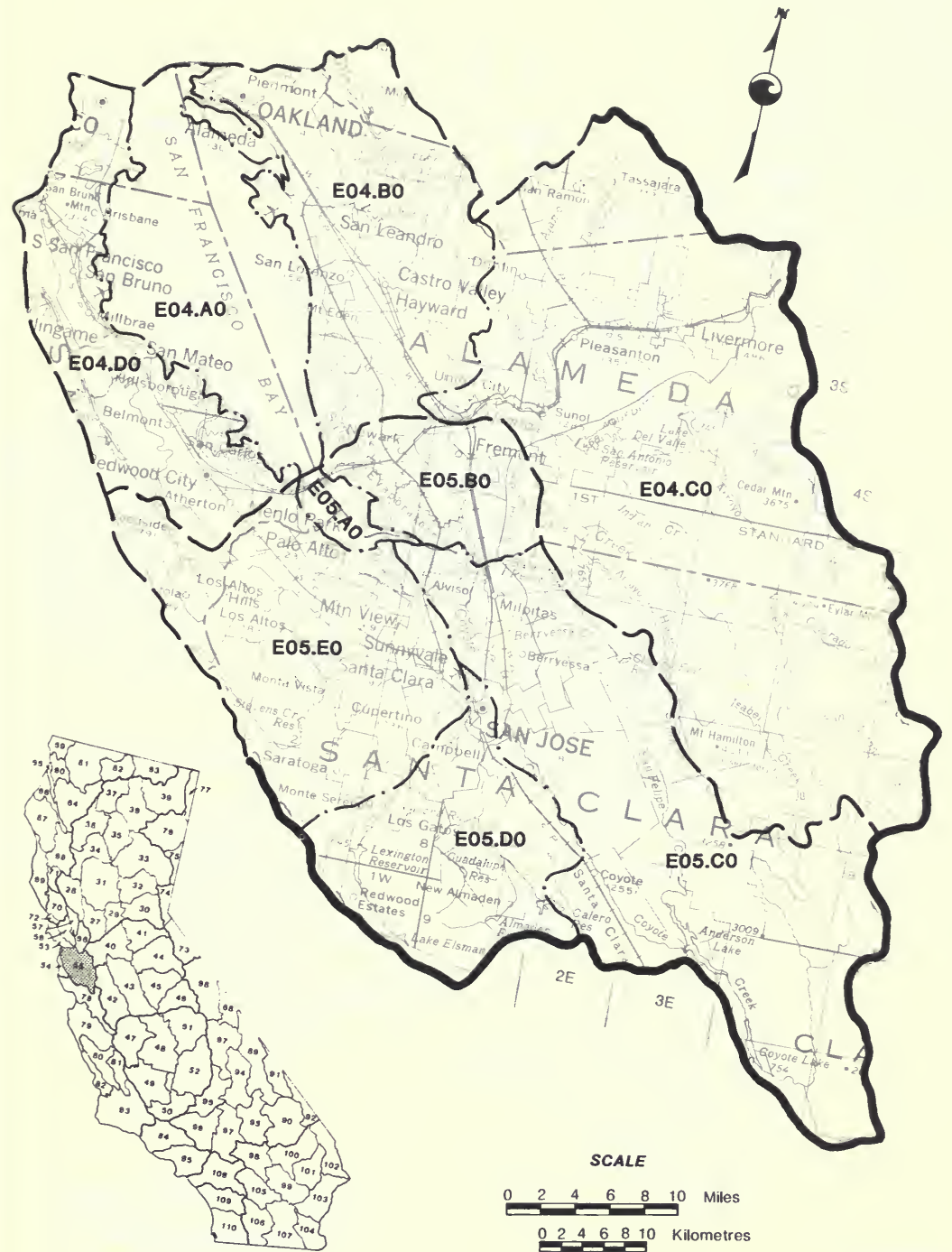

E04 \& E05 


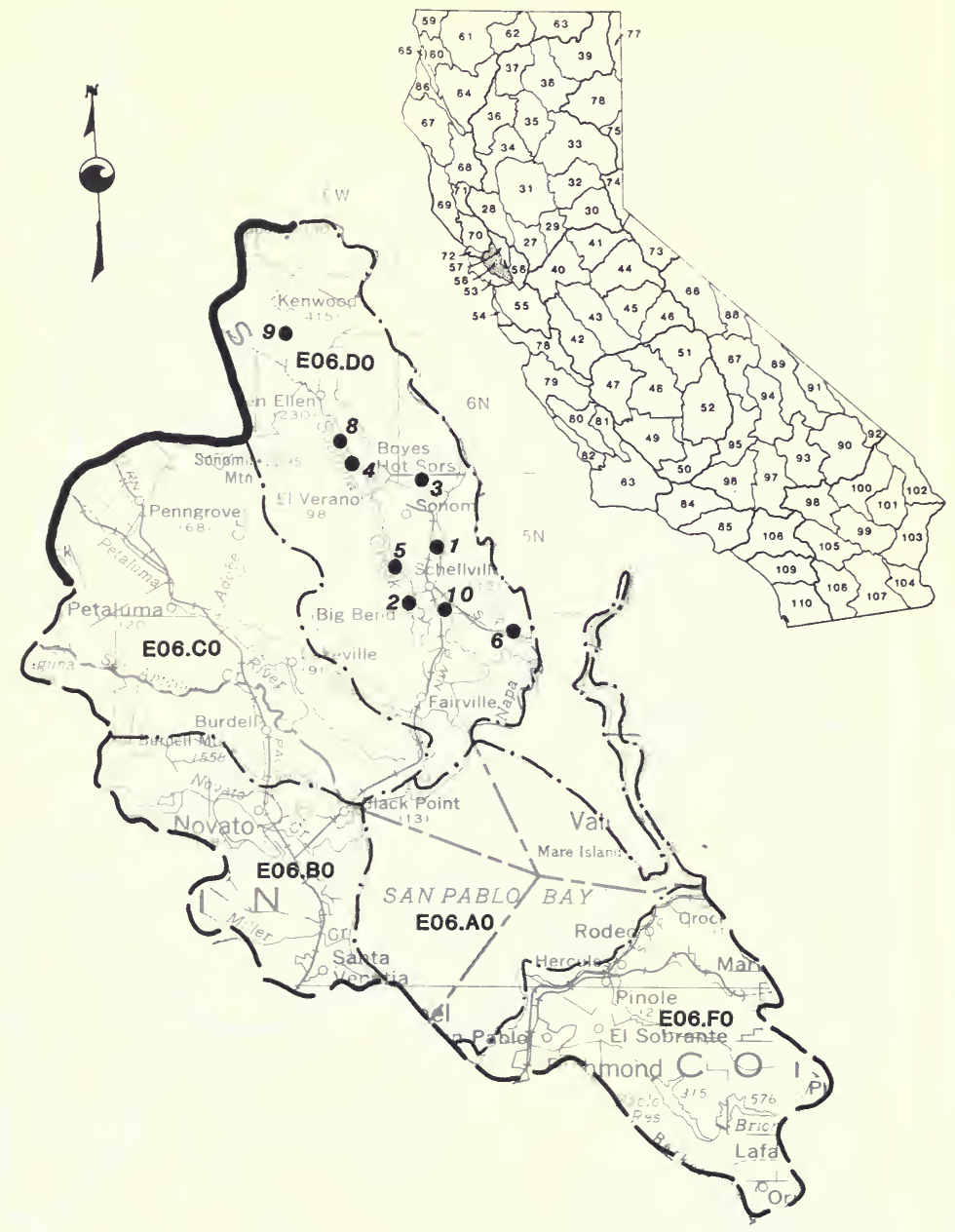

SCALE

E06.A - D \& F

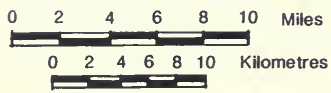




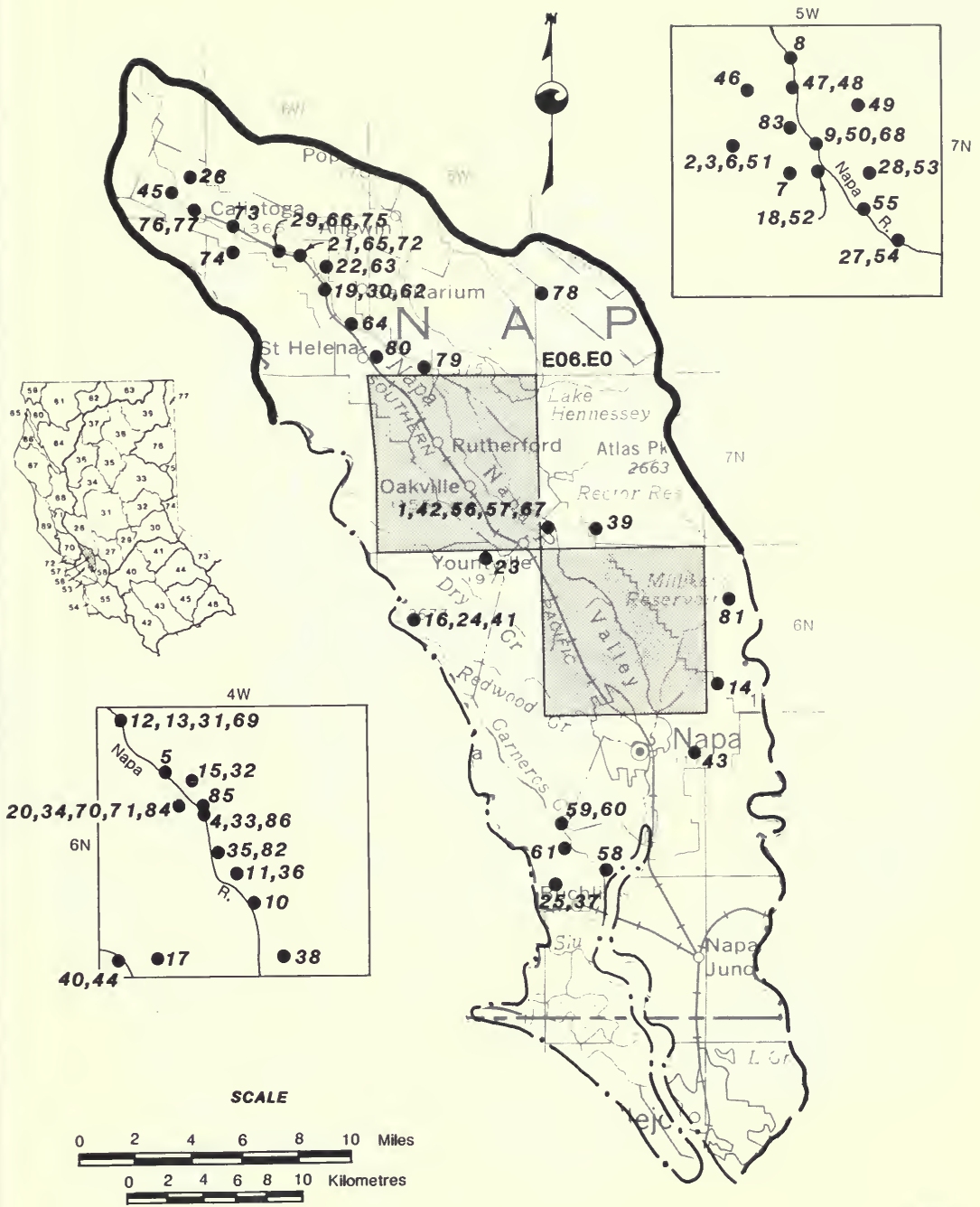



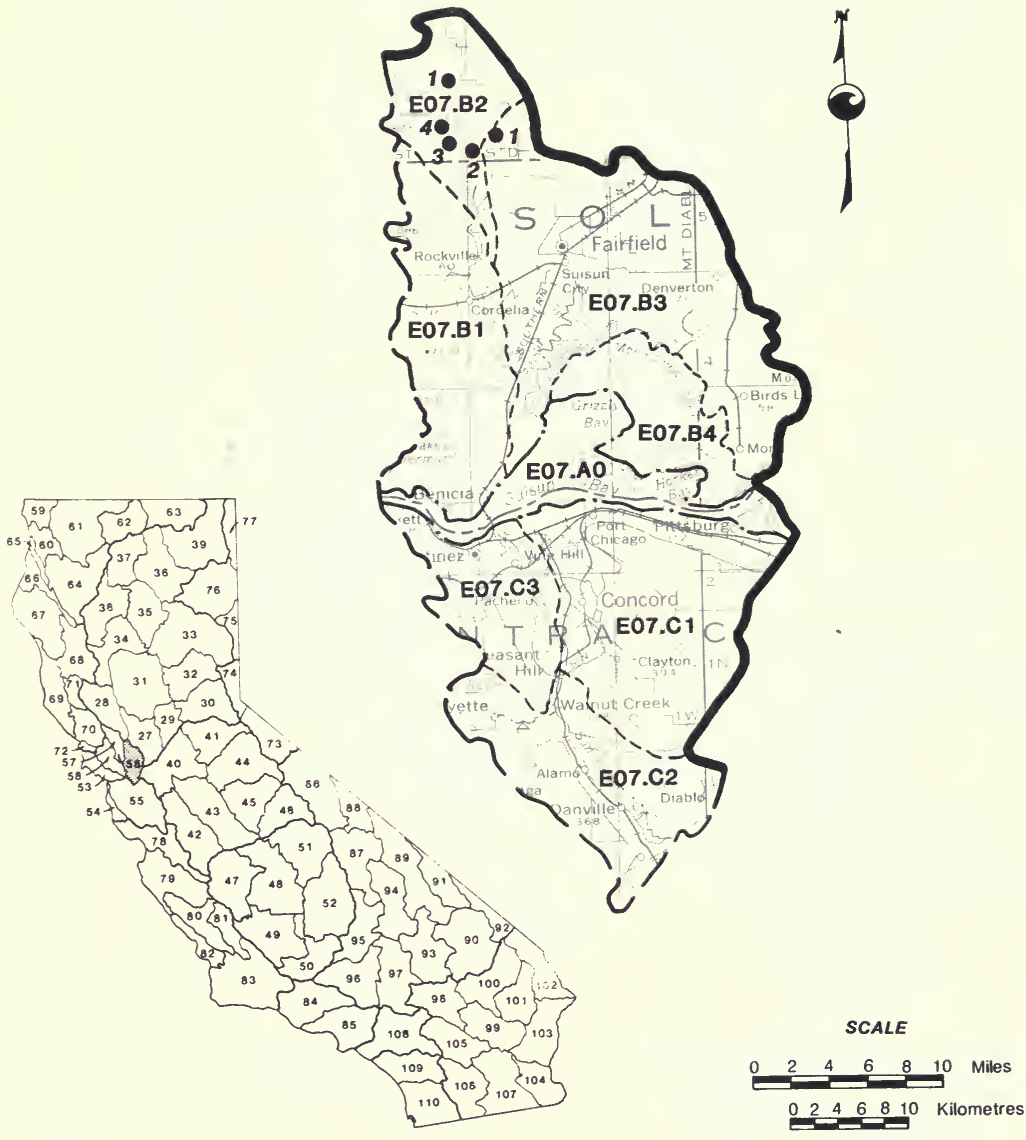

E07 


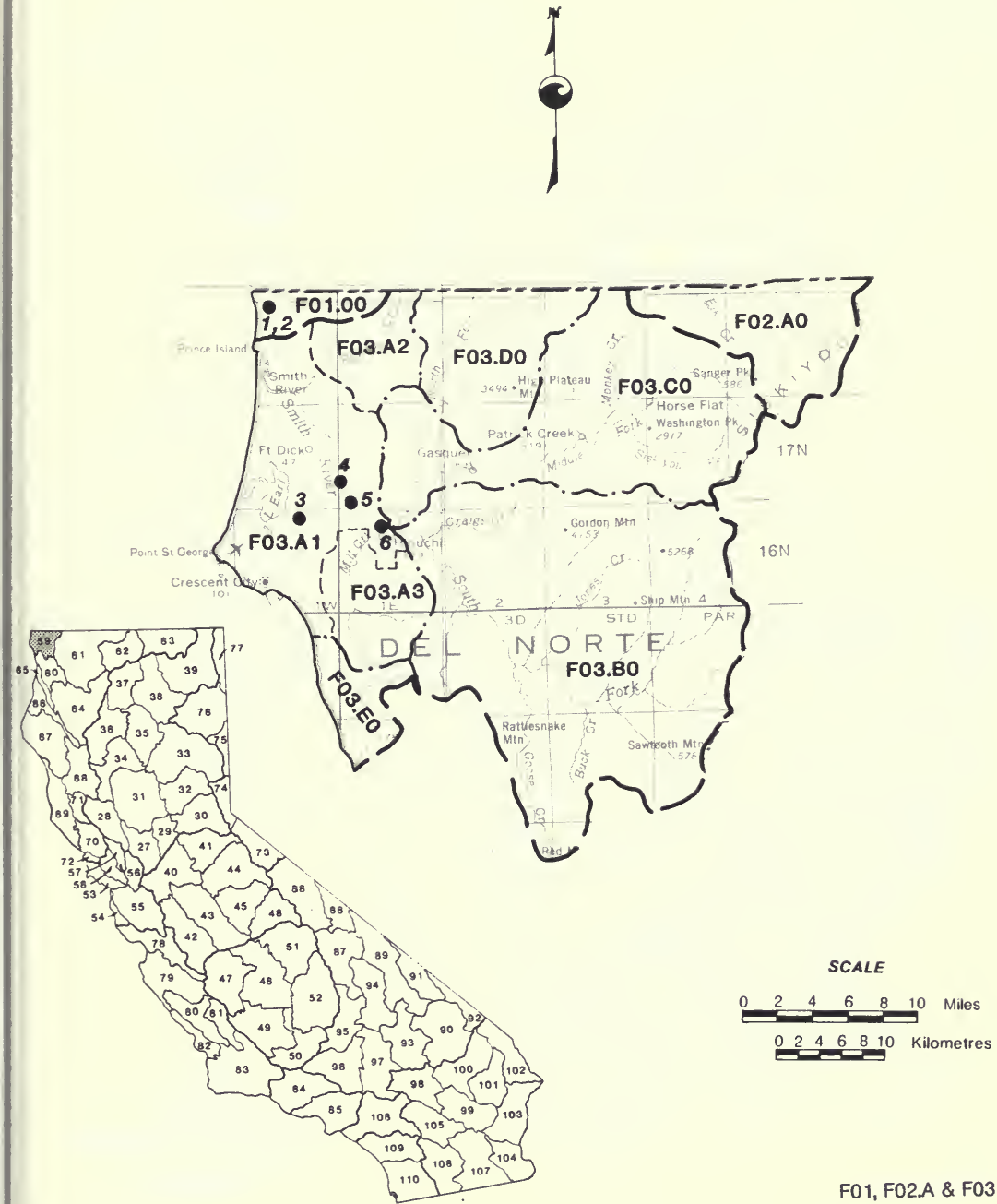




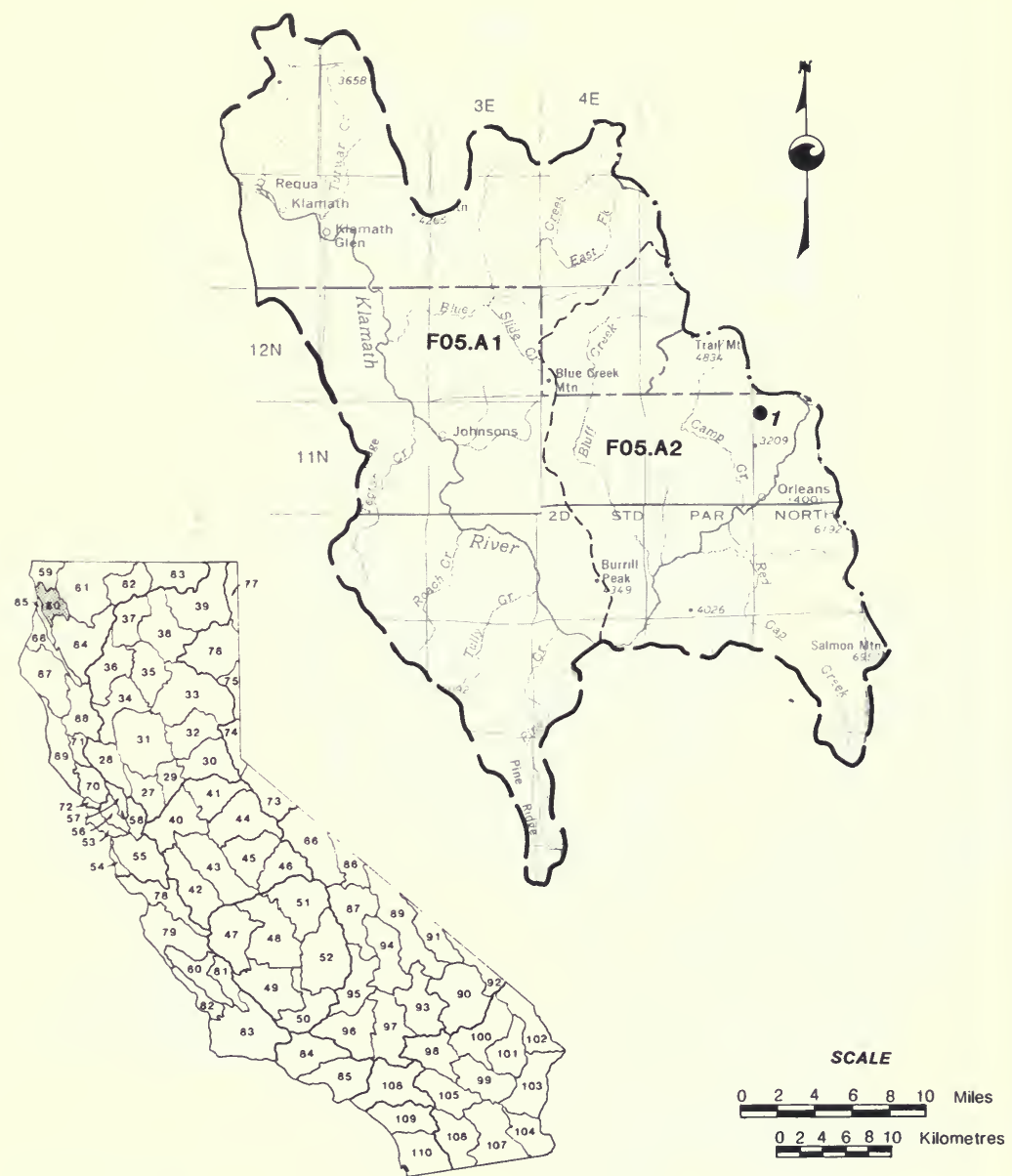

F05.A 


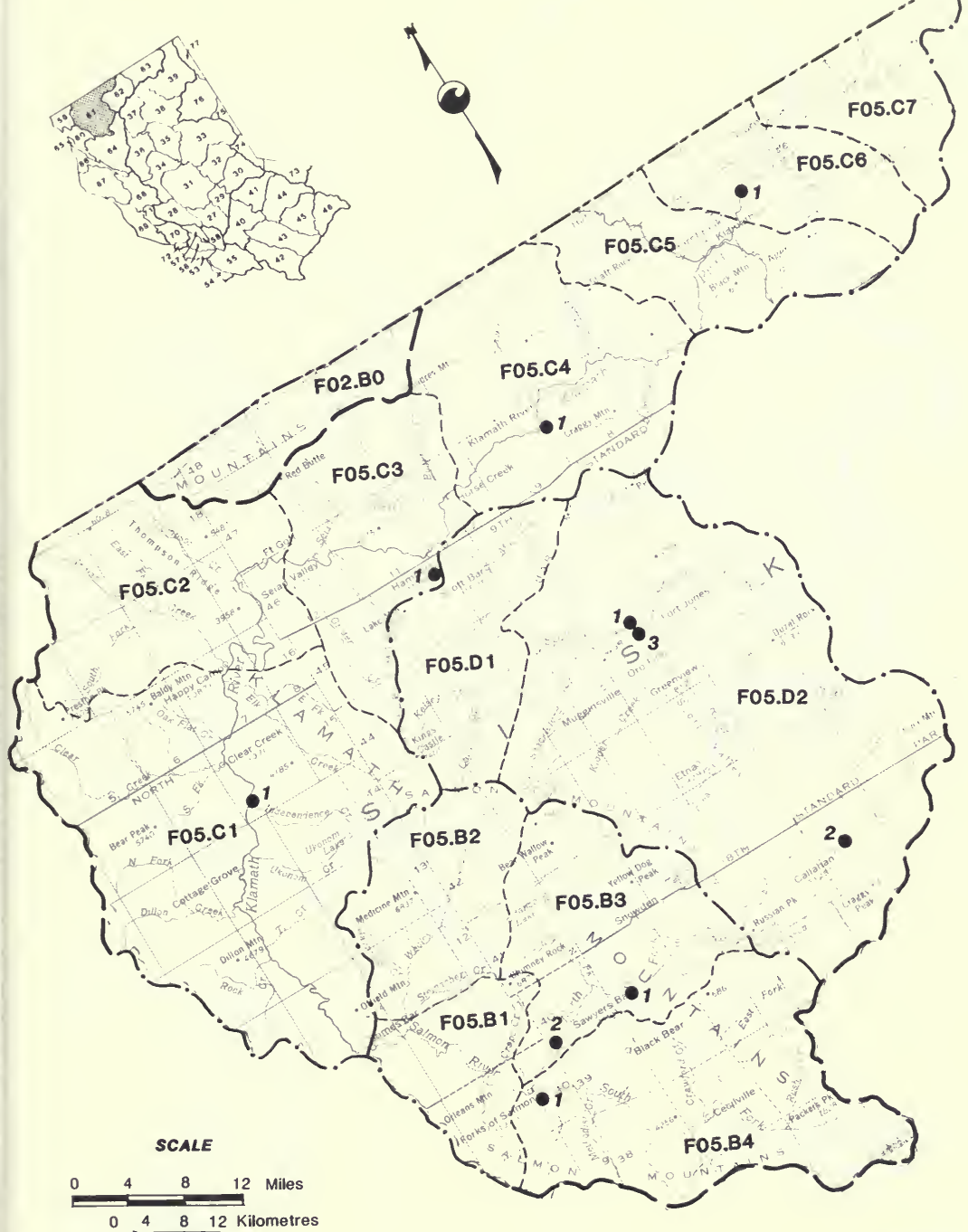

F02.B, F05.B, C \& D 


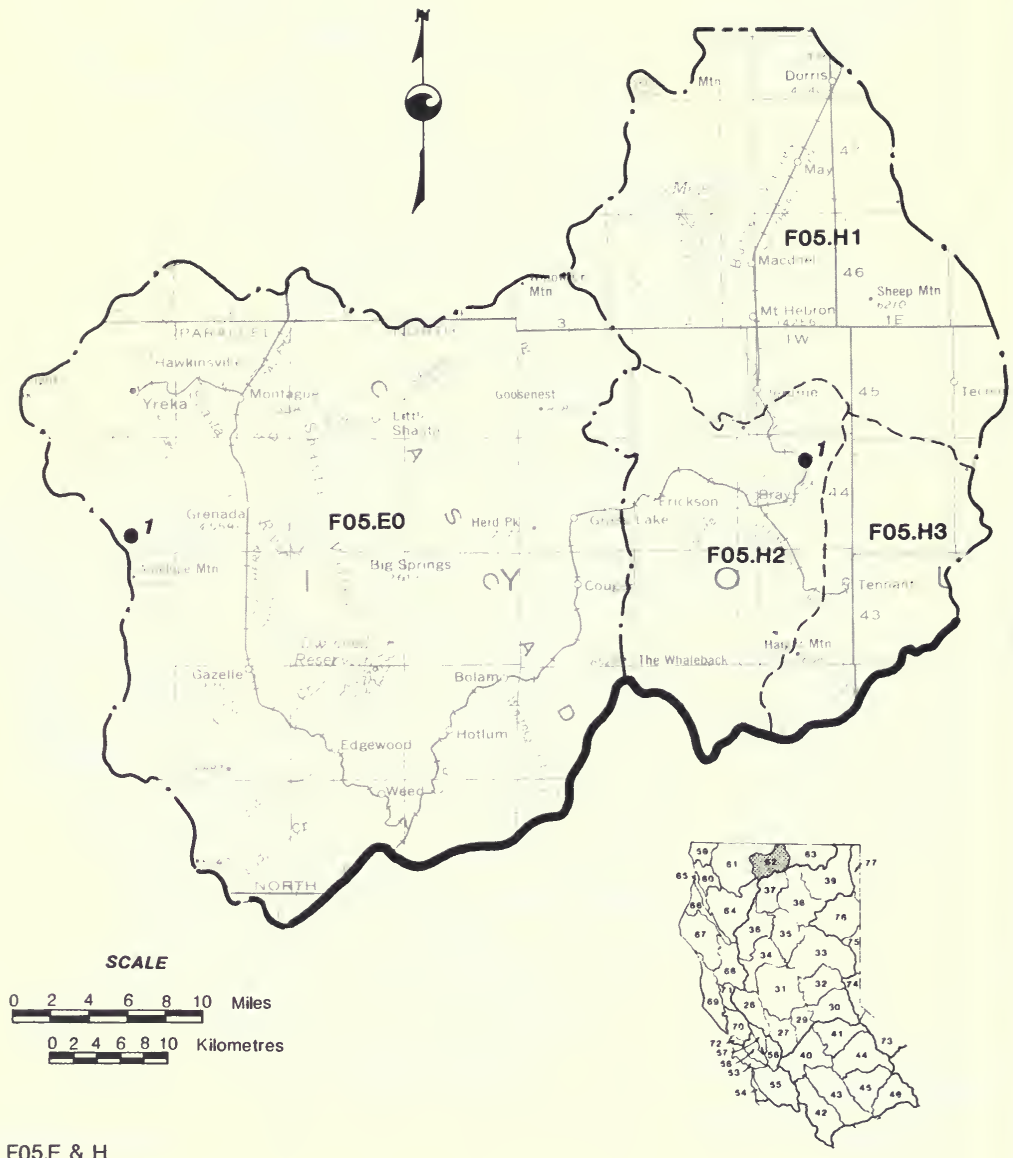

F05.E \& H 


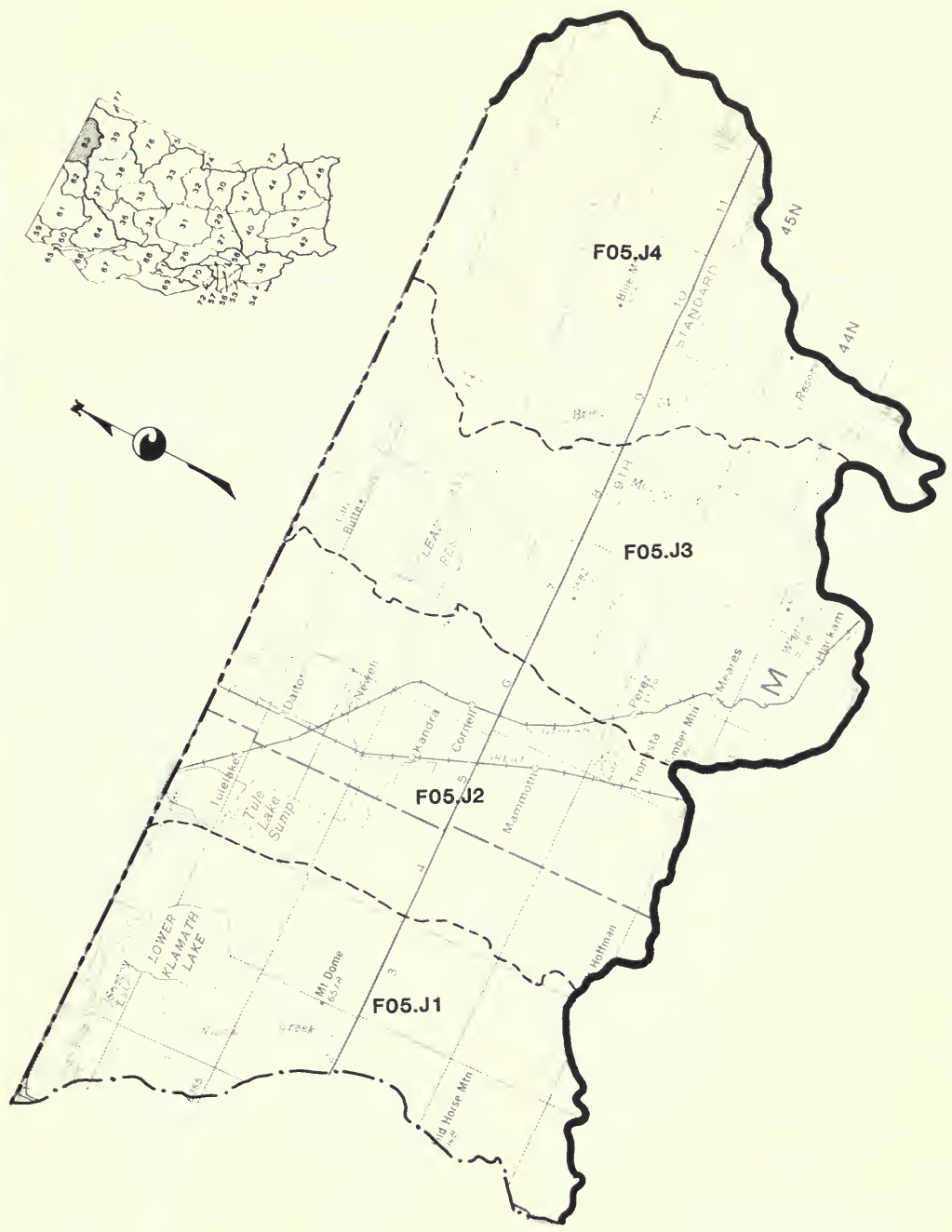

SCALE

\begin{tabular}{llllll}
0 & 2 & 4 & 6 & 8 & 10 \\
\hline
\end{tabular}

F05.J

$\begin{array}{lllllll}0 & 2 & 4 & 6 & 8 & 10 & \text { Kilometres }\end{array}$ 


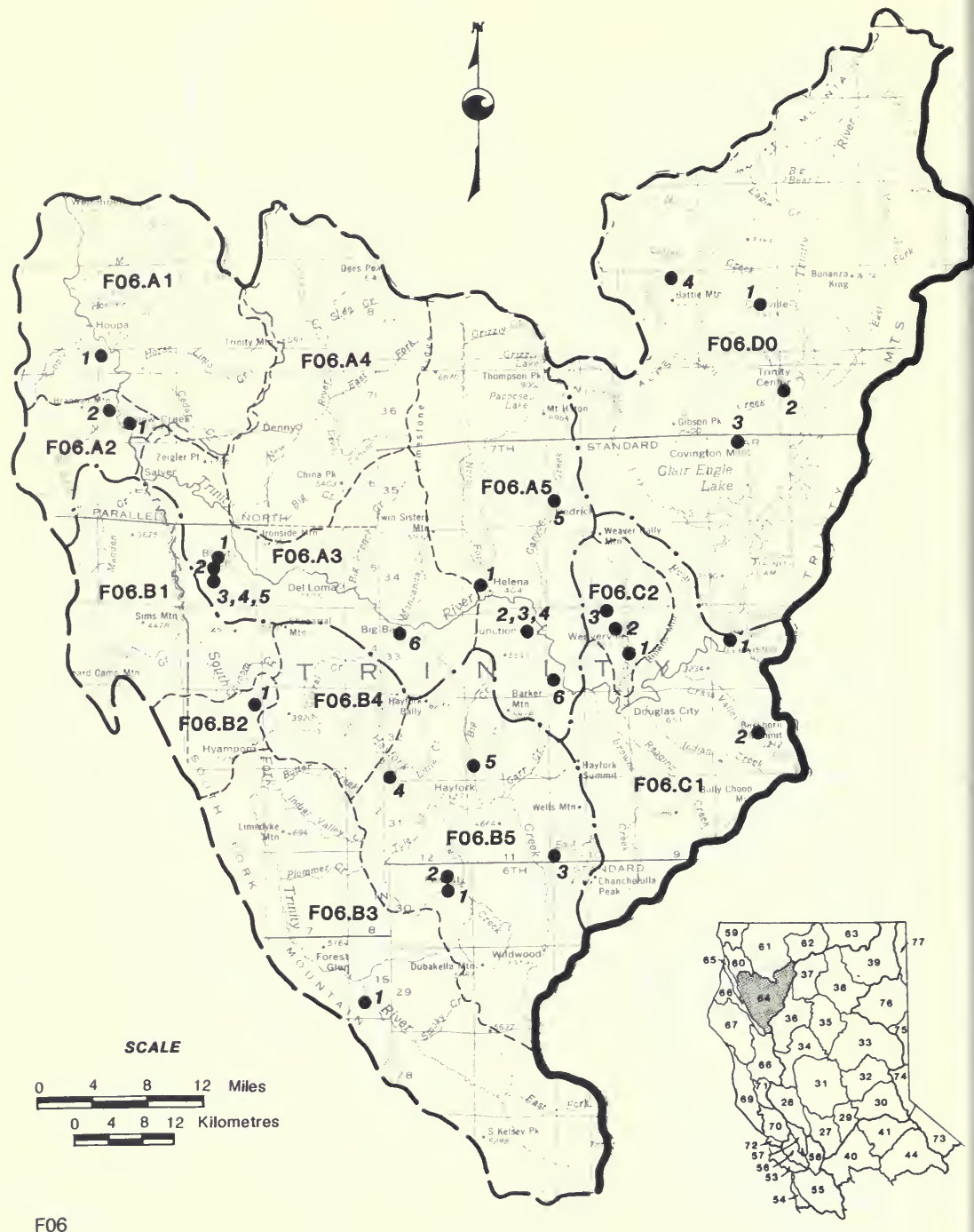




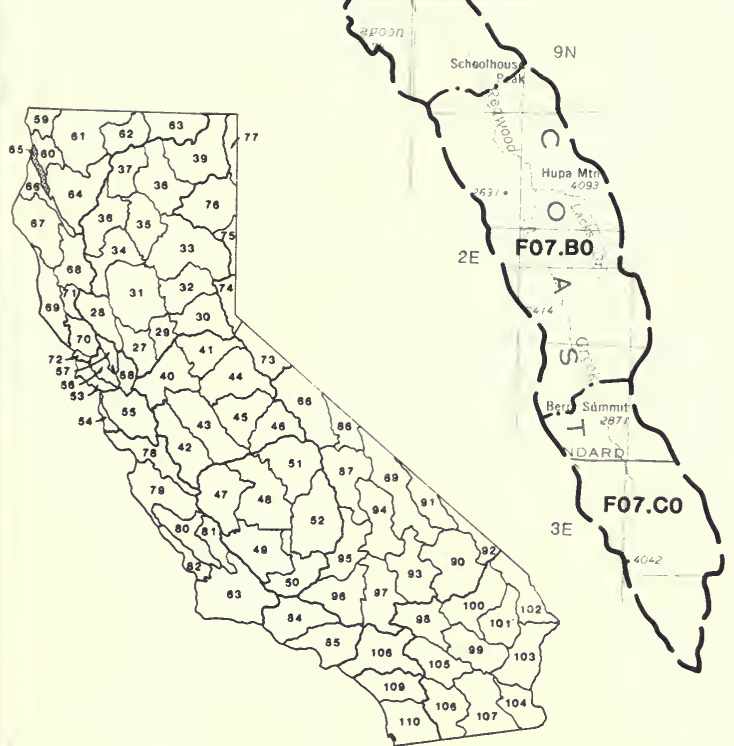

SCALE

\begin{tabular}{lllllll}
0 & 2 & 4 & 6 & 8 & 10 & Miles \\
\hline
\end{tabular}

$024 \quad 6 \quad 8 \quad 10$ Kilometres
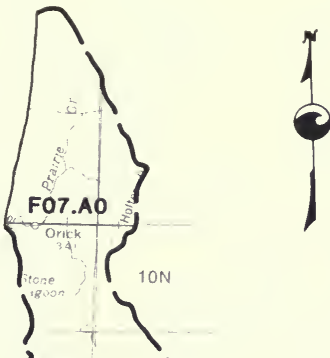


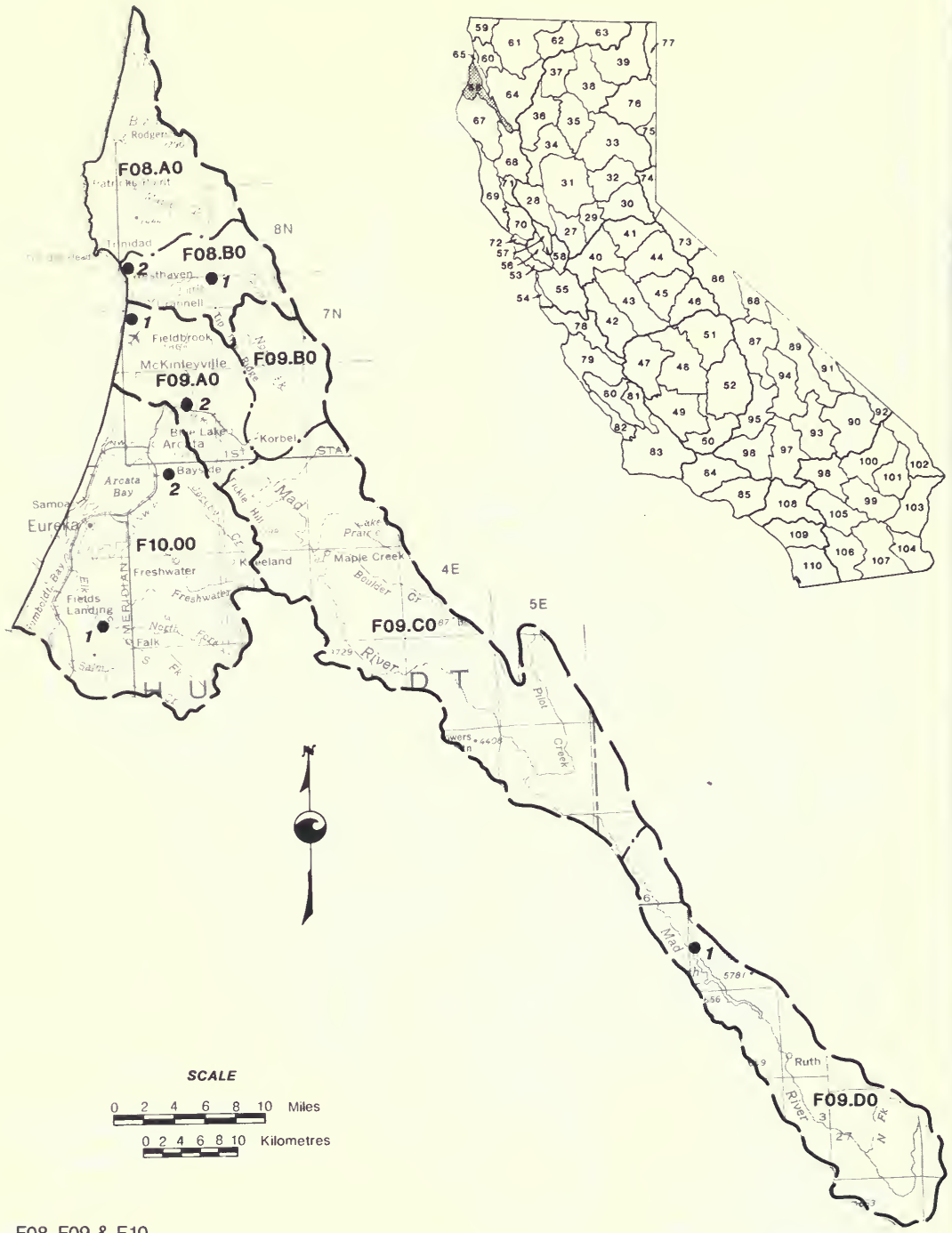

$F 08, F 09 \& F 10$ 


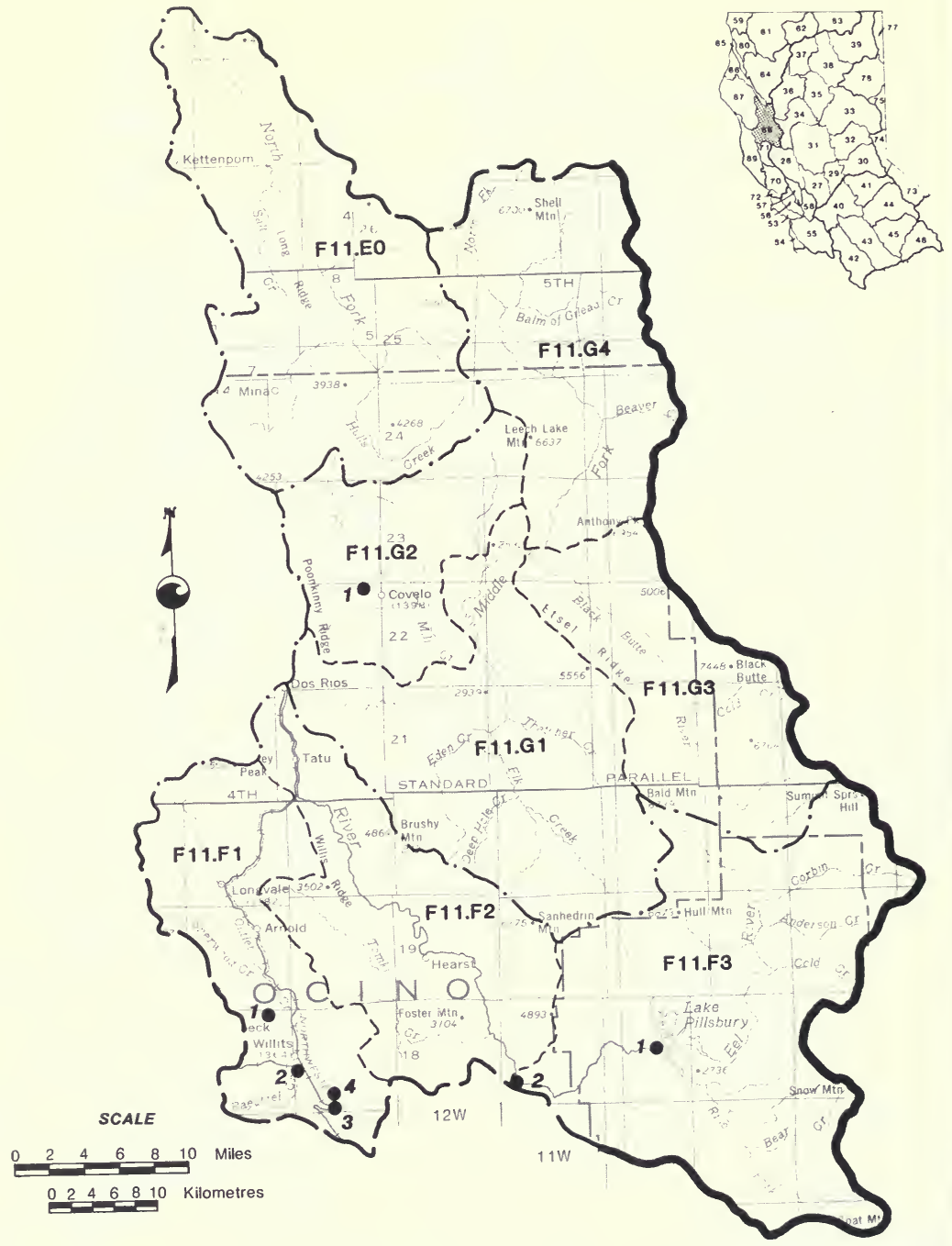

F11.E, F \& G 


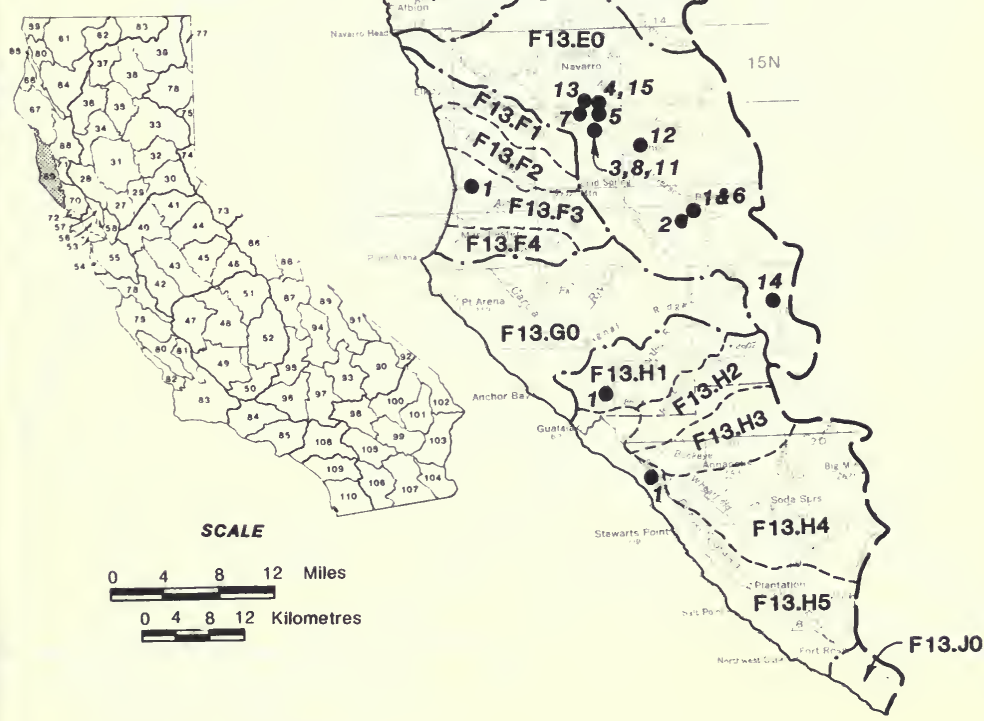




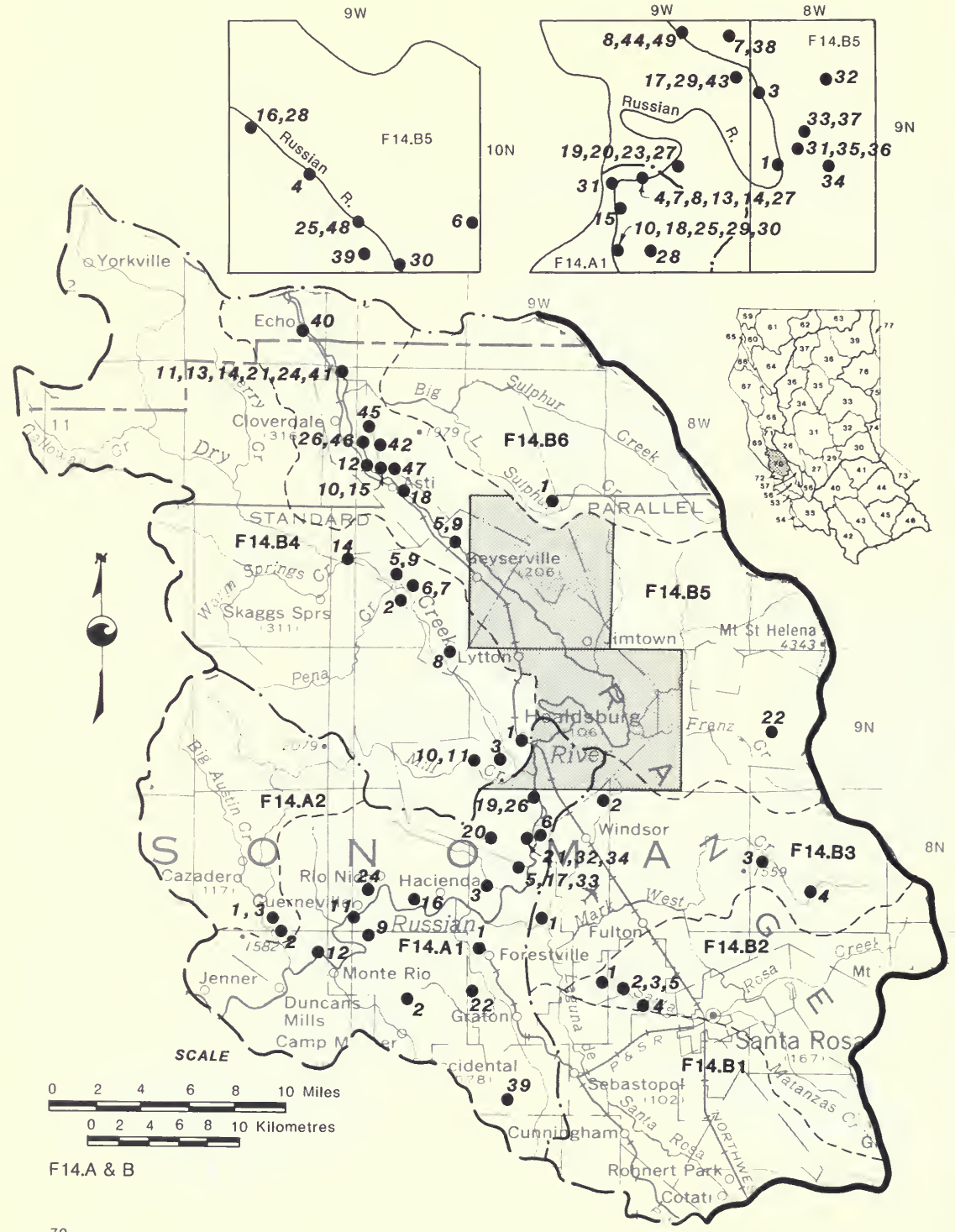




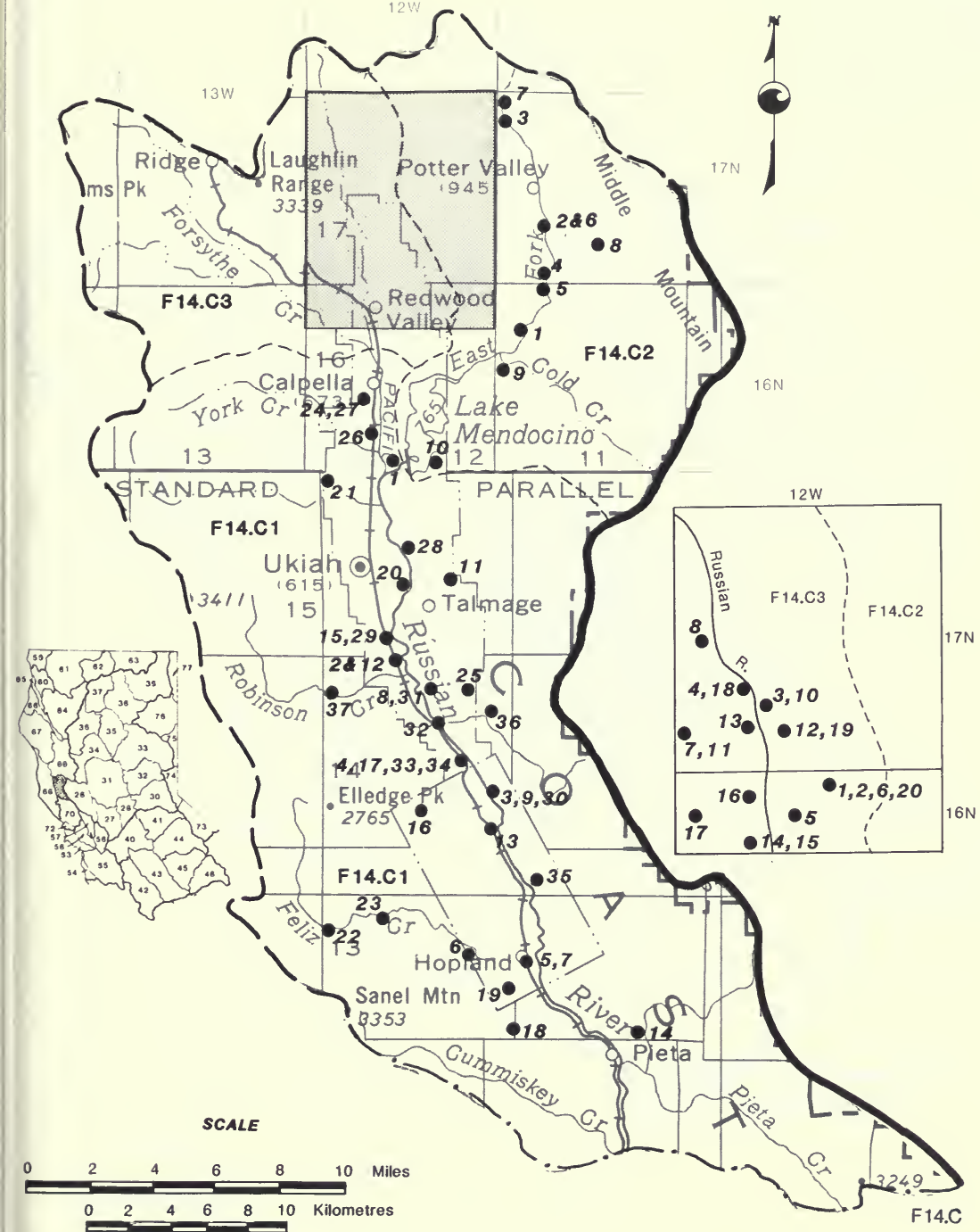




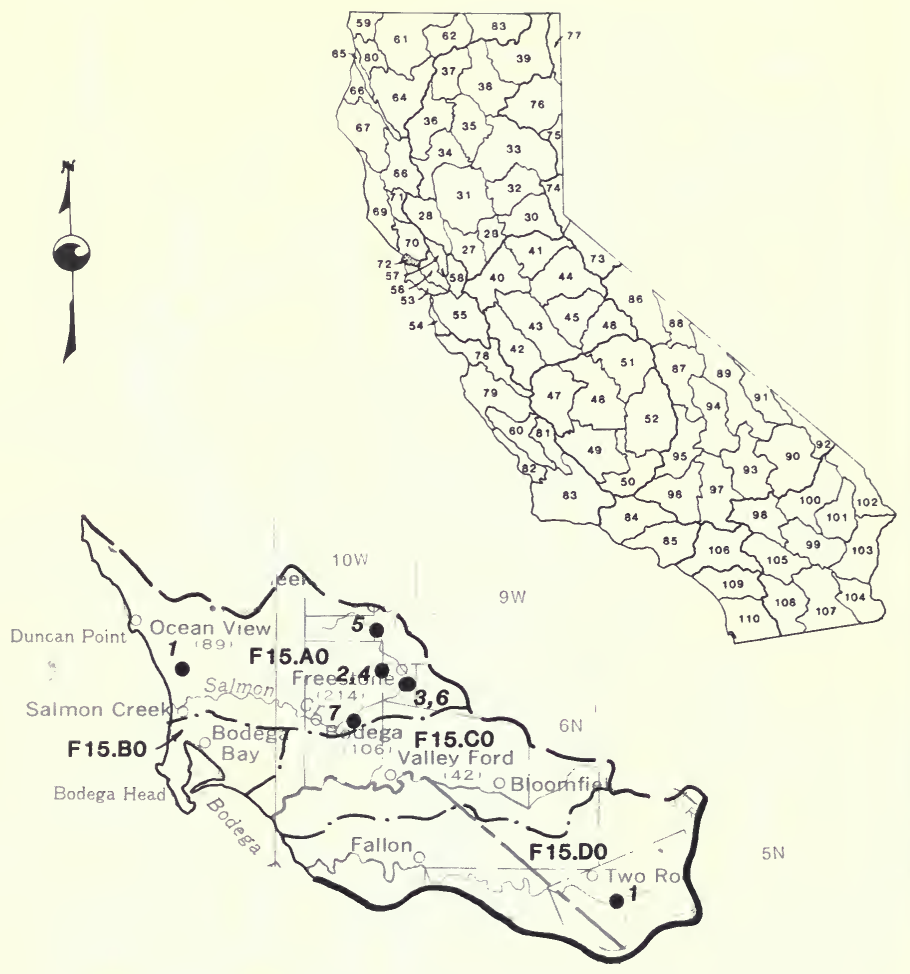

\section{SCALE}

$\begin{array}{lllllll}0 & 2 & 4 & 6 & 8 & 10 & \text { Miles }\end{array}$

$\begin{array}{lllllll}0 & 2 & 4 & 6 & 8 & 10 & \text { Kilometres }\end{array}$ 


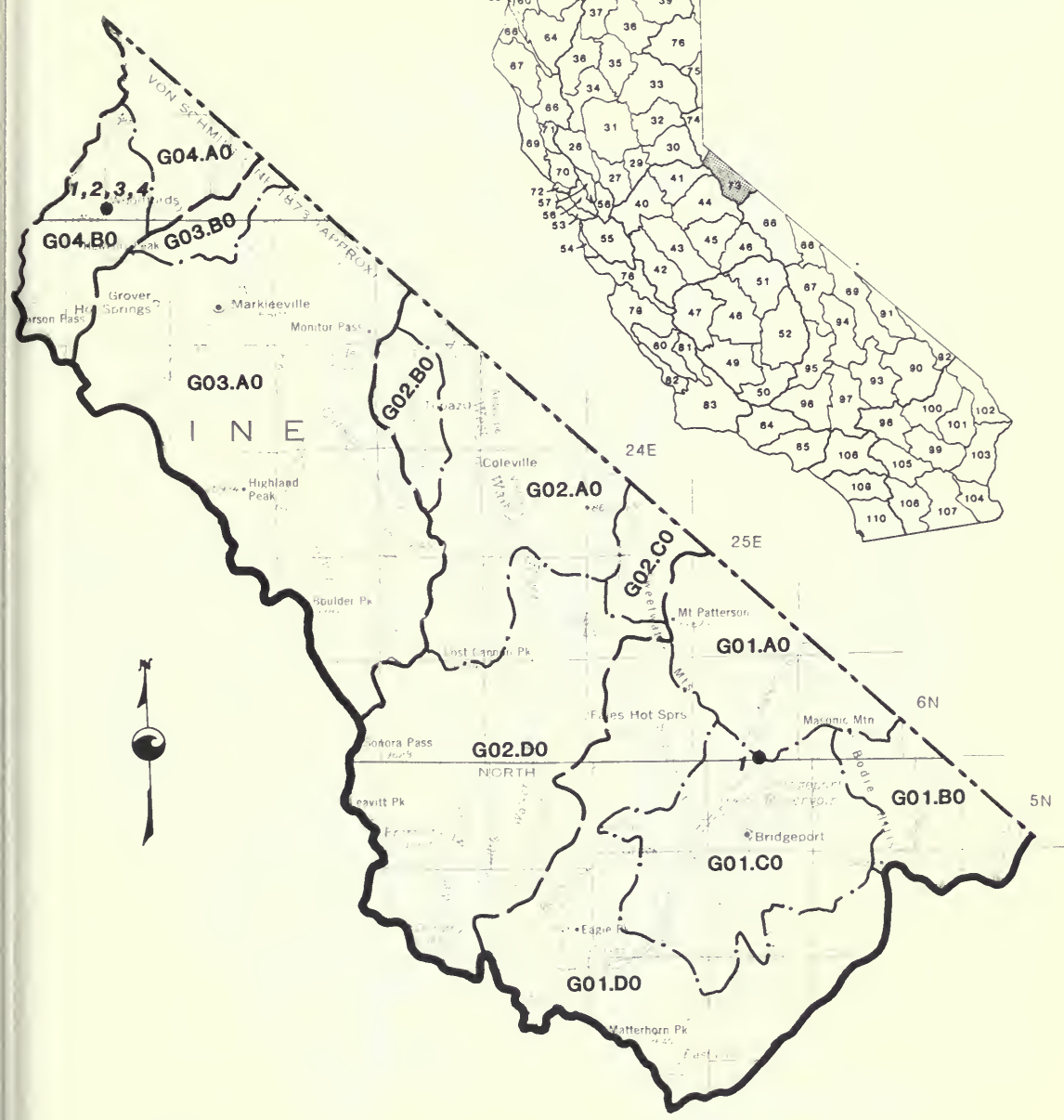

SCALE 


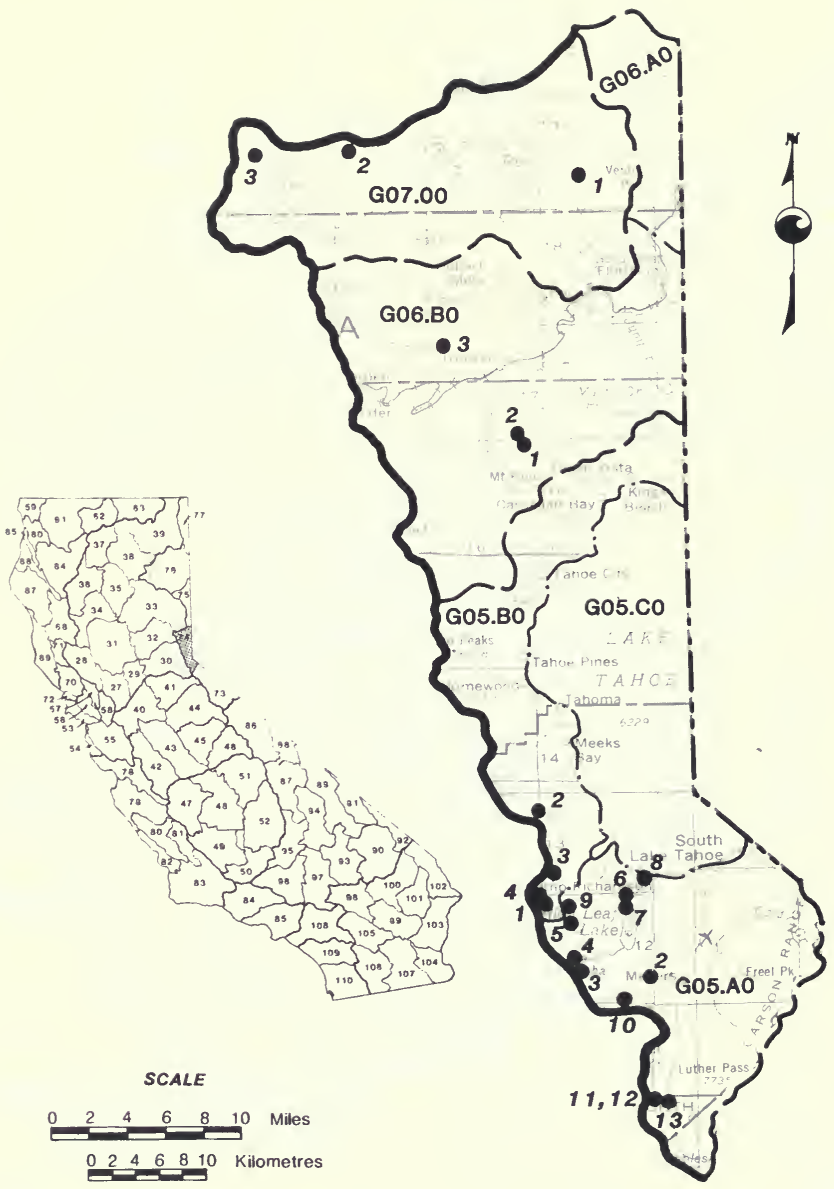

G05, G06 \& G07 


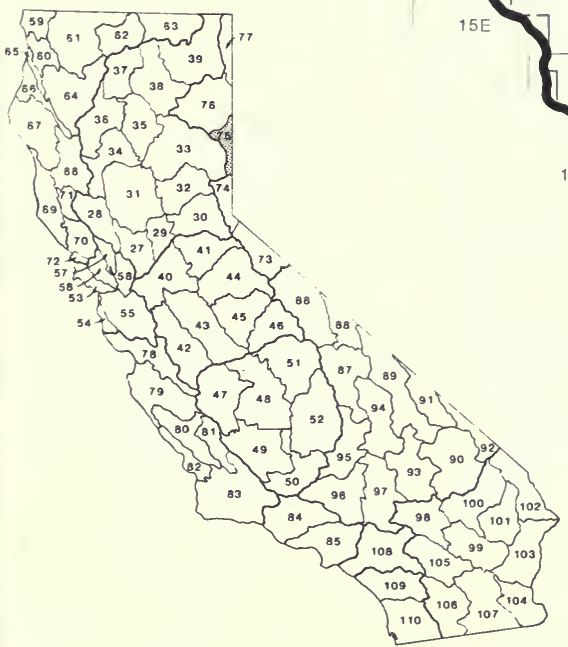

SCALE

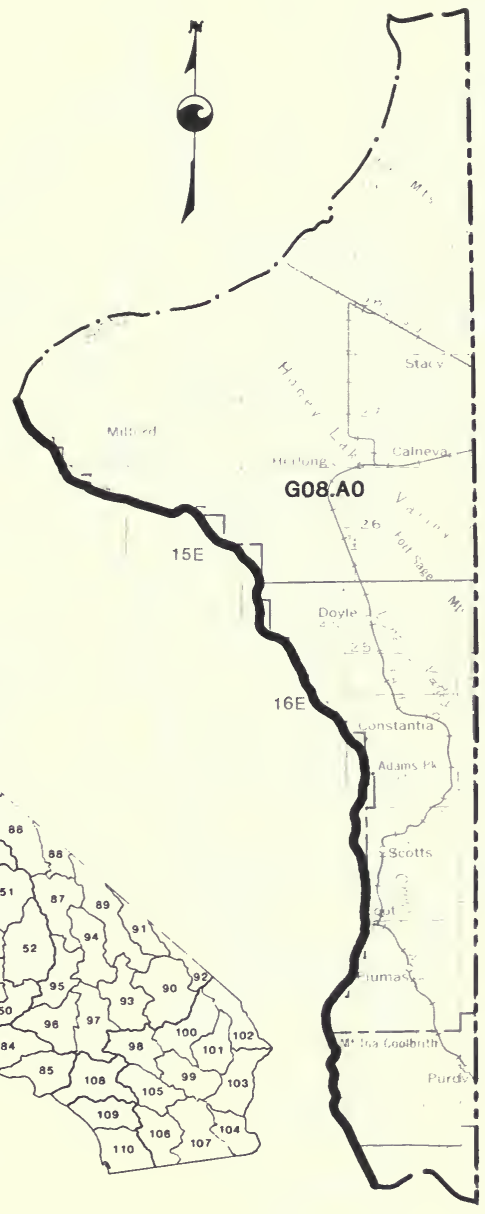

\begin{tabular}{lllllll}
0 & 2 & 4 & 6 & 8 & 10 & Miles \\
\hline
\end{tabular}

$0246 \quad 8 \quad 10$ Kilometres

G08.A 


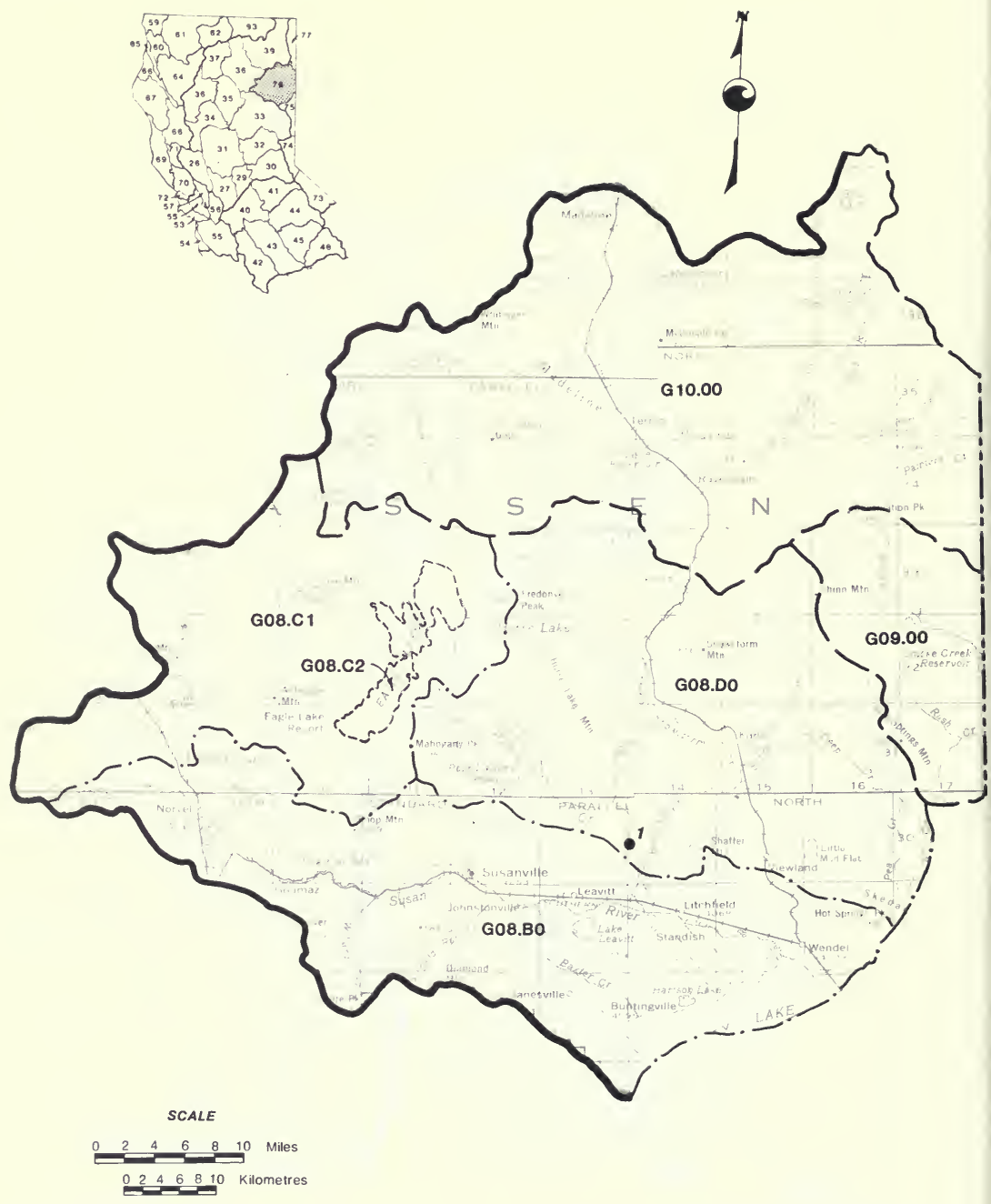

G08B, C, D, G09 \& G10 


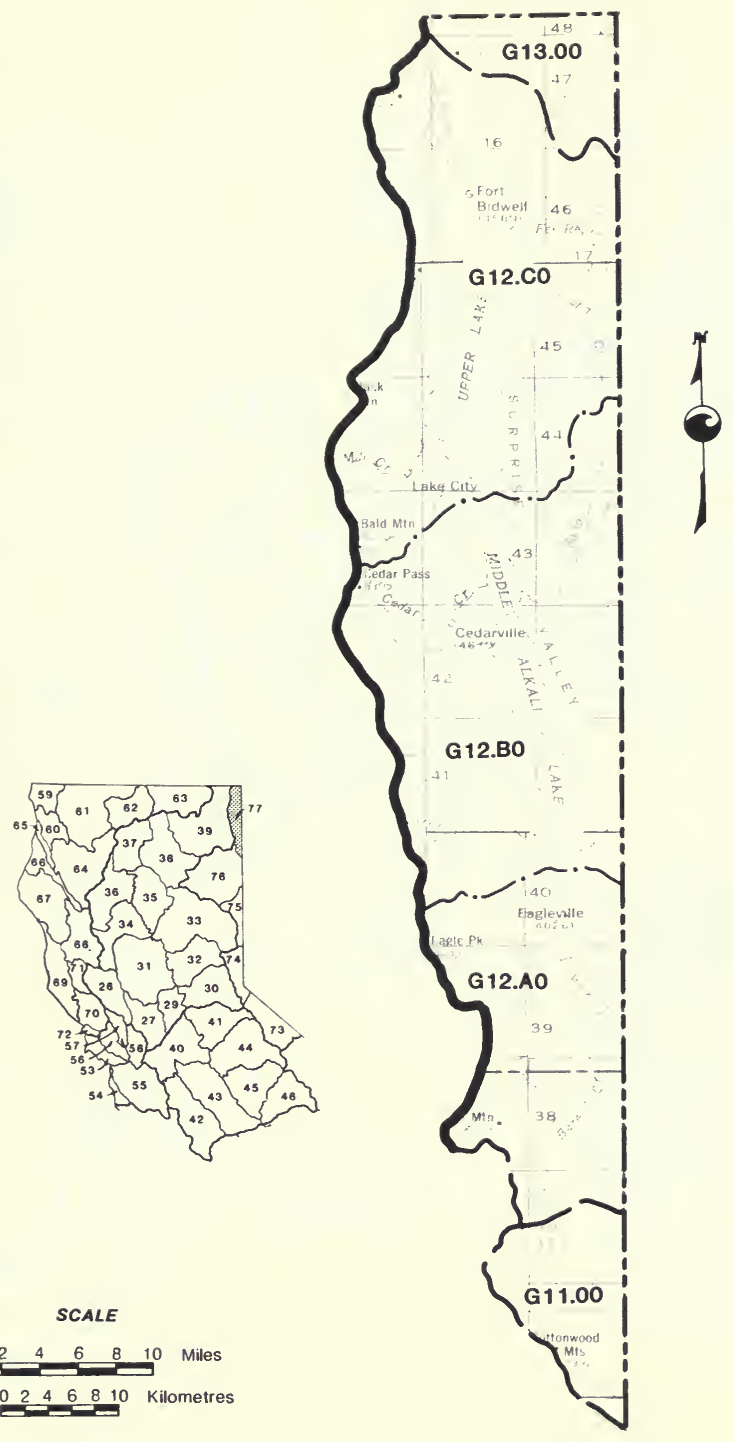

G11, G12 \& G13 


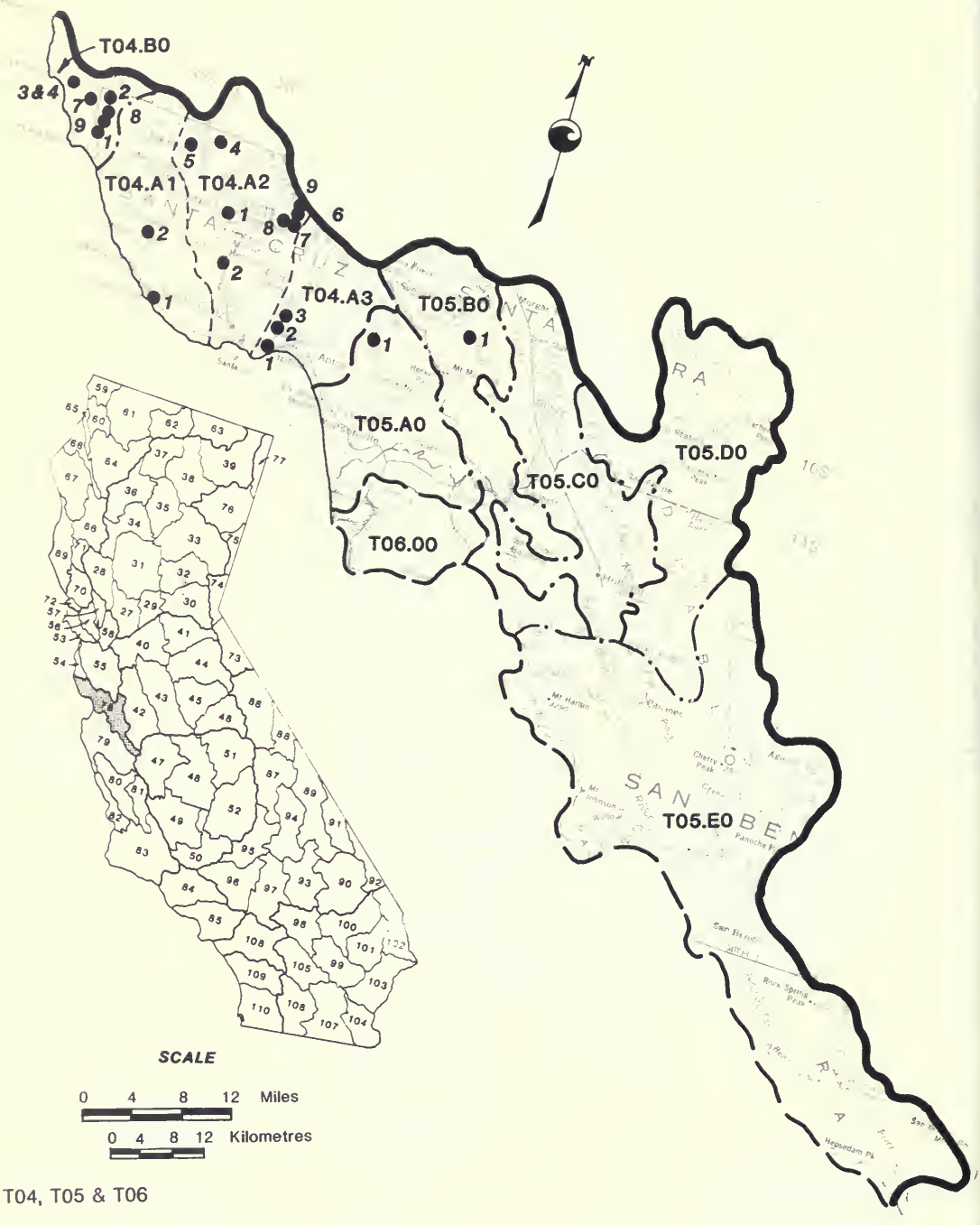




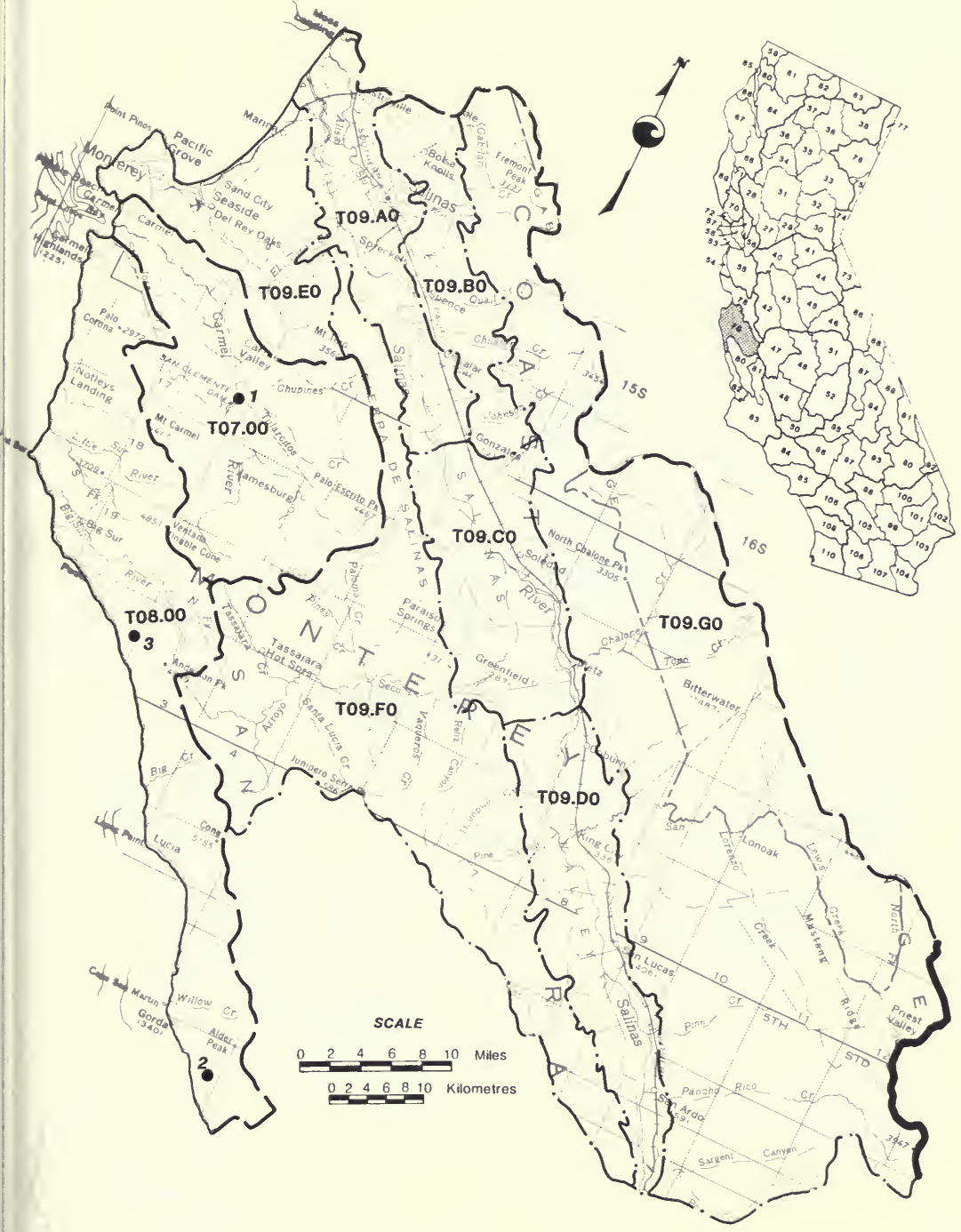

T07, T08, T09.A - G 


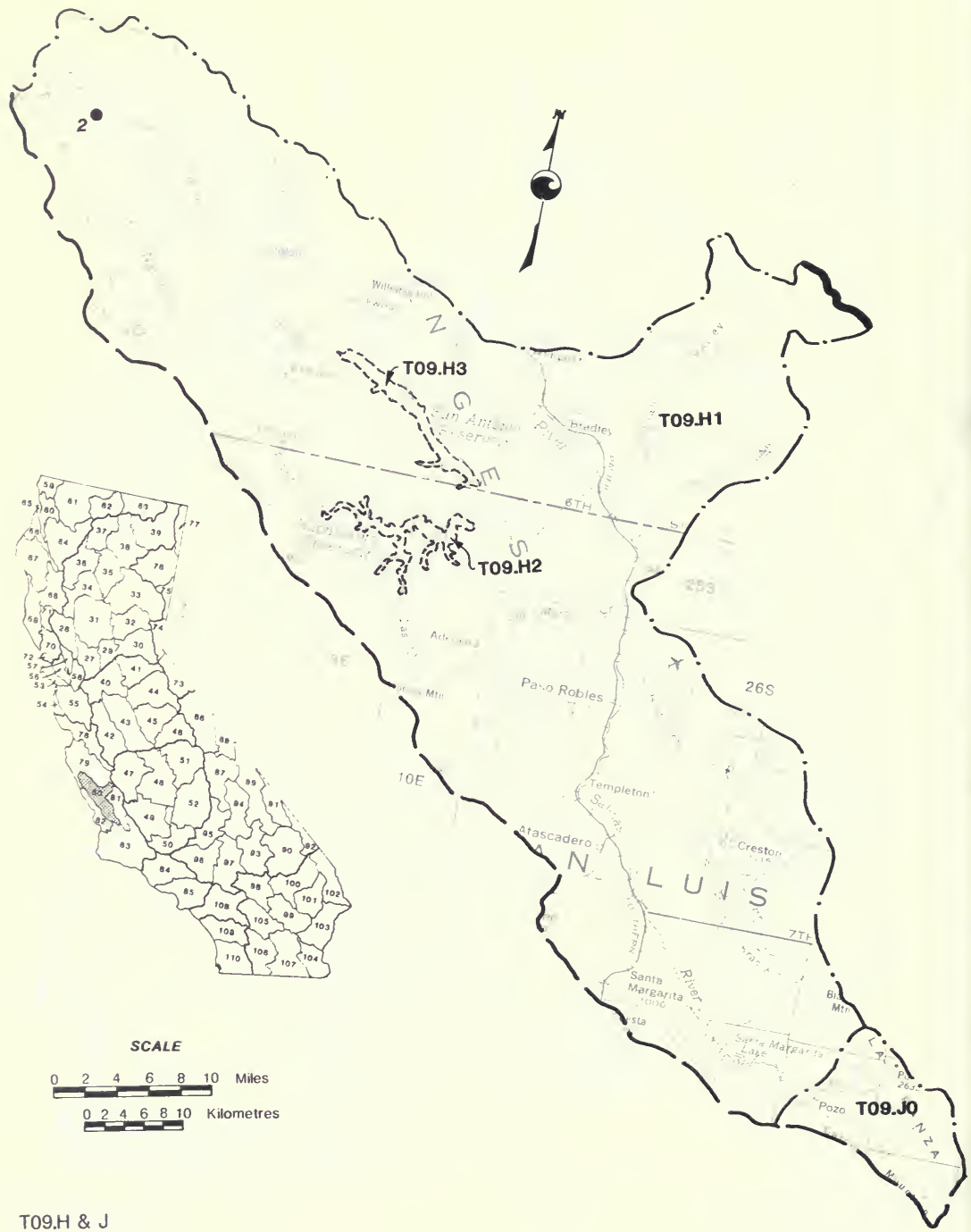




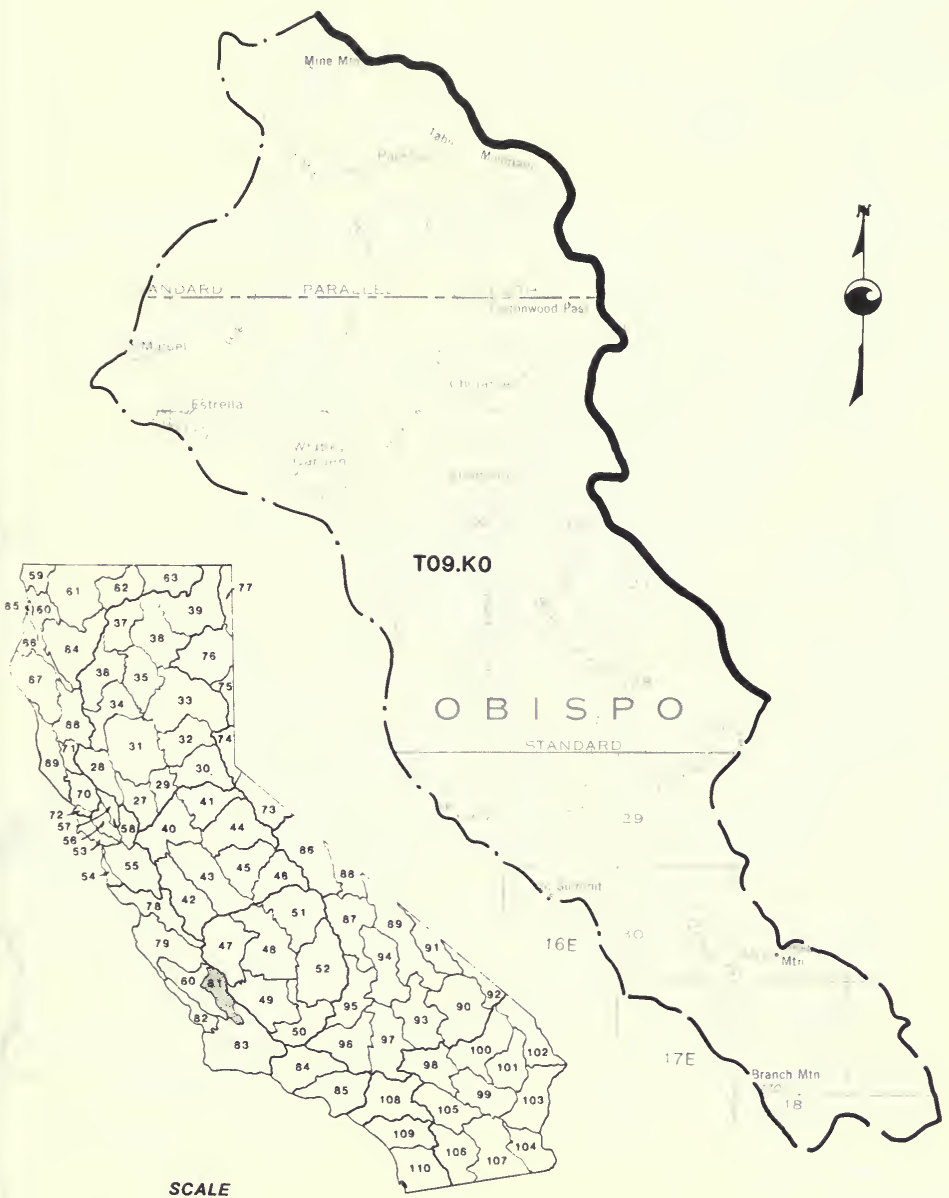

\begin{tabular}{lllllll}
0 & 2 & 4 & 6 & 8 & 10 & Miles \\
\hline & & & & & &
\end{tabular}

\begin{tabular}{lllllll}
0 & 2 & 4 & 6 & 8 & 10 & Kilometres \\
\hline
\end{tabular} 


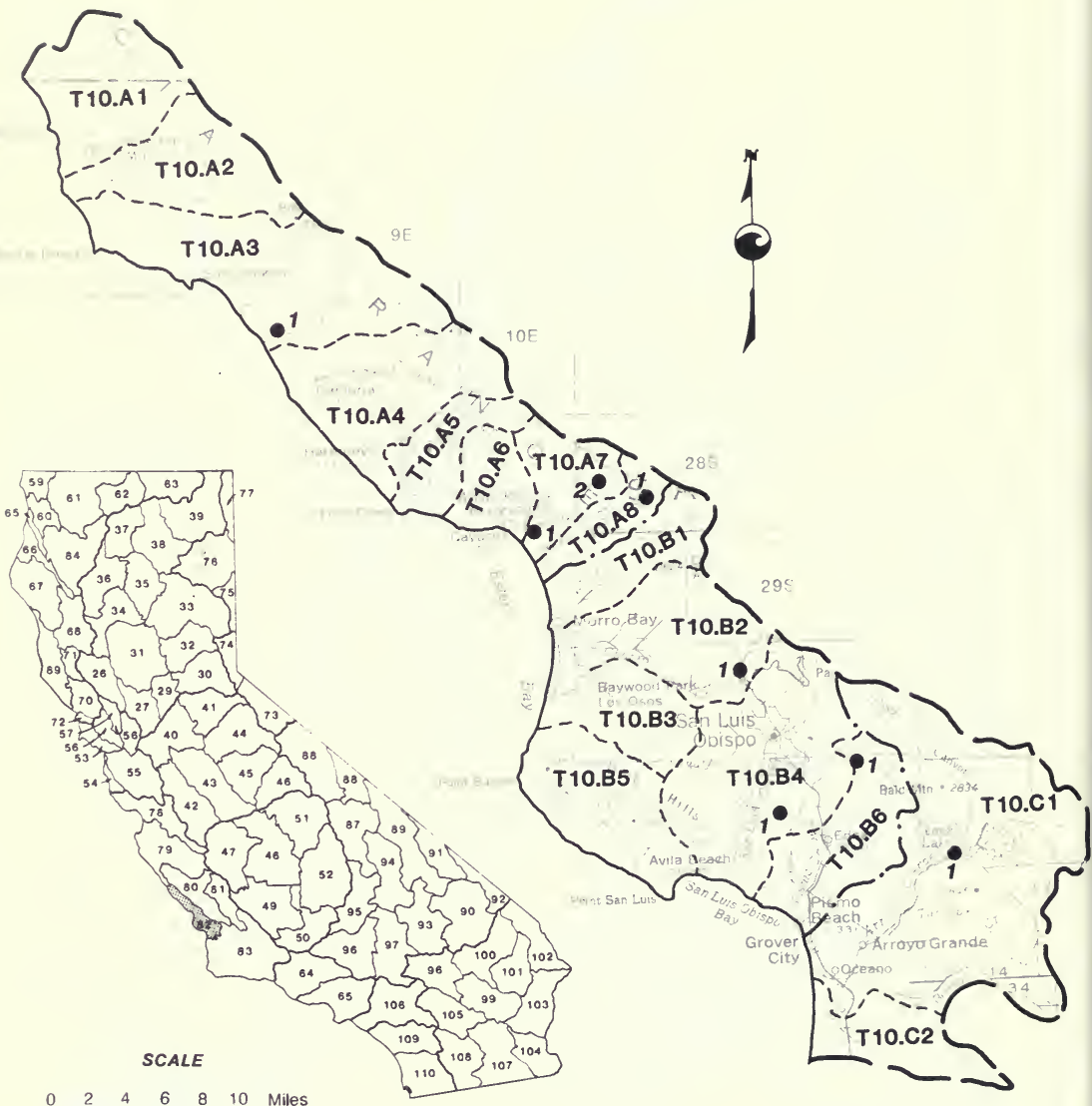

\begin{tabular}{lllllll}
0 & 2 & 4 & 6 & 8 & 10 & Kilometres \\
\hline
\end{tabular} 


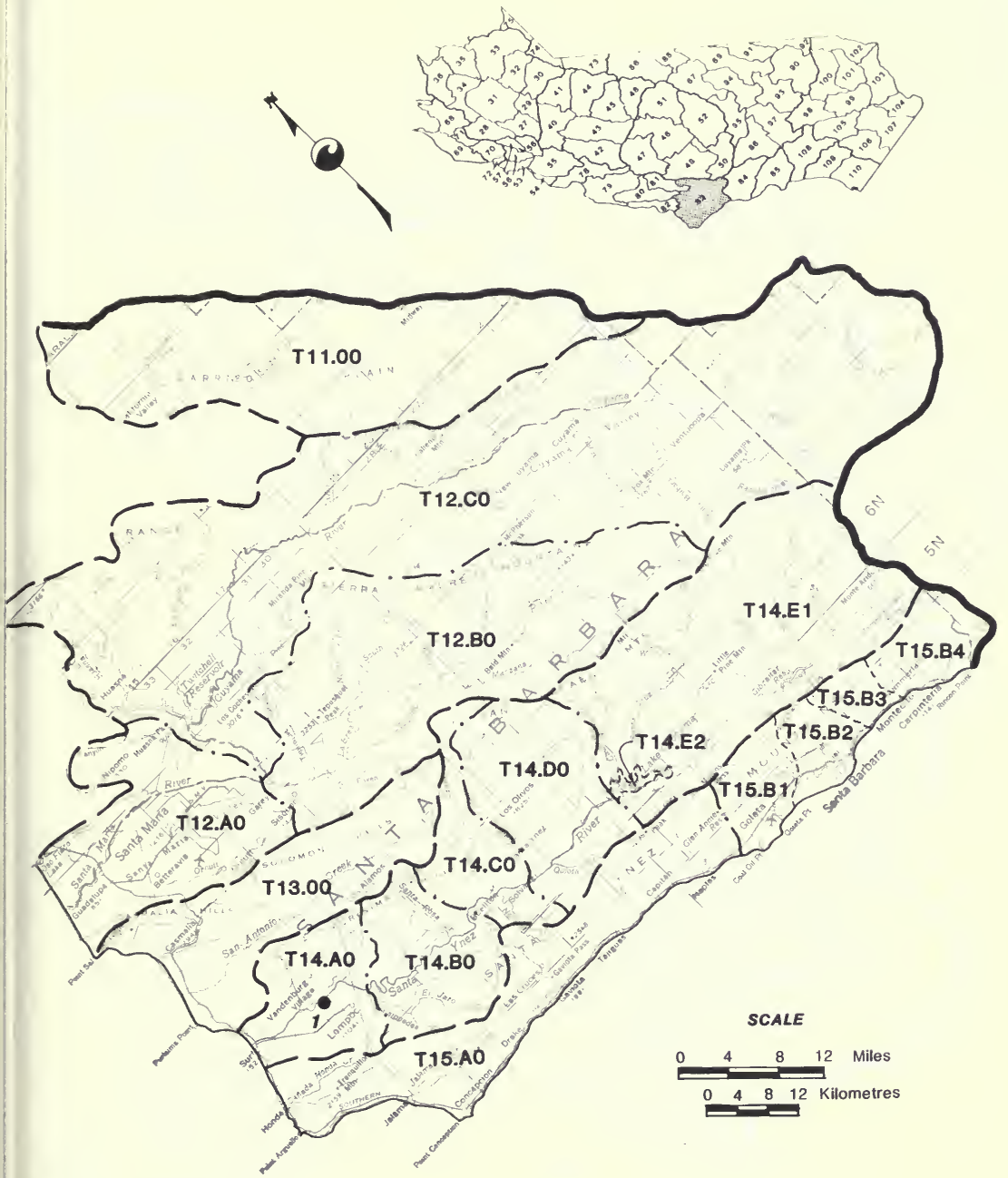

T11 - T15 


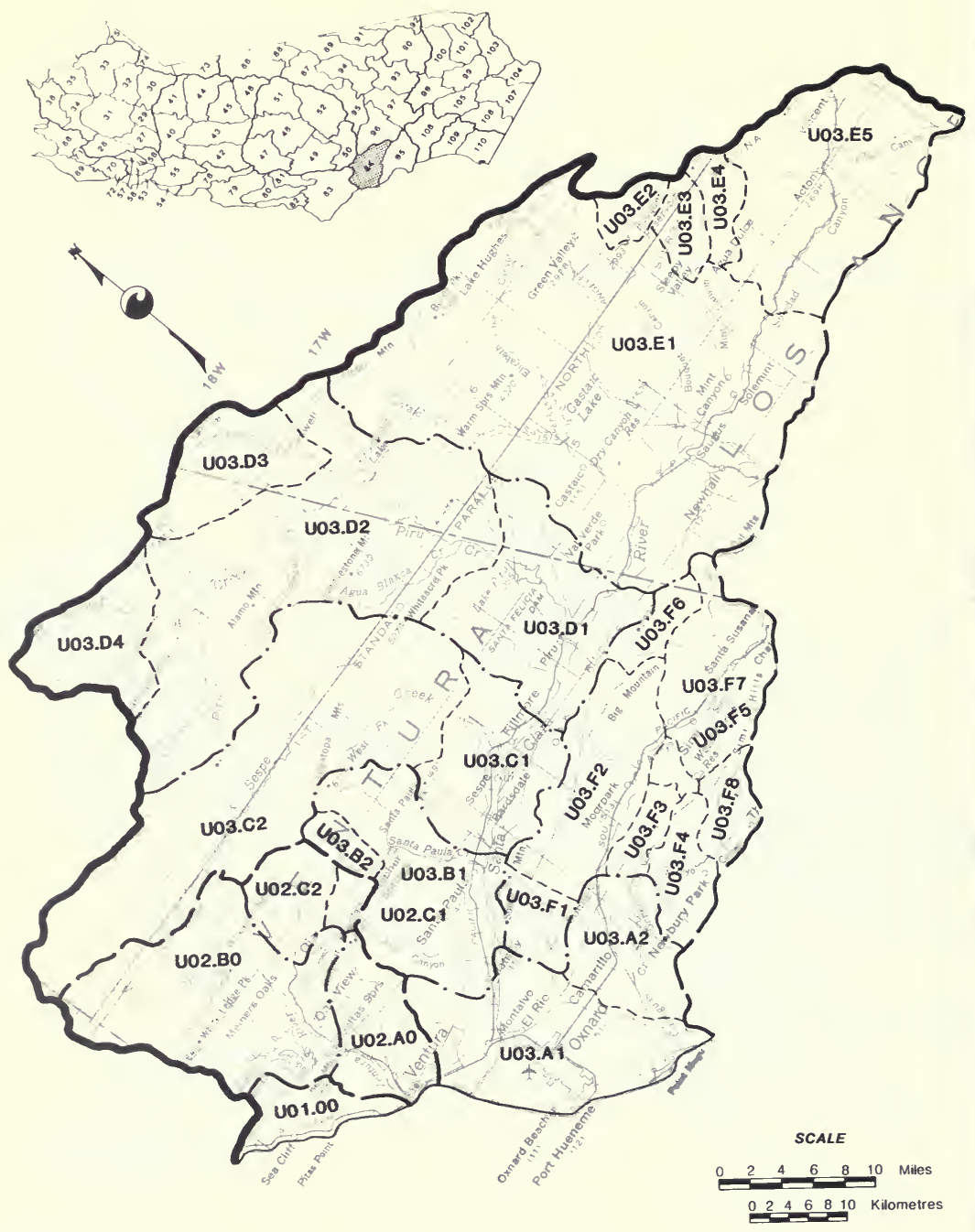

U01, U02 \& U03 


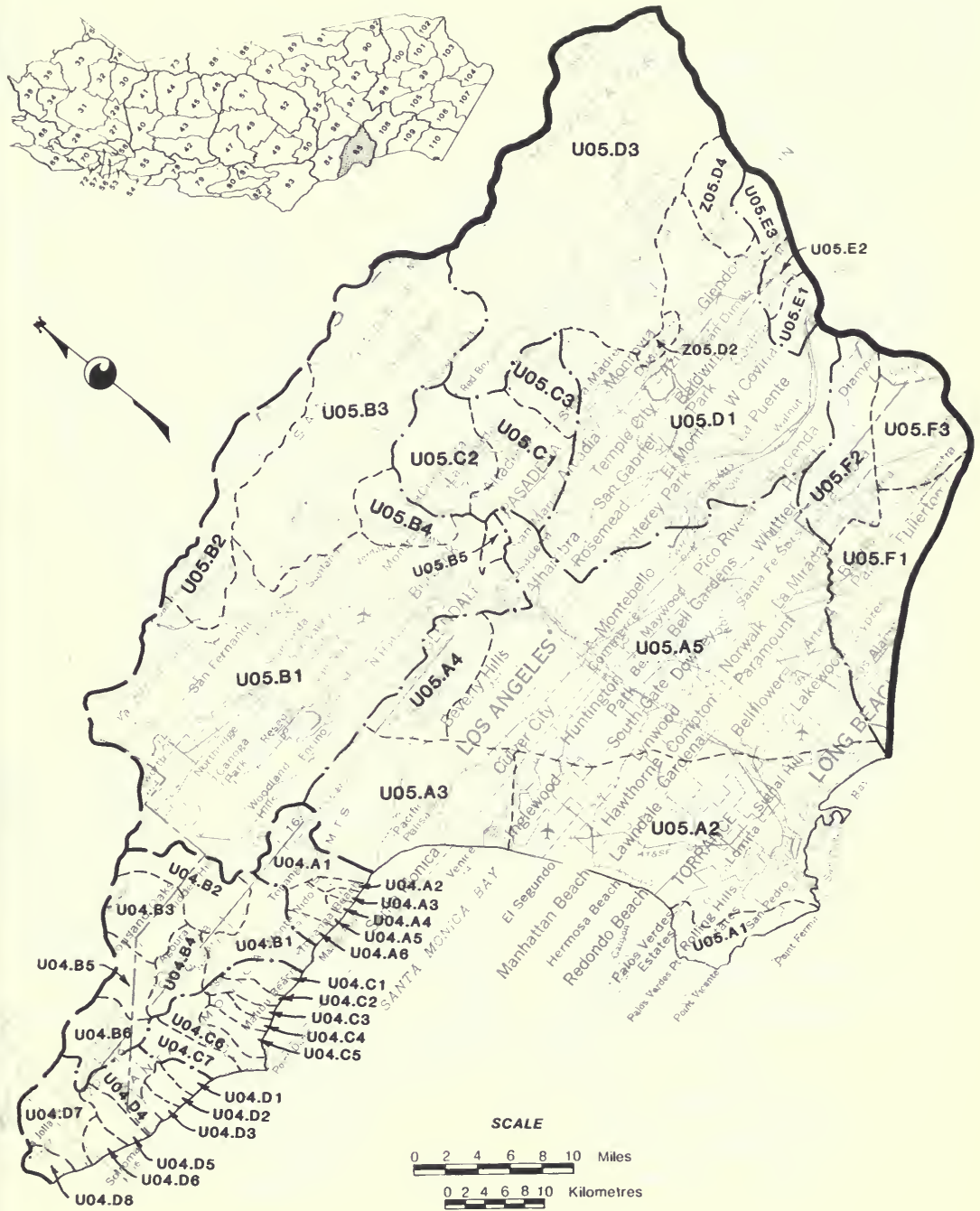

U04 \& U05 


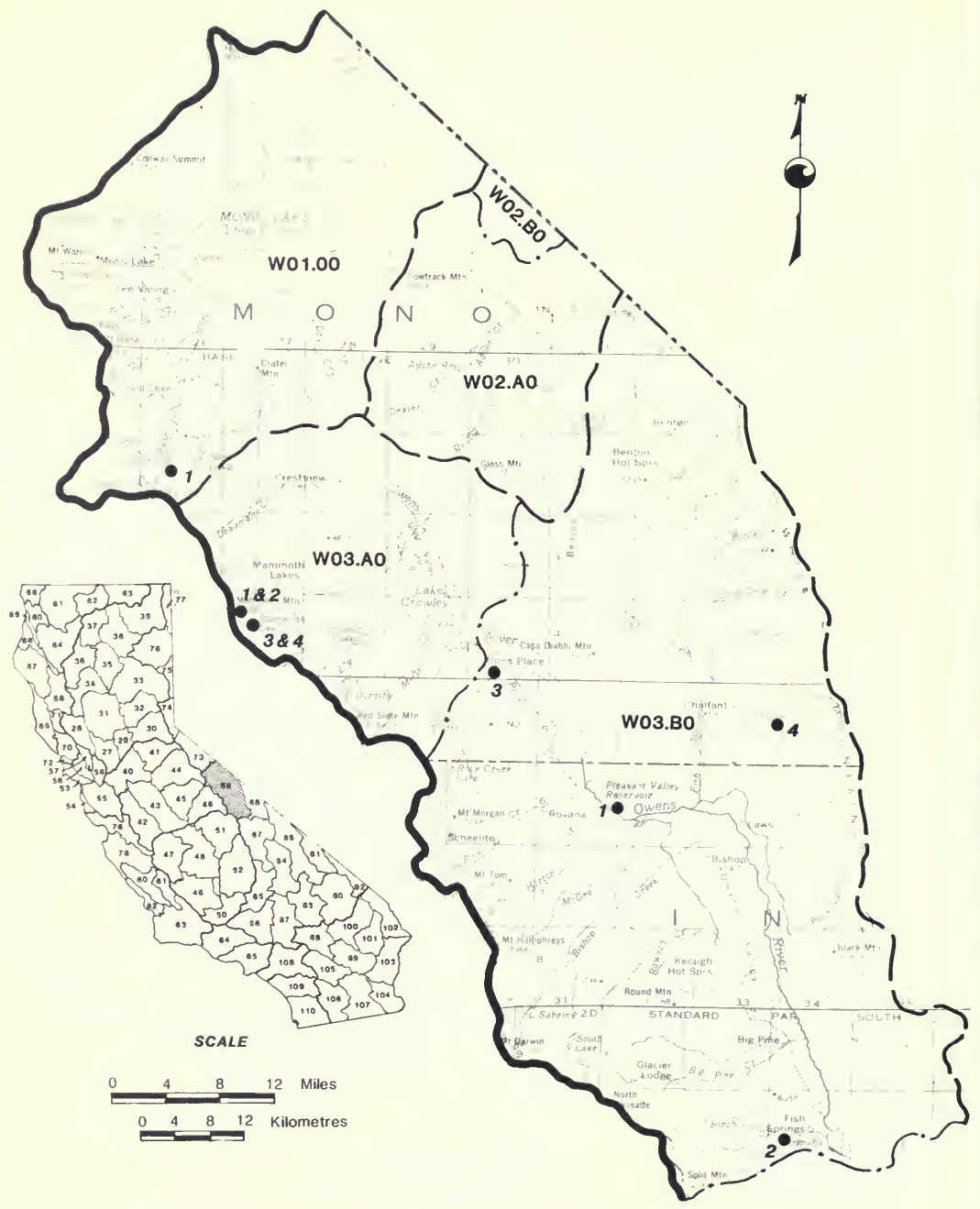

W01, W02, W03.A \& B 


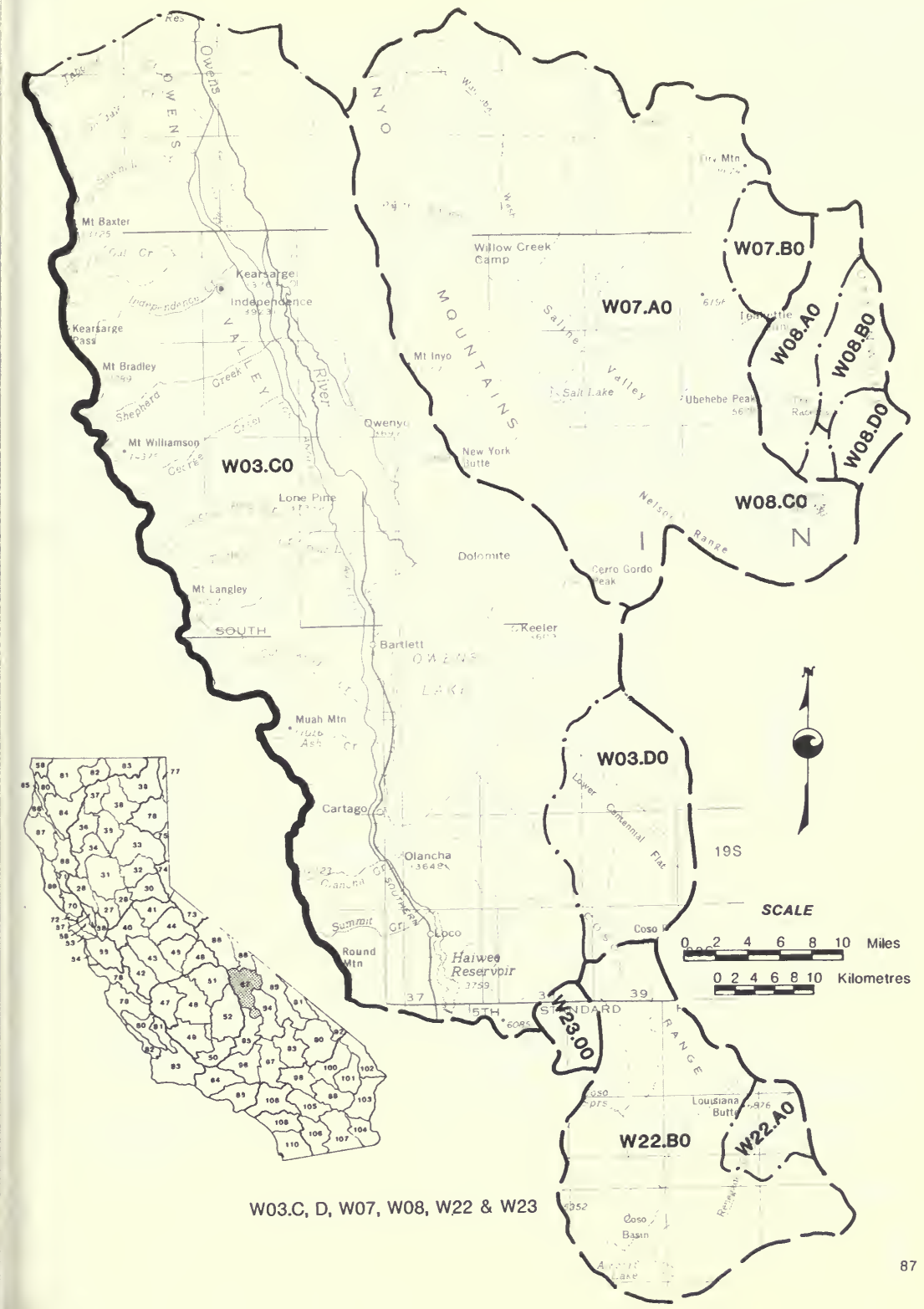




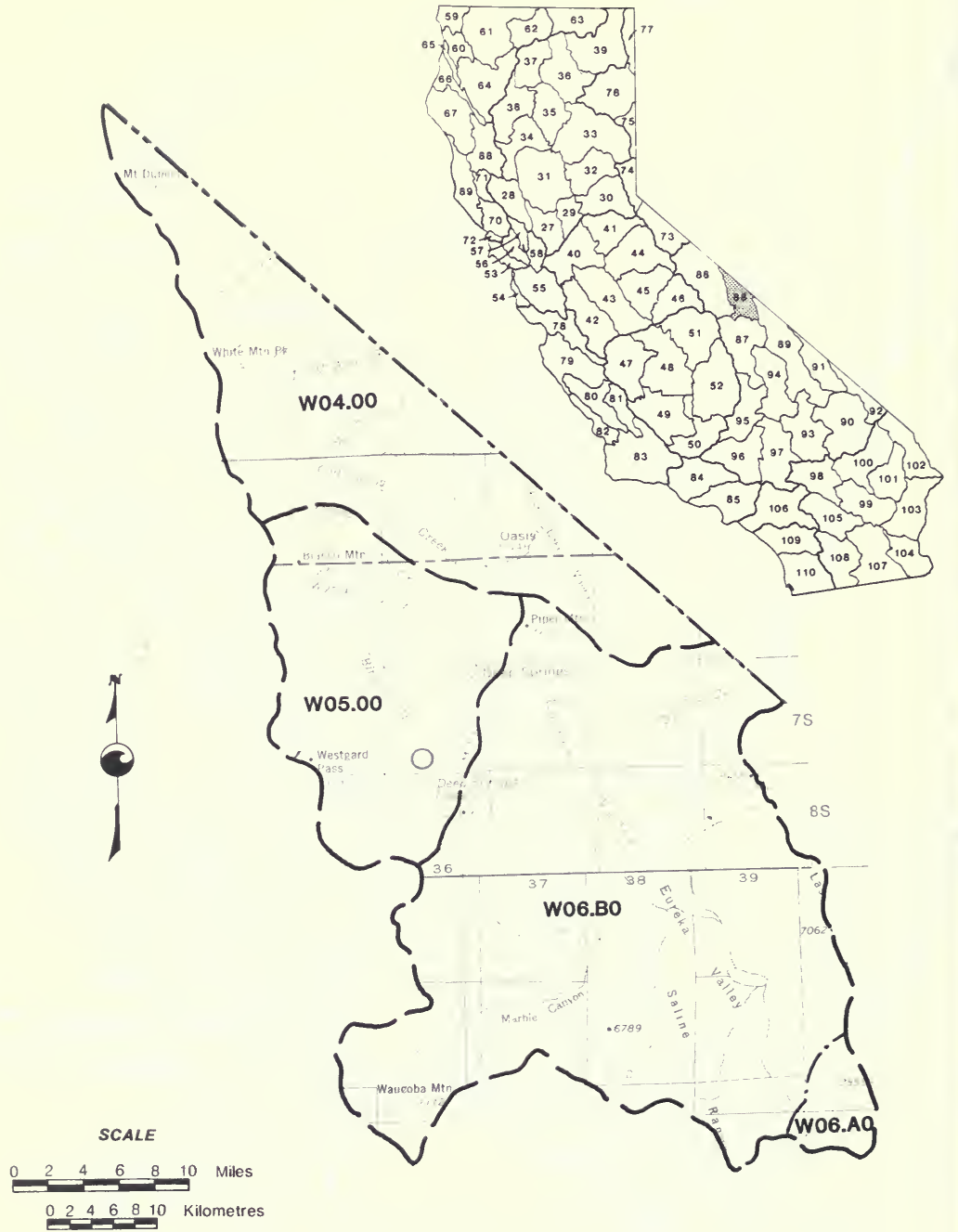

W04, W05 \& W06 


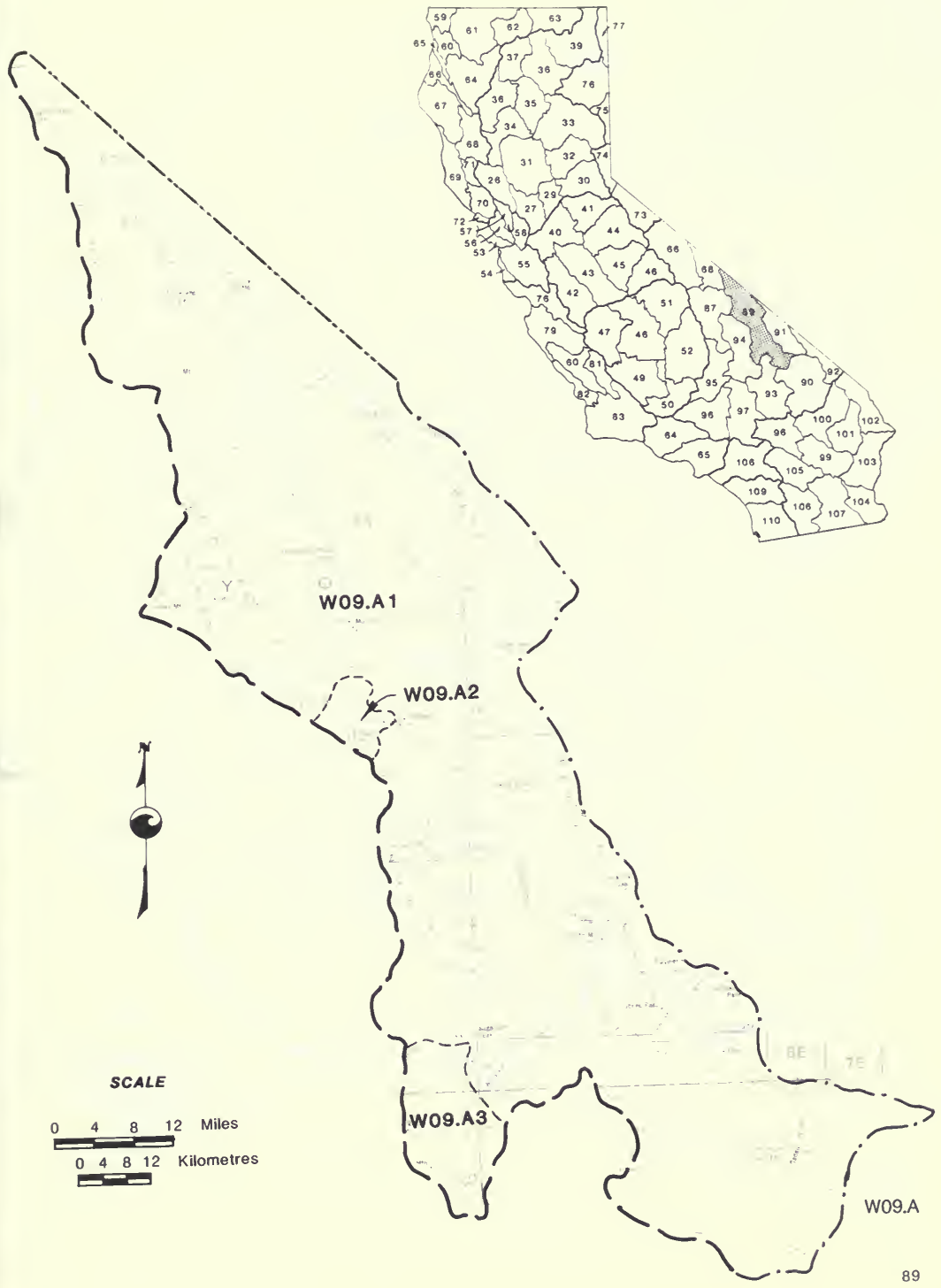




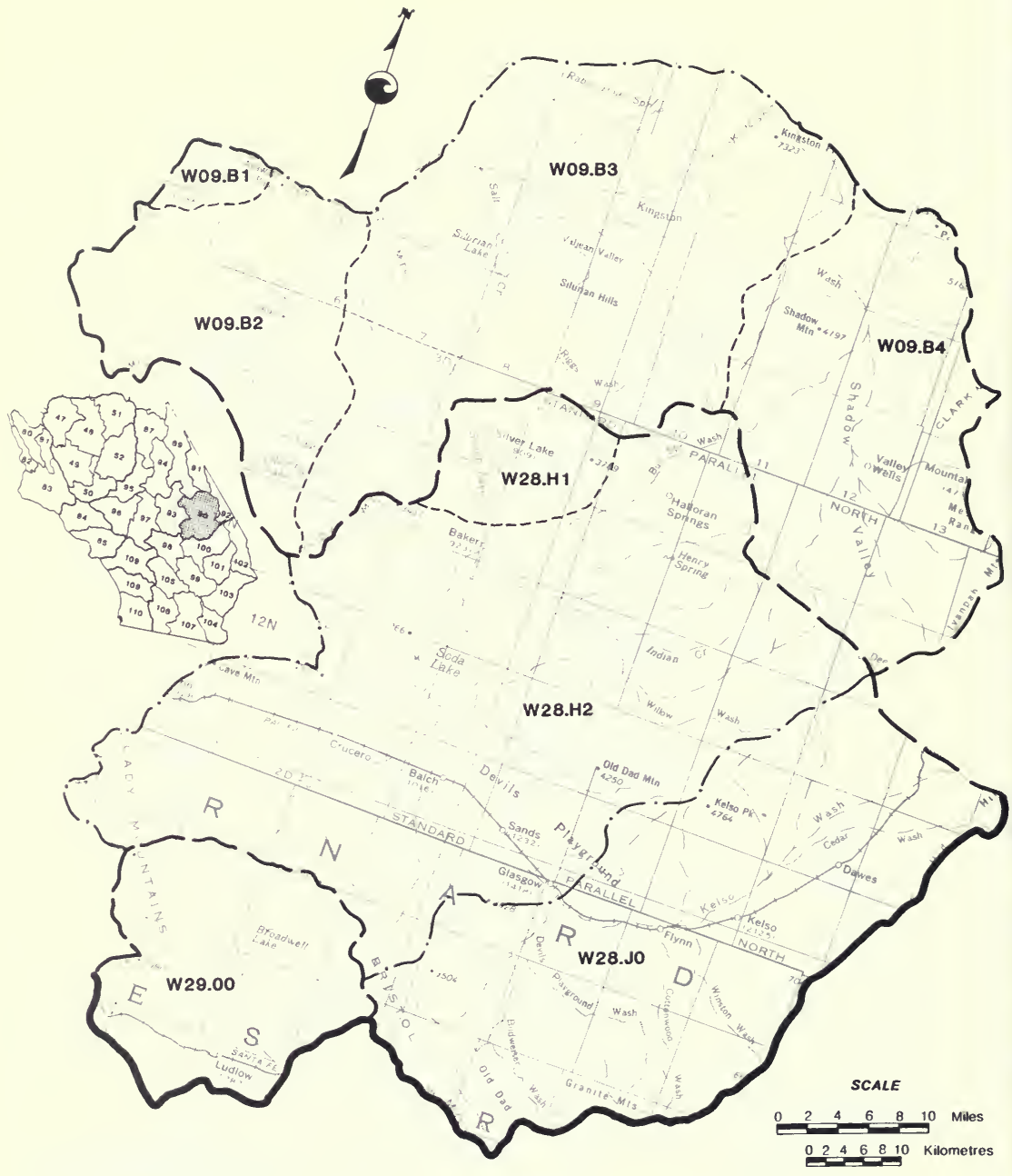

W09.B, W28.H, J \& W29 


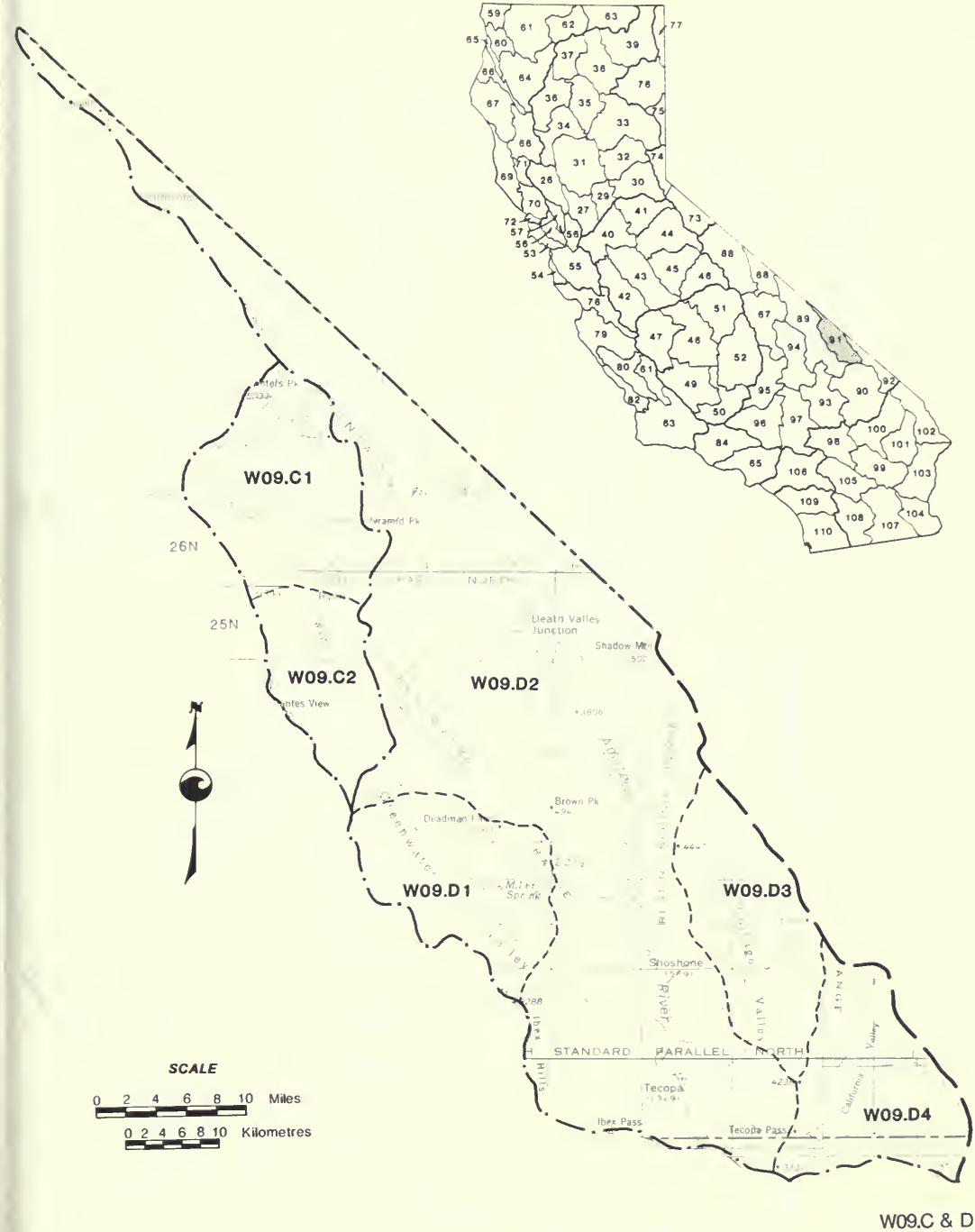




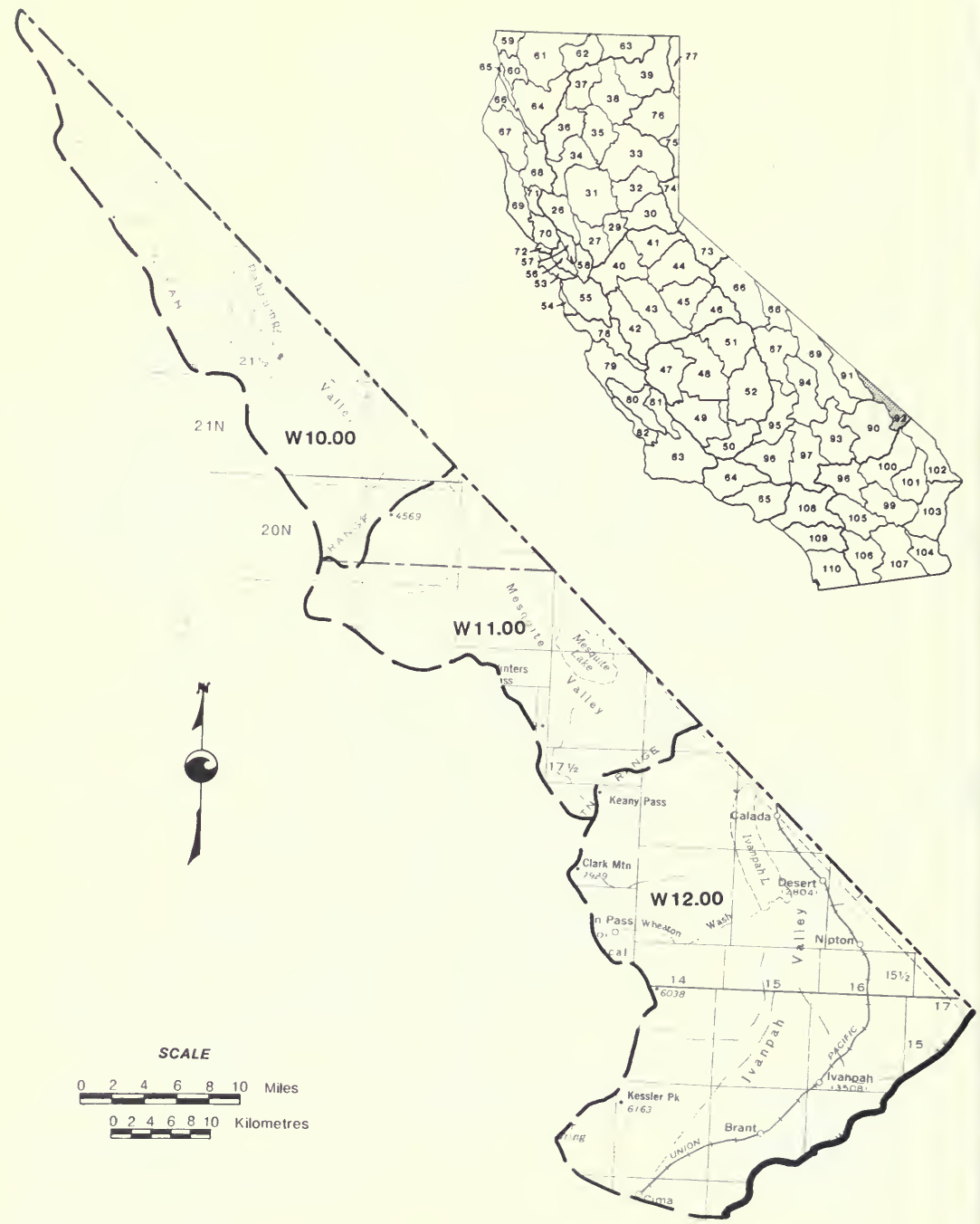

W10, W11 \& W12 


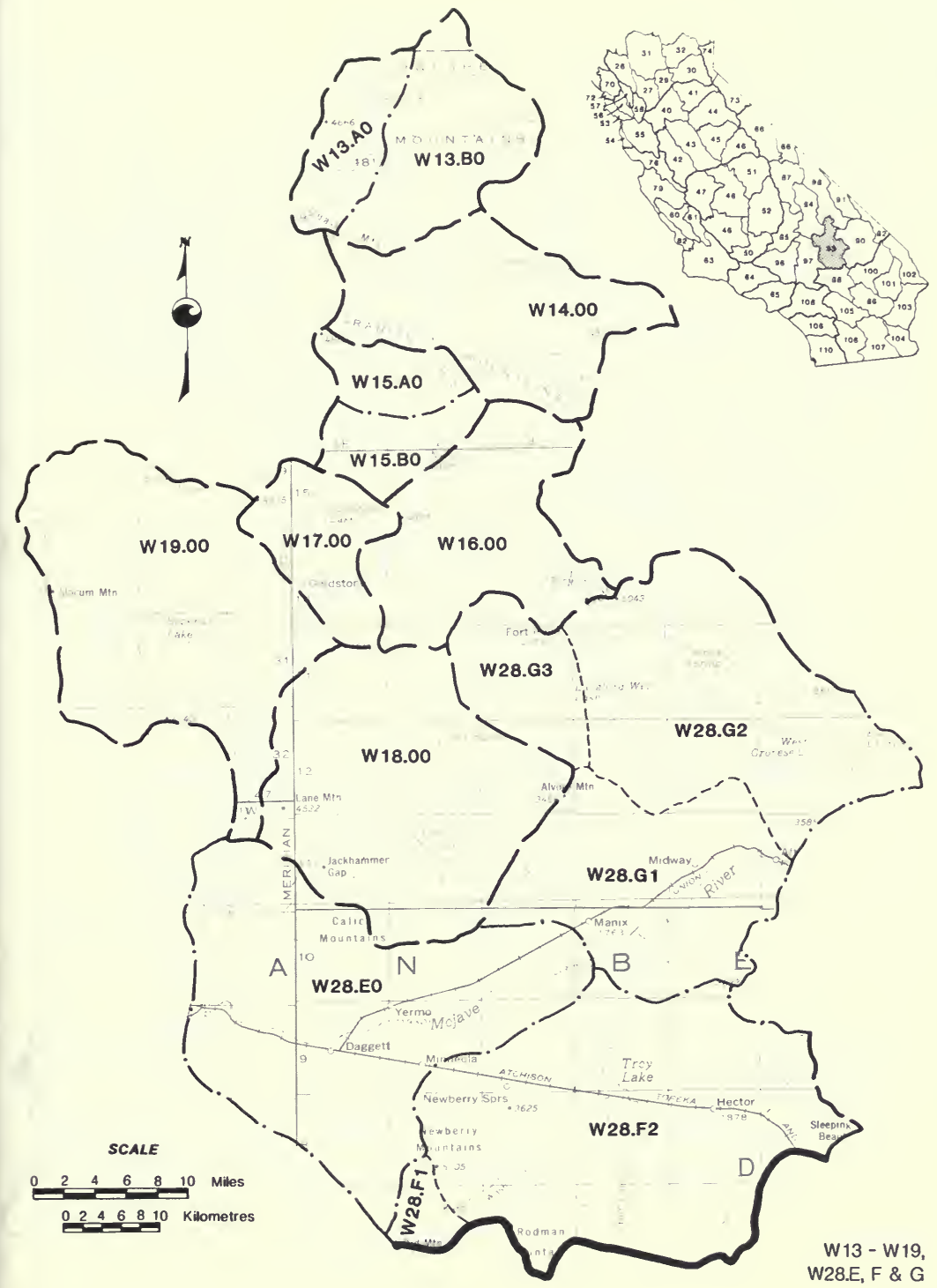




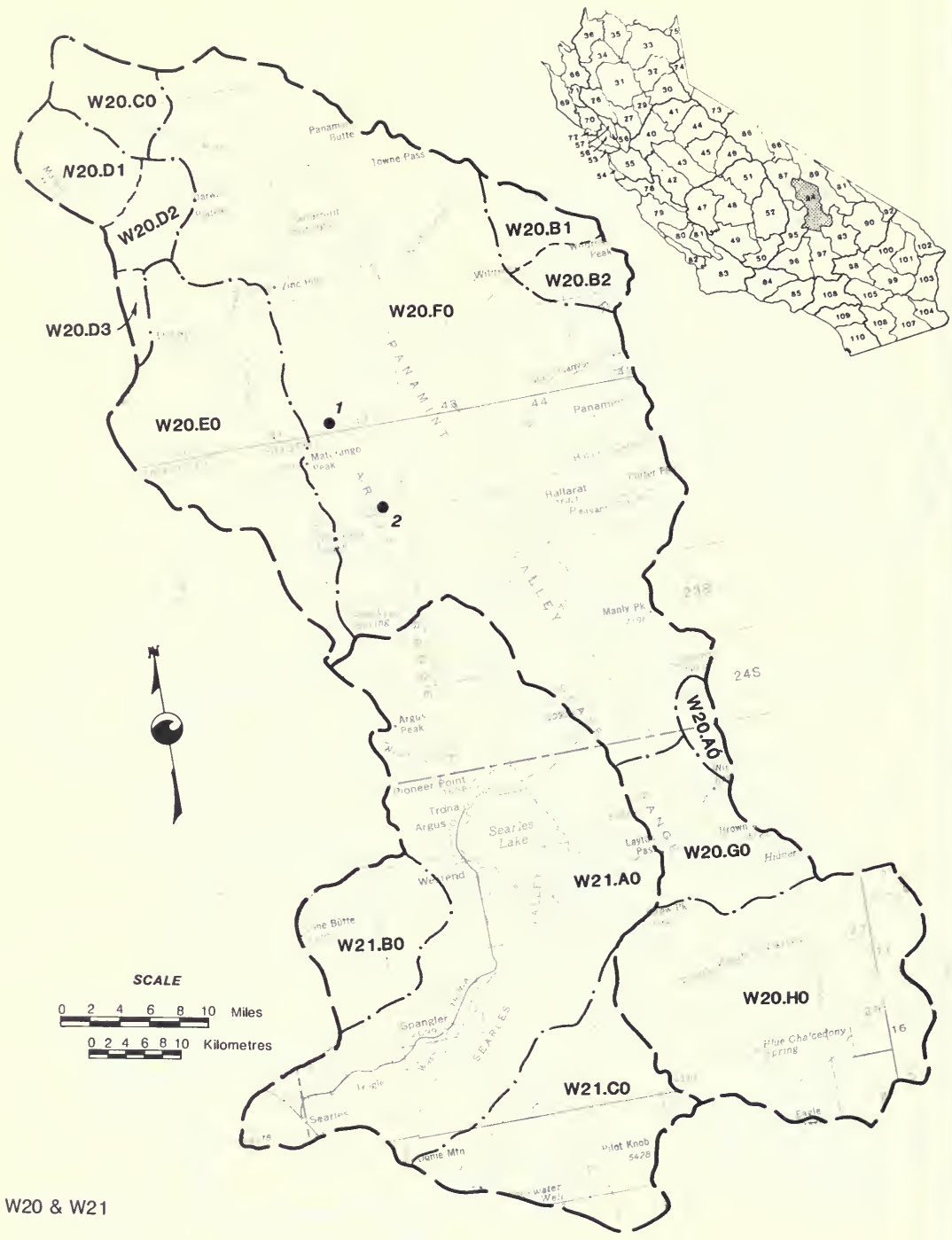




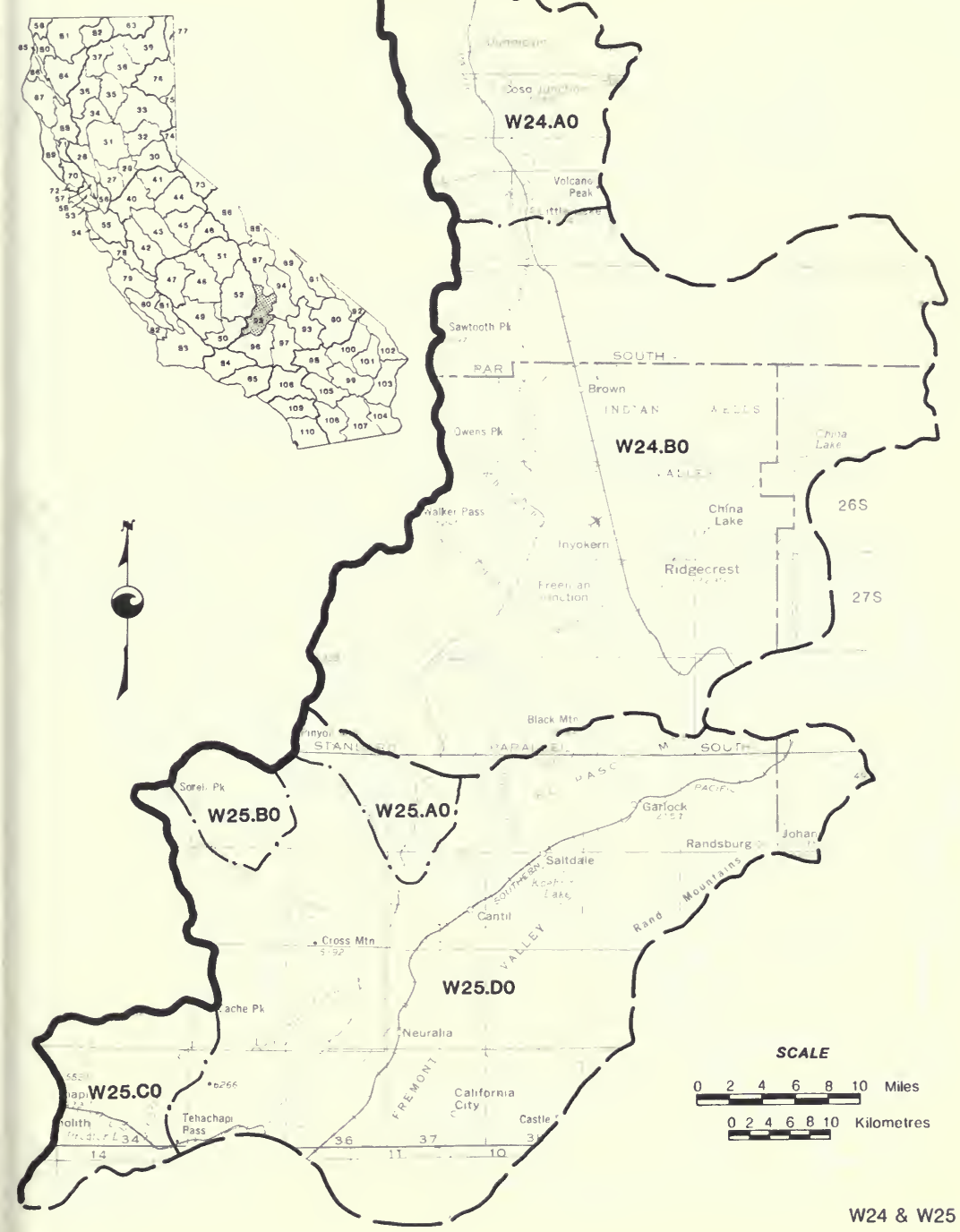




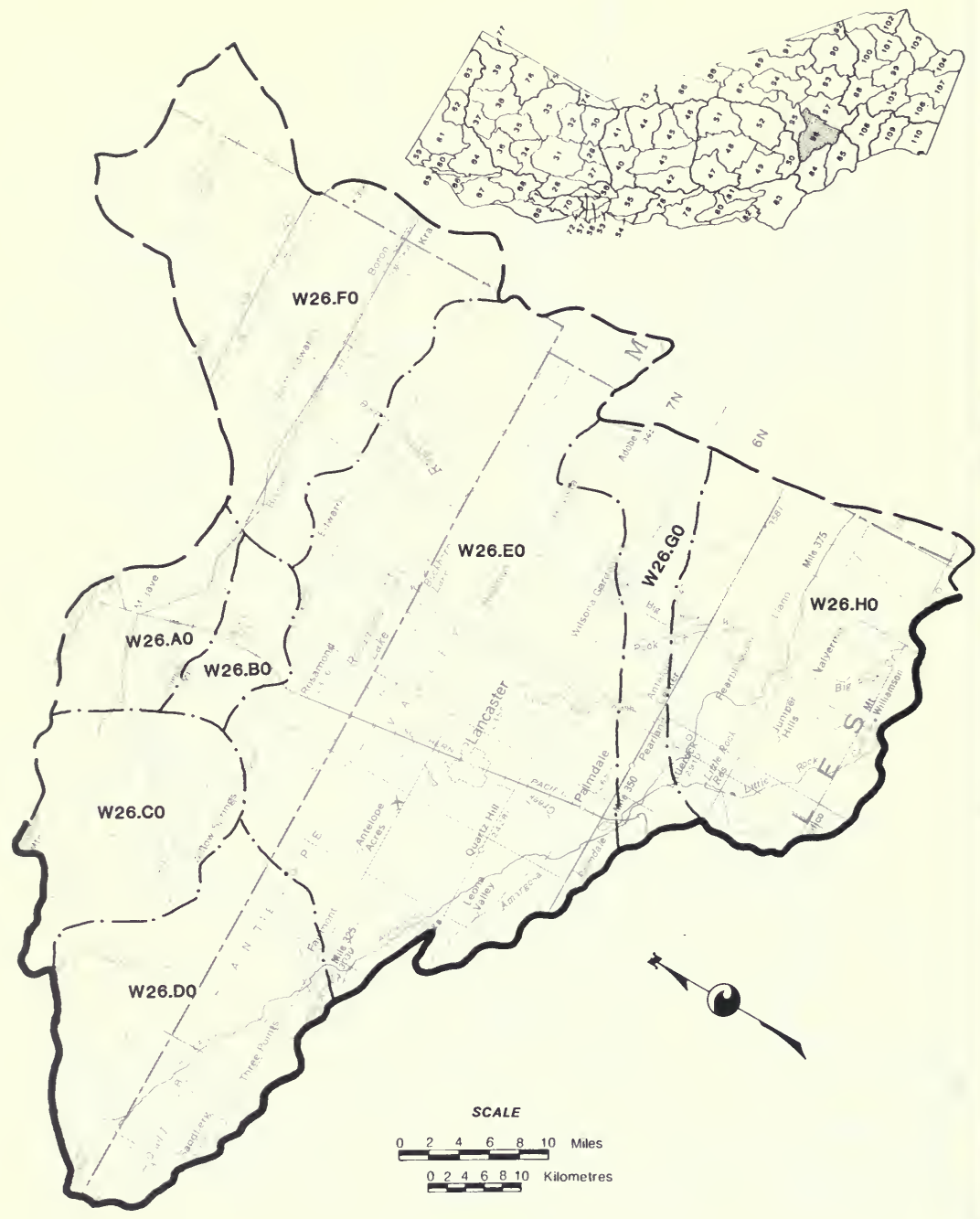

W26 


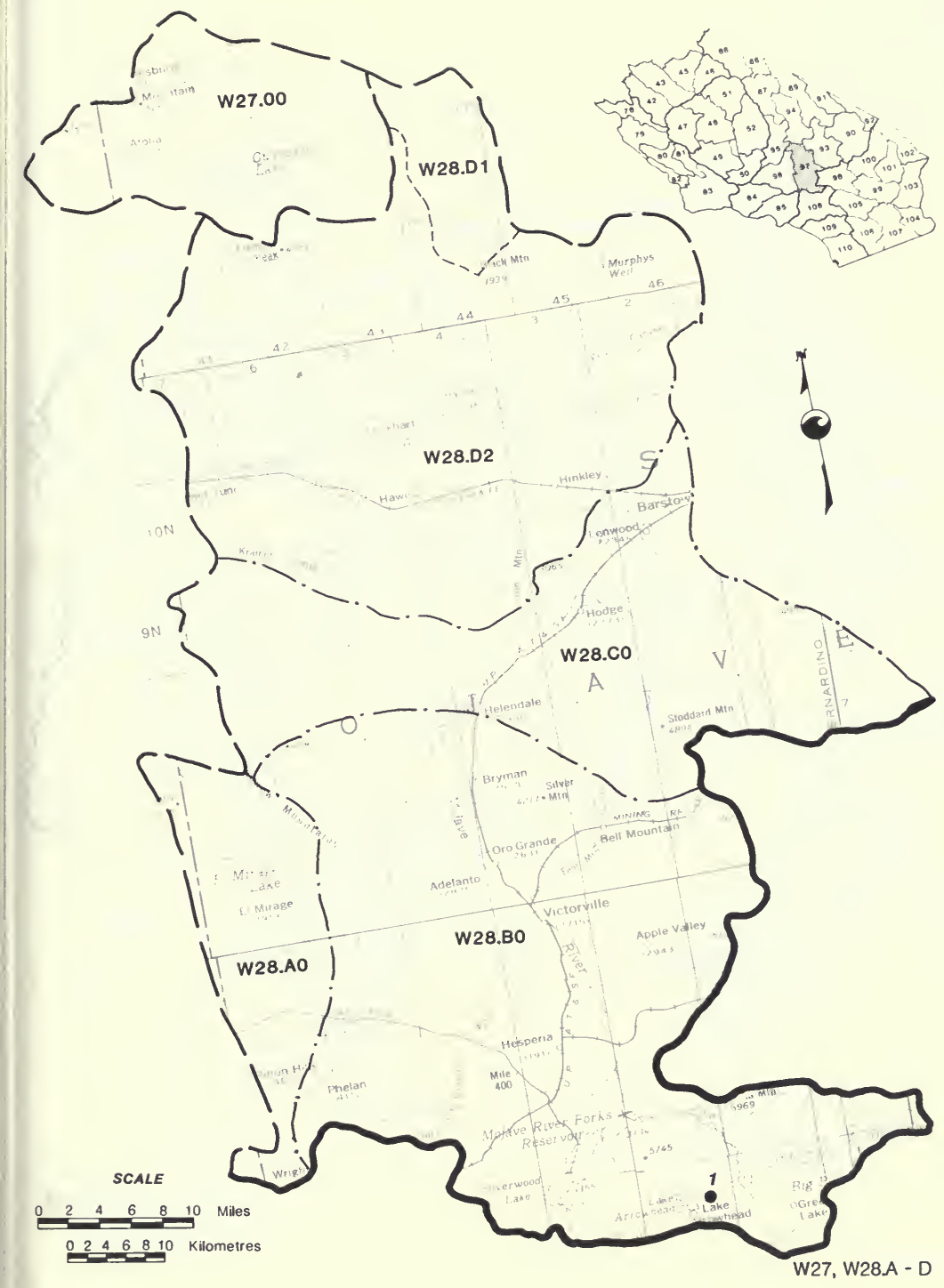




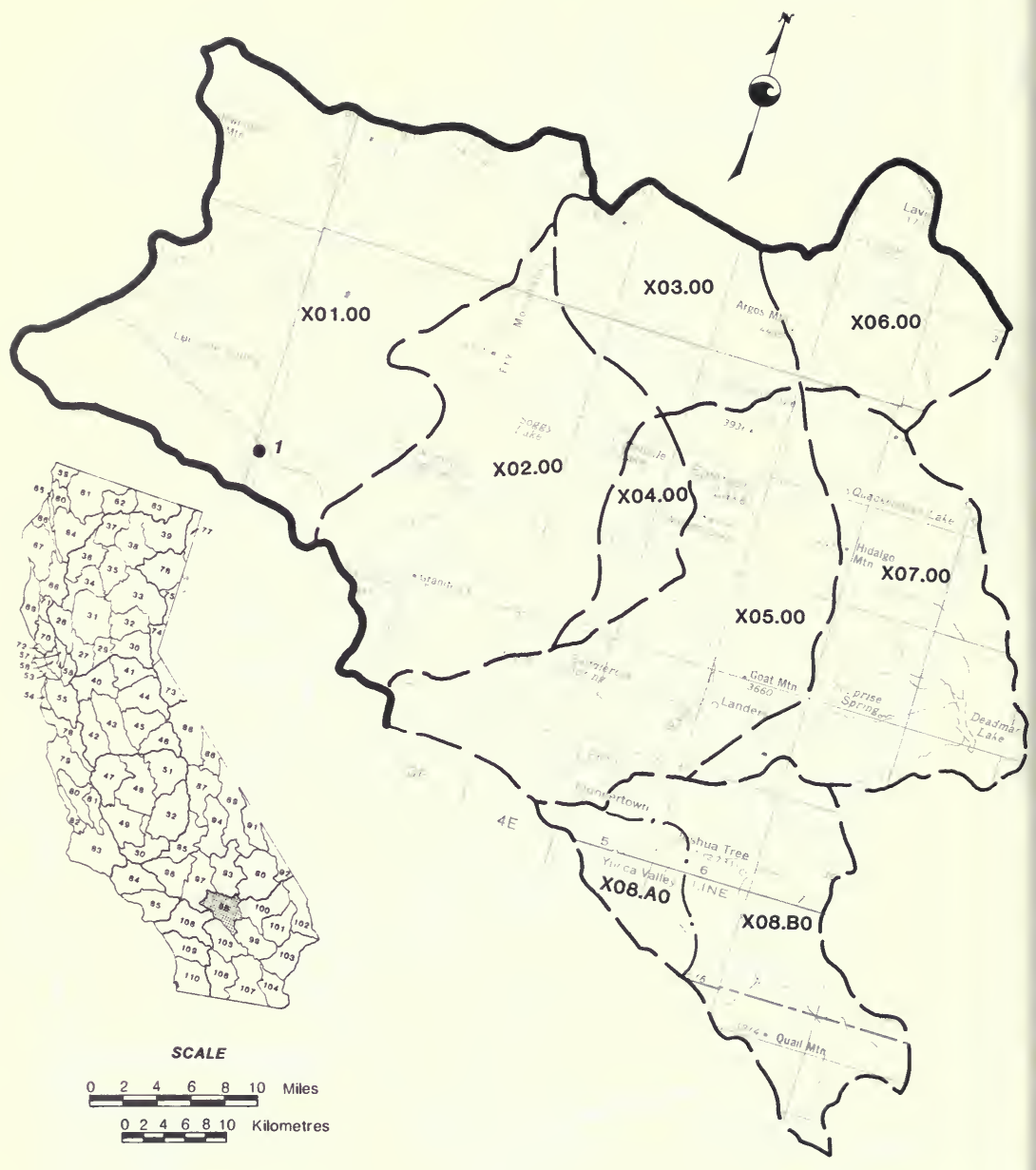

$\mathrm{X} 01-\mathrm{X} 08$ 


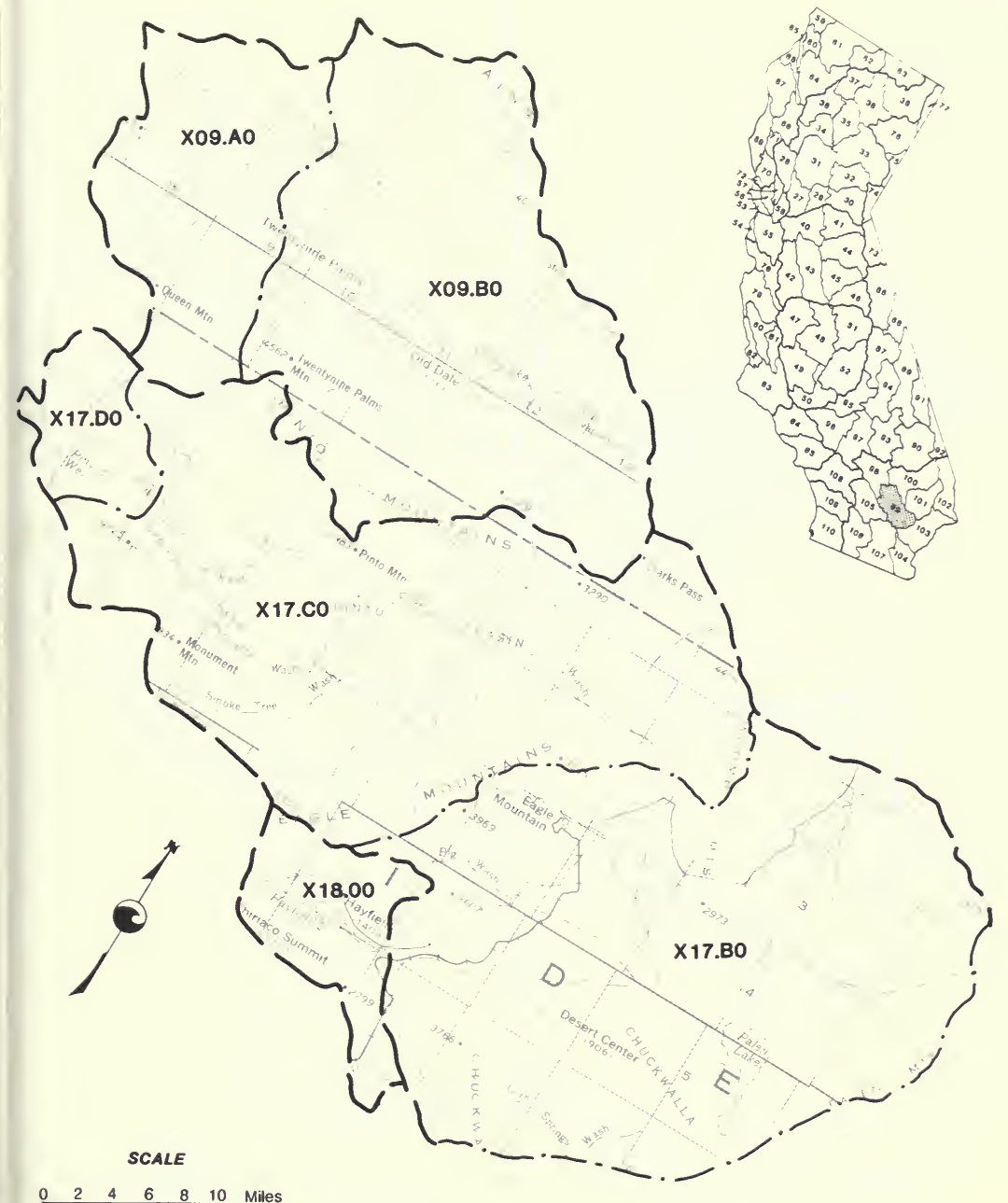

$\begin{array}{lllllll}0 & 2 & 4 & 6 & 8 & 10 & \text { Kilometres }\end{array}$

$X 09, X 17 . B, C, D \& X 18$ 


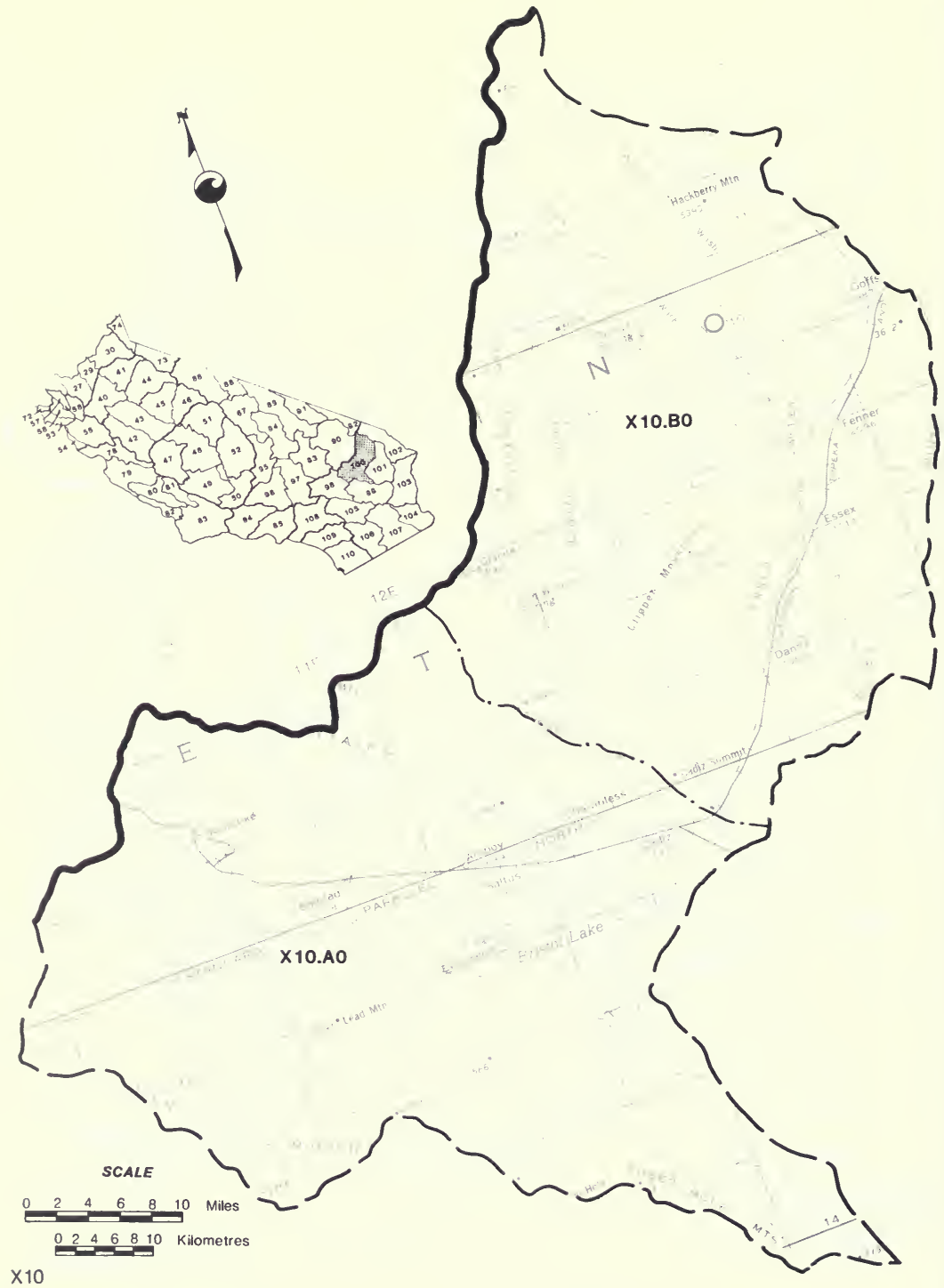




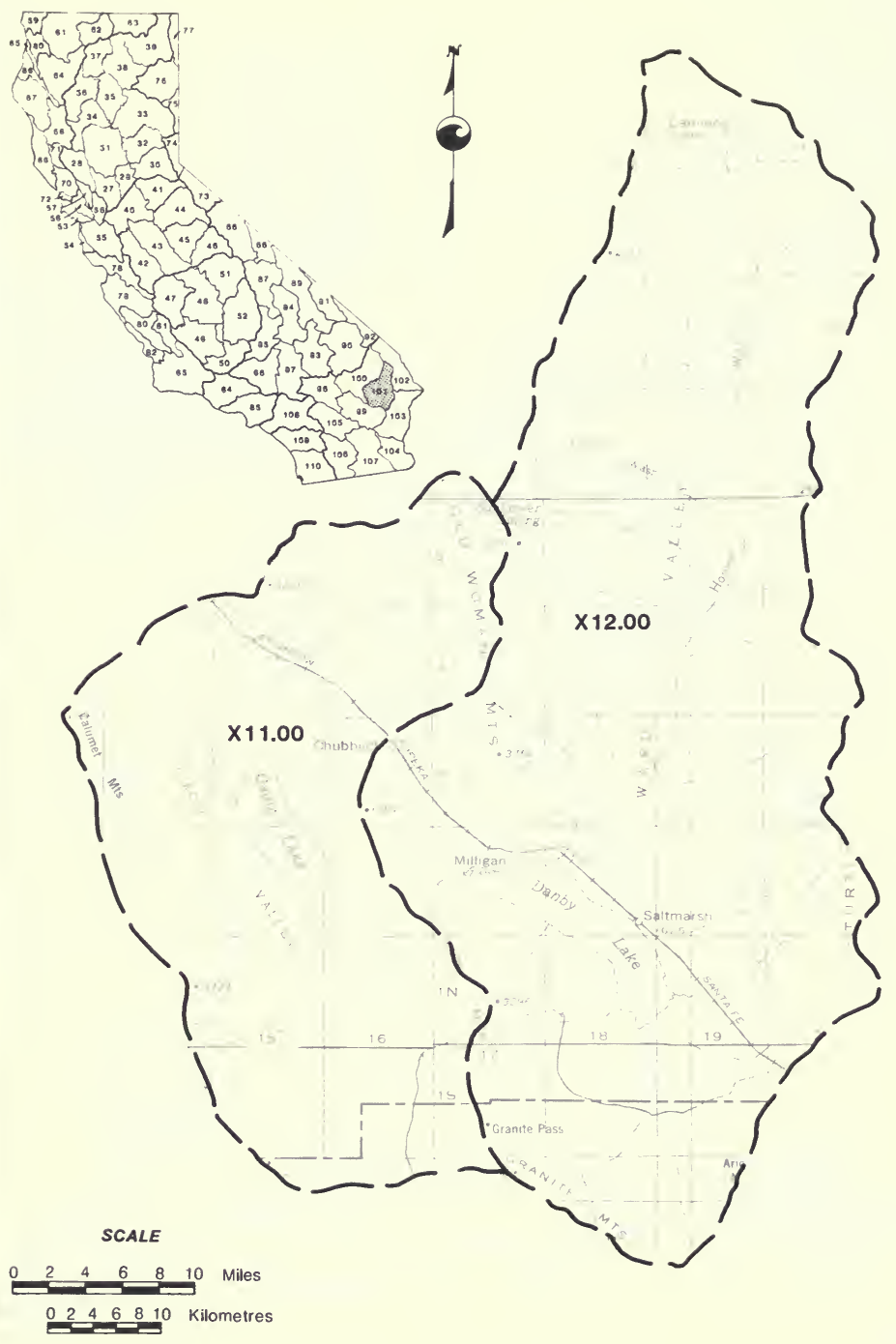

$X 11 \& \times 12$ 


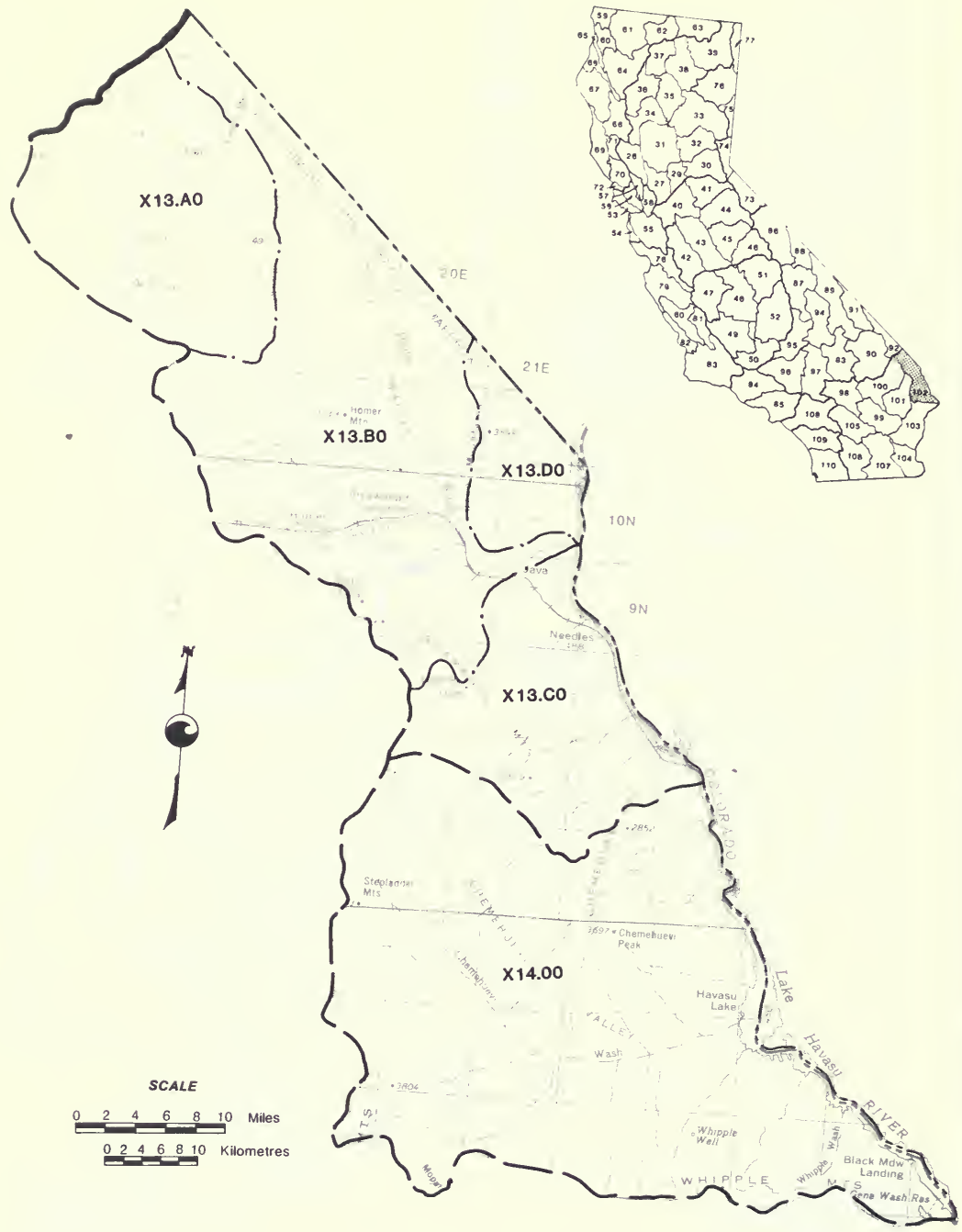

$\times 13 \& \times 14$ 


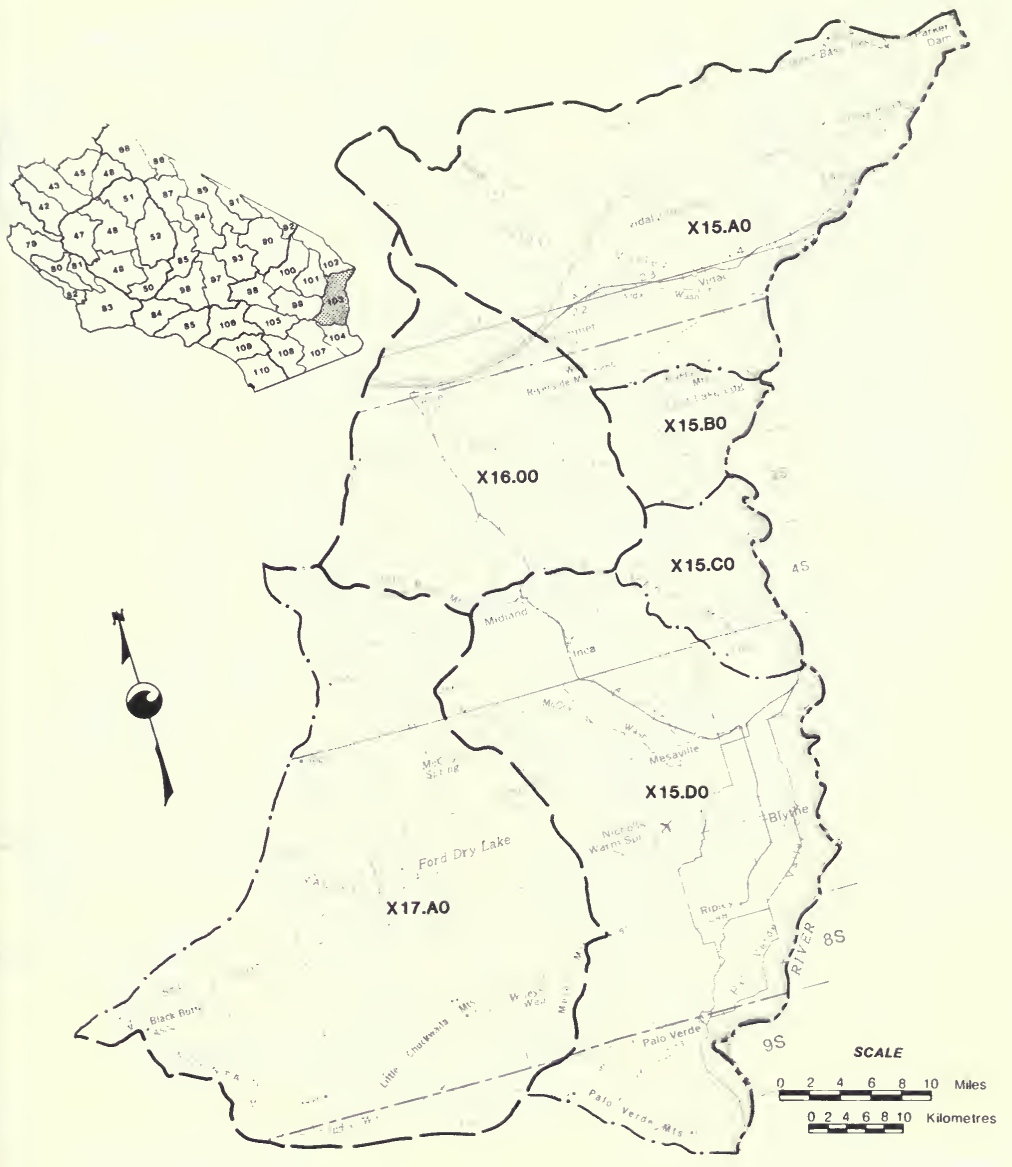

X15.A - D, X16 \& X17.A 


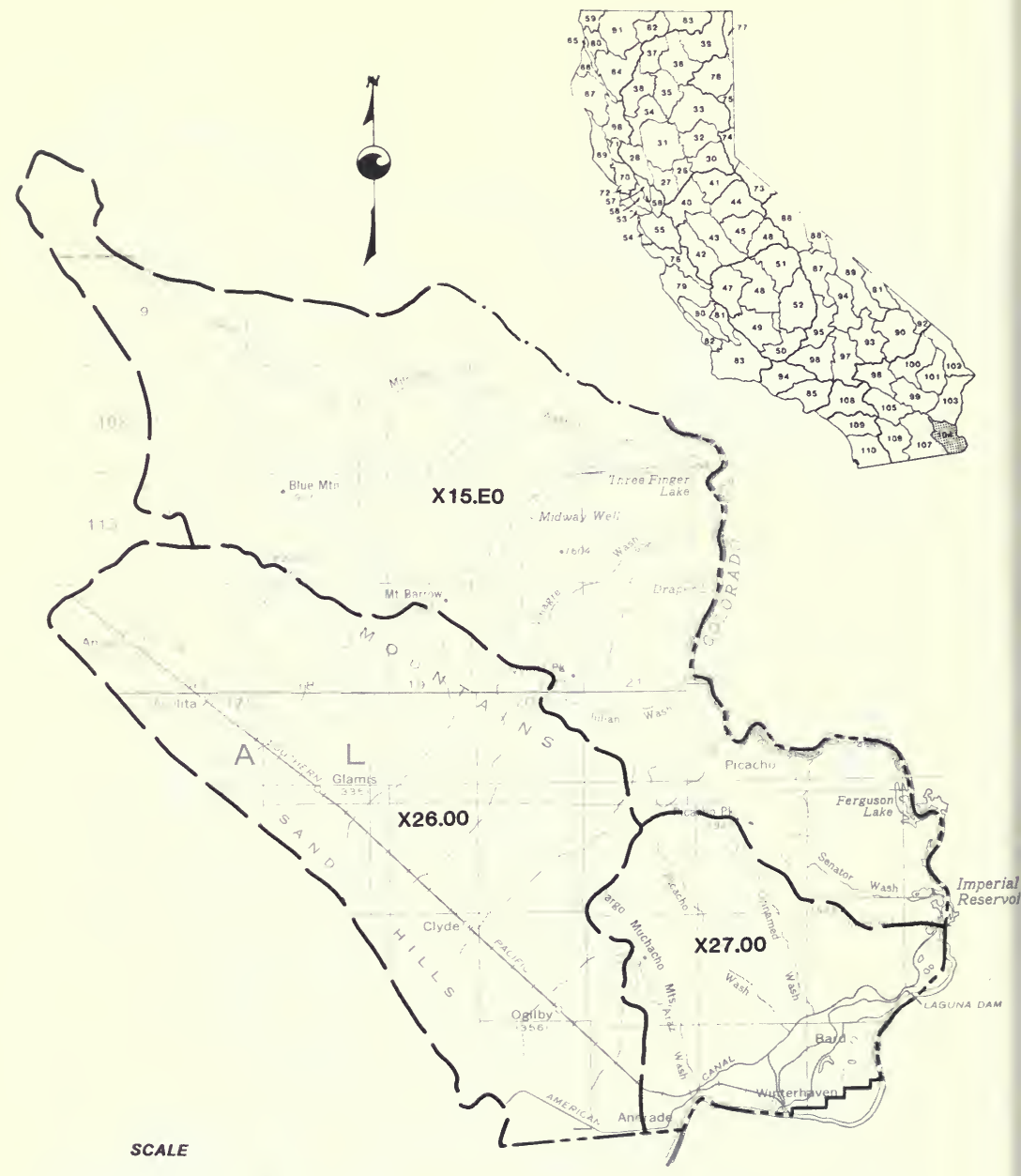

$\begin{array}{lllllll}0 & 2 & 4 & 6 & 8 & 10\end{array}$ Miles

\begin{tabular}{lllllll}
0 & 2 & 4 & 6 & 8 & 10 & Kilometres \\
\hline
\end{tabular}

$X 15 . E, X 26 \&$ X27 


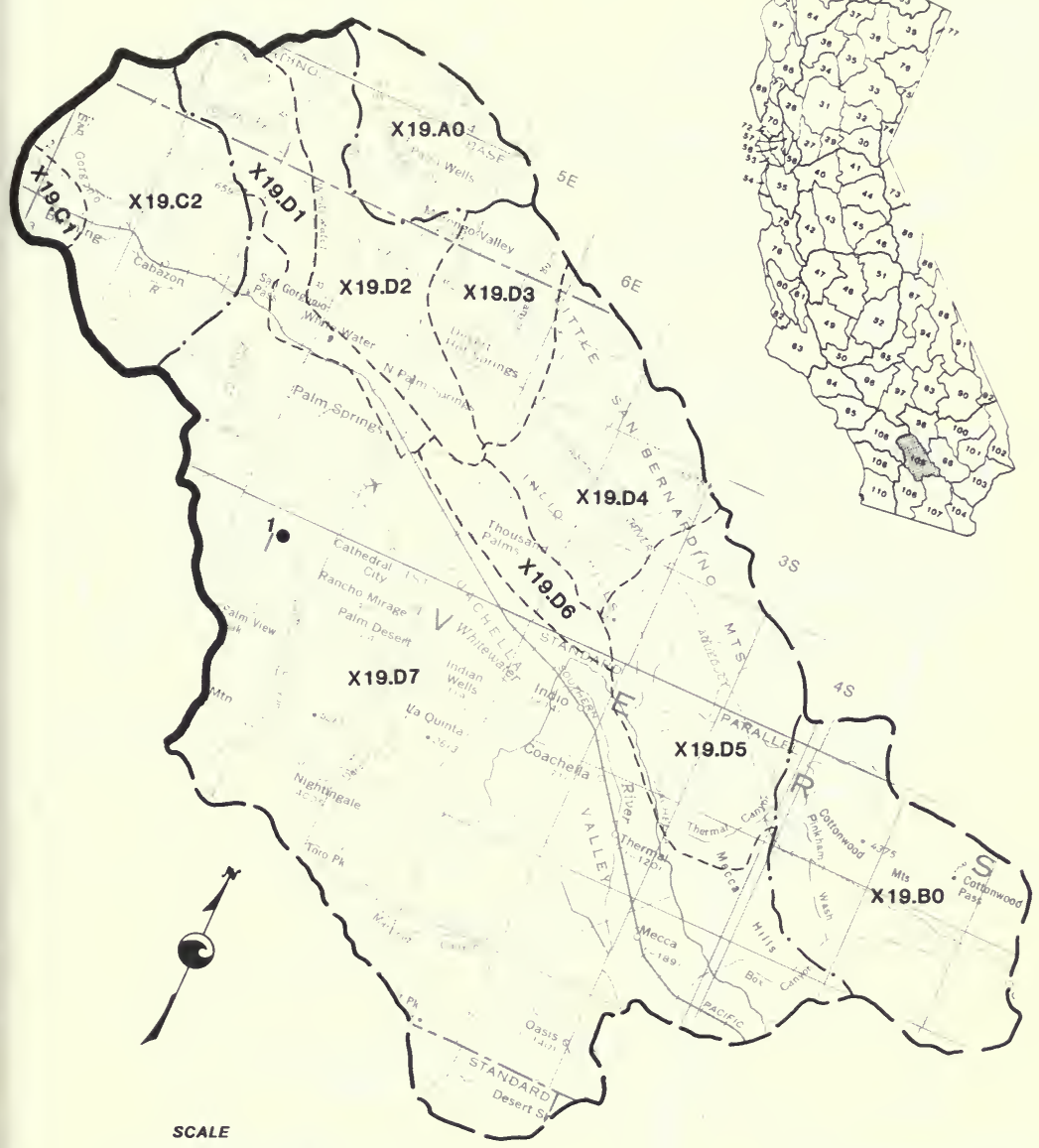

\begin{tabular}{|lcccccc}
0 & 2 & 4 & 6 & 8 & 10 & Miles \\
\hline & 0 & 2 & 4 & 6 & 8 & 10
\end{tabular} 


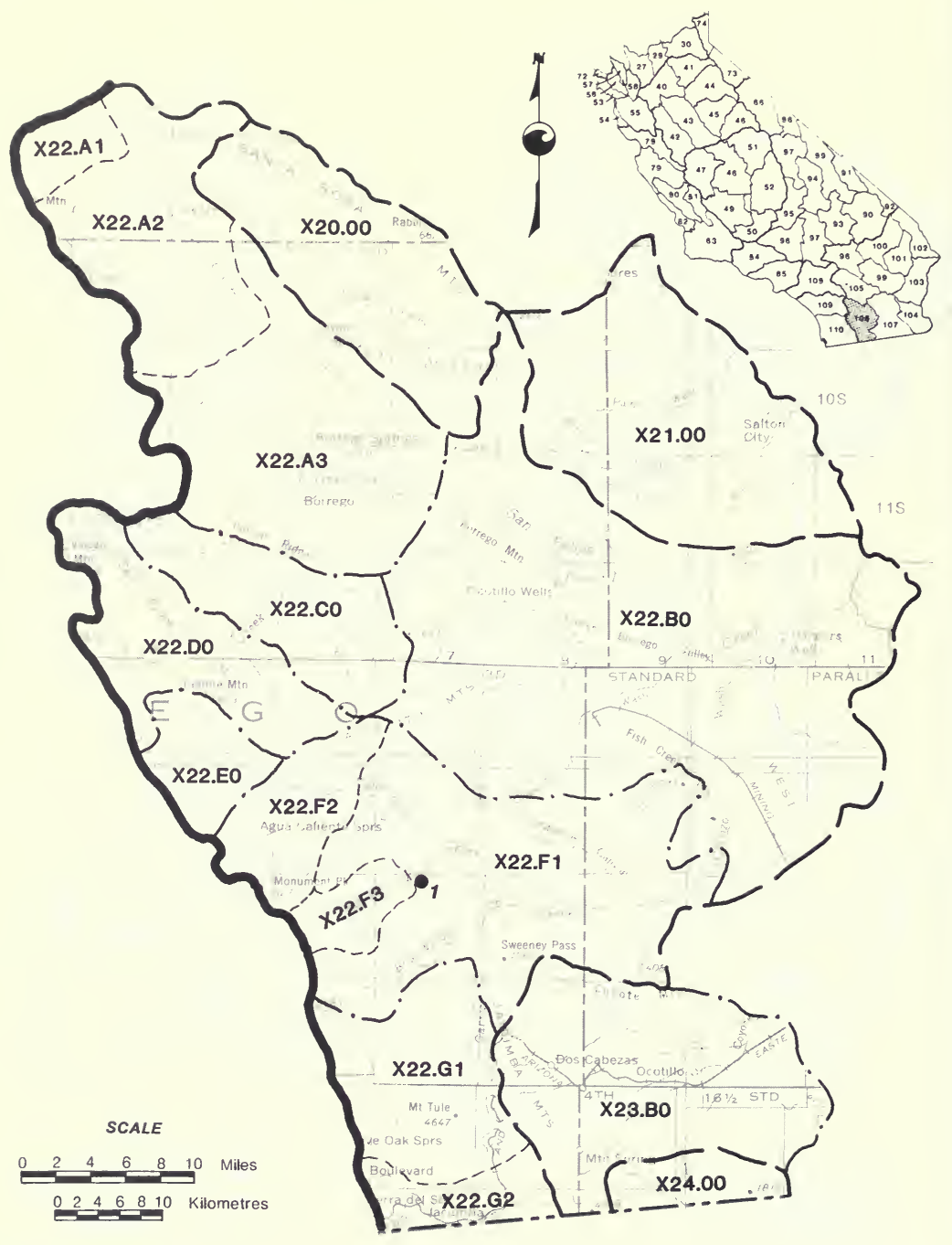

$X 20-X 23 . B \& X 24$ 


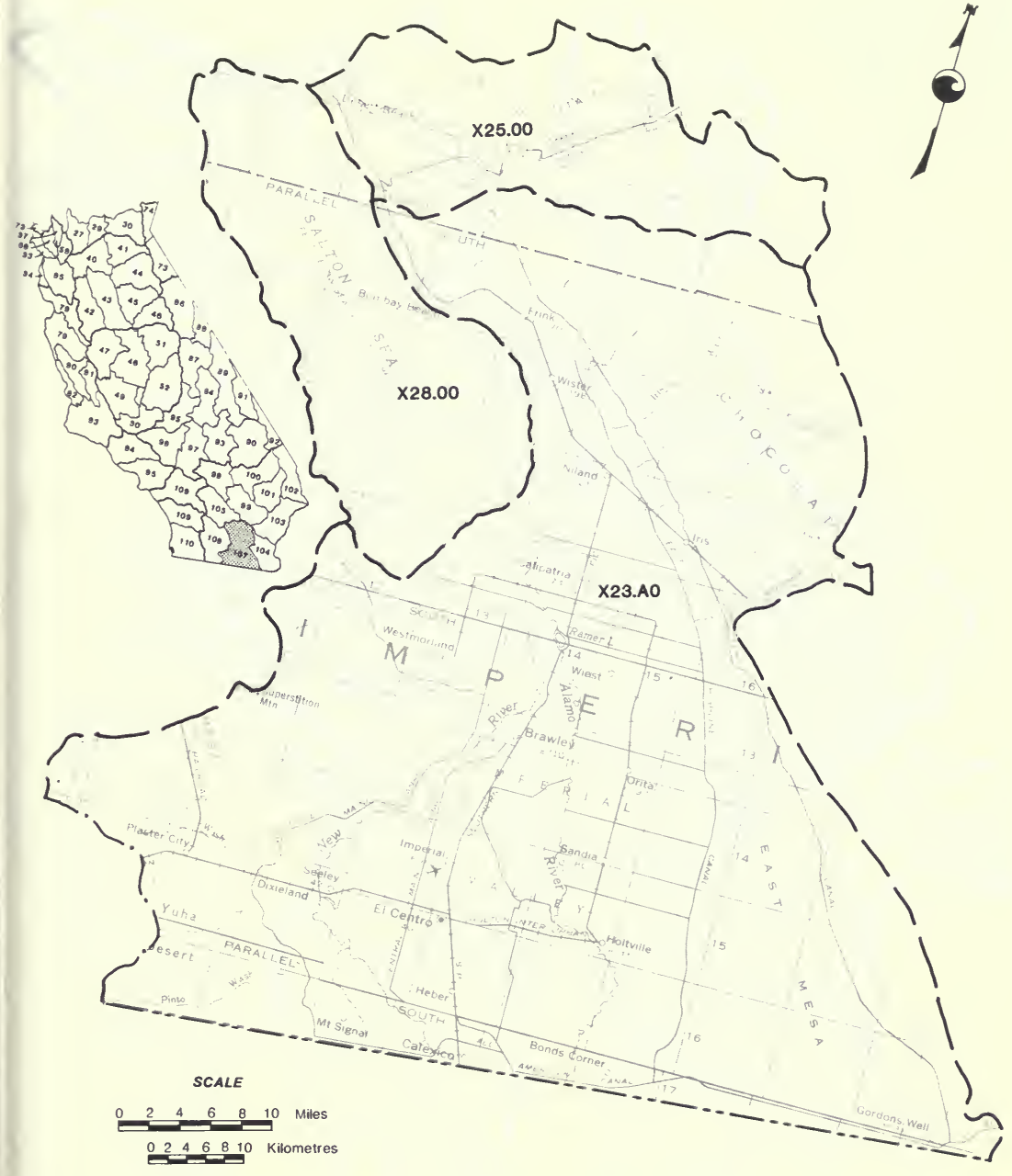

X23.A, X25 \& X28 


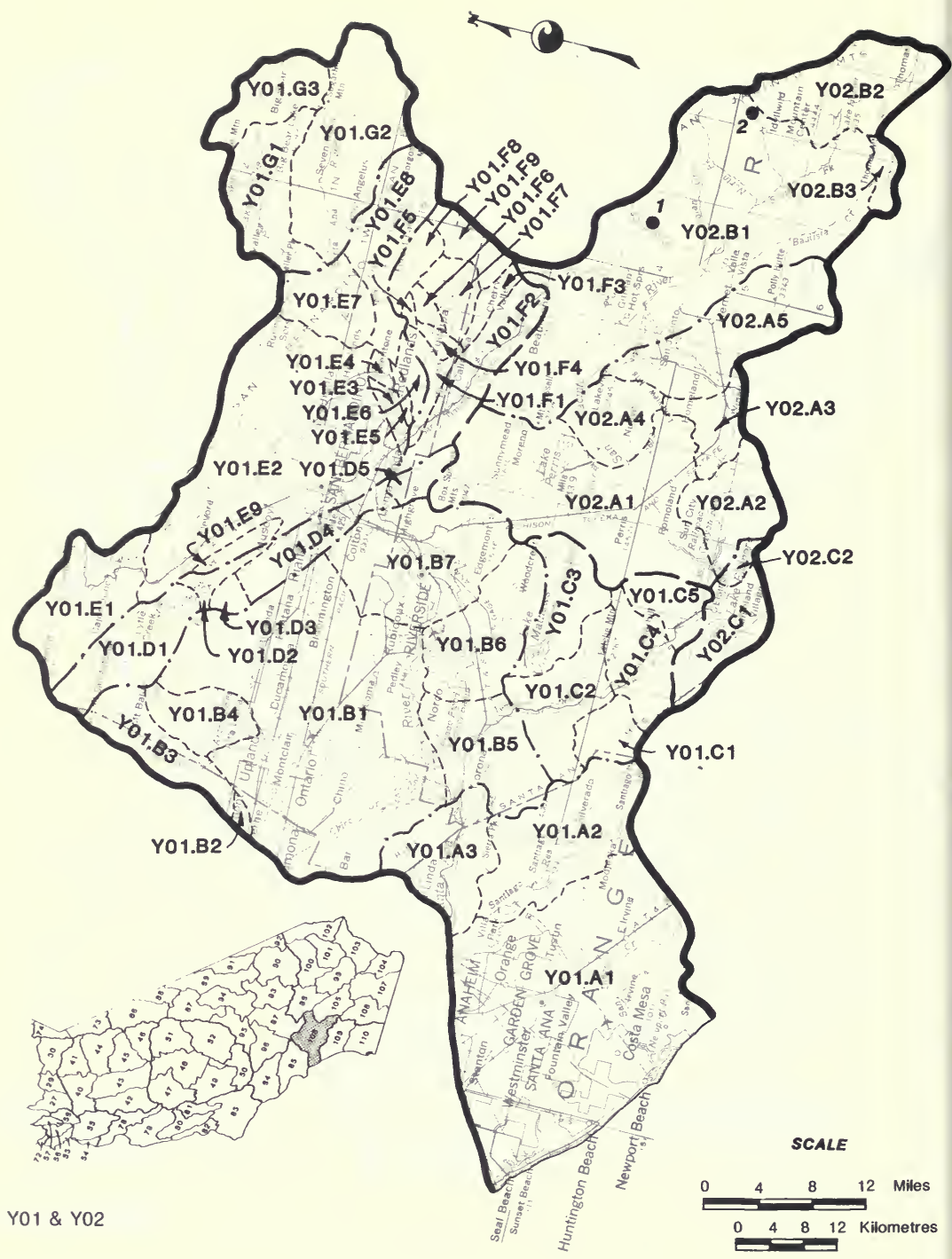




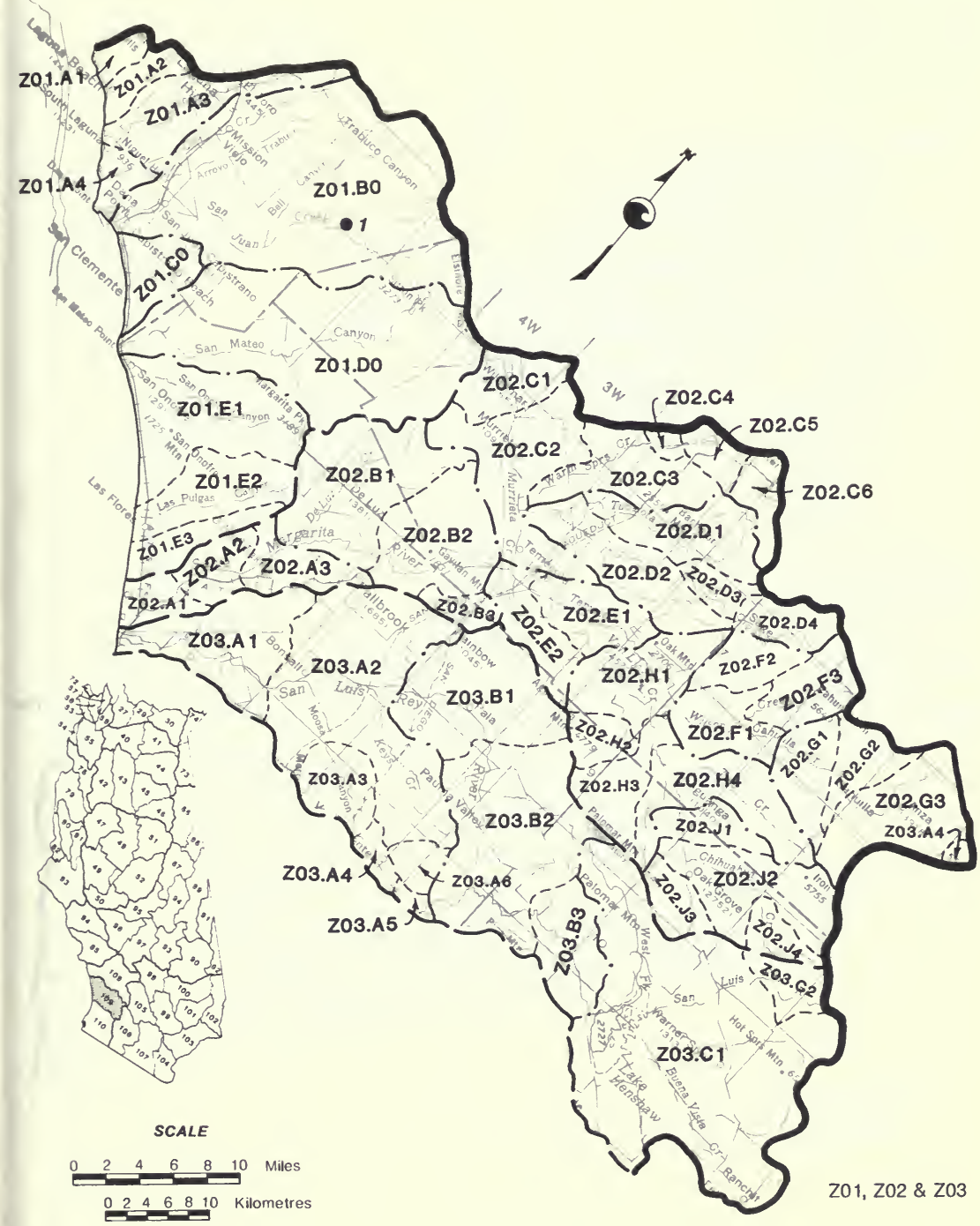




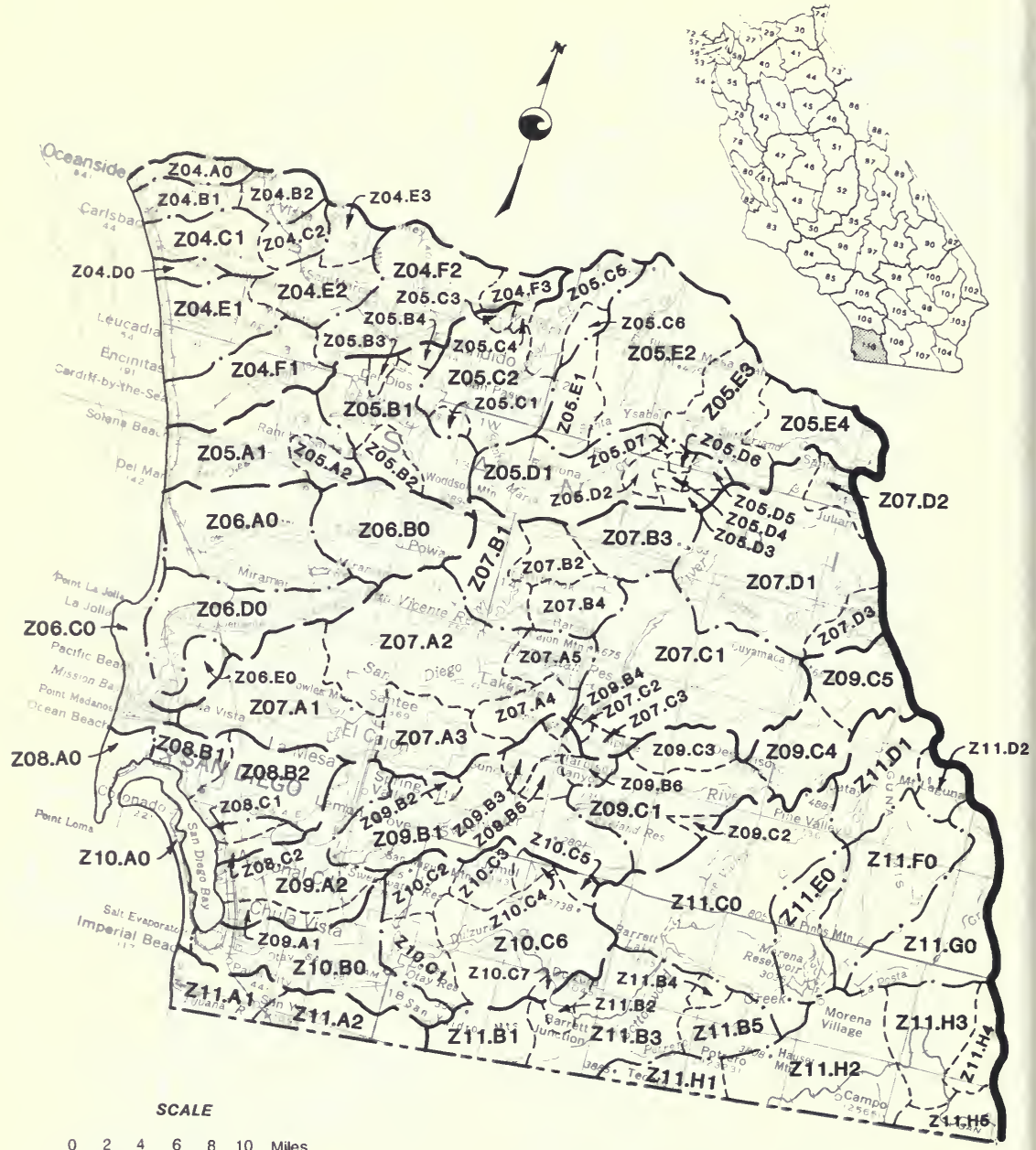

\begin{tabular}{lllllll}
0 & 2 & 4 & 6 & 8 & 10 & Kilometres \\
\hline
\end{tabular} 
Table of

Instream Flow Requirements

Related to

Stream Diversions 


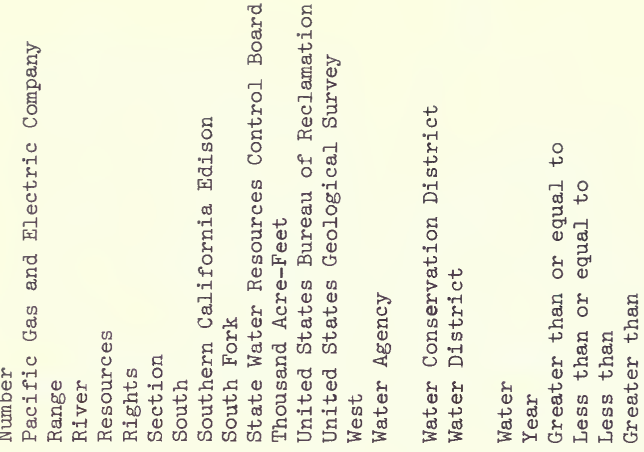

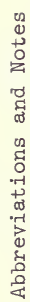

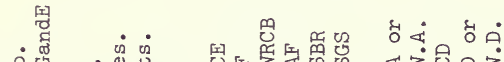

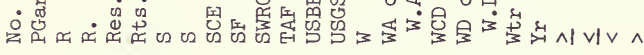

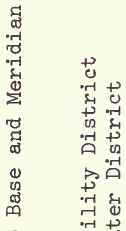

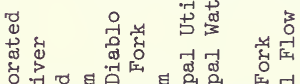

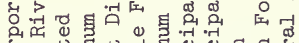
究

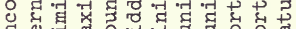

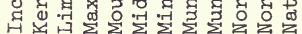

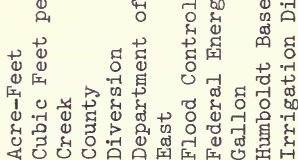

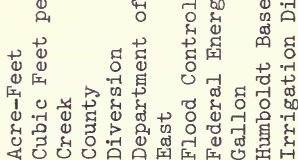

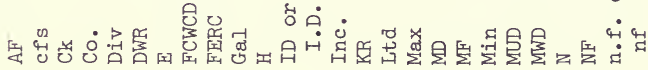



若

嵌 ฮัర్ష

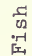

我

3 \&

क्ष

尊

$=$

옹ำ

() 'न

近

\&ै।

4ै⿴囗十)

4

氙罗

光

क्ष ठ․ำ

प1

लै

(1)

के $\stackrel{\infty}{0}$ 过

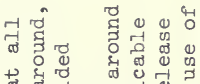

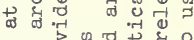

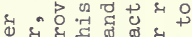

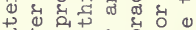

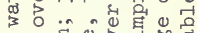

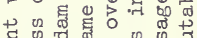

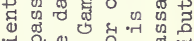

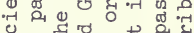

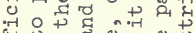
क्षे उ का वै का క उ क स्व न्ل

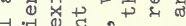
न न 记

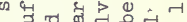

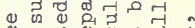
๖ + छี न्र

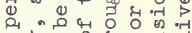
1 $\overrightarrow{0} 0$ है तो

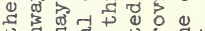

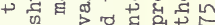

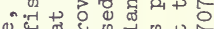
वै क्ष क्ष ठ ळ प

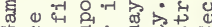

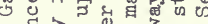
ช สี

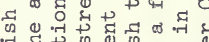

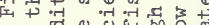

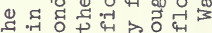
म

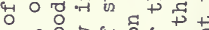
施施出 ๙ิ जू

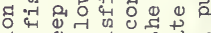
ॠ ผ थ 000 द्व ठำ

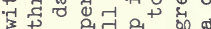
ब थ कू के क्ये क क्ष

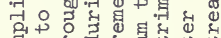
贾

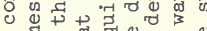

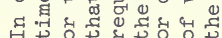


कृ

云高

กั

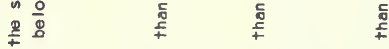

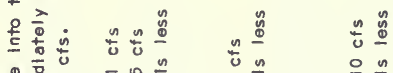

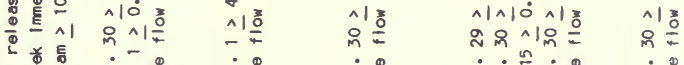

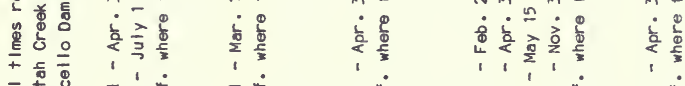

安

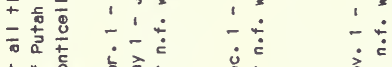

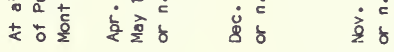

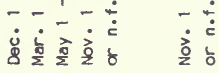

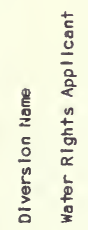

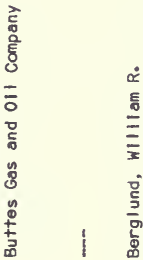

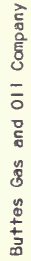

$\stackrel{m}{r}$

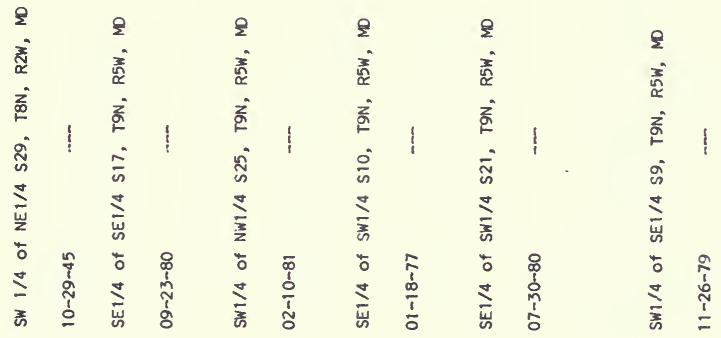

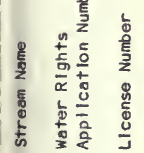

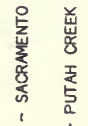

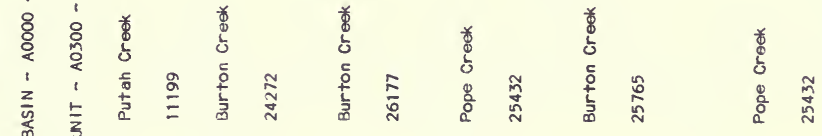

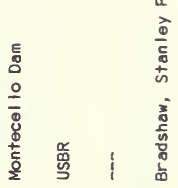

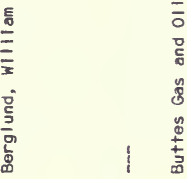

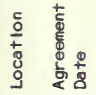

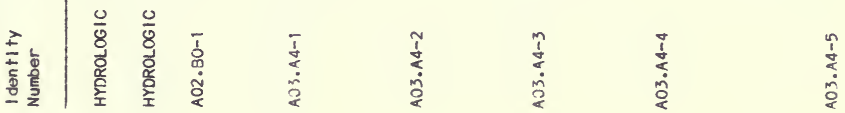




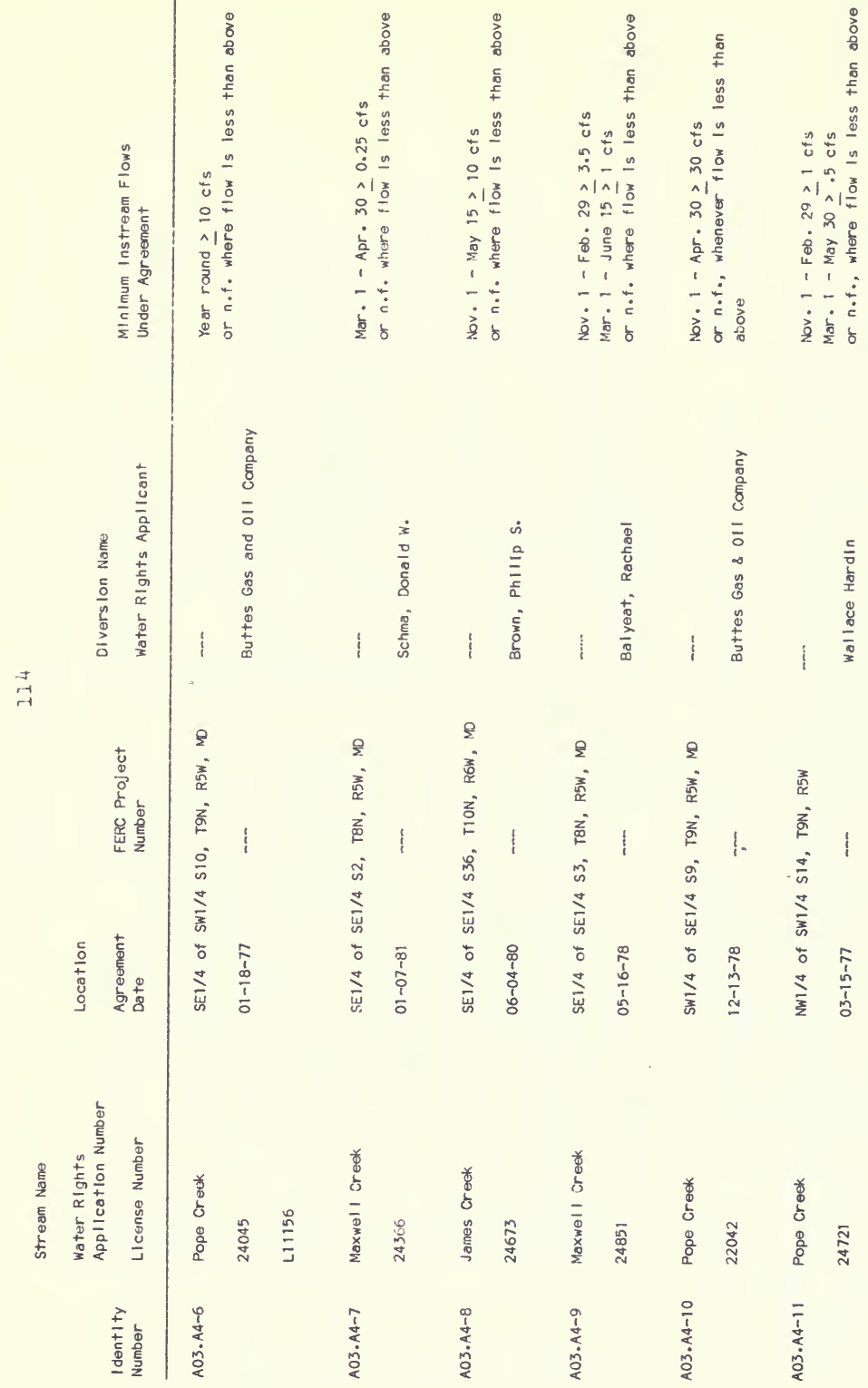




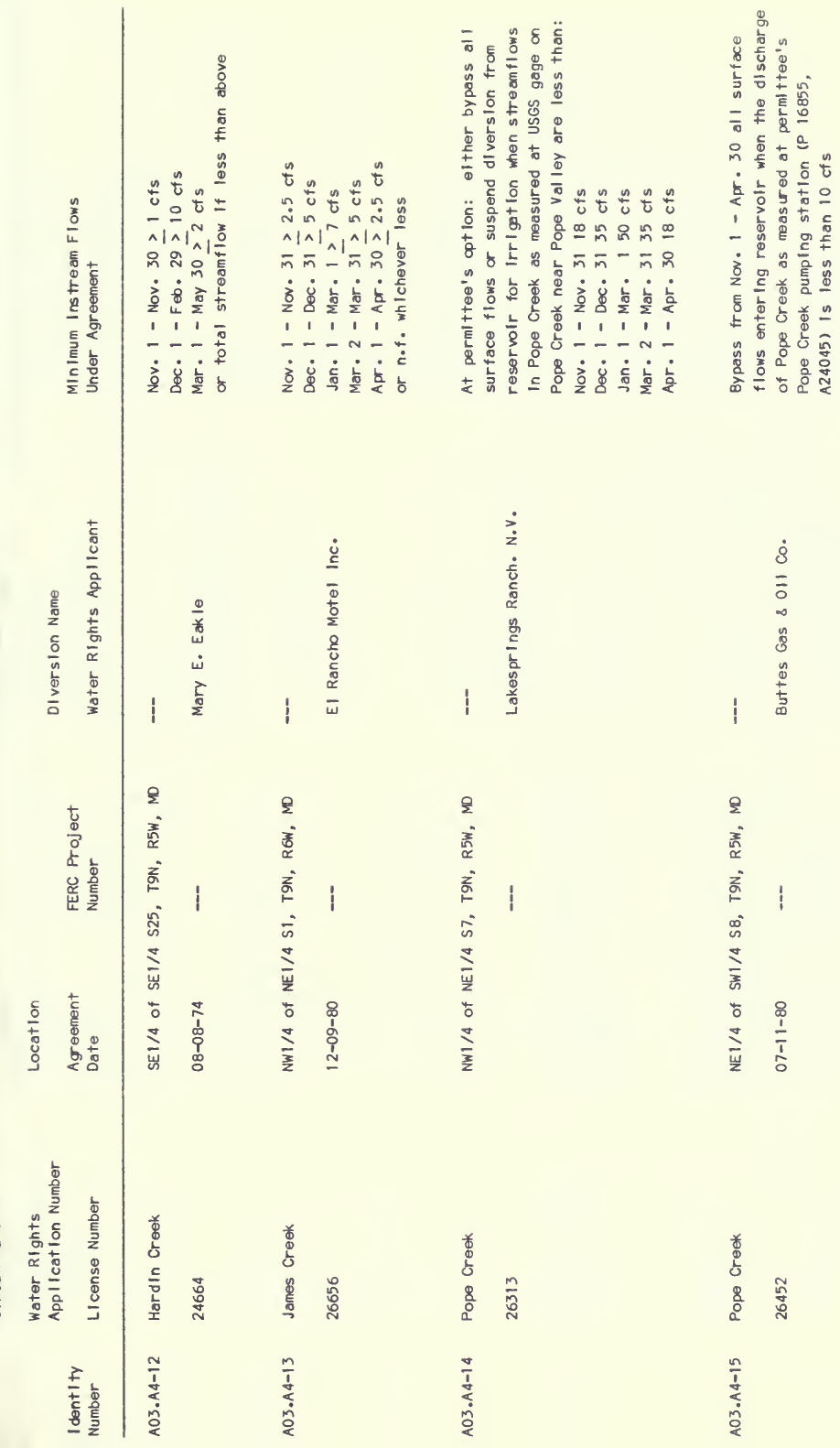




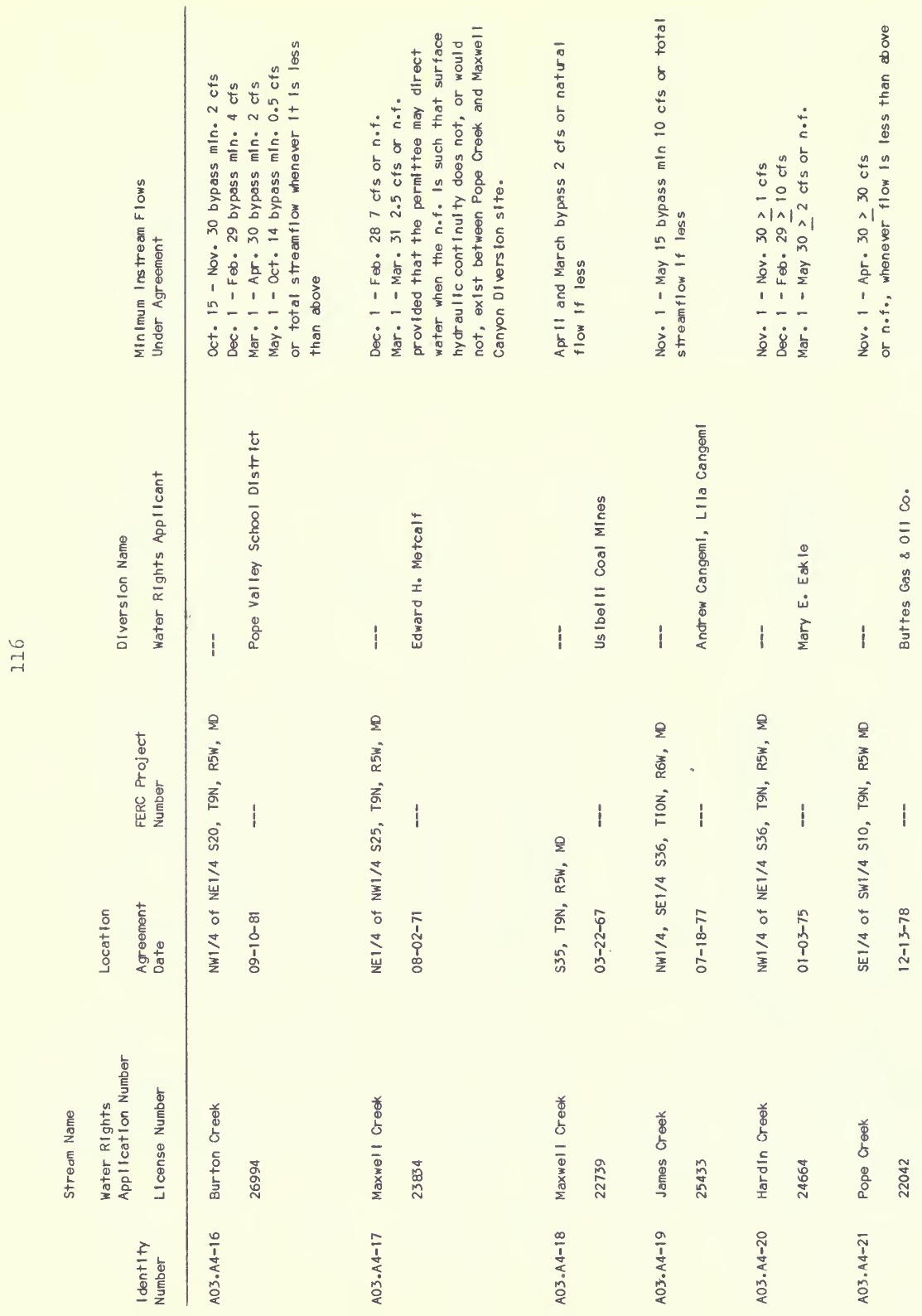




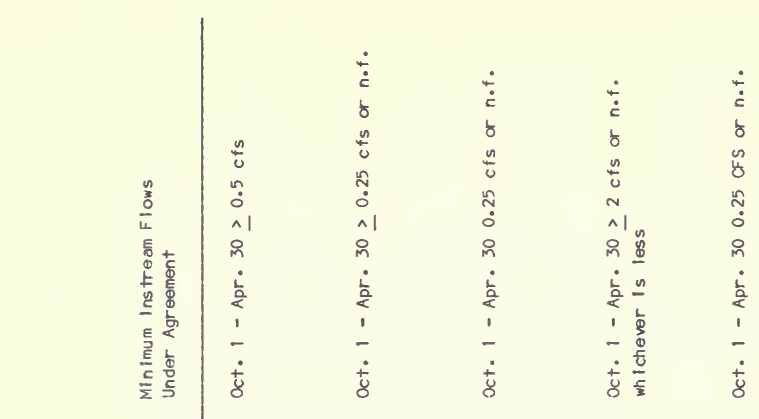

$\stackrel{\infty}{\exists}$
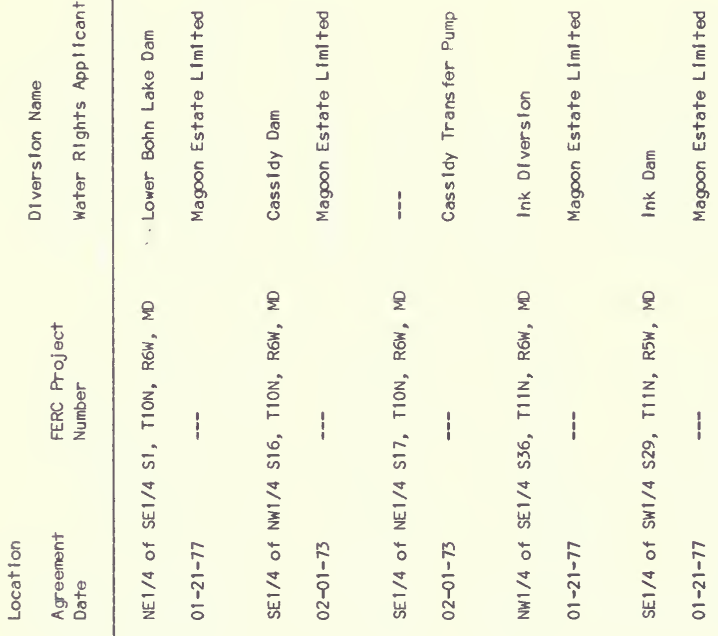

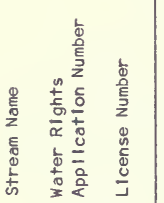

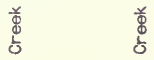

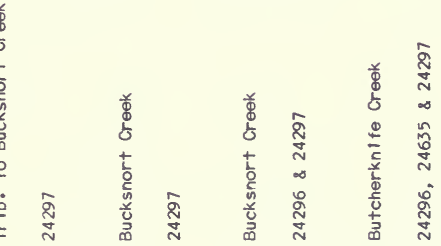

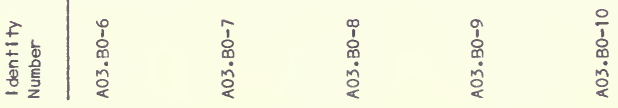




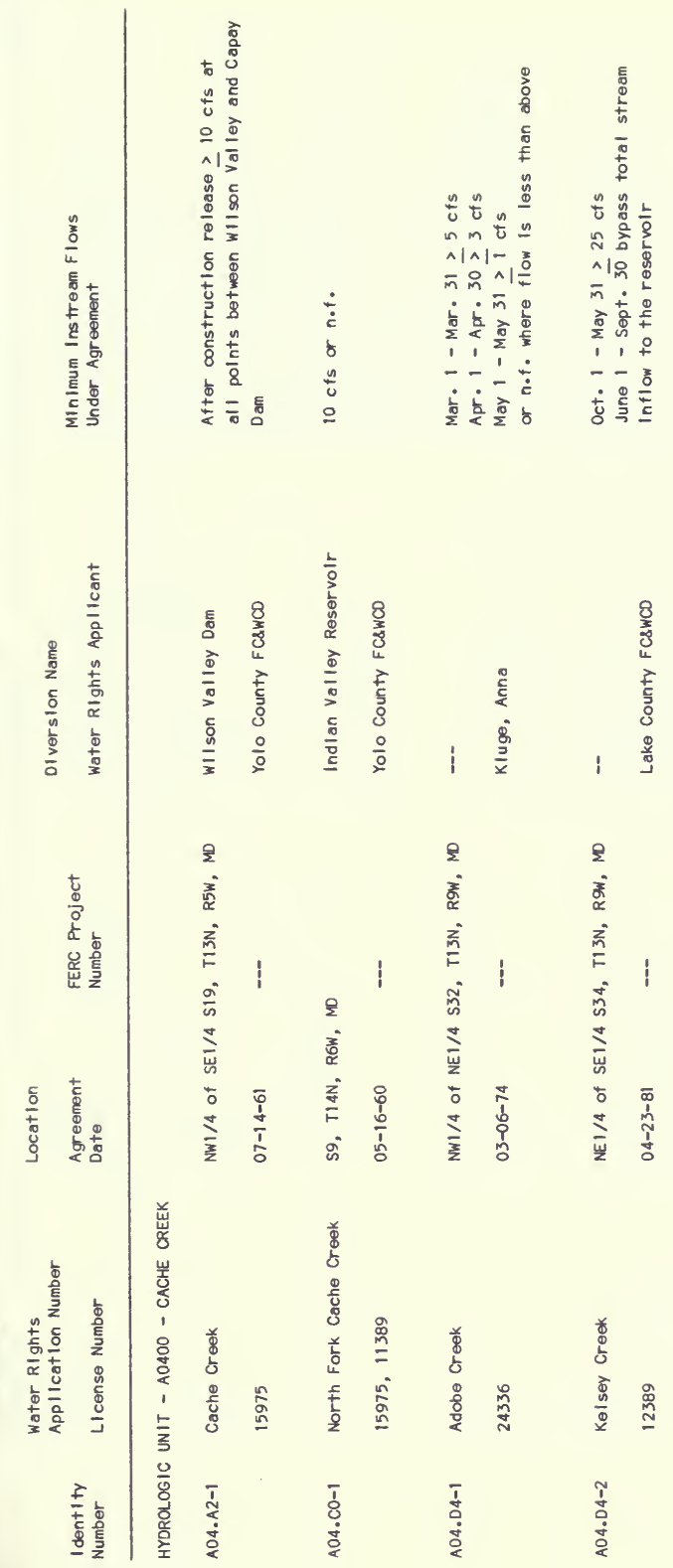




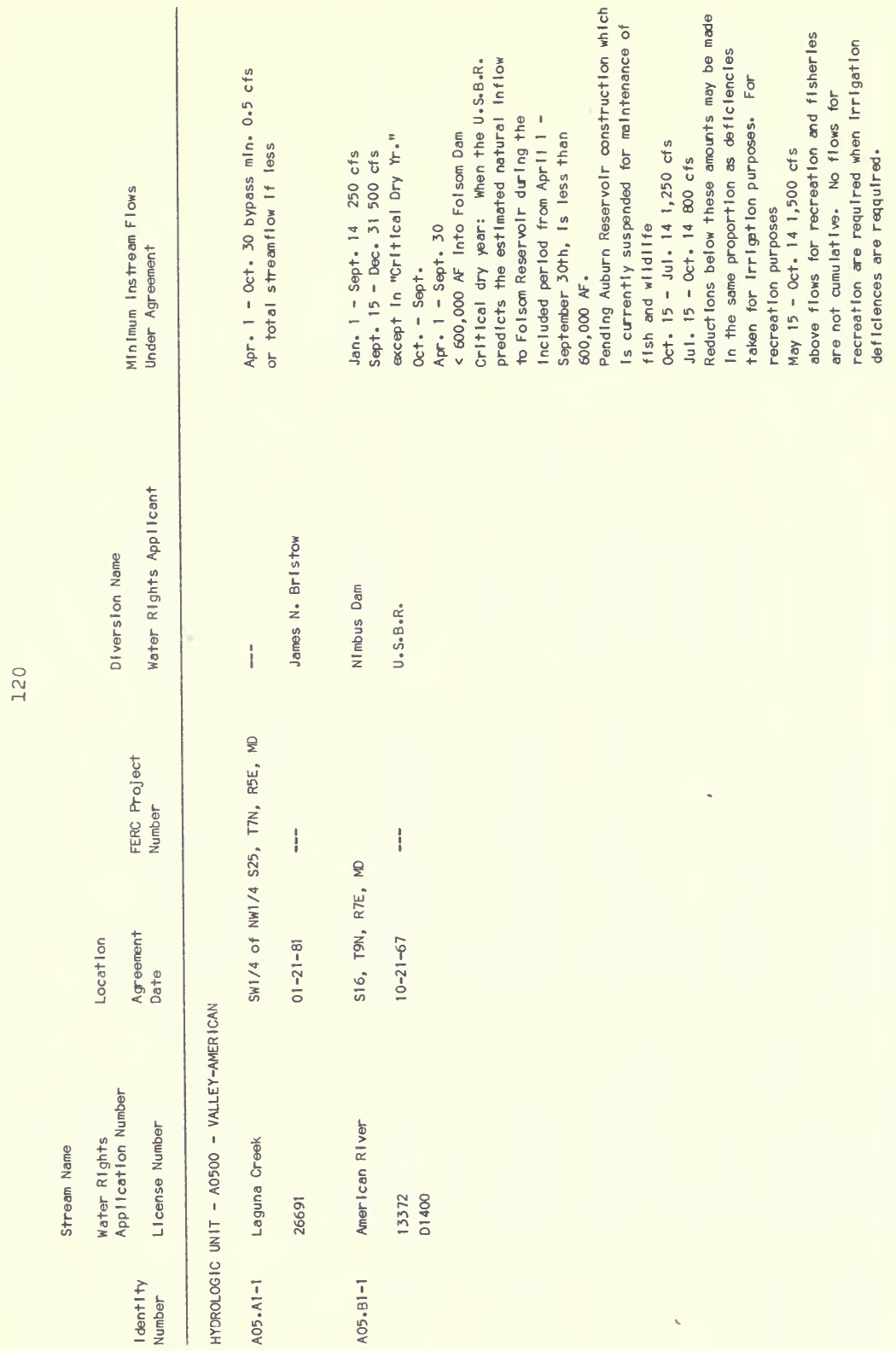




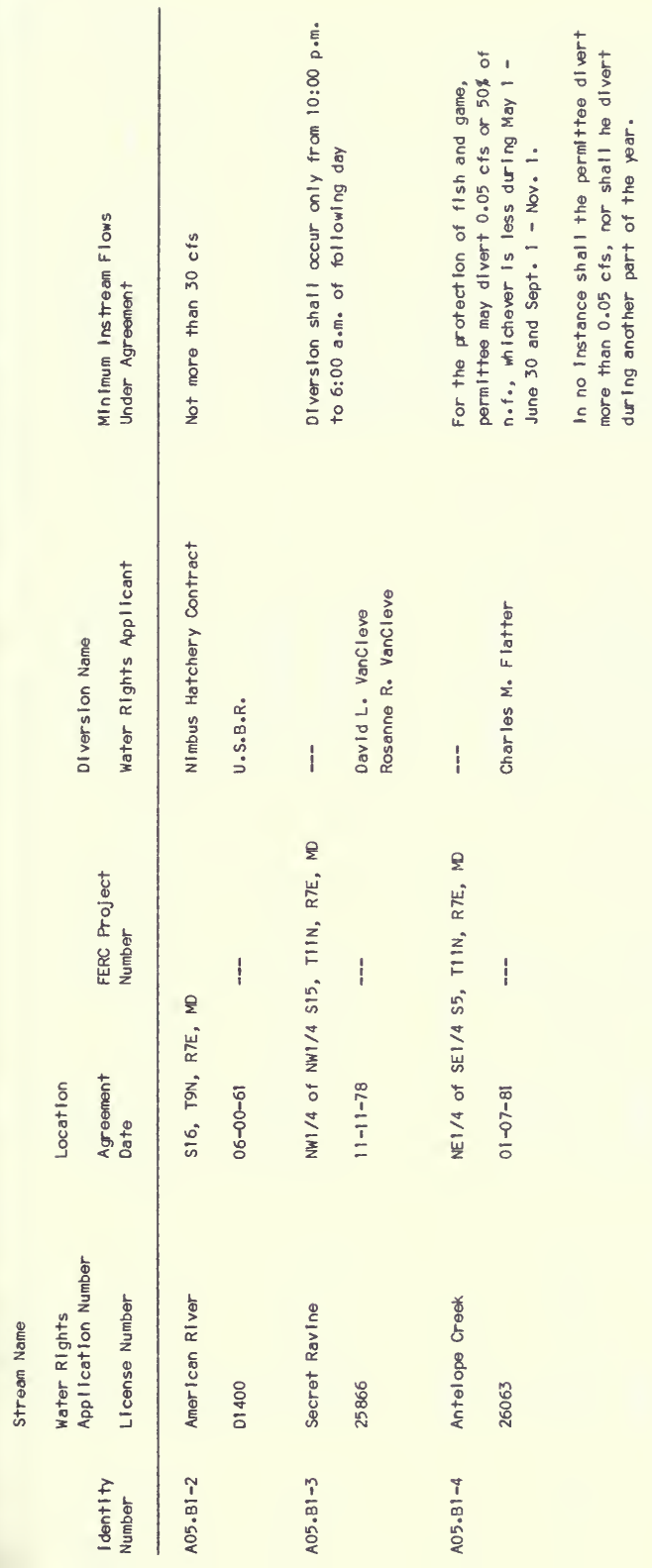




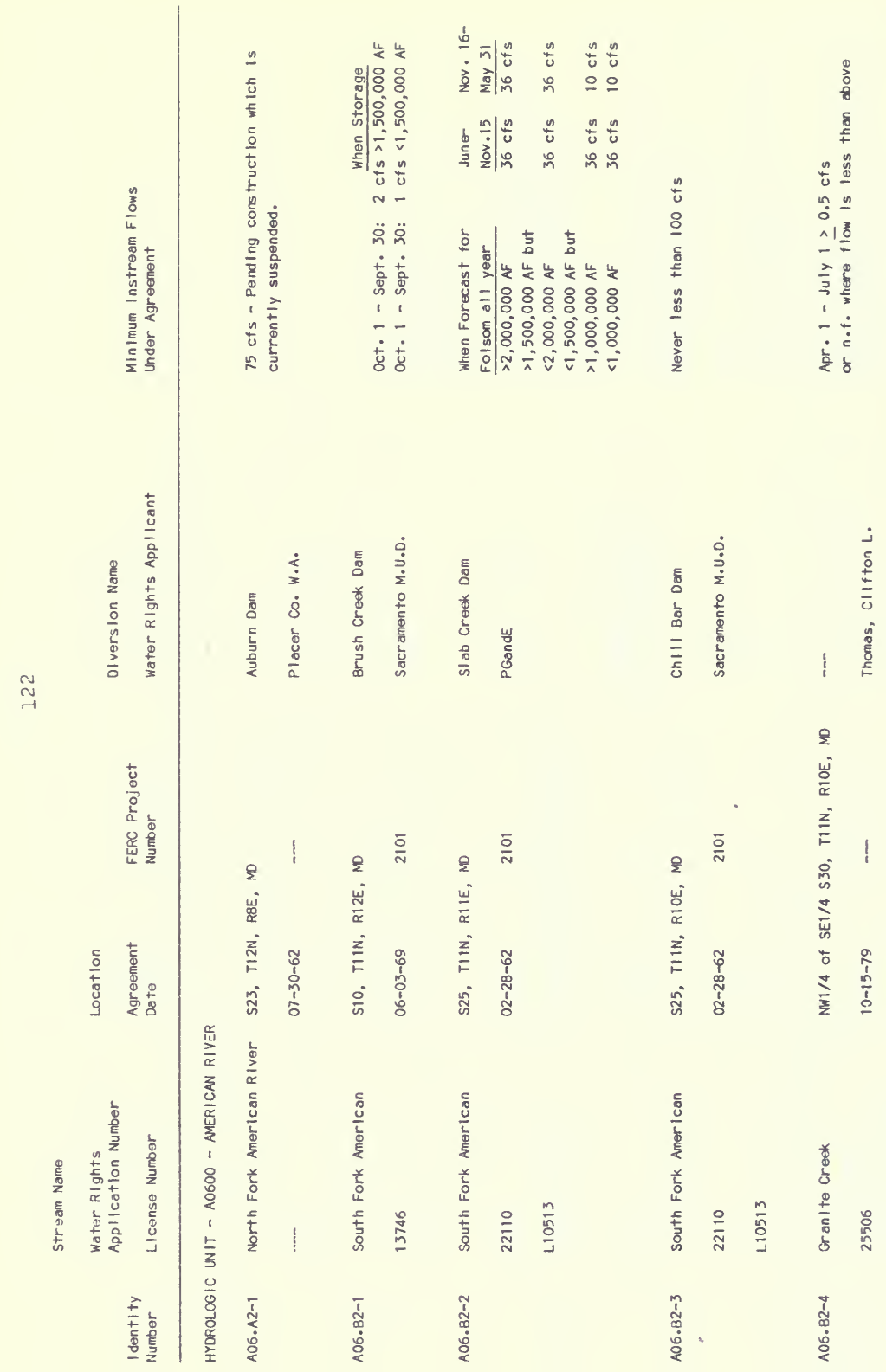




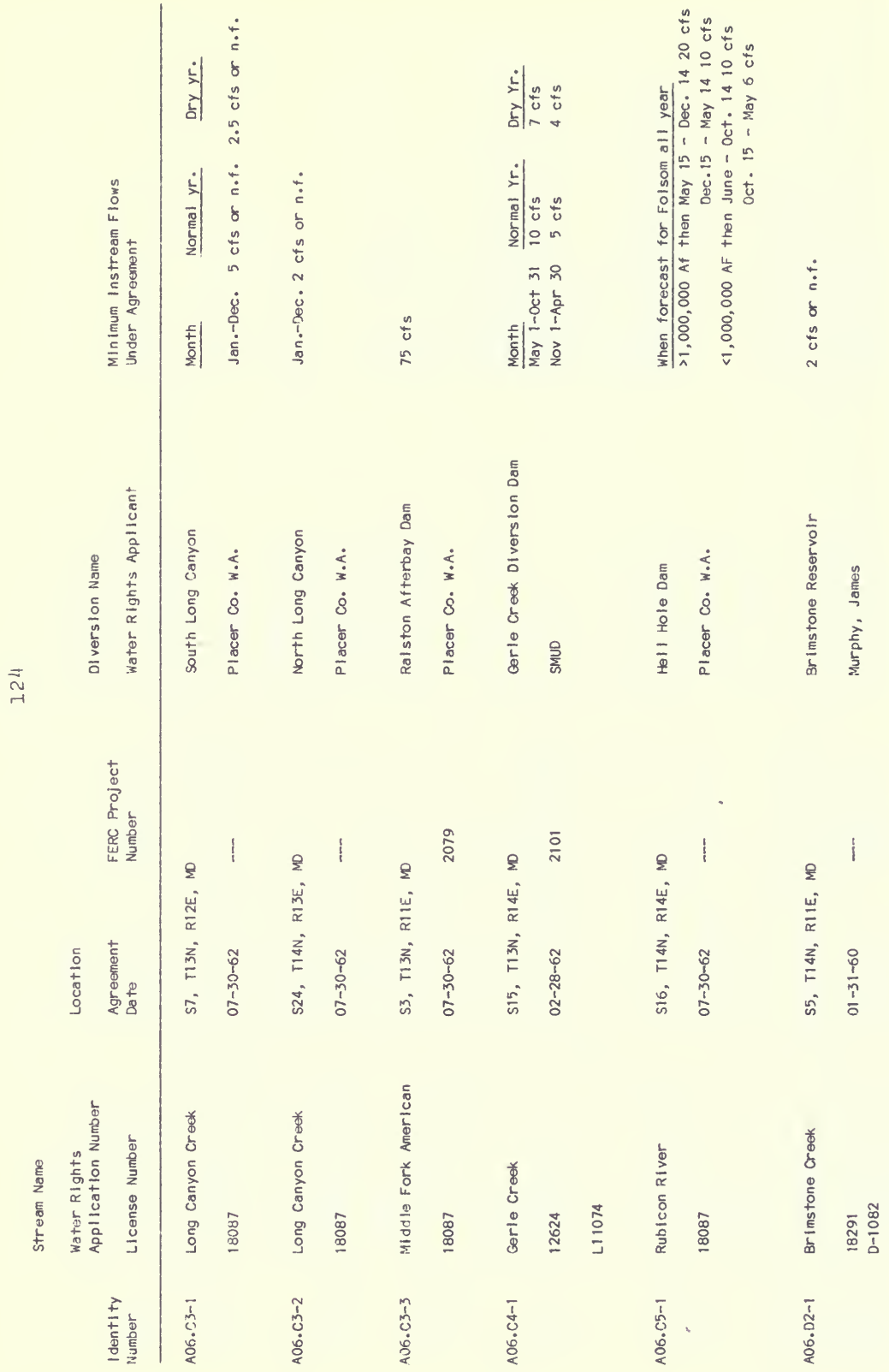




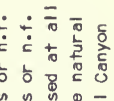

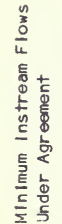

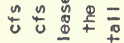

⿰幺𠃌

的齿恕点

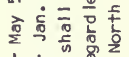

1108

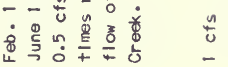

$\frac{n}{6}$

돈

+ 을

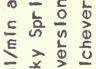

बํํㅇ

ᄂ

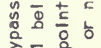

วิำ 웅

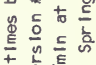

=

世立品桌

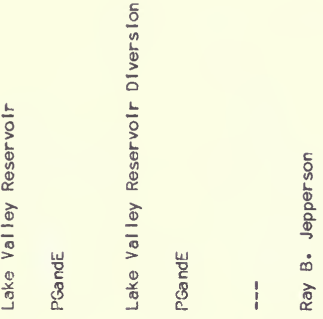

온 올욜

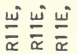

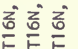

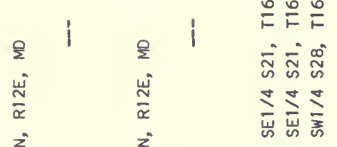

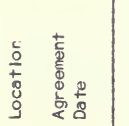

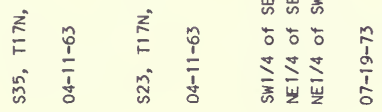

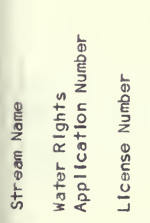

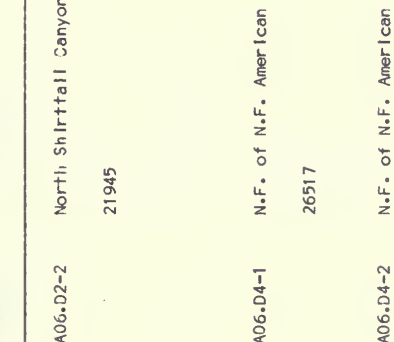

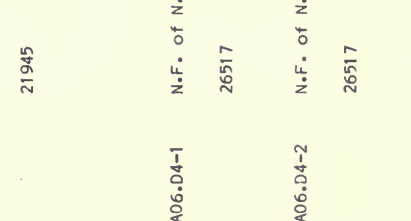

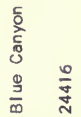




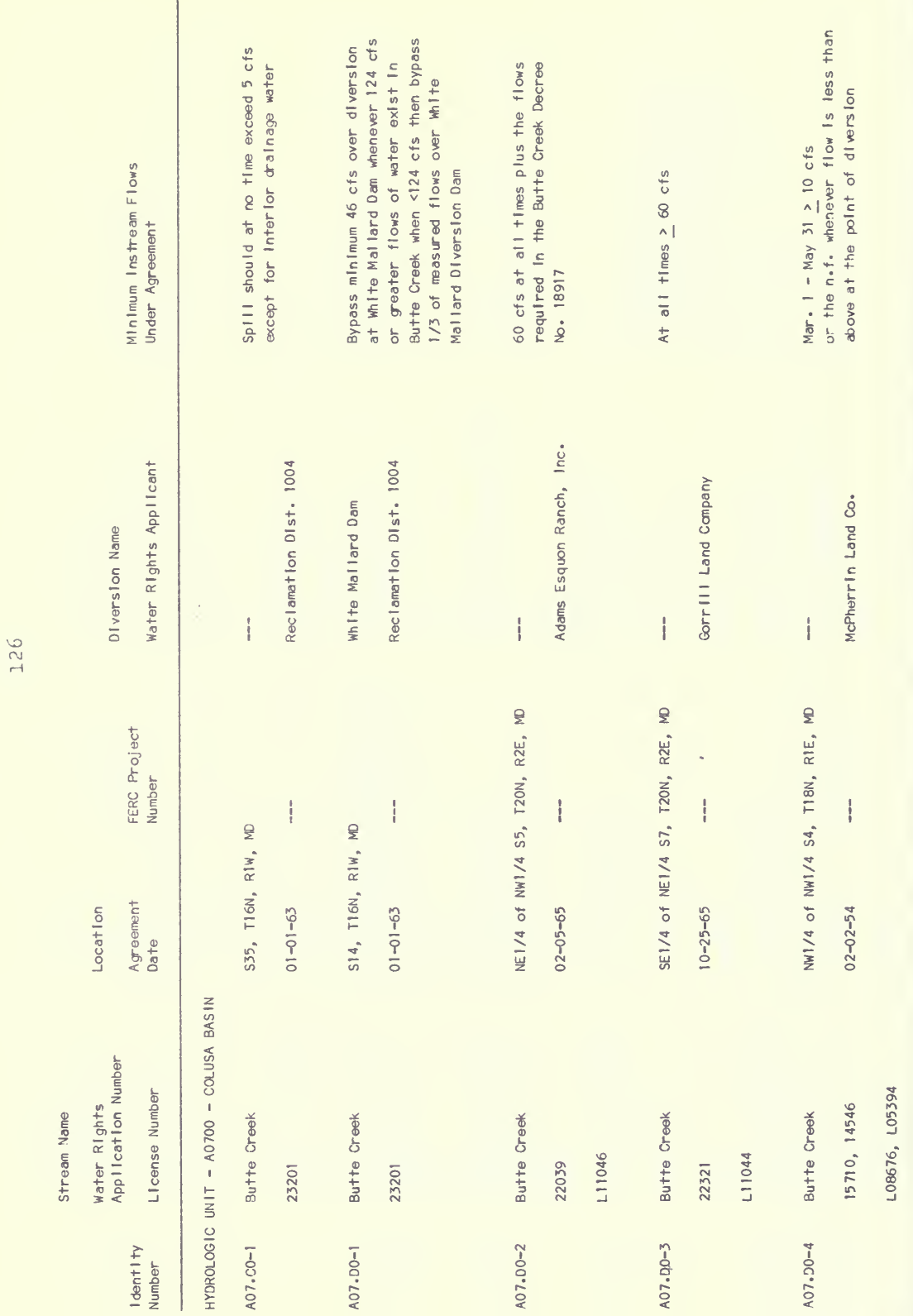




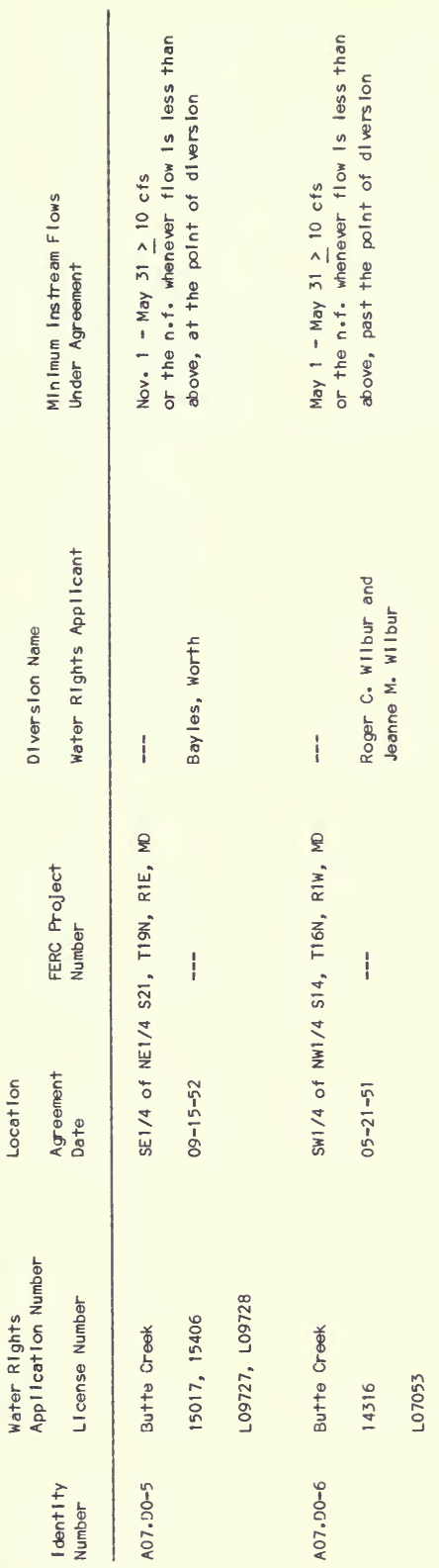




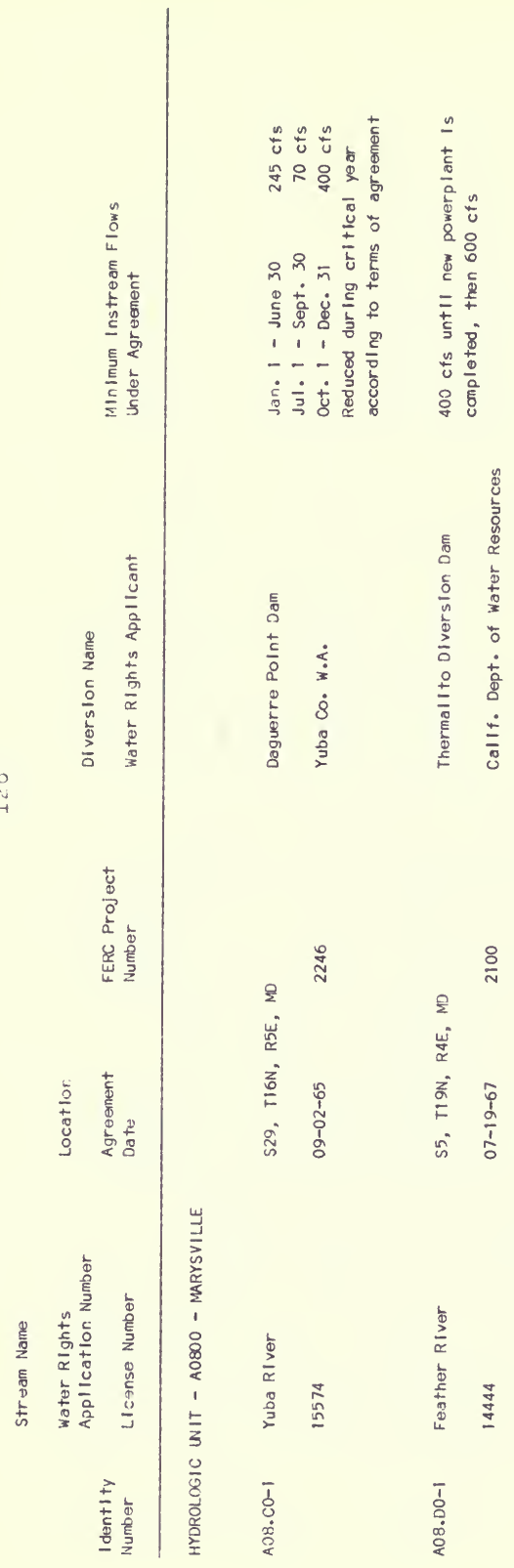




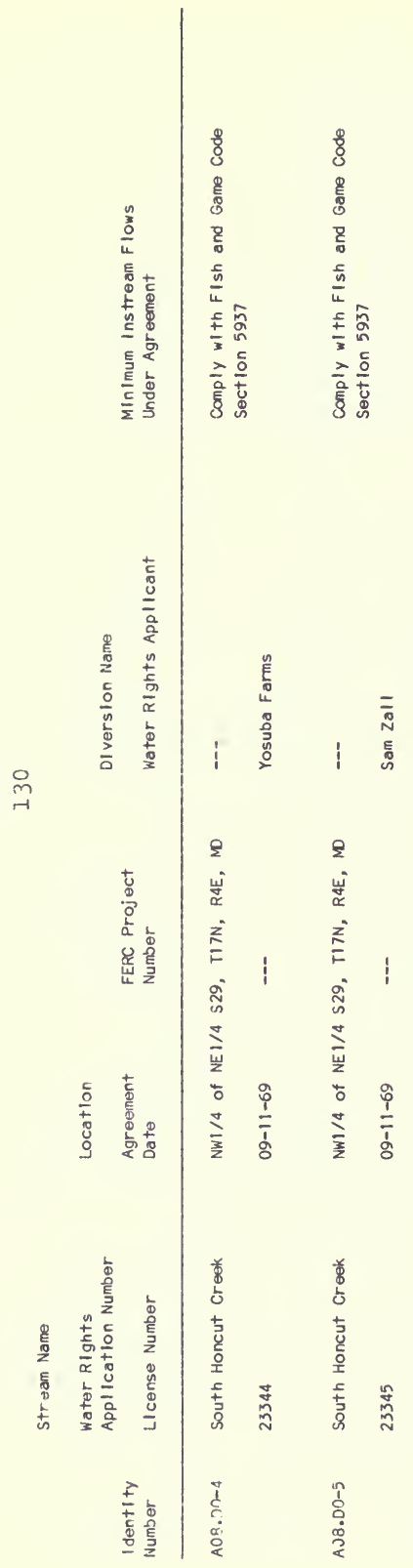




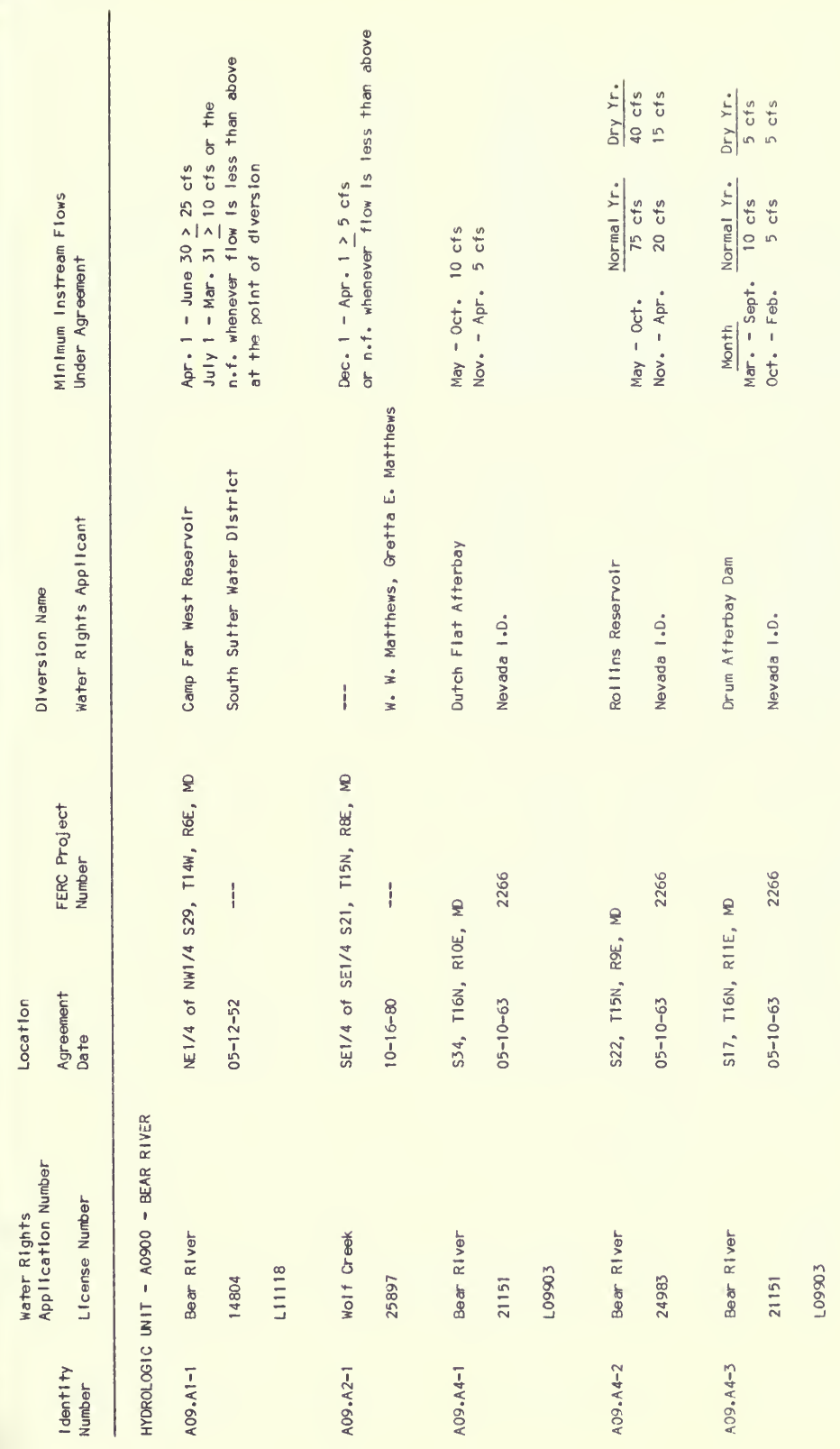




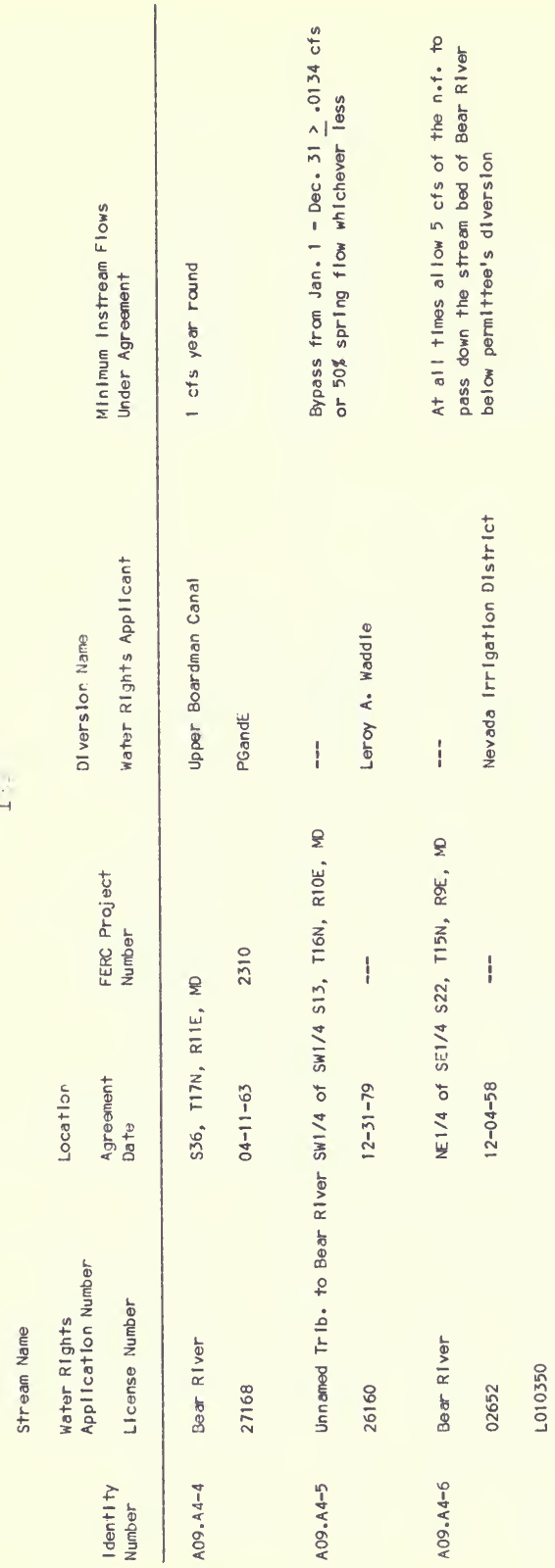




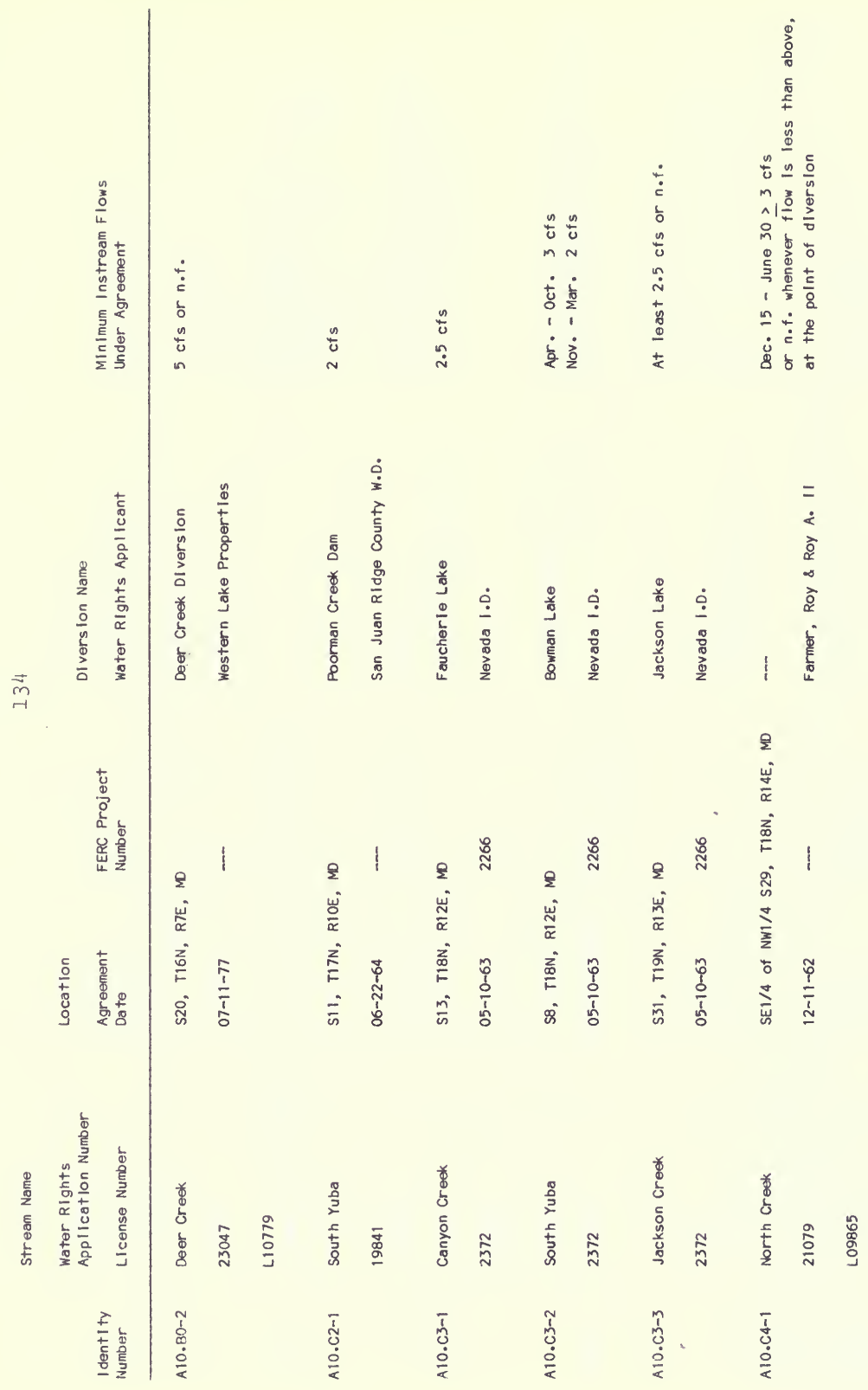




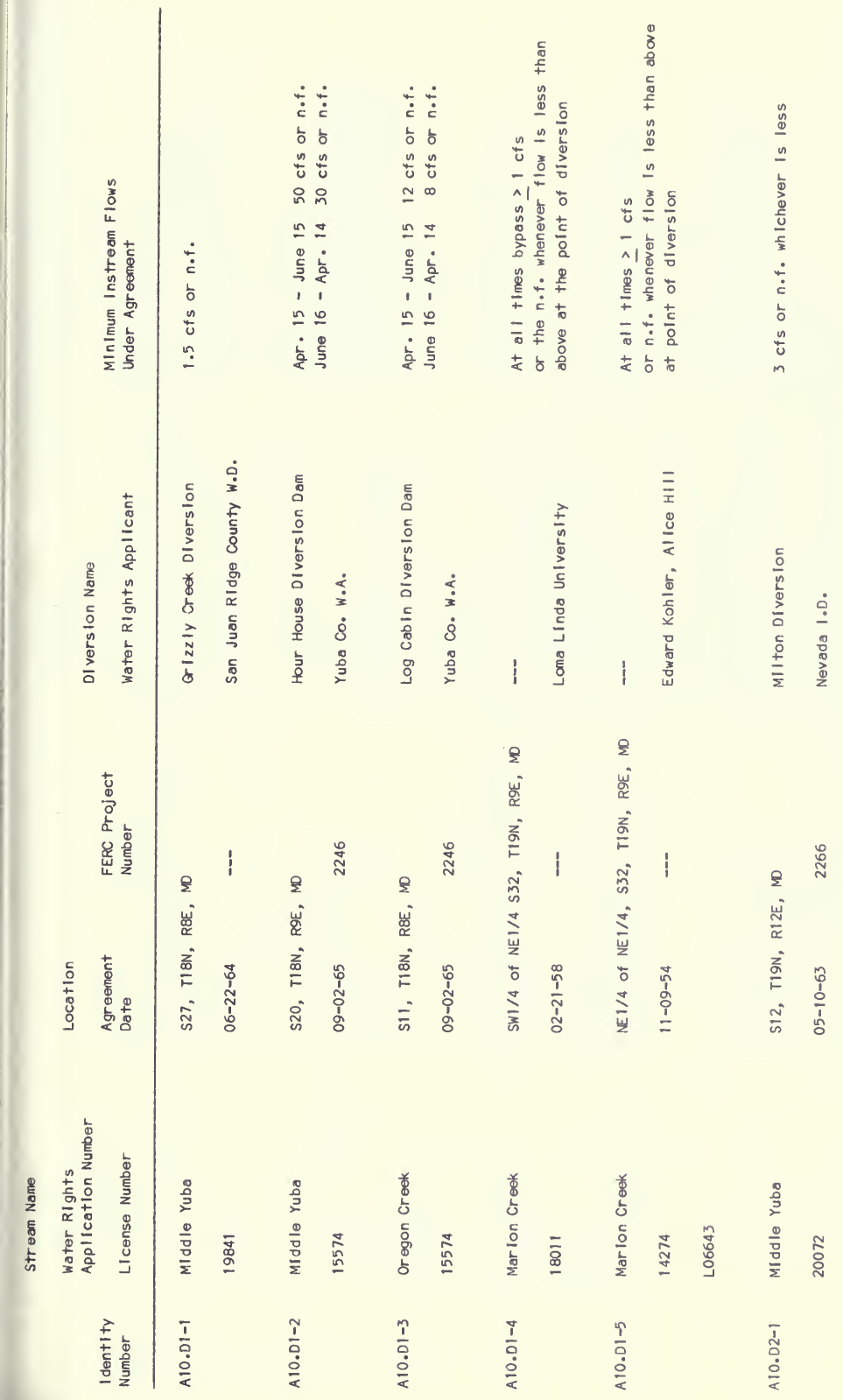

क्षी 


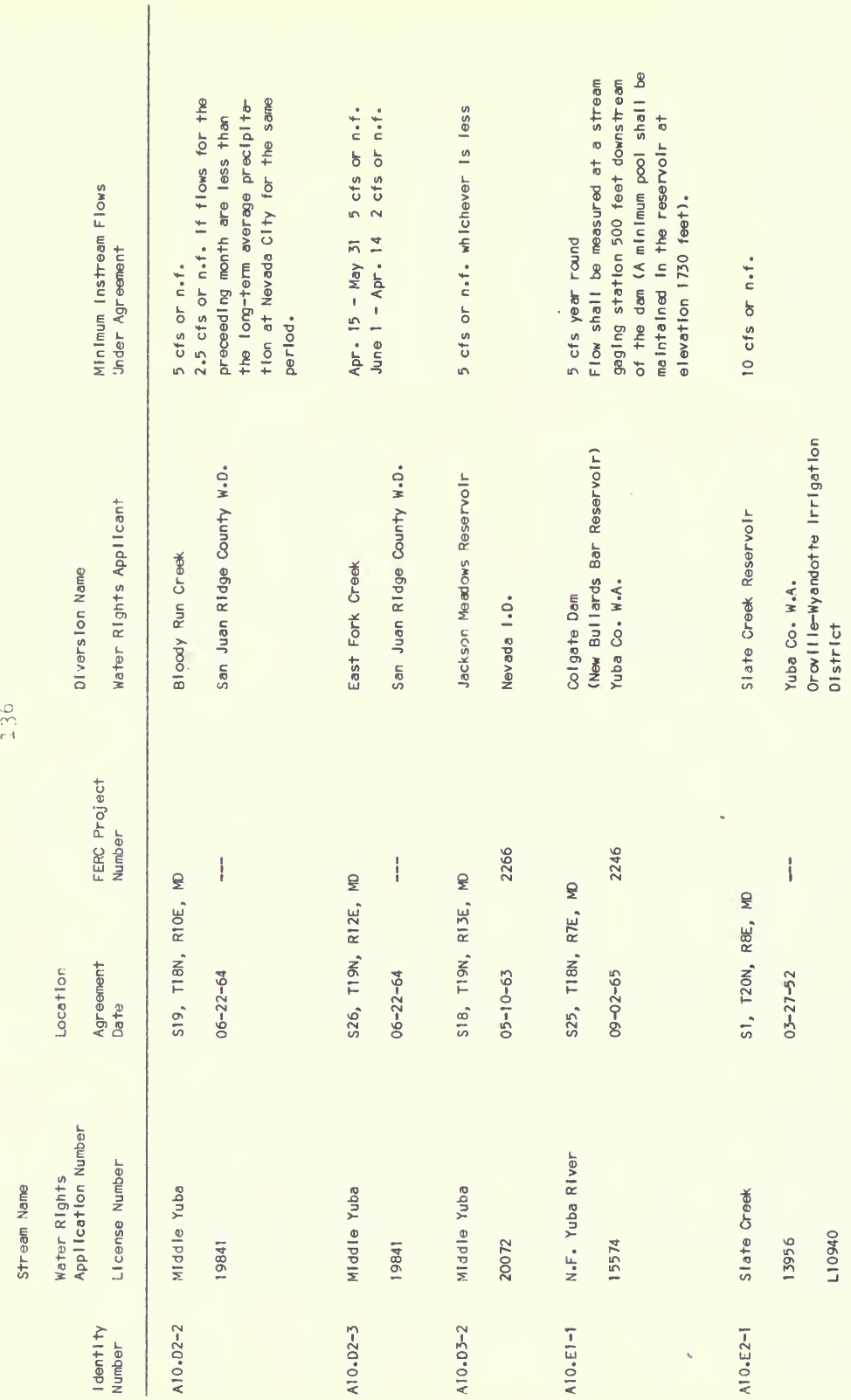




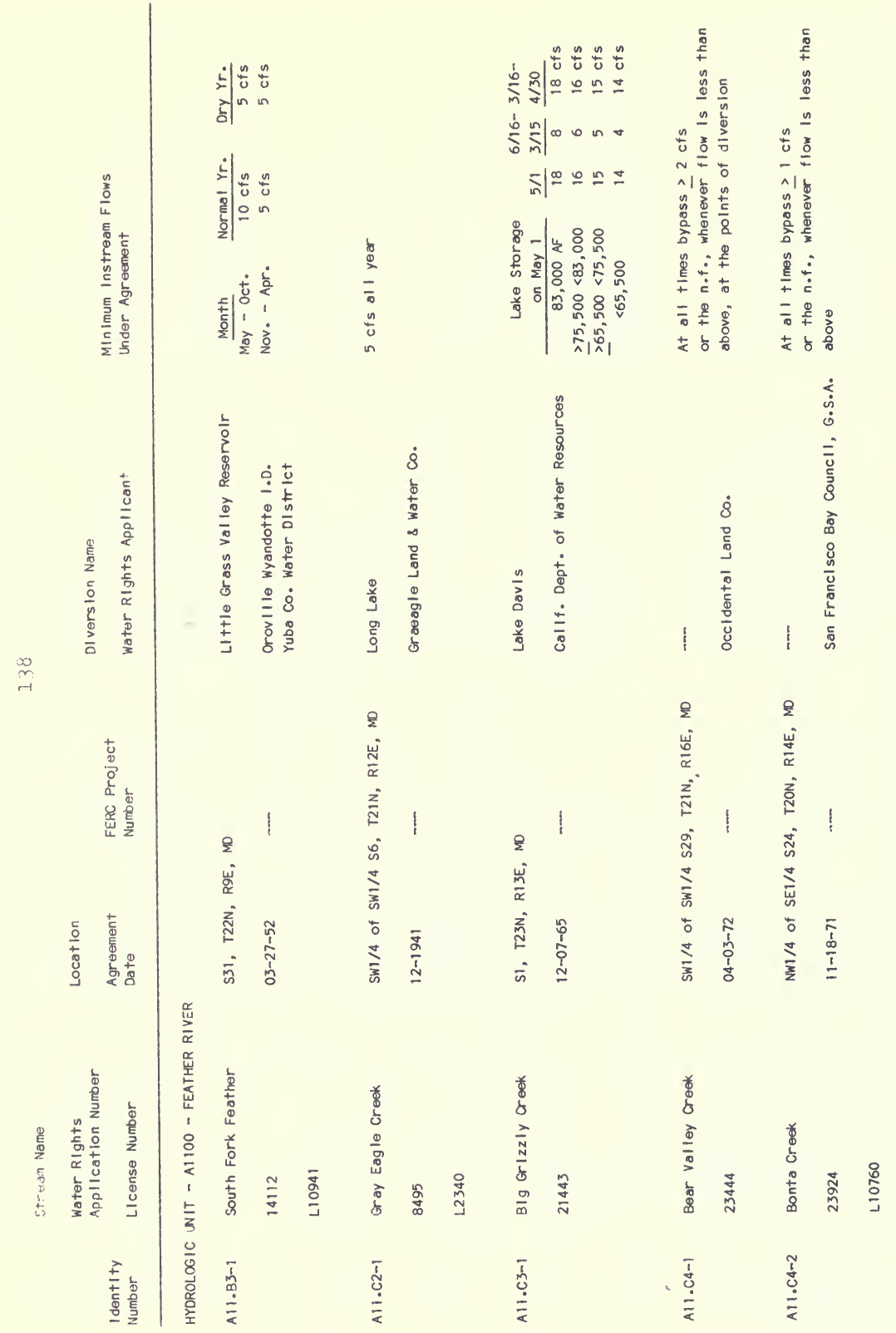




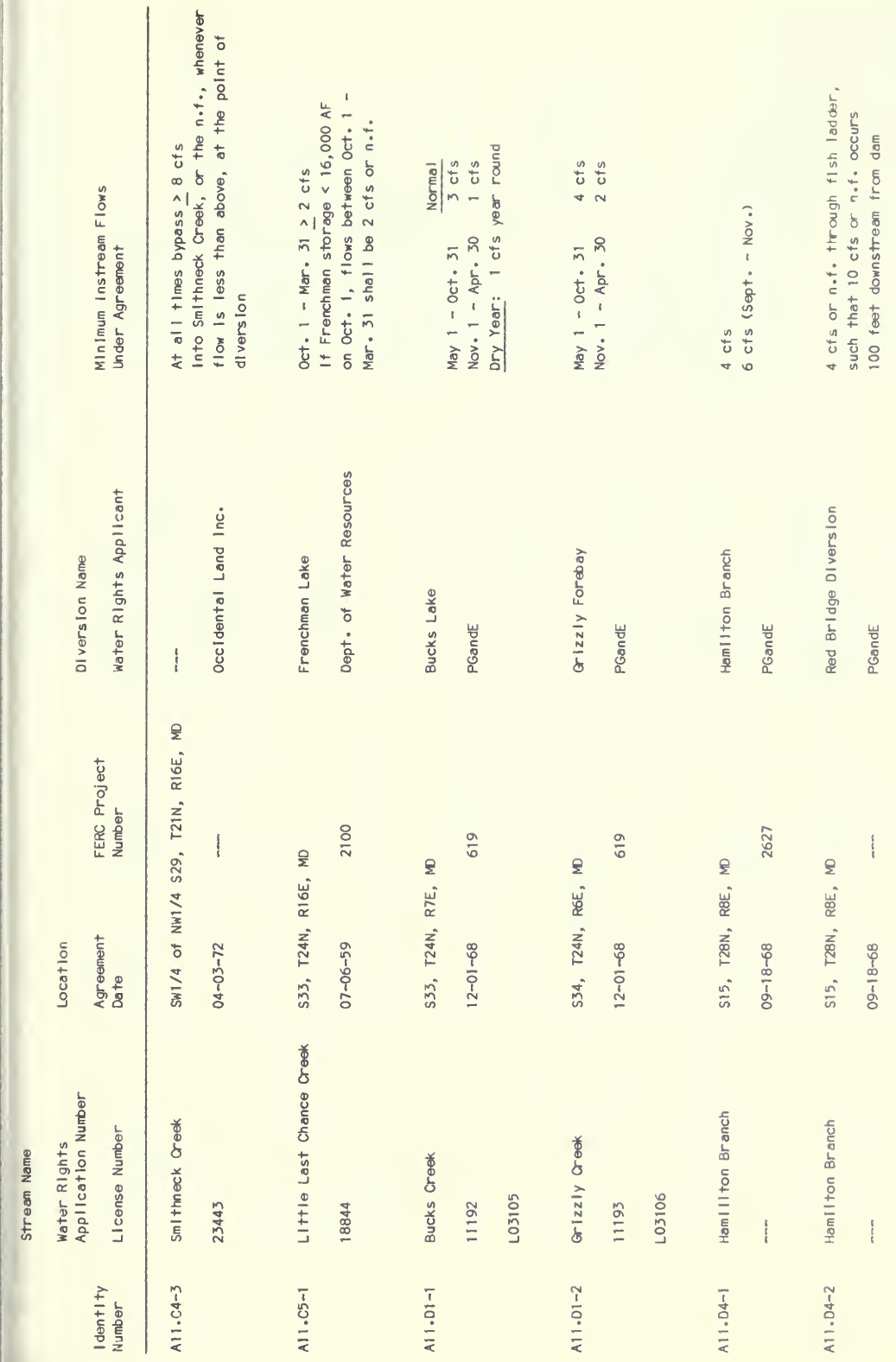




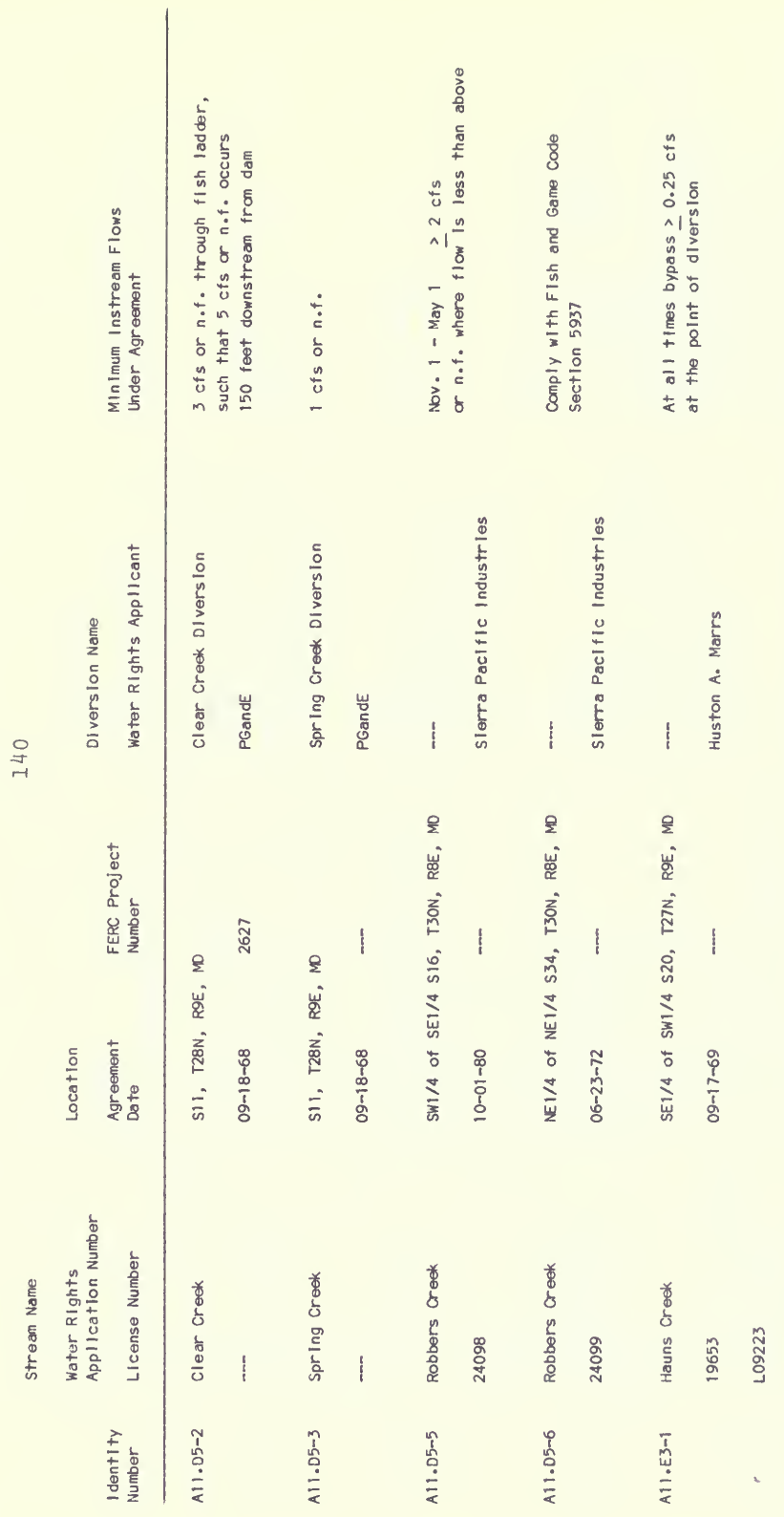




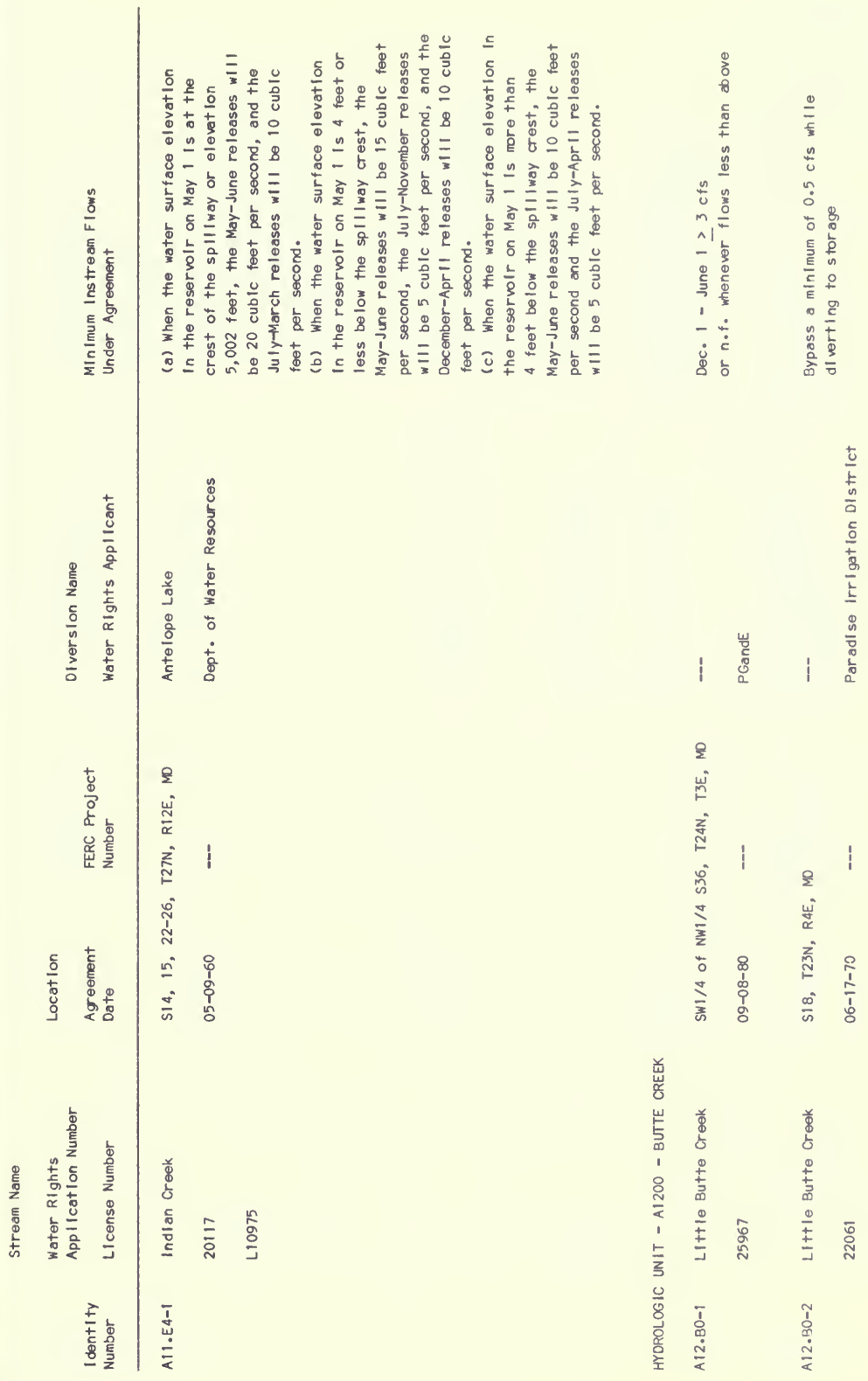




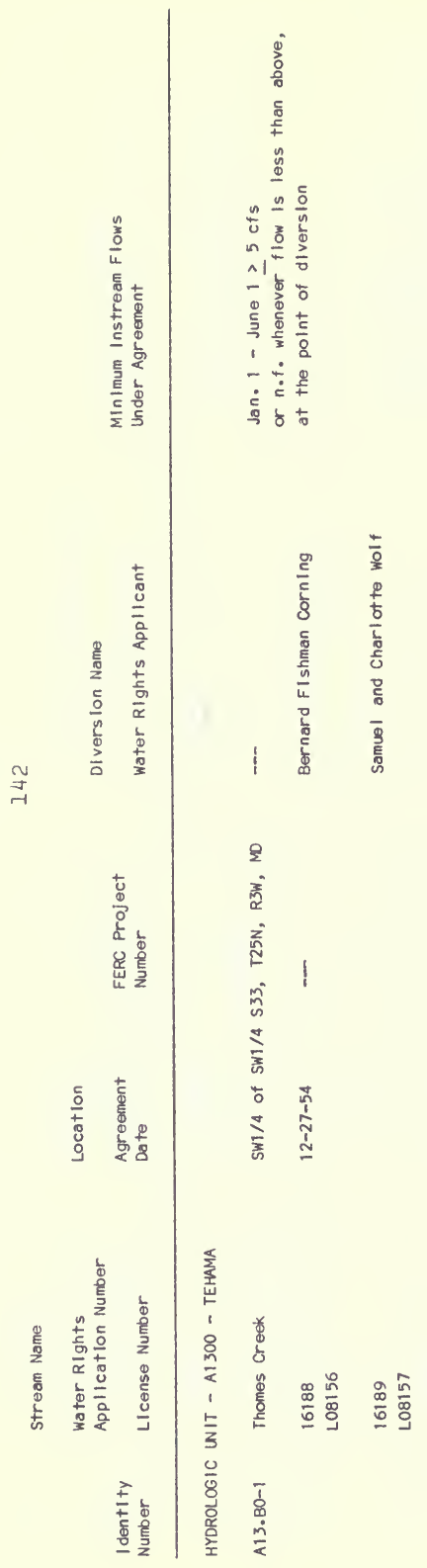




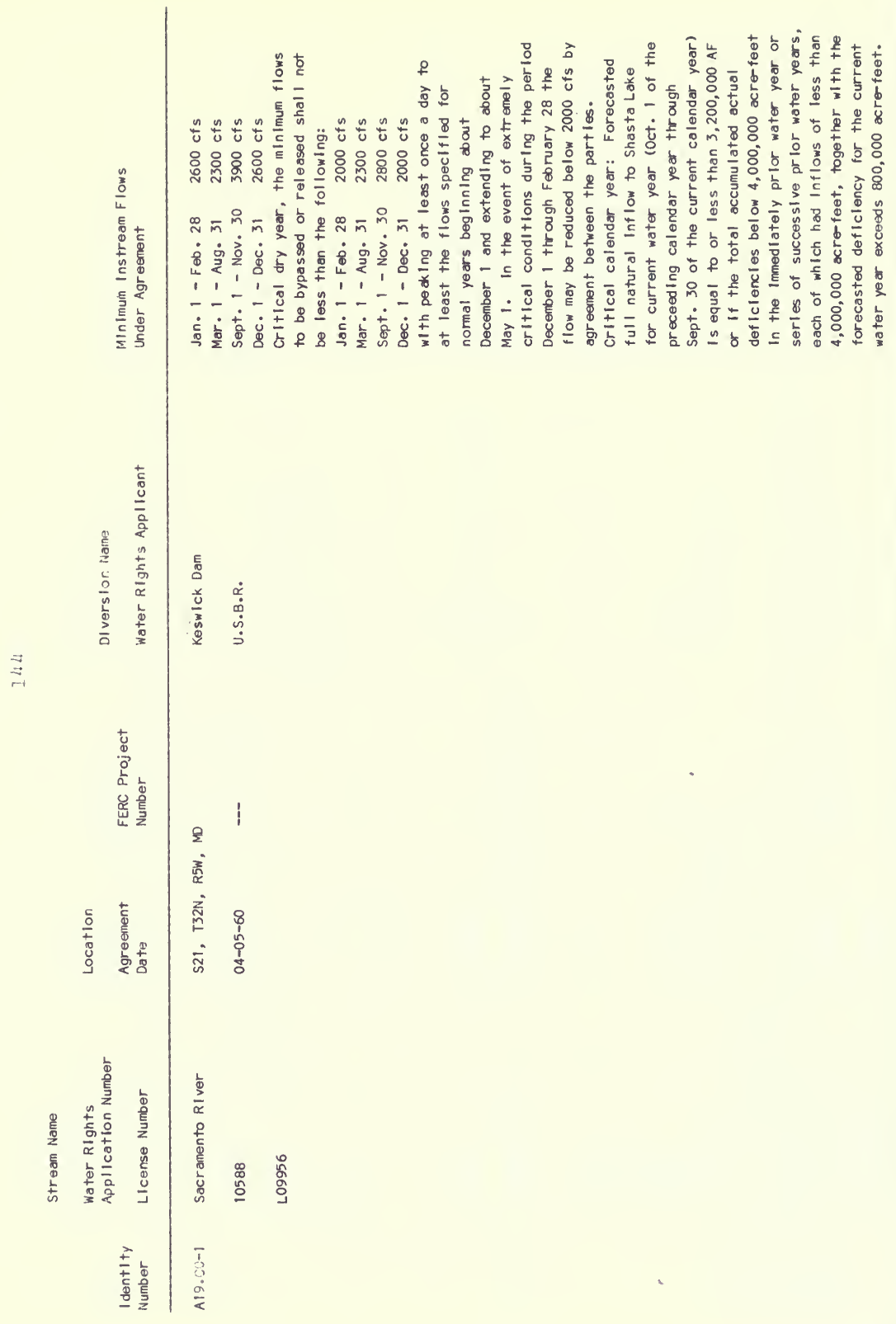




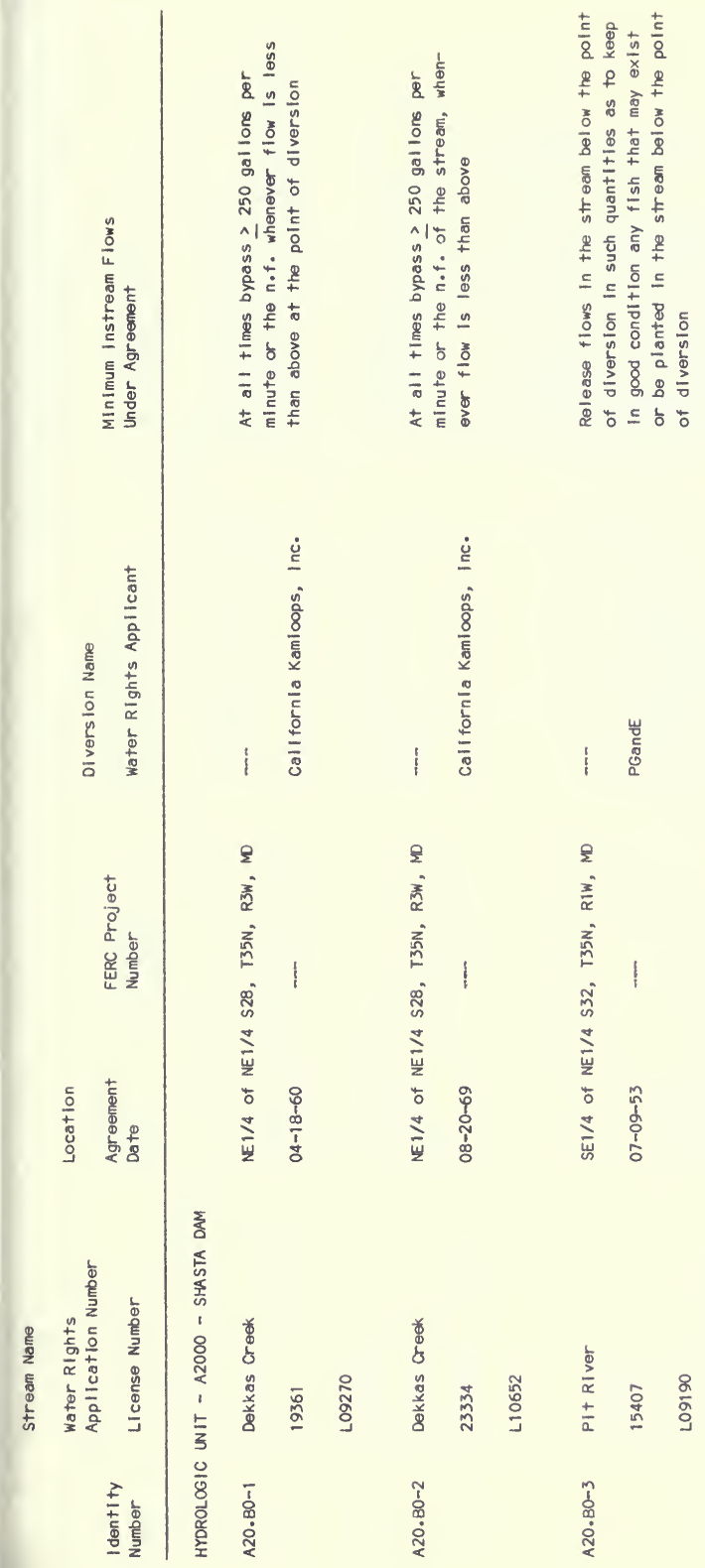




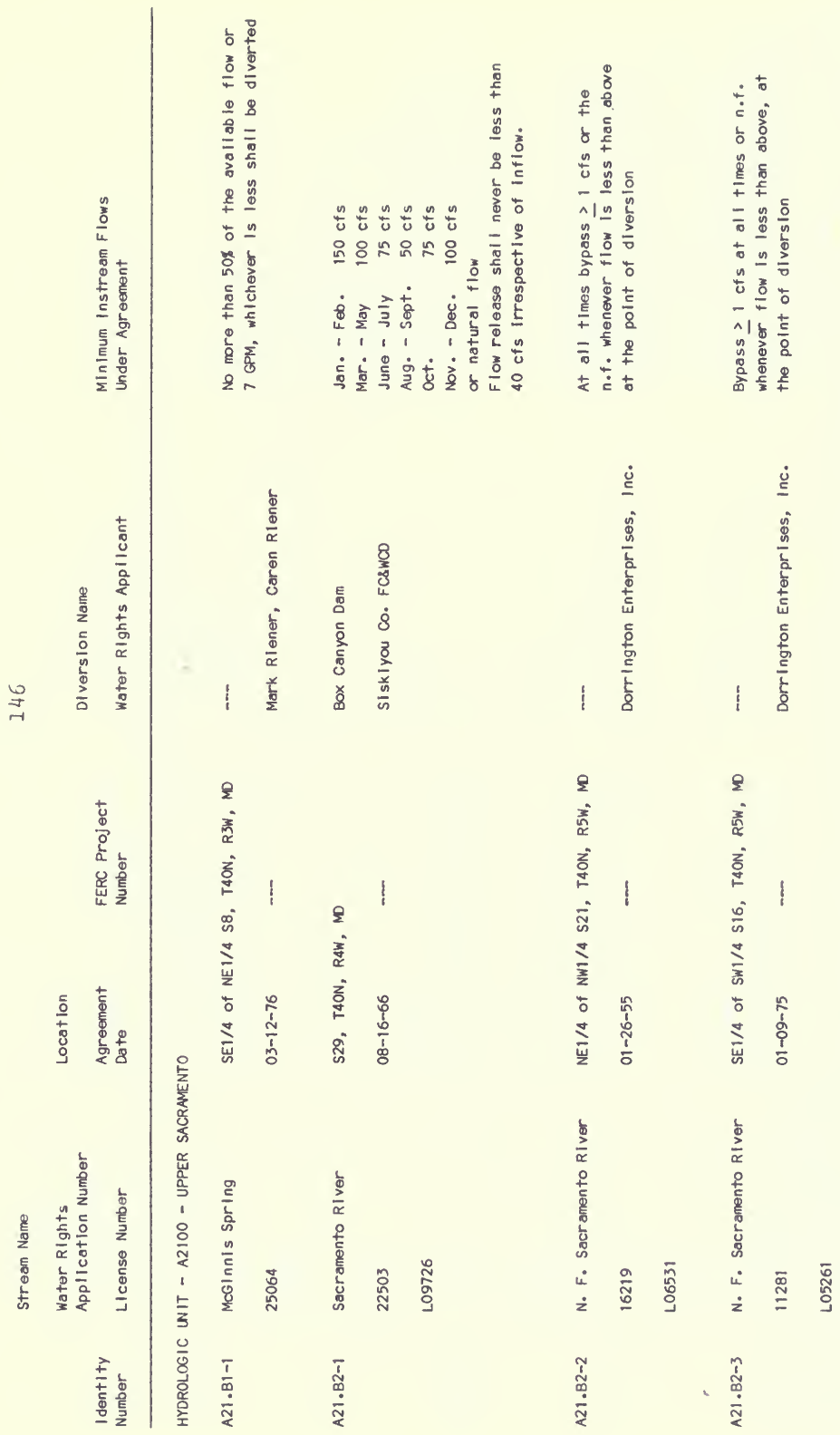




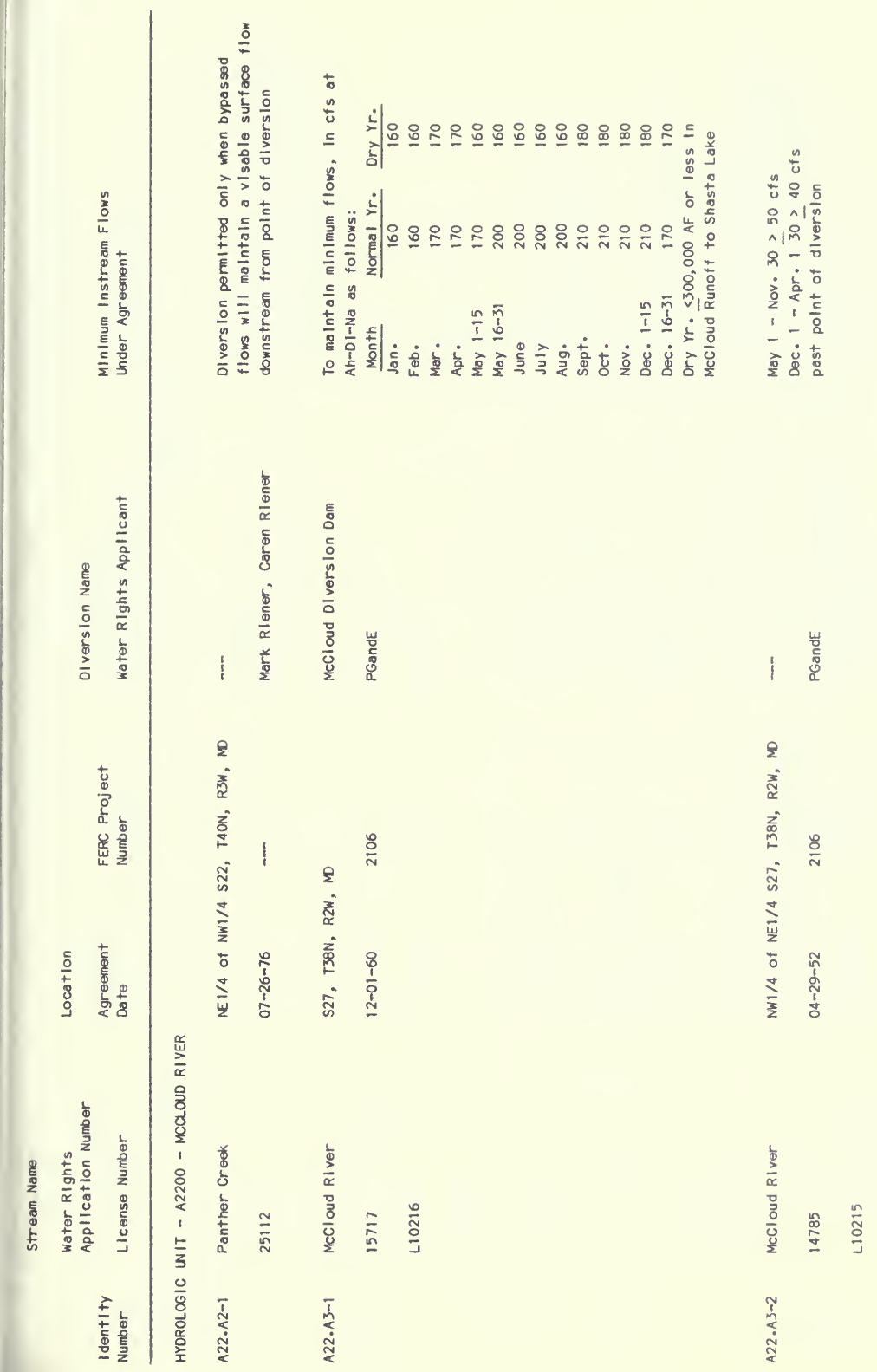




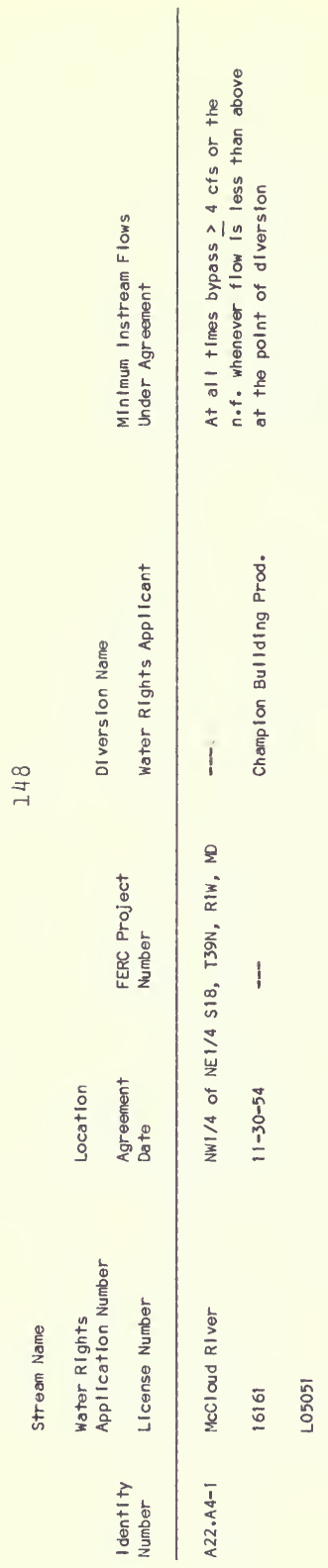




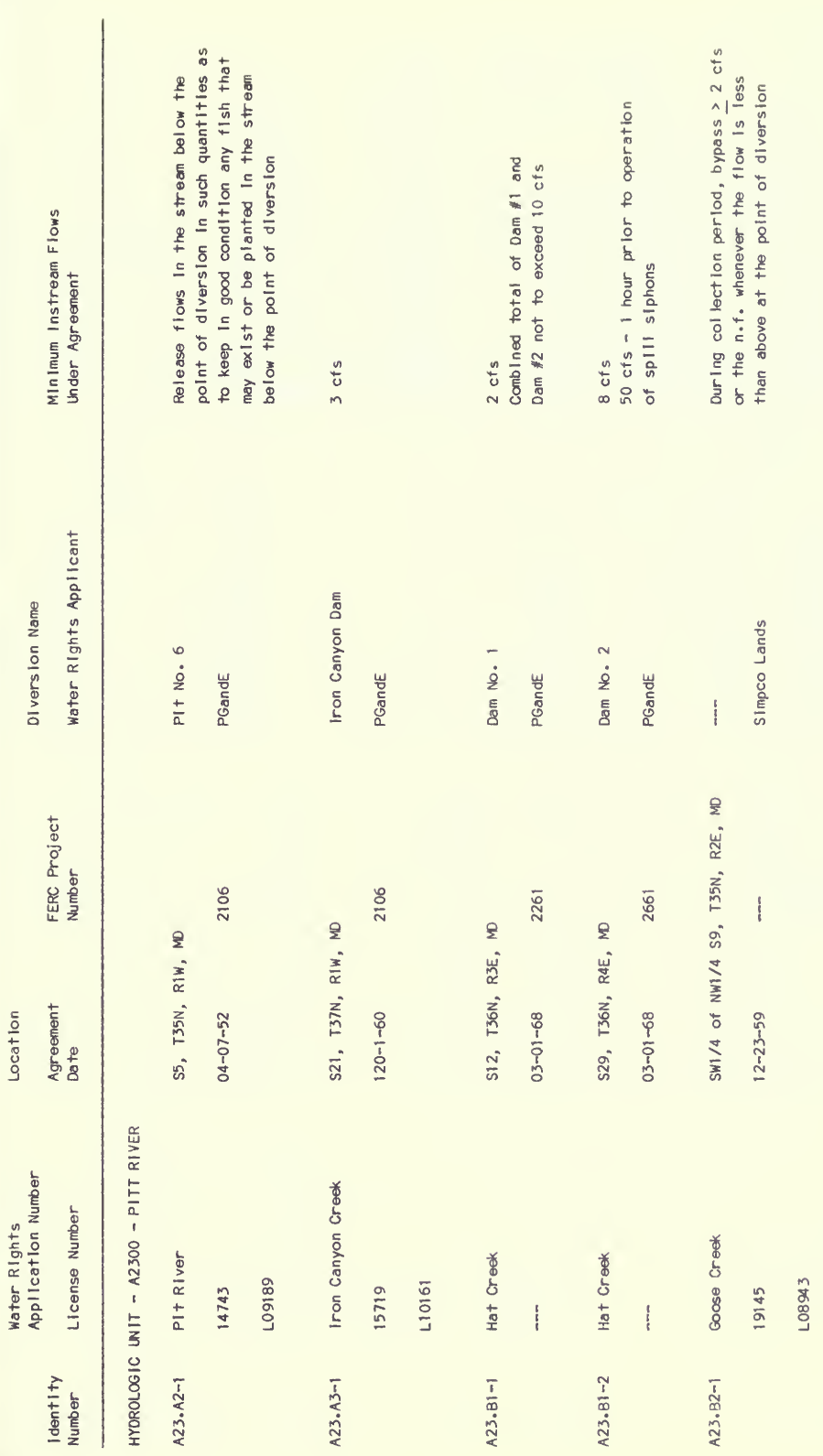




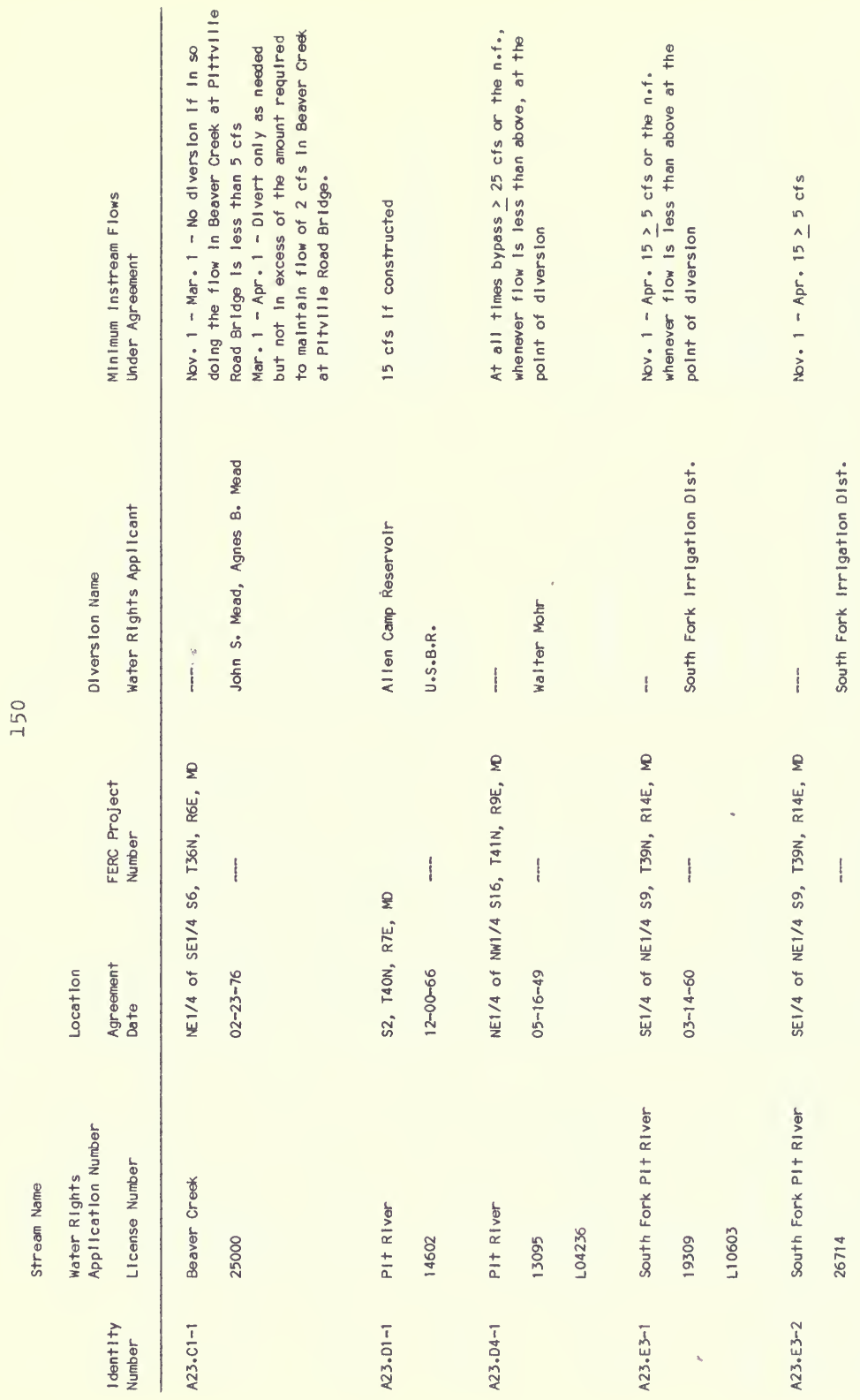




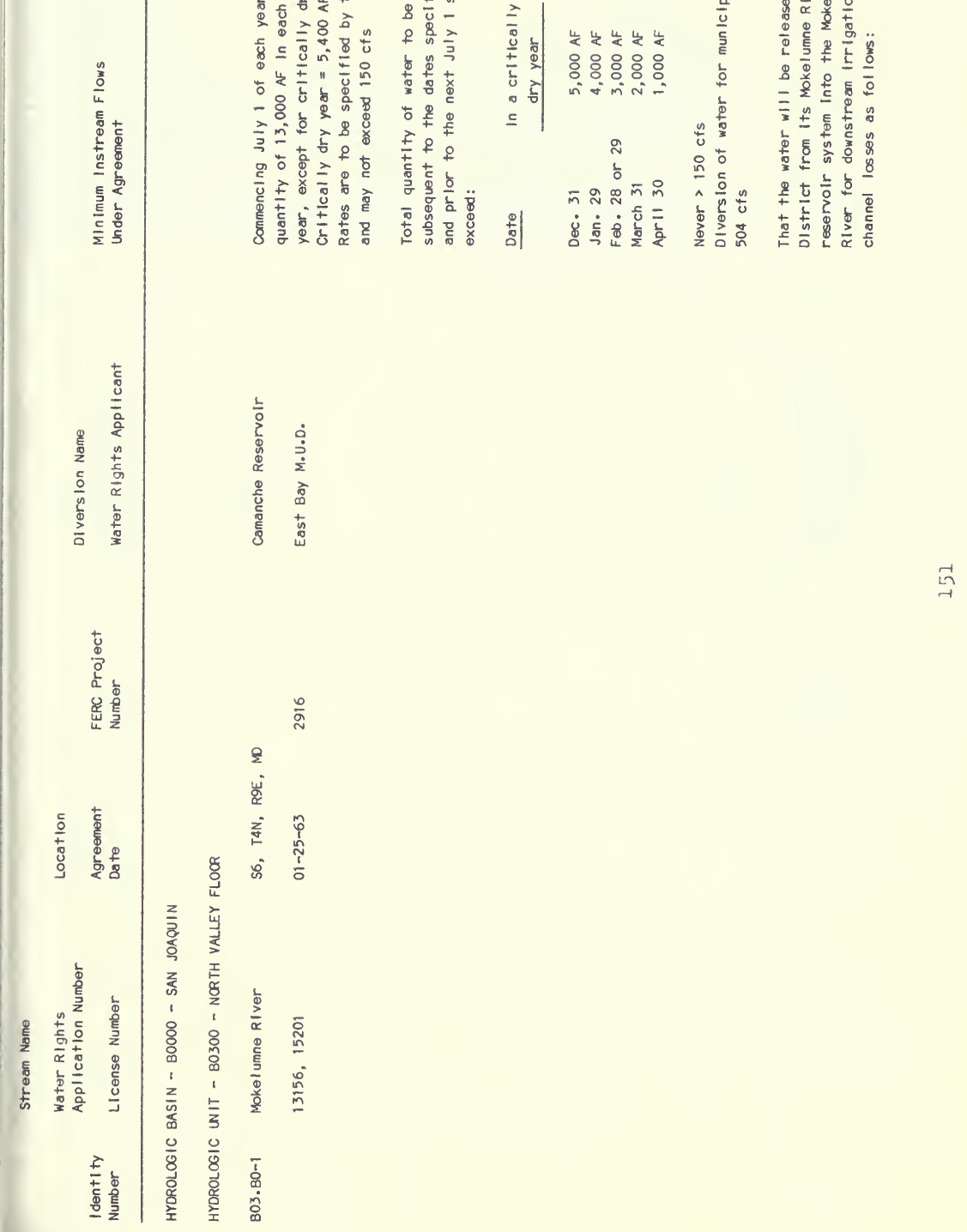




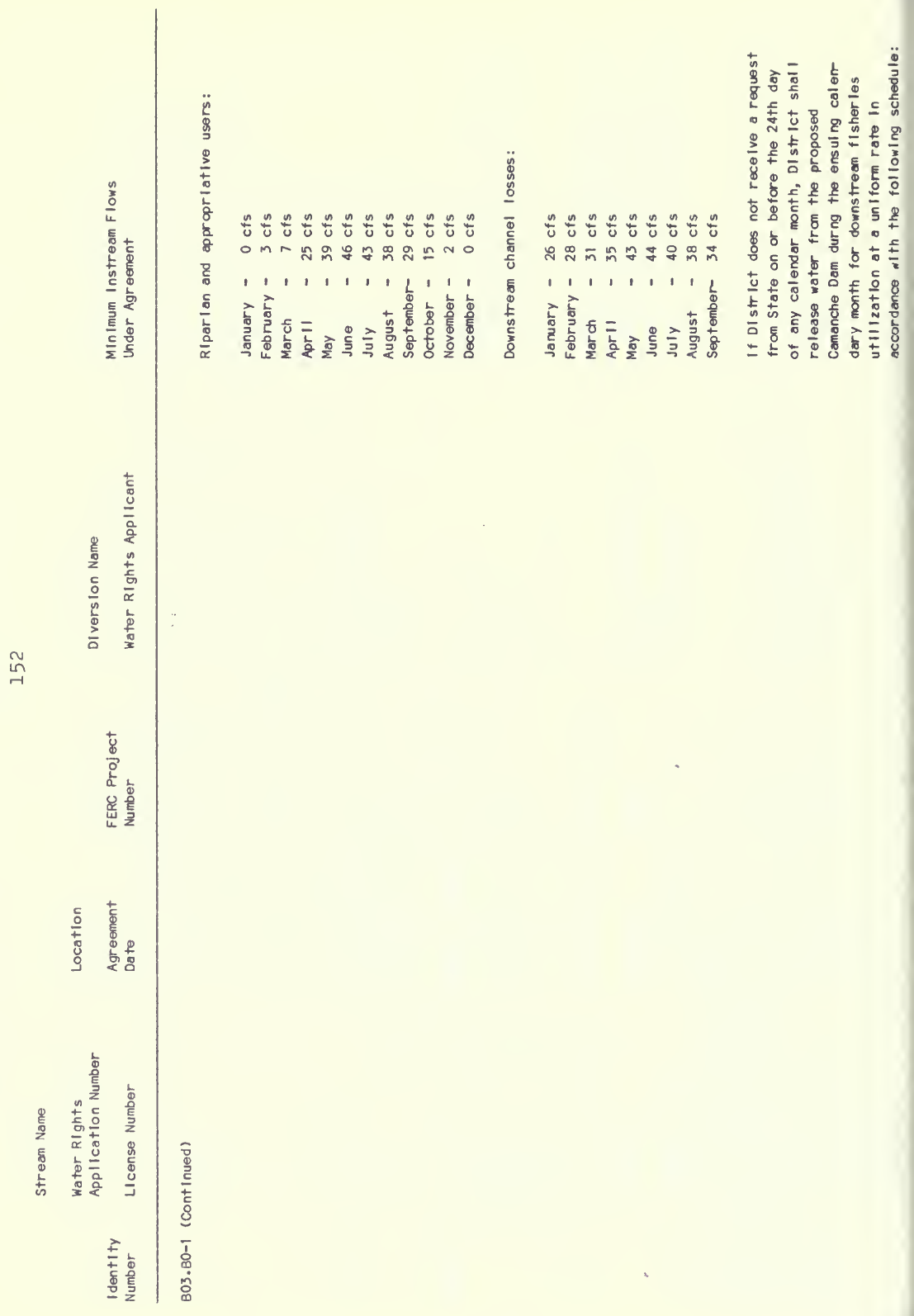




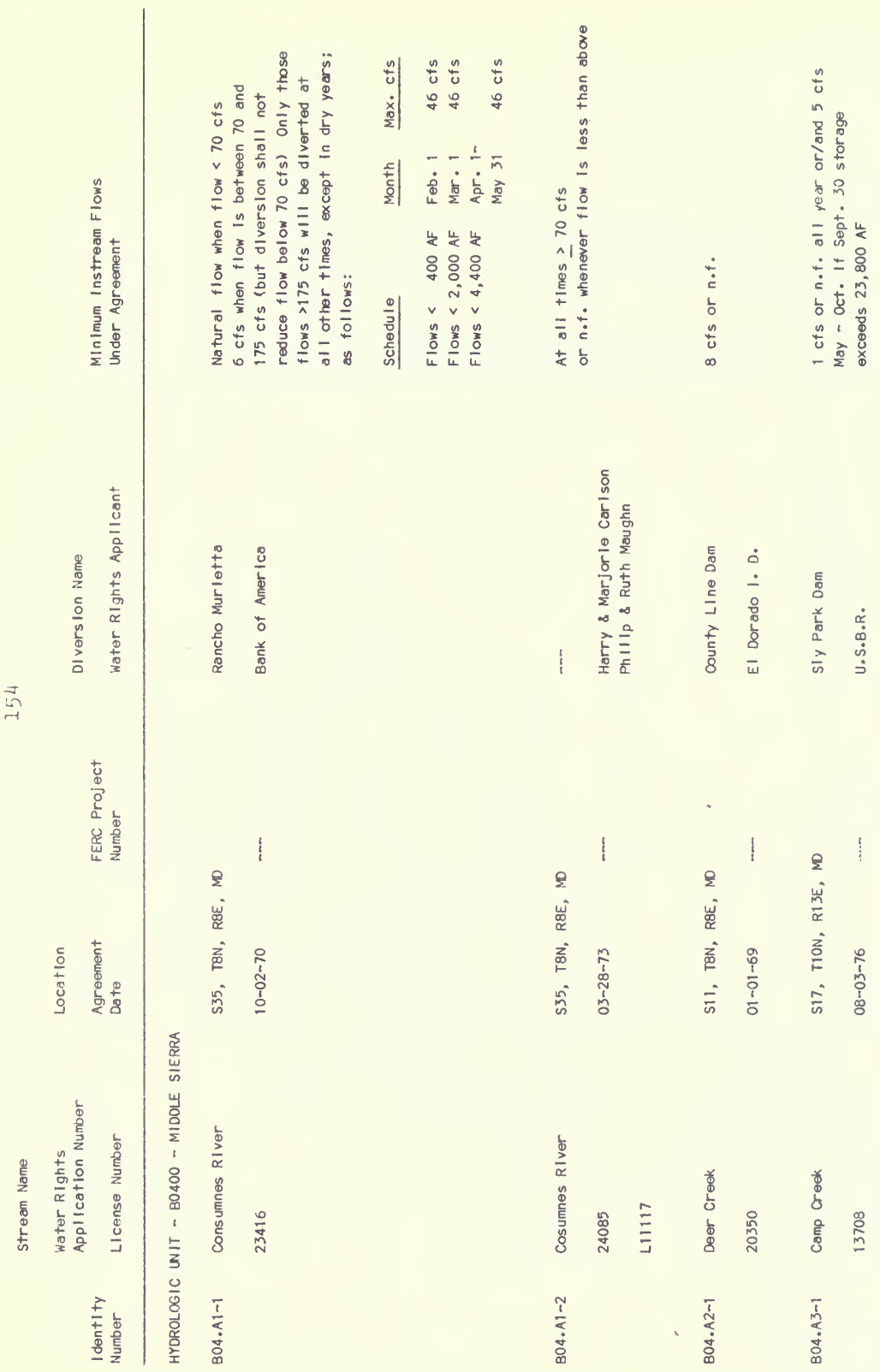




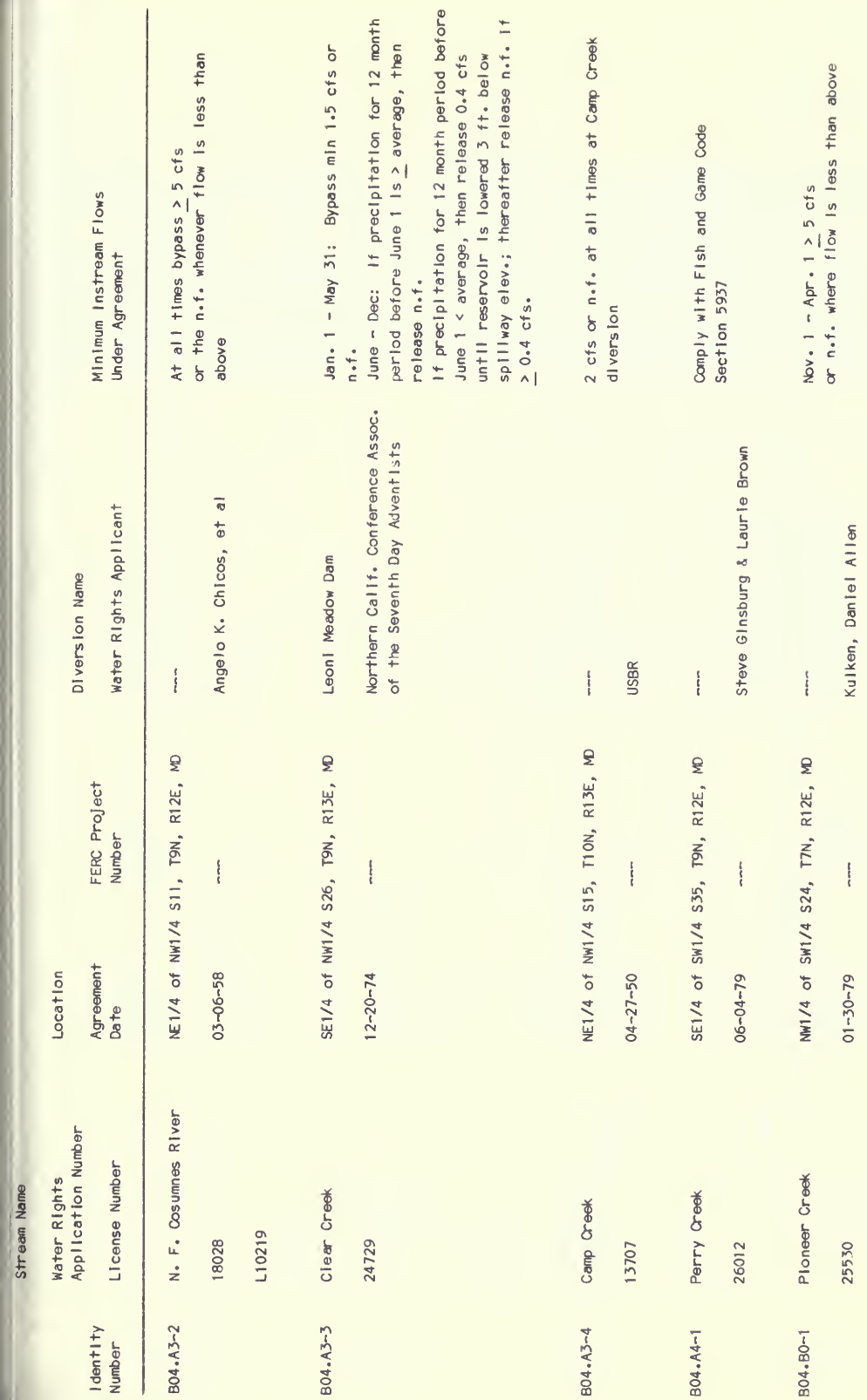

$\stackrel{5}{ก}$ 


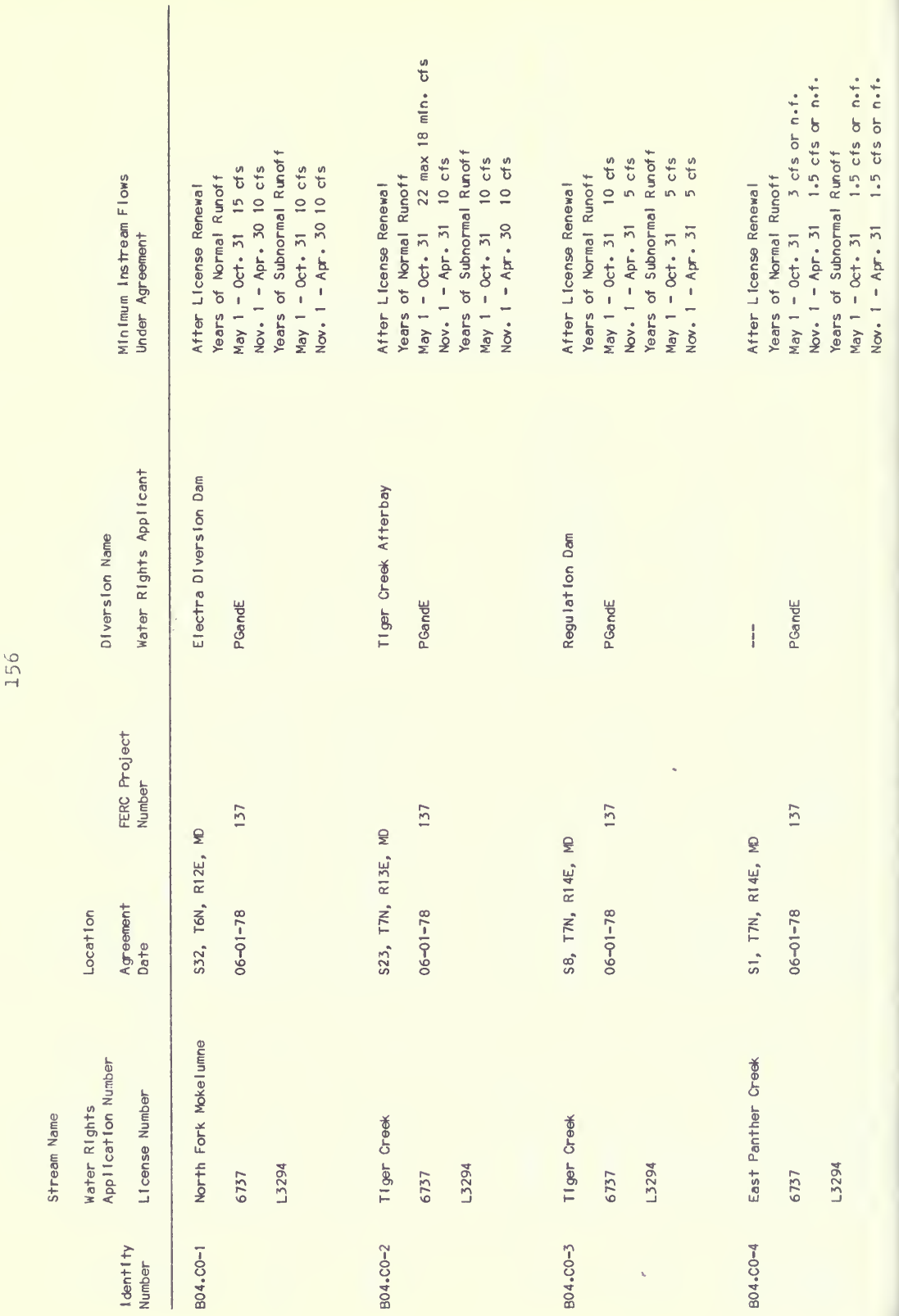




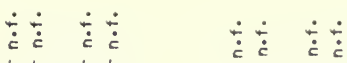

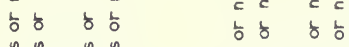

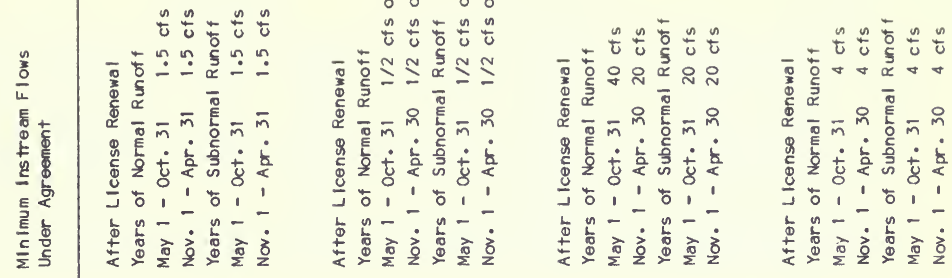

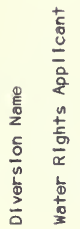

$\begin{array}{r}\text { U⿺ } \\ \text { I } \\ \text { I } \\ \hline\end{array}$

\begin{tabular}{r} 
岁 \\
| \\
\hline \\
0
\end{tabular}

W
|
|
I
0.

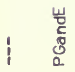

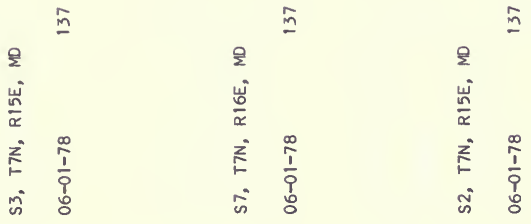

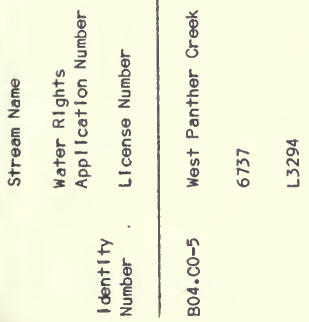

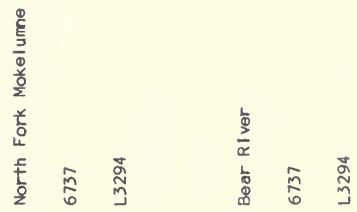

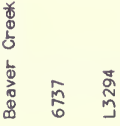

0
$\vdots$
0
$\dot{0}$

$\hat{d}$
$\dot{0}$
$\dot{0}$

$\infty$
$\stackrel{⿱}{0}$
$\dot{d}$
$\dot{0}$ 


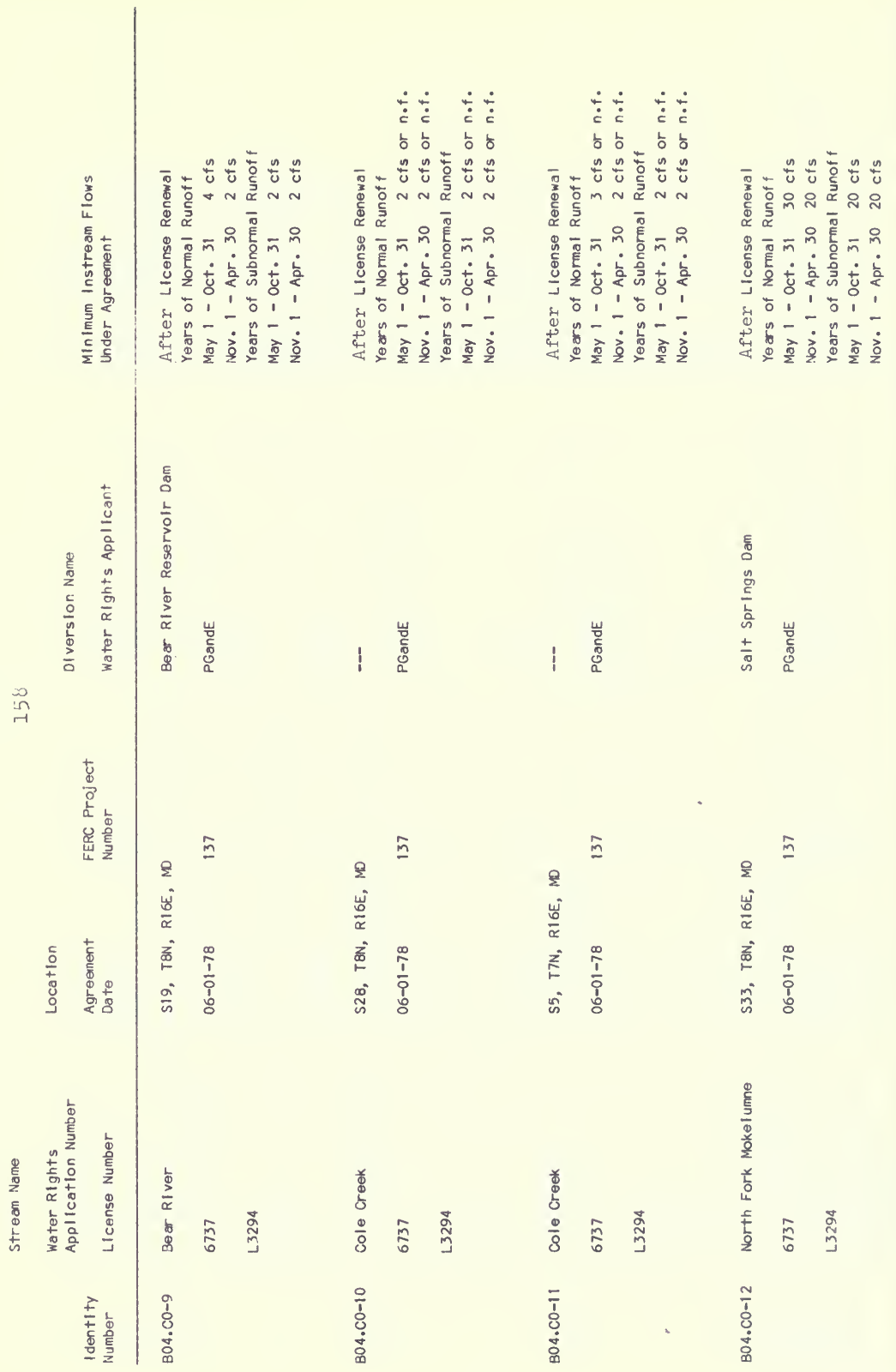




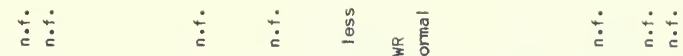

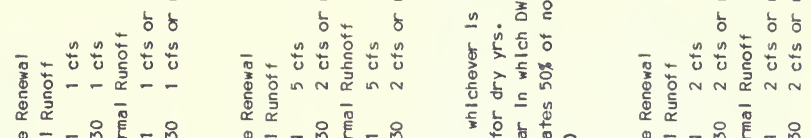

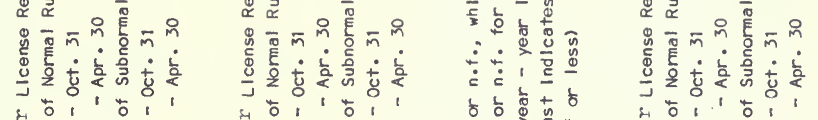

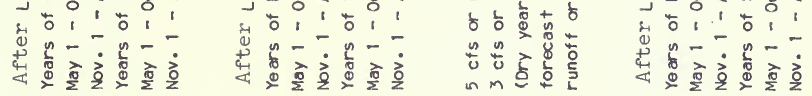
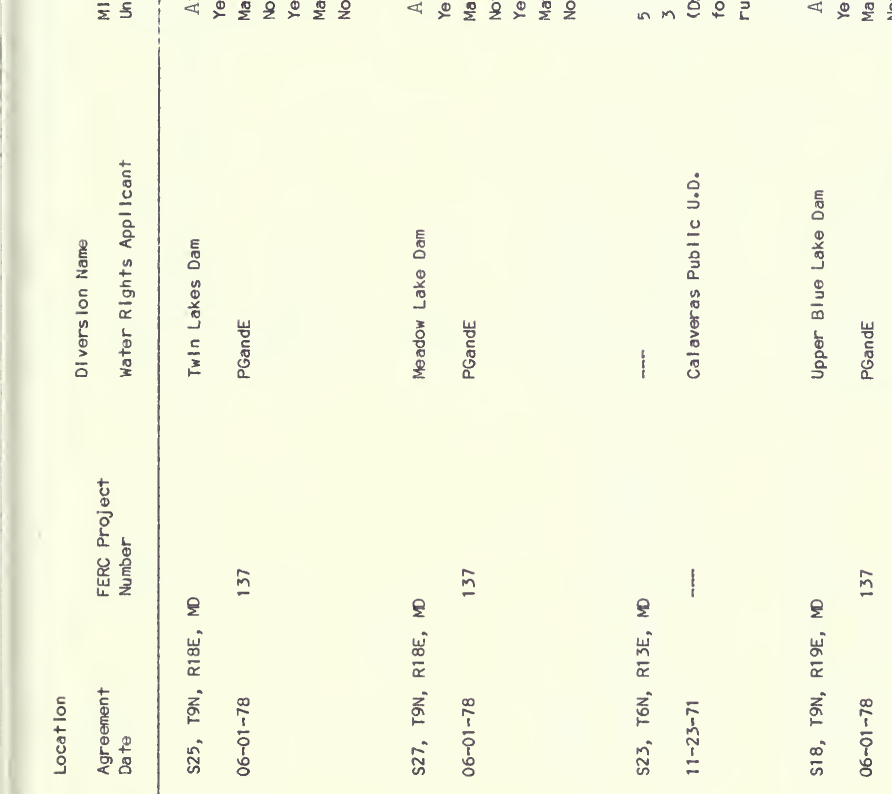

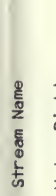
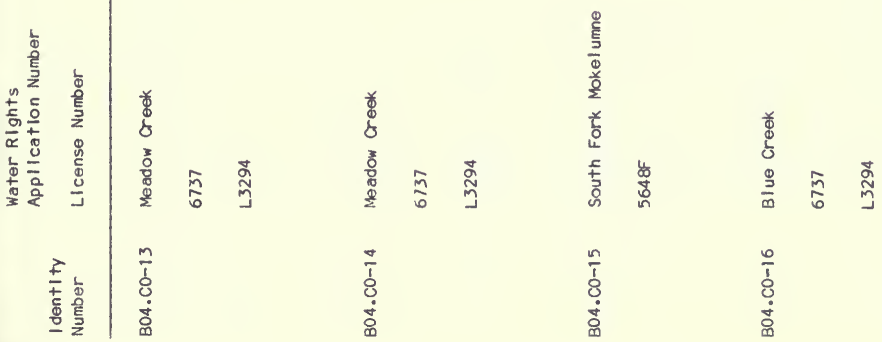

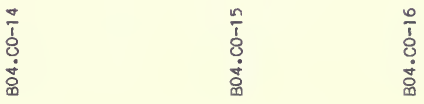




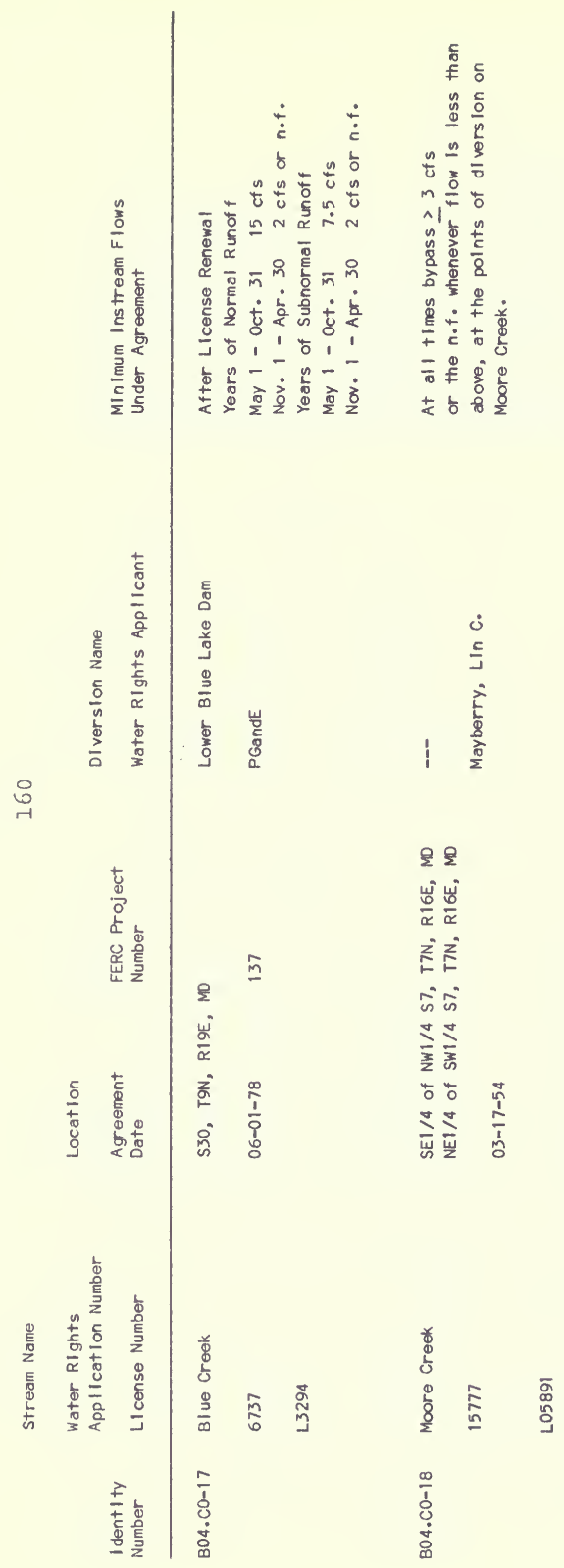




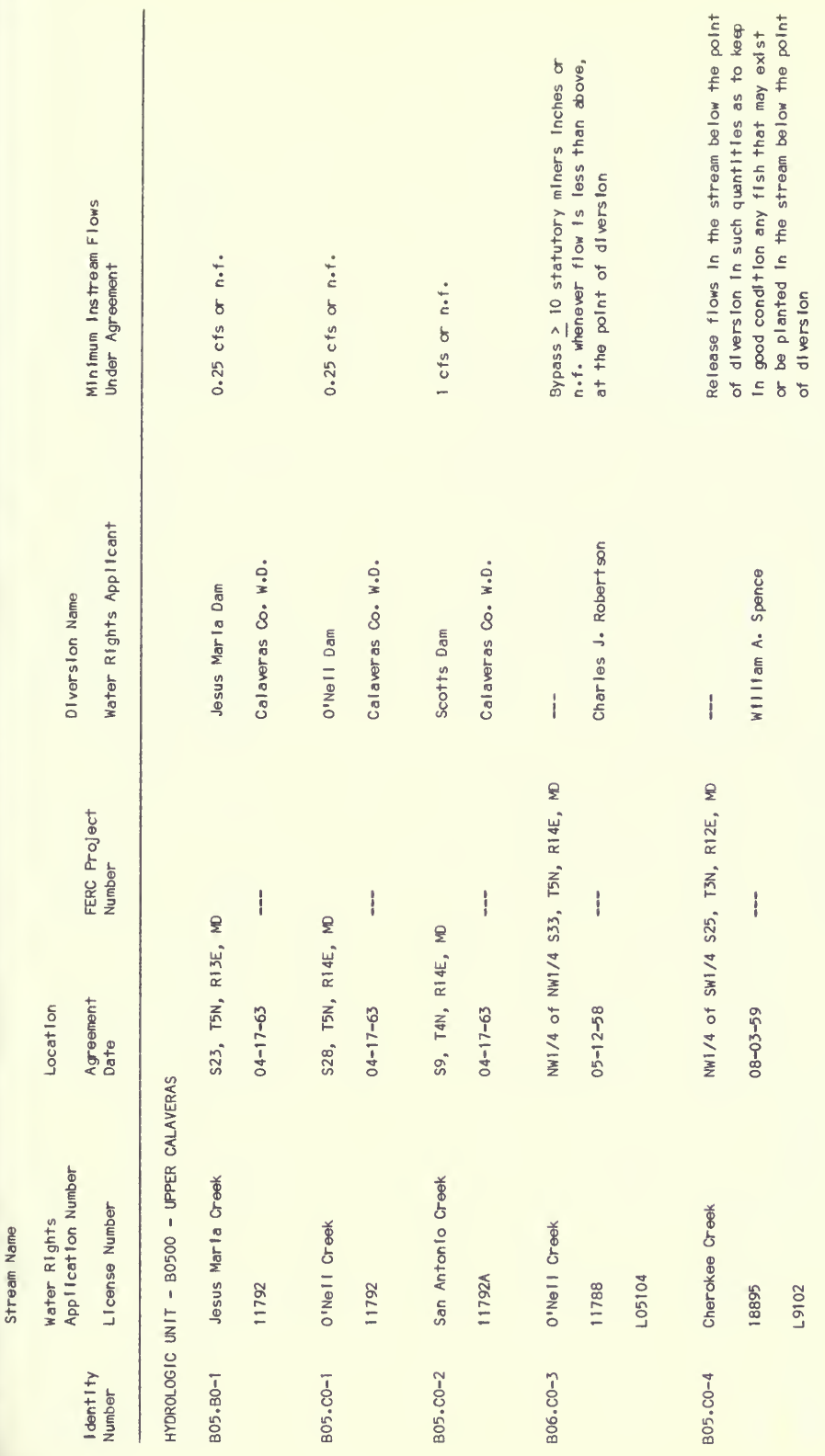

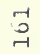




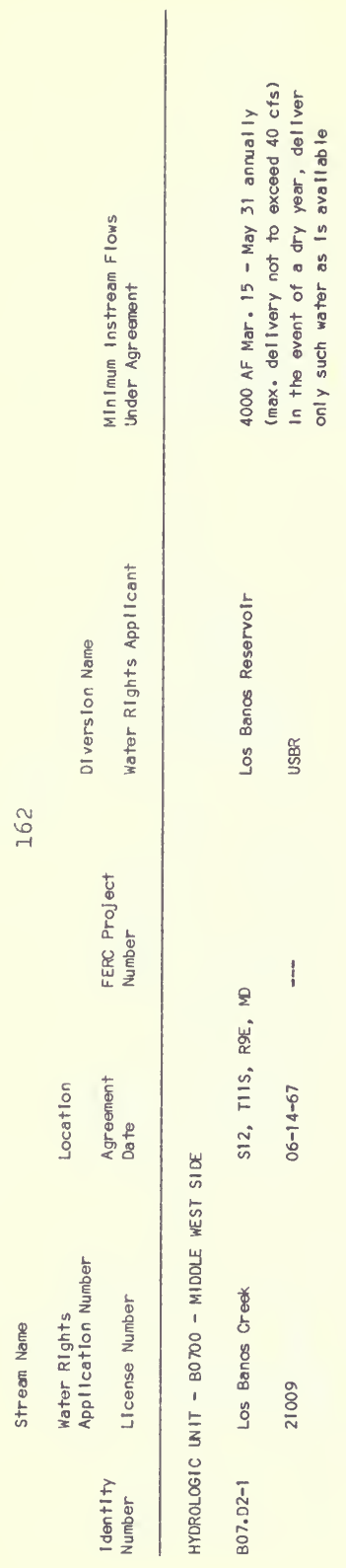




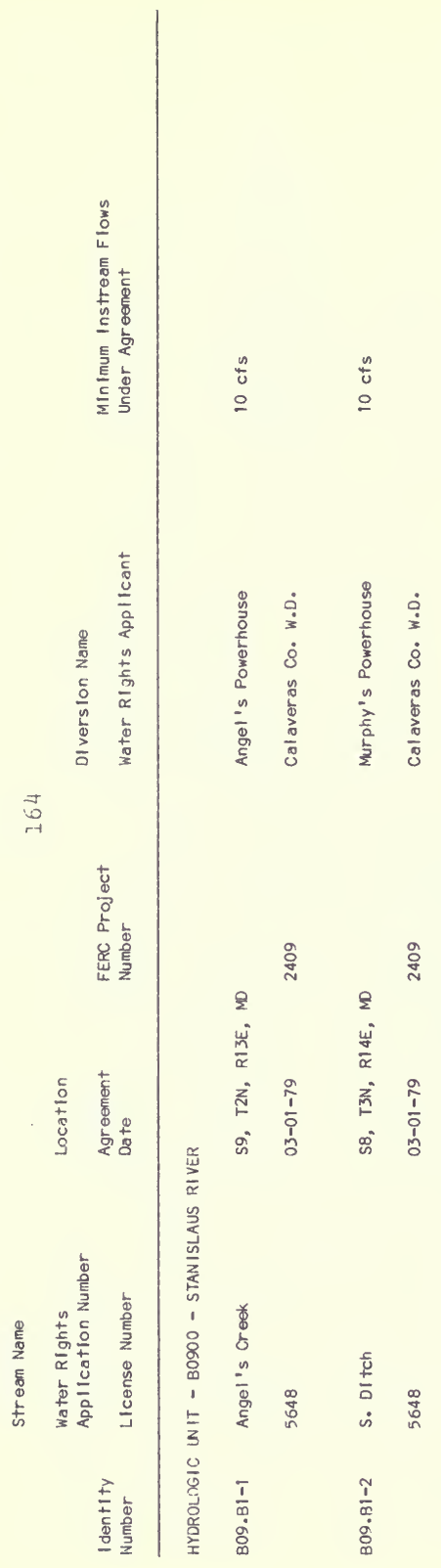




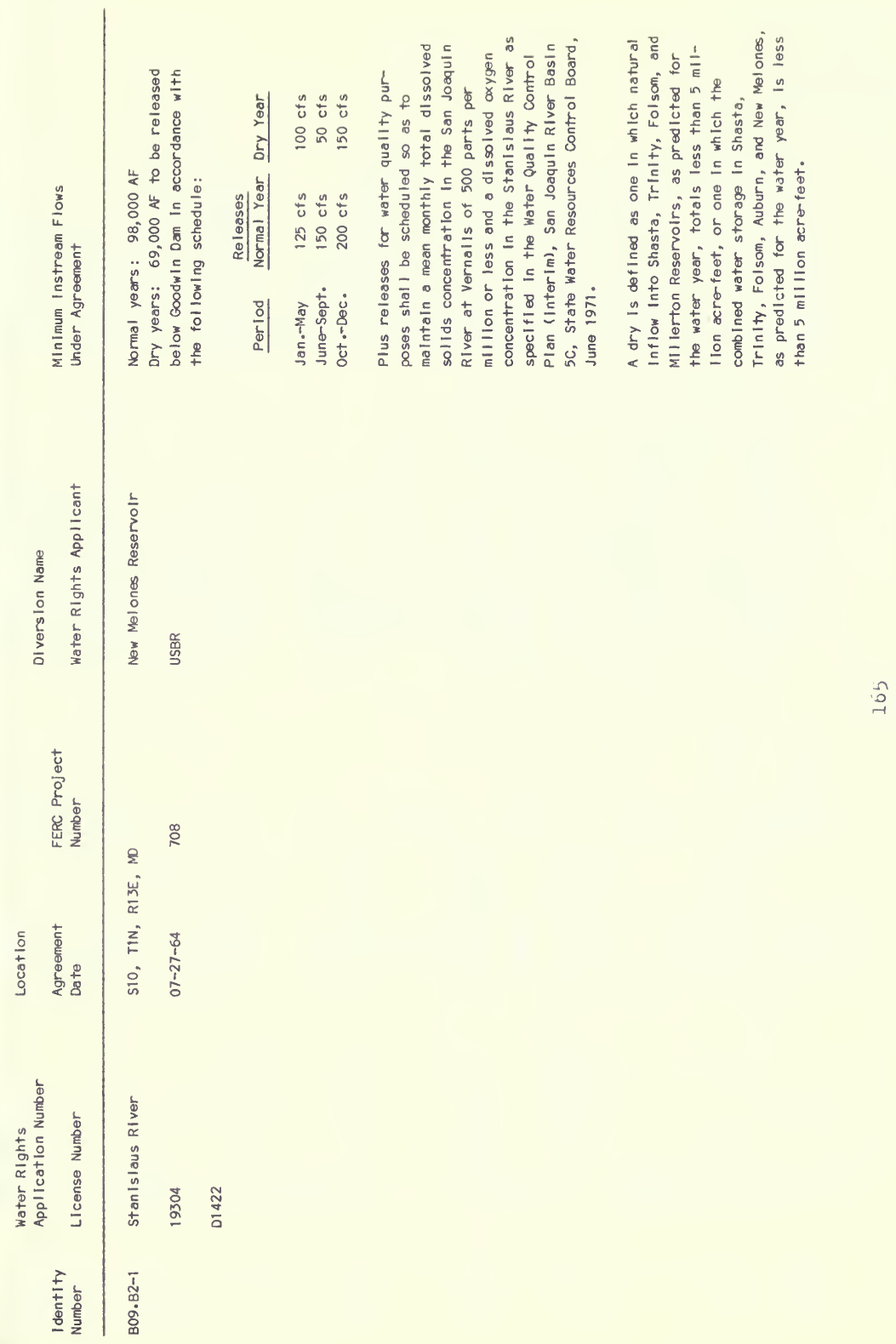




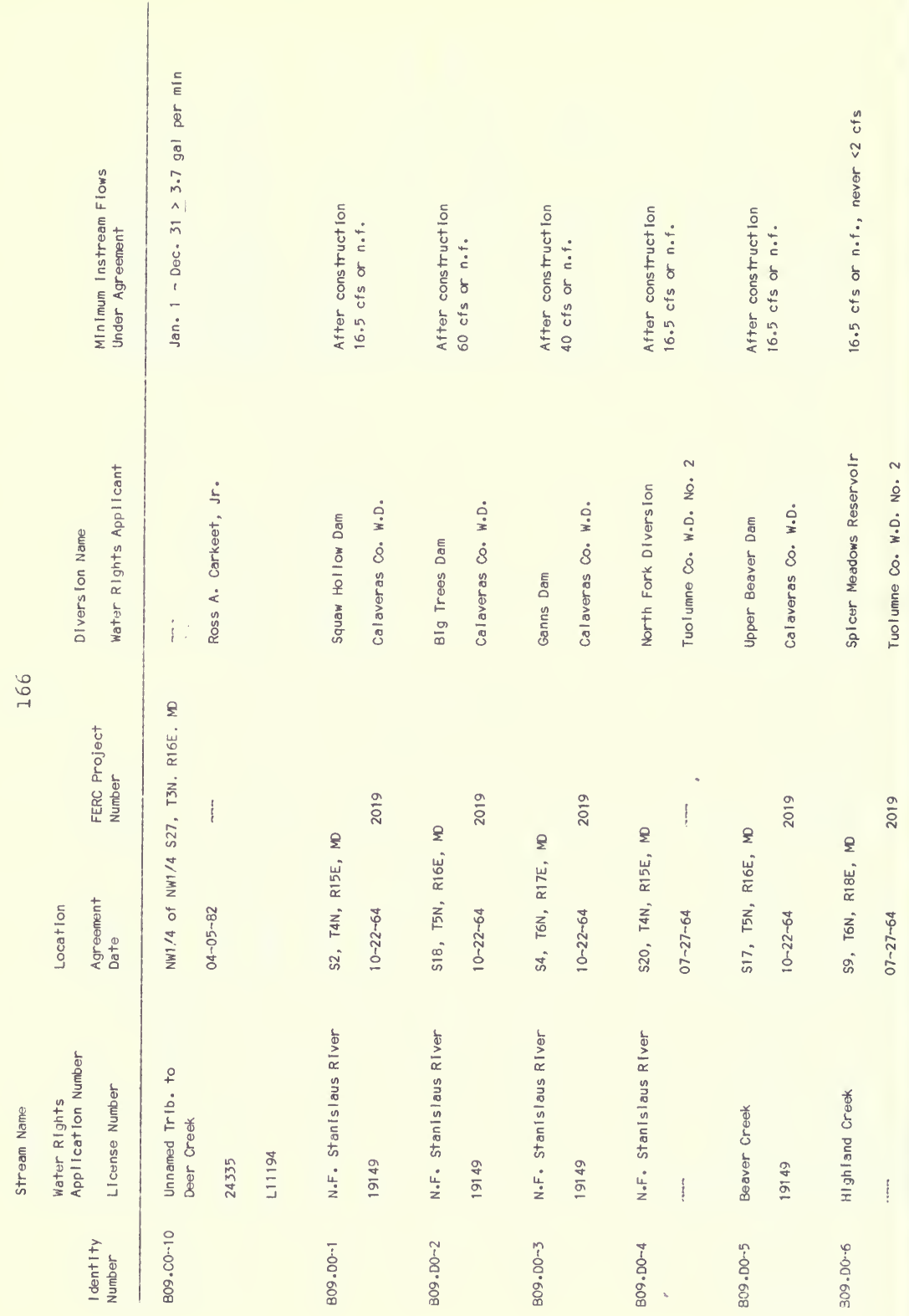




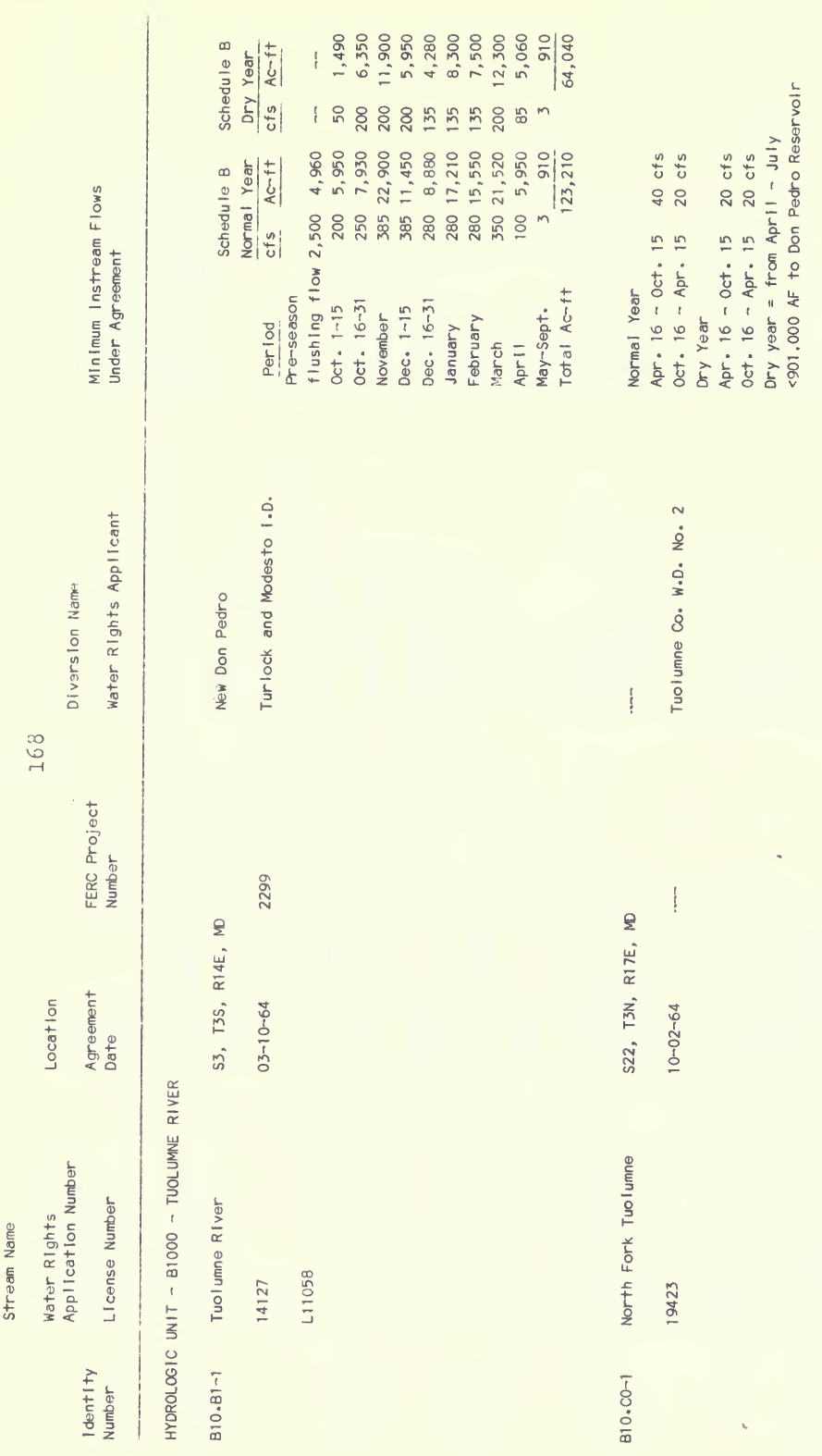




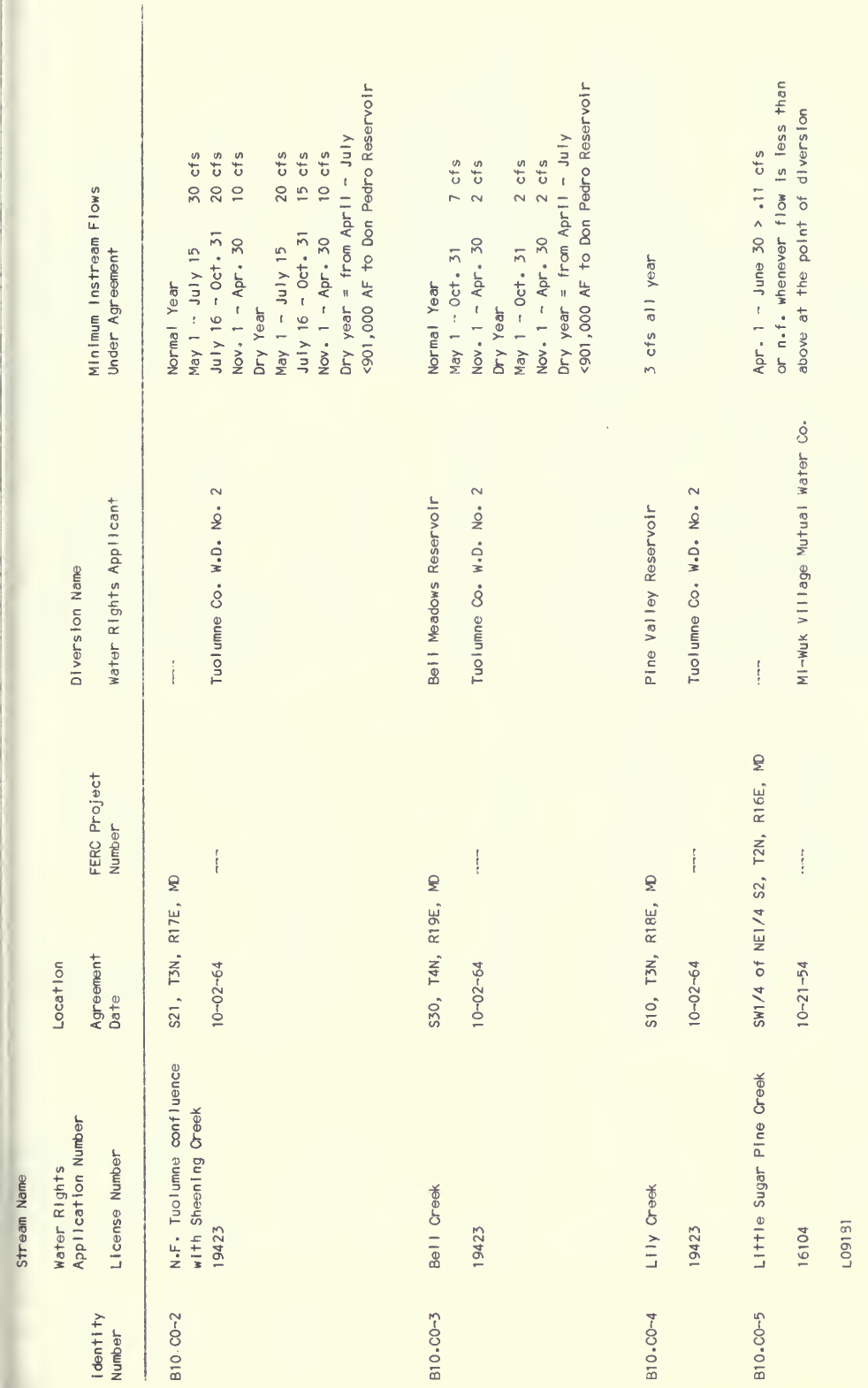




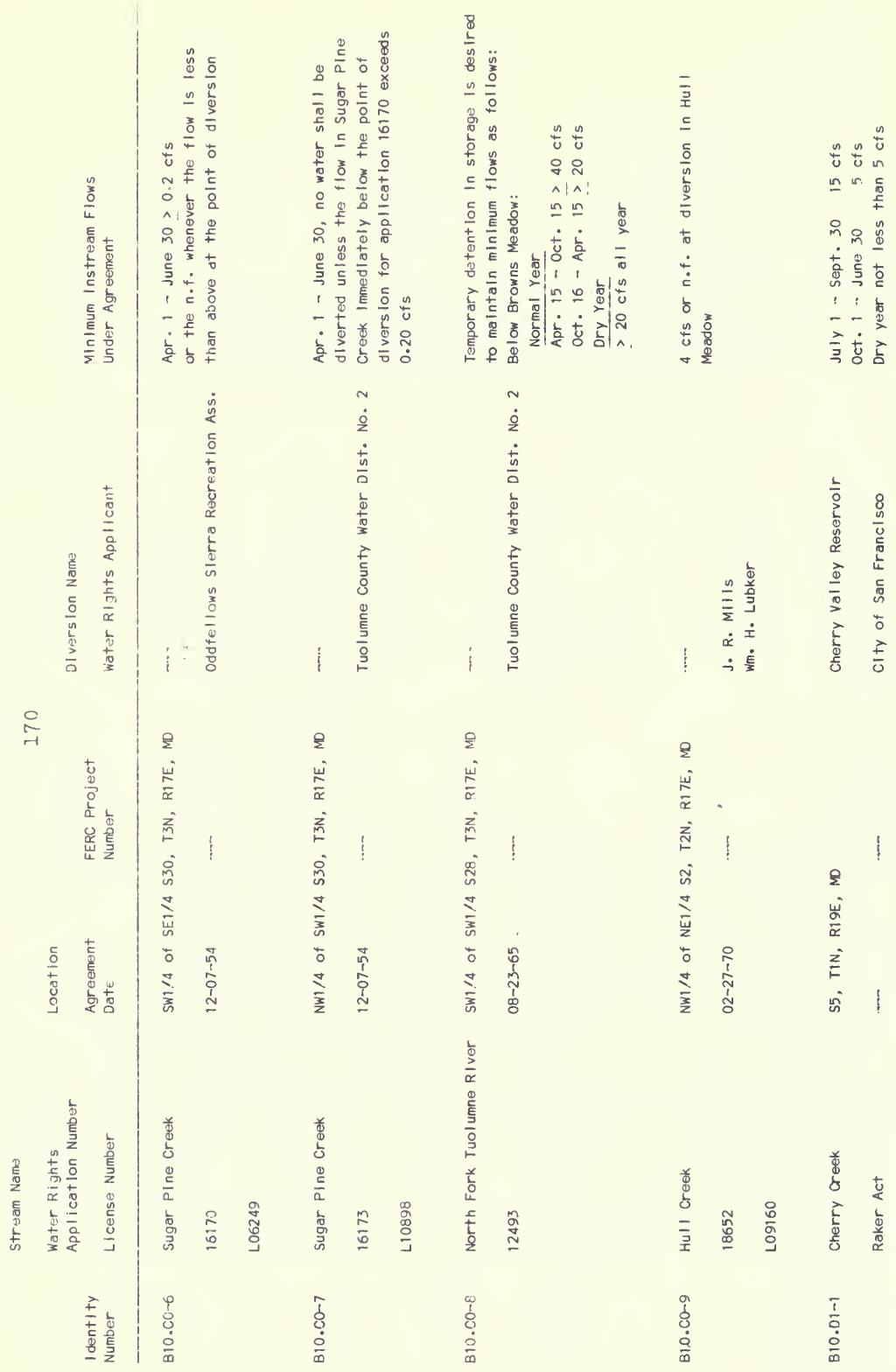




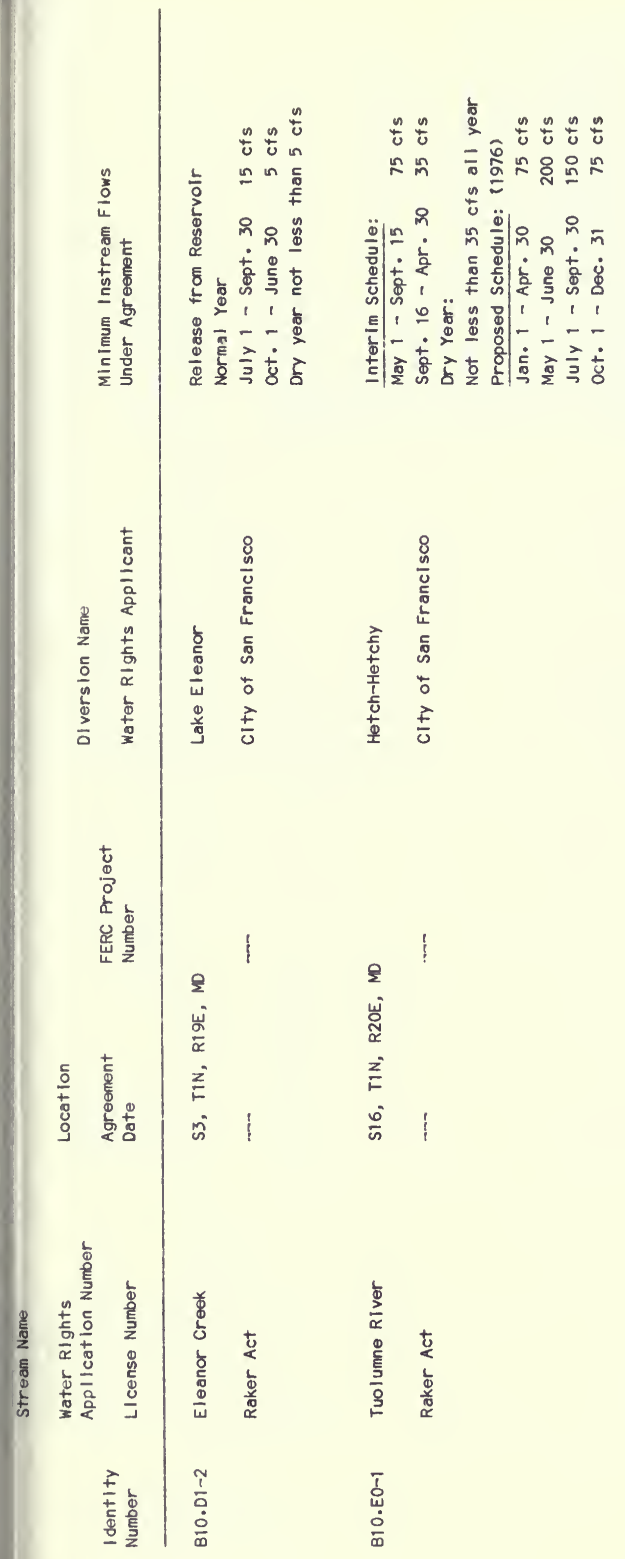

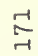




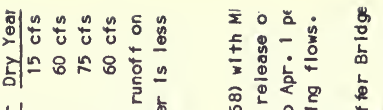

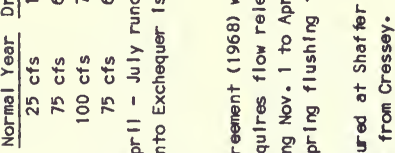

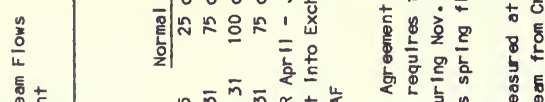

$$
\begin{aligned}
& \text { 离 }
\end{aligned}
$$

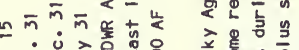

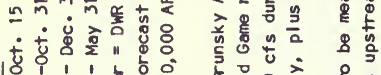

$$
\begin{aligned}
& \text { 罡| }
\end{aligned}
$$

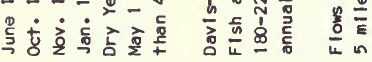

$$
\begin{aligned}
& \stackrel{N}{N}
\end{aligned}
$$

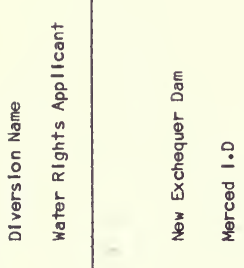

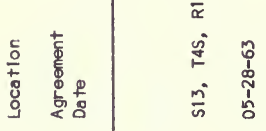

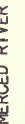

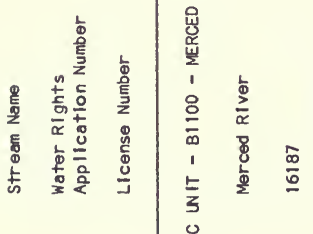

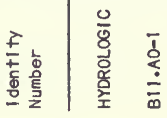




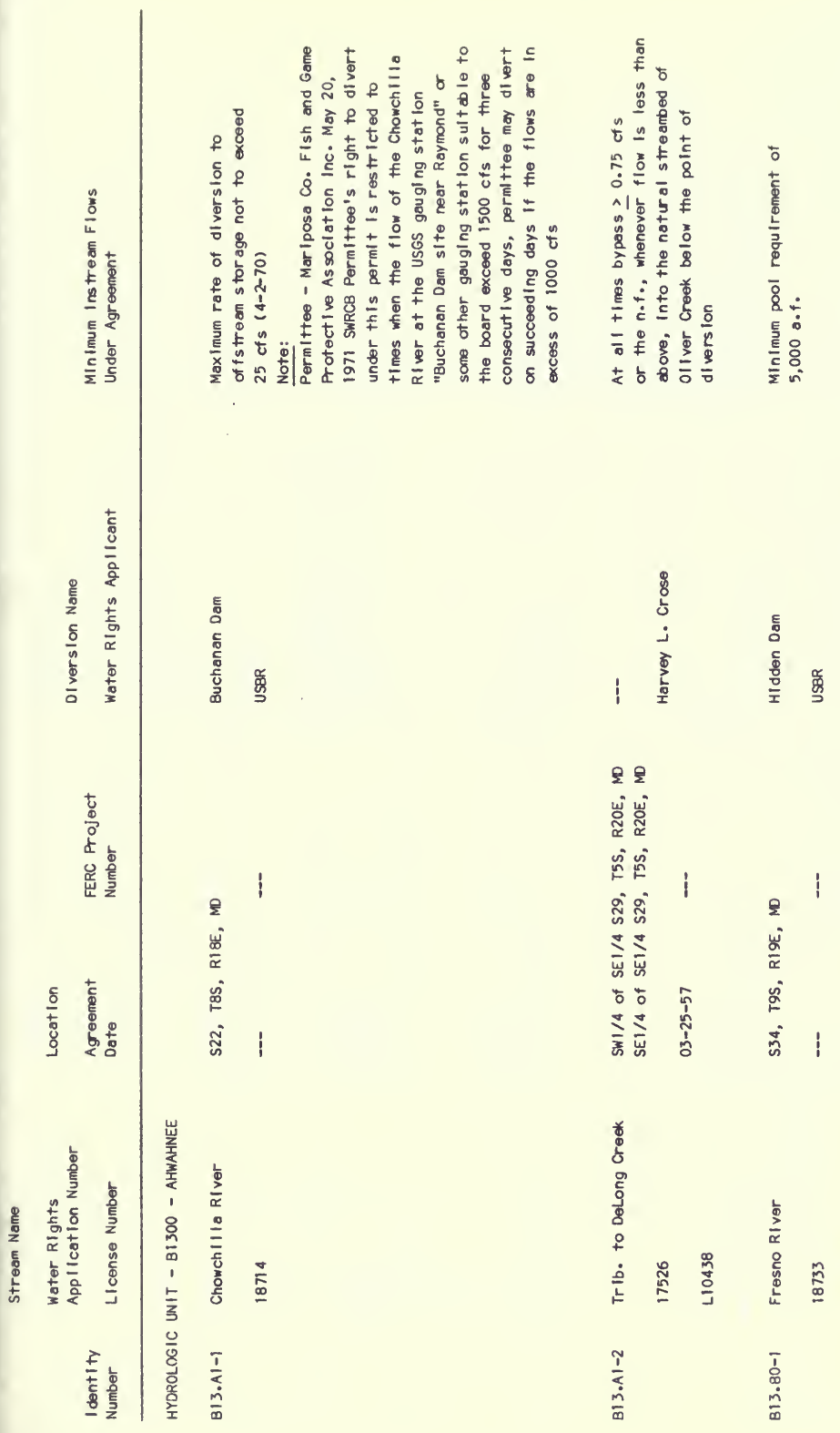




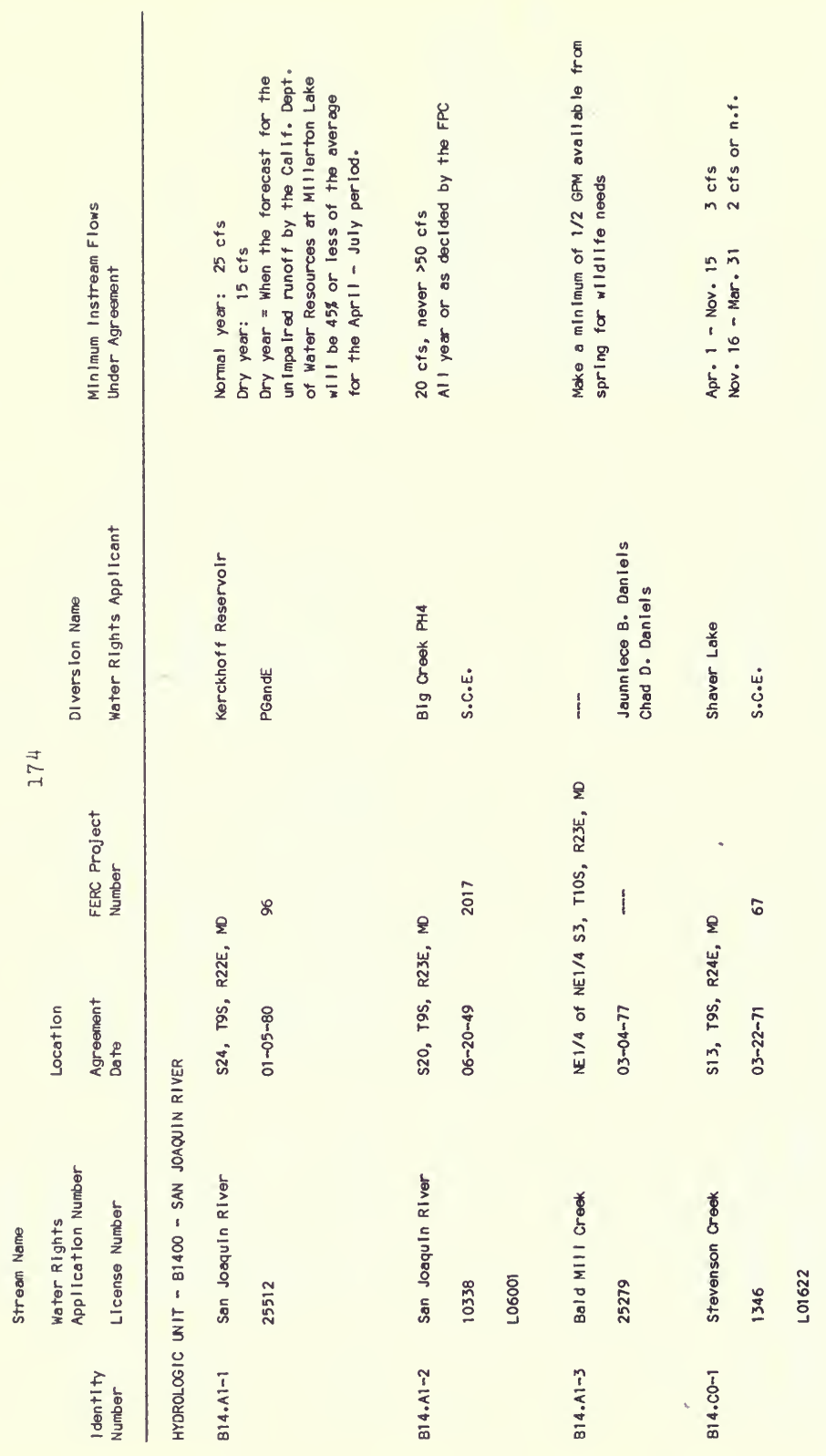




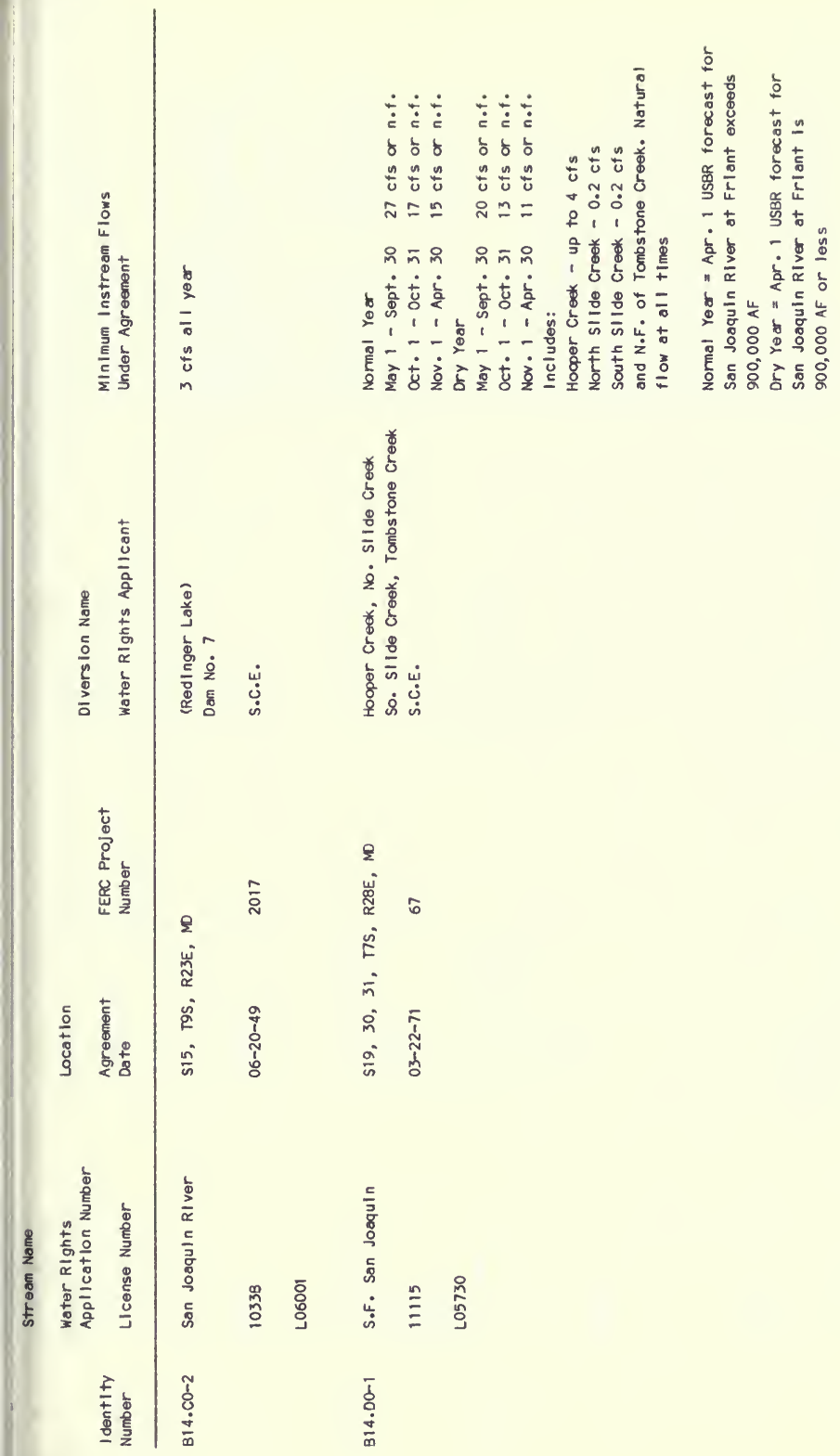




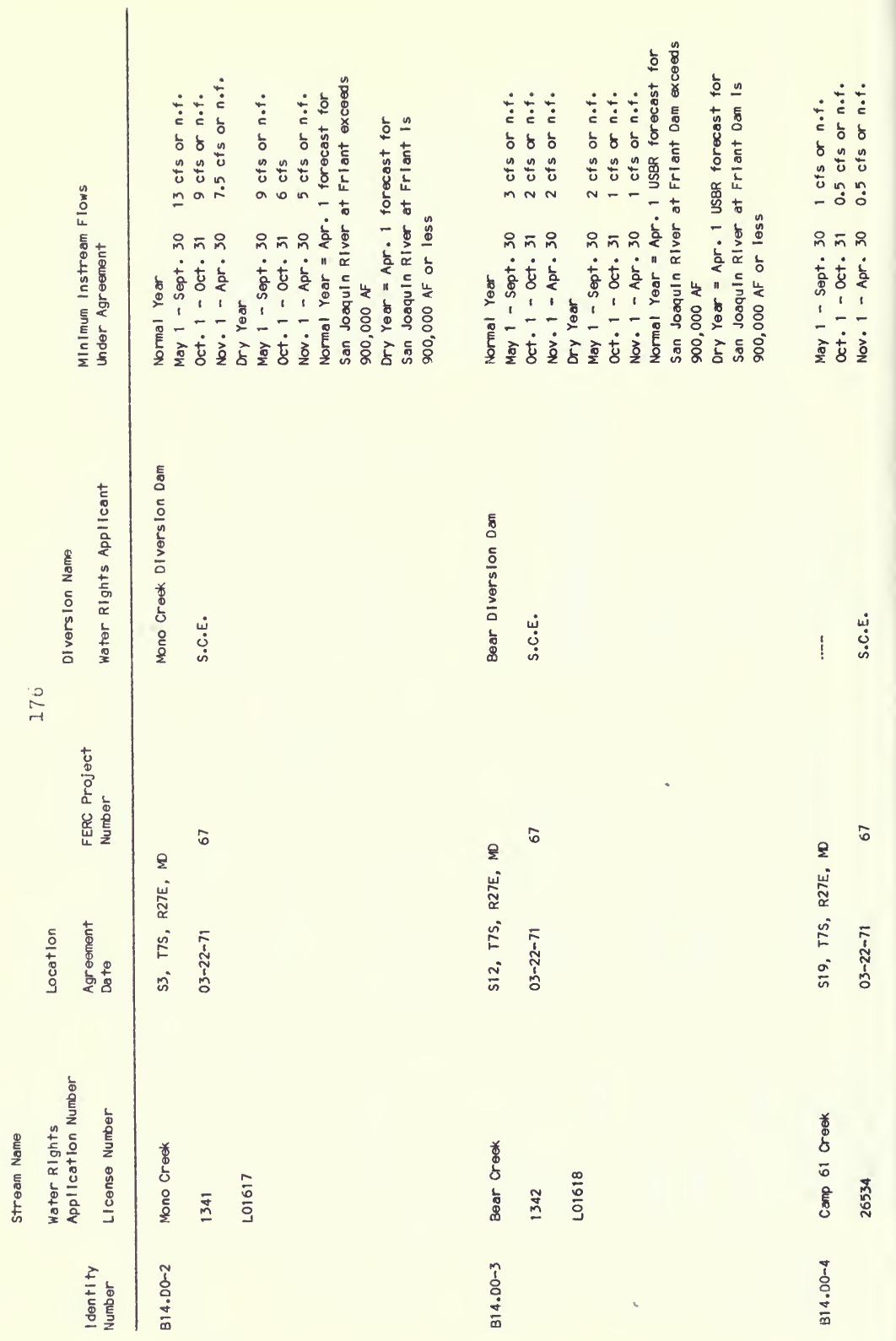




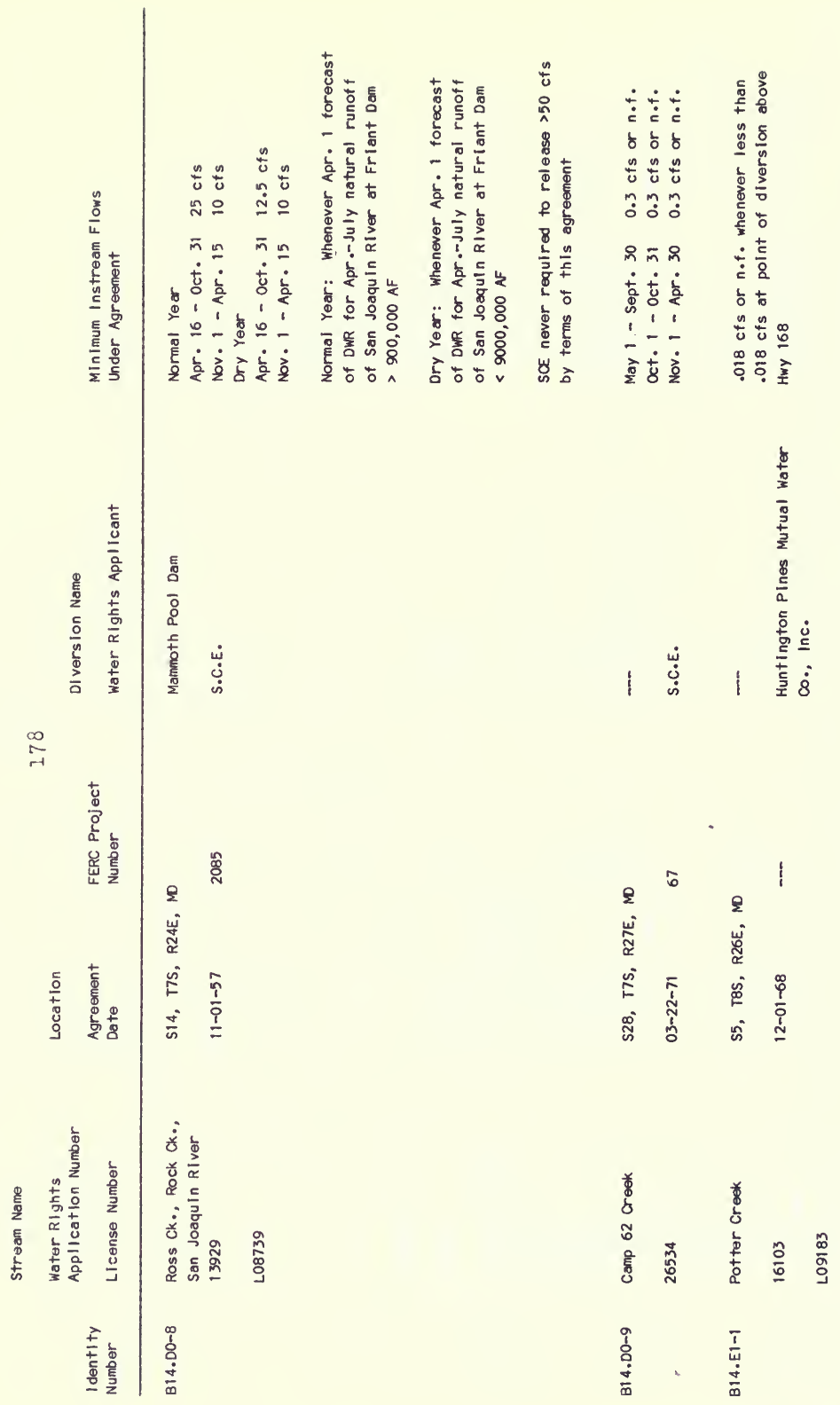




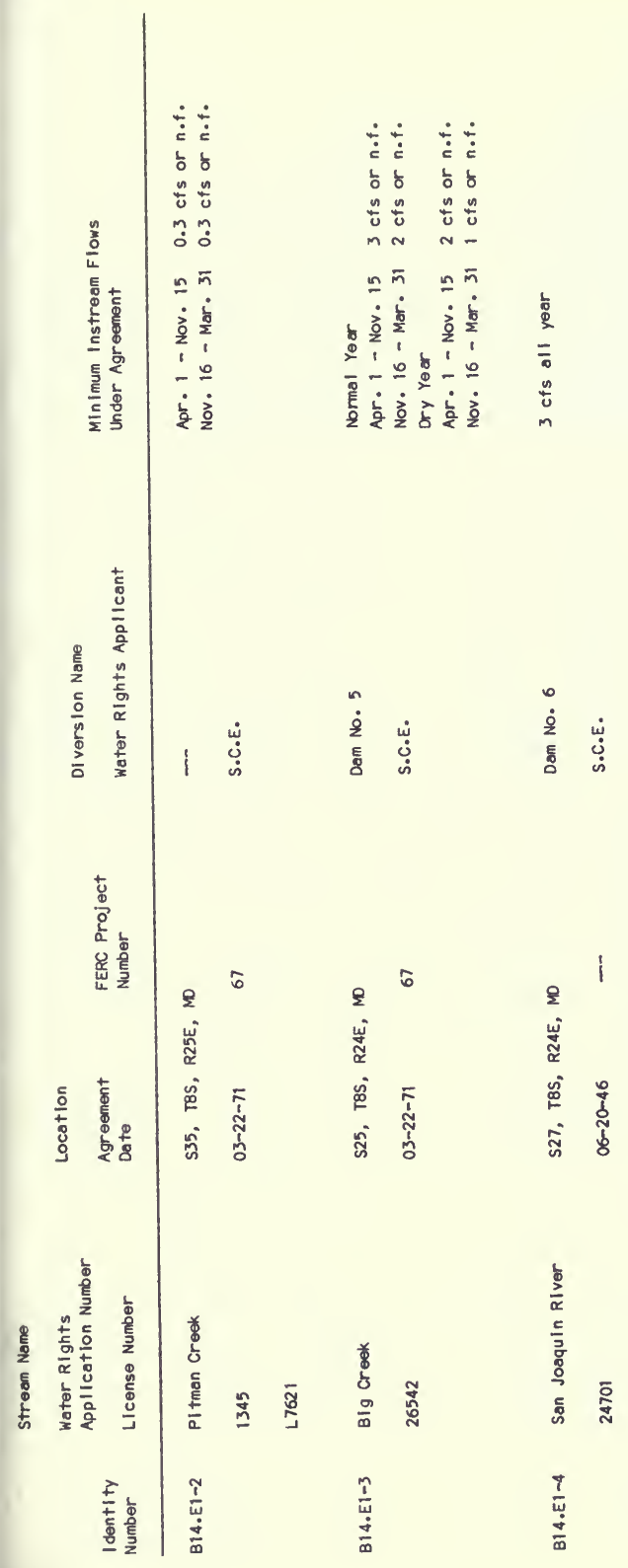

국 


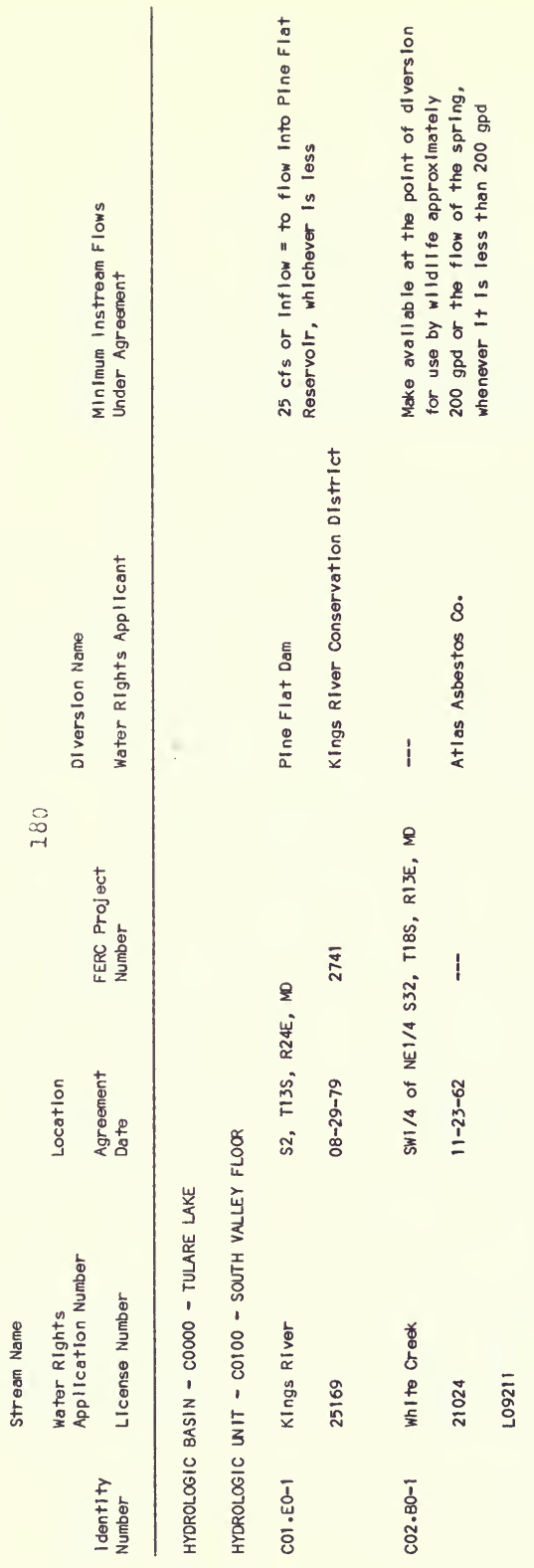





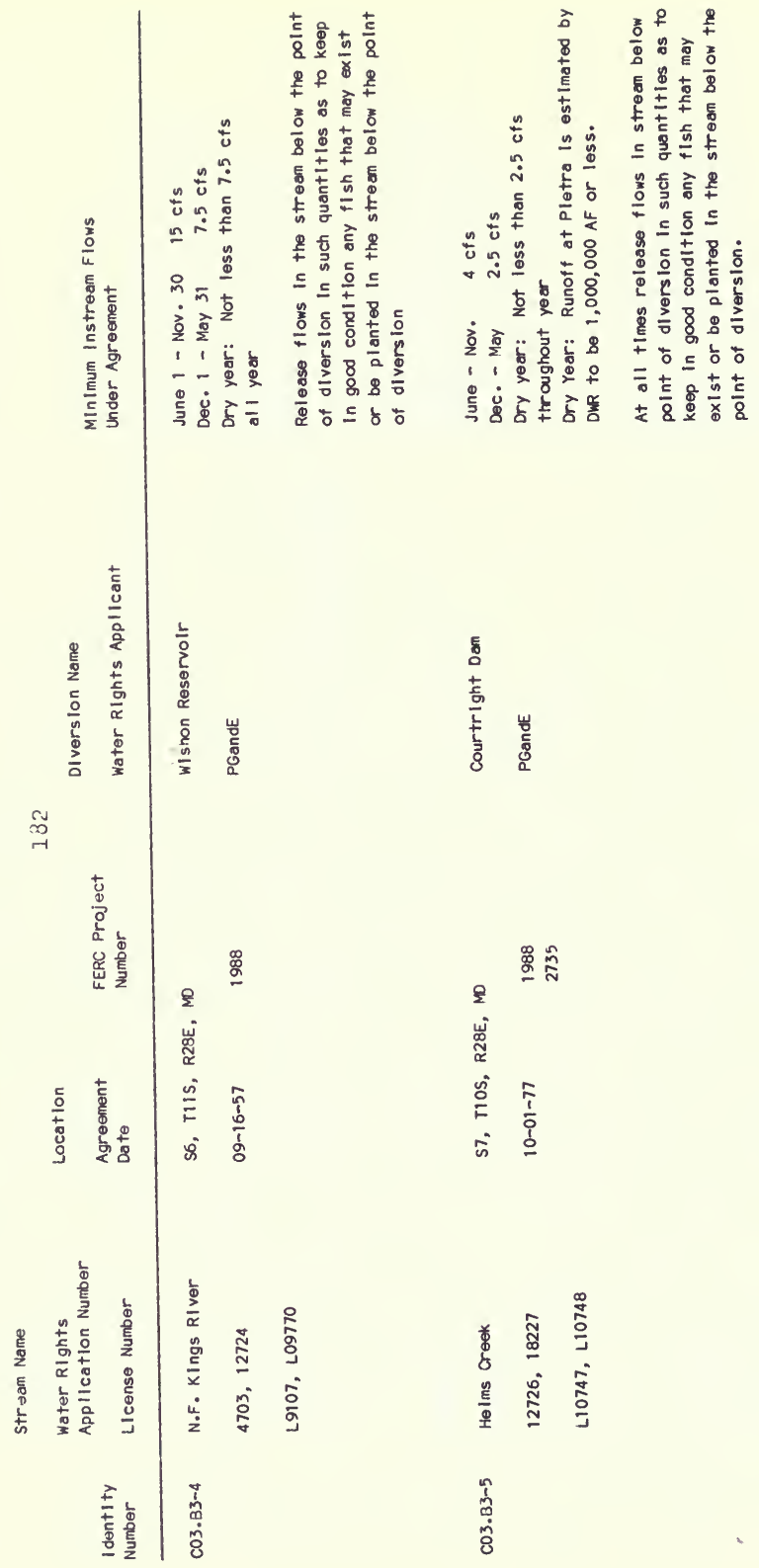




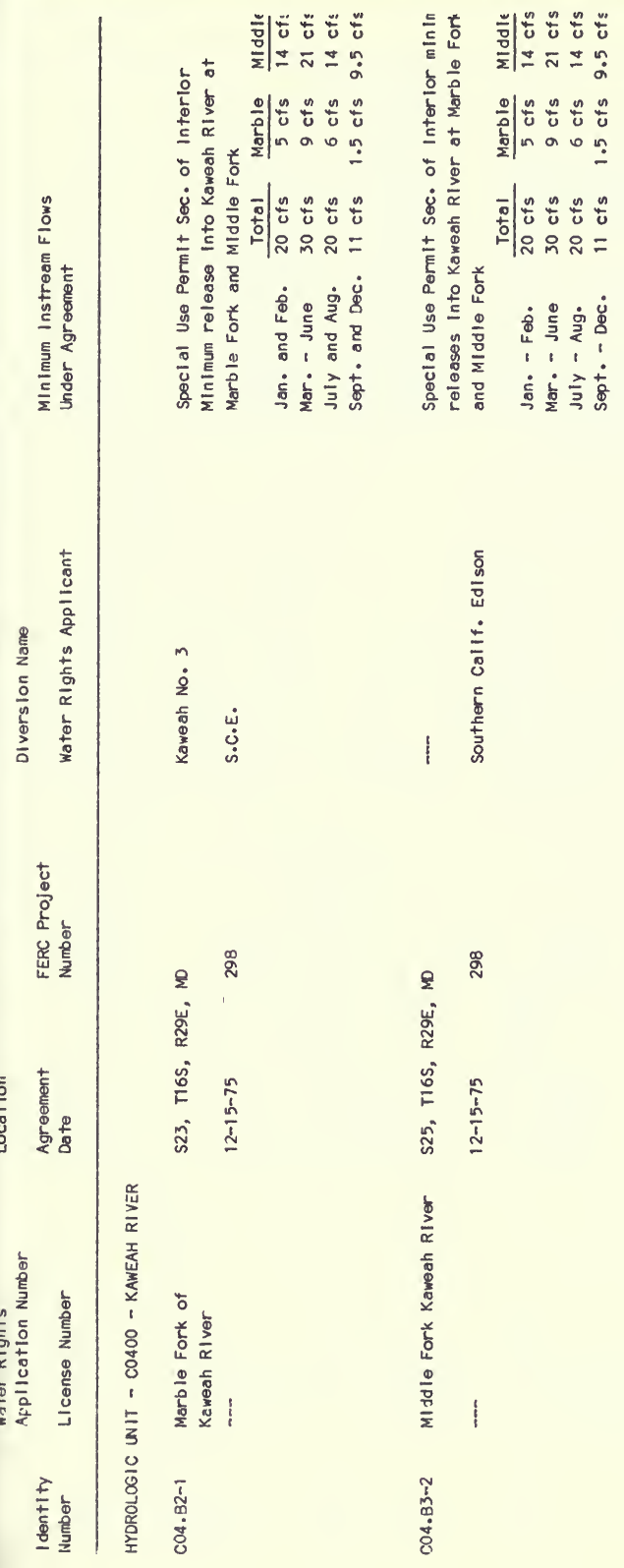

$m$
$\infty$ 


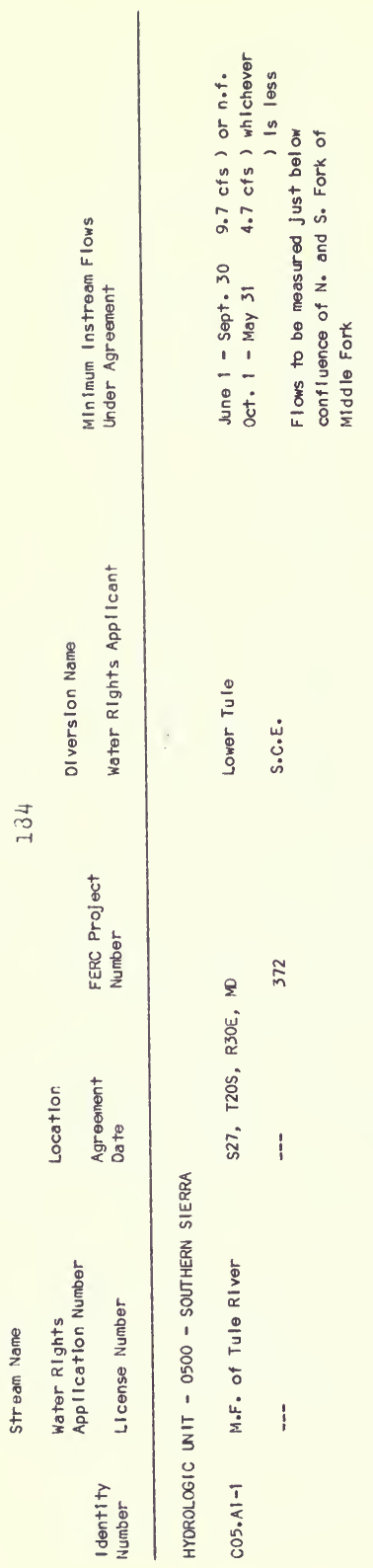





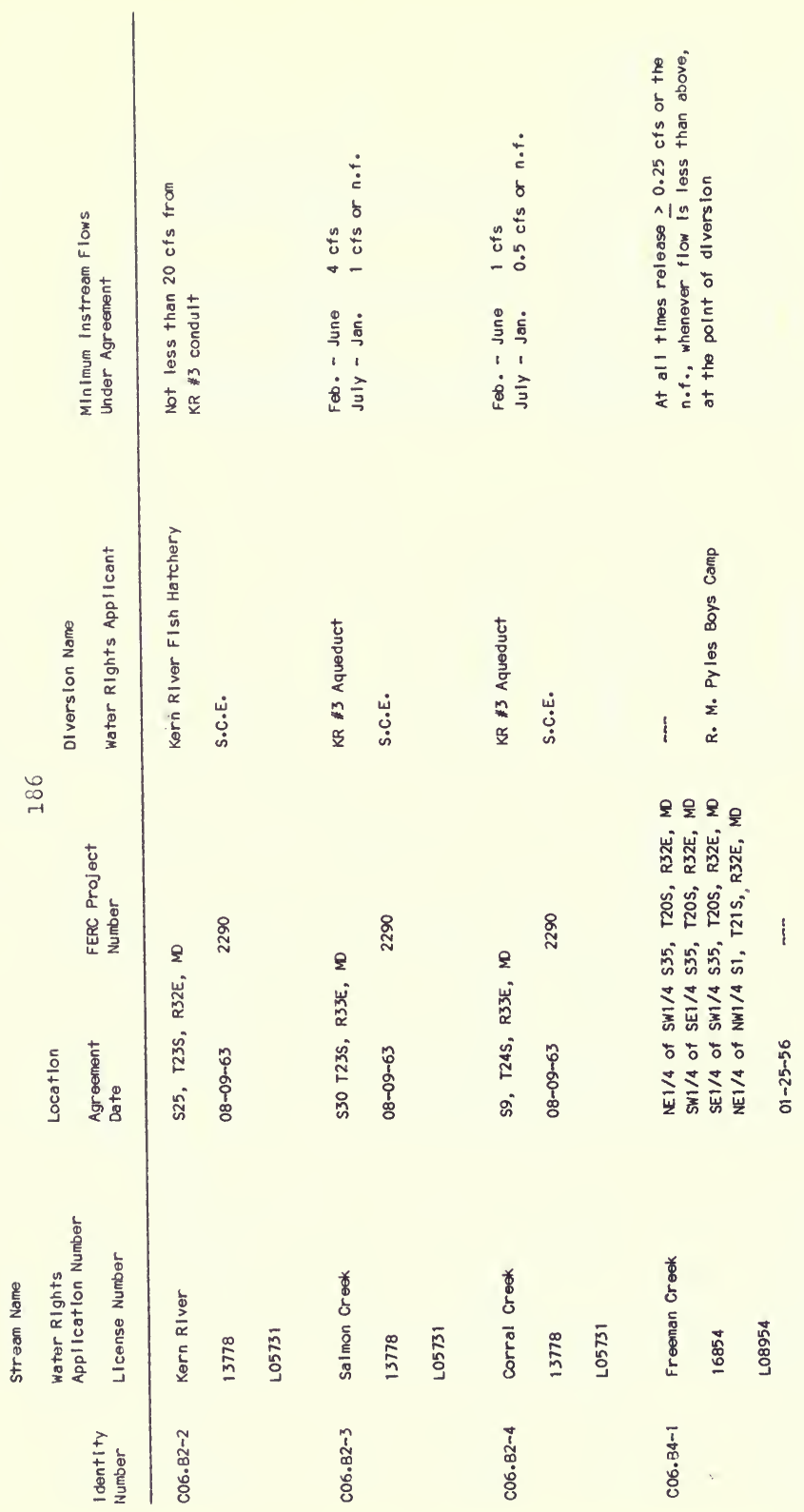




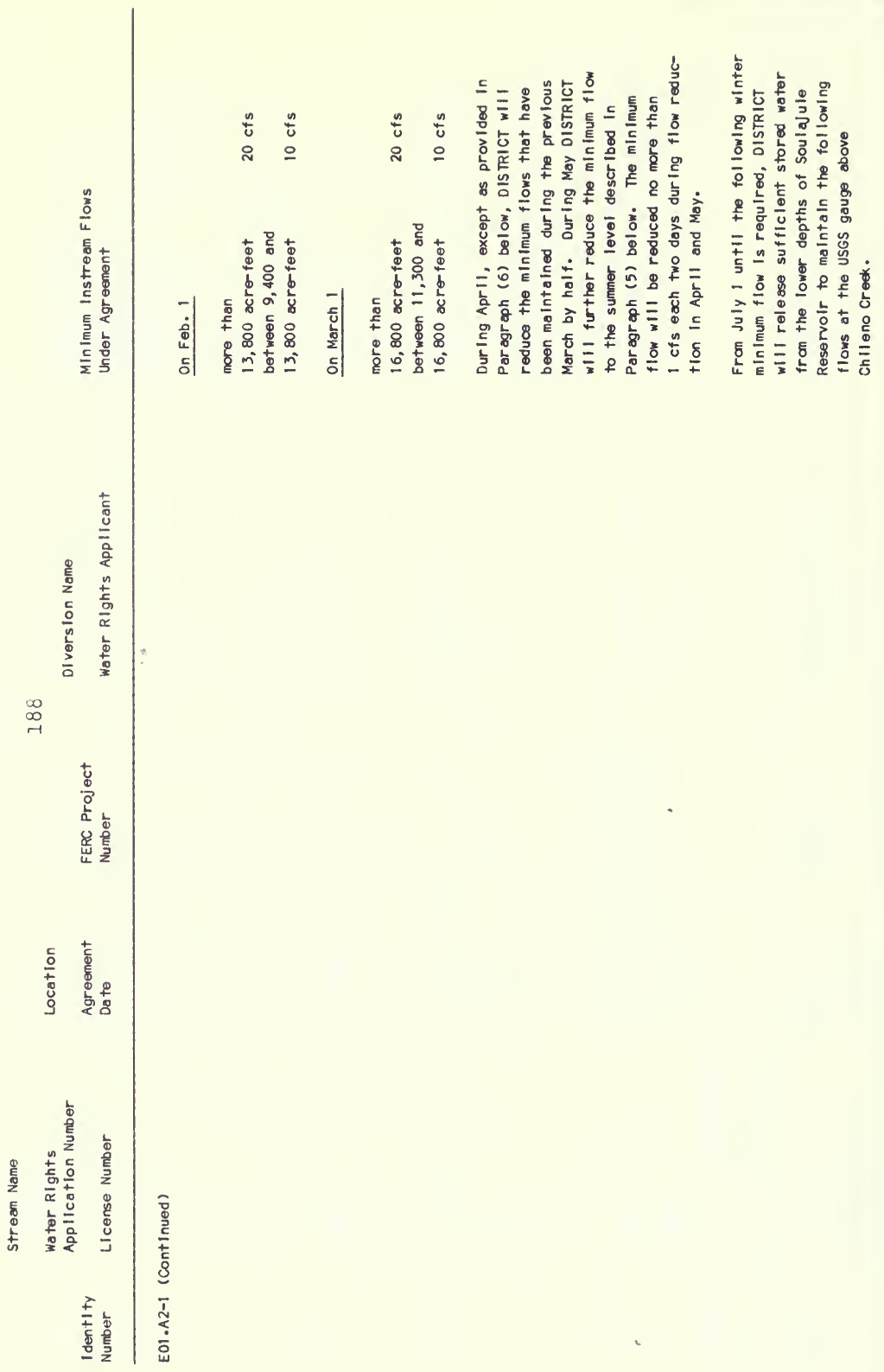




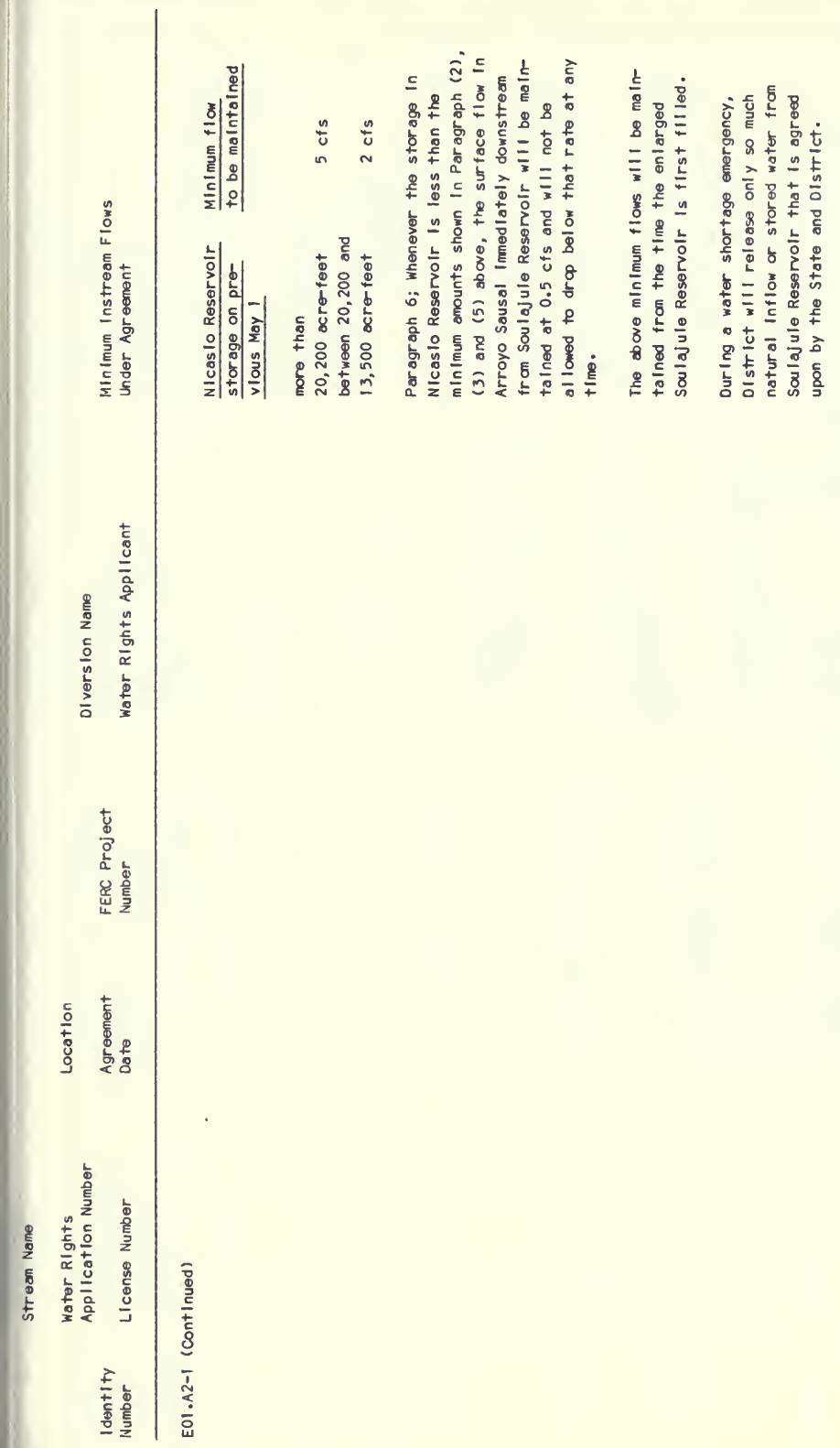




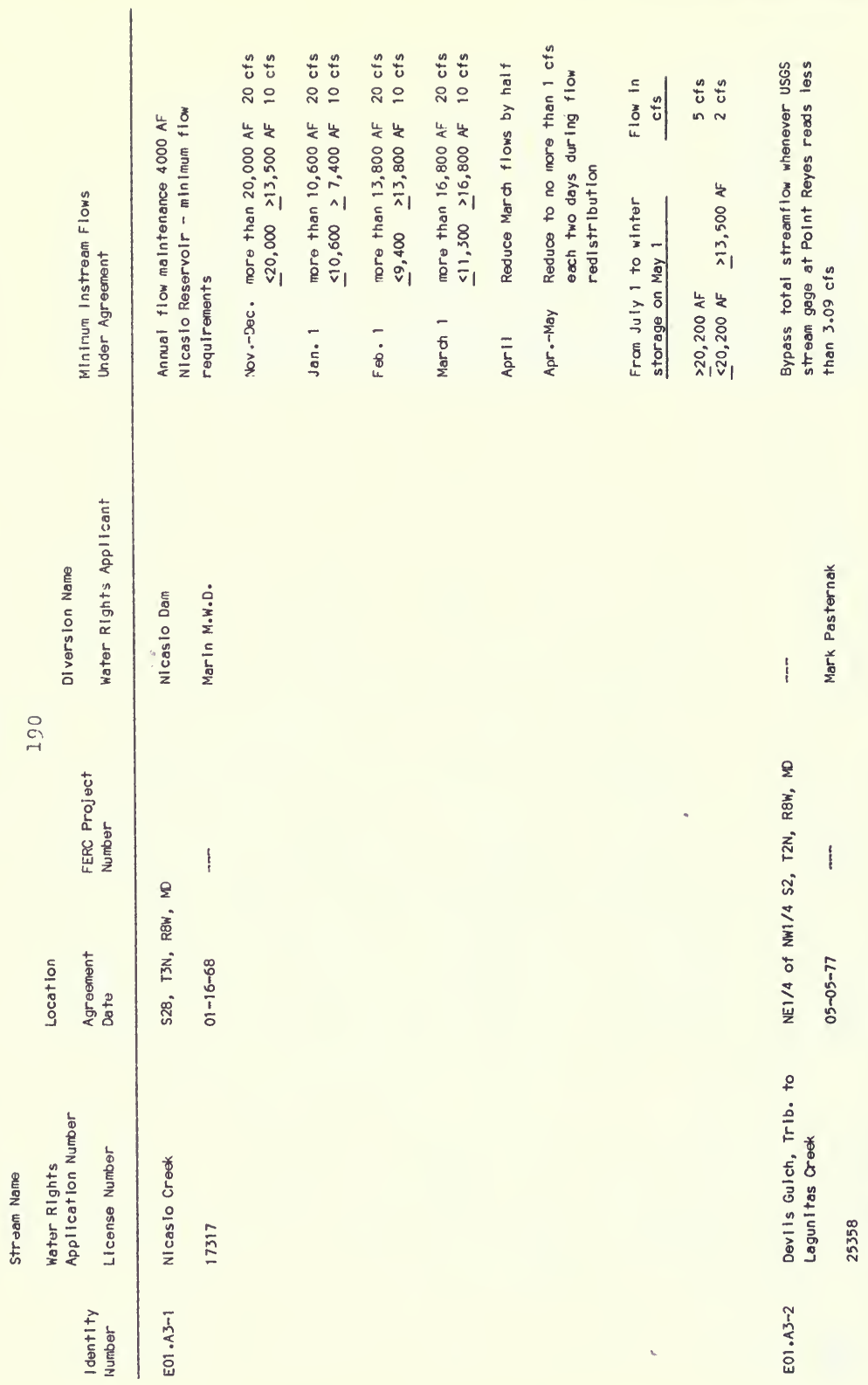




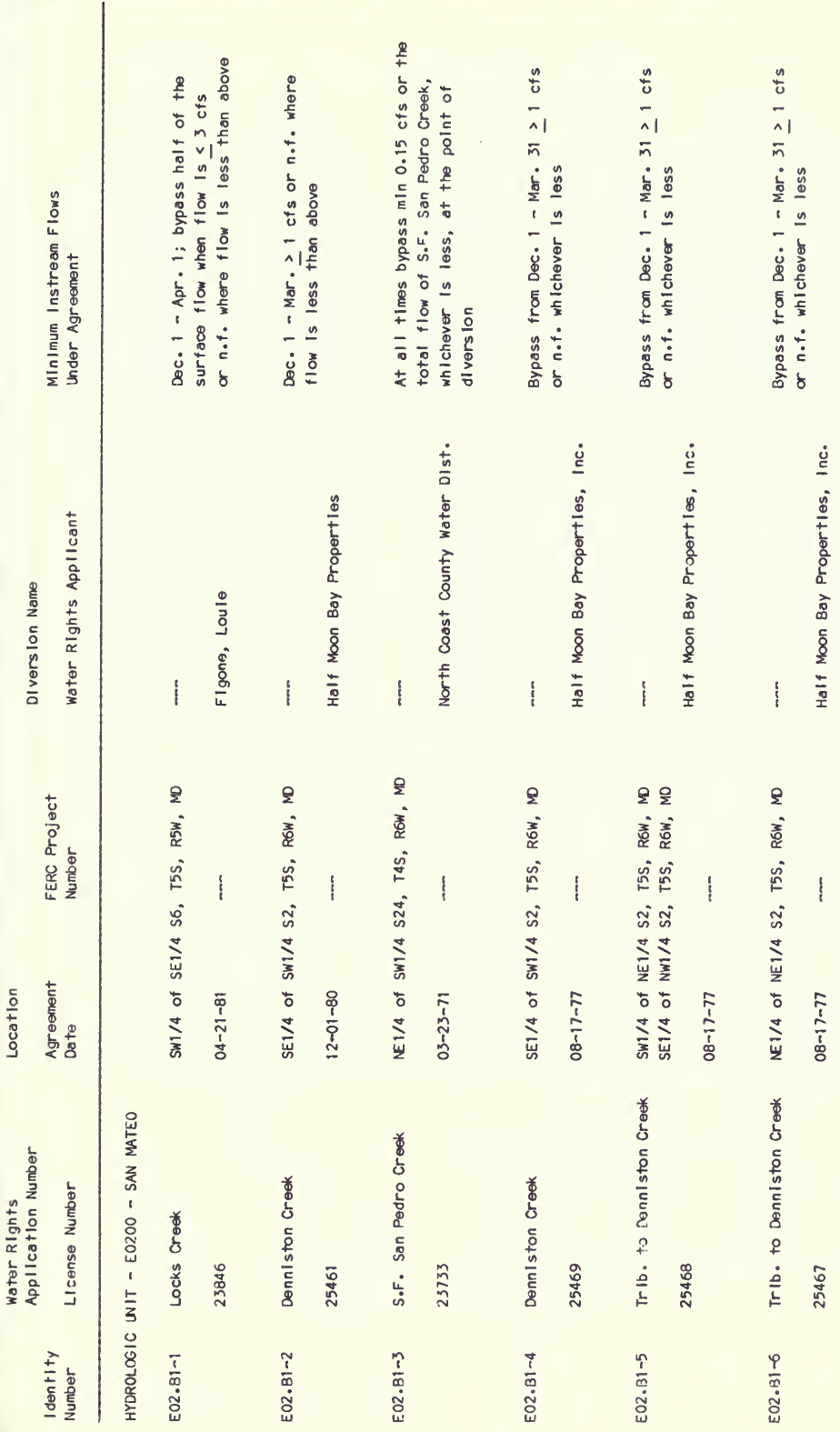




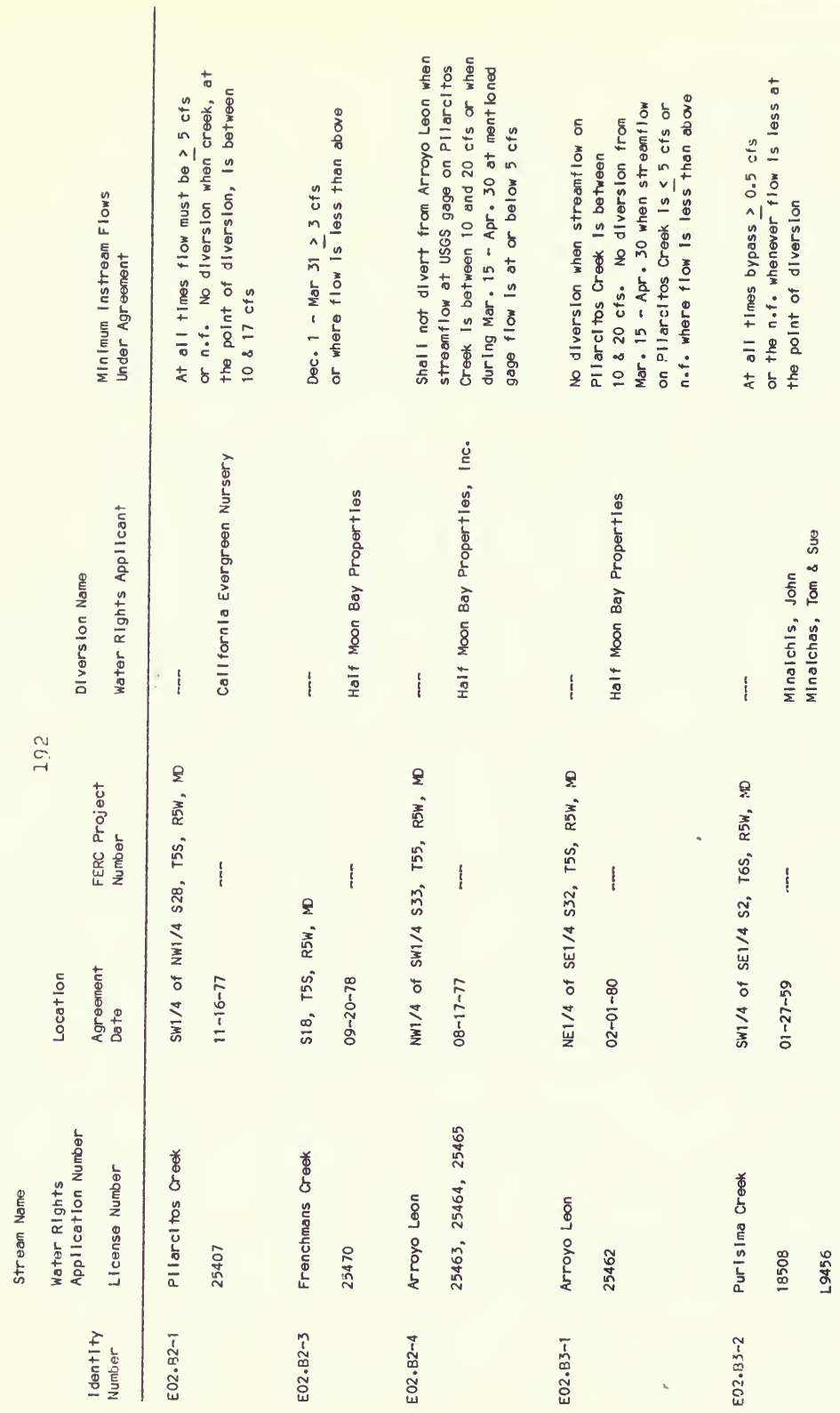




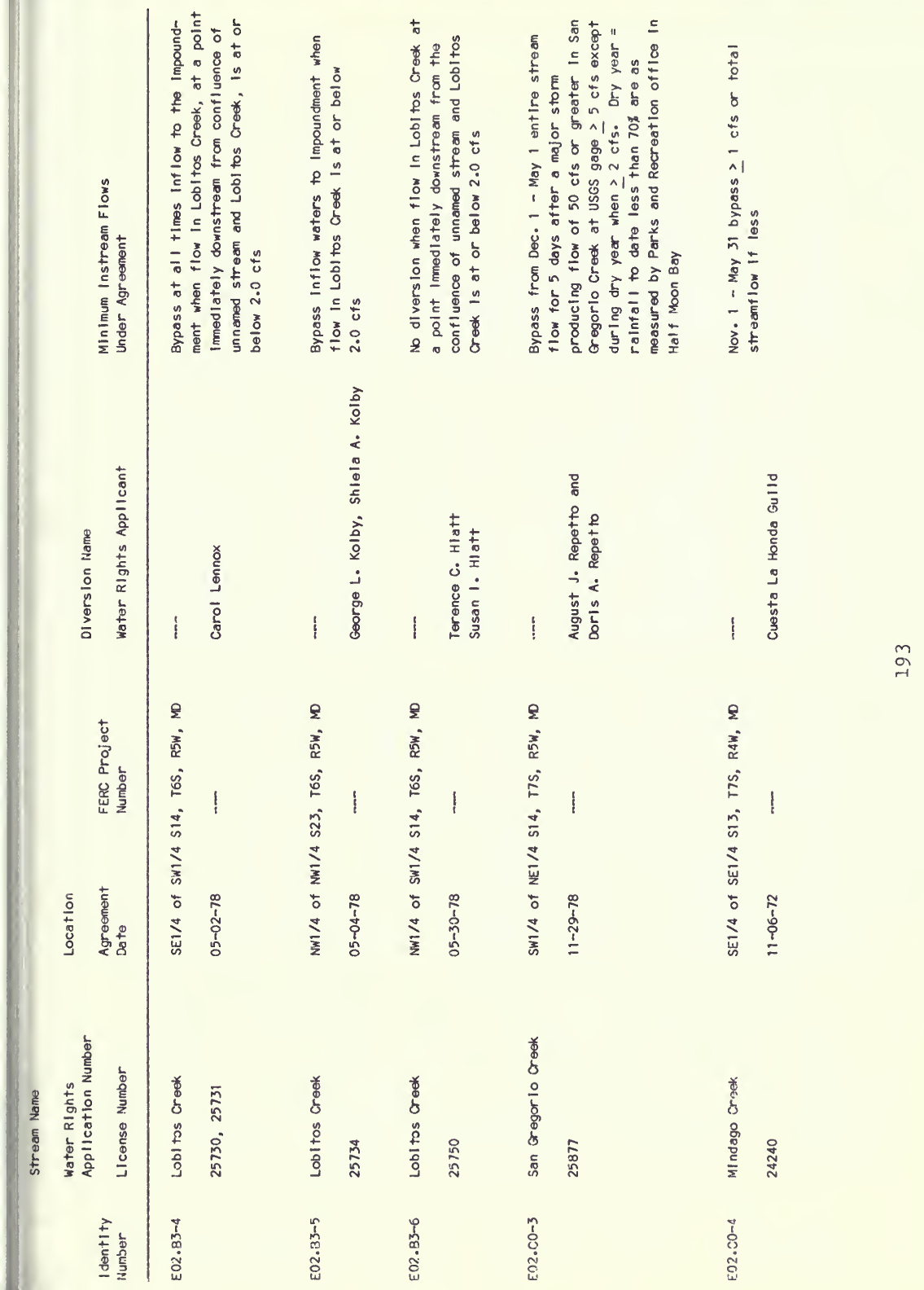



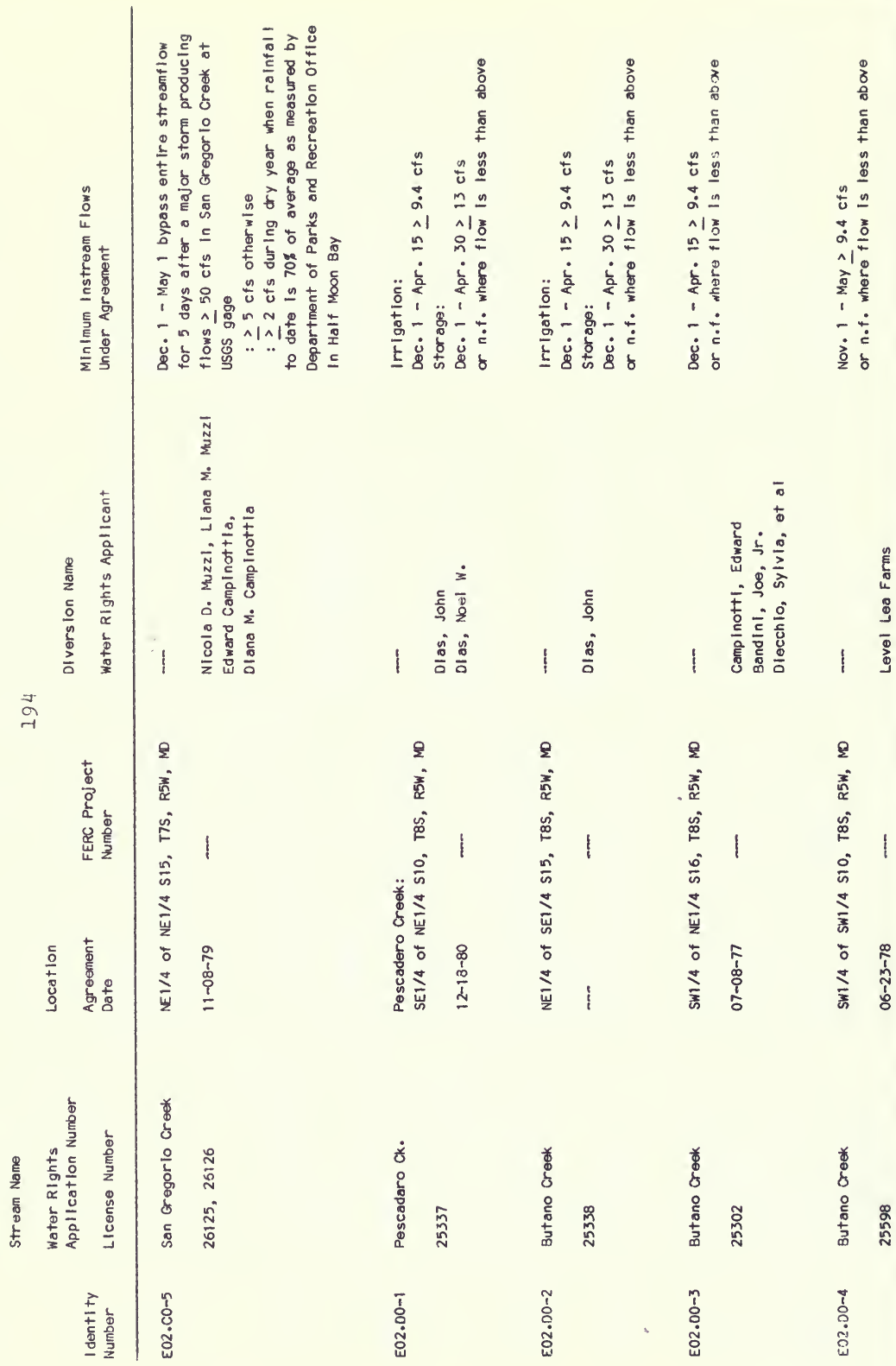


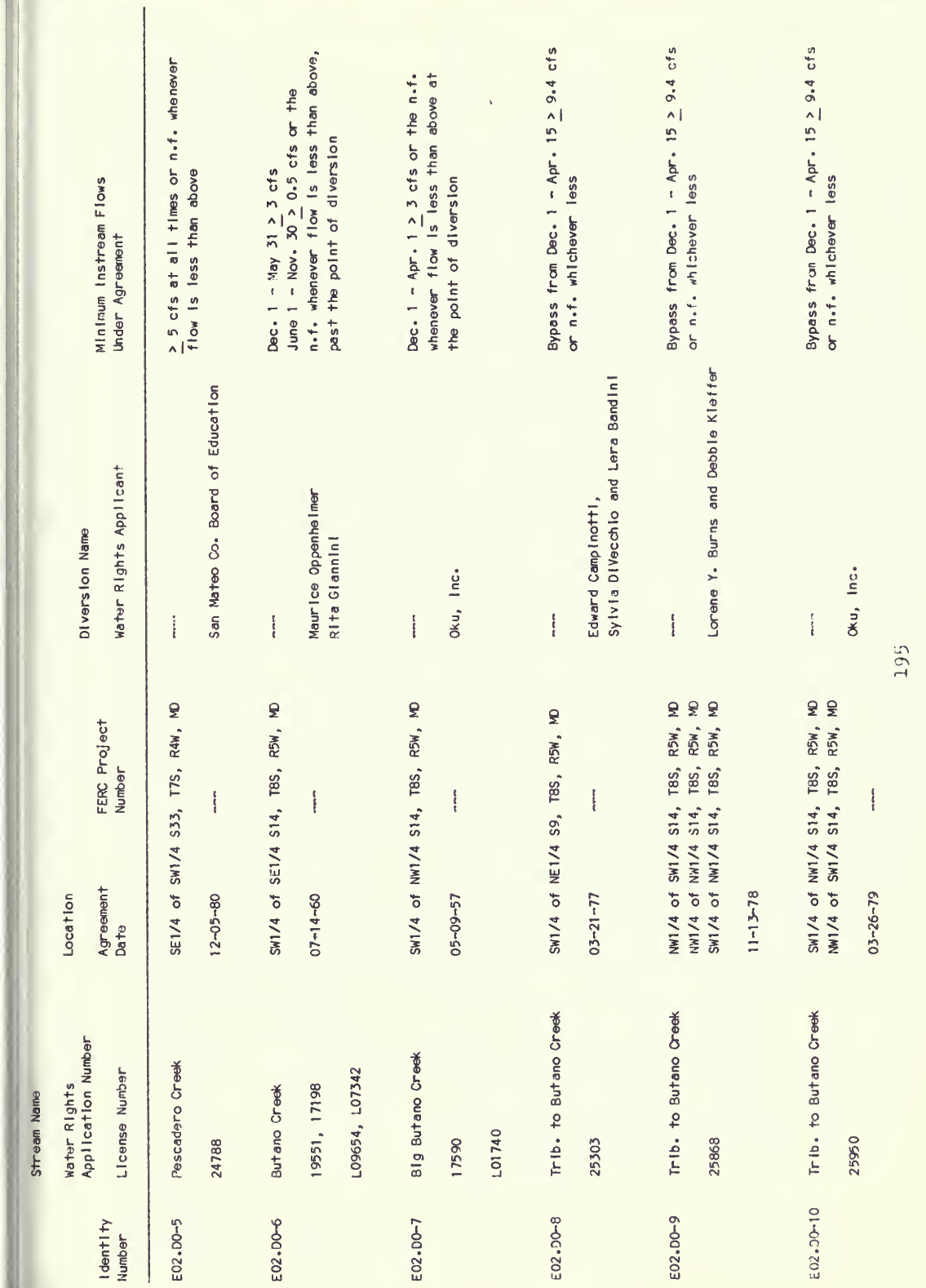




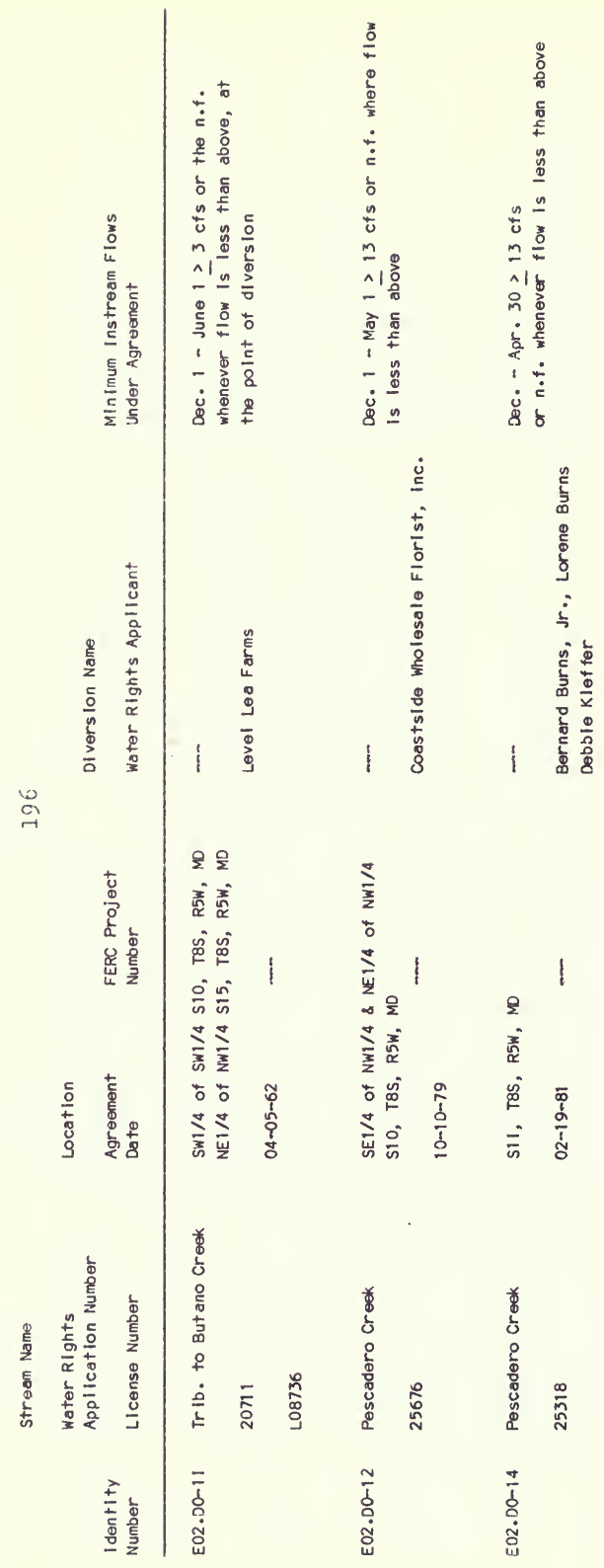




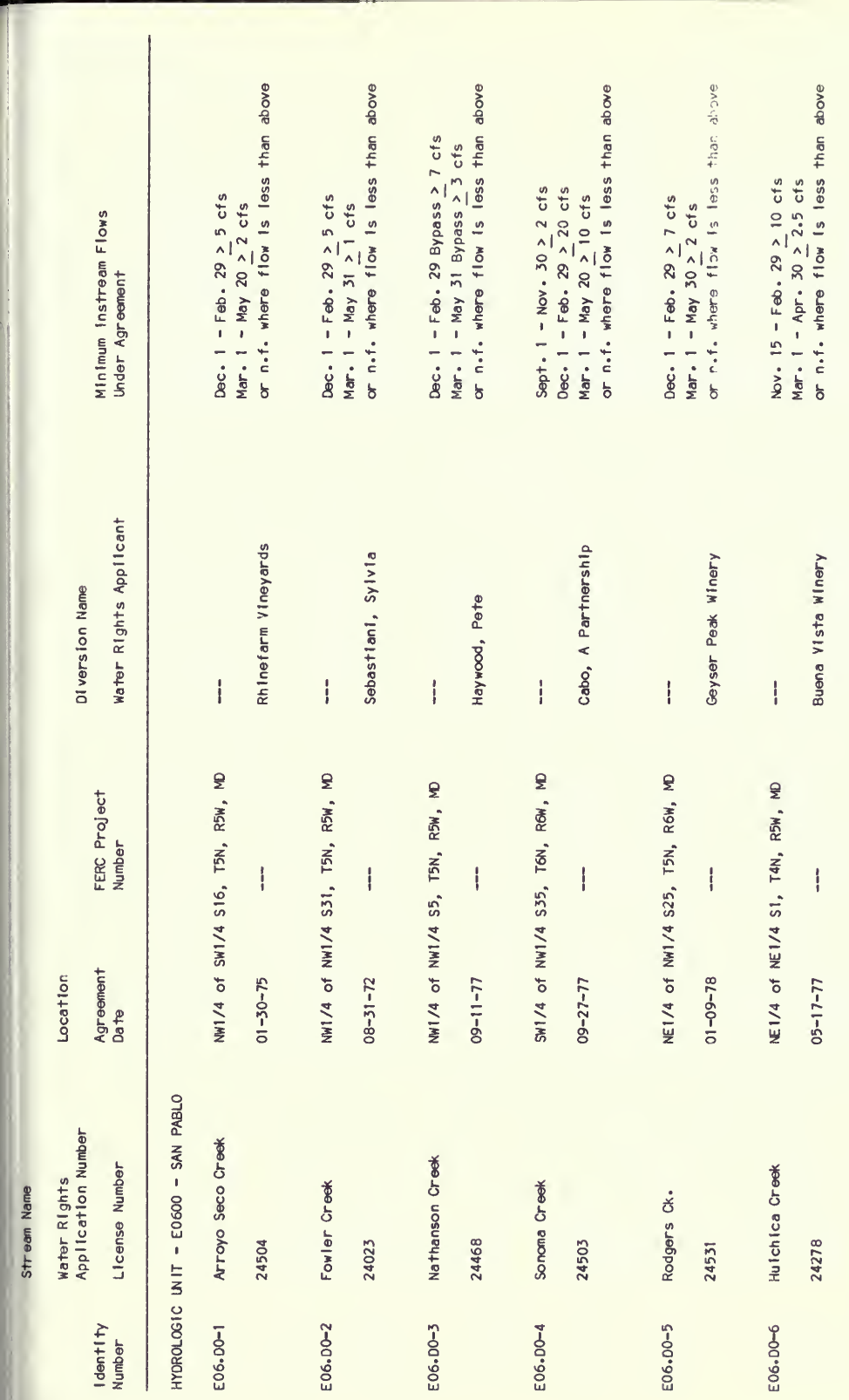

$\Phi$ 


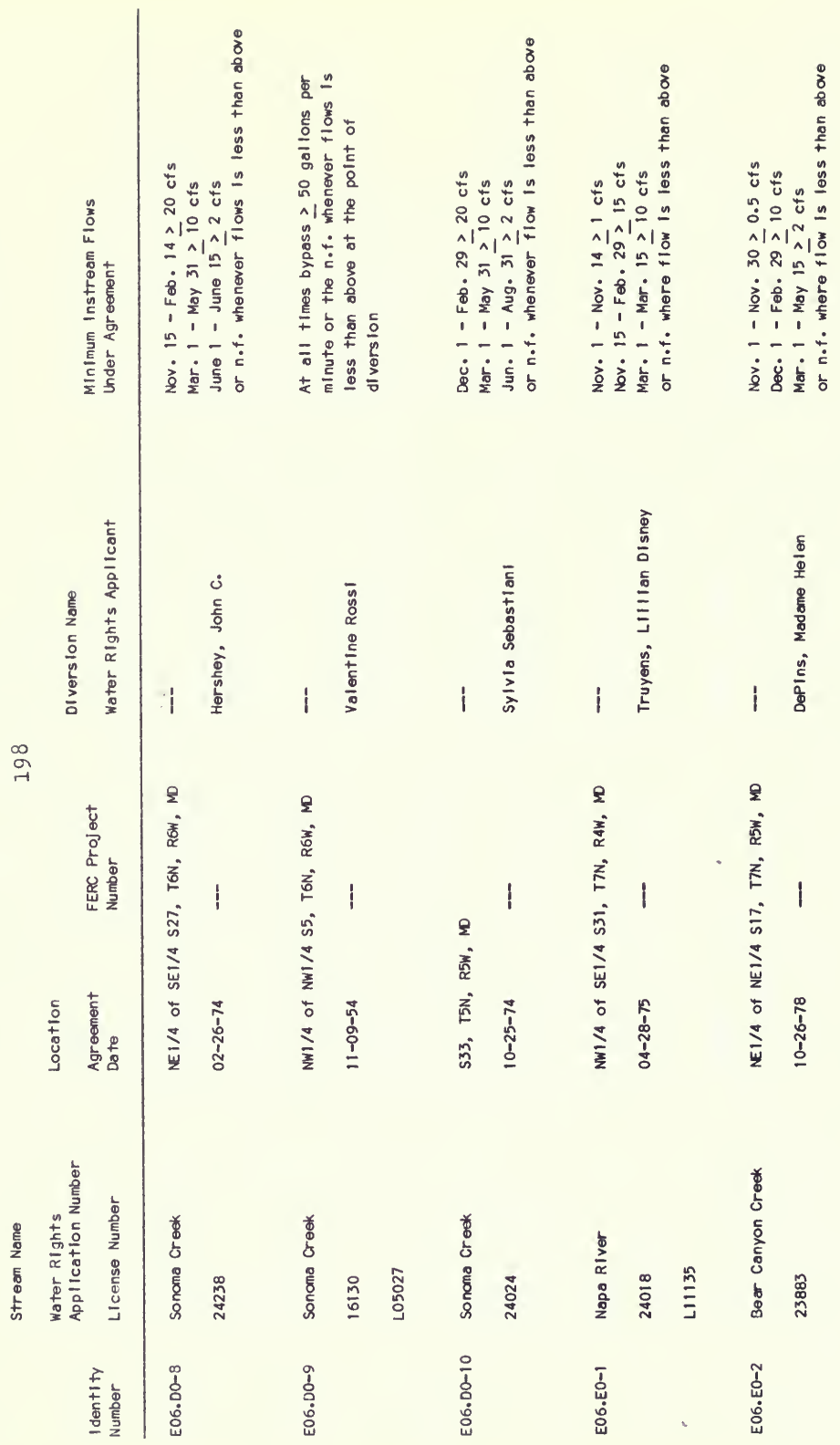




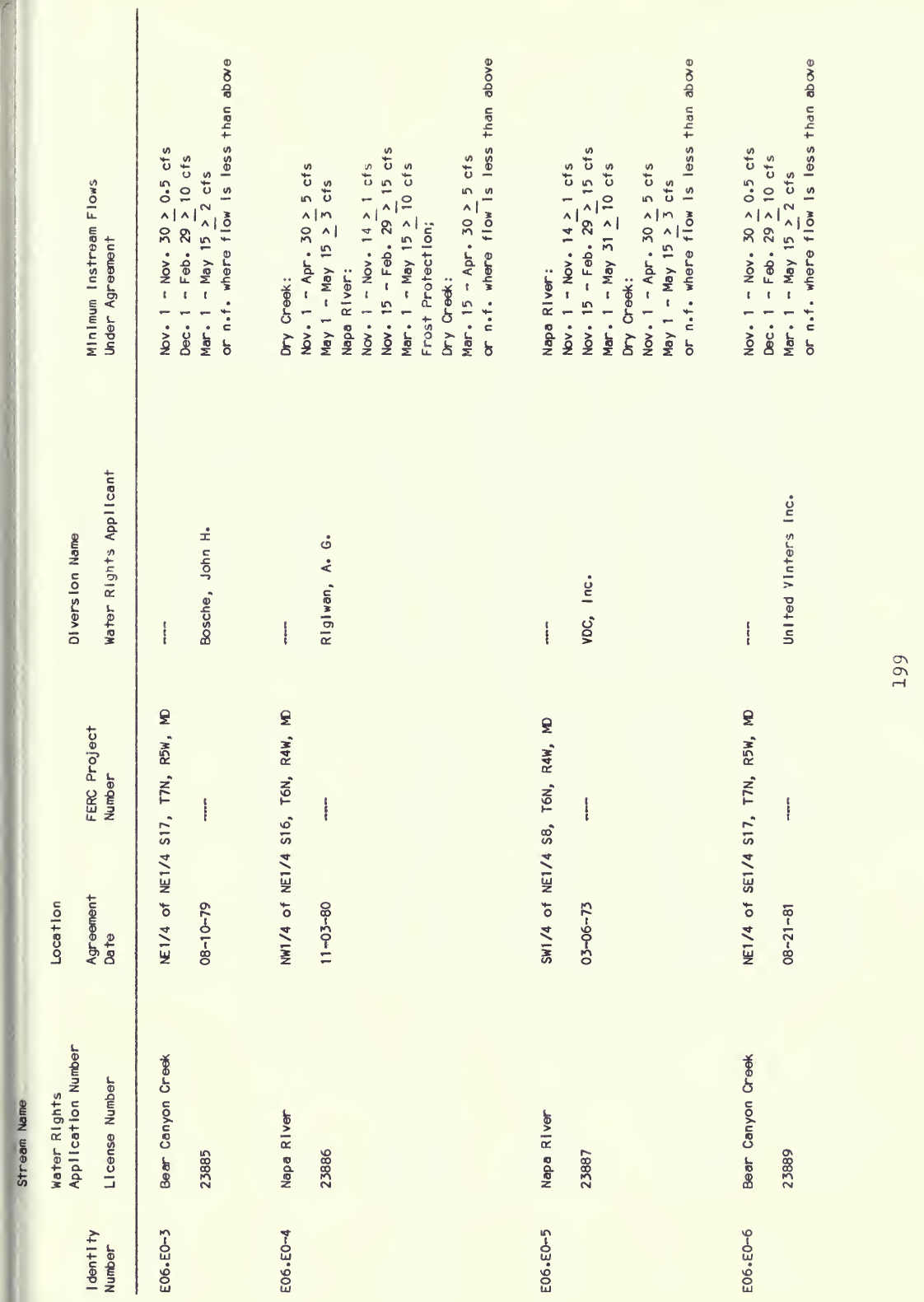




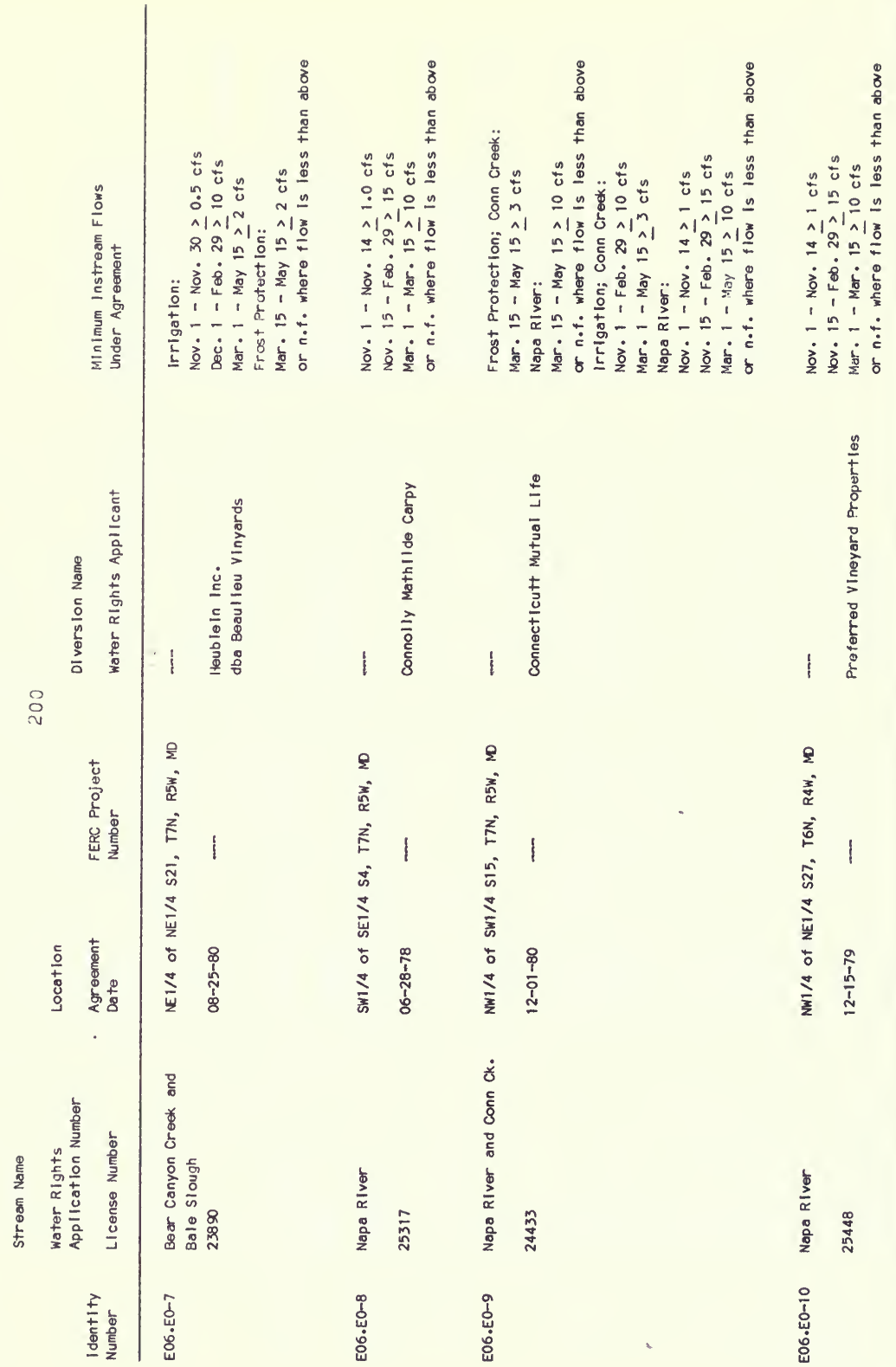





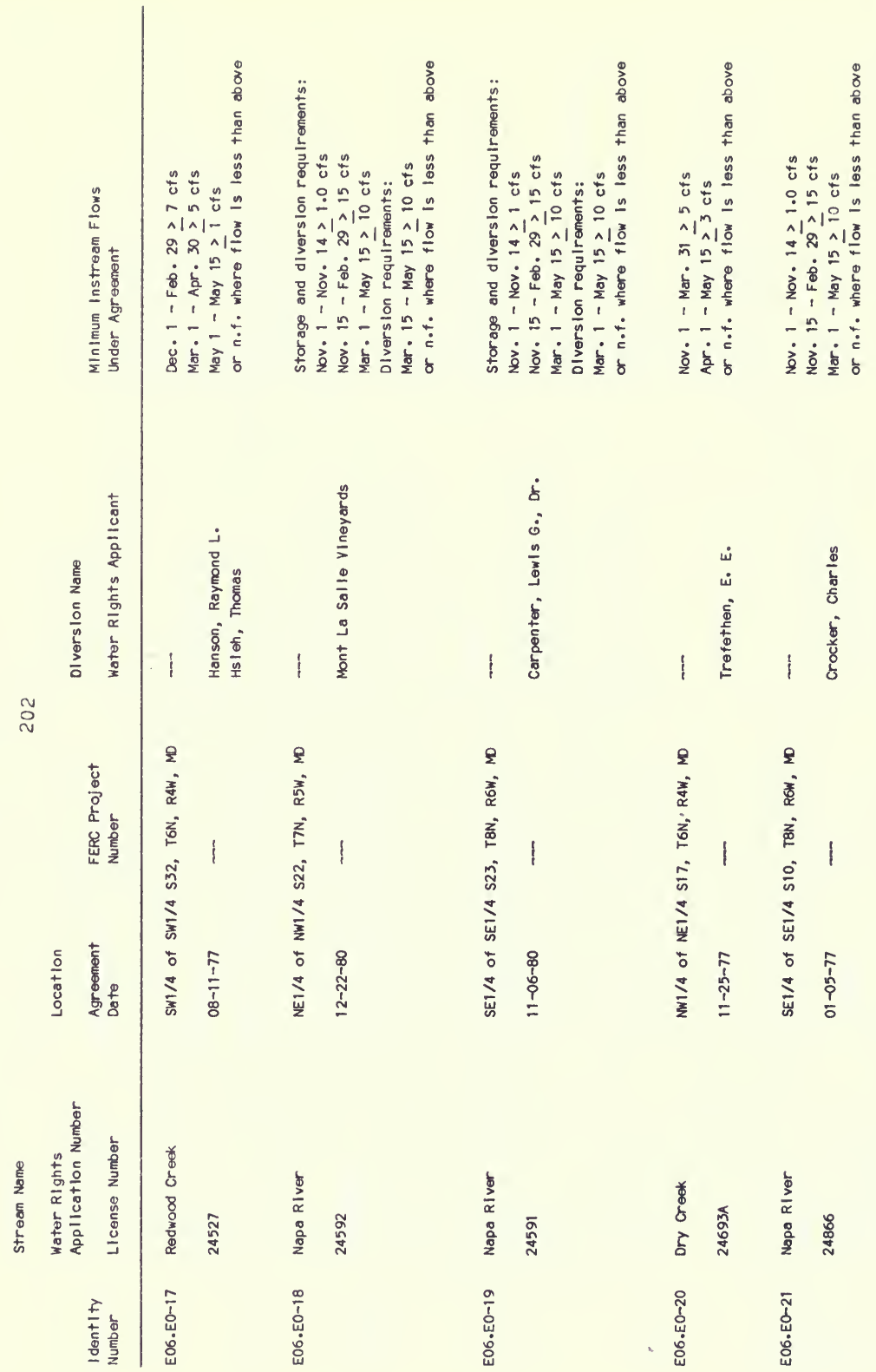




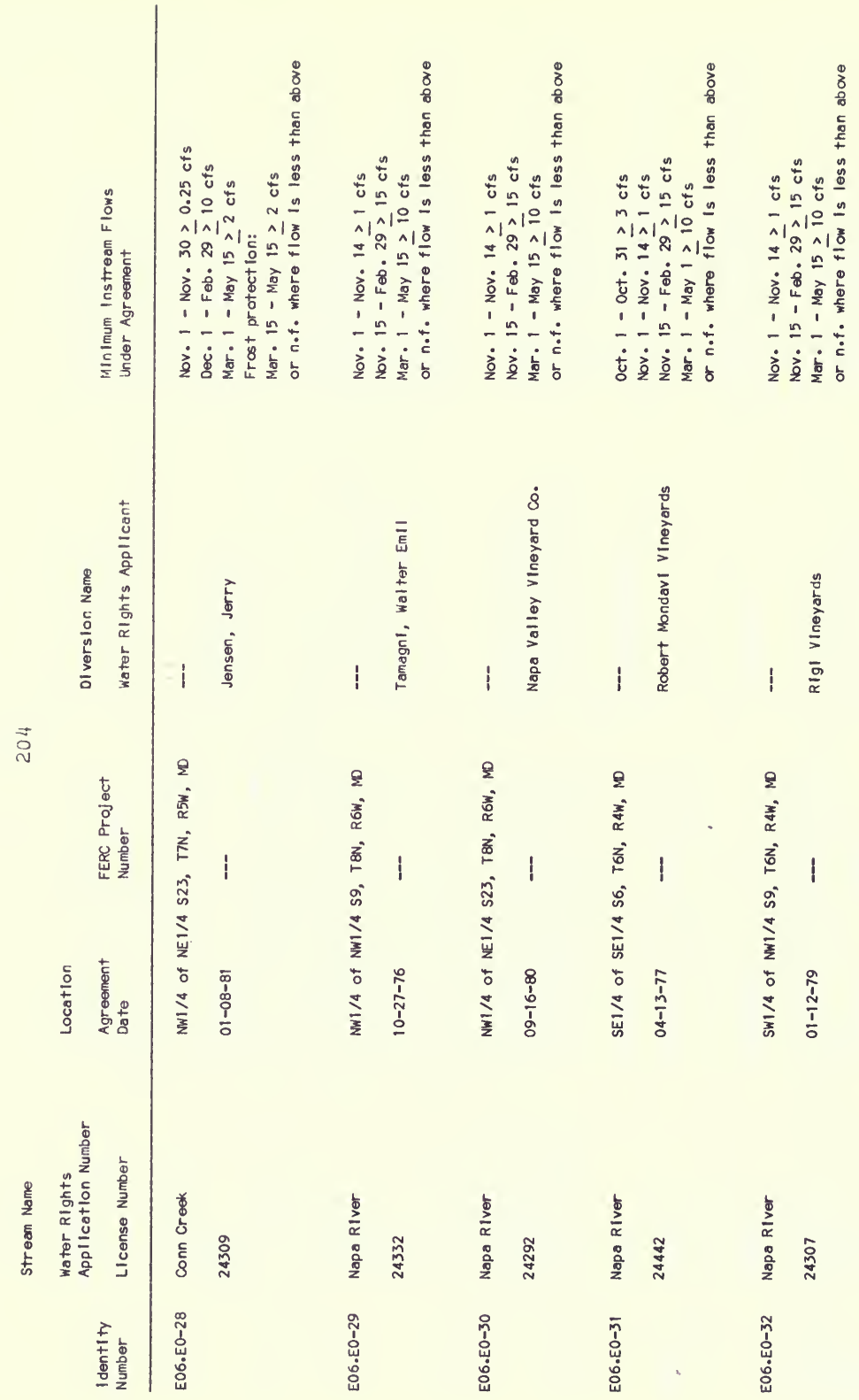




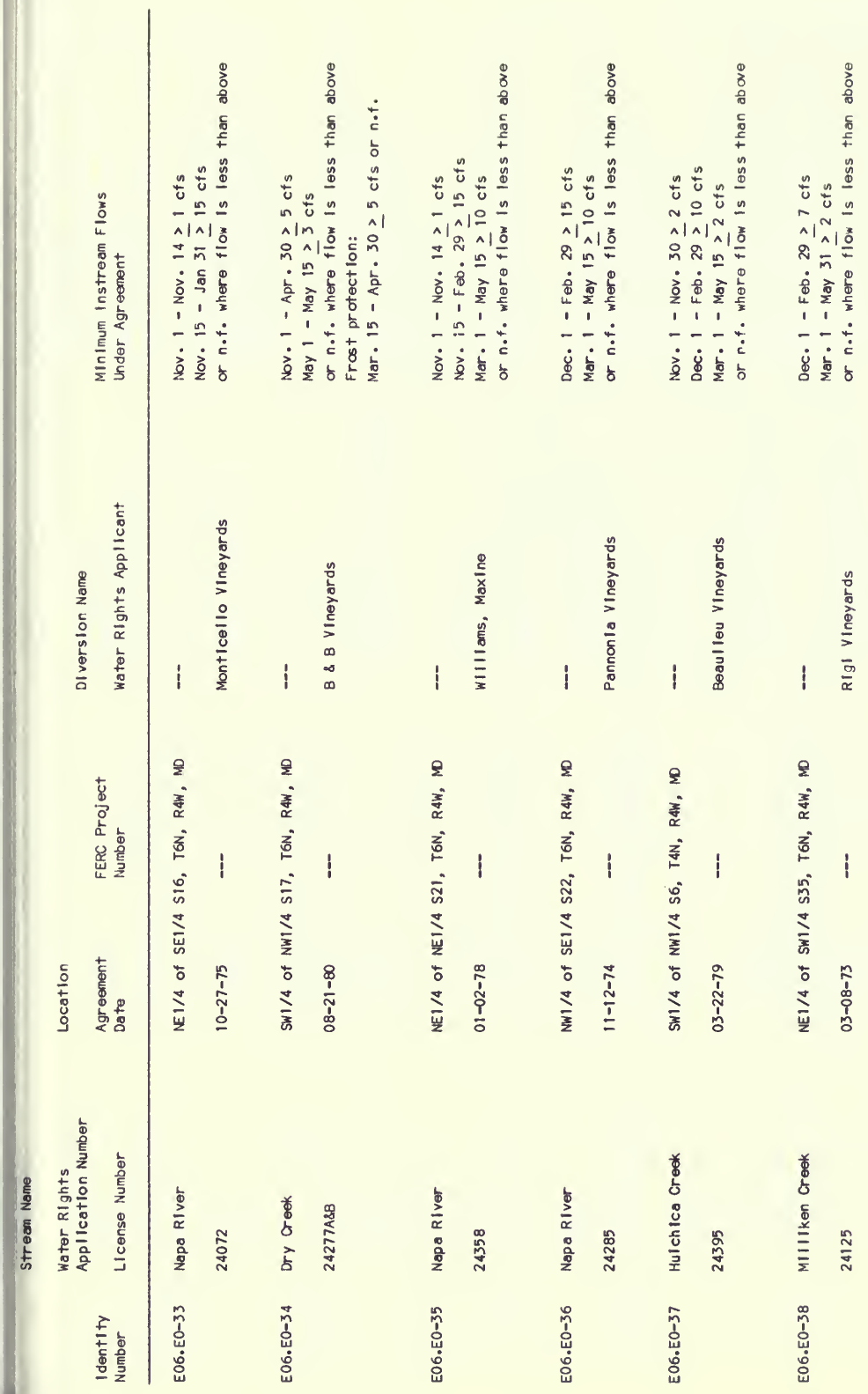

ำ 


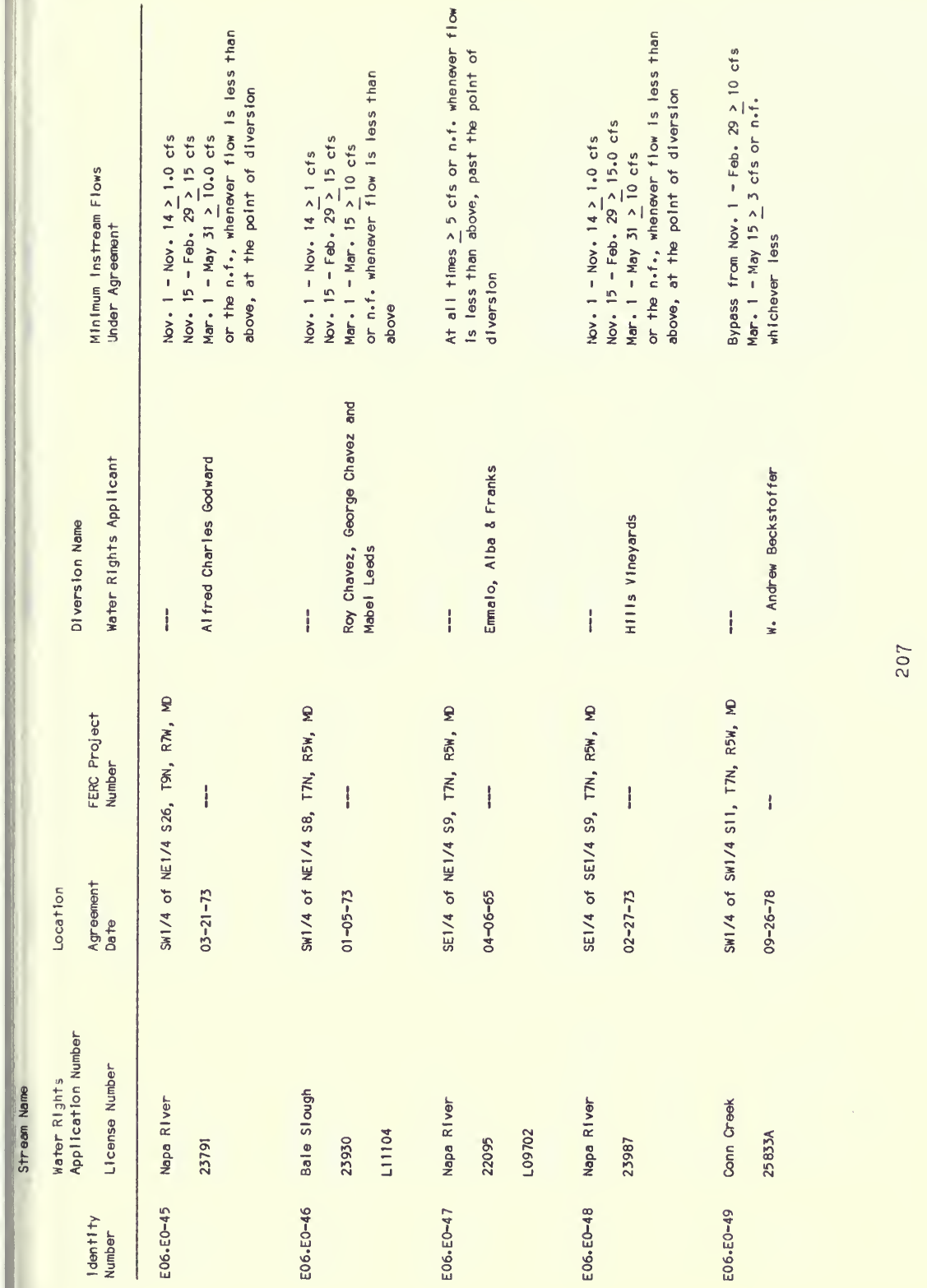




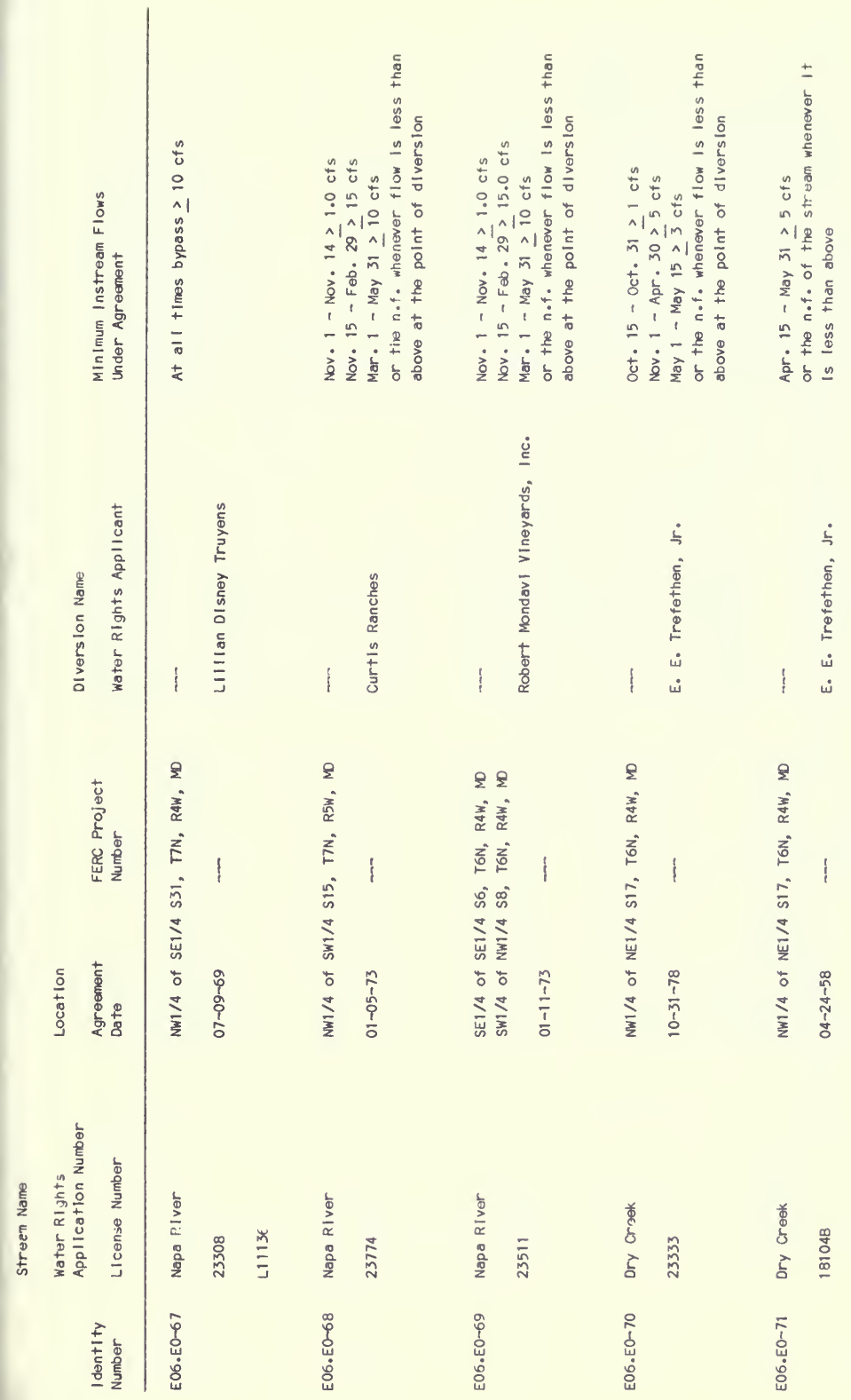




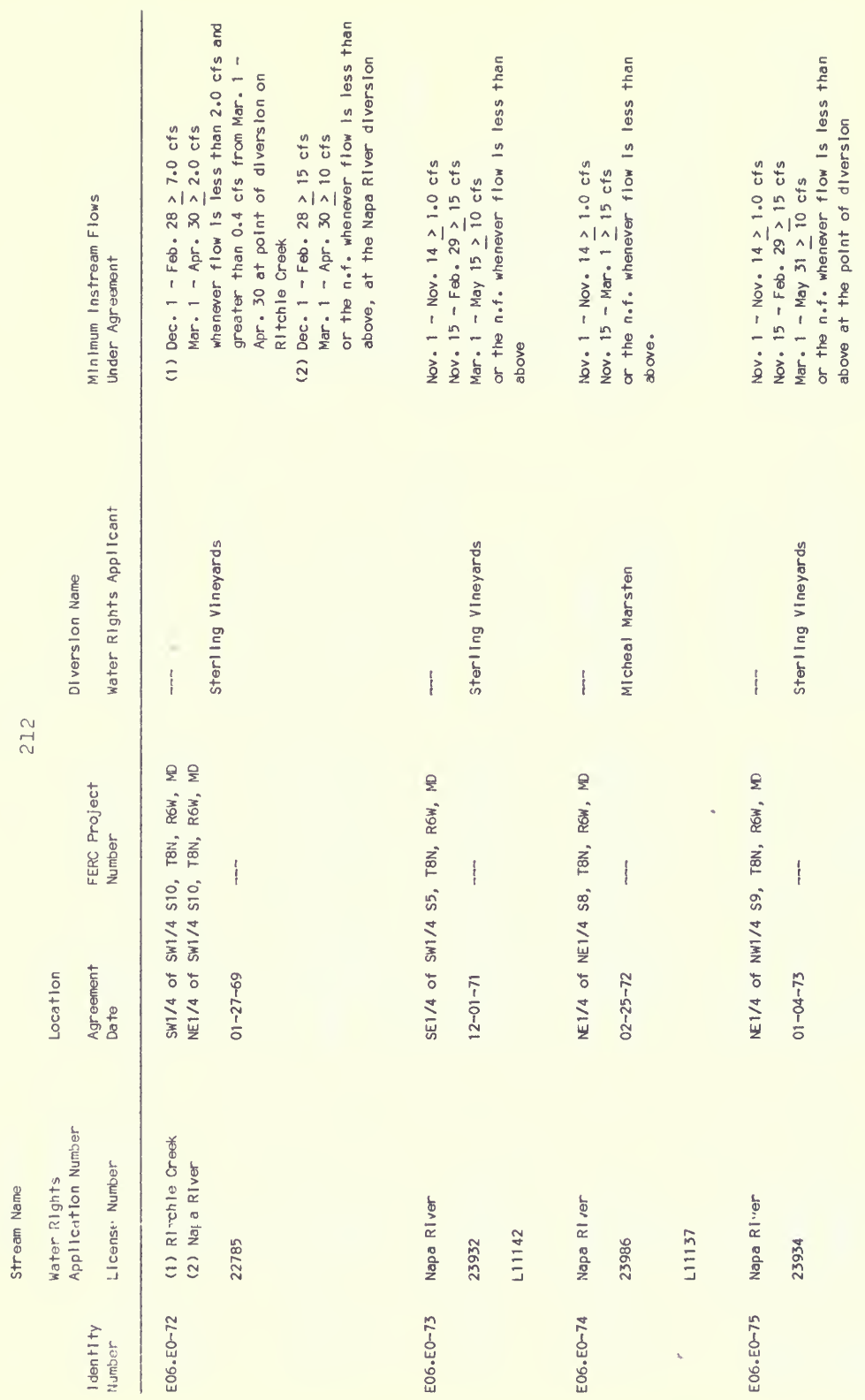




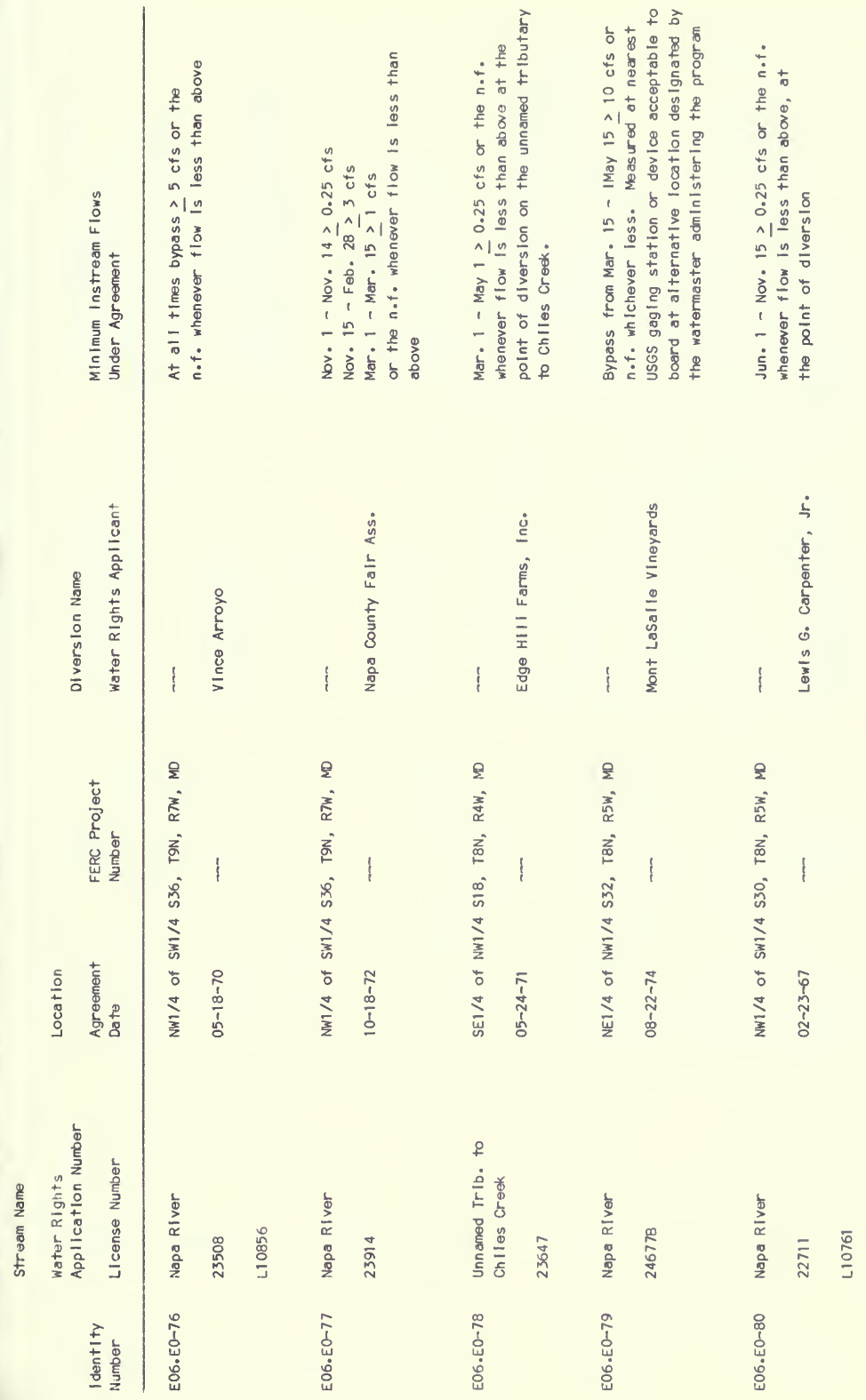




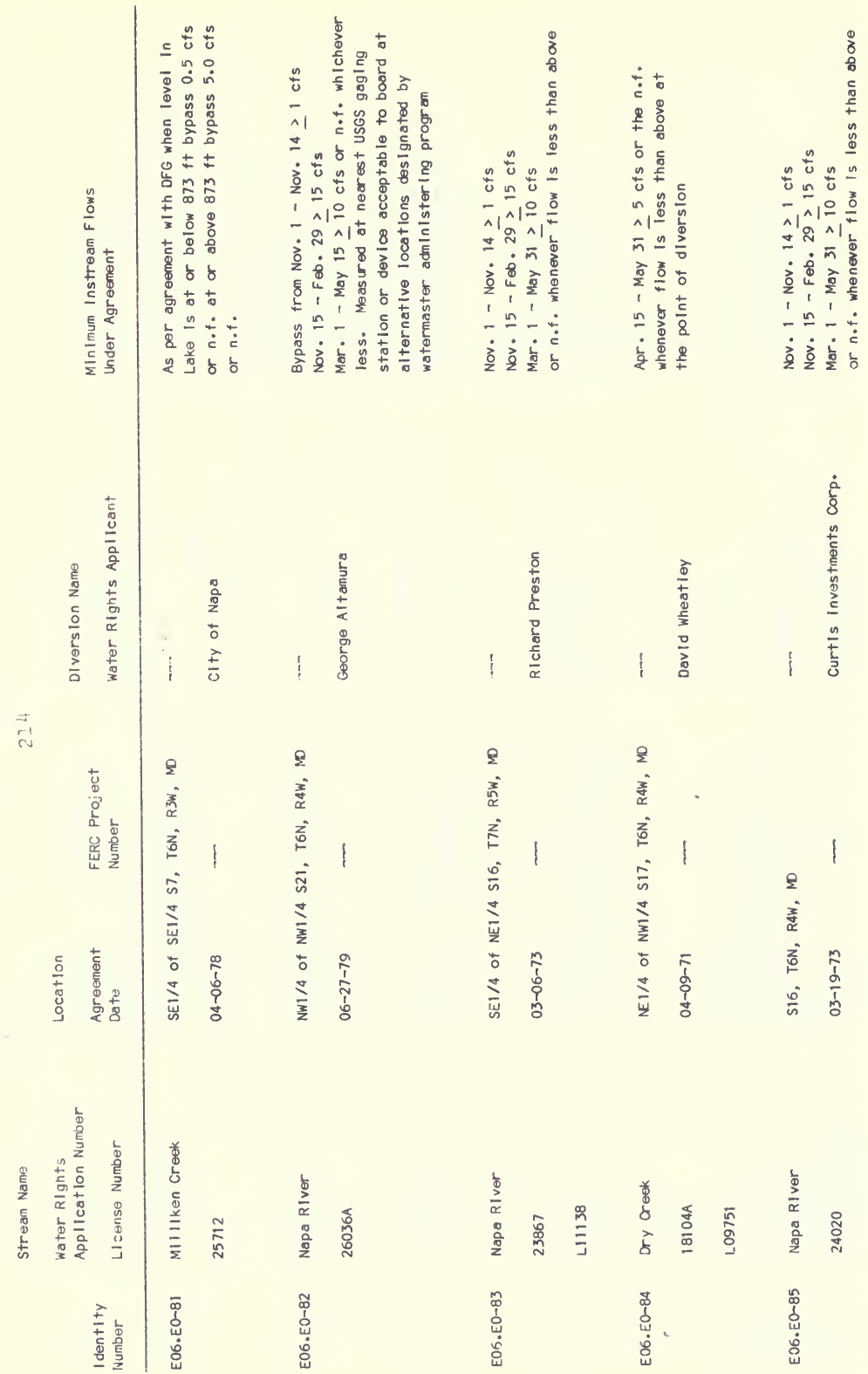




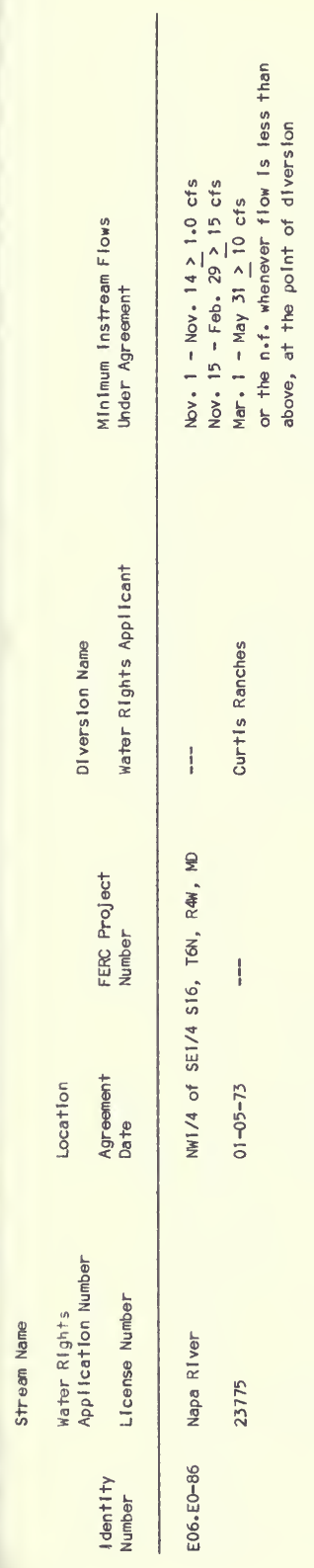




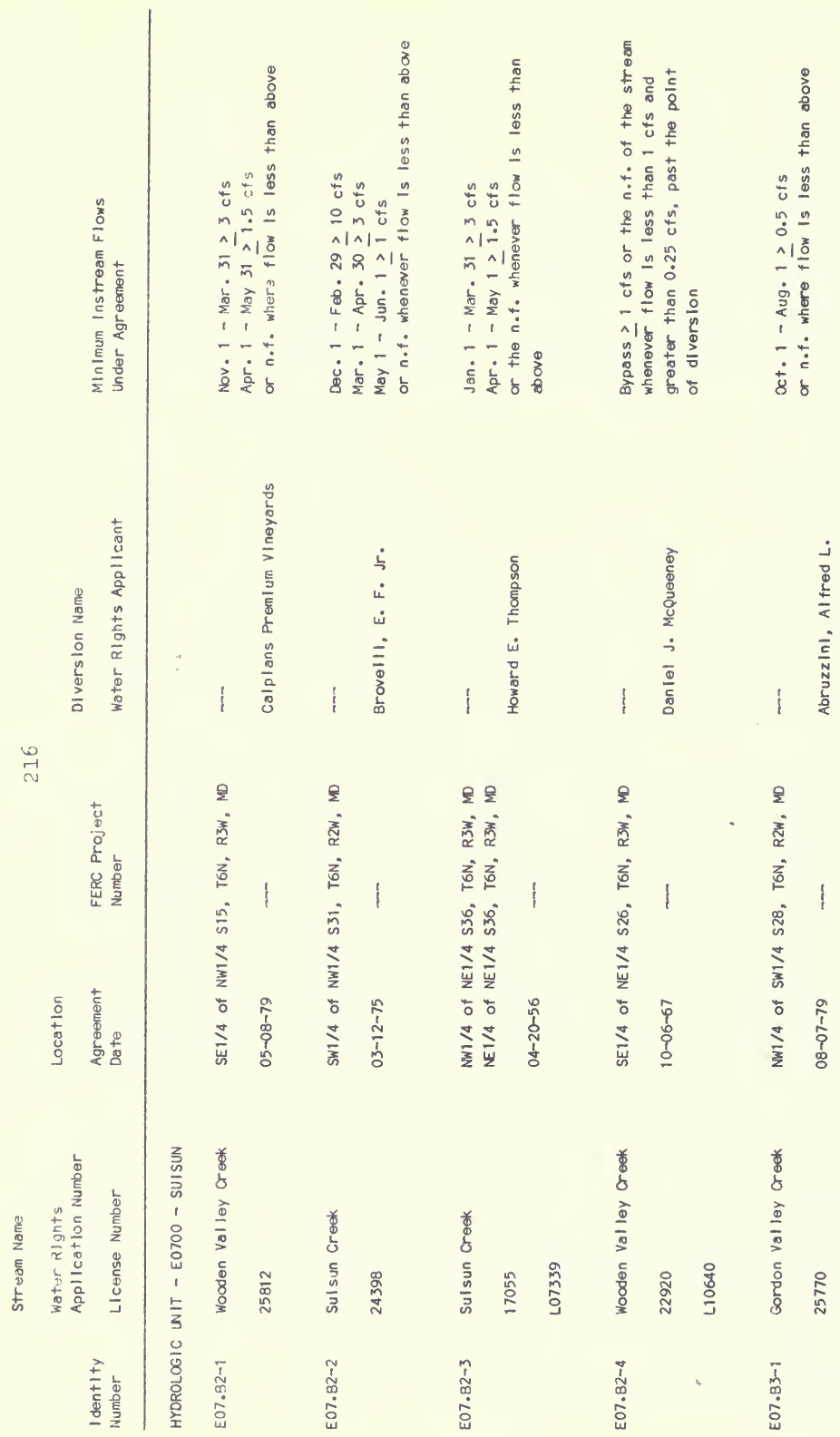




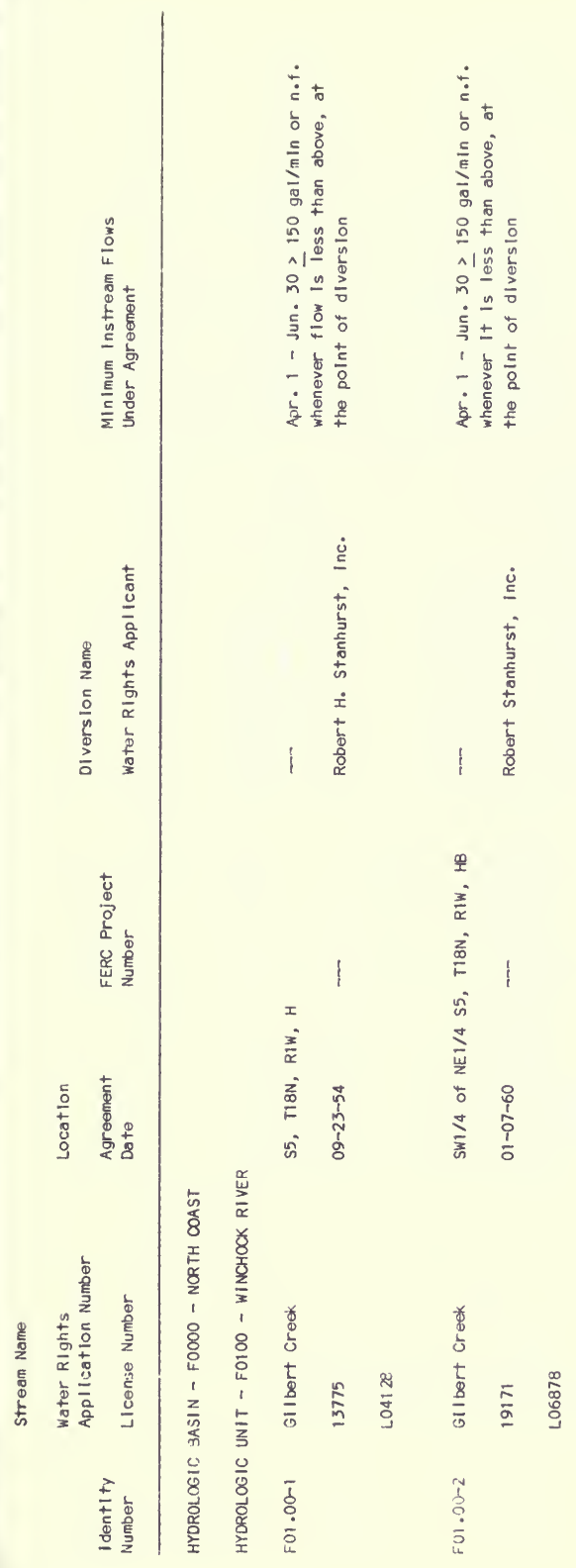




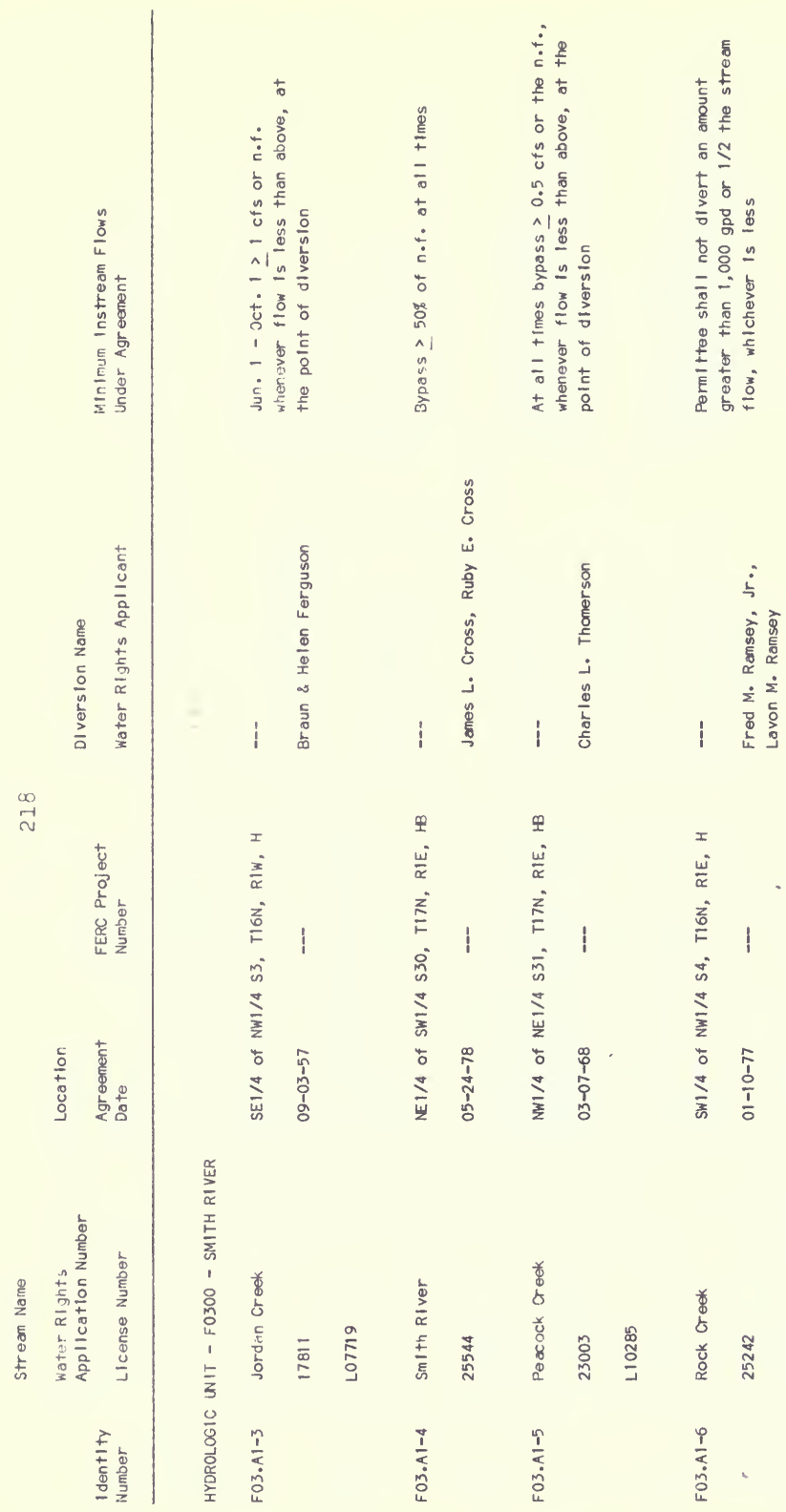




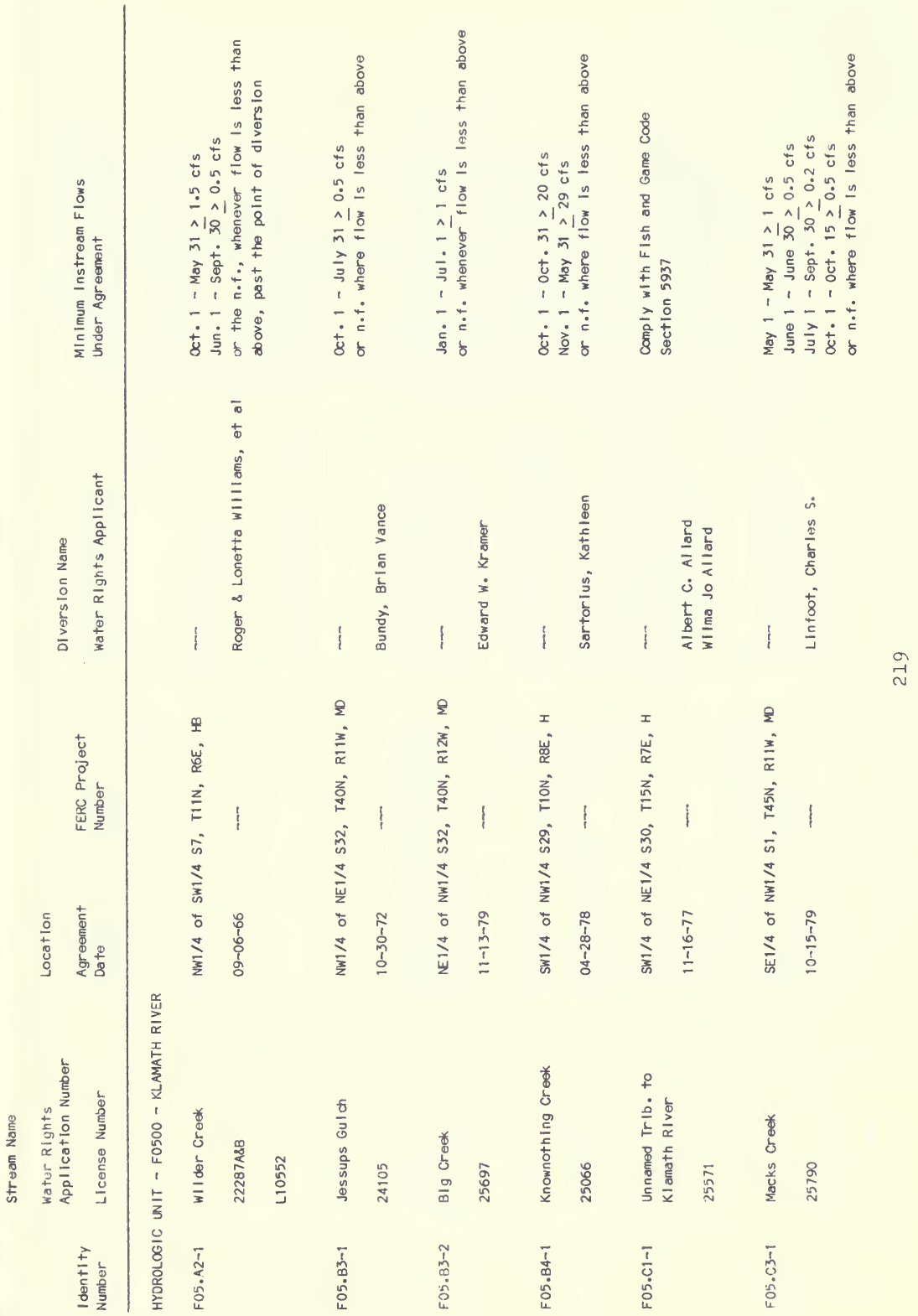




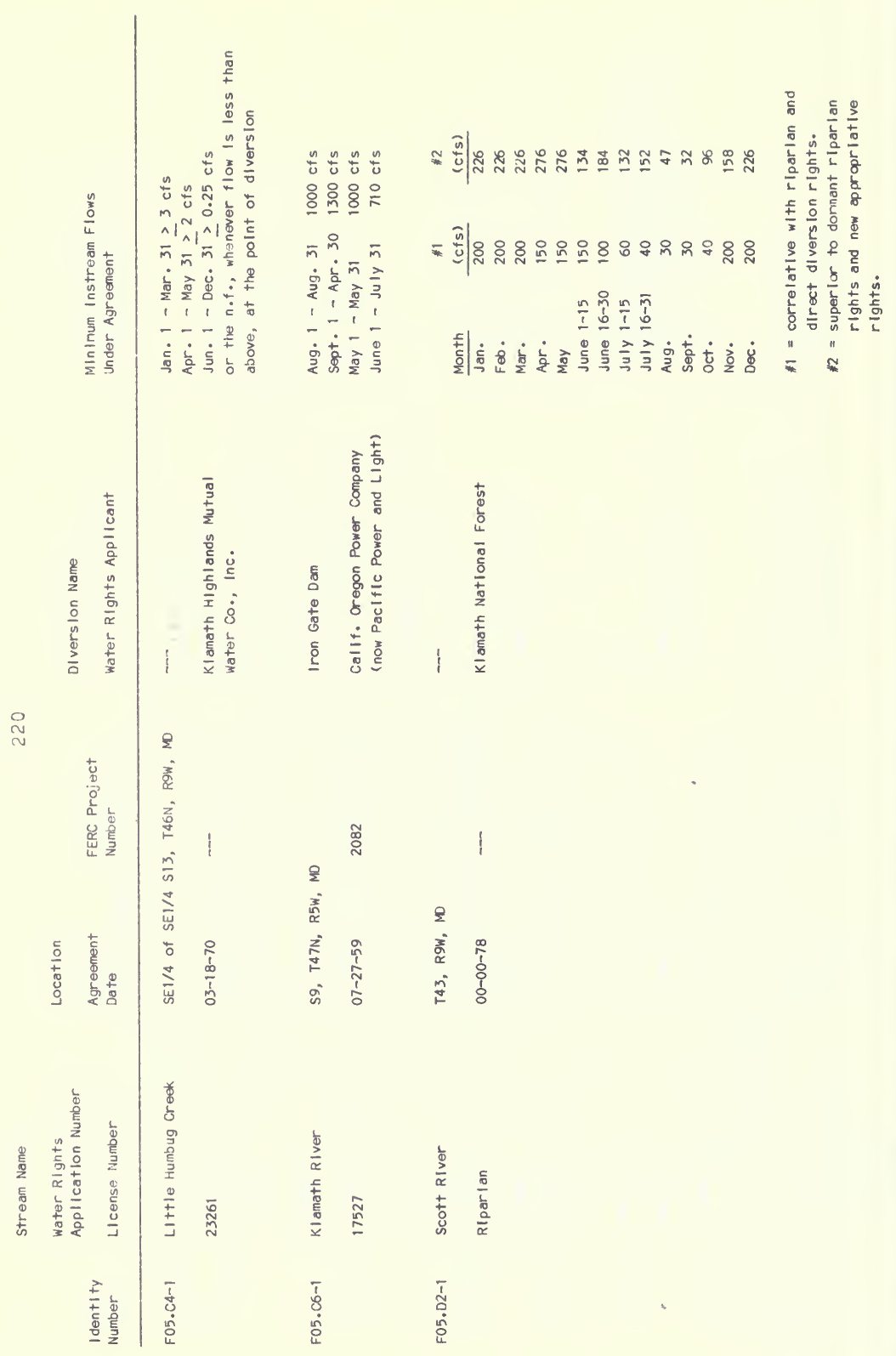




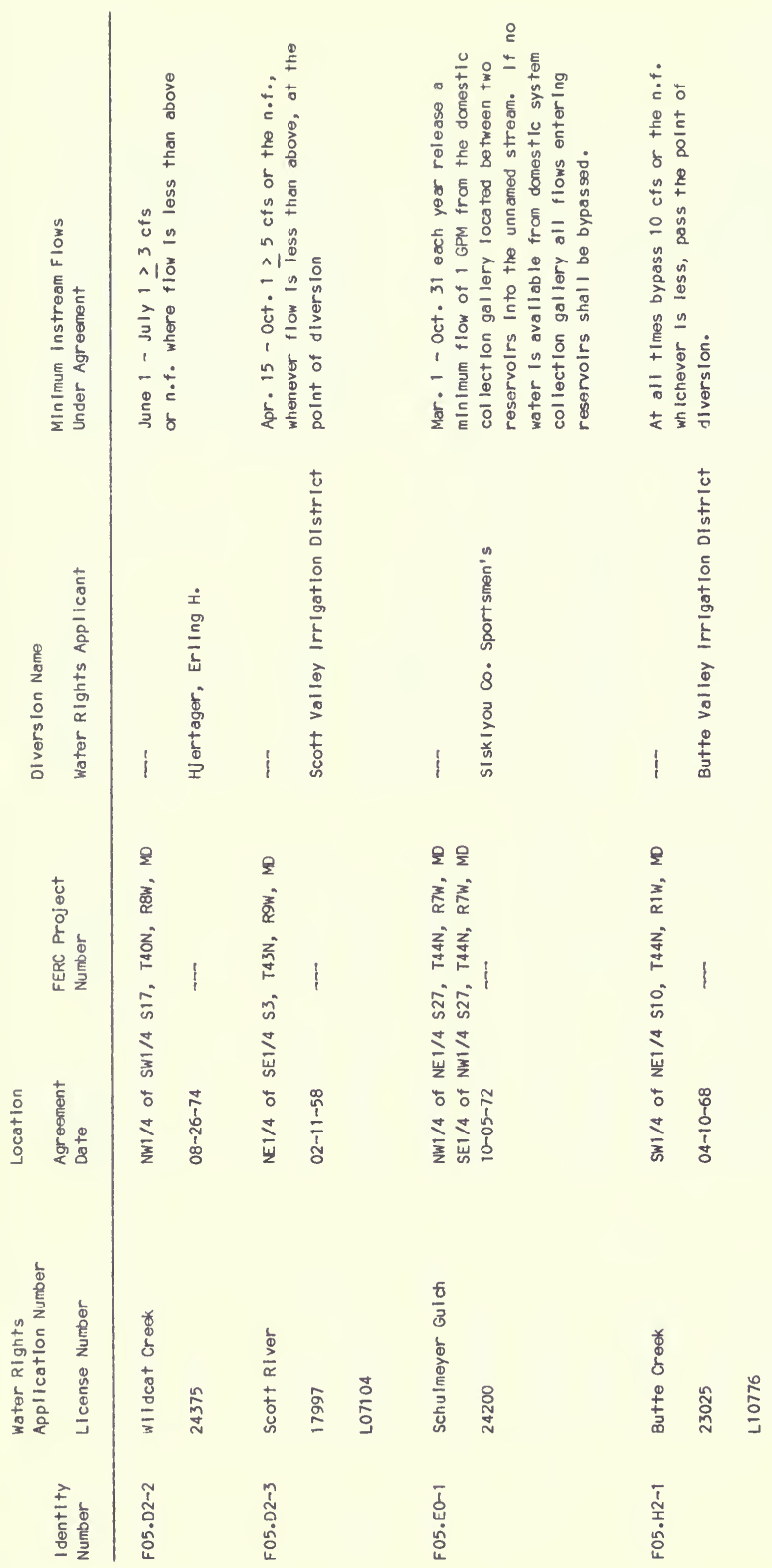




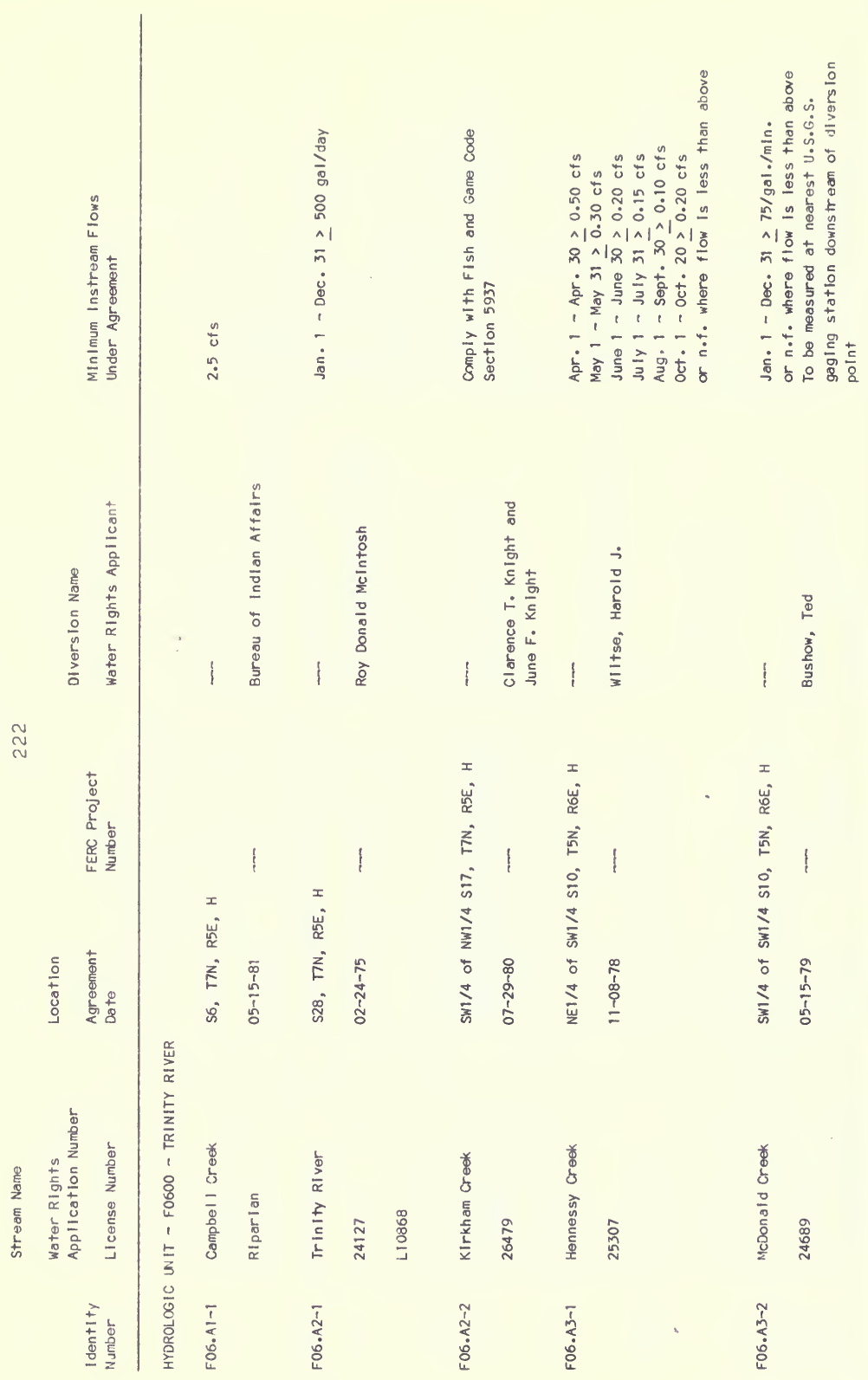




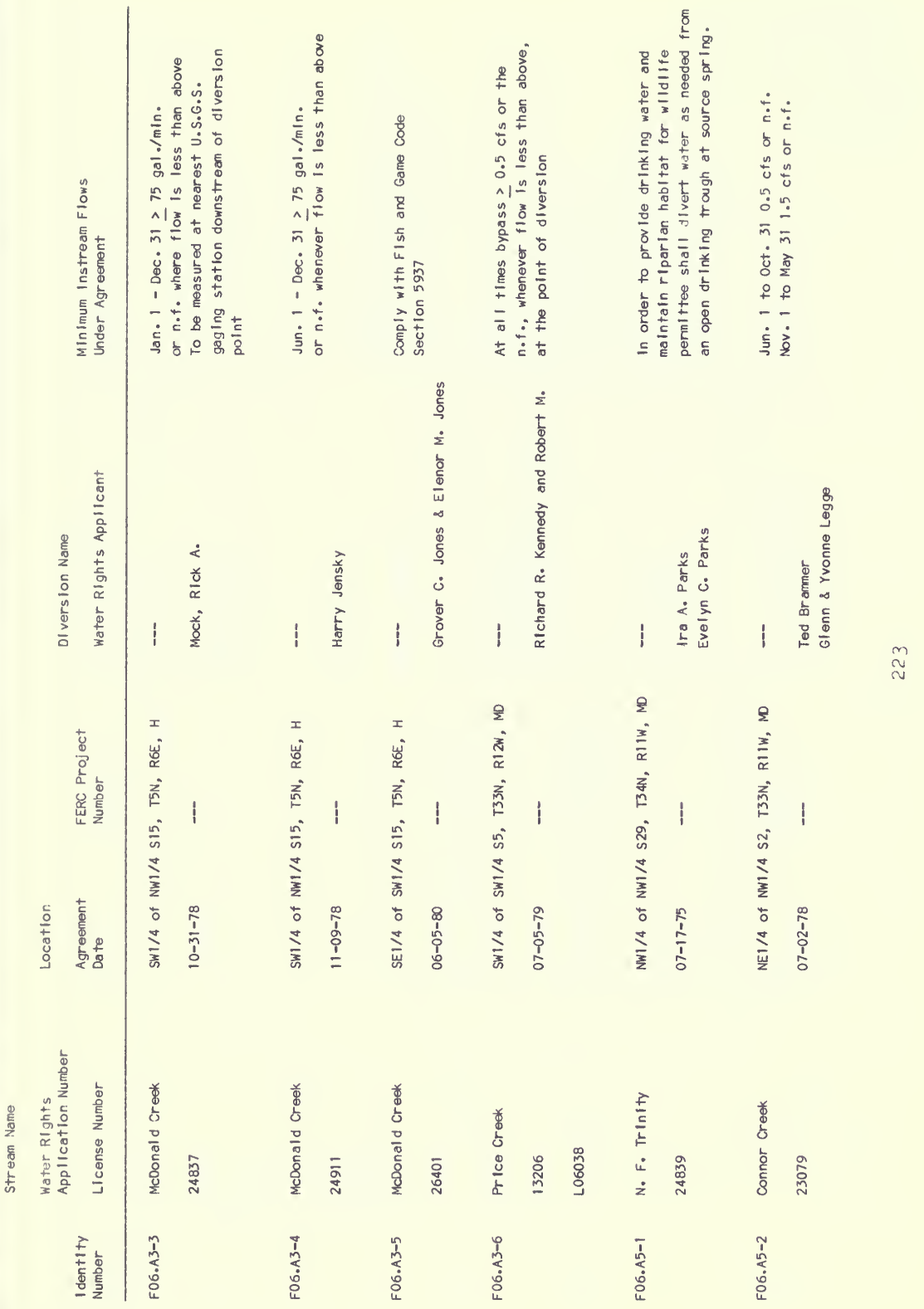




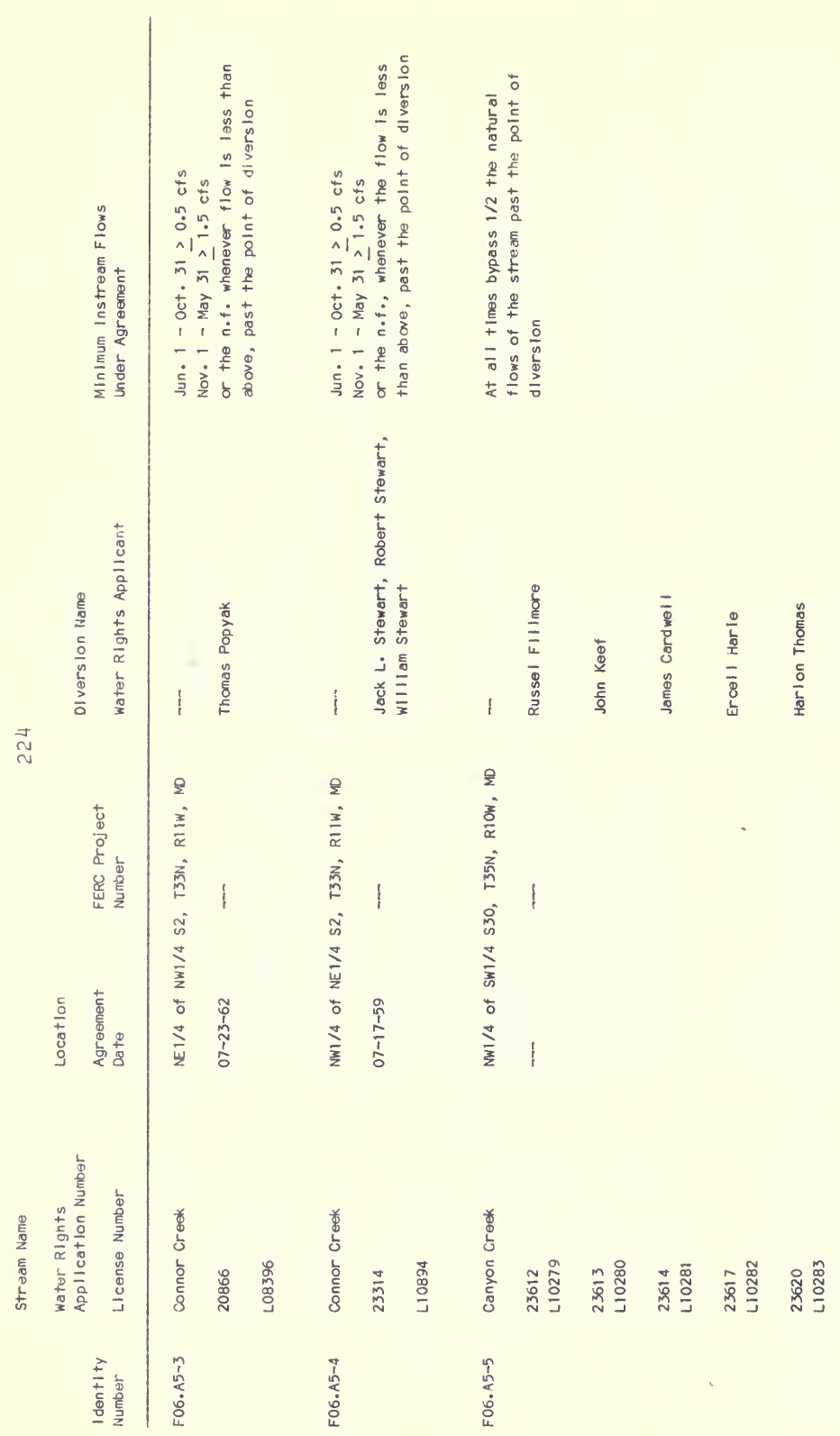




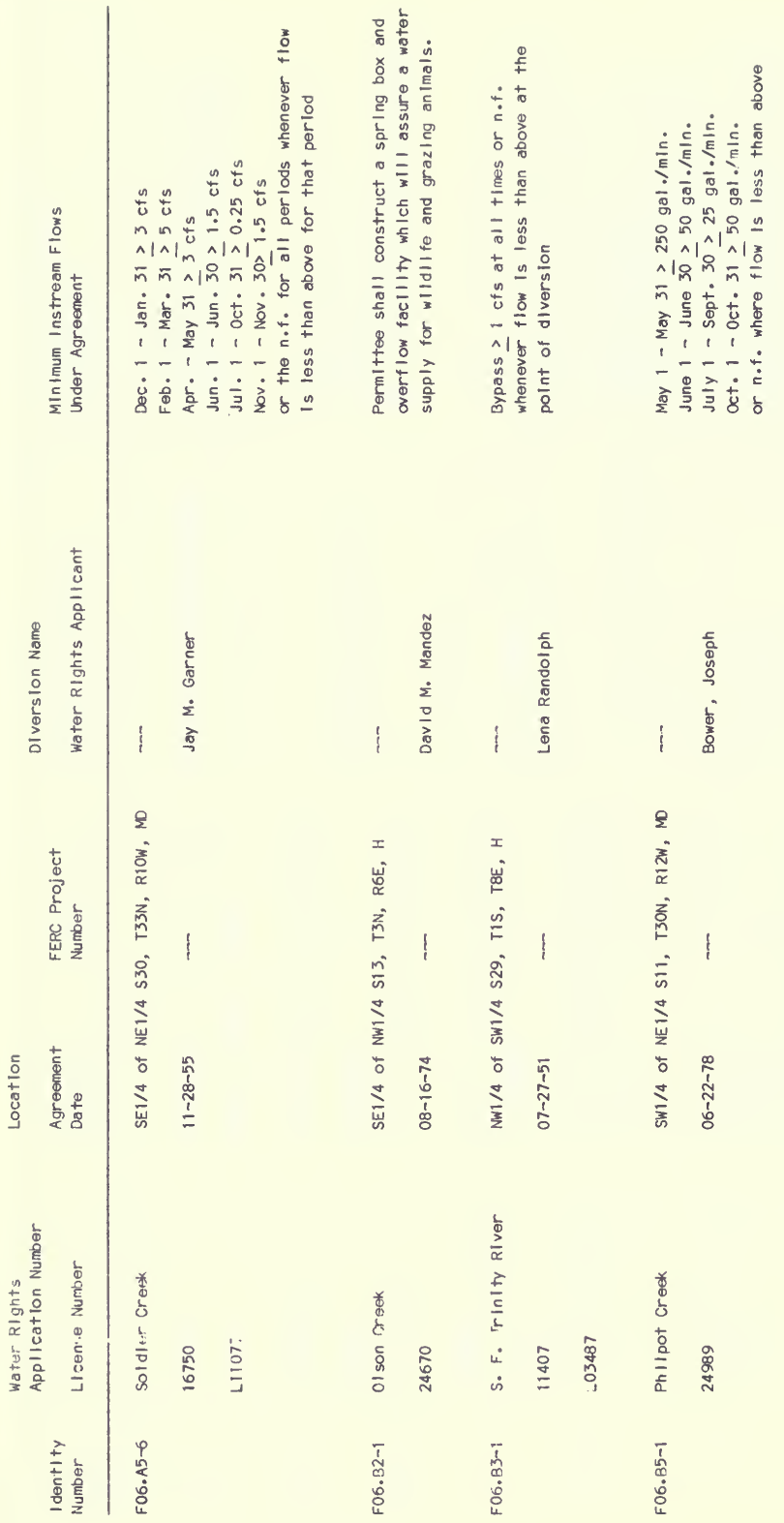




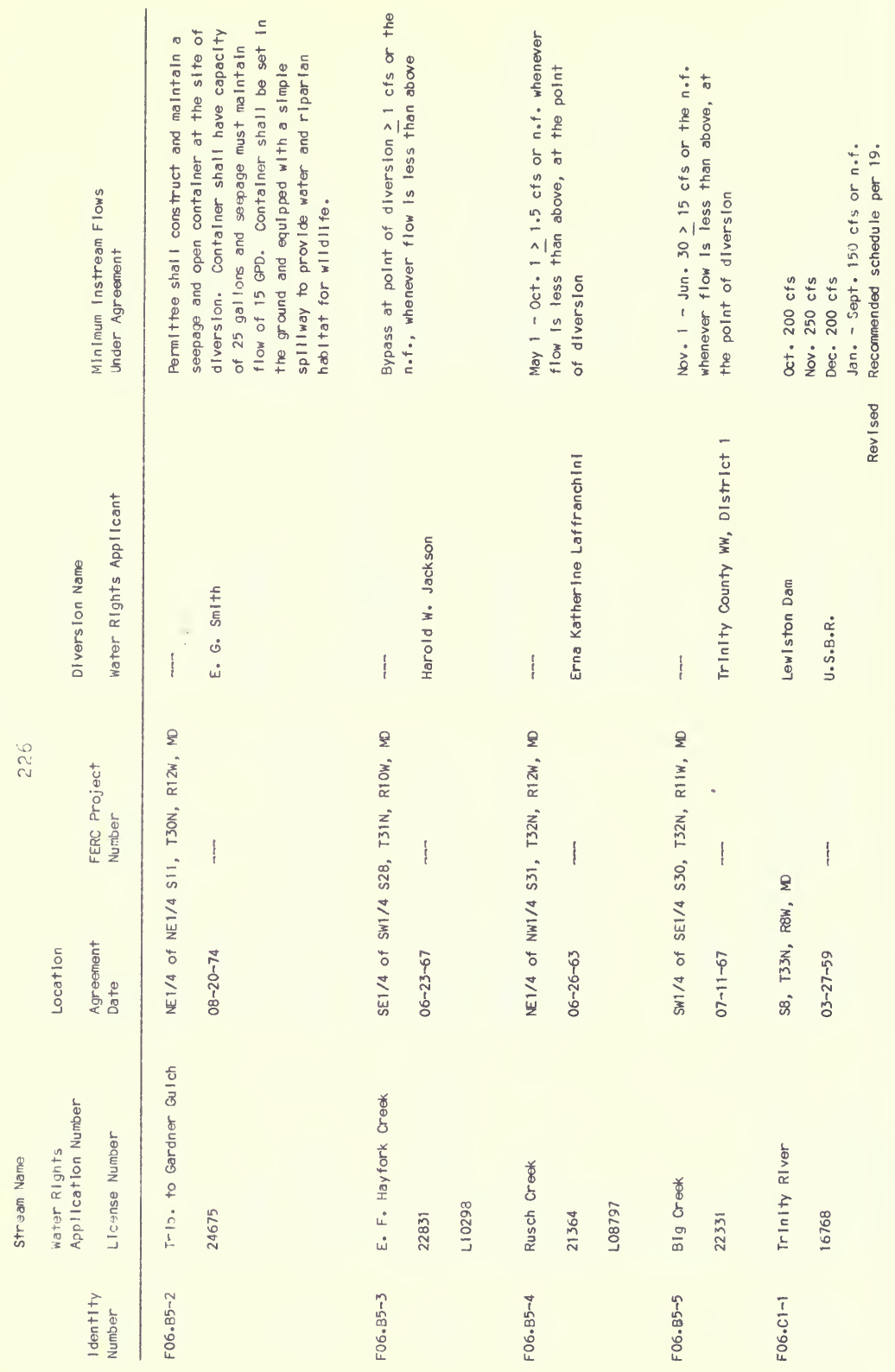




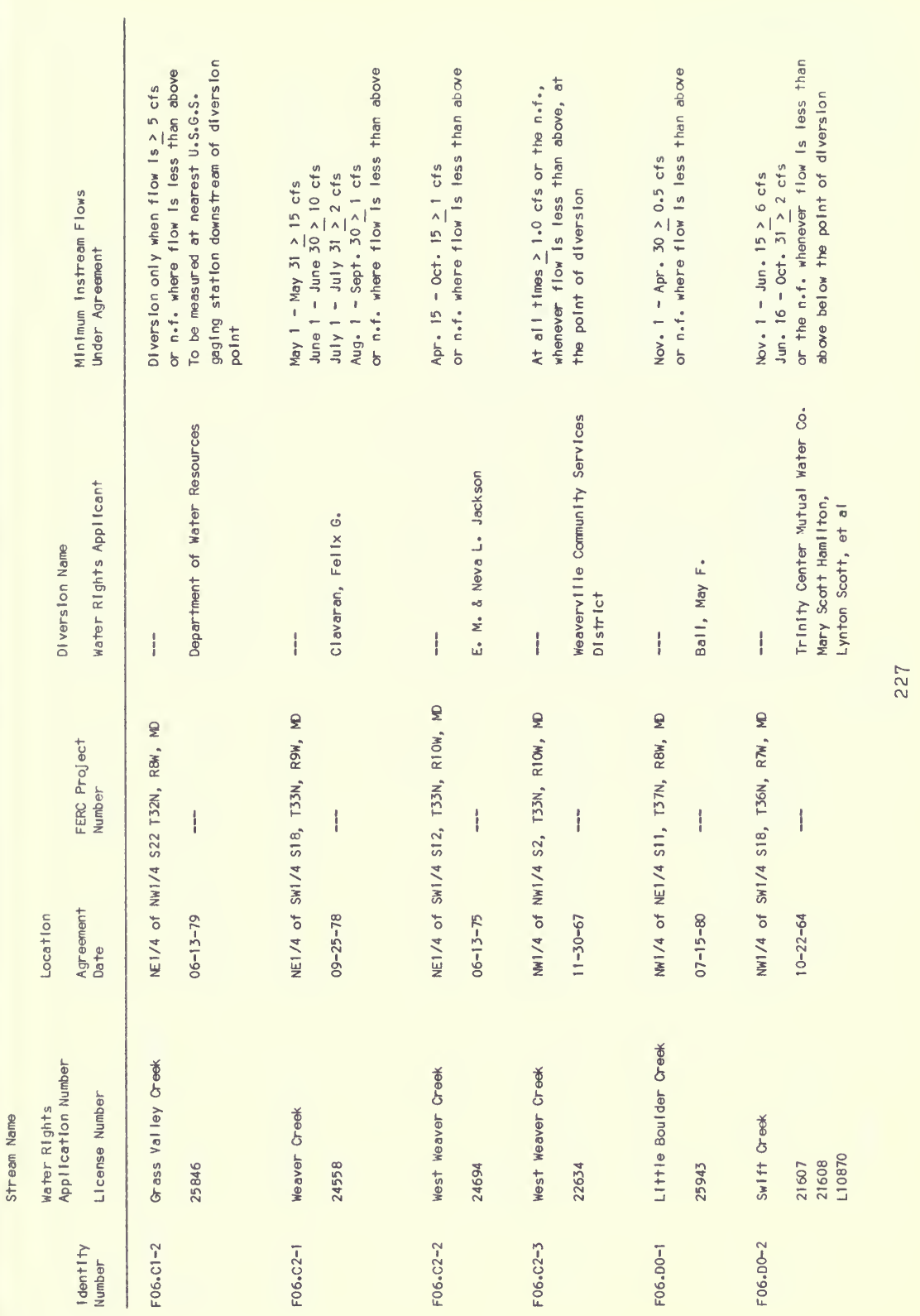




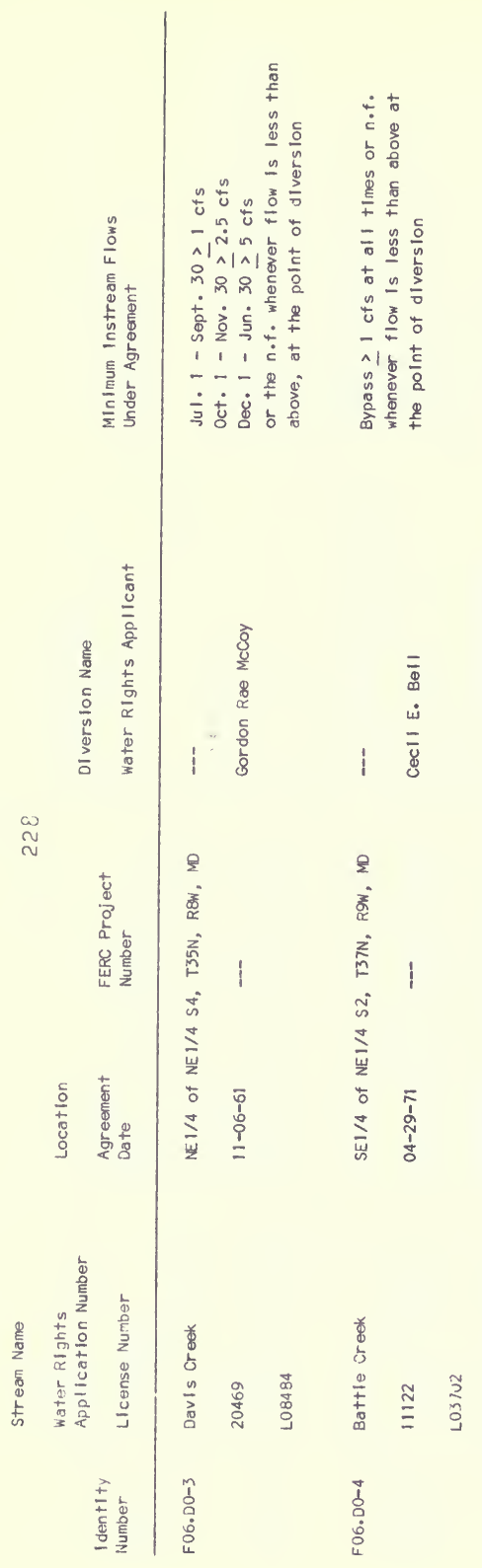




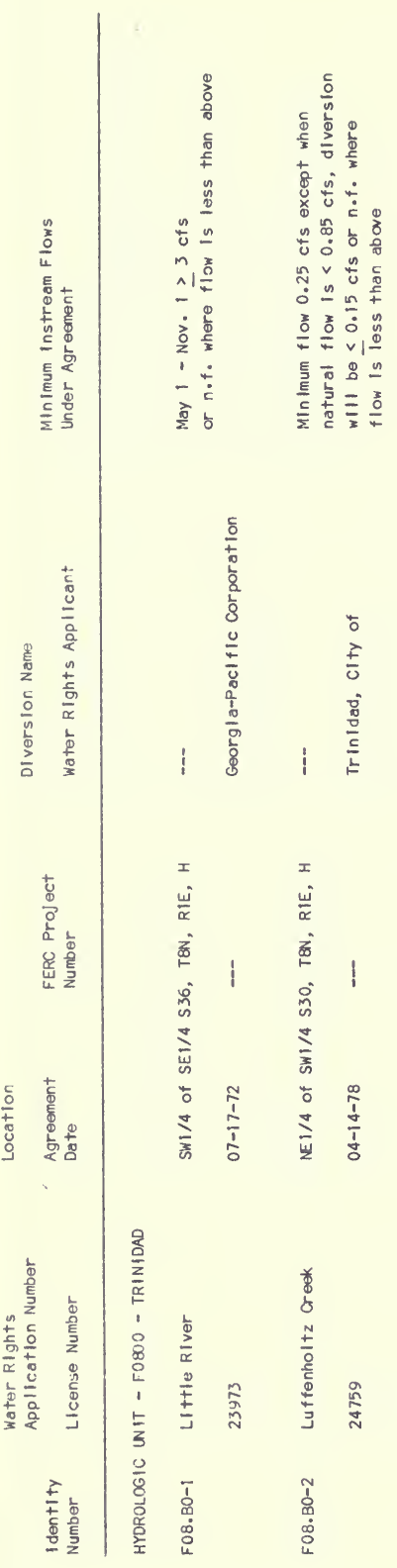




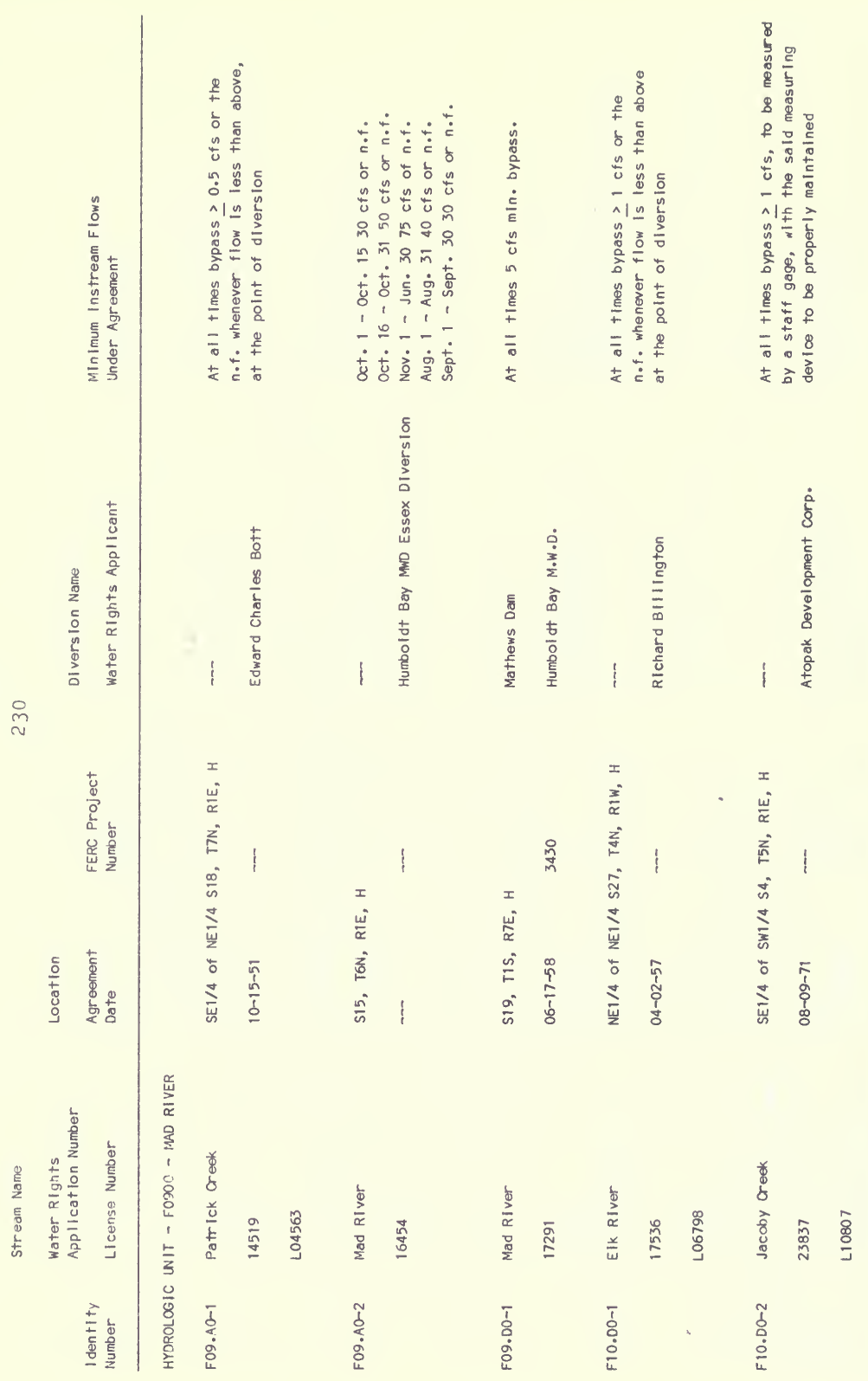




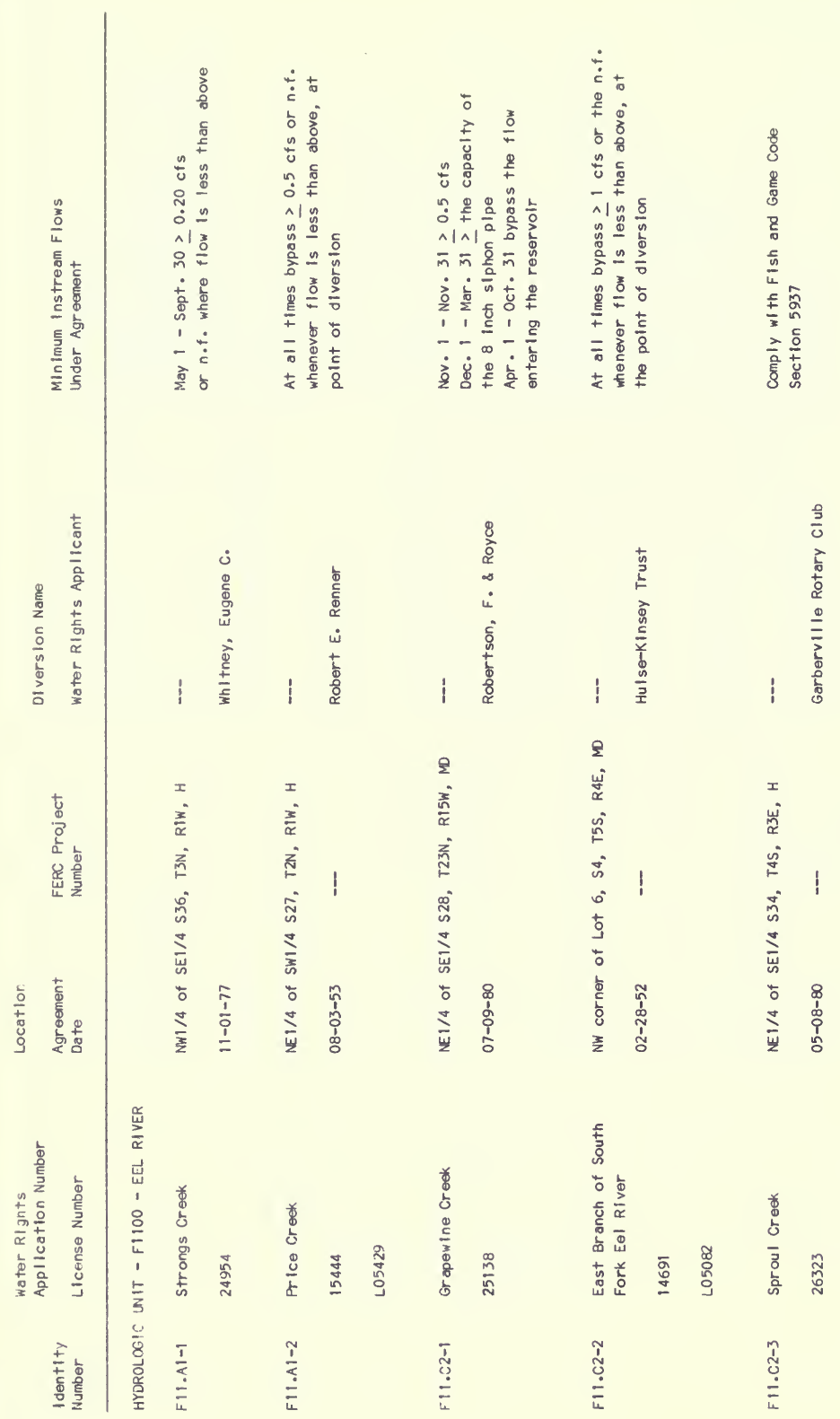




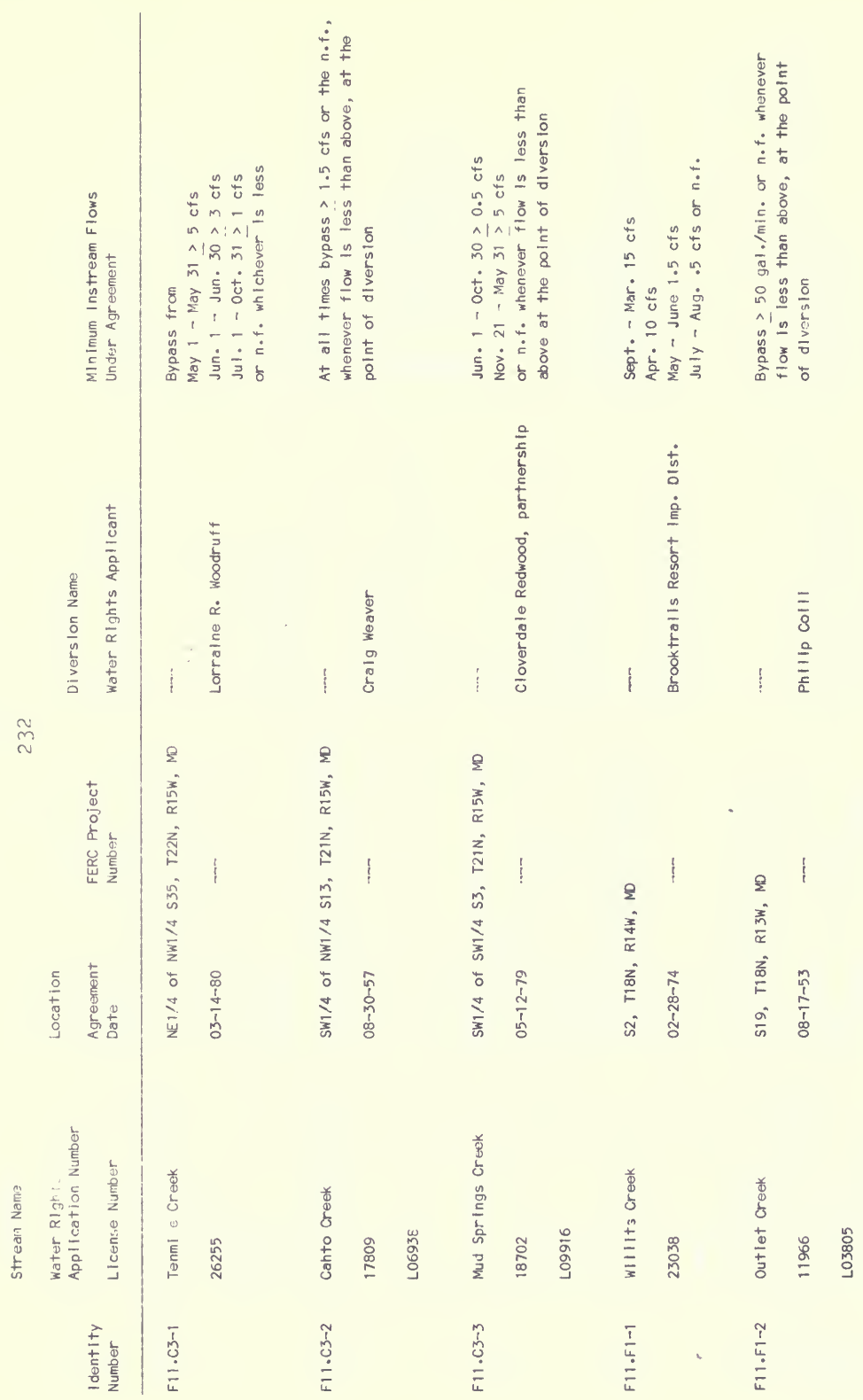




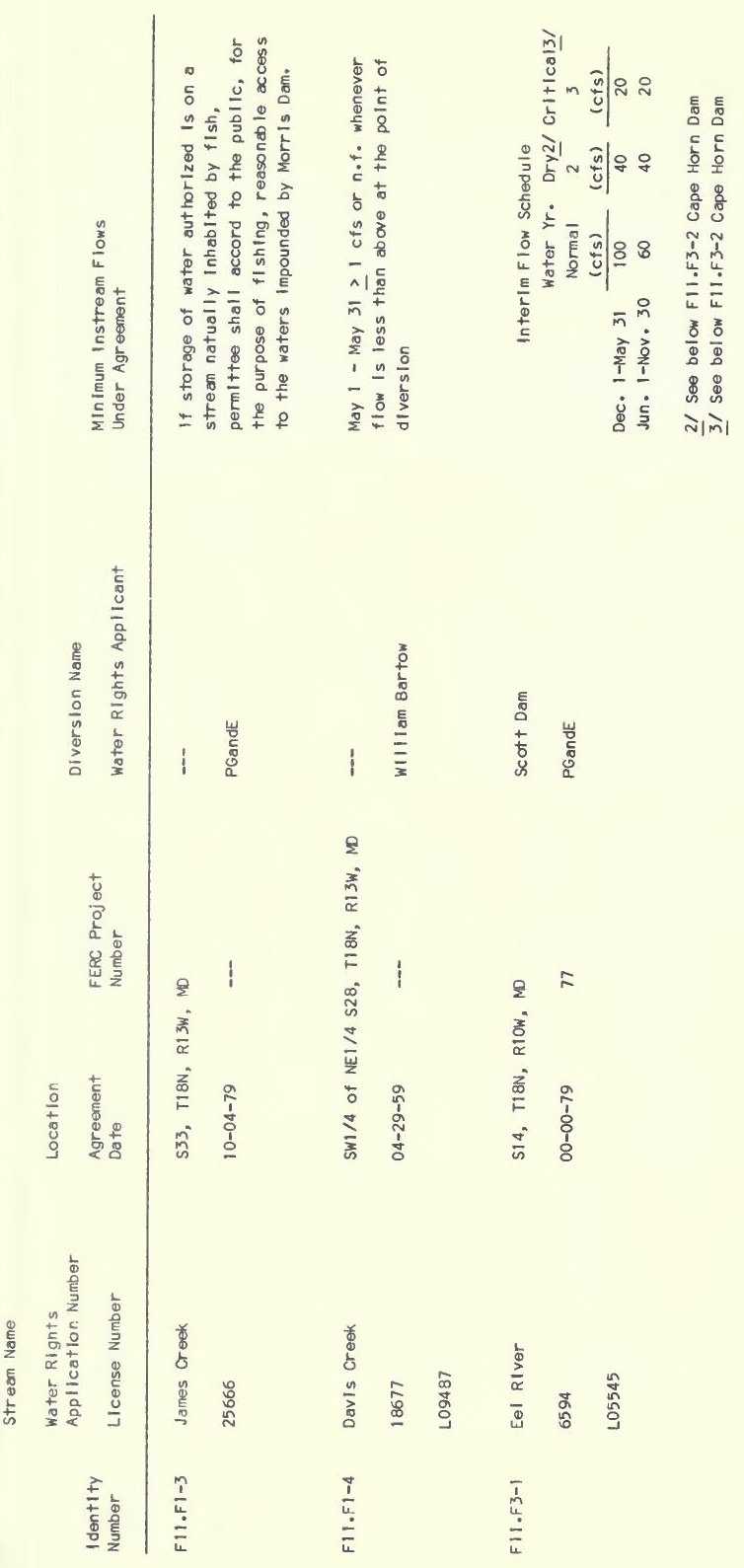

$\stackrel{m}{\sim}$ 


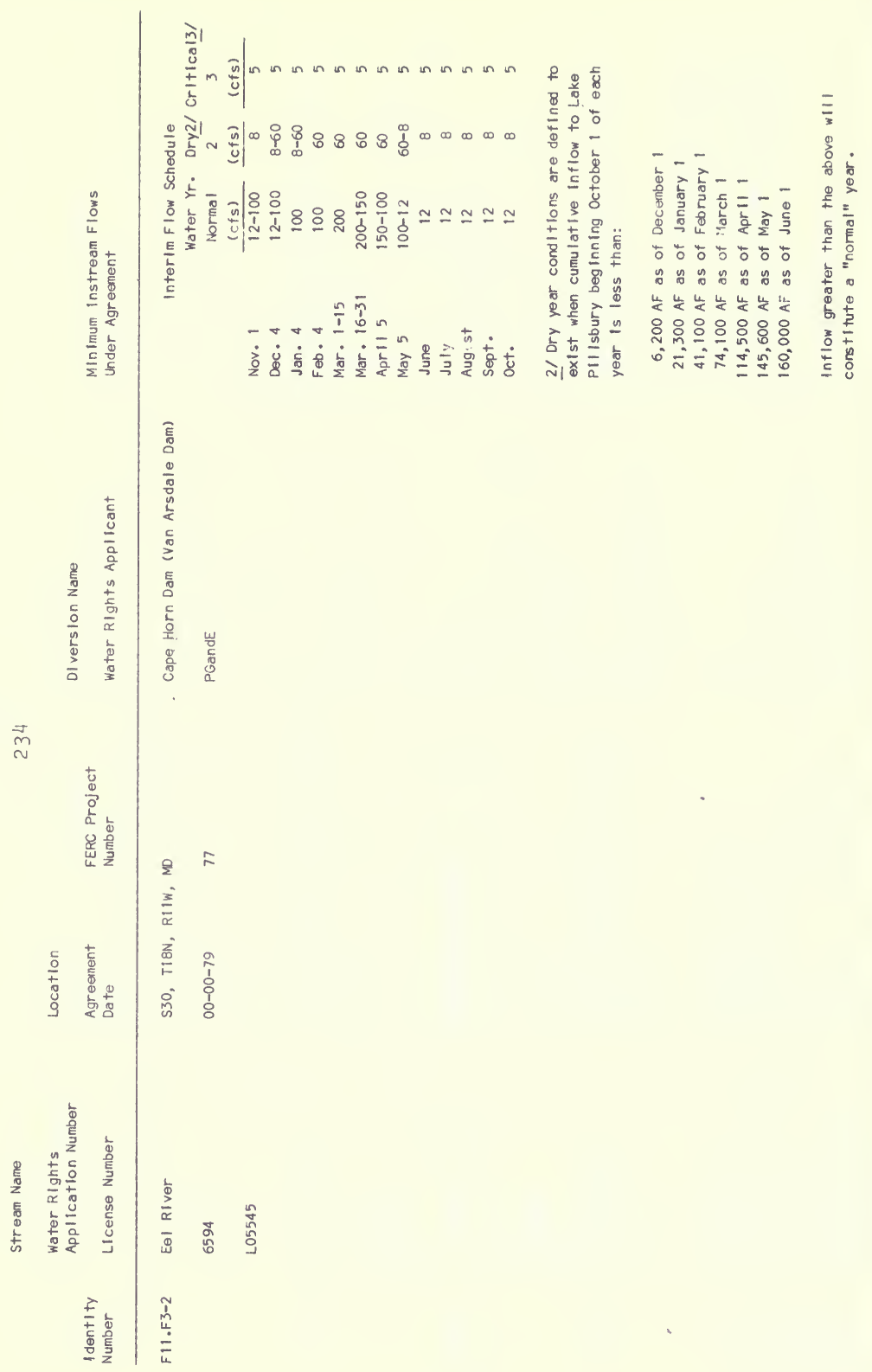




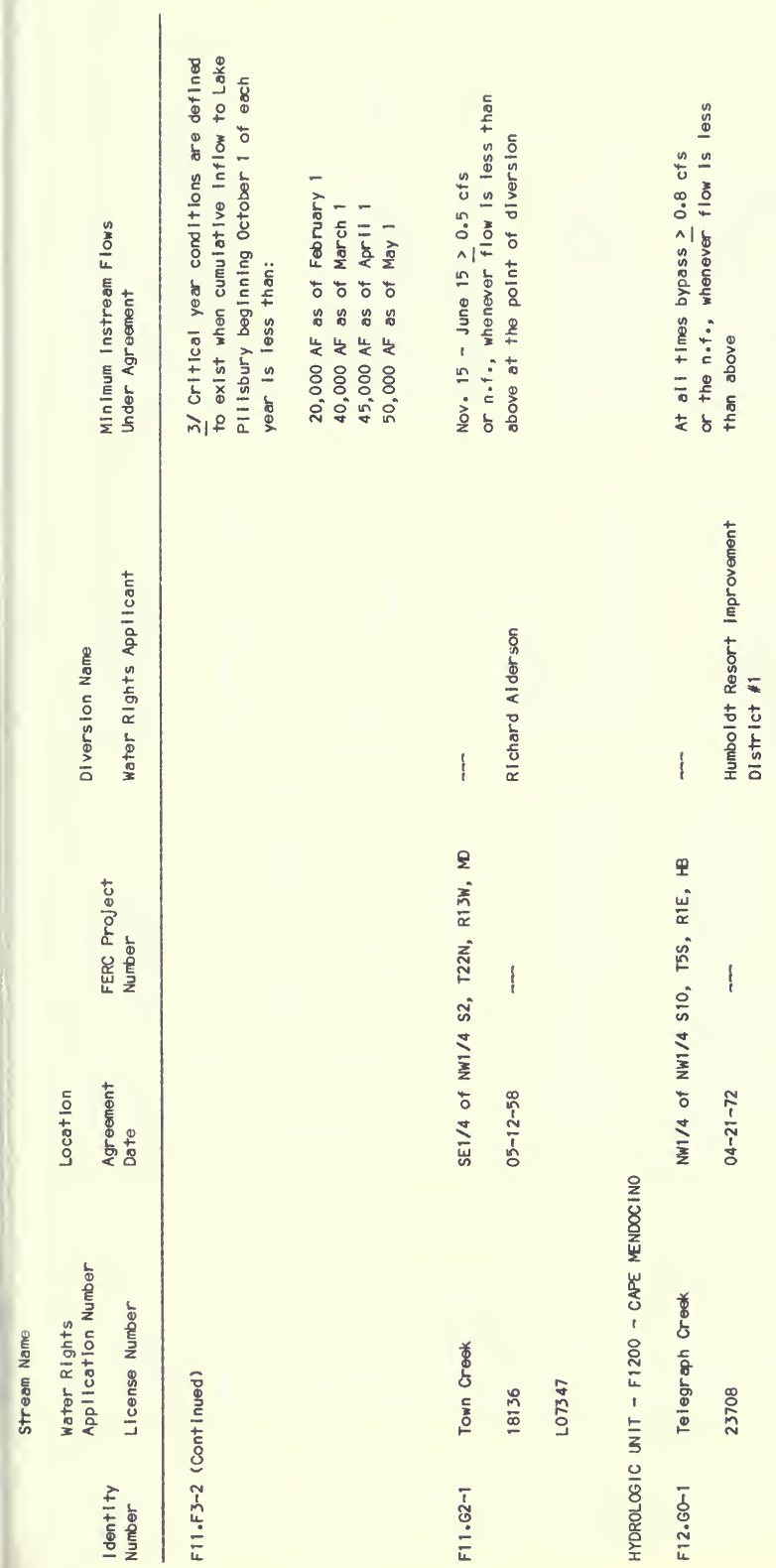

$\stackrel{\leftrightarrow}{\text { g }}$ 


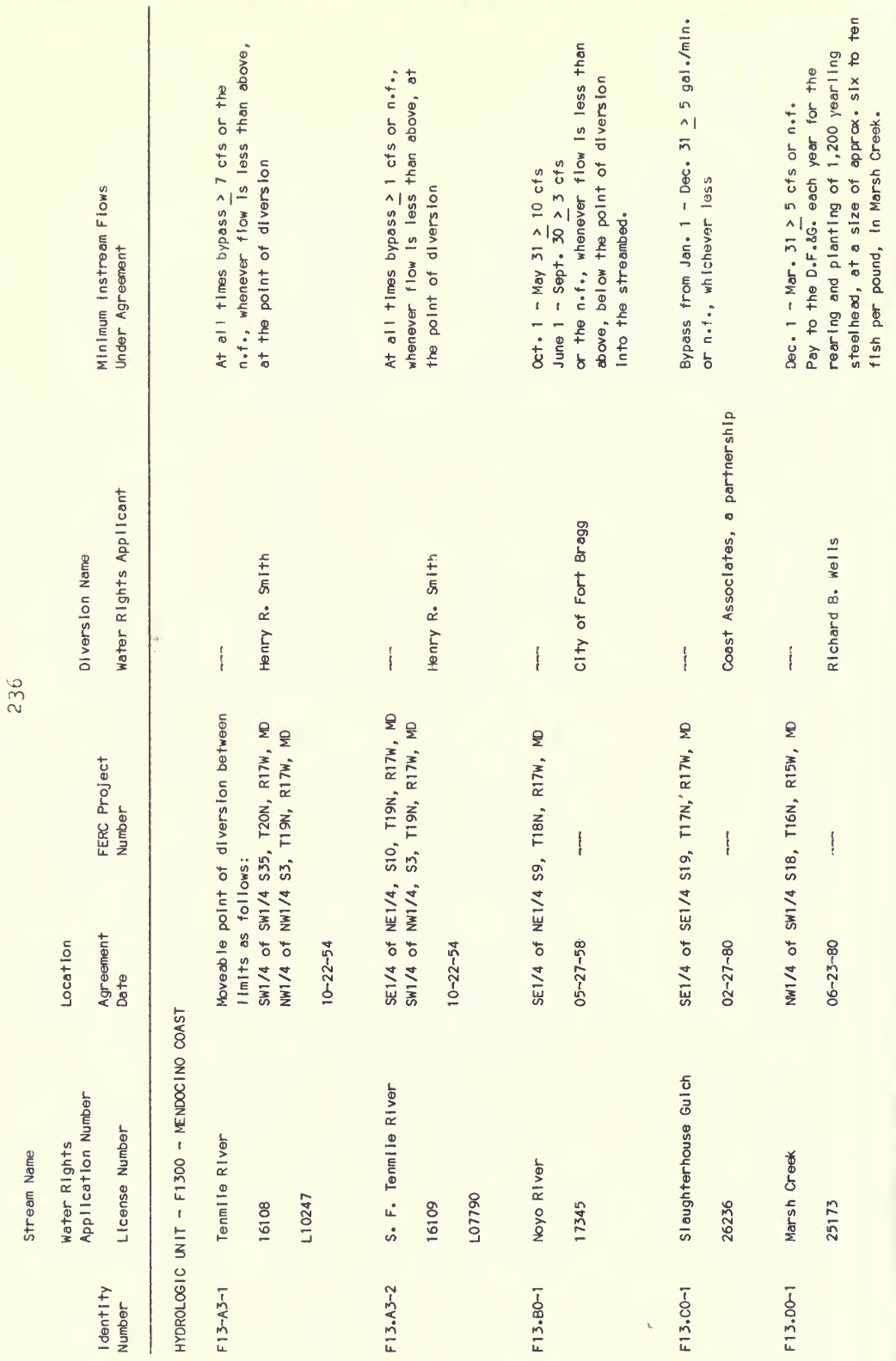




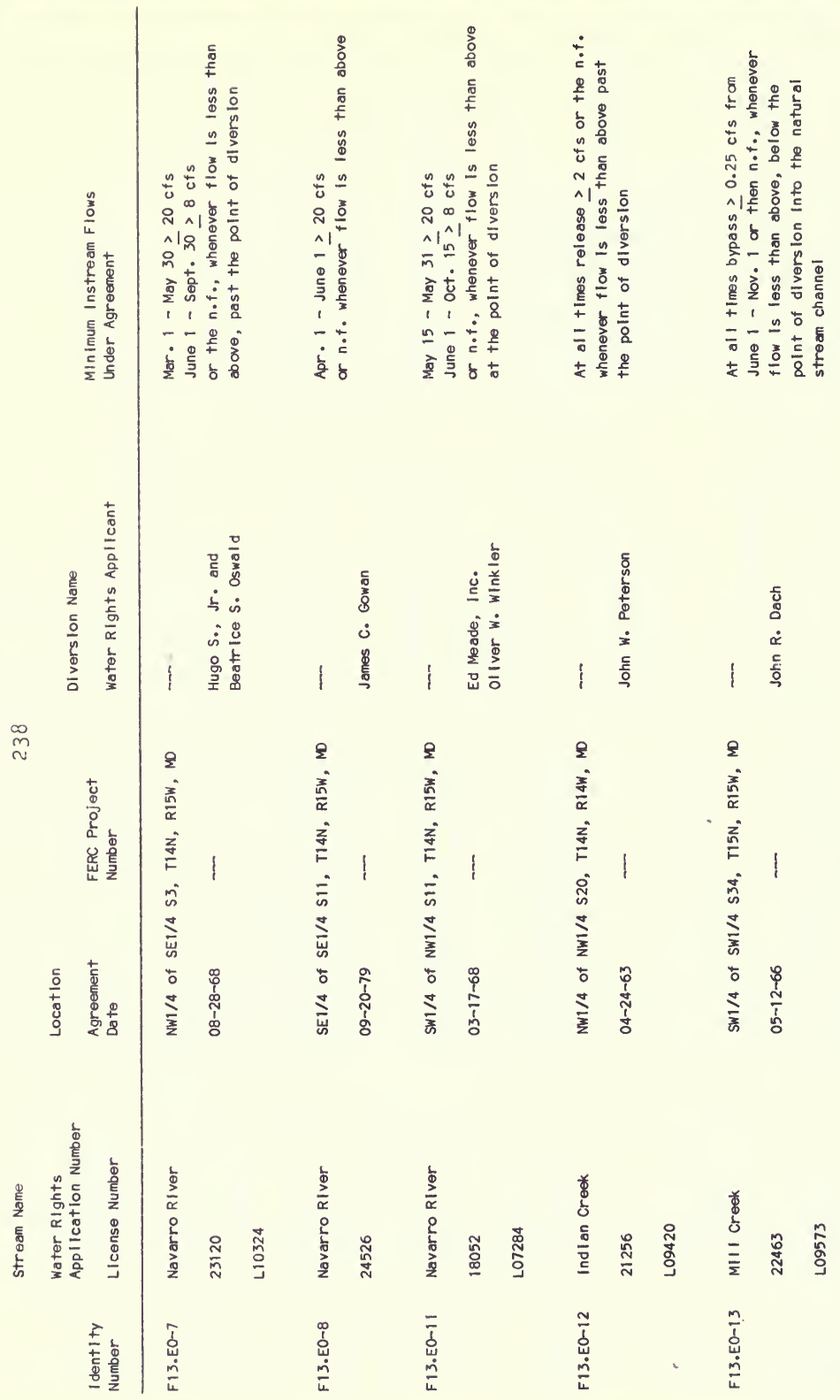




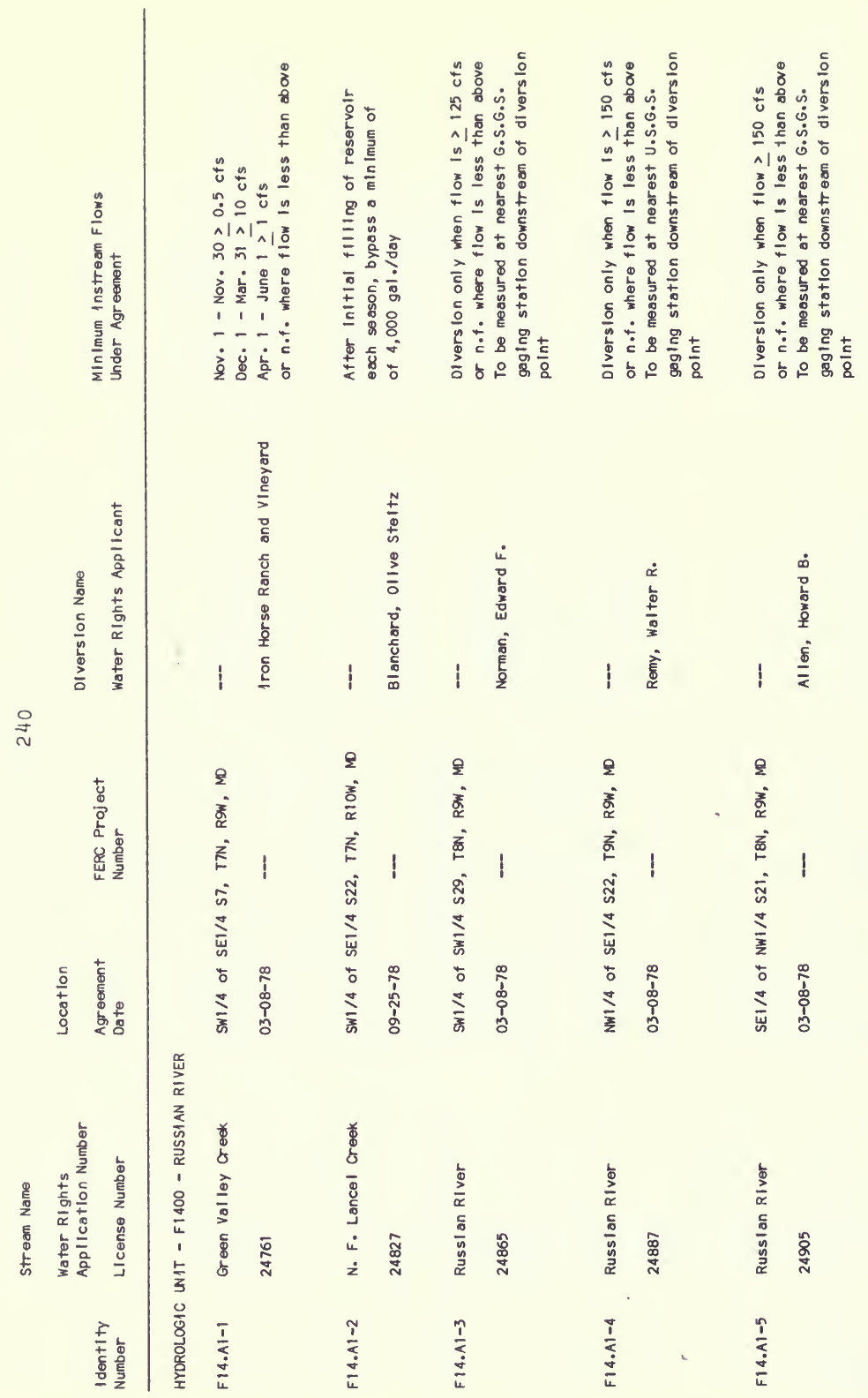





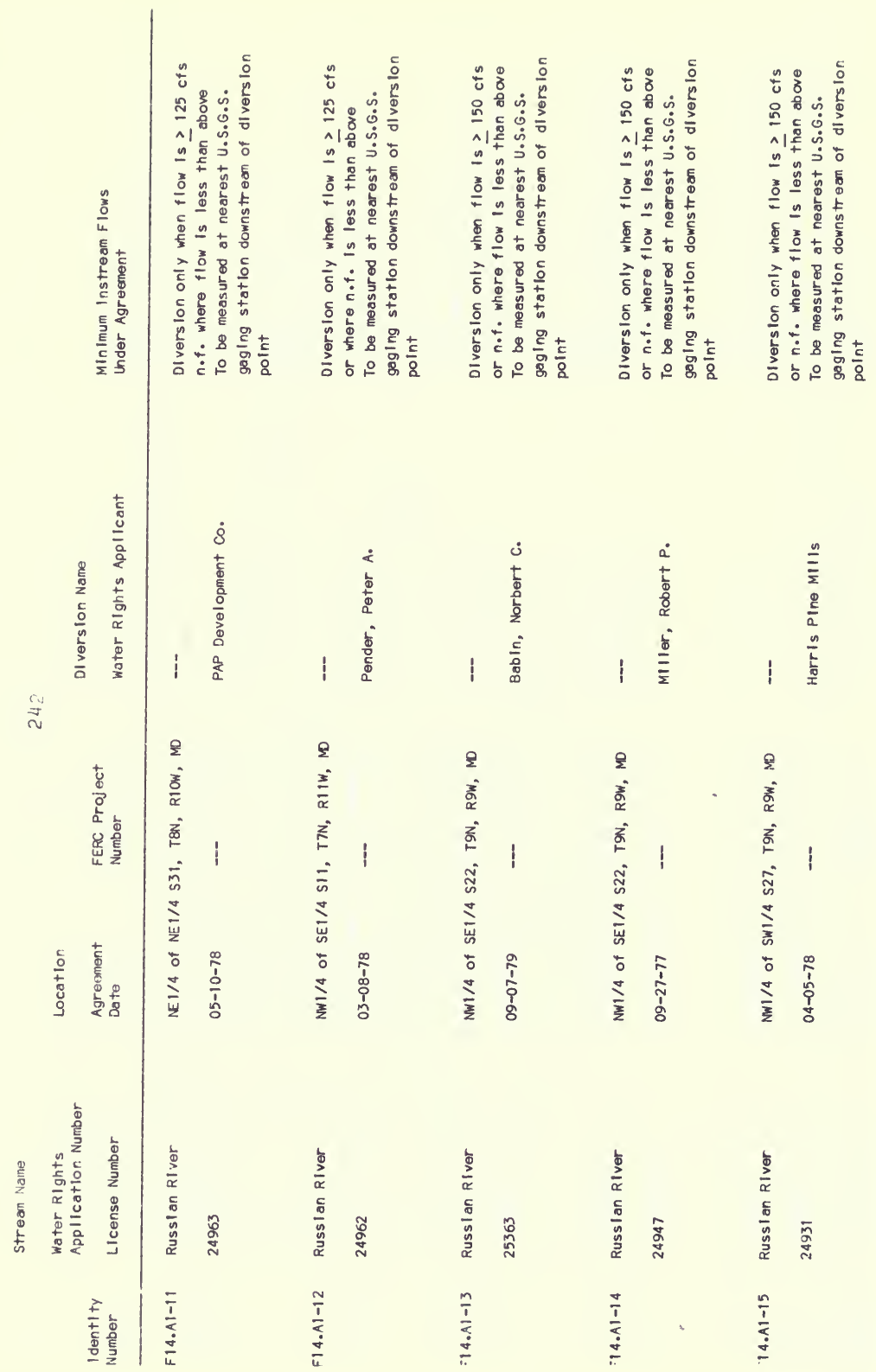




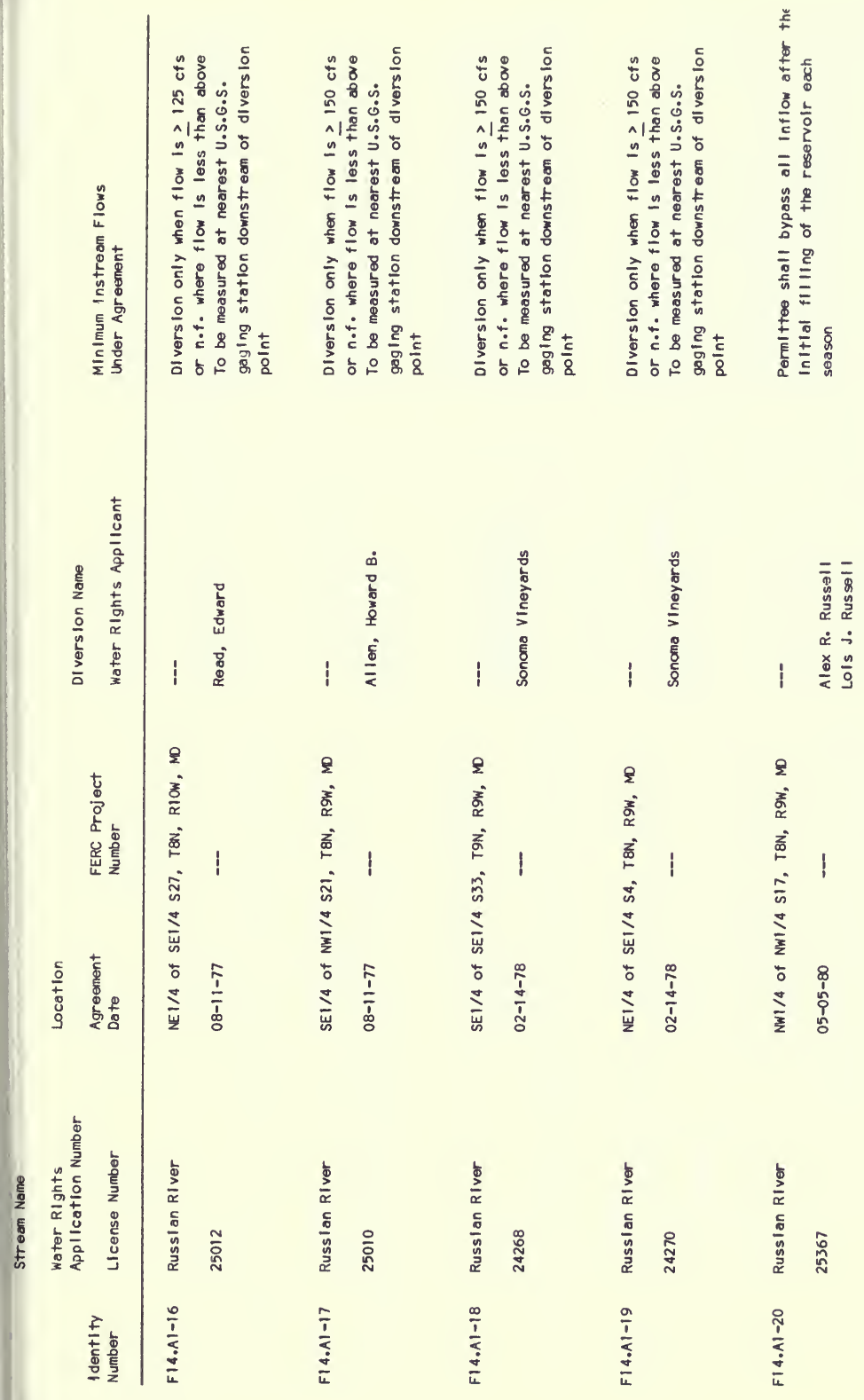




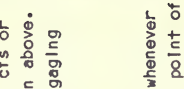

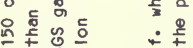

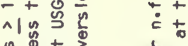

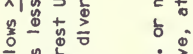

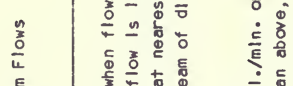

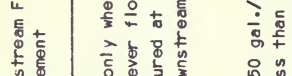

告点

胥

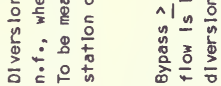

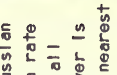

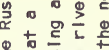

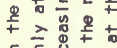

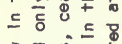

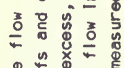

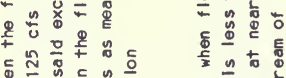

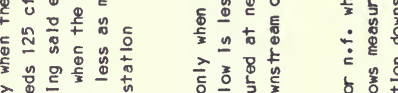

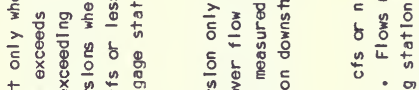

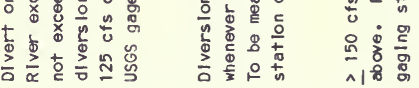

츰

范

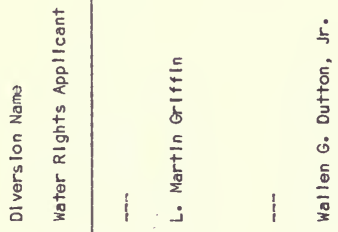

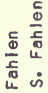

$\dot{\dot{u}}$

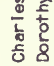

产

产

욜

离

로<smiles>C1=CCC=C1</smiles>

른

志

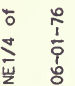

竞

$\frac{t}{0}$

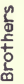

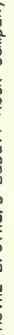

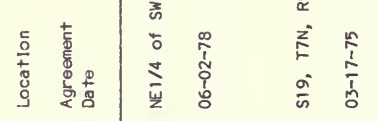

高

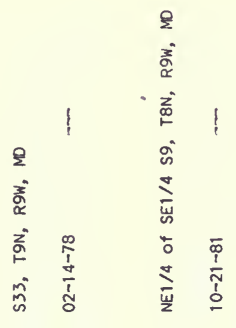

$\frac{2}{\frac{3}{0}}$

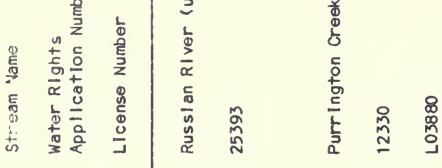

$\frac{5}{\alpha}$

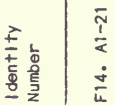

$\frac{\substack{4 \\ \frac{d}{4}}}{\frac{1}{4}}$

$\frac{\frac{\pi}{d}}{\frac{d}{1}}$

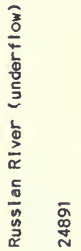

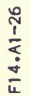




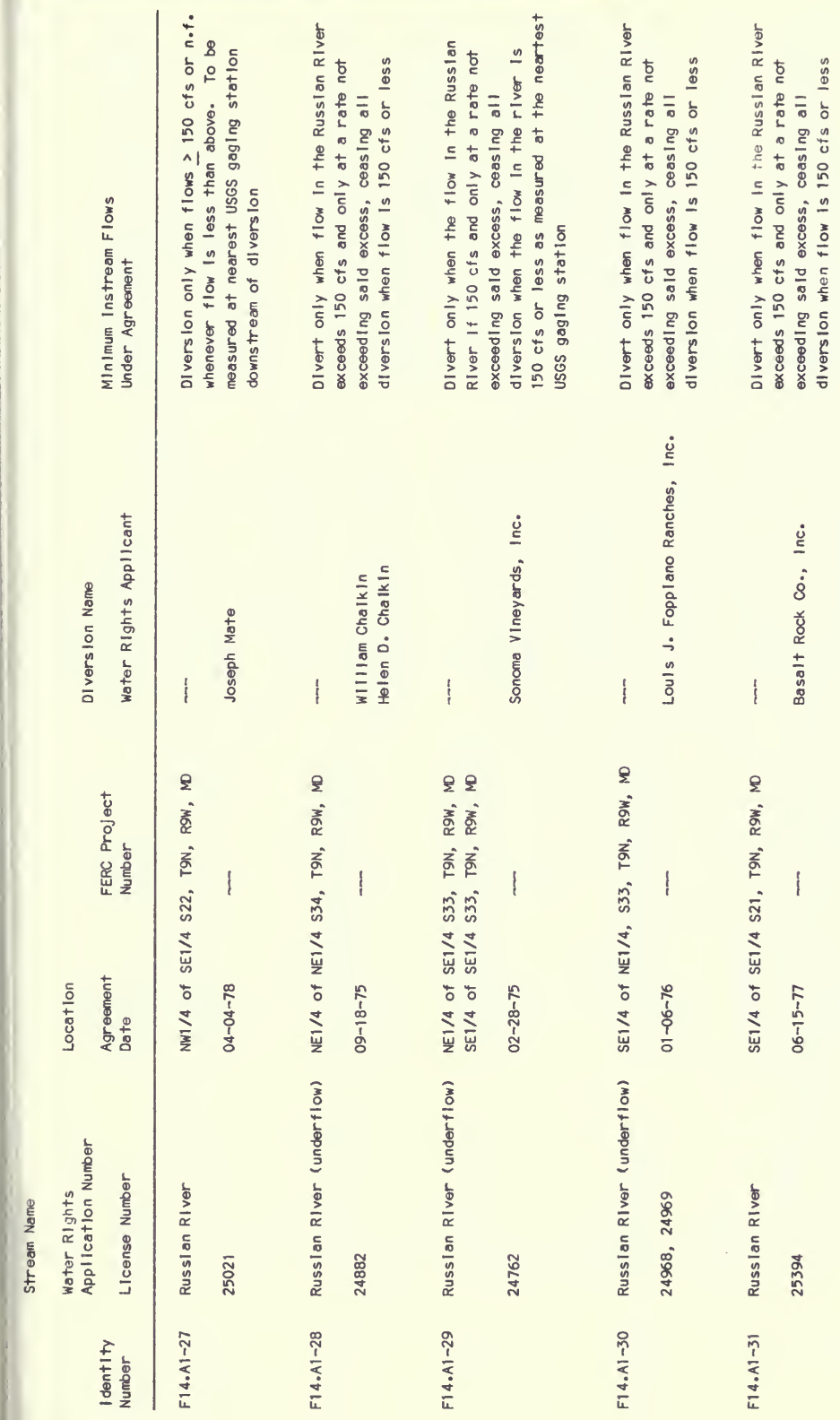

$\tilde{z}$ 


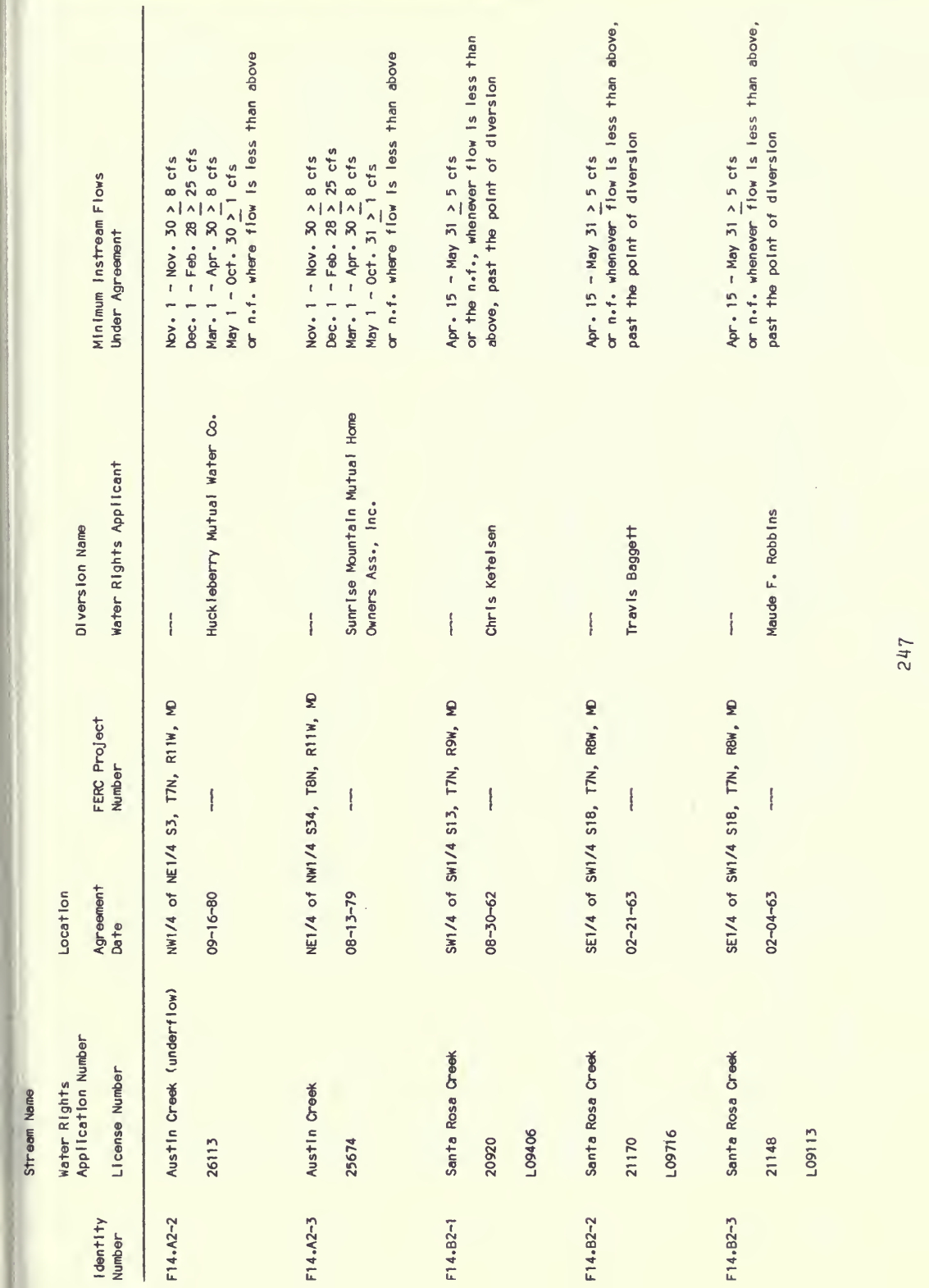




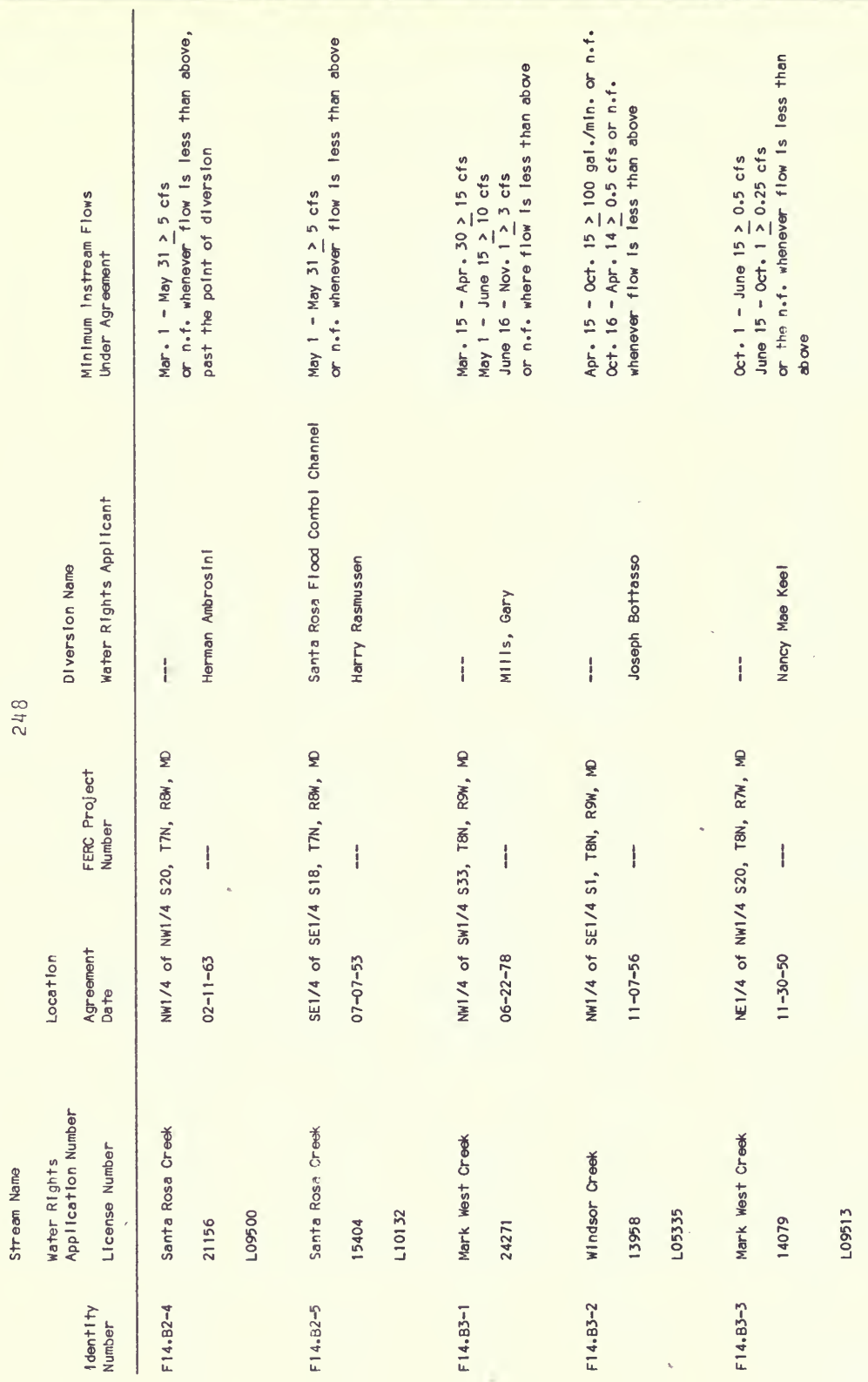




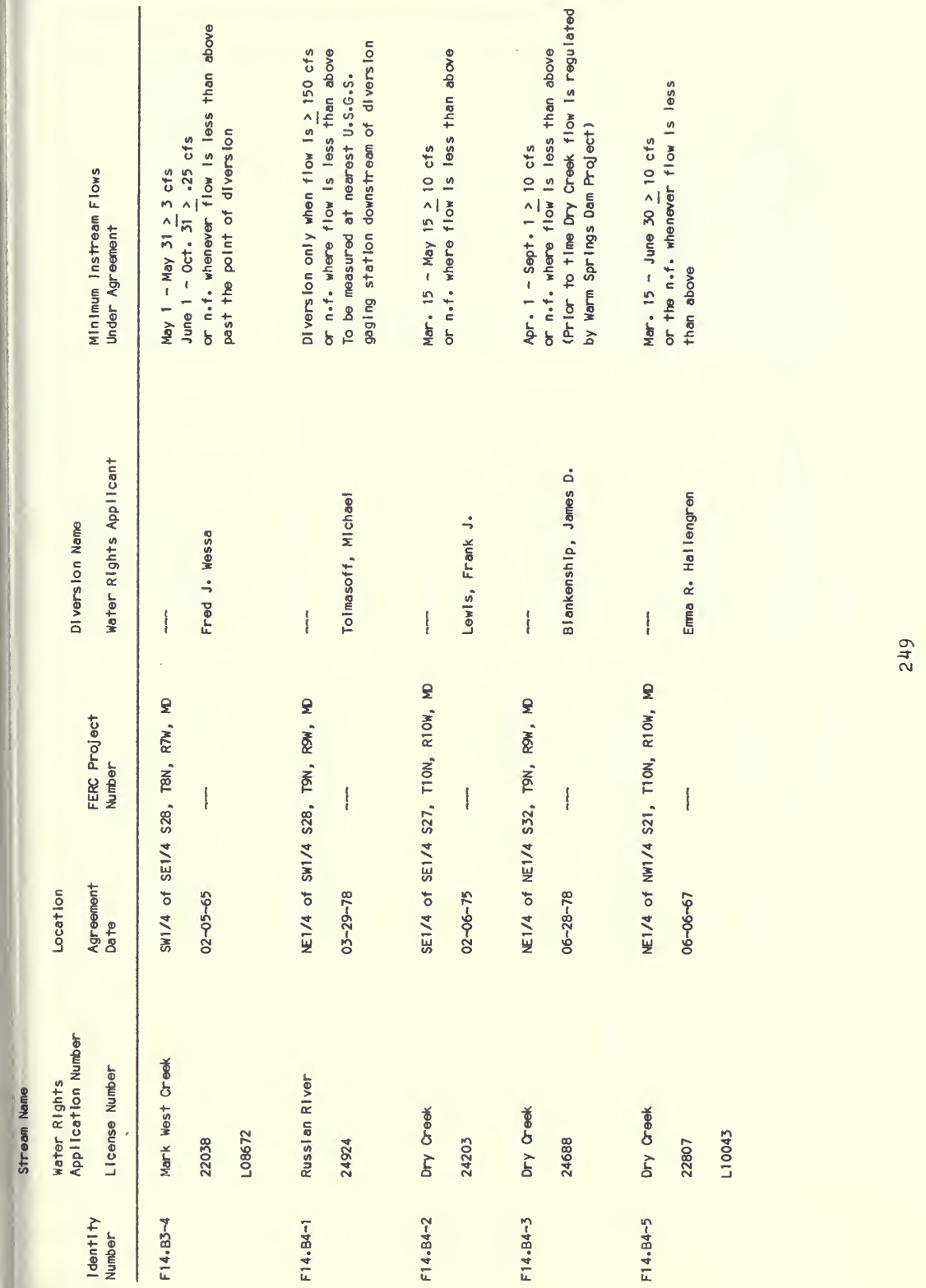




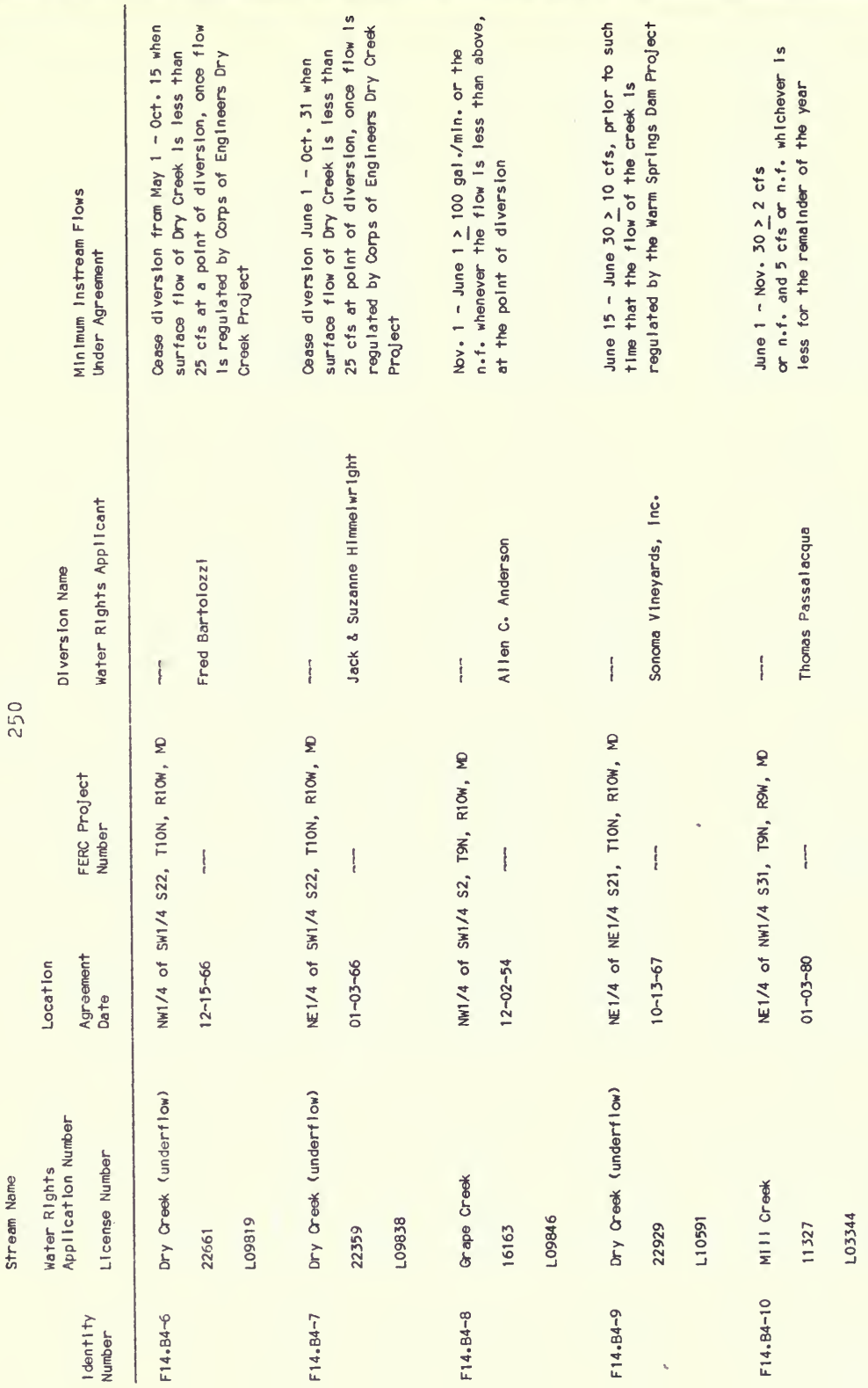




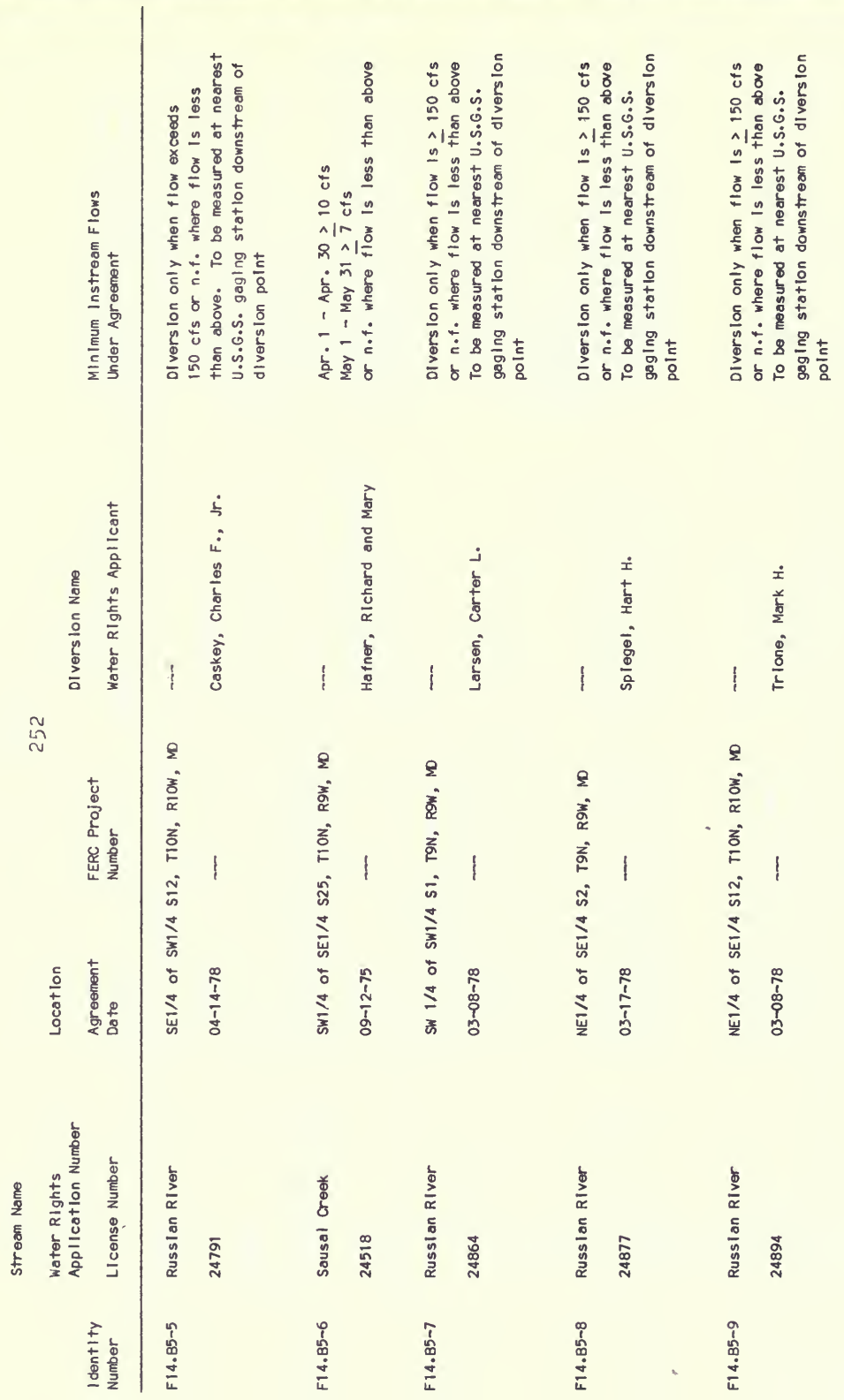




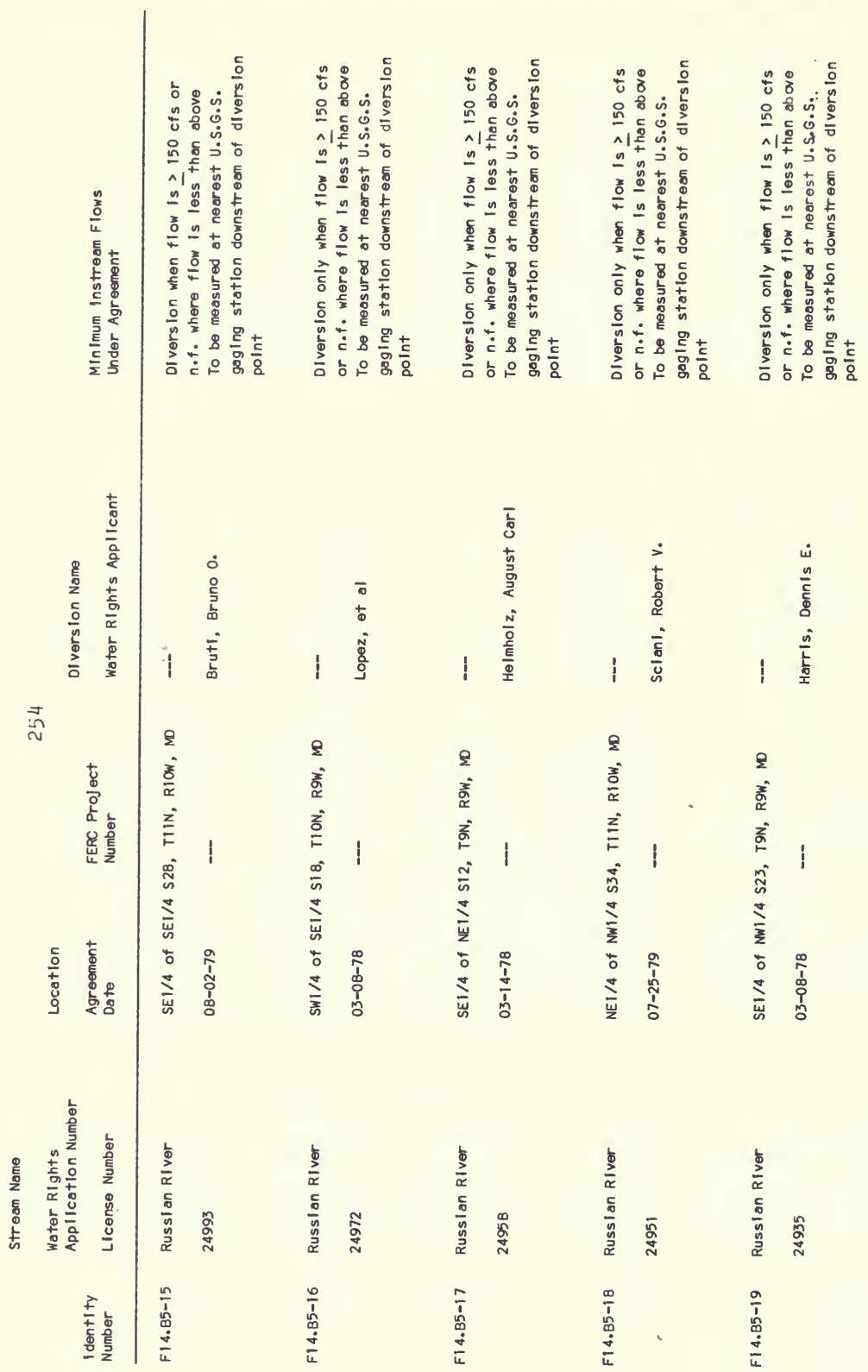




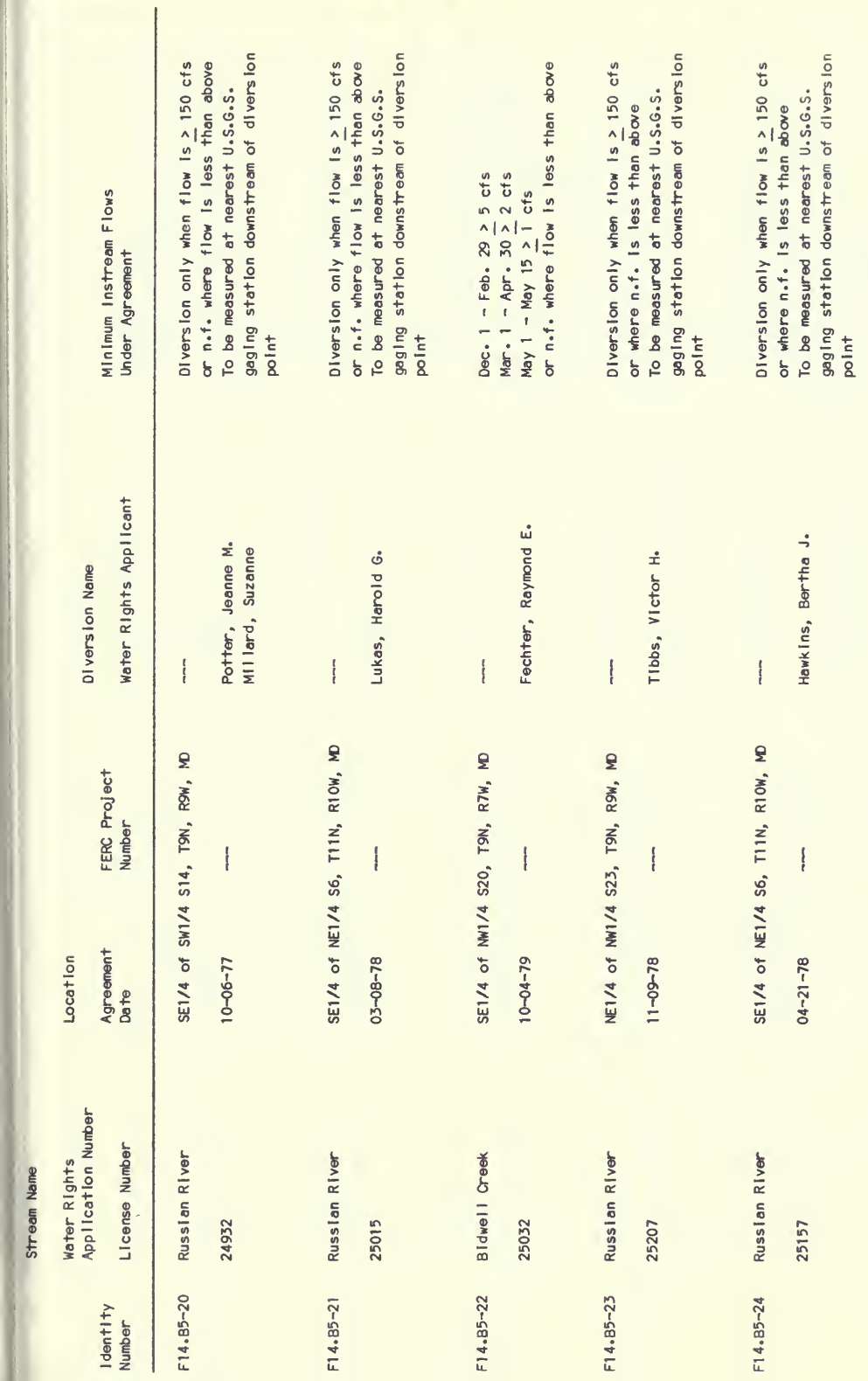

$\stackrel{n}{n}$ 


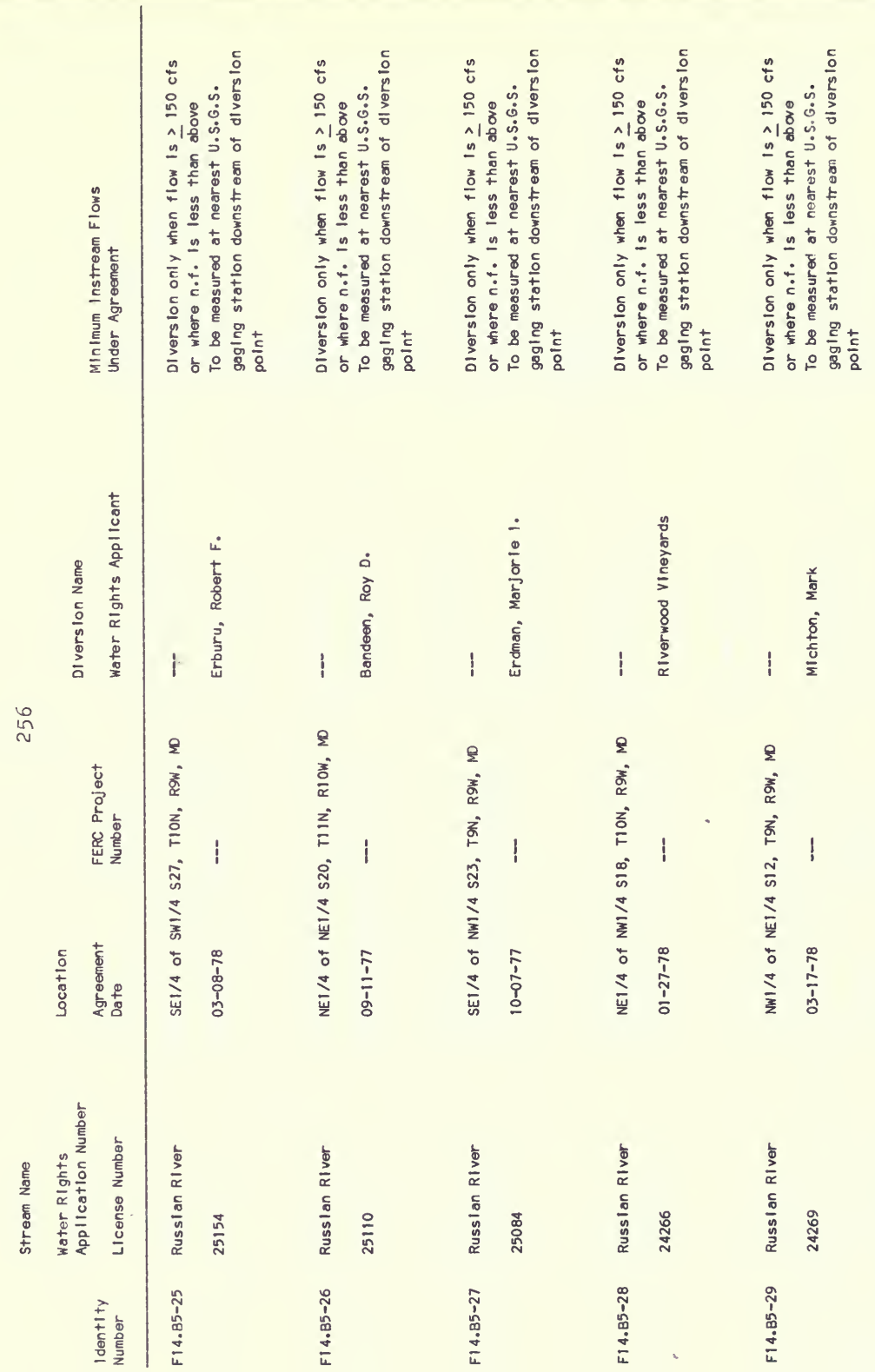




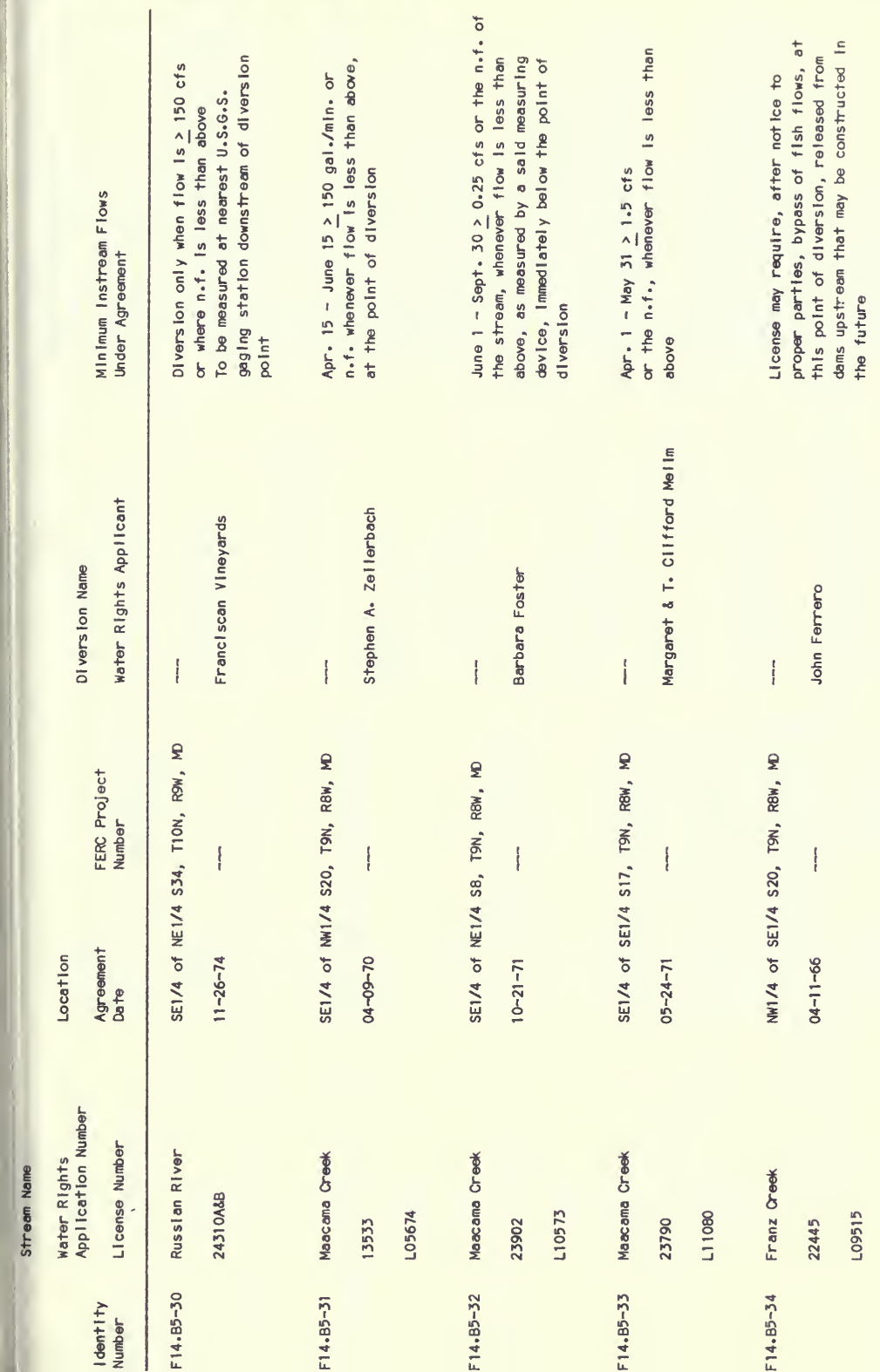

in 


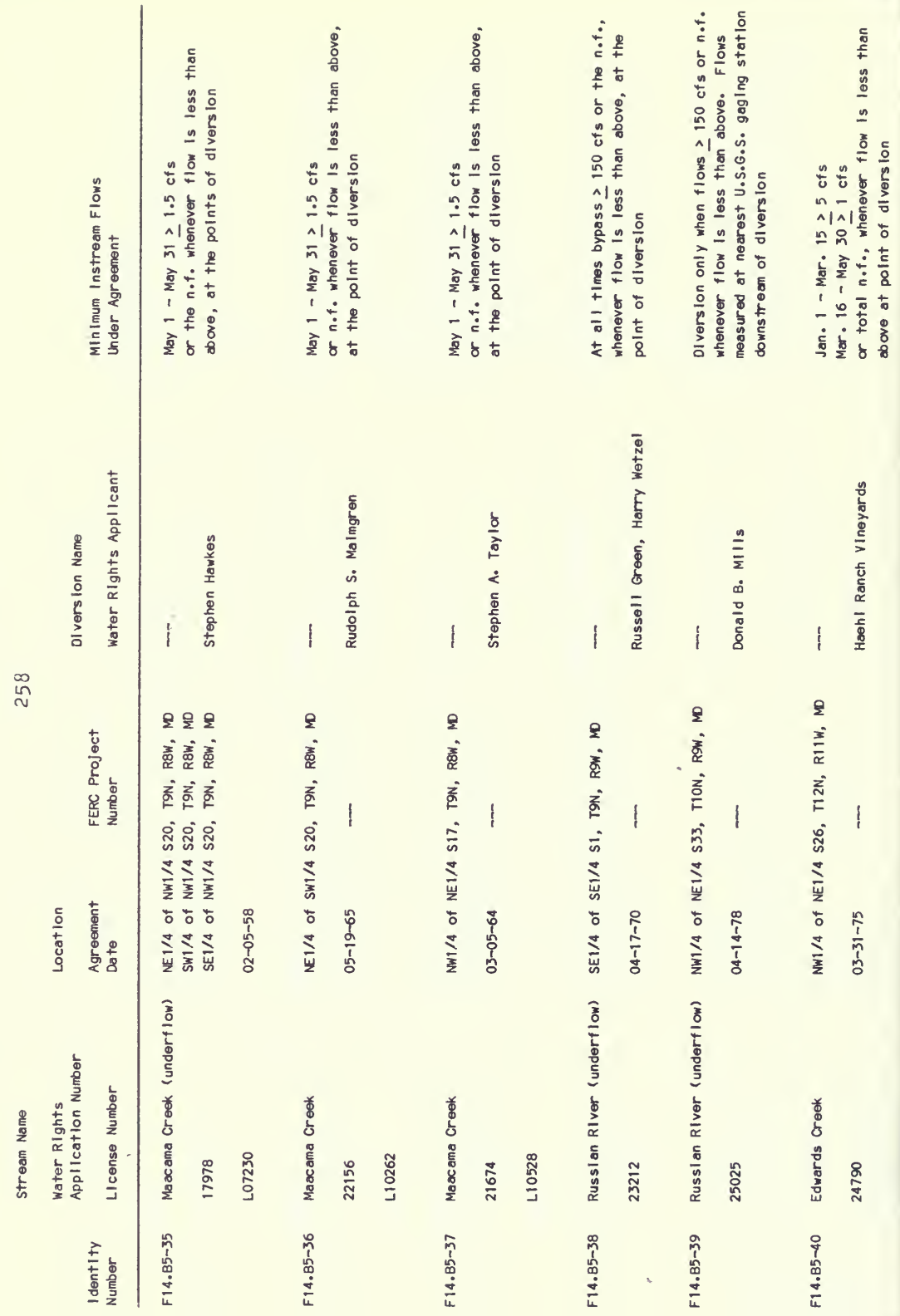




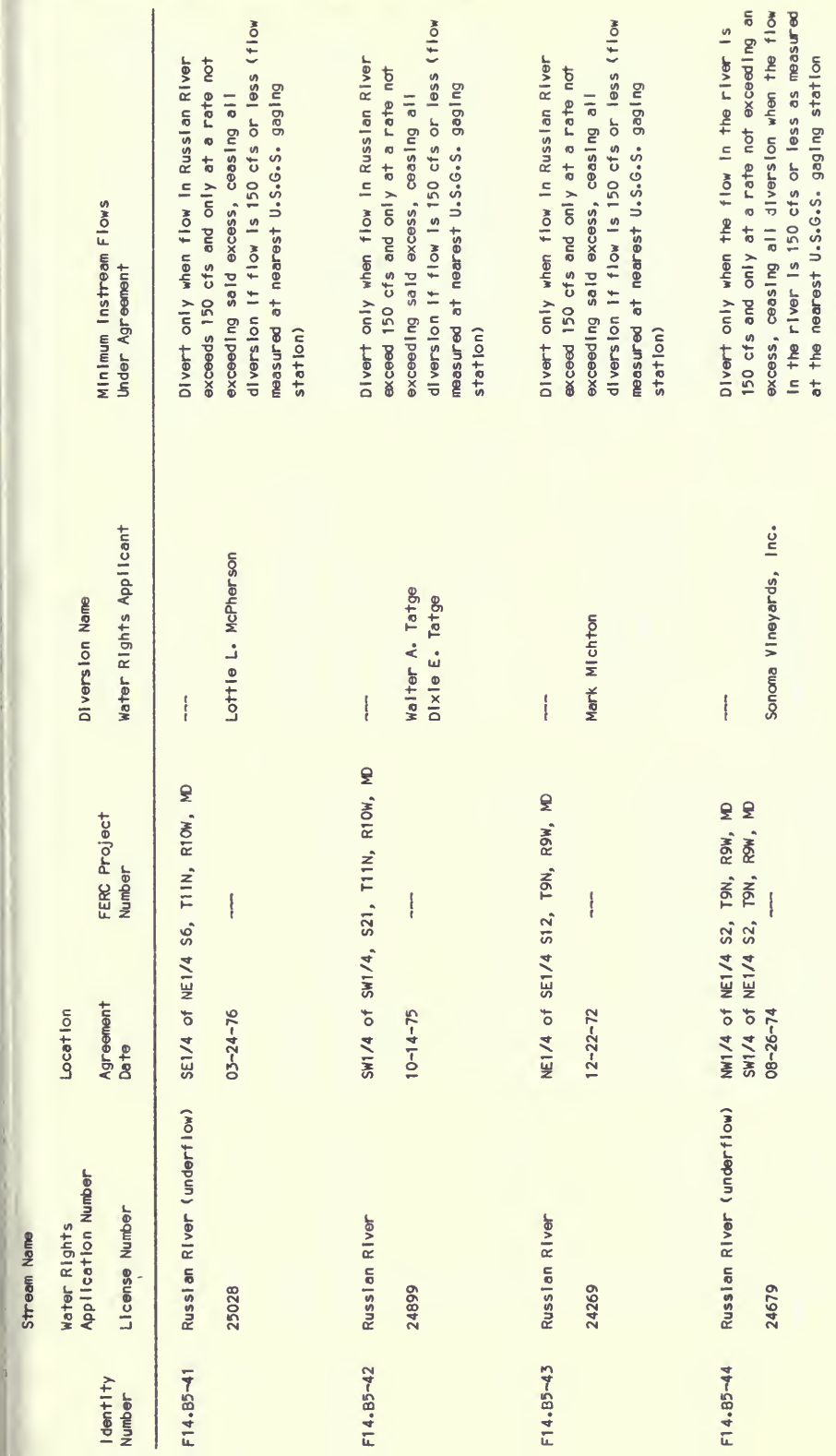




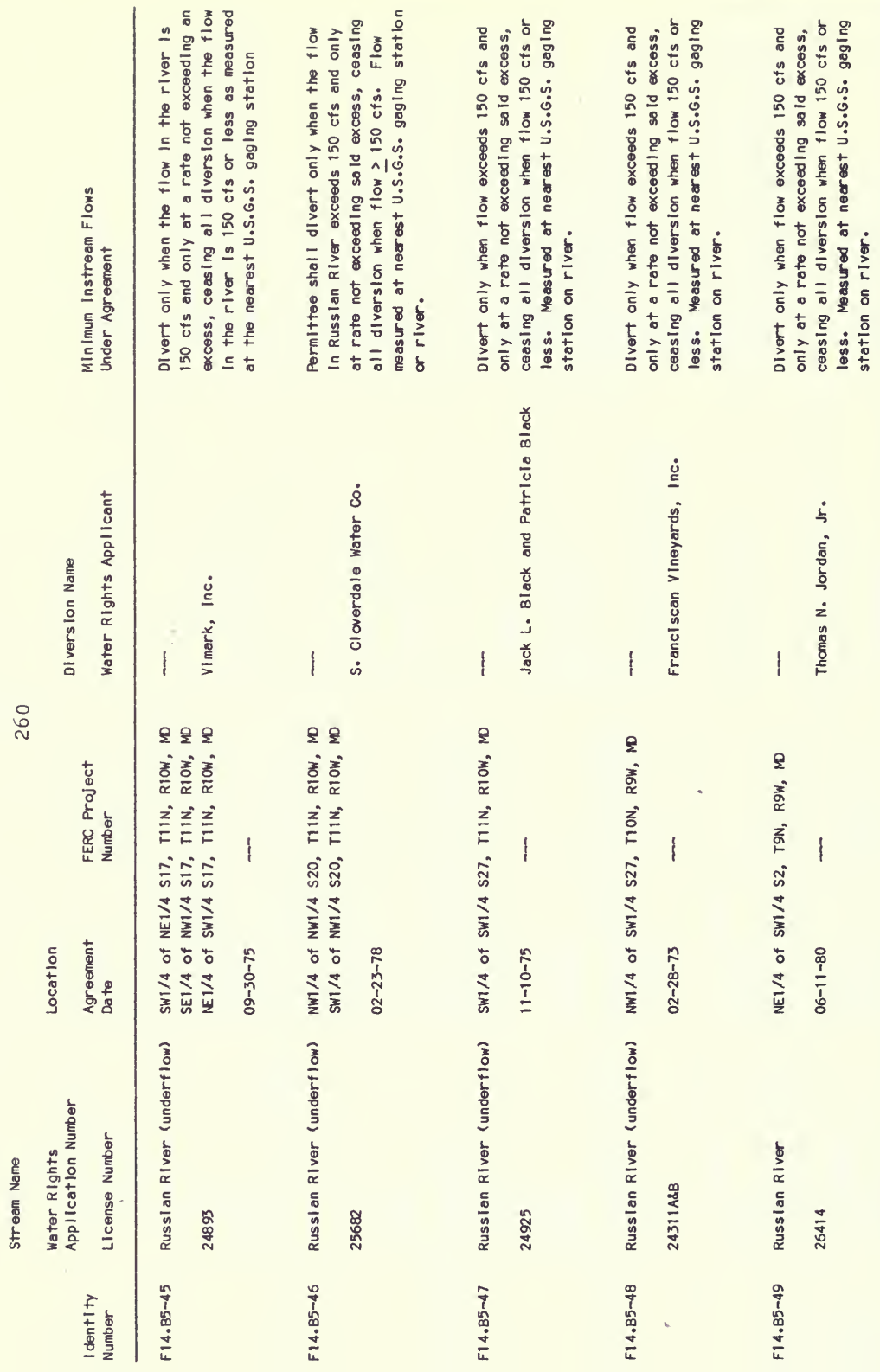




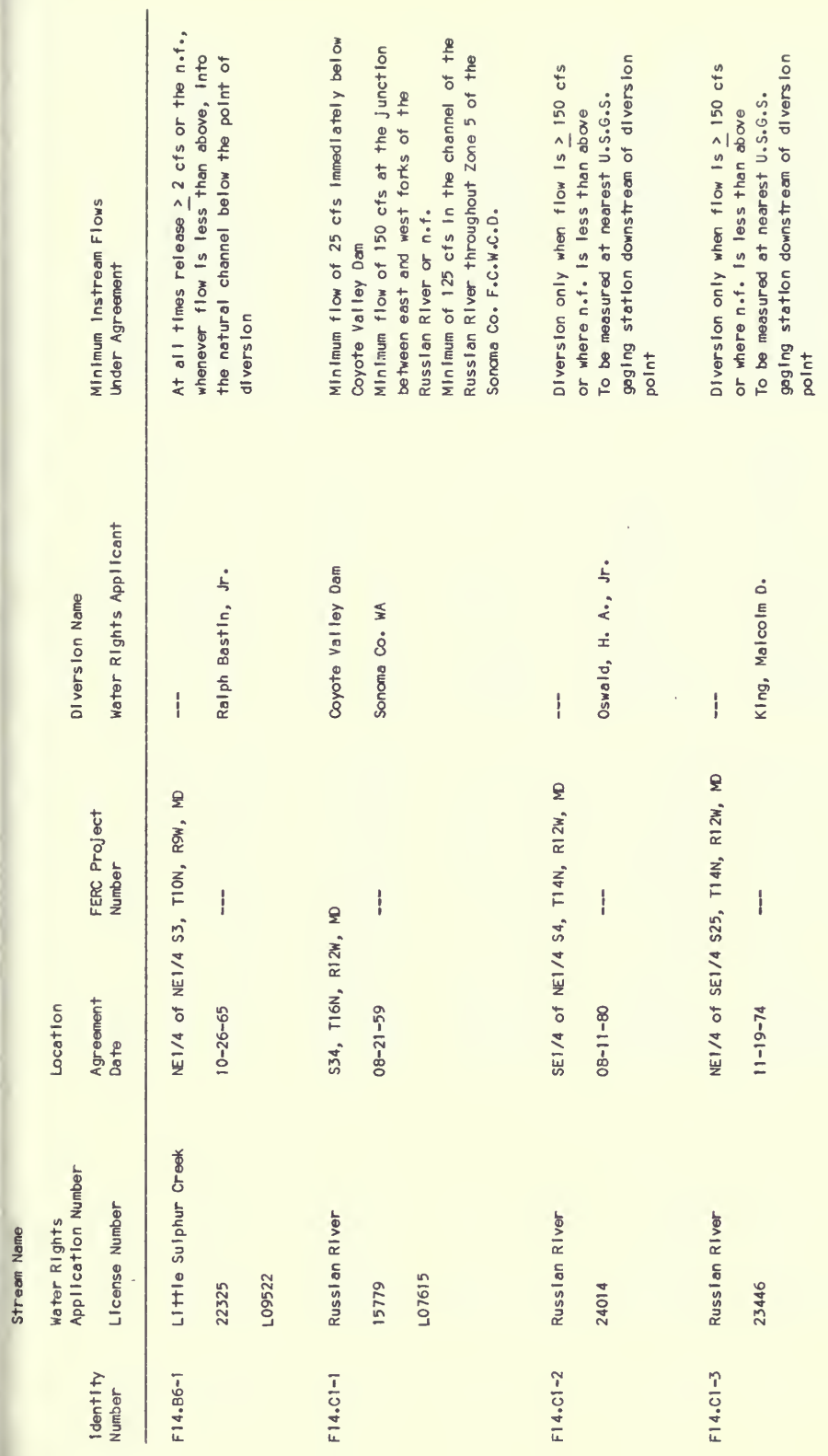

d 


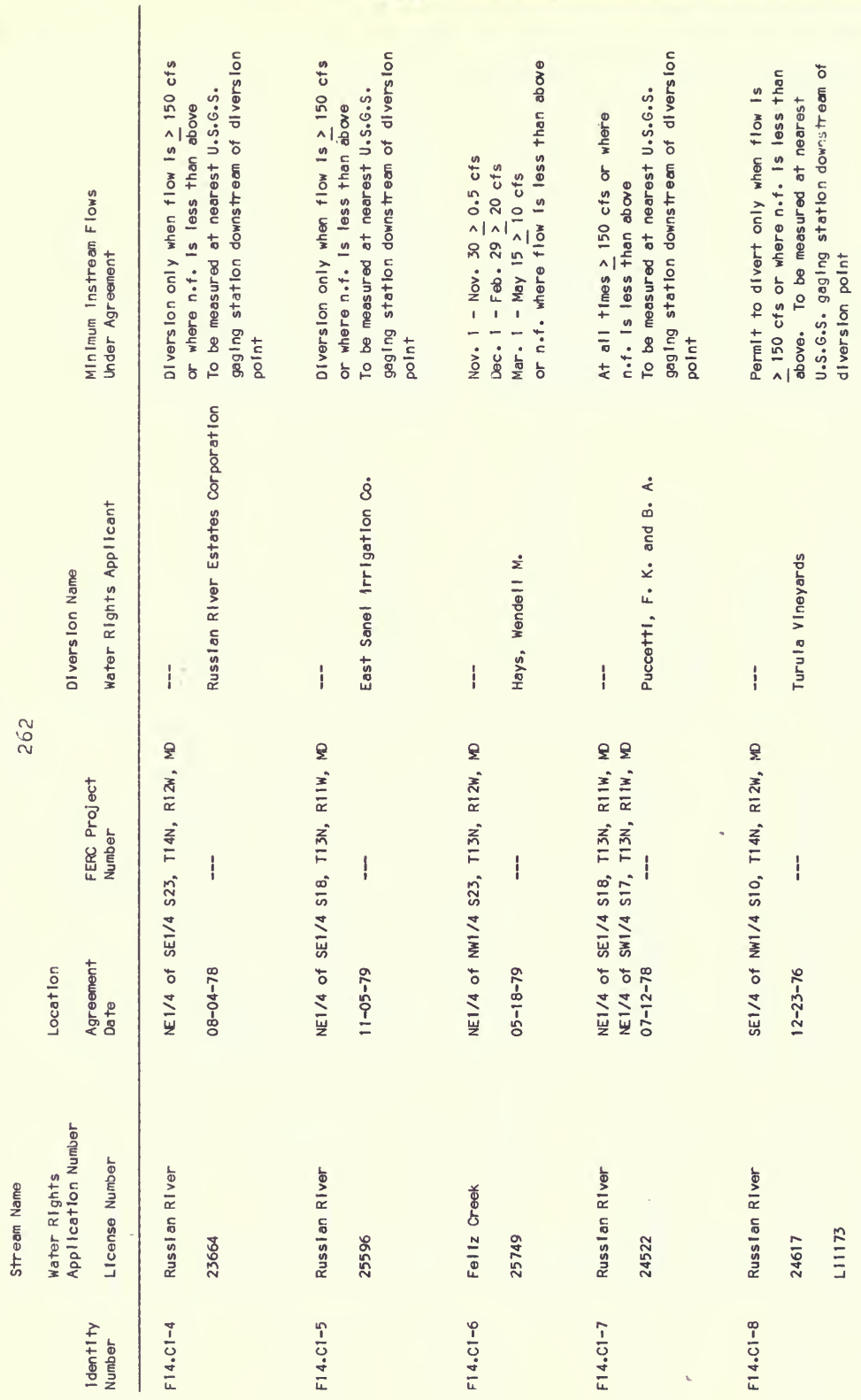




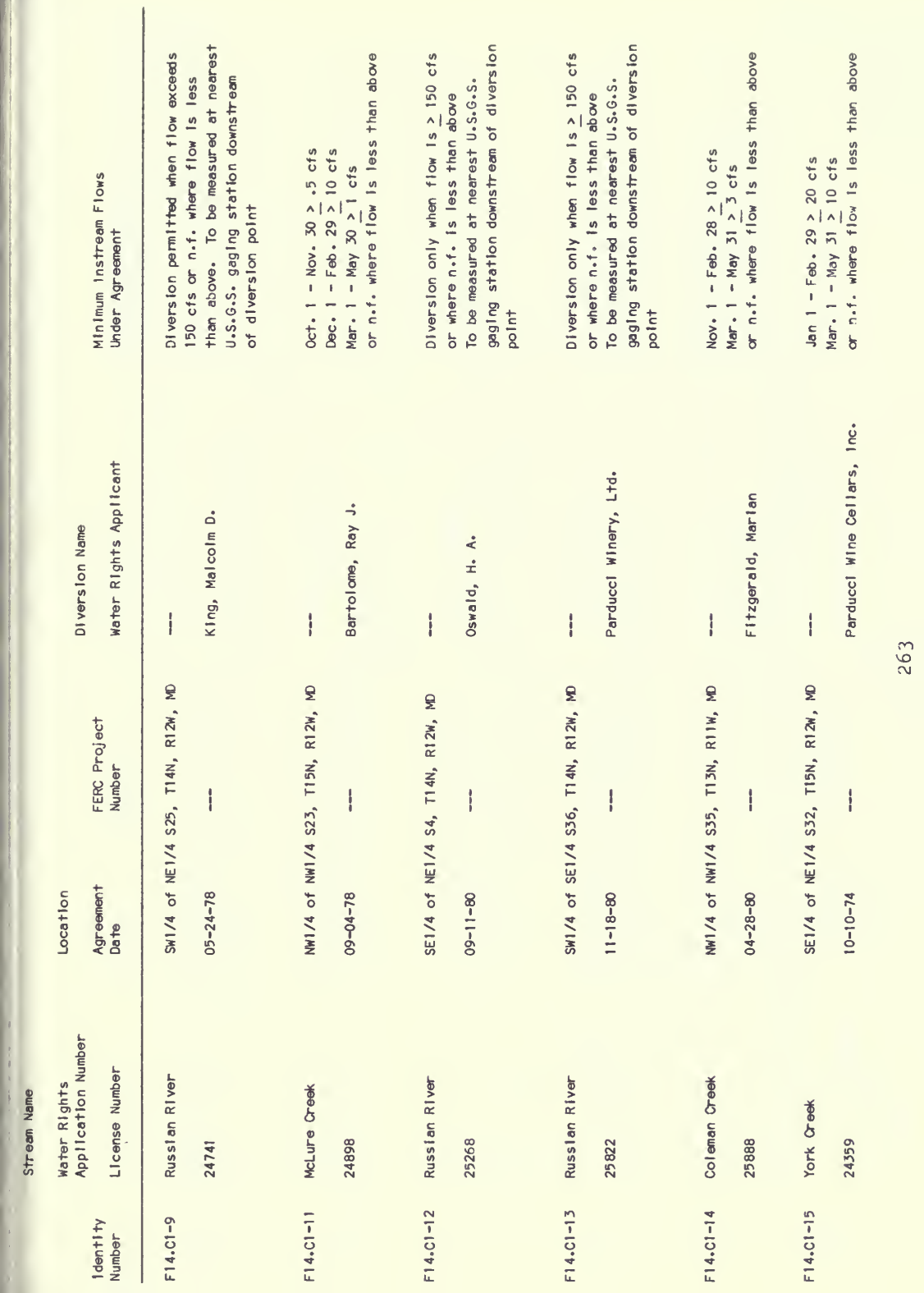




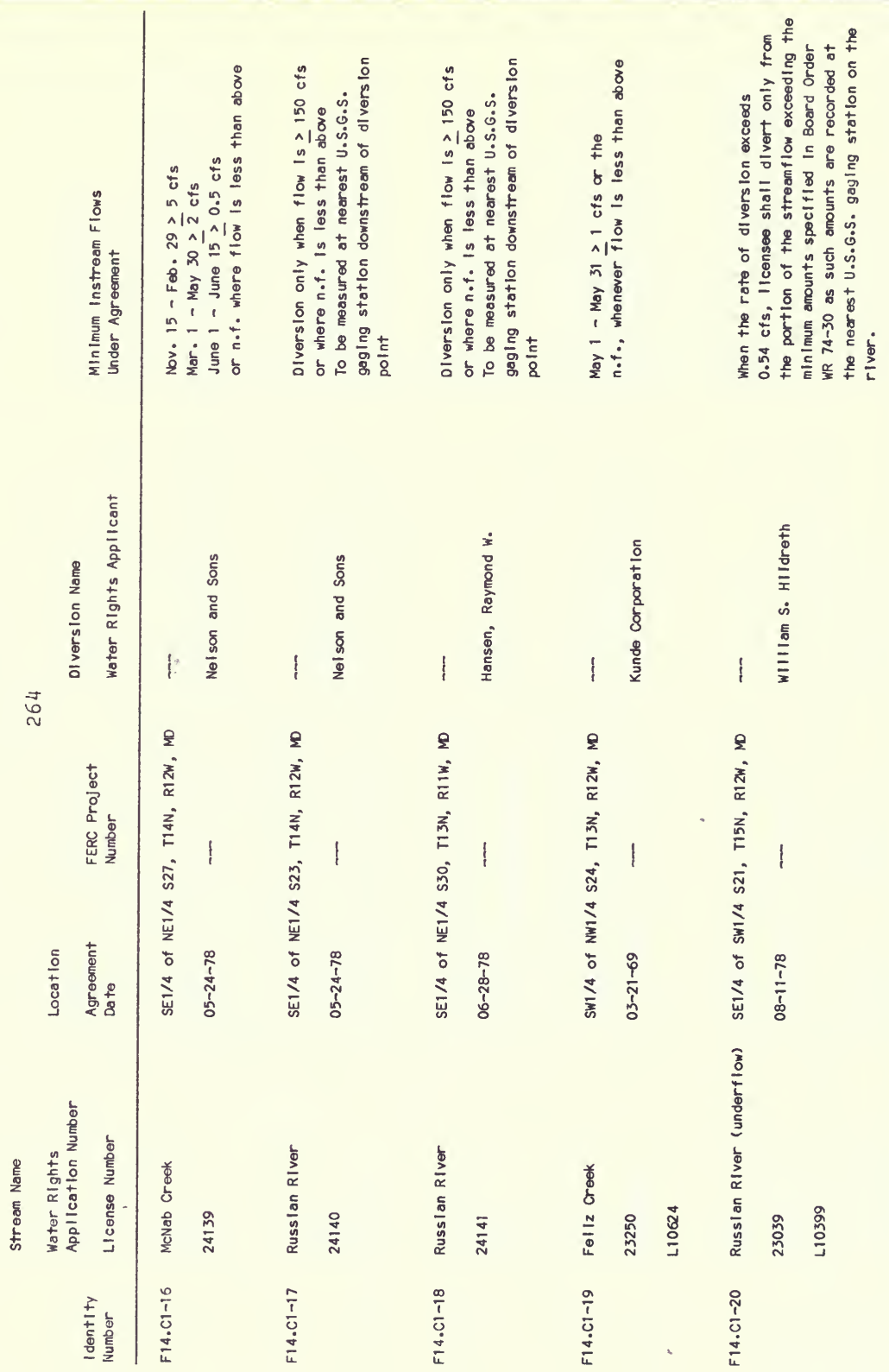




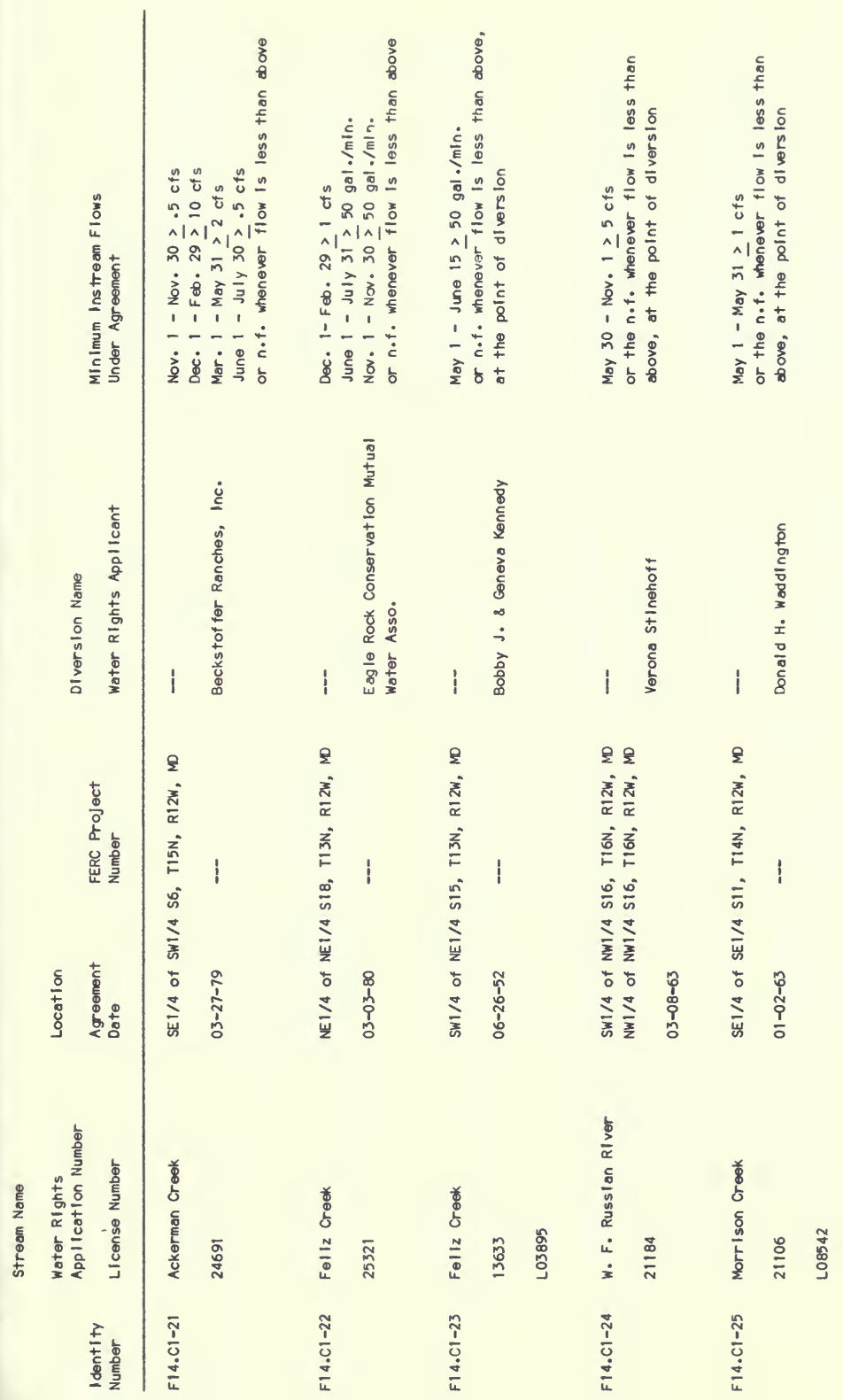

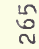




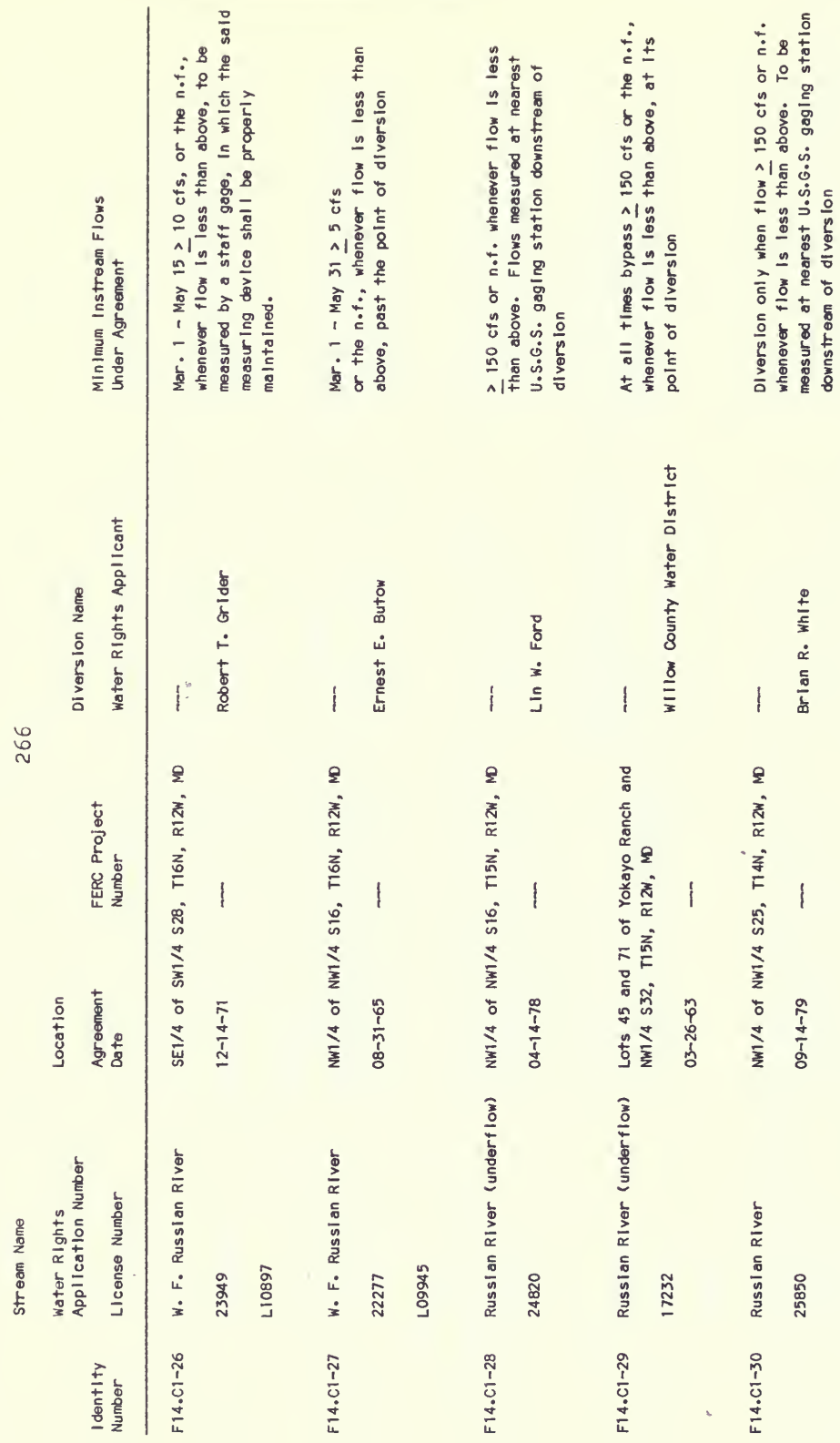




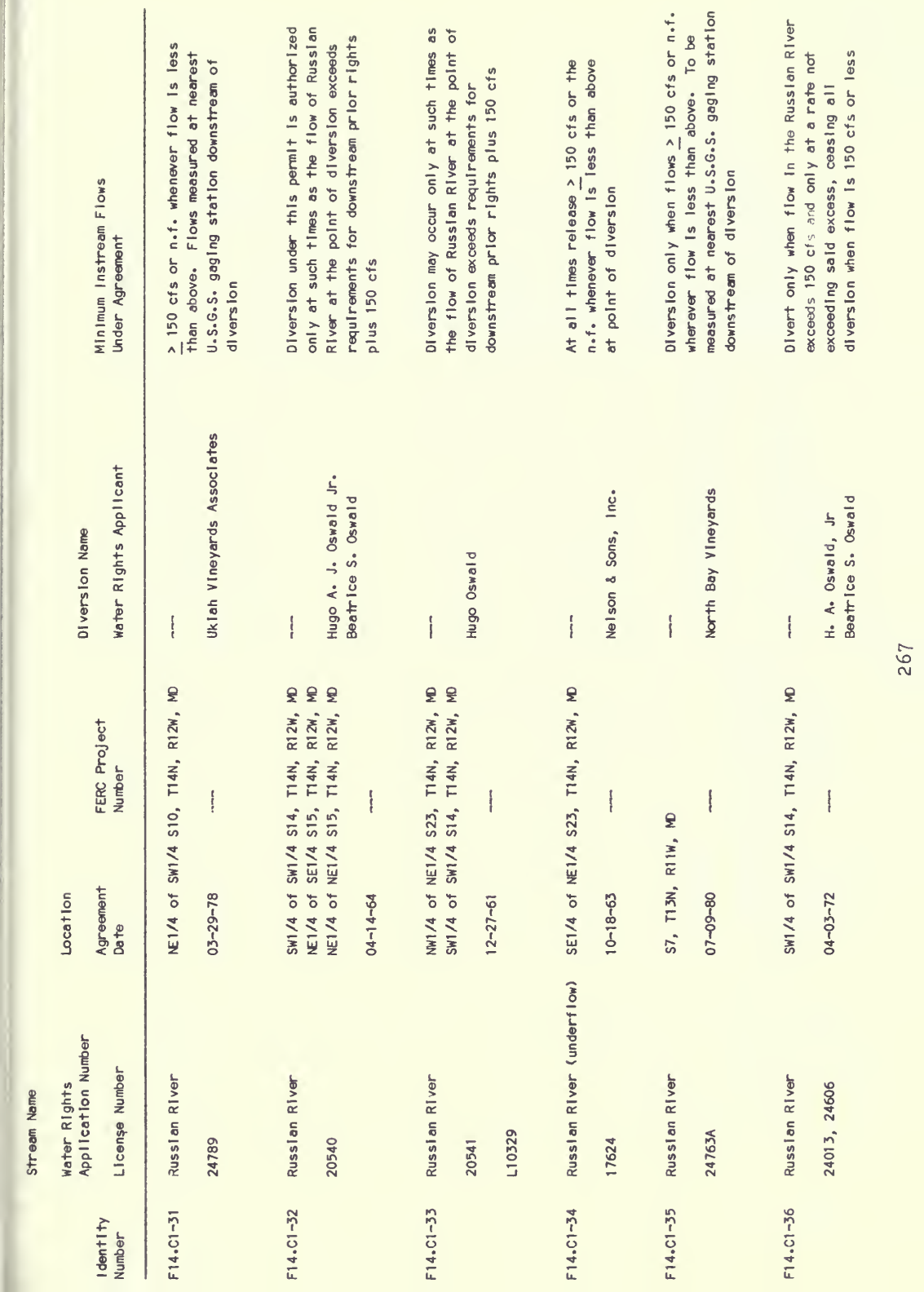




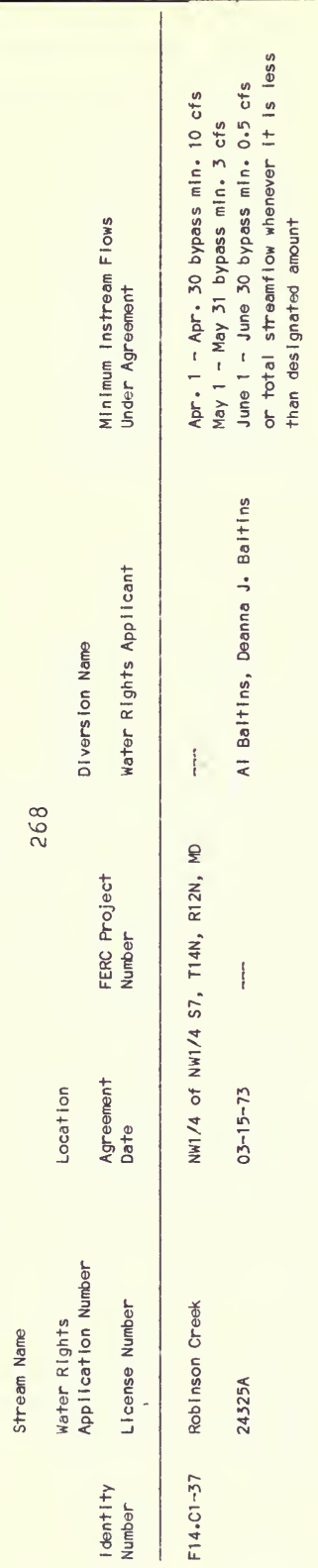




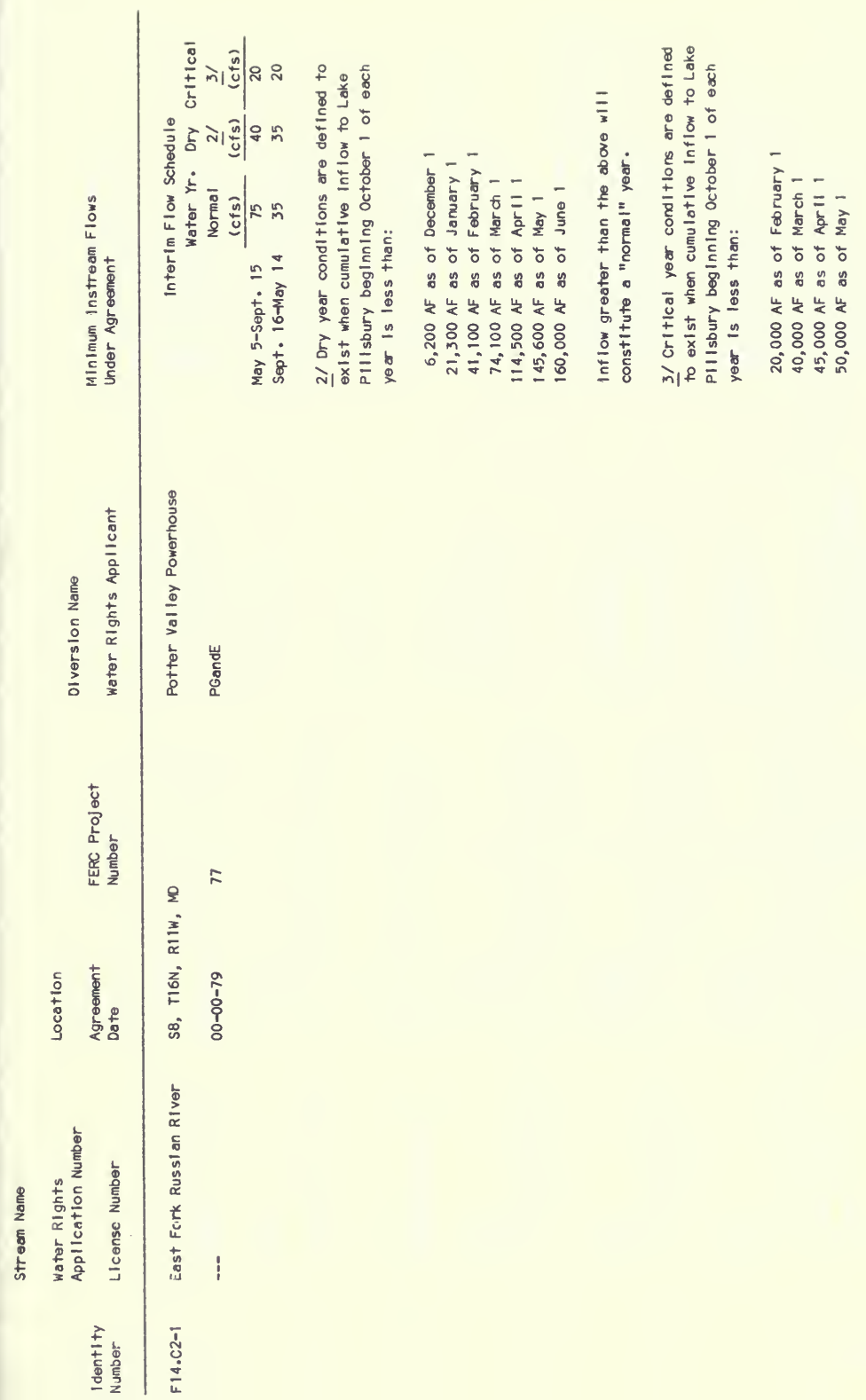




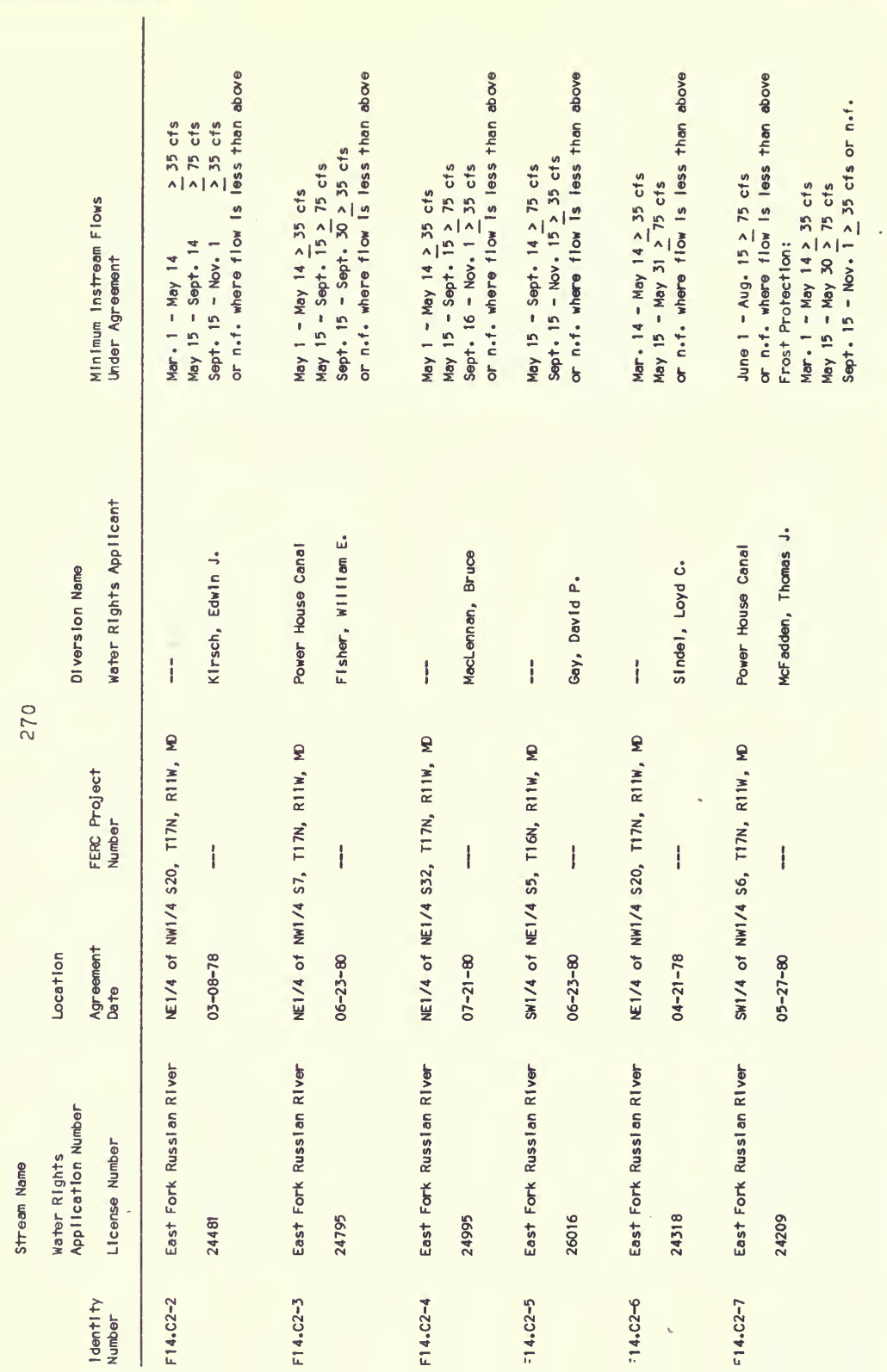




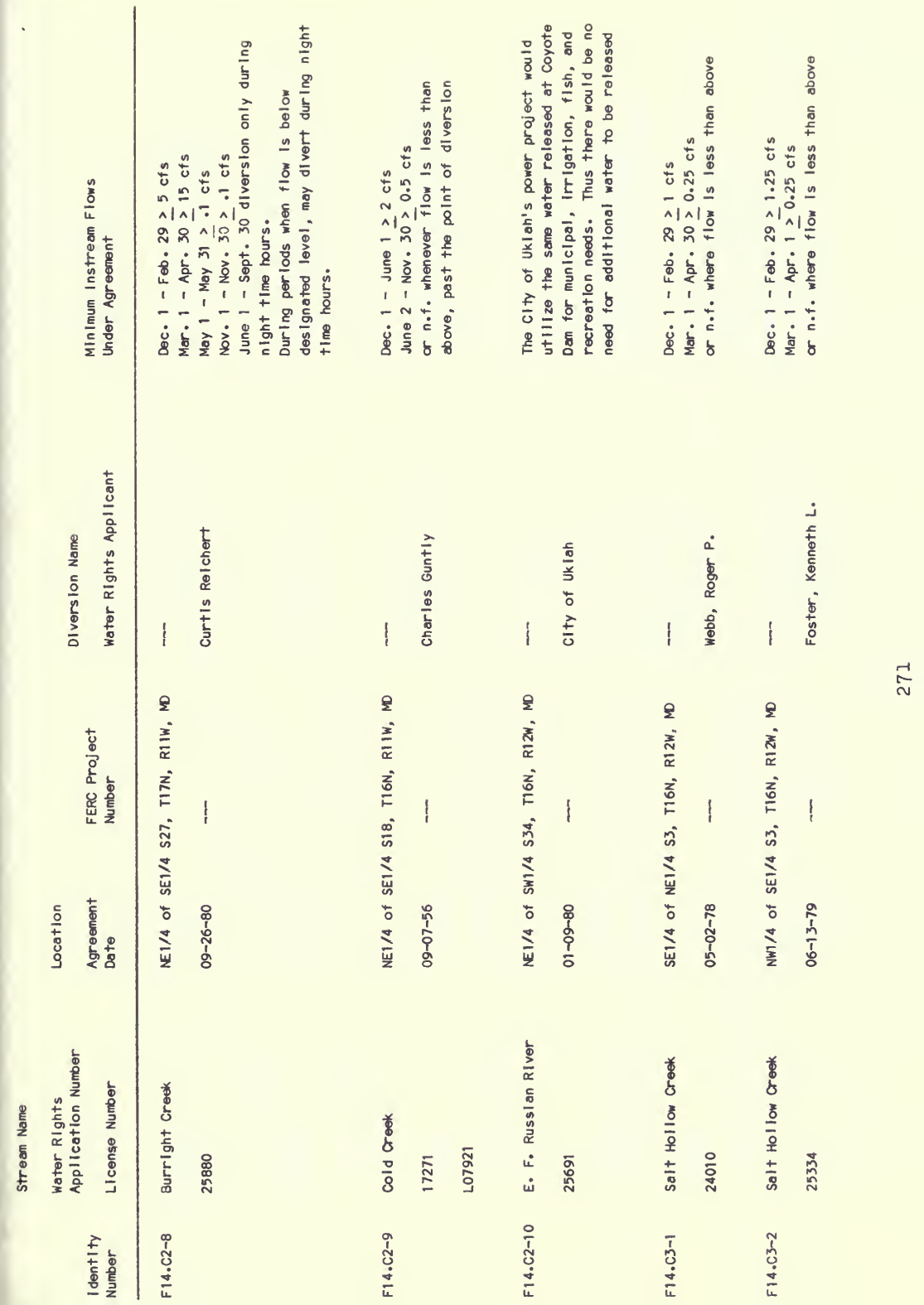




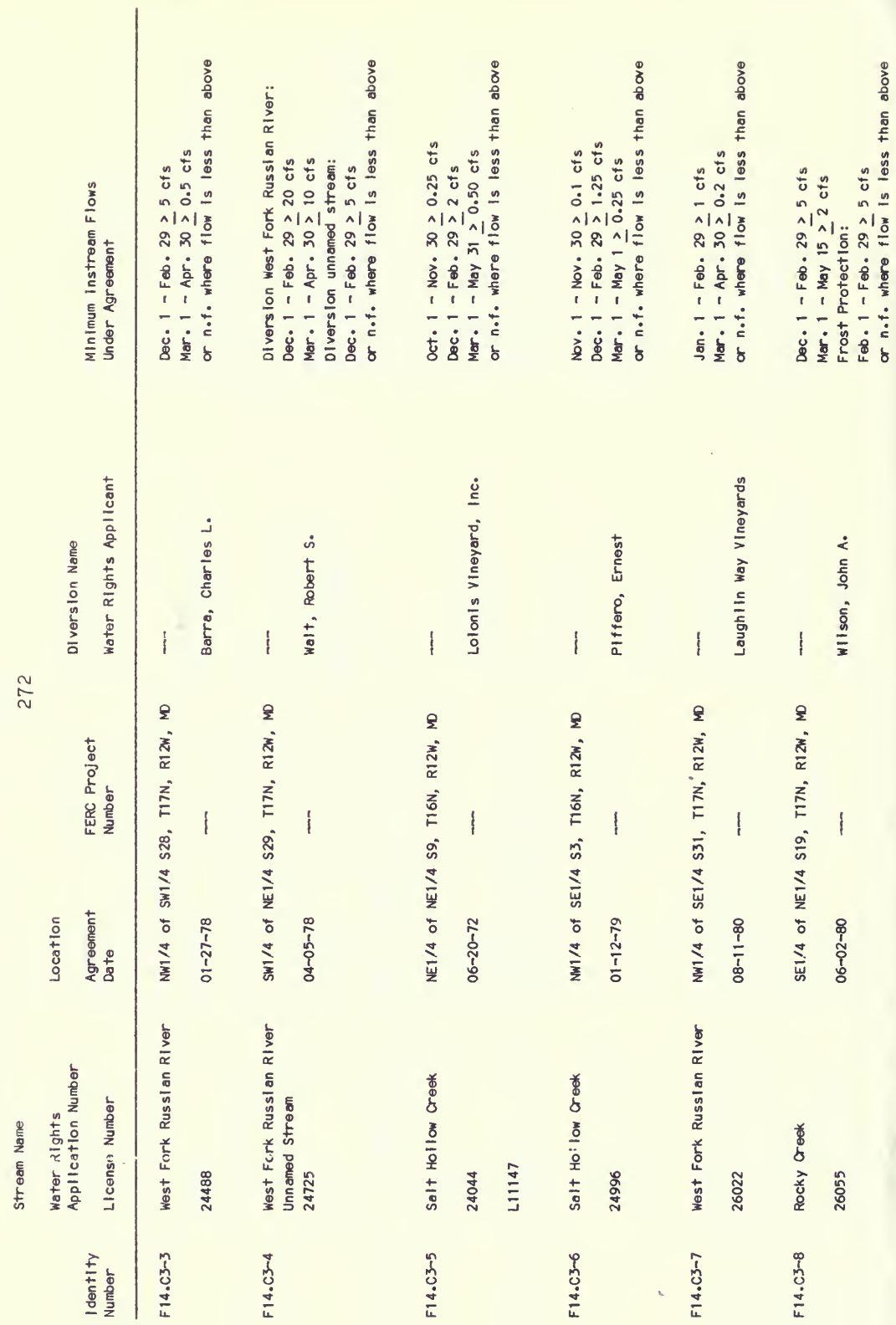




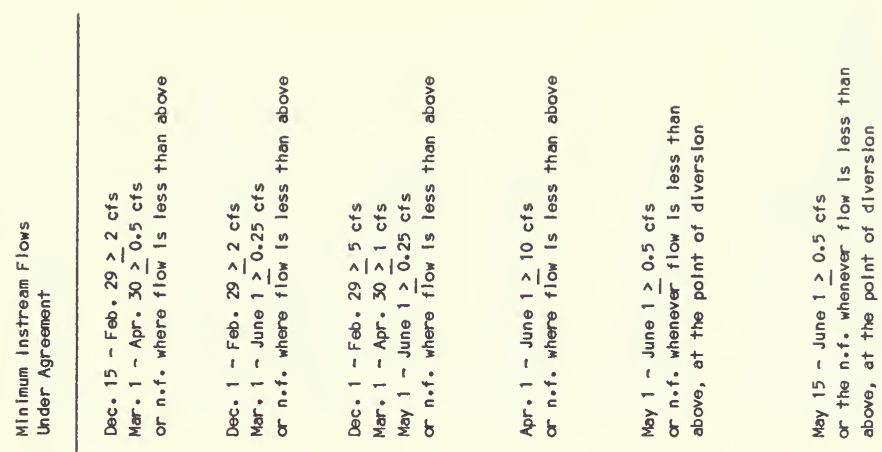

$\stackrel{m}{n}$
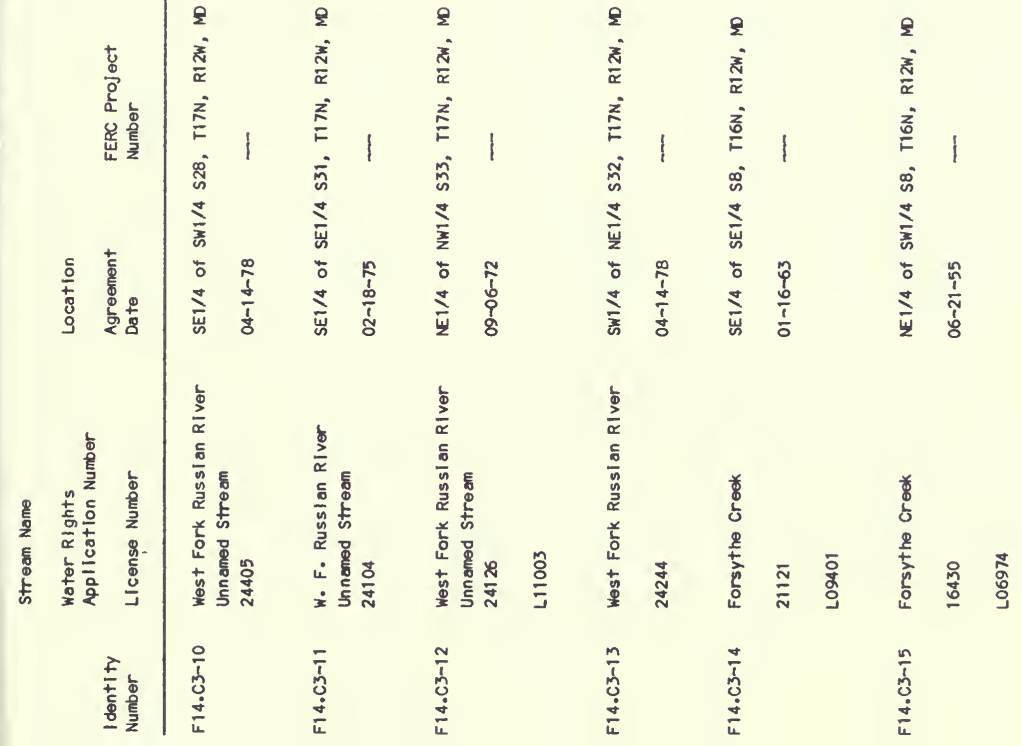


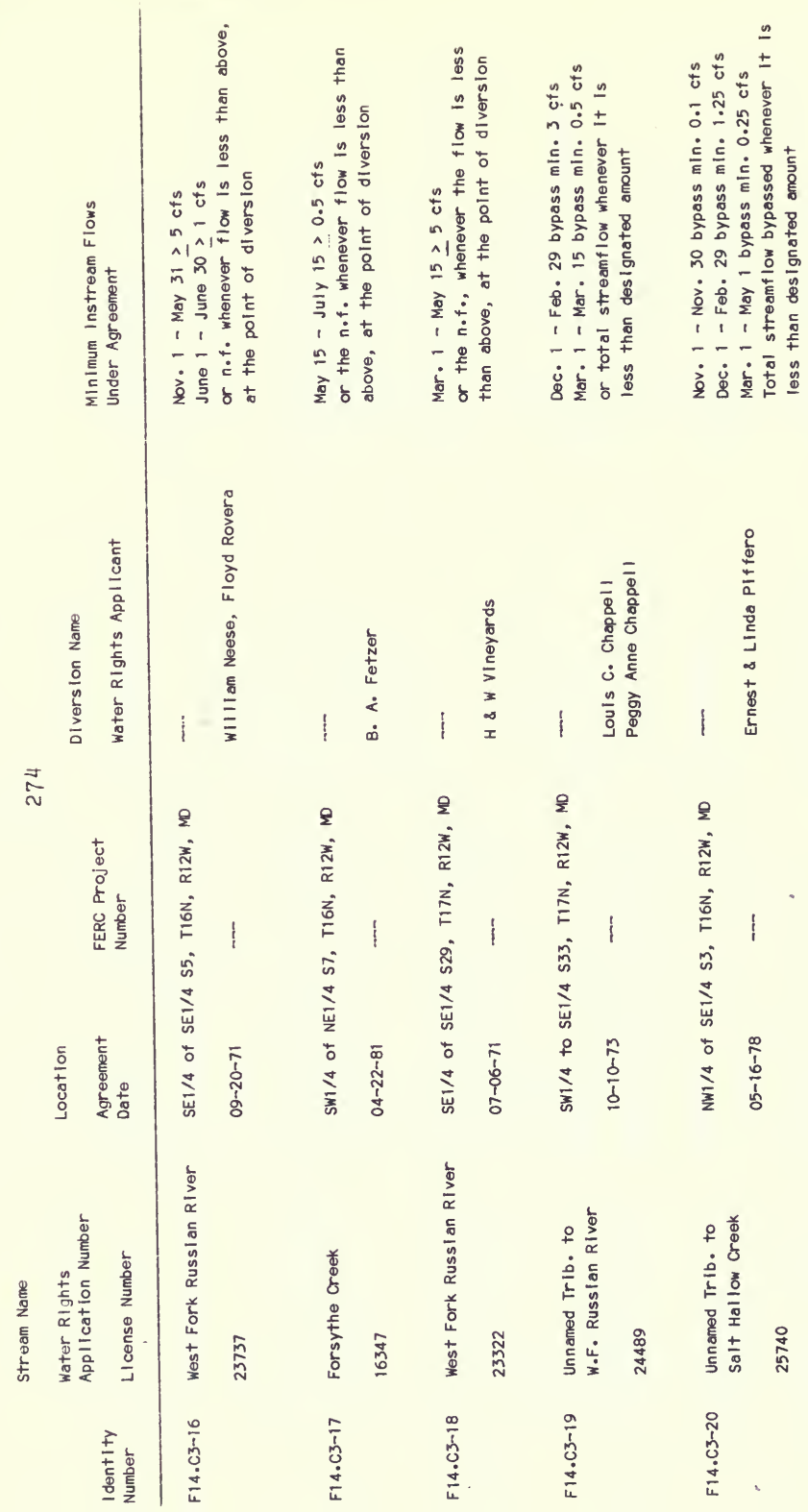




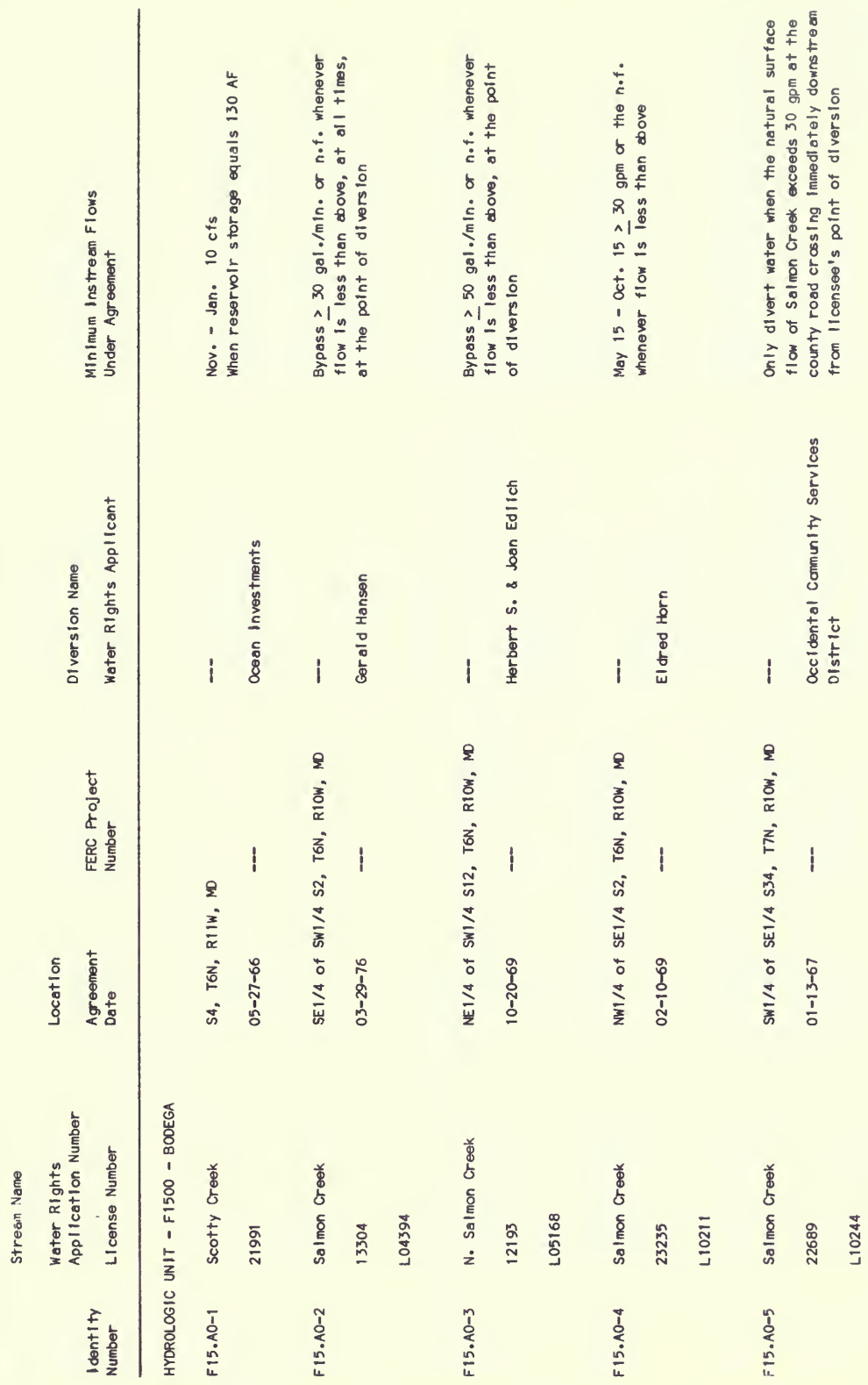

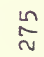




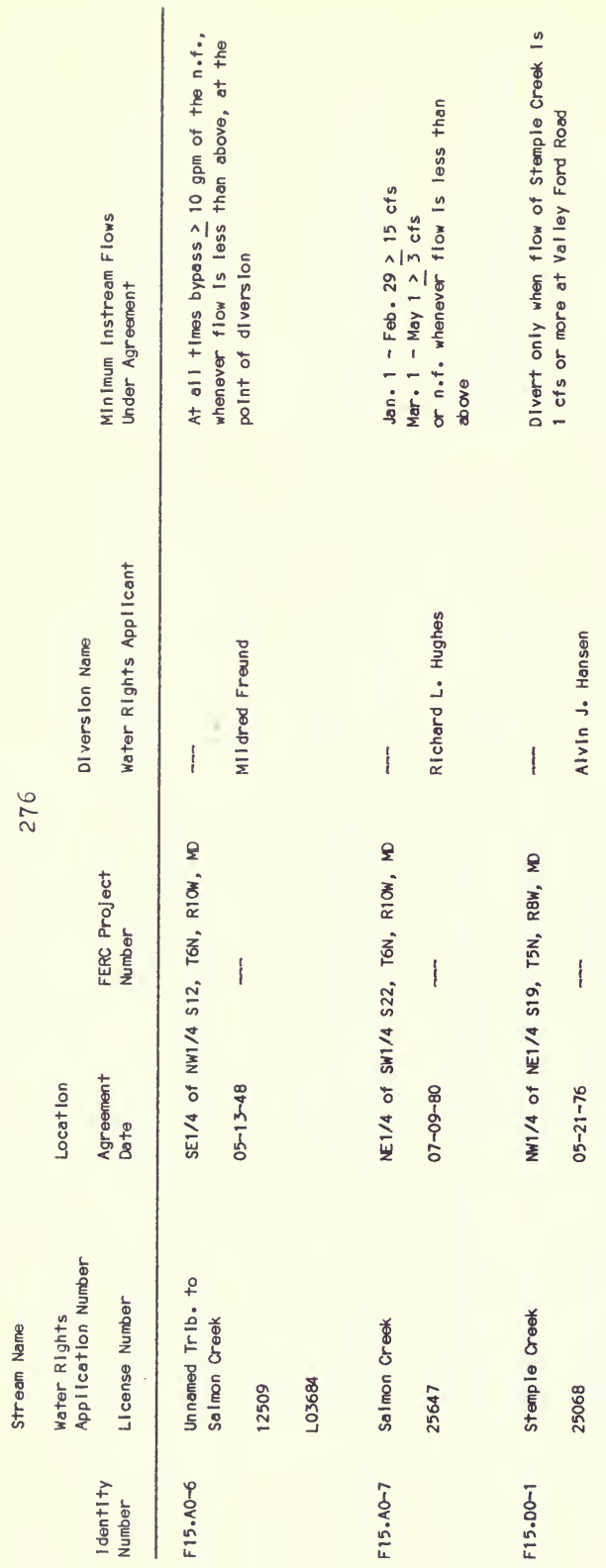



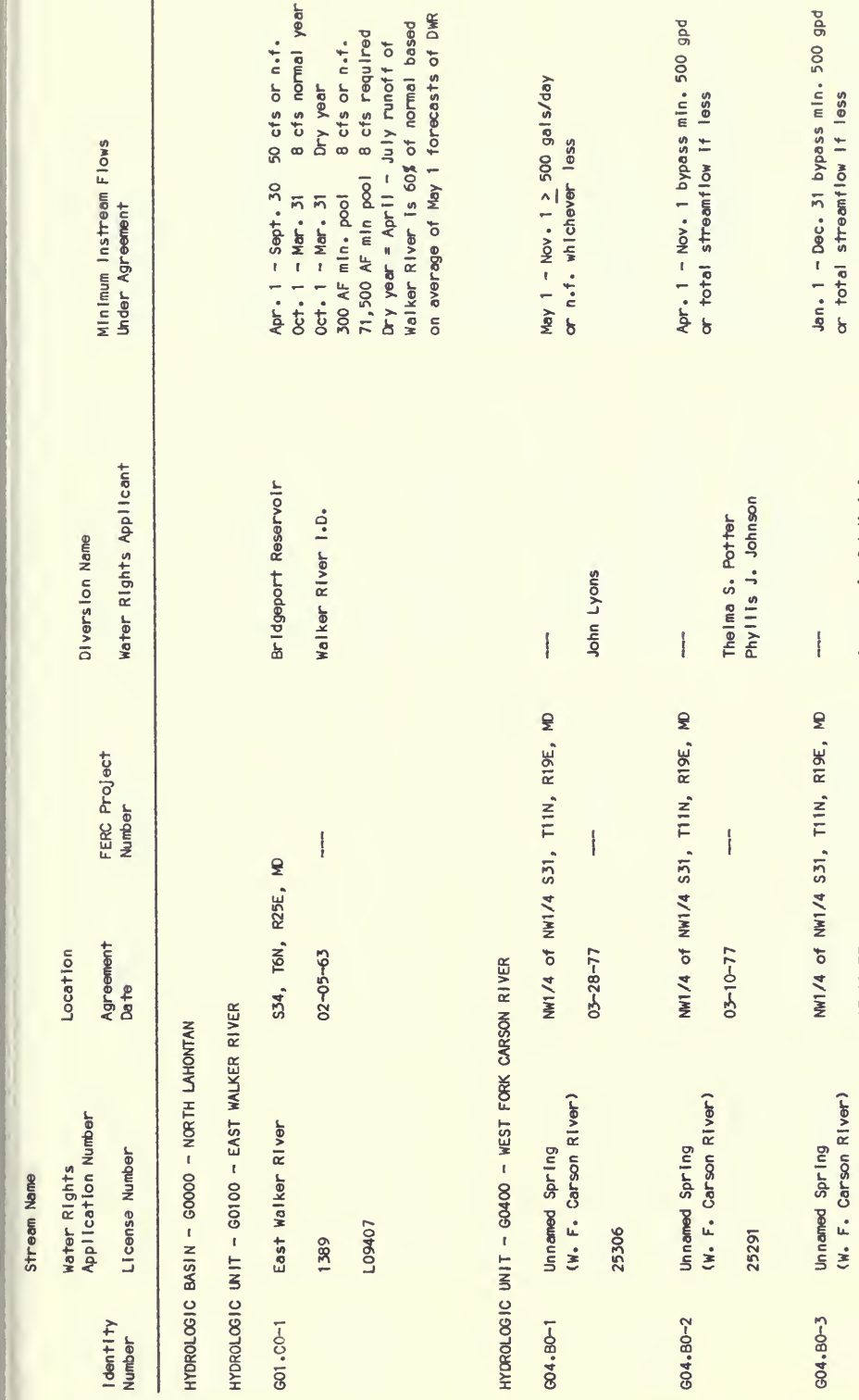

ก 


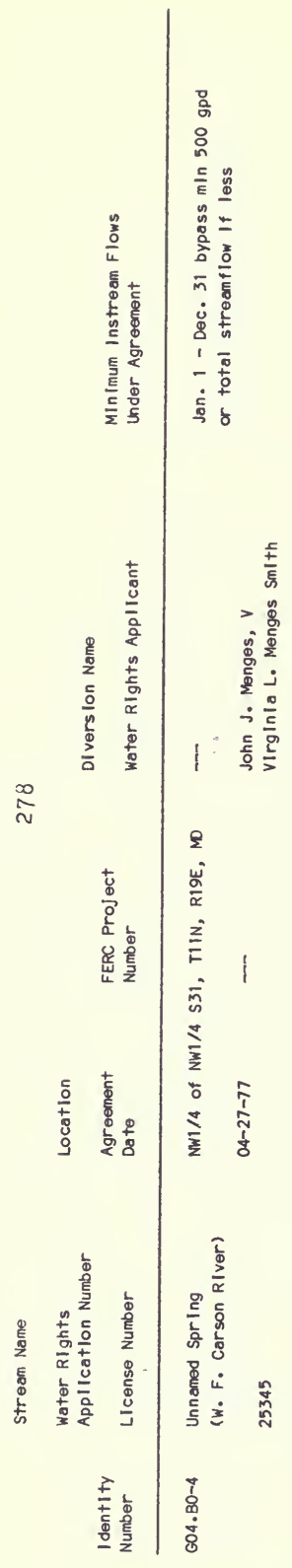




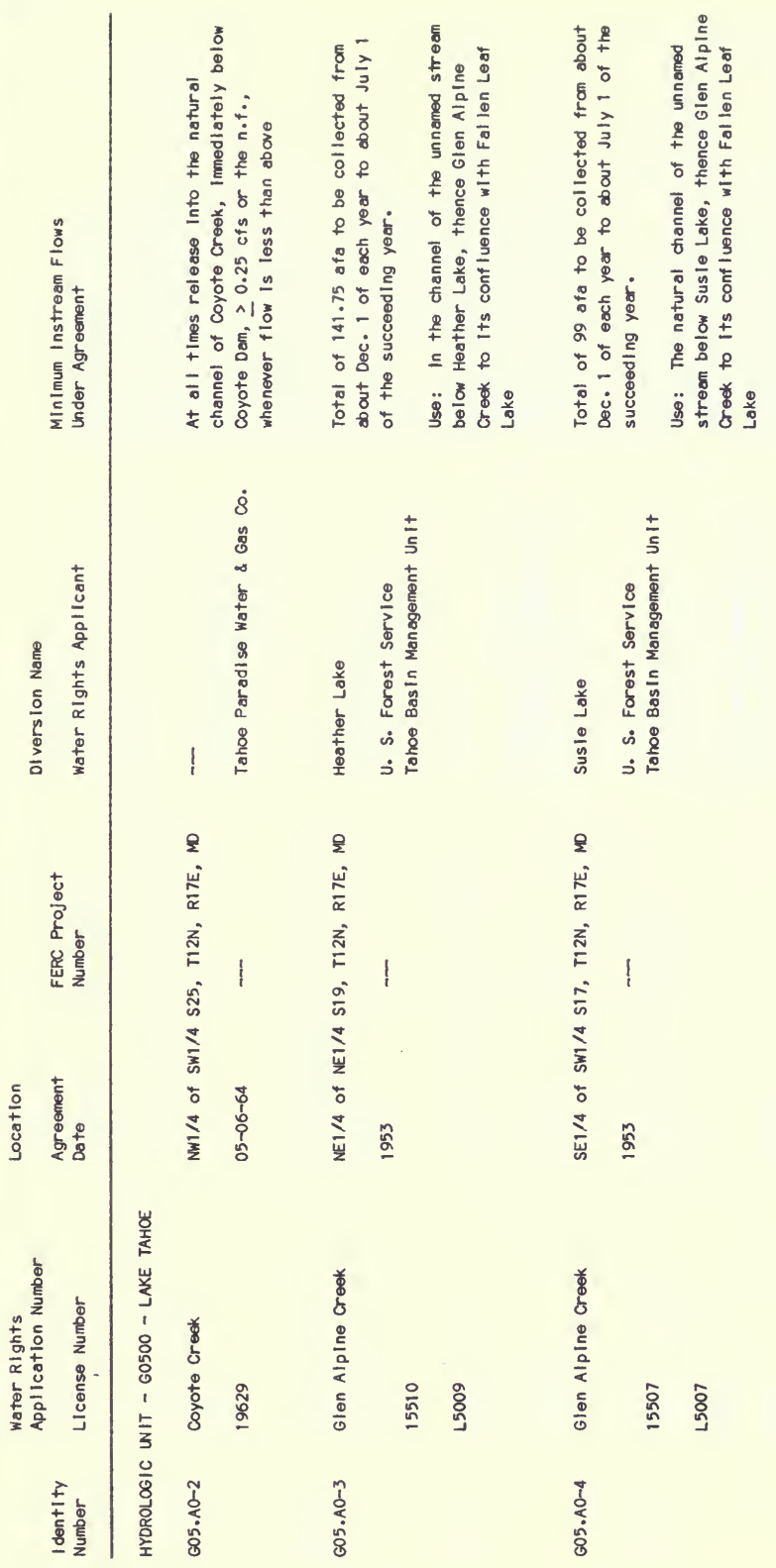

$\stackrel{a}{\sim}$ 


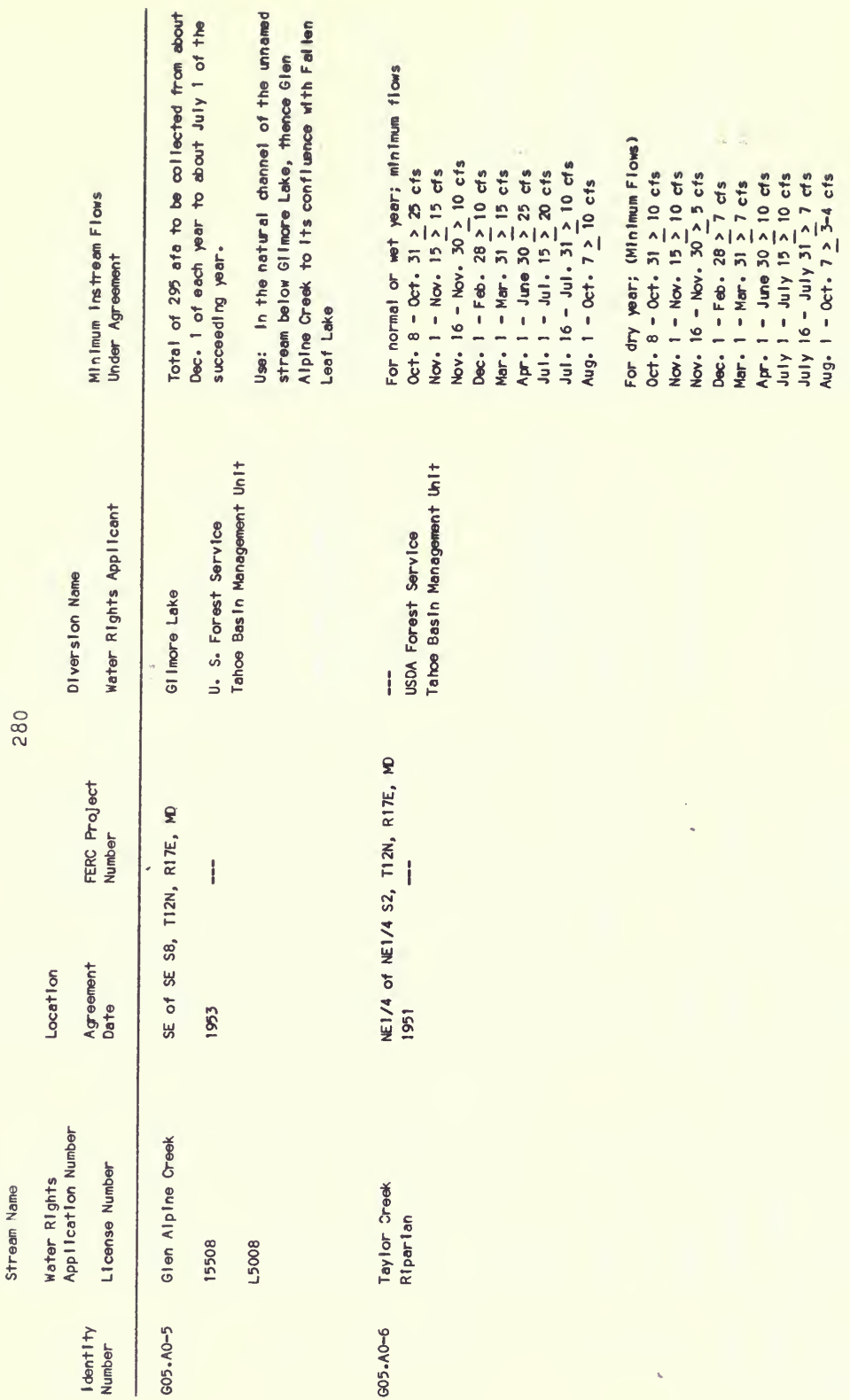




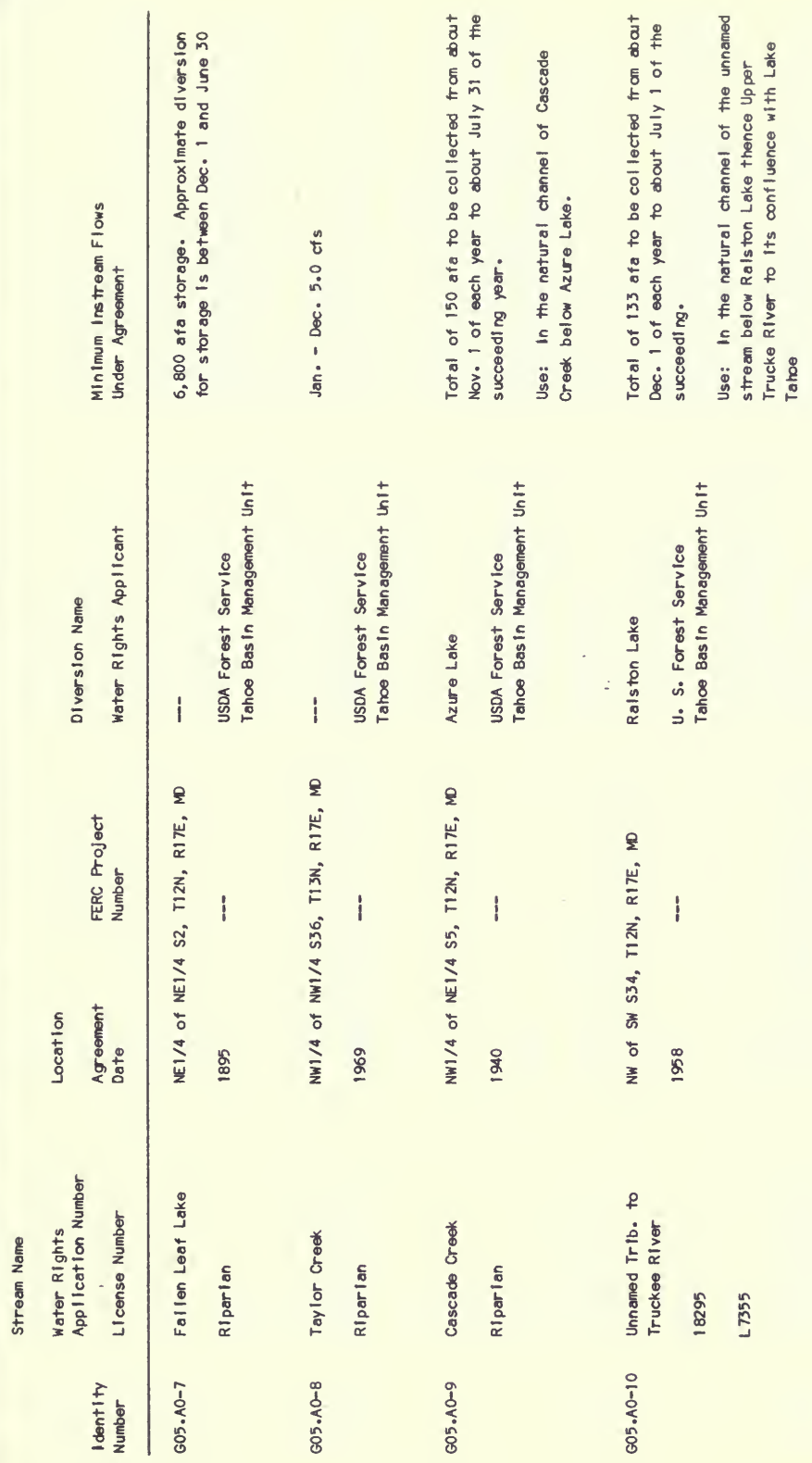




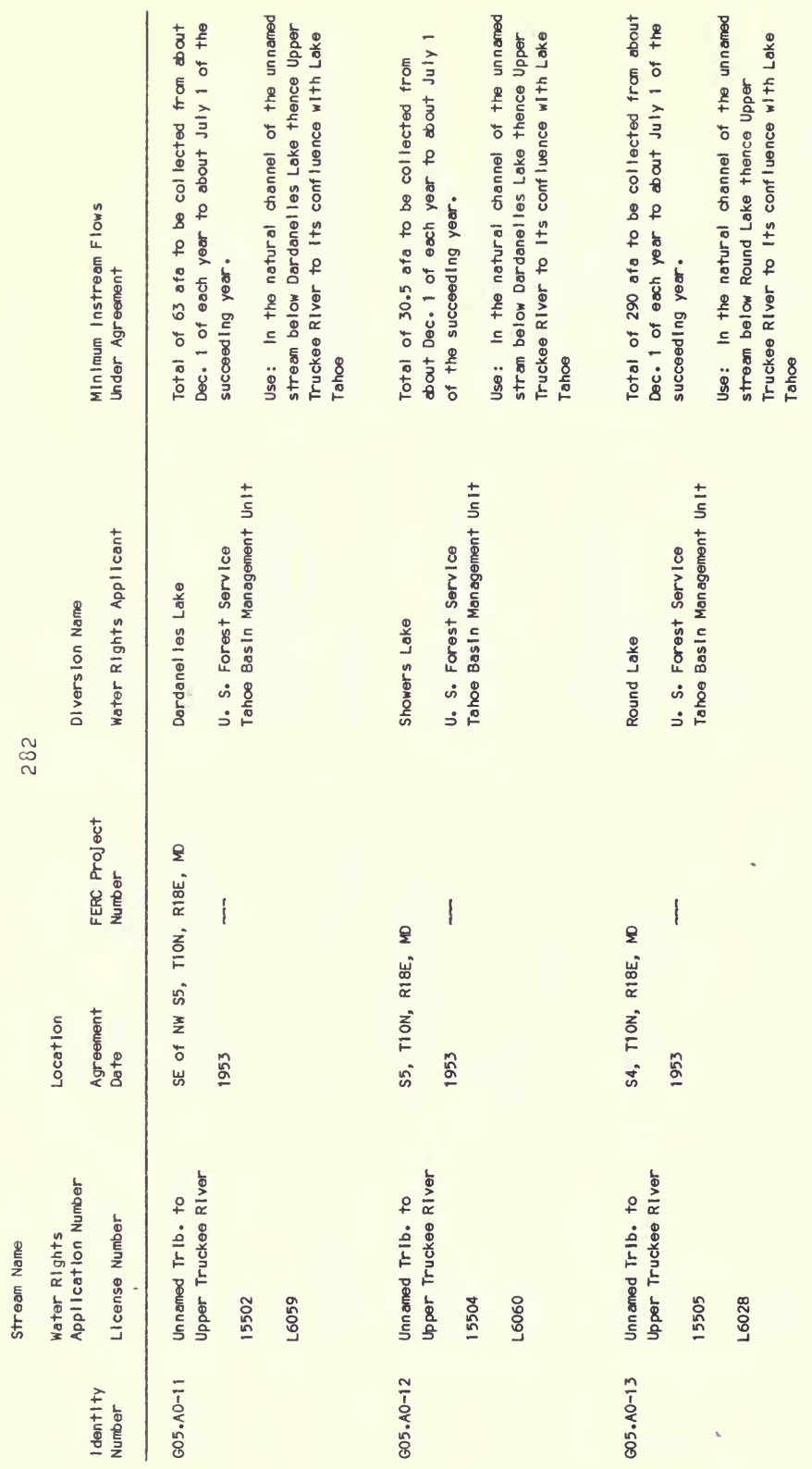




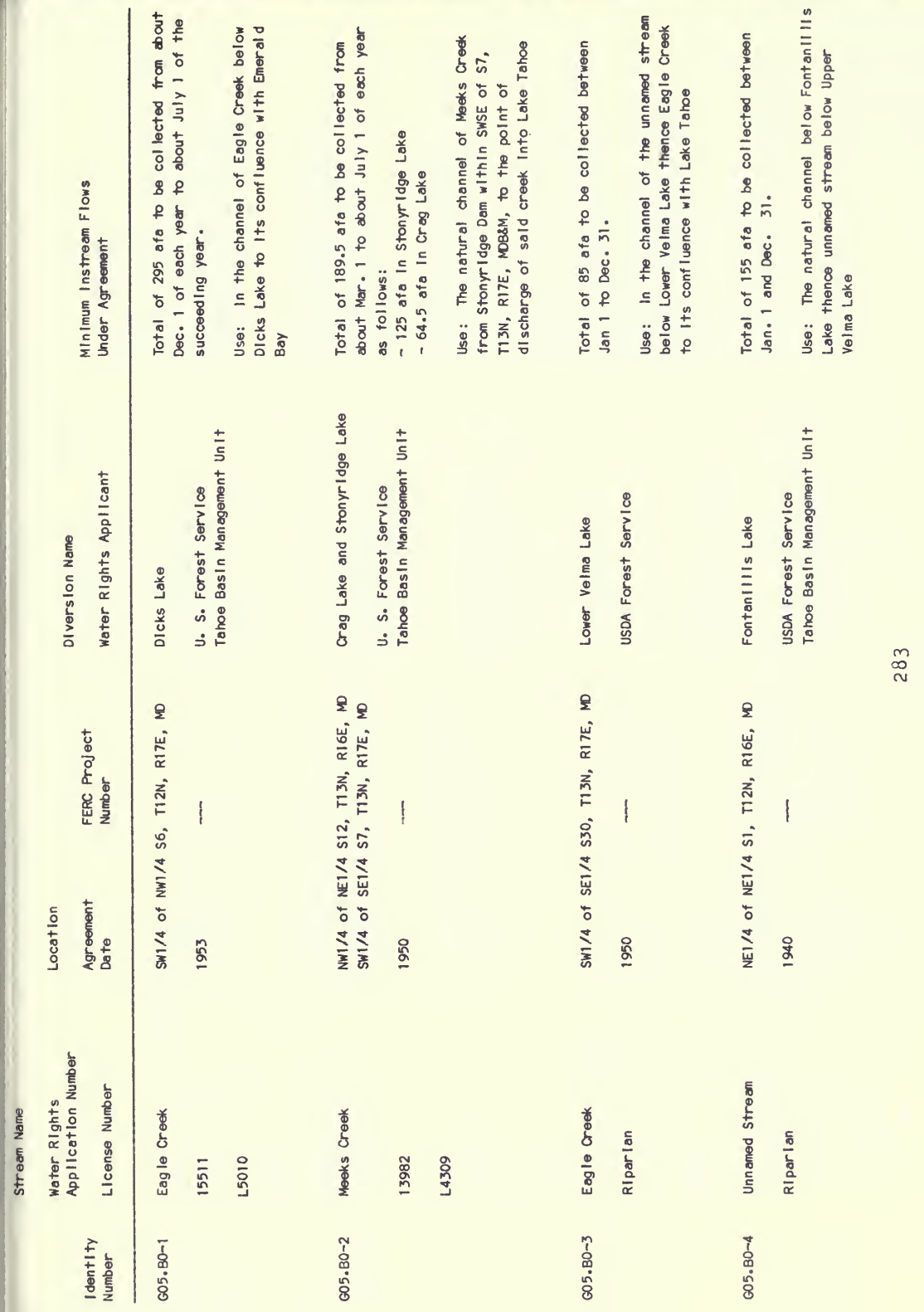




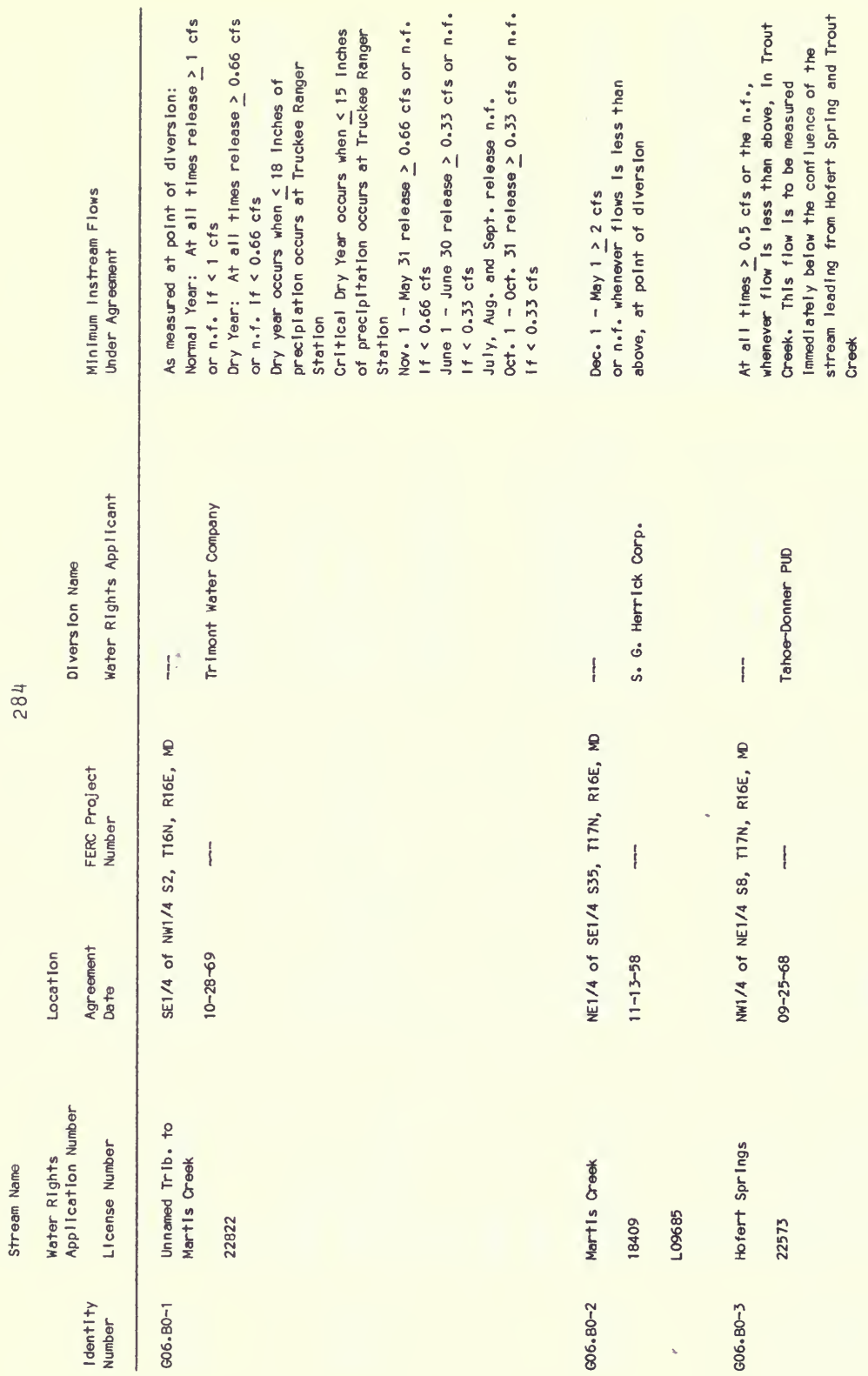




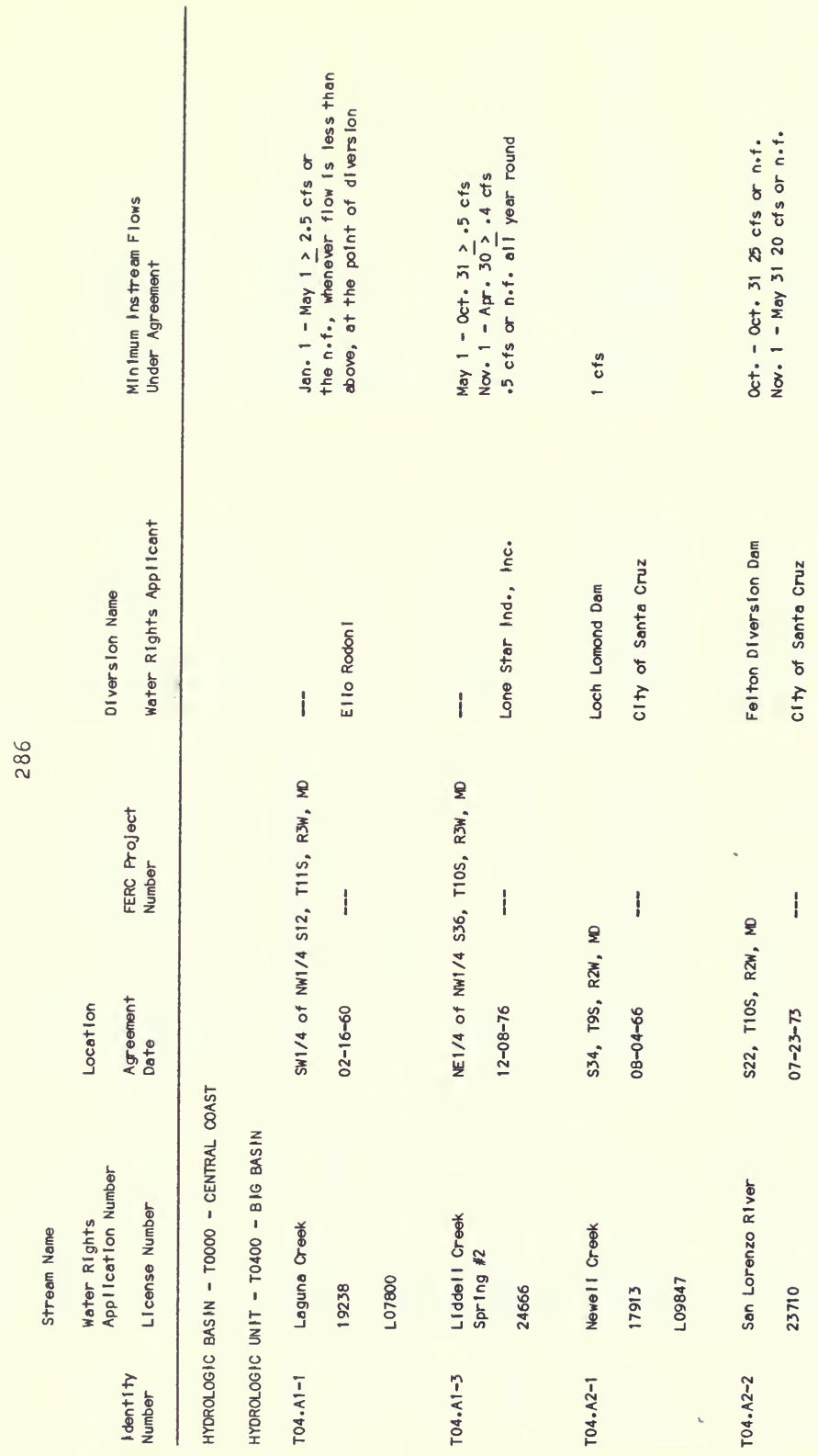




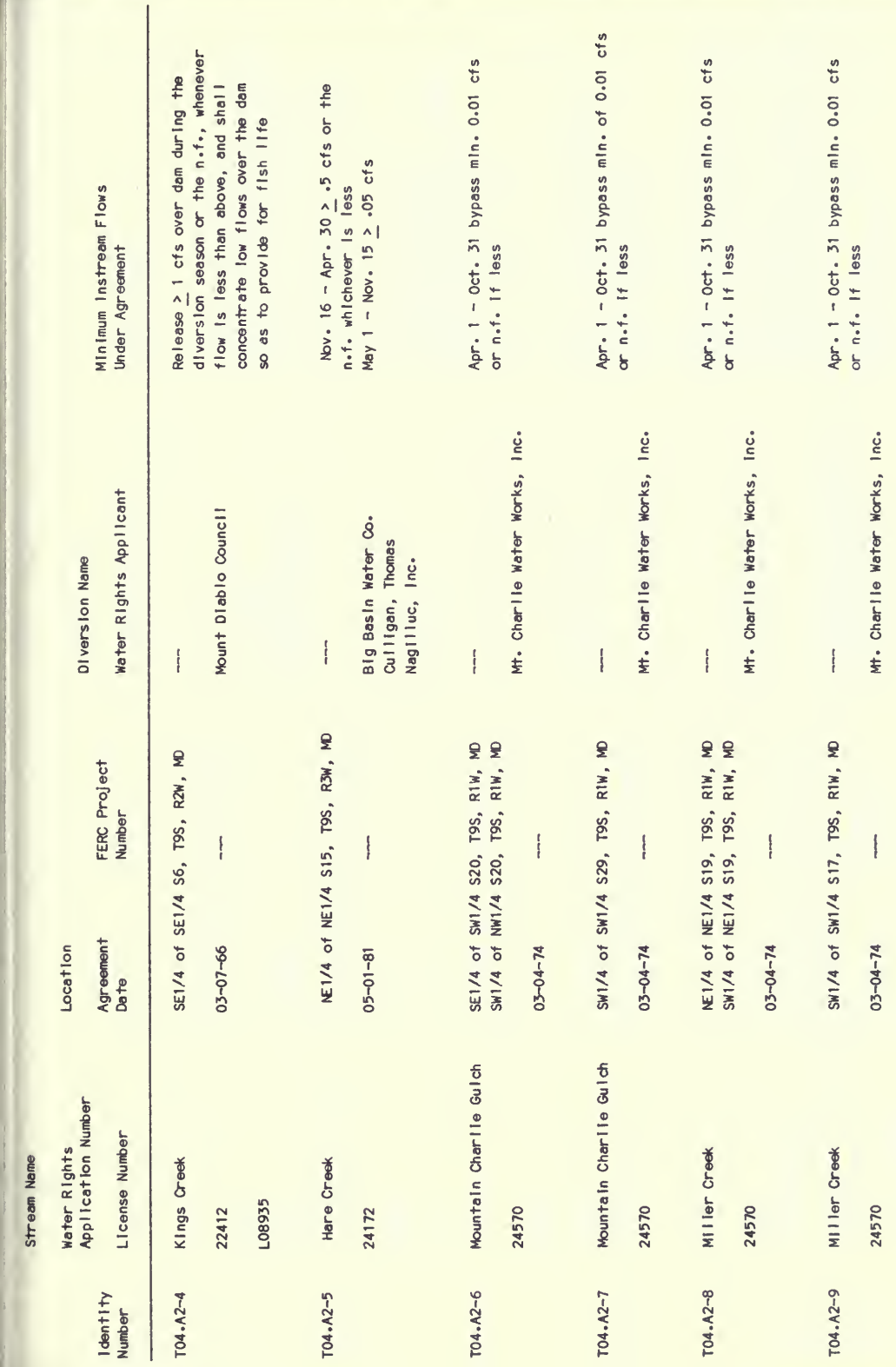




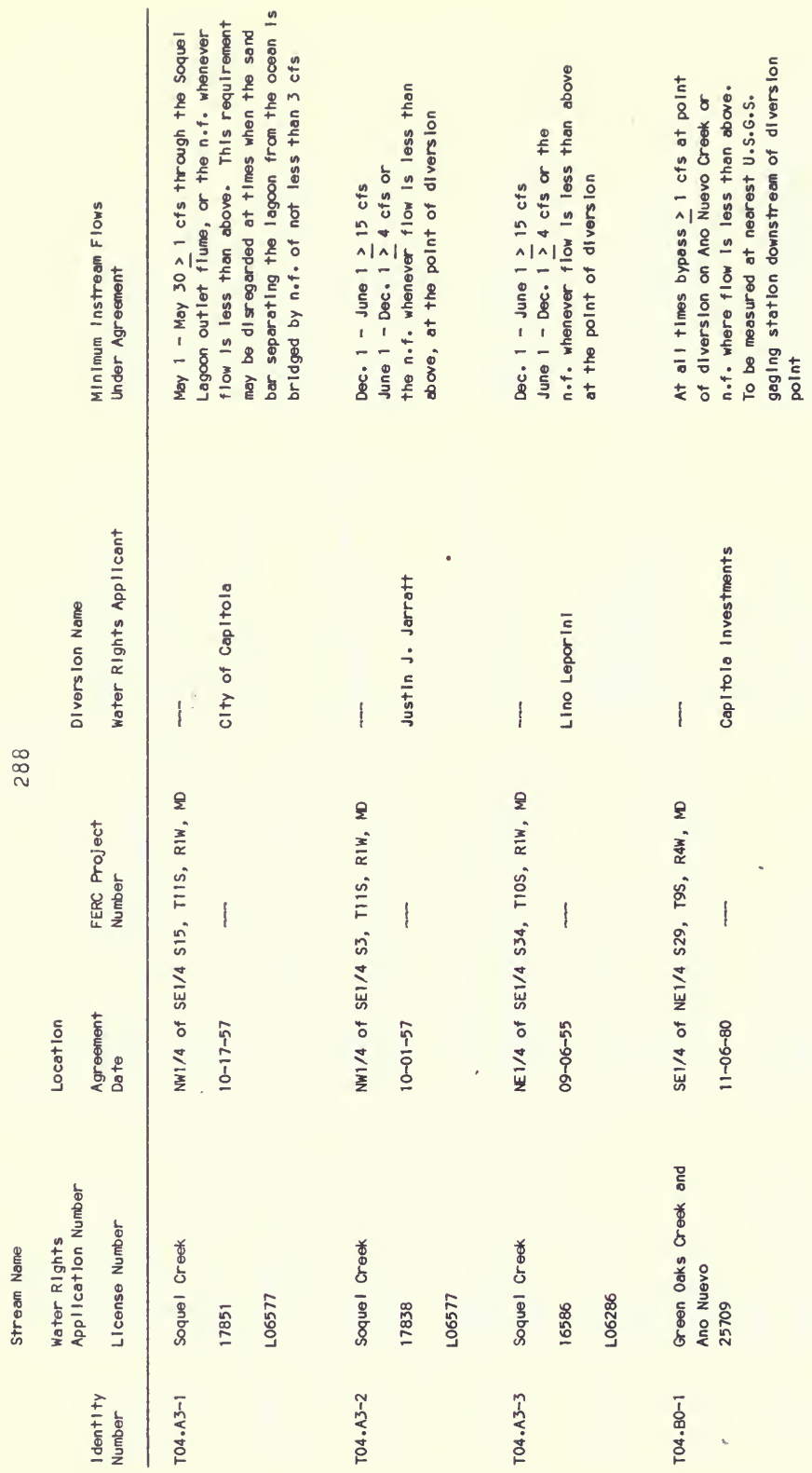




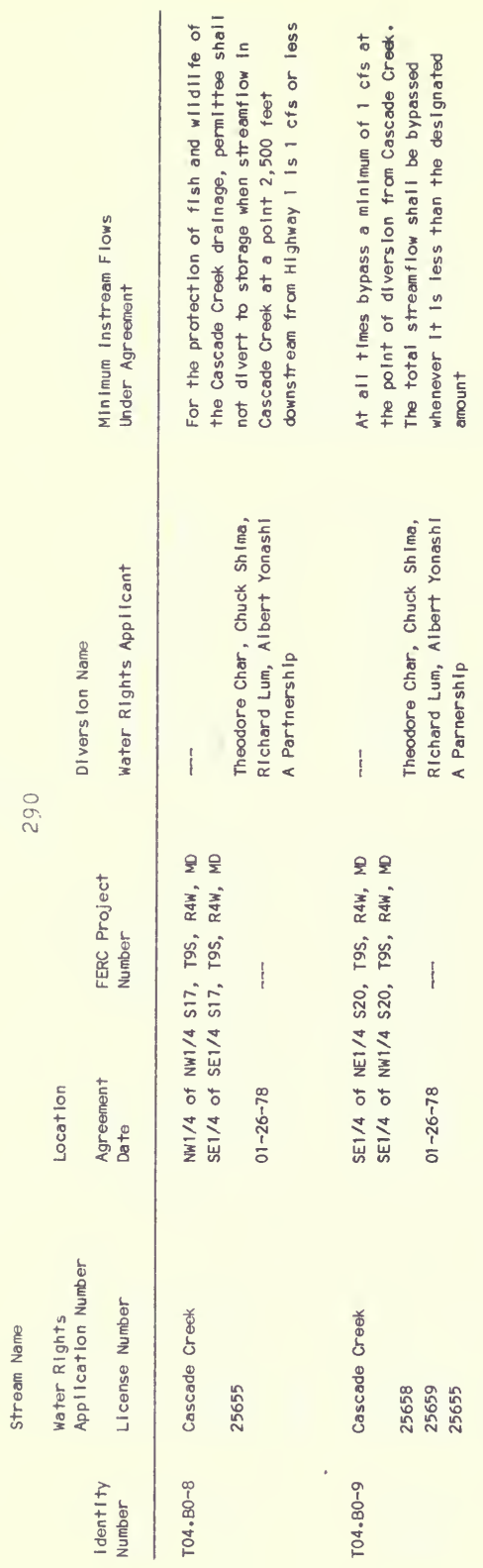




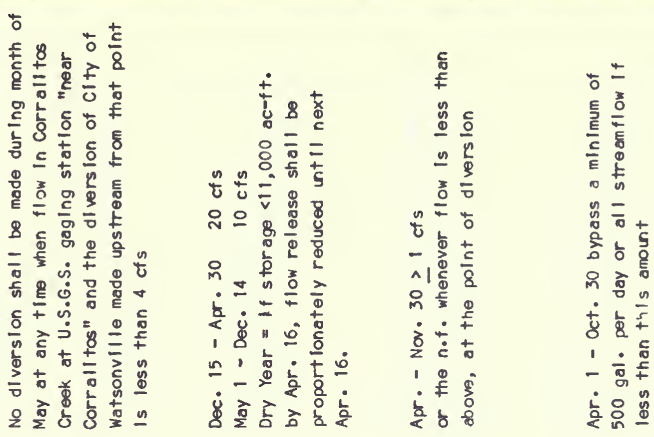




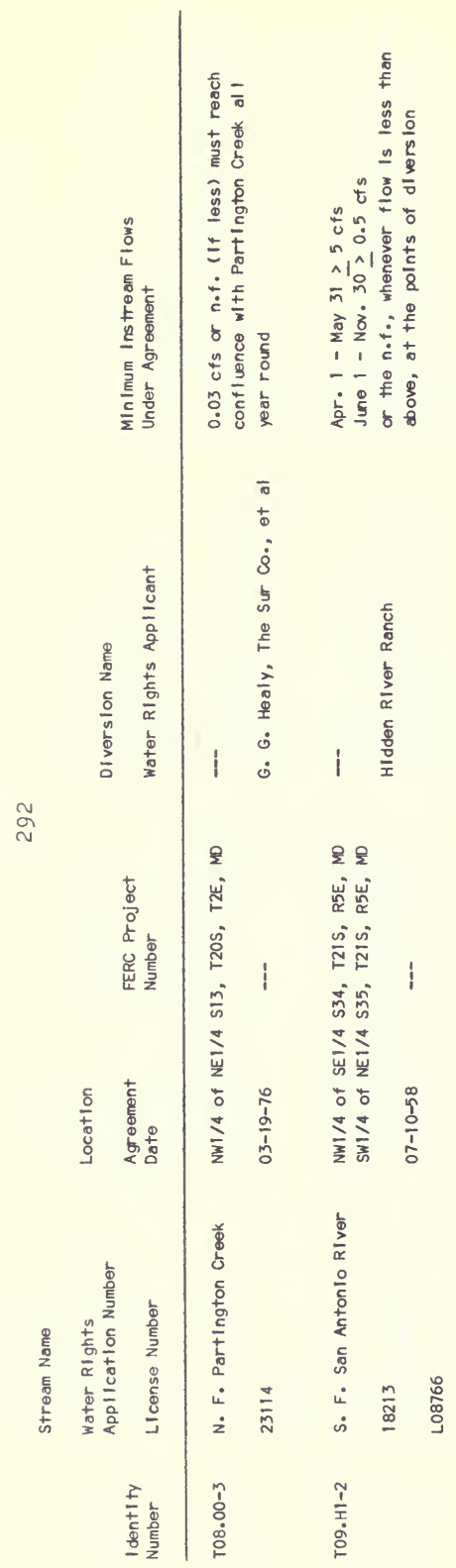




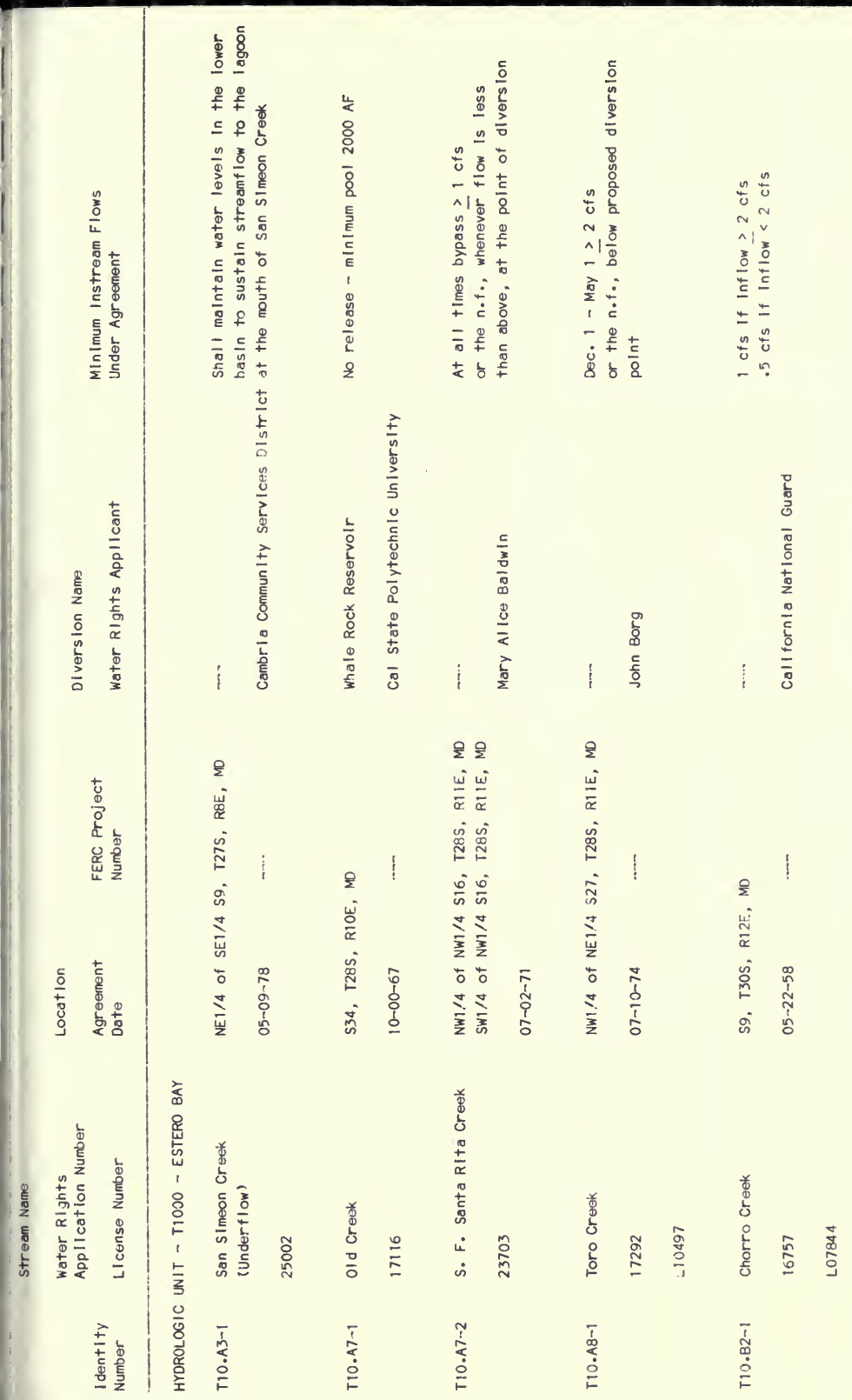




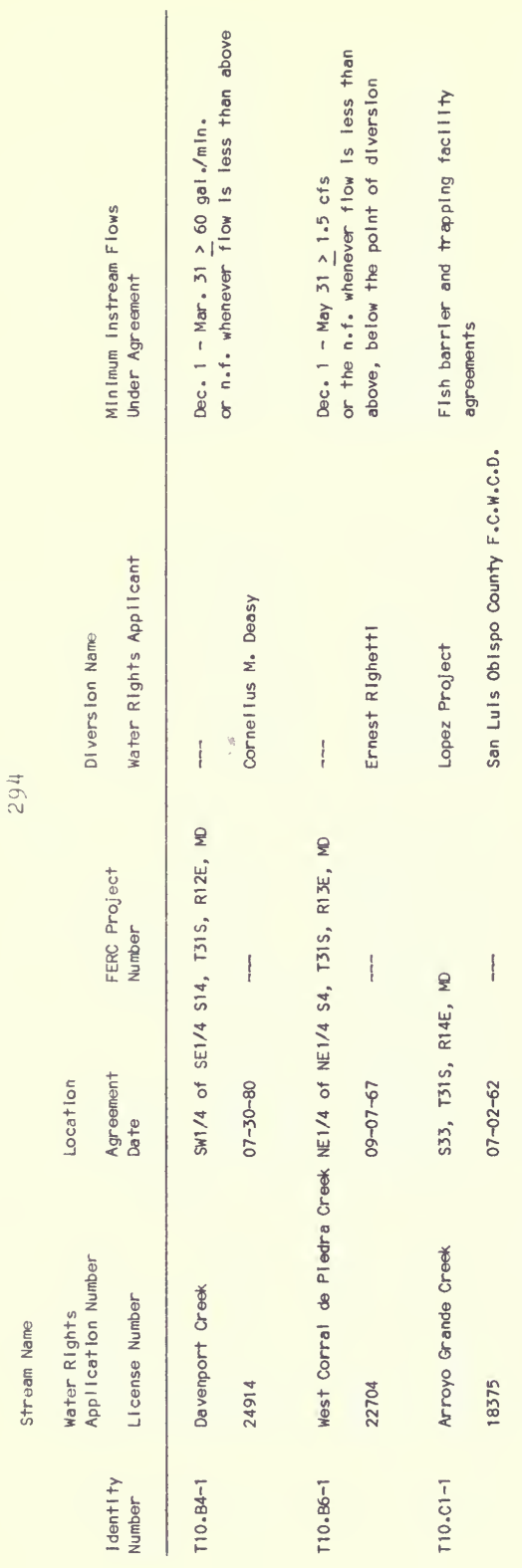




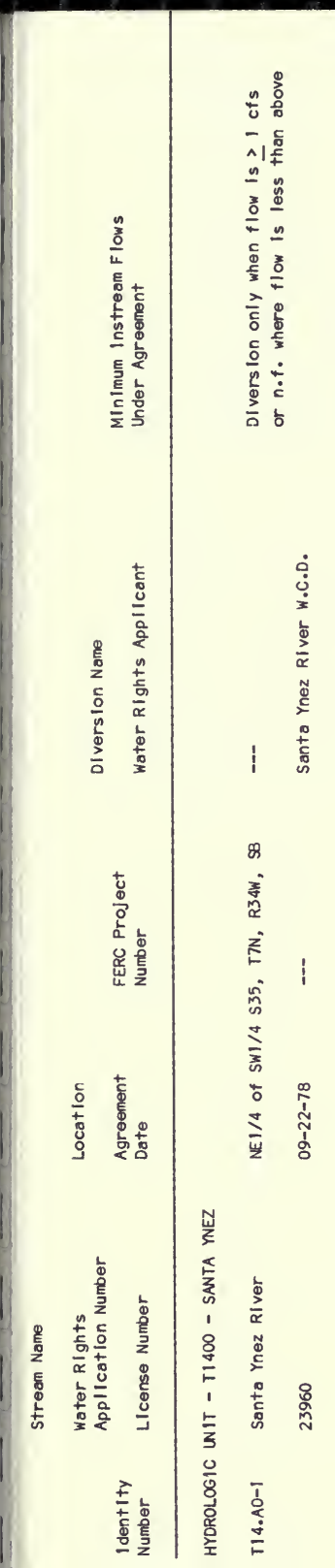

กิ 


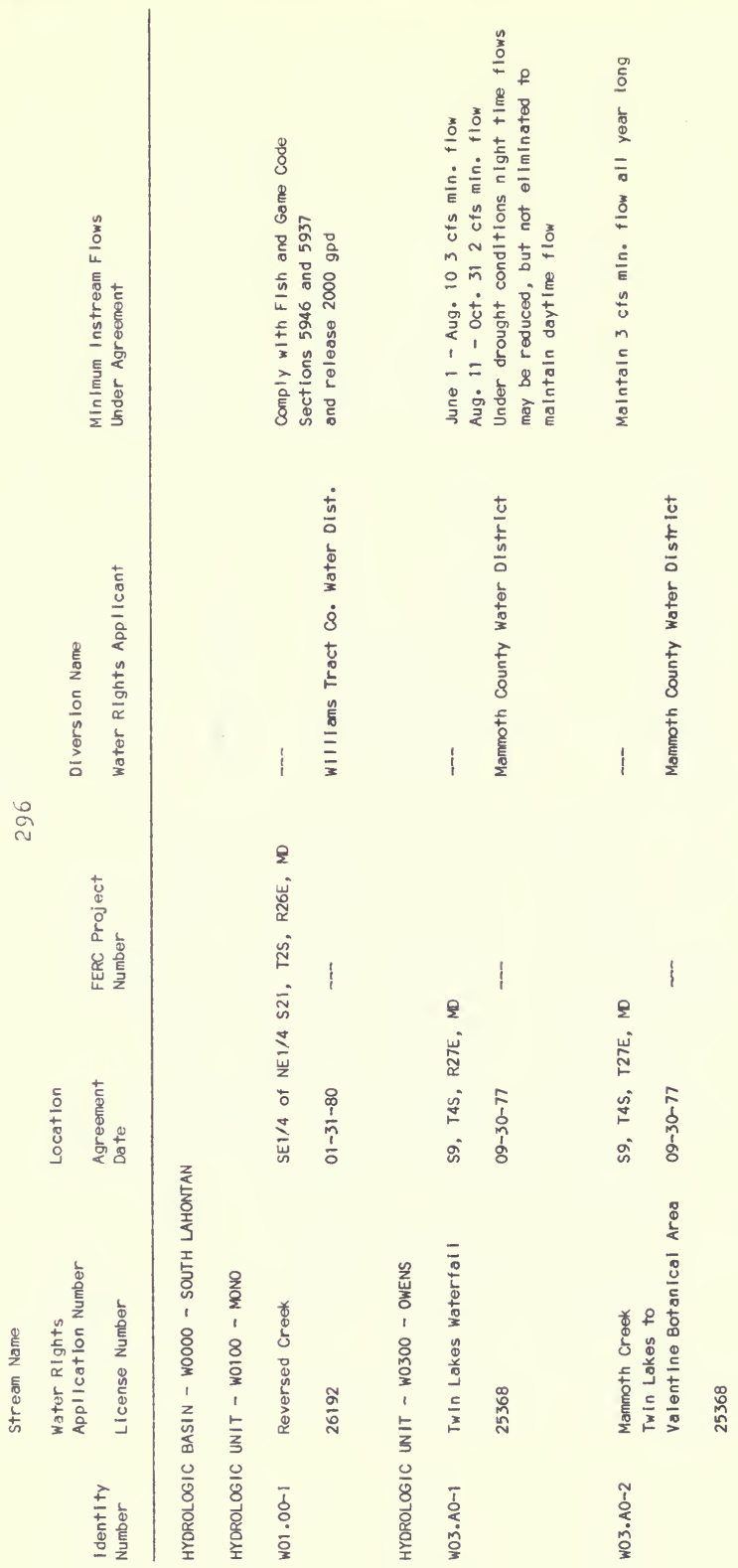




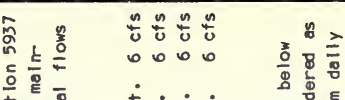

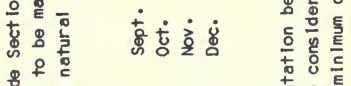

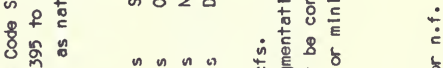

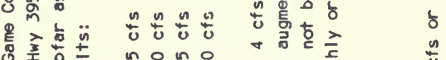

$\stackrel{\text { ๘ }}{+}$

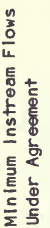

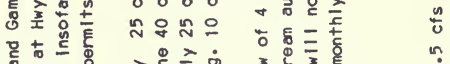

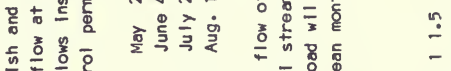

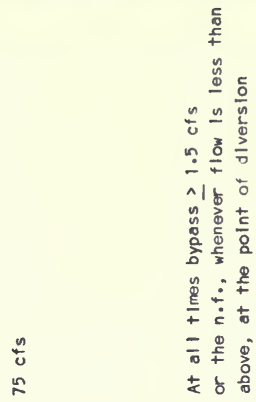

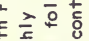

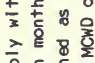

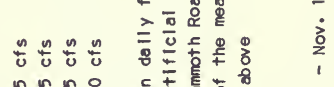

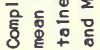

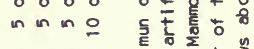

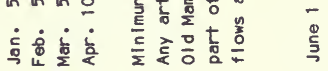

$\stackrel{2}{\sim}$

<

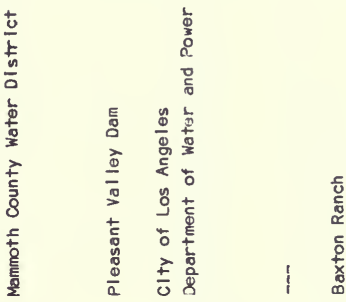

?

울

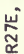

พั้

它

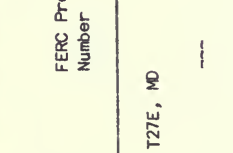

$\frac{6}{n}$

是

ลิ

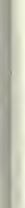

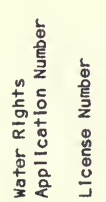

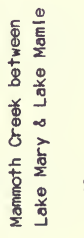

แั

$\frac{5}{2}$

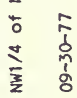

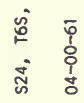

은

ㅇ

这

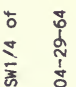

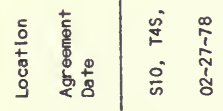

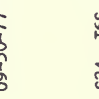

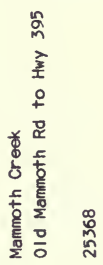

$\frac{1}{\alpha}$

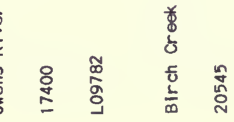




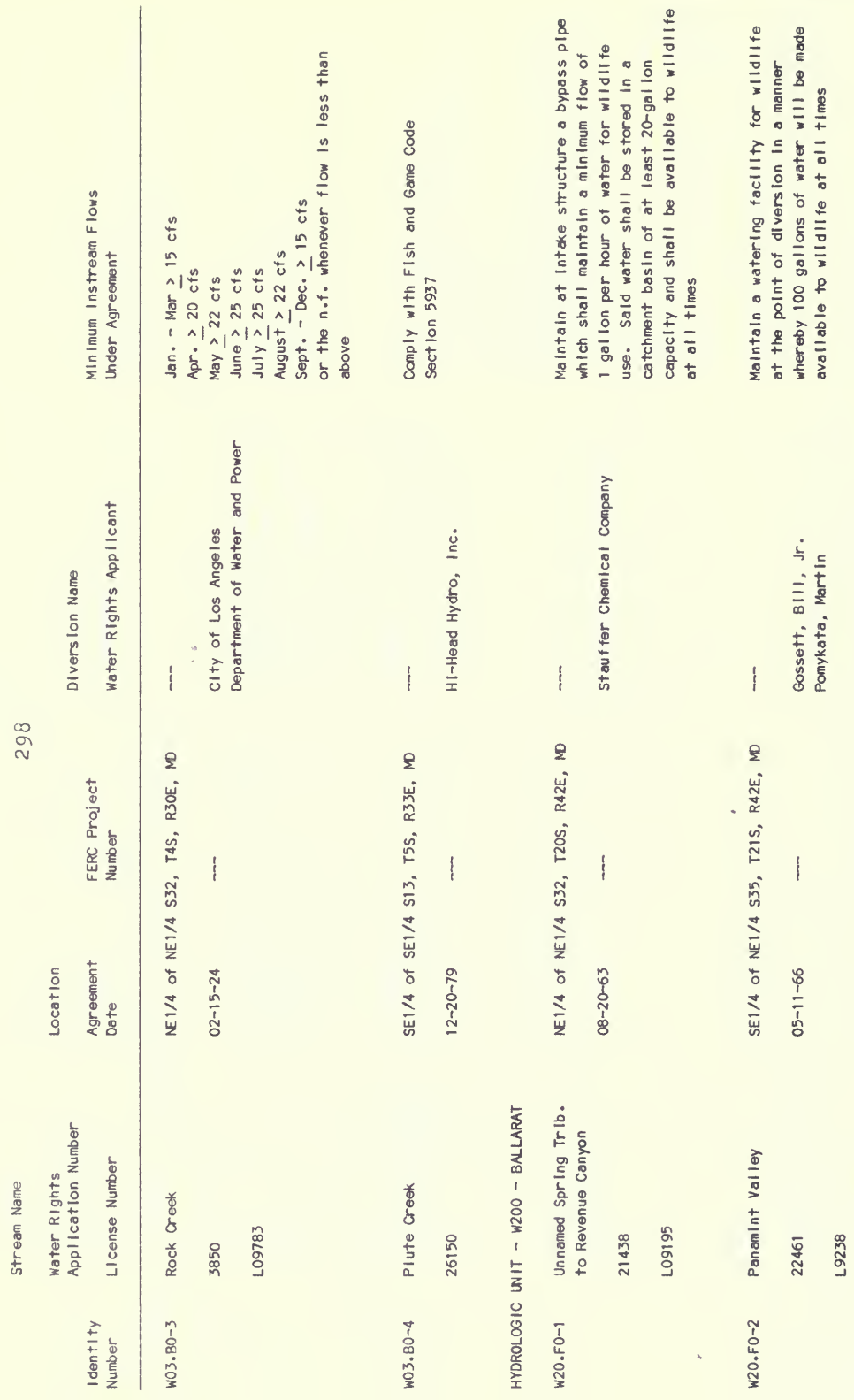




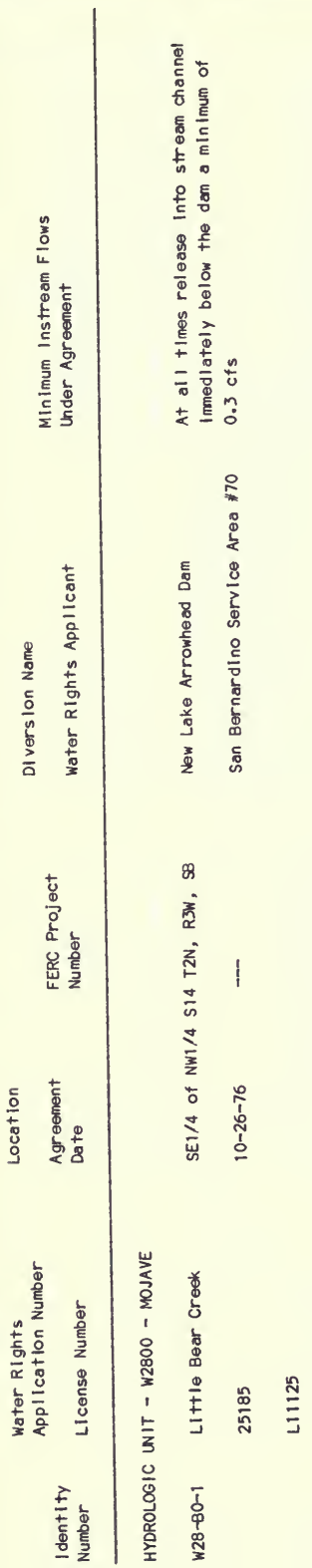




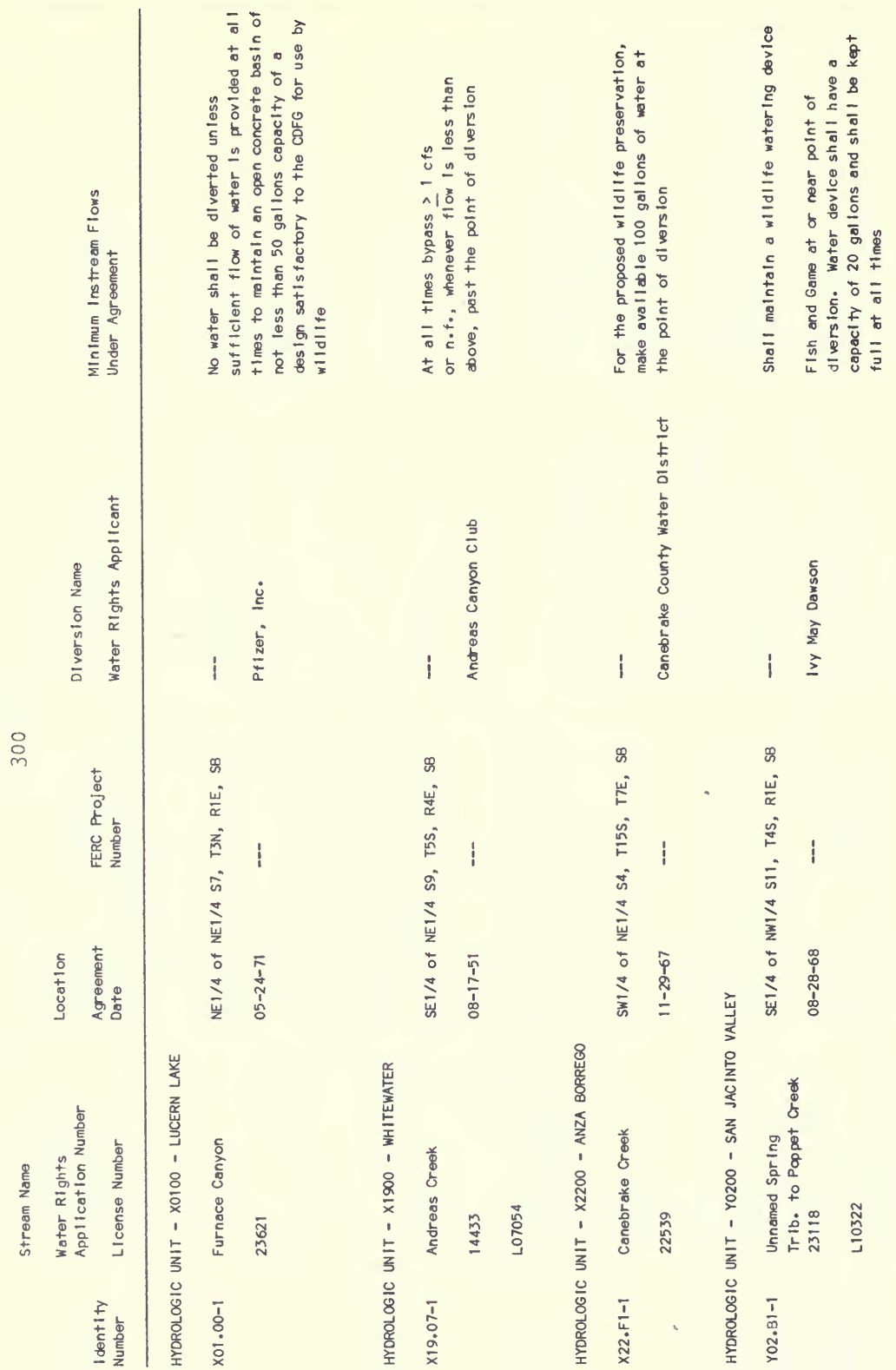




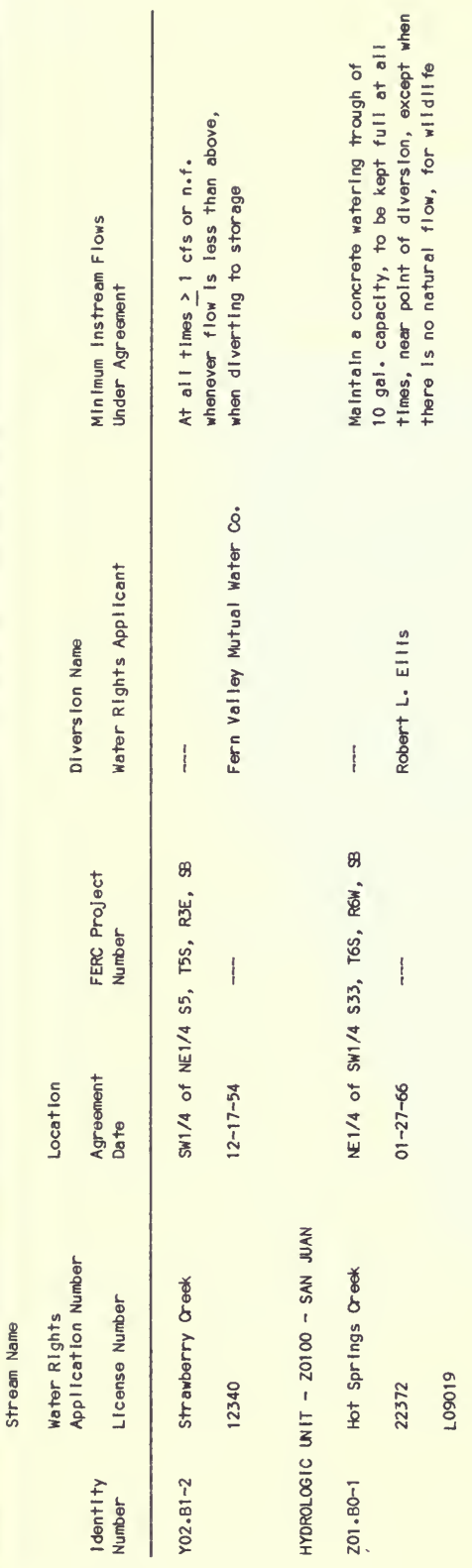

官 


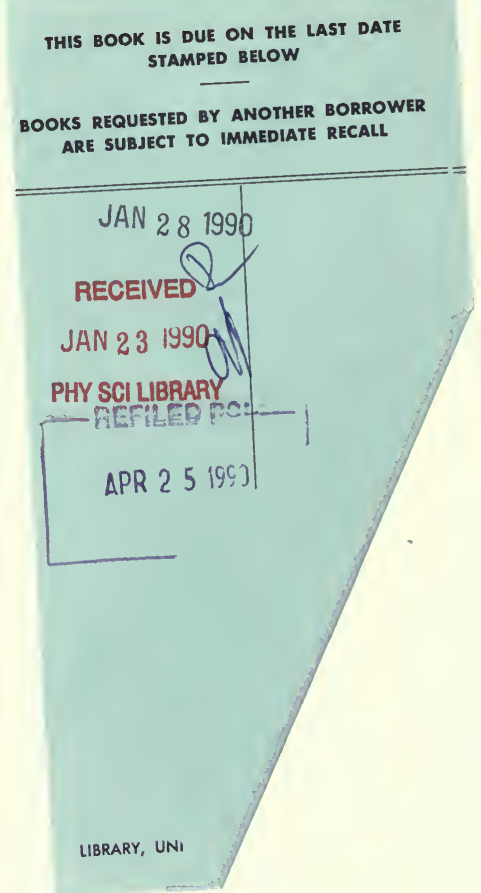


CONVERSION FACTORS

\begin{tabular}{|c|c|c|c|c|}
\hline Quantity & To Convert from Metric Unit & To Customary Unit & $\begin{array}{l}\text { Multiply Metric } \\
\text { Unit By }\end{array}$ & $\begin{array}{l}\text { To Convert to Metric } \\
\text { Unit Multiply } \\
\text { Customary Unit By }\end{array}$ \\
\hline \multirow[t]{4}{*}{ Length } & millimetres $(\mathrm{mm})$ & inches (in) & 0.03937 & 25.4 \\
\hline & centimetres $(\mathrm{cm})$ for snow depth & Inches (in) & 0.3937 & 2.54 \\
\hline & metres $(m)$ & feet $(f t)$ & 32808 & 0.3048 \\
\hline & kilometres (km) & miles (mi) & 0.62139 & 1.6093 \\
\hline \multirow[t]{4}{*}{ Area } & square millimetres $\left(\mathrm{mm}^{2}\right)$ & square inches $\left(\mathrm{in}^{2}\right)$ & 0.00155 & 645.16 \\
\hline & square metres $\left(\mathrm{m}^{2}\right)$ & square feet $\left(\mathrm{ft}^{2}\right)$ & 10.764 & 0.092903 \\
\hline & hectares (ha) & acres (ac) & 2.4710 & 0.40469 \\
\hline & square kilometres $\left(\mathrm{km}^{2}\right)$ & square miles $\left(\mathrm{mi}^{2}\right)$ & 0.3861 & 2.590 \\
\hline \multirow[t]{5}{*}{ Volume } & litres (L) & gallons (gal) & 0.26417 & 3.7854 \\
\hline & megalitres & million gallons $\left(10^{6}\right.$ gal) & 0.26417 & 3.7854 \\
\hline & cubic metres $\left(\mathrm{m}^{3}\right)$ & cubic feet $\left(\mathrm{ft}^{3}\right)$ & 35.315 & 0.028317 \\
\hline & cubic metres $\left(\mathrm{m}^{3}\right)$ & cubic yards $\left(y d^{3}\right)$ & 1.308 & 0.76455 \\
\hline & cubic dekametres (dam³) & acre-feet $(a c-f t)$ & 0.8107 & 1.2335 \\
\hline \multirow[t]{5}{*}{ Flow } & cubic metres per second $\left(\mathrm{m}^{3} / \mathrm{s}\right)$ & $\begin{array}{l}\text { cubic feet per second } \\
\qquad\left(\mathrm{ft}^{3} / \mathrm{s}\right)\end{array}$ & 35.315 & 0.028317 \\
\hline & litres per minute (L/min) & $\begin{array}{l}\text { gallons per minute } \\
\text { (gal } / \mathrm{min})\end{array}$ & 0.26417 & 3.7854 \\
\hline & litres per day (L/day) & gallons per day (gal/day) & 0.26417 & 3.7854 \\
\hline & megalitres per day (ML/day) & $\begin{array}{l}\text { million gallons } \\
\text { per day (mgd) }\end{array}$ & 0.26417 & 3.7854 \\
\hline & $\begin{array}{l}\text { cubic dekometres per day } \\
(\text { dam } 3 / \text { day) }\end{array}$ & $\begin{array}{l}\text { acre-feet per day (ac- } \\
\mathrm{ft} / \text { day) }\end{array}$ & 0.8107 & 1.2335 \\
\hline \multirow[t]{2}{*}{ Mass } & kilograms $(\mathrm{kg})$ & pounds $(i b)$ & 2.2046 & 0.45359 \\
\hline & megagrams (Mg) & tons (short, $2,000 \mathrm{lb}$ ) & 1.1023 & 0.90718 \\
\hline Velocity & metres per second $(\mathrm{m} / \mathrm{s})$ & feet per second $(\mathrm{ft} / \mathrm{s})$ & 3.2808 & 0.3048 \\
\hline Power & kilowatts (kW) & horsepower (hp) & 1.3405 & 0.746 \\
\hline \multirow[t]{2}{*}{ Pressure } & kilopascals (kPa) & $\begin{array}{l}\text { pounds per square inch } \\
\text { (psi) }\end{array}$ & 0.14505 & 6.8948 \\
\hline & kilopascals (kPa) & feet head of water & 0.33456 & 2.989 \\
\hline Specific Capacity & $\begin{array}{l}\text { litres per minute per metre } \\
\text { drawdown }\end{array}$ & $\begin{array}{l}\text { gallons per minute per } \\
\text { foot drawdown }\end{array}$ & 0.08052 & 12.419 \\
\hline Concentration & milligrams per litre (mg/L) & parts per million (ppm) & 1.0 & 1.0 \\
\hline $\begin{array}{l}\text { Electrical Con- } \\
\text { ductivity }\end{array}$ & $\begin{array}{l}\text { microsiemens per centimetre } \\
(\mathrm{uS} / \mathrm{cm})\end{array}$ & micromhos per centimetre & 1.0 & 1.0 \\
\hline Temperature & degrees Celsius $\left({ }^{\circ} \mathrm{C}\right)$ & degrees Fahrenheit ( $\left.{ }^{\circ} \mathrm{F}\right)$ & $11.8 \times{ }^{\circ} \mathrm{C}$ & $\left({ }^{\circ} \mathrm{F}-32\right) / 1.8$ \\
\hline
\end{tabular}


State of California-Resources Agency

Department of Water Resources

P.O. Box 388

Sacramento

95802 


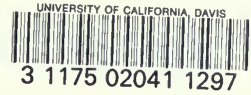


